\title{
Driving biomineralisation using soft templation
}

\author{
by \\ Conrad Douglas Lendrum
}

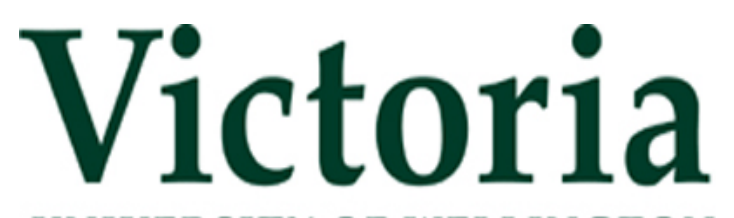

UNIVERSITY OF WELLINGTON

Te Whare Wānanga

o te Ūpoko o te Ika a Māui

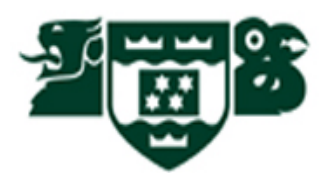

\begin{abstract}
A thesis
submitted to the Victoria University of Wellington in fulfilment of the requirements for the degree of

Doctor of Philosophy in Chemistry.

Victoria University of Wellington




\section{Abstract}

Sea shells, bones and teeth are three examples of Nature's unrivalled ability to produce complex hierarchical structures from simple inorganic materials. Unlike the synthetic approach of using 'exotic' materials to introduce functionality, Nature has employed structural control to maximise properties. Key to this control is the use of an organic framework to guide inorganic nucleation and growth. The question of how structural information is transferred from the organic framework to the inorganic crystal has inspired many studies in the field of biomineralisation, yet our understanding remains limited. One aspect that has received considerable attention is the molecular recognition process that occurs at the organic/inorganic interface. Unlocking the mysteries of the intermolecular interactions associated with molecular recognition using a model Langmuir monolayer system is the aim of this research.

Elucidation of the molecular recognition process requires an understanding of host/guest chemistry, double layer theory, Langmuir monolayer chemistry, and crystallisation theory, with the added complexity that both the host and guest are dynamic and constantly changing. This level of complexity demands a holistic approach to accommodate the many interacting parameters, therefore this study consists of a comparative analysis of calcium carbonate crystallisation under twelve subtly altered surfactant monolayer systems. Based around the acid and alcohol moieties, commonly explored in biomineralisation studies, these monolayer systems involve: mixtures of octadecanoic acid and octadecanol, hydroxyl-, carboxyl-, bromine- and methyl- substituted octadecanoic acids. By making minor chemical modifications to the membrane molecules we can subtly alter the electronic landscape presented to the supersaturated subphase and probe how the mix of intermolecular forces changes the interfacial interaction.

In order to understand the monolayer/subphase interaction and therefore build up 
a picture of the crystallising system each monolayer was probed on pure water, calcium chloride and sodium bicarbonate subphases. The understanding gleaned from these experiments fed into the elucidation of the significantly more complex calcium carbonate crystallising subphase/monolayer interaction. Information about monolayer and subphase behaviour was obtained from surface pressure isotherms, surface potential measurements, Brewster Angle Microscopy, grazing incidence Xray diffraction (GIXD) and X-ray reflectivity (XRR). This information was correlated with crystal properties such as the nucleation face and gross morphology to develop a picture of the interfacial interaction.

Results show that monolayer surface charge and ion-ion electrostatic interactions are important but do not dictate crystal orientation. The manipulation of the head group chemistry highlighted the influence of head group spacing and therefore lattice matching in crystal orientation. Further it was found that a high degree of interfacial matching not only facilitated face-selective nucleation but also has a significant impact of crystal morphology. GIXD results show the rearrangement of the monolayer structure upon nucleation for the first time. Combined with X-ray reflectivity generated electron density profiles this has lead to a significant improvement in our understanding of the interfacial interaction.

As such this body of work has culminated in the proposition of a cation-mediated hydrogen-bonded soap network facilitated by the presence of the bicarbonate anion as an intermediate entity for crystal nucleation under Langmuir monolayers. Such a network accounts for the influence of electrostatics, lattice, symmetry and spatial geometry matching that contribute to face-selective nucleation and more generally the molecular recognition process in biomineralisation. However the evidence presented here for a monolayer/subphase network is largely qualitative and the hypothesis requires more direct validation. 


\section{Acknowledgements}

First and foremost, Cynthia, thanks just does not cut it. Without your support this would never have happened. What a time we've had, me absent much of it and you giving birth to two children, sadly losing your Dad and generally having to run the household single handed. You are marvellous, and your support has been fantastic. I guess now I've run out of excuses for those jobs around the house......

To my supervisor, mentor, proof reader, and friend, Kate. Thanks muchly. Although it is too late now to change anything but I hope I haven't been too much of a burden. I have really enjoyed my time in your tenure and have learnt heaps. Perhaps if/when I am ever worthy we could work together as colleagues.

I would also like to thank IRL for supporting me through my $\mathrm{PhD}$. Ian Brown, Graham Weir and Mike Arnold, thanks for your support and advice during the various stages of my project.

A big thanks to Mark Bowden, who has provided distraction and assistance when needed. If you do head off to the US, all the best but it just wont be the same discussing Liverpool's woes via email.

Finally my fellow suffragettes who share Laby 105 thanks and all the best. 


\section{Table of Contents}

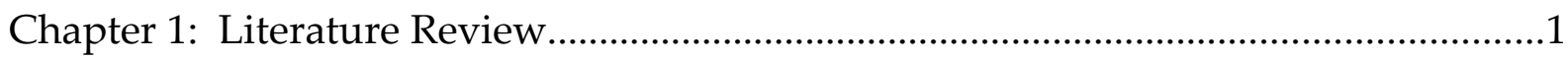

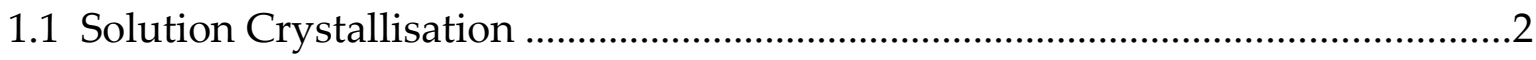

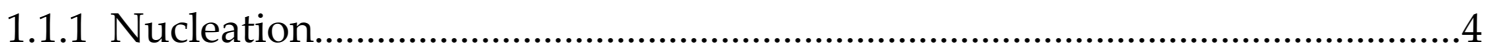

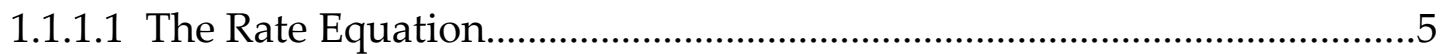

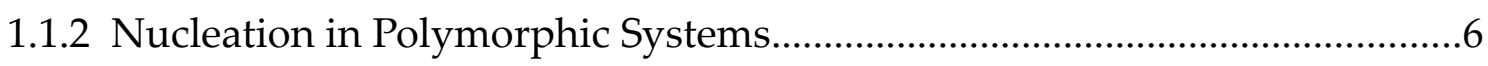

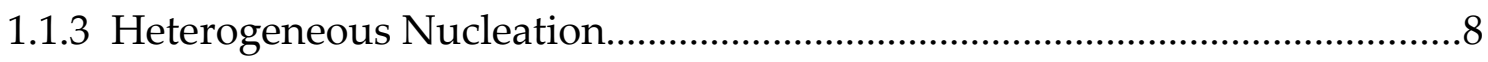

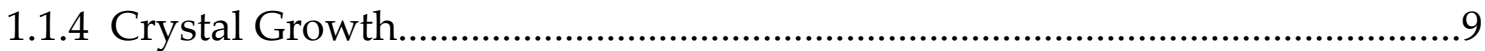

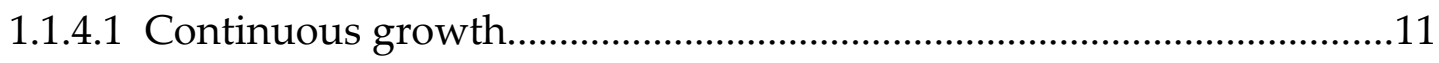

1.1.4.2 Surface Nucleation.......................................................................11

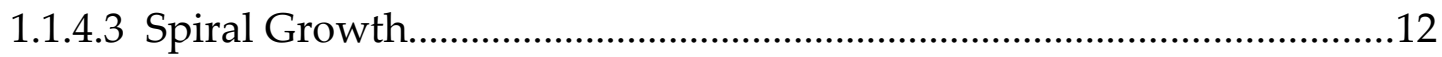

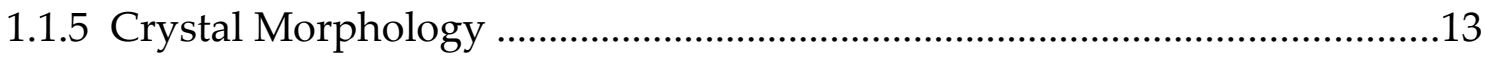

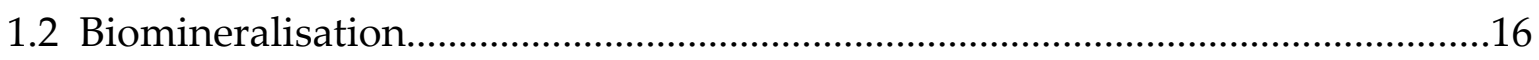

1.3 Introduction to Langmuir Monolayers............................................................25

1.3.1 Chemical Structure and Varieties...........................................................26

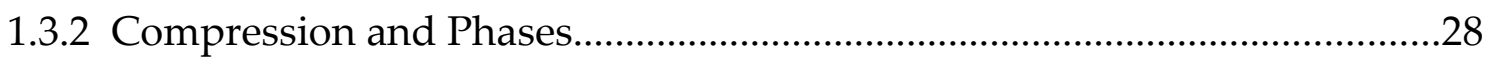

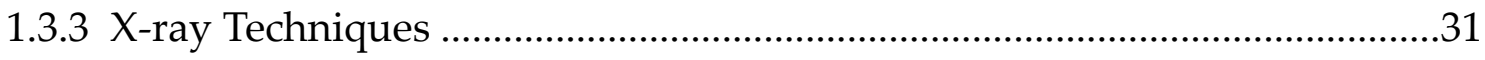

1.3.3.1 Grazing Incidence X-ray Diffraction (GIXD) …..................................32

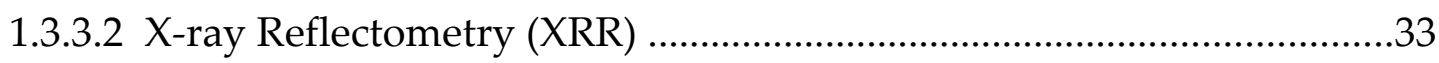

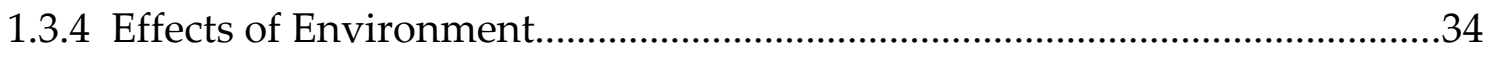

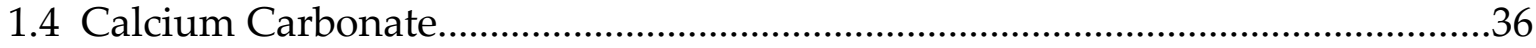

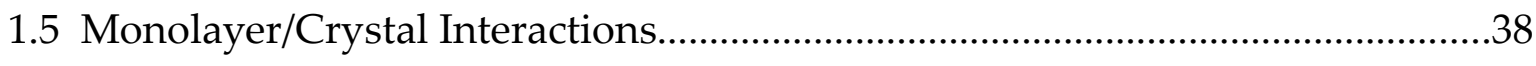

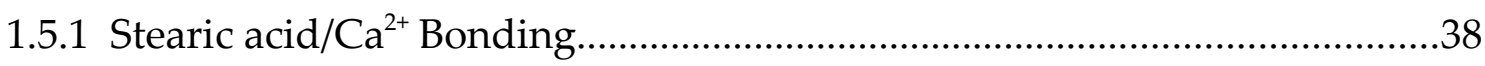

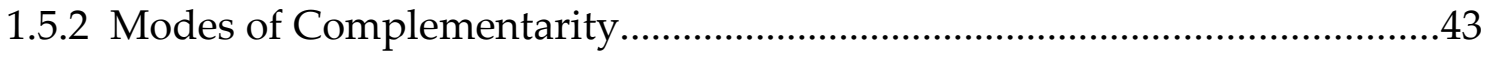

1.5.2.1 Lattice Geometry and Symmetry......................................................43

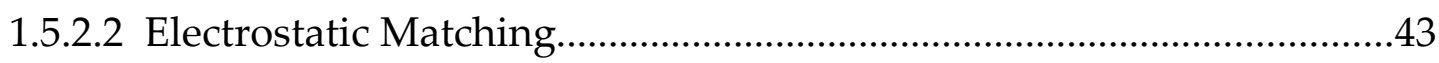


1.5.2.3 Stereochemical Matching............................................................................43

1.5.2.4 Topography ………………………………………………………….....4

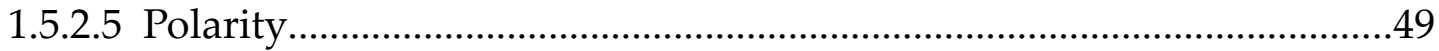

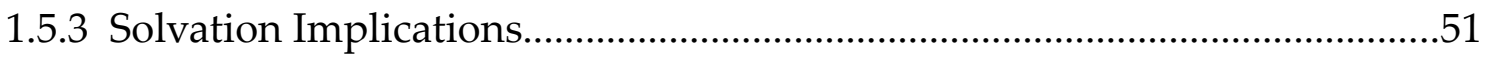

1.5.4 Current Understanding .............................................................................52

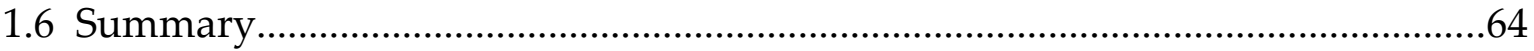

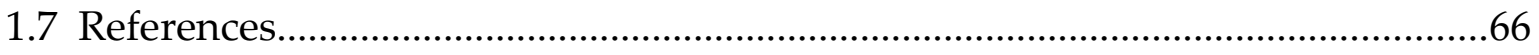

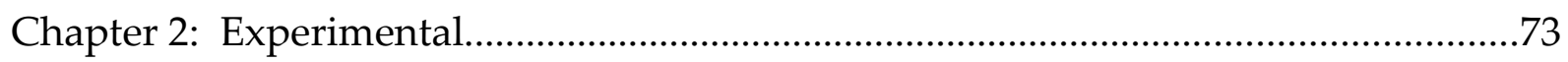

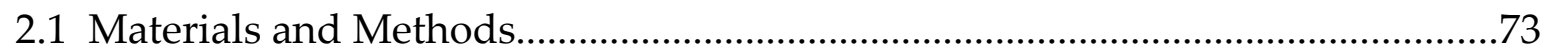

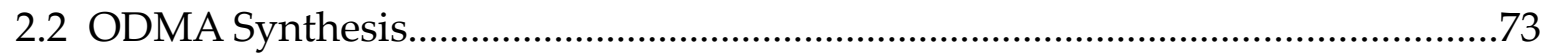

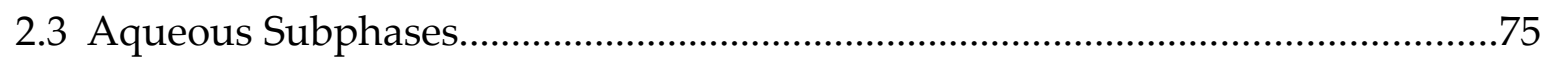

2.3.1 pH Measurements.......................................................................................76

2.4 Surface Pressure-Area Isotherms...........................................................................77

2.4.1 Surface Potential...........................................................................................

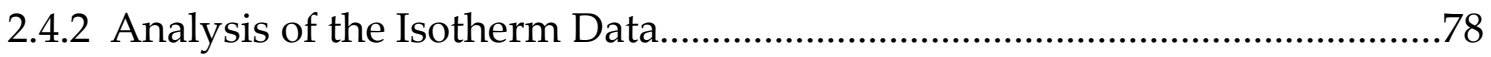

2.4.3 Reproducibility ...........................................................................................79

2.4.4 Brewster Angle Microscopy................................................................................8

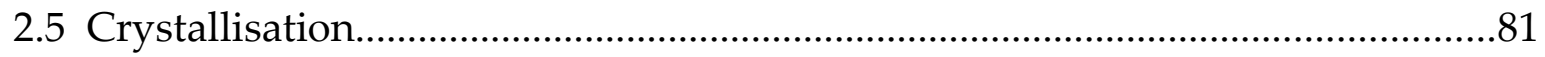

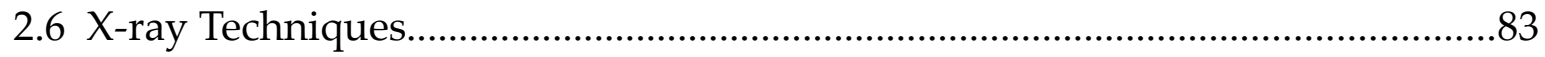

2.6.1 GIXD Measurement and Analysis..................................................................83

2.6.1.1 Symmetry and Peak $(h k)$ Assignment .....................................................84

2.6.1.2 Lattice Parameter Determination ...........................................................86

2.6.1.3 Tilt and Azimuth .................................................................................... 88

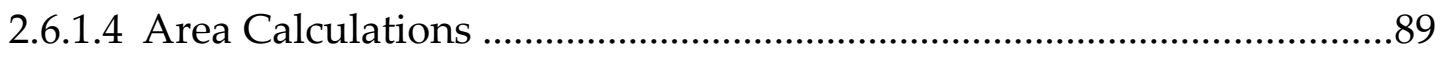

2.6.1.5 Long Range Order …………………………………………………..... 89

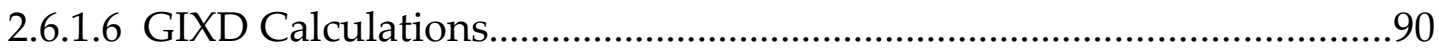

2.6.2 XRR Measurements and Calculations........................................................90 
2.6.2.1 XRR Analysis.......................................................................................90

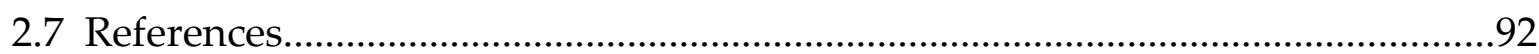

Chapter 3: Mixed Octadecanoic Acid/ Octadecanol Monolayers....................................95

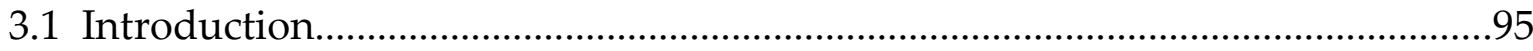

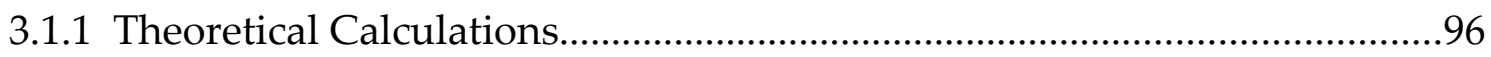

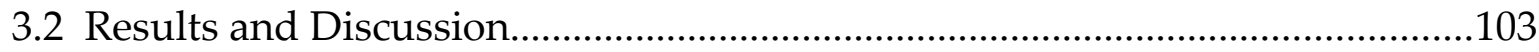

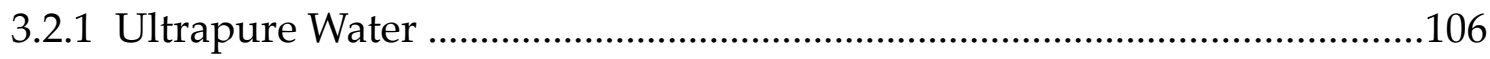

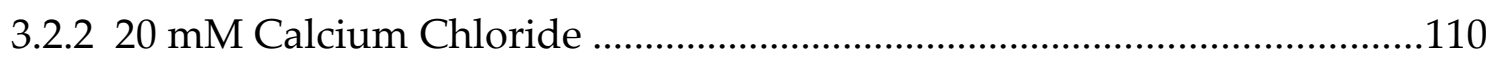

3.2.2.1 Low-alcohol content..............................................................................111

3.2.2.2 High-alcohol content..............................................................................114

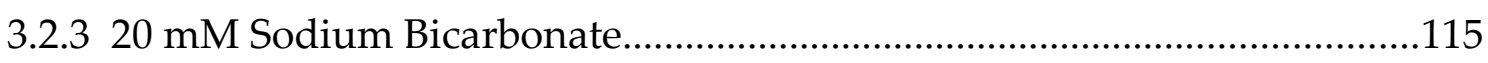

3.2.3.1 A Hydrogen-Bonded Network ..............................................................117

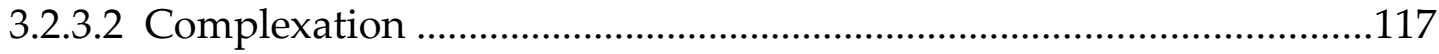

3.2.3.3 Hofmeister Related Interactions ………………………………….......118

3.2.4 10 mM Calcium Carbonate Crystallising Subphase.....................................121

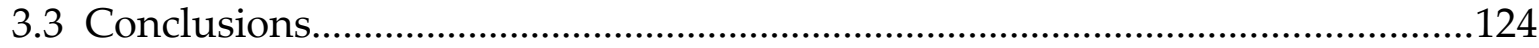

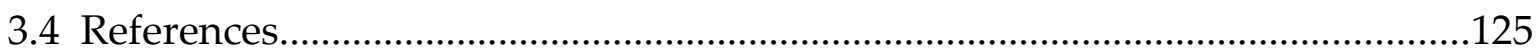

Chapter 4: Morphology and Orientation for Mixed Monolayer Systems....................129

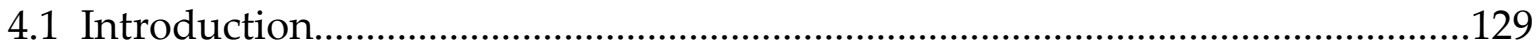

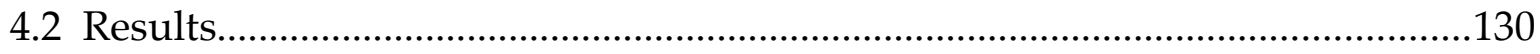

4.2.1 Monolayer Behaviour..................................................................................133

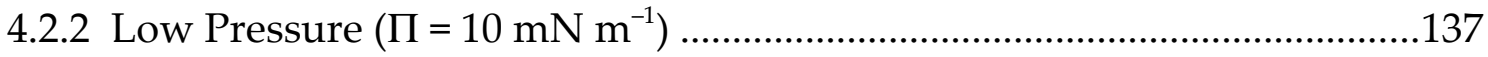

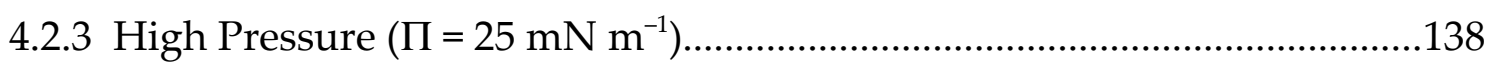

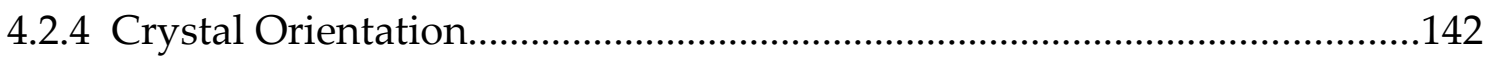

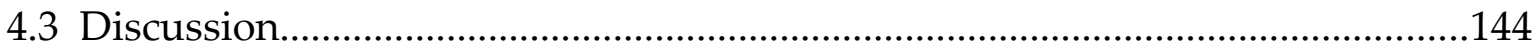

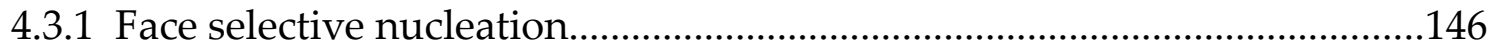

4.3.1.1 Lattice Matching $\ldots \ldots \ldots \ldots \ldots \ldots \ldots \ldots \ldots \ldots \ldots \ldots \ldots \ldots \ldots \ldots \ldots \ldots \ldots \ldots \ldots \ldots \ldots \ldots \ldots \ldots \ldots \ldots \ldots \ldots \ldots \ldots \ldots \ldots \ldots . .148$ 
4.3.1.2 Spatial Geometry Matching....................................................................149

4.3.1.3 Electrostatics.......................................................................................

4.3.2 Morphology ………………………………………………………………....151

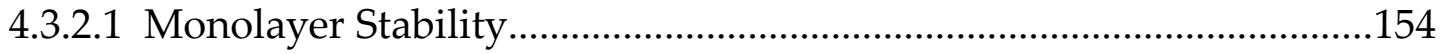

4.3.2.2 Perturbation.............................................................................................

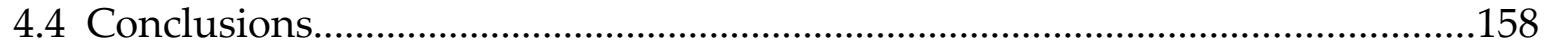

4.5 References.....................................................................................................159

Chapter 5: 2-Hydroxyoctadecanoic Acid Monolayers ......................................................163

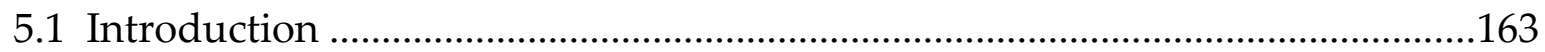

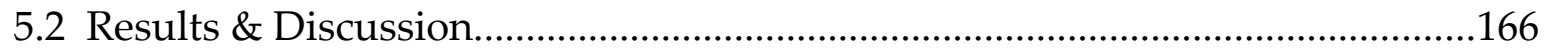

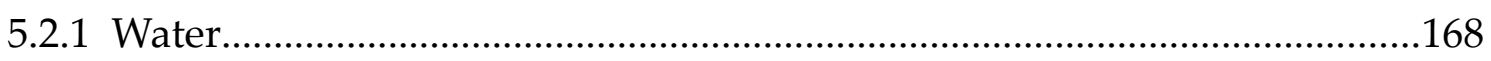

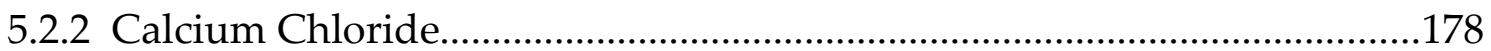

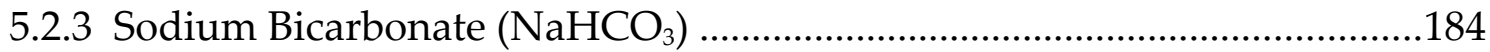

5.2.4 Sodium Chloride...........................................................................................191

5.2.5 Calcium Carbonate Crystallising Subphase (CCCS)..................................193

5.2.6 Cation Mediated Hydrogen Bonding Network............................................196

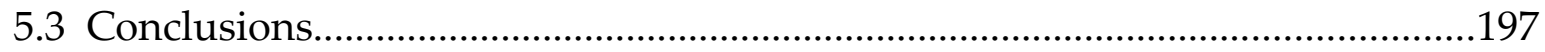

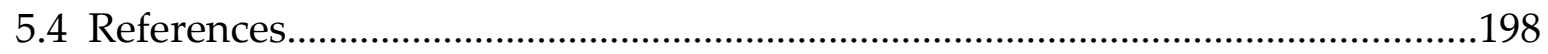

Chapter 6: Calcite Crystallisation Under 2-HSA Monolayers.........................................201

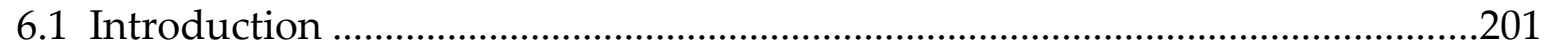

6.2 Experimental............................................................................................202

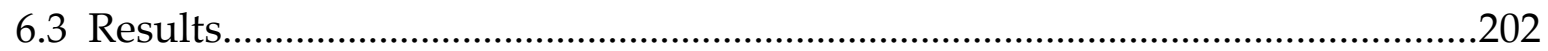

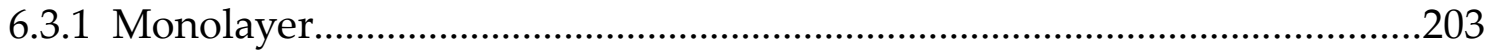

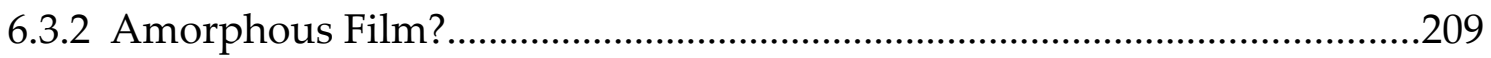

6.3.3 Crystallisation............................................................................................210

6.3.3.1 Ex situ Crystallisation Studies..............................................................212

6.4 Conclusions 


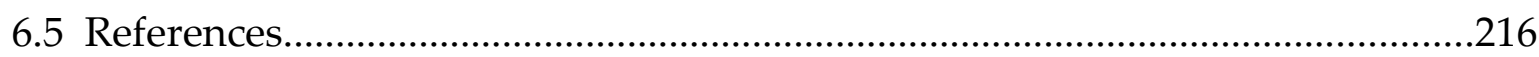

Chapter 7: Substituted Acids...................................................................................217

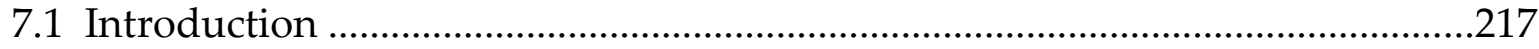

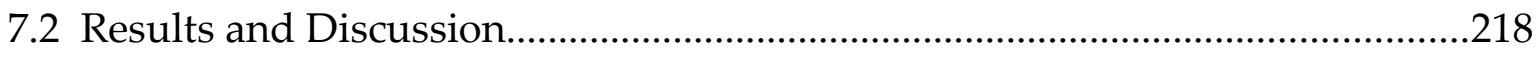

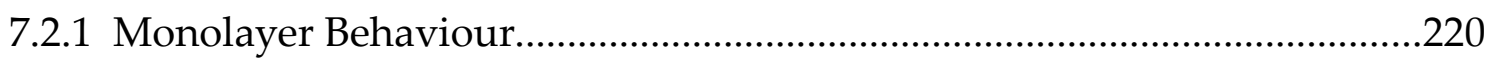

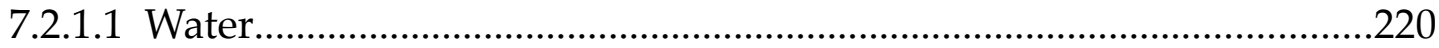

7.2.1.2 Calcium Chloride..................................................................................226

7.2.1.3 Sodium Bicarbonate...............................................................................230

7.2.1.4 Calcium Carbonate Crystallising Subphase...........................................234

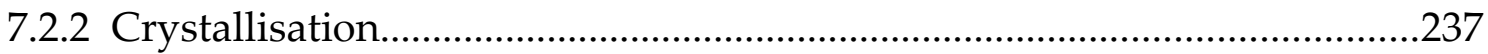

7.3 Conclusions

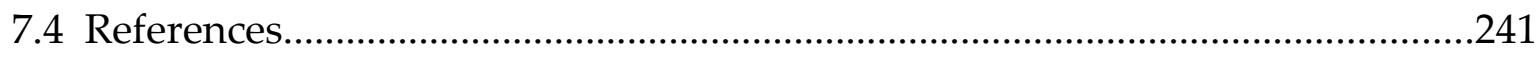

Chapter 8: Conclusions and Future Work....................................................................243

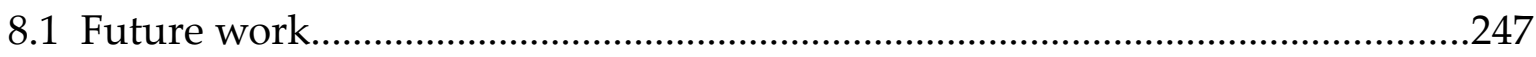

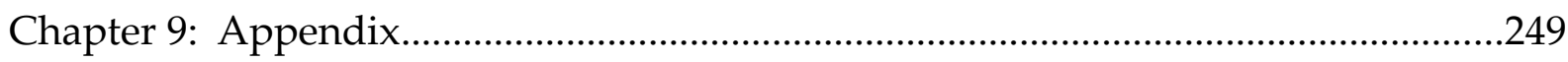

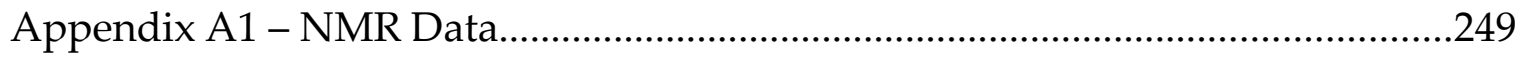

Appendix A2 - Mixed Monolayer Trend Plots...…………………………………....254

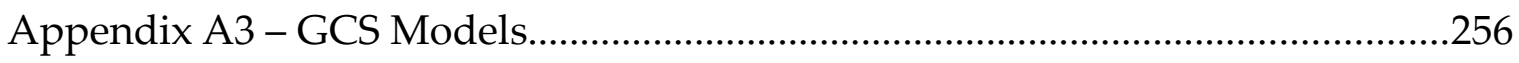

Appendix A4 - Crystal Orientations..........................................................................264

Appendix A5 - GIXD Analysis.............................................................................278

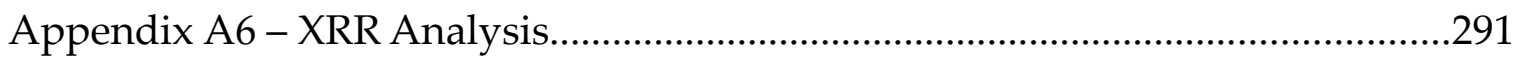




\section{Illustration Index}

Figure 1.1 A generalised solubility plot. A supersaturated solution is achieved by either: (a) by cooling the solution, or (b) by solvent evaporation[1]..............4

Figure 1.2 A solubility temperature plot for a hypothetical dimorphic system[1].......7

Figure 1.3 Three possible nucleation rate combinations for a hypothetical dimorphic system[1].

Figure 1.4 A schematic of a crystal surface showing potential growth kink (K), step

(S) and face (F) sites[1].

Figure 1.5 A schematic illustrating the generation of nuclei by way of surface nucleation[1]

Figure 1.6 An atomic force microscope (AFM) image of two newly formed spiral growth hillocks grown on the calcite (10.4) face. (Taken from Teng et al.[6])

Figure 1.7 An example of the effect of supersaturation on the growth rate of particular paracetamol crystal faces[1]..... .14

Figure 1.8 A schematic illustrating the effect of selective adsorption of impurities onto particular crystal faces and its subsequent affect on morphology[1].15

Figure 1.9 A generalised scheme of biomineralisation in organisms. The key aspect is the spatial barrier, which separates the mineralisation process from the external environment. This compartmentalisation allows the regulation of the physicochemical and biochemical properties in such a way to facilitate controlled nucleation and growth of biominerals[17].

Figure 1.10 Crystallisation pathways via thermodynamic (A) or kinetic (B) controlled routes[52]. .22

Figure 1.11 An extended three-pathway model used to describe the interaction between an organic matrix and a precipitating inorganic phase that leads 
to oriented crystallisation. Pathway A is the more conventional view, where aqueous cations bind to the matrix, followed by counter-anions. The achievement of critical cluster size results in oriented nucleation on a specific crystallographic face. Pathway B involves the binding of solution crystallised particles on a specific crystal face. Pathway $\mathrm{C}$ involves the formation of an intermediate amorphous phase either in solution or on the matrix interface. This amorphous primary particle then transforms via a matrix-mediated mesophase transition resulting in oriented crystallisation[52].

Figure 1.12 Examples of some common surfactants: (a) palmitic acid; (b) methyl palmitate; (c) diacylphosphatidylethanolamine; (d) diacylphosphatidylcholine; and (e) diacylphosphatidylcholine with an aliphatic branch........27

Figure 1.13 A schematic illustrating the monolayer ordering that occurs on the application of surface pressure.

Figure 1.14 (a) A generic monolayer phase diagram[56]. (b) A pressure isotherm for a stearic acid monolayer on four different subphases. As the pressure is increased the monolayer passes through phase transitions, which can be plotted against temperature.

Figure 1.15 A schematic of the GIXD and specular reflectivity geometry from a horizontal surface. $K_{i}$ and $K_{f}$ are the incident and reflected wave vectors, with angles $\alpha_{\mathrm{i}}$ and $\alpha_{\mathrm{f}}$ between the beam and the surface, respectively. $Q$ is the diffracted wave vector with components $Q_{z}$ and $Q_{x y}$.

Figure 1.16 BAM images of uncompressed monolayers $\left(\Pi=0 \mathrm{mN} \mathrm{m}^{-1}\right)$ of (a) palmitic acid; (b) lignoceric acid; and compressed monolayers of (c) lignoceric acid at $\Pi=29 \mathrm{mN} \mathrm{m}^{-1}$ and $(\mathrm{d})$ triacontanoic acid at a pressure of $27 \mathrm{mN} \mathrm{m}^{-1}$. Scale bar $=0.5 \mathrm{~mm}[70]$. 35

Figure 1.17 The typical crystal habits of calcium carbonate. The important faces are 
labelled and the crystal lattice arrangement is shown[75]. In the calcite (001) schematic the carbonate groups are actually displaced into the page relative to the calcium ions. All the atoms in the calcite (110) and (104) planes are in the plane of the page. In the aragonite schematic the two different oxygen atoms are represented as filled and unfilled spheres.....39

Figure 1.18 The four main types of metal ion/carboxylate interactions observed: (a) an uncoordinated anion, (b) a monodentate ligand, (c) a bidentate chelate and (d) a bridging bidentate interaction.

Figure 1.19 Suggested mechanism of surface complexation of dicarboxylates on a calcite surface[40]. .41

Figure 1.20 Suggested mechanism of surface complexation of $\alpha$-hydroxy carboxylates on a calcite surface[40]. 42

Figure 1.21 Four crystal faces of calcite, showing the, symmetry, spacing and composition of the face..

Figure 1.22 A schematic of the influence of surface topography on the spatial charge distribution and therefore controlled crystallisation[19].... 47

Figure 1.23 A schematic of the monolayer buckling or protrusions postulated by Kmetko[80] to explain small secondary oscillations in grazing incidence $\mathrm{X}$ ray diffraction Bragg rod studies of $\mathrm{MnCl}_{2}$ and $\mathrm{MgCl}_{2}$ films precipitated under heneicosanoic acid. 49

Figure 2.1 A scheme for the synthesis of octadecylmalonic acid (ODMA). .74

Figure 2.2 (a) A picture of the Trek 320-H-CE electrostatic voltmeter with a 3250 vibrating plate probe used in experimentation. (b) A schematic of the likely experimental set up, based on the work completed by Vogel and Möbius $\{5,6\}$ .78

Figure 2.3 A photograph of the NIMA 102 Langmuir-Blodgett trough that was used for crystallisation experiments. In the centre of the picture is the Wilhelmy 
plate based surface pressure sensor.

Figure 2.4 An illustration of the three different monolayer packing symmetries. In the hexagonal arrangement the monolayers are untilted, and equally spaced. The centred rectangular lattice is a distorted hexagonal arrangement, due to tilt along the bonding direction. The rectangular lattice can be manifested as either a NN or NNN tilted phase. Finally, the oblique lattice reflects a further distortion from the hexagonal lattice, due to an intermediate tilting direction .86

Figure 2.5 A schematic defining the polar tilt angle (t) and the tilt direction or azimuth $(\Psi)$ 88

Figure 2.6 XRR and electron density profiles for 2-HSA on $\mathrm{CaCl}_{2}$ at $\Pi=25 \mathrm{mN} \mathrm{m}^{-1}$. (a) In the reflectivity profile the solid line reflects the model fit of the experimentally determined data (points). (b) The electron density profile the dashed line represents the model boxes and the solid line is the smoothed box model. Highlighted in colour is the three box model with the model parameters labelled. .93

Figure 3.1 A schematic of the combined Demchak-Fort/Gouy Chapman Stern numerical model employed to elucidate the experimental surface potential data. .97

Figure 3.2 Mean area per molecule of the mixed ODA and ODOH system as a function of the $\mathrm{ODOH}$ added on four different subphases at $\sim 20^{\circ} \mathrm{C}$. Depicted are examples of the four general trends utilized to describe the mixed monolayer behaviour. a) Condensation behaviour on water at $\mathrm{pH}$ 5.6, b) ROM behaviour: $20 \mathrm{mM} \mathrm{CaCl}_{2}$ at $\mathrm{pH} \sim 5.5$, c) alcohol-like behaviour: $20 \mathrm{mM} \mathrm{NaHCO}$ plus $\mathrm{CO}_{2}(\mathrm{~g})$ at $\mathrm{pH} \sim 6.0$ and $\mathrm{d}$ ) acid-like behaviour: $10 \mathrm{mM}$ CCCS plus $\mathrm{CO}_{2}(\mathrm{~g})$ at $\mathrm{pH} \sim 5.8$. In all graphs the solid line represents the rule of mixtures trend and the dashed line is drawn to 
guide the eye to the assigned monolayer behaviour.

Figure 3.3 Representative surface pressure isotherms of pure octadecanoic acid on the different subphases investigated. The inflection point in the water, $\mathrm{CaCl}_{2}$, and $\mathrm{NaHCO}_{3}$ isotherms is associated with a tilted $L_{2}$ to untilted $L S$ transition. This transition is absent for the CCCS where the monolayer is untilted at all pressures. 106

Figure 3.4 Surface potential measurements for the mixed monolayers on water. The solid line represents ROM behaviour and the dashed line is added to highlight the acid-like behaviour of the data.

Figure 3.5 Surface potential plot for the mixed systems for a $20 \mathrm{mM} \mathrm{CaCl}_{2}$ subphase. The solid line reflects ROM behaviour, which is followed in the lowalcohol content monolayer (up to $\sim 40$ mol.\%). The data shows significant negative deviation away from ROM for higher alcohol content, highlighted by the dashed line added to guide the eye.

Figure 3.6 Variation of surface potential as a function of alcohol substitution for the $20 \mathrm{mM} \mathrm{NaHCO}{ }_{3}$ subphase. The solid line represents the rule of mixtures trend and the dashed line guides the eye to the acid-like behaviour of the experimental results. 116

Figure 3.7 A 2-dimensional representation of the chain structure of crystalline sodium bicarbonate, viewed down the $a$ and $b$ axes. The large spheres represent the sodium ions, with the bicarbonate layer in between. The dashed lines depict the $\mathrm{O}-\mathrm{H} \cdots \mathrm{O}$ hydrogen bond between adjacent bicarbonate molecules.

Figure 3.8 Surface potential plot for the mixed systems for the CCCS. The solid line reflects $\mathrm{ROM}$ behaviour.

Figure 4.1 Nucleation at the air/water interface in the absence of a monolayer. The product is randomly oriented rhombohedral calcite crystals with very 
little elongation.

Figure 4.2 The changing morphology of calcite. (a) The classical rhombohedra, (b) the truncated rhombohedra (type III) with the insert showing the monolayer side (the insert scale bar represents $10 \mu \mathrm{m}$ ), (c) type II calcite, (d) an elongated type II calcite, with arrows indicating the two sides of the crystal morphology, and (e) type I calcite, the arrow indicates the surface presented to the subphase, the insert shows an enlargement of a type I crystal (the insert scale bar represents $10 \mu \mathrm{m}$ ).

Figure 4.3 Evidence for the absence and presence of tilt in the mixed monolayers on CCCS. The red dashed line guides the eye to the tilting transition from the tilted $L_{2}$ phase to the untilted $L S$ phase for the mixed monolayer systems. Brewster angle microscopy images are included as inserts to illustrate the absence and presence of tilt. The image of the untilted LS phase (upper left) for the CCCS at low alcohol contents lacks contrast due to the absence of tilt. In contrast, the presence of the tilted $L_{2}$ phase is reflected in the contrasting domains (lower right). 134

Figure 4.4 (a) The change in area per molecule with time (monolayer stability) during the crystal growth experiments at a constant surface pressure of $25 \mathrm{mN} \mathrm{m}^{-1}$. (b) A comparison of the loss in area after 4 hours of crystal growth for the two pressures $\left(\mathrm{LP}=10 \mathrm{mN} \mathrm{m}^{-1}\right.$ and $\left.\mathrm{HP}=25 \mathrm{mN} \mathrm{m}^{-1}\right) \ldots 135$

Figure $4.510 \mathrm{~mol} \% \mathrm{ODOH}$ on water, bubbled calcium chloride and calcium carbonate crystallising subphases at a pressure of $25 \mathrm{mN} \mathrm{m}^{-1}$. The best stability being achieved on the carbon dioxide bubbled calcium chloride subphase.

Figure 4.6 Overviews of the changes in crystal morphology as the octadecanol content of the monolayer is increased at a constant pressure of $10 \mathrm{mN} \mathrm{m}^{-1}$.
(a) $100 \mathrm{~mol} . \% \mathrm{ODOH}$,
(b) $75 \mathrm{~mol} . \% \mathrm{ODOH}$,
(c) $50 \mathrm{~mol} . \% \mathrm{ODOH}$,
(d) 25 
mol.\% ODOH, (e) $10 \mathrm{~mol} \% \mathrm{ODOH}$, and (f) 0 mol.\% ODOH. The inserts show enlarged images of a typical crystal (the scale bar $=10 \mu \mathrm{m})$.......

Figure 4.7 Overviews of the changes in crystal morphology as the octadecanol content of the monolayer is increased at a constant pressure of $25 \mathrm{mN} \mathrm{m}^{-1}$. (a) $100 \mathrm{~mol} . \% \mathrm{ODOH}$, (b) $75 \mathrm{~mol} \% \mathrm{ODOH}$, (c) $50 \mathrm{~mol} . \% \mathrm{ODOH}$, (d) 25 mol.\% ODOH, (e) $10 \mathrm{~mol} . \% \mathrm{ODOH}$, and (f) 0 mol.\% ODOH. The inserts show enlarged images of a typical crystal (the scale bar $=10 \mu \mathrm{m}) \ldots$

Figure 4.8 SEM images illustrating the textured upper crystal surface, the surface in contact with the monolayer.

Figure 4.9 Overview of the low and high pressure nucleation face assignment data, showing the most prominent nucleation faces for the different mixed monolayer systems. Charge density is based upon a full ionised monolayer (given a surface $\mathrm{pH}$ of 7 at the point of crystallisation) and an average $\mathrm{A}_{\mathrm{m}}$ of $20 \AA^{2} /$ molecule.

Figure 4.10 Computer generated (SHAPE for Window V7.2.2) calcite rhombohedrons oriented with the (11.15) face in the plane of the page. The red polygon represents the nucleation face for different distances from the centre of the crystal (m), highlighting the range of morphologies that result. .152

Figure 4.11 A schematic illustrating the interrelationships occurring between monolayer stability and crystal morphology. The (-) or red lines depict an inverse relationship whereas the $(+)$ or black lines depict a positive relationship. 154

Figure 4.12 The correlation between monolayer stability and morphology. In order of decreasing monolayer stability $(a)>(d)>(b)>(e)$, correlating to the black, olive, teal and orange lines as shown in (c). The crystals (a) and (b) are grown at $\Pi=10 \mathrm{mN} \mathrm{m}^{-1}$ whereas (d) and (e) were grown at 
$\Pi=25 \mathrm{mN} \mathrm{m}^{-1}$

Figure 4.13 SEM images of the variation in nucleation density with surface pressure of pure octadecanoic acid monolayers after 1 hour of growth, (a) $\Pi=$ $10 \mathrm{mN} \mathrm{m}^{-1}$, and (b) $\Pi=30 \mathrm{mN} \mathrm{m}^{-1}$ 157

Figure 5.1 A schematic of DL-2-hydroxyoctadecanoic acid molecule with the hydroxyl (red) and acid (blue) functional groups highlighted. 163

Figure 5.2 Surface pressure isotherms for 2-hydroxyoctadecanoic acid on different subphases: water at $\mathrm{pH} 5.6, \mathrm{CaCl}_{2}$ at $\mathrm{pH} 5.5, \mathrm{NaHCO}_{3}$ at $\mathrm{pH} 6.0$ and a calcium carbonate crystallising subphase at $\mathrm{pH} 5.8$

Figure 5.3 The overall trends in the surface pressure and potential isotherms for the different subphases. Note, there was no transition apparent on the water subphase hence the absence of AT and PT columns, also the maximum surface potential for the $\mathrm{NaHCO}_{3}$ subphase was $-30.6 \pm 12.0 \mathrm{mV}$ and therefore is not shown. (Key: $\mathrm{AO}=$ the $\mathrm{A}_{\mathrm{m}}$ at onset, $\mathrm{A}_{10}=$ the $\mathrm{A}_{\mathrm{m}}$ at $\Pi=$ $10 \mathrm{mN} \mathrm{m}^{-1}, \mathrm{AT}=$ the $\mathrm{A}_{\mathrm{m}}$ at the phase transition, $\mathrm{PT}=\Pi$ at the phase transition, $\mathrm{A}_{25}=$ the $\mathrm{A}_{\mathrm{m}}$ at $\Pi=25 \mathrm{mN} \mathrm{m}^{-1}$, and $\mathrm{SP}=$ the maximum surface potential). 167

Figure 5.4 A BAM image showing the large islands present on water in the absence of pressure. Apparent is the lack of contrast pointing to a homogeneous, but as it turns out disordered monolayer. The scale bar $=1 \mathrm{~mm}$. (Each of the BAM images is of an area of monolayer 4.128 by $3.616 \mathrm{~mm}$, based on a 640x480 image and a x-scale of 5.65 microns/pixel and a y-scale of 8.60 microns/pixel.)

Figure 5.5 GIXD results for 2-HSA on water at $\Pi=0 \mathrm{mN} \mathrm{m}^{-1}$. (a) A 3-D contour plot illustrating the diffraction arc. (b) and (c) The fitting of two and three peak models, respectively, to the summed $Q_{x y}$ profile, with the three-peak model providing the best match. (d) The summed $\mathrm{Q}_{z}$ profile and fit. ...172 
Figure 5.6 2-D contour plots of the behaviour of 2-HSA on water with increasing surface pressure. (a) 0, (b) 5, (c) 10, (d) 17, (e) 25, and (f) $30 \mathrm{mN} \mathrm{m}^{-1}$......173

Figure 5.7 GIXD analysis of 2-HSA on water at $\Pi=0 \mathrm{mN} \mathrm{m}^{-1}$. (a) Variation of the phase composition of the monolayer with pressure, lines drawn to guide the eye. (b) Changes in the lattice parameters $a$, and $b$ with pressure for the three phases. 174

Figure 5.8 Surface potential profiles for 2-HSA on subphases of water, calcium chloride, sodium bicarbonate and CCCS. .176

Figure 5.9 A 2-D contour plot of the GIXD pattern for 2-HSA on CCCS in the absence of applied pressure.

Figure 5.10 A BAM image of a 2-HSA monolayer on a $20 \mathrm{mM} \mathrm{CaCl}_{2}$ subphase at $0 \mathrm{mN} \mathrm{m}^{-1}$ pressure. The islands appear to be multi-domain based on the contrast and are very rigid. .180

Figure 5.11 Indicative structures of 2-HSA head groups in response to cation binding, the precise conformation will vary with surface pressure and chelation. The top structure is consistent with a four-membered ring chelation as opposed to the five-membered ring of the lower structure. 180

Figure 5.12 XRR reflectivity and electron density profiles for 2- $\mathrm{HSA}$ on $\mathrm{CaCl}_{2}$ at $\Pi=$ $10 \mathrm{mN} \mathrm{m}^{-1}$. In the reflectivity profile the solid line reflects the model fit of the experimentally determined data (points). In the electron density profile the dashed line represents the model boxes and the solid line is the smoothed box model.

Figure 5.13 XRR data and electron density profiles for 2-HSA on $\mathrm{CaCl}_{2}$ at $\Pi=$ $25 \mathrm{mN} \mathrm{m}^{-1}$. In the reflectivity profile the solid line reflects the model fit of the experimentally determined data (points). In the electron density profile the dashed line represents the model boxes and the solid line is the smoothed box model. 
Figure 5.14 A BAM image illustrating the presence of two phases in a monolayer of 2-HSA on an aqueous $\mathrm{NaHCO}_{3}$ subphase. The scale bar represents $1 \mathrm{~mm}$.

Figure 5.15 The ratio of NN-tilted and untilted (U) phases in a 2-HSA monolayer on a sodium bicarbonate subphase. 186

Figure 5.16 The variation of the lattice parameters with pressure. The $b$ lattice parameters coincide for the two phases. The lines are added to guide the eye. 186

Figure 5.17 Isotherm of 2-HSA on a $\mathrm{NaHCO}_{3}$ subphase. Overlaid are BAM images illustrating the changes in monolayer structure that occur with the application of pressure. The first image at zero pressure, before onset, shows the phase coexistence that arises immediately on surfactant addition. As pressure is applied the relatively rigid domains come together to form a coherent monolayer. The second image shows the persistence of two phases, as indicated by the regions of differing contrast. The inflection in the isotherm is associated with a phase change and the loss of all phase contrast in the BAM images. Finally a small kink in the profile is associated with a brittle collapse event. The scale bar represents $1 \mathrm{~mm}$. 188

Figure 5.18 (a) 2-D contour plot of the GIXD diffraction pattern for a 2-HSA monolayer on a $\mathrm{NaHCO}_{3}$ subphase at a $\Pi=17 \mathrm{mN} \mathrm{m}^{-1}$. (b) The summed $\mathrm{Q}_{x y}$ profile with a fitted 2-peak model. (c) The summed $\mathrm{Q}_{z}$ profile also with a fitted 2-peak model. 190

Figure 5.19 GIXD results for 2-HSA on $\mathrm{NaHCO}_{3}$ at $\Pi=25 \mathrm{mN} \mathrm{m}^{-1}$. (a) A contour plot of the diffraction pattern, interestingly diffuse scattering is evident at low $\mathrm{Q}_{\mathrm{xy}}$. (b) The summed $\mathrm{Q}_{\mathrm{xy}}$ profile with a two-peak model fit. (c) The equivalent summed $Q_{z}$ profile also with a two-peak model fit. 191 
Figure 5.20 2-D contour plots illustrating the behaviour of 2-HSA on a $\mathrm{NaCl}$ subphase at different surface pressures: (a) 5, (b) 10, (c) 17, (d) 25, and (e) $30 \mathrm{mN} \mathrm{m}^{-1}$

Figure 5.21 A BAM image of the phase coexistence that occurs for 2-HSA on CCCS immediately upon addition of the surfactant. The scale bar represents 1 $\mathrm{mm}$.

Figure 6.1 GIXD data for 2-HSA on $\mathrm{CaCl}_{2}$ (top) and CCCS (bottom). A comparison of the two systems shows a subtle loss of order and an extension in $Q_{z}$ for CCCS. 205

Figure 6.2 Monolayer rearrangement in response to subphase nucleation and growth events. The solid lines reflect the fitting of Lorentzian peaks to the scattered experimental data. The sequence of images (a) to (f) represents $\sim 1-1.5$ hours in experimental time, culminating in very small but intense Bragg spots in (f). 207

Figure 6.3 The change in peak properties with increasing crystallisation time. The symbols plot peak intensity variation and the solid lines plot the shift in $\mathrm{Q}_{\mathrm{xy}}$. (Note the large gap in the data between $\mathrm{T} \sim 50$ and $\mathrm{T} \sim 130$ mins corresponds to the period the trough was opened to induce crystallisation.). .208

Figure 6.4 Debye-Scherrer rings for different d-spacings of calcite, aragonite, vaterite, ikaite and monohydrocalcite. Overlaid are the observed diffraction peaks. Note that the majority of peaks are paired reflecting the refraction of the incoming beam.

Figure 6.5 Representative SEM images of crystals grown under 2-HSA monolayers. (a)-(c) Were grown at a monolayer surface pressure of $10 \mathrm{mN} \mathrm{m}^{-1}$. (d)-(e) Were grown at a monolayer pressure of $25 \mathrm{mN} \mathrm{m}^{-1}$. 215

Figure 7.1 Semi-empirical PM3 calculations of the electron density distribution for 
neutral and deprotonated substituted C6 acids.

Figure 7.2 Surface pressure isotherms for the substituted octadecanoic acid monolayers on pure water at $\mathrm{pH}=5.6$. The insert is a magnified view of a low-pressure inflection in the isotherm of the 2-HSA monolayer.

Figure 7.3 Surface potential profiles for the different substituted octadecanoic acids on water. The irregular profile at higher areas is consistent with a coexistence of two phases, one being gas-like. Colour coded ellipses highlight the plateau regions of the potential isotherm.

Figure 7.4 Isotherm characteristics for the substituted octadecanoic acids on a water subphase. Note, only the ODMA monolayer displayed a transition, hence the absence of AT and PT columns for the other surfactants. The negative surface potential for ODMA exceeds the ordinate scale, thus an insert is included to highlight the differences in $\Delta \mathrm{V}_{\max }$ for the substituted acids. (Key: $\mathrm{AO}=$ the $\mathrm{A}_{\mathrm{m}}$ at onset, $\mathrm{A}_{10}=$ the $\mathrm{A}_{\mathrm{m}}$ at $\Pi=10 \mathrm{mN} \mathrm{m}^{-1}, \mathrm{AT}=$ the $\mathrm{A}_{\mathrm{m}}$ at the phase transition, $\mathrm{PT}=\Pi$ at the phase transition, $\mathrm{A}_{25}=$ the $\mathrm{A}_{\mathrm{m}}$ at $\Pi=$ $25 \mathrm{mN} \mathrm{m}^{-1}$, and $\mathrm{SP}=$ the maximum surface potential). 223

Figure 7.5 Surface pressure isotherms of the substituted octadecanoic acids on a 20 $\mathrm{mM}$ calcium chloride subphase.

Figure 7.6 General isotherm trends for the substituted acids on a $20 \mathrm{mM}$ calcium chloride subphase. The insert summarises the surface potential results which exceed the ordinate-scale. (Key: $A O=$ the $A_{m}$ at onset, $A_{10}=$ the $A_{m}$ at $\Pi=10 \mathrm{mN} \mathrm{m}^{-1}, \mathrm{AT}=$ the $\mathrm{A}_{\mathrm{m}}$ at the phase transition, $\mathrm{PT}=\Pi$ at the phase transition, $\mathrm{A}_{25}=$ the $\mathrm{A}_{\mathrm{m}}$ at $\Pi=25 \mathrm{mN} \mathrm{m}^{-1}$, and $\mathrm{SP}=$ the maximum surface potential). 228

Figure 7.7 Surface potential profiles for the substituted acids on a $20 \mathrm{mM}$ calcium chloride subphase. 229

Figure 7.8 Surface pressure (top) and potential (bottom) isotherms for the 
substituted acids on $20 \mathrm{mM}$ sodium bicarbonate. The colour coded ellipses highlight the plateau region, absent in 2-HSA and ODMA profiles.

Figure 7.9 The overall characteristics of the substituted acids on a sodium bicarbonate aqueous subphase. The surface potential data exceeds the ordinate scale, thus an insert is included to highlight the differences in $\Delta \mathrm{V}_{\max }$ for the substituted acids. (Key: $A O=$ the $A_{m}$ at onset, $A_{10}=$ the $A_{m}$ at $\Pi=10 \mathrm{mN}$ $\mathrm{m}^{-1}, \mathrm{AT}=$ the $\mathrm{A}_{\mathrm{m}}$ at the phase transition, $\mathrm{PT}=\Pi$ at the phase transition, $\mathrm{A}_{25}$ $=$ the $\mathrm{A}_{\mathrm{m}}$ at $\Pi=25 \mathrm{mN} \mathrm{m}^{-1}$, and $\mathrm{SP}=$ the maximum surface potential)...233

Figure 7.10 Surface pressure (top) and potential (bottom) isotherms of the substituted acids on CCCS. .235

Figure 7.11 Substituted acid monolayer characteristics on CCCS. The surface potential data exceeds the ordinate scale, thus an insert is included to highlight the differences in $\Delta \mathrm{V}_{\max }$ for the substituted acids. (Key: $\mathrm{AO}=$ the $A_{m}$ at onset, $A_{10}=$ the $A_{m}$ at $\Pi=10 \mathrm{mN} \mathrm{m}^{-1}, A T=$ the $A_{m}$ at the phase transition, $\mathrm{PT}=\Pi$ at the phase transition, $\mathrm{A}_{25}=$ the $\mathrm{Am}$ at $\Pi=25 \mathrm{mN} \mathrm{m}^{-1}$, and $\mathrm{SP}=$ the maximum surface potential).

Figure 7.12 Representative SEM images of the crystals produced under the substituted acids at $\Pi=10 \mathrm{mN} \mathrm{m}^{-1}$. (a) 2-HSA, (b) 2-MODA, (c) 2-BODA, (d) 3-HSA, (e) ODMA, and (f) a surfactant free control. The inserts illustrate typical crystals where the scale bar is $1 \mu \mathrm{m}$. .239

Figure 7.13 Representative crystal morphologies for the substituted acids at a constant surface pressure of $\Pi=25 \mathrm{mN} \mathrm{m}^{-1}$. (a) 2-HSA, (b) 2-MODA, (c) 2-BODA, (d) 3-HSA, (e) ODMA, and (f) a control free of surfactant. The inserts illustrate typical crystals where the scale bar is $1 \mu \mathrm{m}$. 240 


\section{Index of Tables}

Table 1 Crystallographic characteristics of the three crystalline phases of calcium carbonate found in nature.

Table 2 Fitting parameter ranges for monolayer potential in the Demchak Fort/Gouy Chapman Stern model. 100

Table 3. Selected properties of the dominant nucleation faces as determined by interedge angle measurements.

Table 4 The best fitting models for 2-HSA on water at $\Pi=10$ and $25 \mathrm{mN} \mathrm{m}^{-1}$. .175

Table 5 A summary of the parameters of the different 2-HSA monolayers as determined by GIXD.

Table 6 A list of the calcium carbonate d-spacings that lie in the vicinity of the observed GIXD crystal peaks. .209 


\section{Abbreviations}

2-BODA

2-D

2-HHA

2-HSA

2-MODA

3-D

3-HSA

$\mathrm{A}_{10}$

$\mathrm{A}_{25}$

ACC

AFM

$\mathrm{A}_{\mathrm{m}}$

$\mathrm{AO}$

APS

AT

ATR-FTIR

BAM

CCCS

DF

$\Delta \mathrm{V}_{\text {max }}$

ESP

$\mathrm{EtOH}$

FSN

FTIR

FWHM 2-bromooctadecanoic acid

Two-dimensional

2-hydroxyhexadecanoic acid

2-hydroxyoctadecanoic acid

2-methyloctadecanoic acid

Three-dimensional

3-hydroxyoctadecanoic acid

Area per molecule at $10 \mathrm{mN} \mathrm{m}^{-1}$

Area per molecule at $25 \mathrm{mN} \mathrm{m}^{-1}$

Amorphous calcium carbonate

Atomic force microscopy

Area per molecule

Area per molecule at onset

Advanced photon source

Area per molecule at the transition

Attenuated total reflection-Fourier transform infrared

Brewster angle microscopy

Calcium carbonate crystallising subphase

Demchak-Fort

Maximum change in the surface potential

Equilibrium spreading pressure

Ethanol

Face selective nucleation

Fourier-transform infrared

Full width half maximum 
GC

GCS

GIXD

HDMS

$\mathrm{HP}$

HPA

IR

$L_{2}$

$L_{2}^{\prime}$

LB

LP

LS

NN

NNN

ODA

ODMA

$\mathrm{ODOH}$

PA

PT

PTFE

ROM

SAM

SEM

SP

U

XRR
Gouy Chapman

Gouy Chapman Stern

Grazing incidence X-ray diffraction

Hexamethyldisilazane

High pressure

2-hydroxypalmitic acid

Infrared

Tilted liquid-like phase

Tilted liquid-condensed phase

Langmuir-Blodgett

Low pressure

Untilted condensed phase

Nearest neighbour

Next nearest neighbour

Octadecanoic acid

octadecylmalonic acid

Octadecanol

Palmitic acid

Pressure at the phase transition

Polytetrafluroethylene

Rule of mixtures

Self-assembled monolayer

Scanning electron microscopy

The maximum surface pressure

Untilted

X-ray reflectivity 


\section{Chapter 1: Literature Review}

The development and improvement of materials is synonymous with being Human, whether it be the shaping of wood into clubs and spears or the search for room temperature superconducting materials. Much of the property improvement of the last couple of centuries was attained through compositional changes, where rare and at times toxic elements/molecules (which are often expensive), were used. However the demand for greater performance, has in many situations, exhausted the capacity for improvement achievable solely through composition change. Thus the push for improvement has focussed on Nanotechnology where size-related effects are captured. At the nano-scale the manipulation of size and shape is difficult, this fabrication of such materials requires techniques such as lithography and self assembly. However our ability to design, fabricate and use materials at this level is limited.

Nature provides a method for addressing these limitations through the design and fabrication of structures on multiple length scales, so-called hierarchical materials. The processes developed over millions of years of evolution have led to the development of structures and processes that produce materials that capture the property improvements associated with control at the nano-scale with macro-scale usability. Through multi-level control Nature is able to achieve significant property improvement from the most basic and common of materials, chalk, limestone or calcium carbonate.

Simply put, this hierarchical control is achieved through the use of organic frameworks that influence the nucleation and growth of inorganic materials. Understanding this process is difficult, involving many disciplines. The work 
outlined in this thesis is aimed at elucidating one small, but important, aspect of this process: the molecular recognition or information transfer processes across the organic/inorganic interface that leads to structural control.

Before exploring the subtleties of the interfacial interaction and elucidating how nature achieves such great control of crystal shape, size, orientation and polymorph, it helps to understand classical crystallisation theory.

\subsection{Solution Crystallisation}

Crystallisation is a supramolecular process involving the formation of an ordered 3-D molecular array from a solution where the ions or atoms are randomly dispersed[1]. Similarly, Adamson and Gast[2] described crystallisation, in the absence of foreign surfaces, to involve the clustering of molecules or ions, which grow by accretion to the point of forming crystallites. Typically, for this to occur the solution concentration has to be well beyond the saturation point. In contrast, classical crystallisation theory describes crystallisation as simply, a phase transition from a high energy solvated state to the low energy crystalline state[3]. Although this view is correct it is oversimplified and therefore somewhat misleading, particularly as it ignores the important role of kinetics. While thermodynamics is the basis for classical crystallisation theory, which is concerned with the interfacial energy of a system, and defines the final equilibrium phase that is crystallised, kinetics determine the rate of nucleation and growth. Davey and Garside[1] referred to crystallisation as a kinetic process, driven by the level of supersaturation.

The presence of a supersaturated solution is the prerequisite for crystallisation to occur. Supersaturation $(\sigma)$ is defined as the ratio of the solution activities in the supersaturated state $\left(a_{s s}\right)$ to the activities at equilibrium $\left(a_{e q}\right)$

$$
\sigma=\ln \left(\frac{a_{s s}}{a_{e q}}\right) .
$$

For an ideal solution, or one where the activity coefficients are independent of 
concentration, Equation 1 simplifies to a ratio of molar concentrations, $x_{s s}$ and $x_{e q r}$

$$
\sigma=\ln \left(\frac{x_{s s}}{x_{e q}}\right) .
$$

However, merely having a supersaturated solution will not guarantee crystallisation. Figure 1.1 illustrates a generalised solubility plot, highlighted in this plot is a metastable region. In this region the supersaturation driving force is insufficient to overcome the surface energy barrier to nucleation. Therefore within the metastable region there is no new nucleation, only continued growth of existing crystals. To understand how nucleation is initiated we turn to classical nucleation theory. 


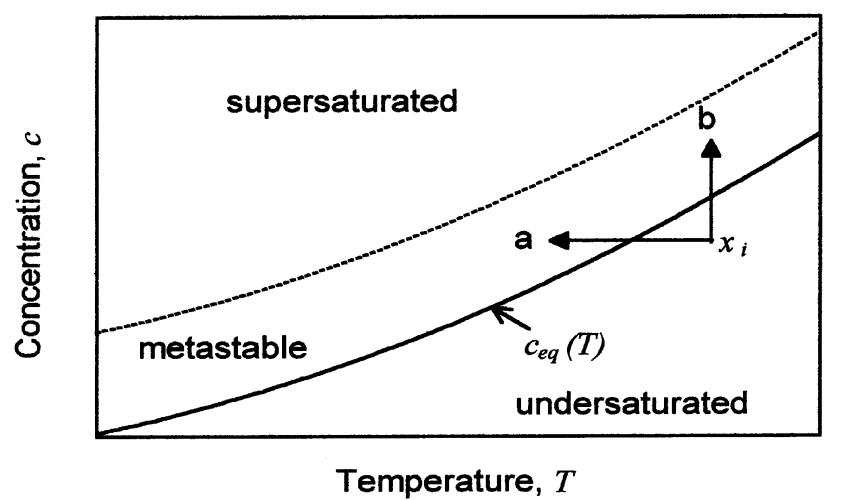

Figure 1.1 A generalised solubility plot. A supersaturated solution is achieved by either: (a) by cooling the solution, or (b) by solvent evaporation[1].

\subsubsection{Nucleation}

Classical crystallisation theory uses the concept of a critical nucleus size $\left(z_{c}\right)$ to help describe the nucleation process. Due to high interfacial energies a nucleus smaller than the critical size will be unstable and dissolve. In contrast, nuclei larger than the critical size, for that particular system, are stable and will continue to grow. This concept of a critical nucleus does not prescribe if, or how, nucleation will occur, if a solution is supersaturated it will precipitate out regardless, rather the issue with the critical nucleus size is purely one of kinetics. However, nucleation is the result of a sufficient number of atoms or molecules clustering together to exceed the critical size, therefore the probability of nucleation is affected by the value of the critical size. The ability to modify the critical size by changing the interfacial energy of the system means that the probability of nucleation can be controlled. Consequently, the likelihood and rate of nucleation are extremely dependent on the interfacial energy and supersaturation[3]. This is highlighted in the term derived for the Gibbs energy of nuclei formation (on a per molecule basis):

$$
\Delta G=-z k T \sigma+\beta \gamma z^{\frac{2}{3}}
$$

where: $z=$ the number of atoms or molecules in the nuclei,

$$
k=\text { Boltzmann's constant, }
$$


$T=$ temperature $(\mathrm{K})$,

$\sigma=$ supersaturation,

$\beta=$ an area shape factor, and

$\gamma=$ the interfacial tension.

By rearranging Equation 3 the size or number of molecules in the nuclei necessary to give a negative $\Delta G$ (i.e. a spontaneous process), defined as the critical nuclei size $\left(z_{c}\right)$, can be calculated. In general, the probability of forming nuclei larger than $z_{c}$ will depend on the height of the energy barrier relative to the available thermal energy $(k T)$. Equation 3 shows that as the level of supersaturation increases the energy barrier $(\Delta G)$ decreases. Alternatively, increased supersaturation reduces the nuclei size $(z)$ required to achieve a given value of $\Delta G$, therefore the critical nuclei size $\left(z_{c}\right)$ will also decrease[1].

\subsubsection{The Rate Equation}

Given this thermodynamic model for crystal nucleation it is necessary to derive an equation for the rate of nucleation $(J)$. To do so it is assumed that the nuclei form by stepwise aggregation. Thus for a nucleus, containing $A$ molecules having reached the critical size $z_{c}$, the overall reaction can be expressed as:

$$
z_{c} A \rightarrow A_{c}
$$

and the equilibrium constant $\left(K_{z}\right)$ for this reaction is:

$$
K_{z}=\frac{\left[A_{c}\right]}{[A]^{z_{c}}} .
$$

The resulting expression for the rate of nucleation is (on a per mole basis, for the full derivation of this expression refer to Davey and Garside[1], or Mullin[4]):

$$
J=K_{J} \exp \left(-\frac{16 \gamma^{3} v_{c}^{2}}{3 R^{3} T^{3} \sigma^{2}}\right)
$$

where: $\quad K_{J}=P[A]^{z_{c}}$

$P=$ the probability of a critical nucleus growing into a mature crystal, 
$v_{c}=$ the molar volume of the crystal, and

$R=$ gas constant.

Equation 6 accounts for the metastable region, at low supersaturation levels the interfacial energy term dominates and there is insufficient driving force to create new nuclei[1]. The equation also highlights the importance of the interfacial energy, supersaturation and temperature on the rate of nucleation. Interfacial energy and supersaturation are especially important in that they can potentially be influenced or controlled by an organic framework.

\subsubsection{Nucleation in Polymorphic Systems}

In polymorphic systems where more than one solid phase can crystallise out, such as calcium carbonate, Ostwald's law of phases may apply. The theory states that a crystallising system would move from a supersaturated state to the most stable equilibrium state in stages. Thus in a polymorphic system all of the possible phases would crystallise out, if only momentarily, on the way to the equilibrium phase. For example, a supersaturated calcium carbonate solution would first precipitate out amorphous $\mathrm{CaCO}_{3}$, which would transform to vaterite and then to calcite. At temperatures above $40^{\circ} \mathrm{C}$, or in the presence of $\mathrm{Mg}^{2+}$, vaterite would transform to aragonite[5].

Ostwald's law of phases can be investigated by exploring the dimorphic system shown in Figure 1.2.

A solution with the composition $x_{i}$ at temperature $T_{i}$ is supersaturated with respect to both phases I and II. Therefore two supersaturation equations can be defined, both in regards to phase II: the initial supersaturated solution $\left(\sigma_{i}\right.$, Equation $7)$, and a saturated phase I solution ( $\sigma_{x}$, Equation 8) 


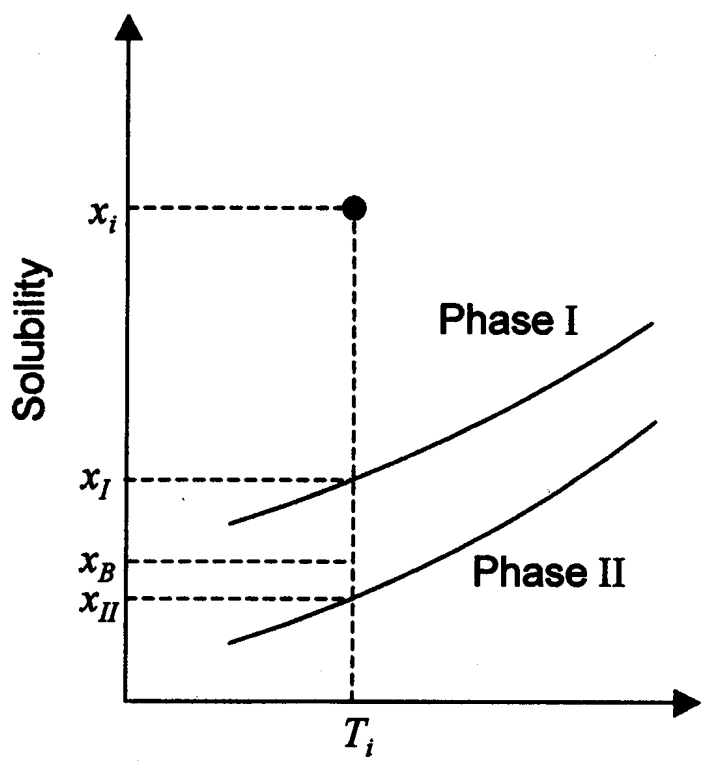

Temperature

Figure 1.2 A solubility temperature plot for a hypothetical dimorphic system $\{1\}$.

Likewise the two nucleation rate equations can be written:

$$
\begin{gathered}
J_{I}=K_{J, I} \exp \left[-B_{I} /\left(\sigma_{i}-\sigma_{x}\right)^{2}\right], \text { and } \\
J_{I I}=K_{J, I I} \exp \left[-B_{I I} / \sigma_{i}^{2}\right],
\end{gathered}
$$

where $B_{\mathrm{I}}$ and $B_{\mathrm{II}}$ are given by appropriate values of $\frac{16 \pi \gamma^{3} V^{2}}{3 R^{3} T^{3}}$ and both $K_{\mathrm{J}, \mathrm{I}}$ and $K_{\mathrm{J}, \mathrm{II}}$ are functions of the equilibrium concentration $\left(x_{\text {eq }}\right)$ and temperature[1].

Solving the two rate equations simultaneously allows the nucleation behaviour of a system to be explored. It can be shown that for a dimorphic system that there are three possible situations.

If $K_{\mathrm{J}, \mathrm{I}}>K_{\mathrm{J}, \mathrm{II}}$ then above some level of supersaturation phase I nucleates at a faster rate than phase II. However this is reversed below this same level of supersaturation (Figure 1.3[1]).

If $K_{J, I I}>K_{J, I}$ and $(1-a / c)^{3}<b$, then the stable phase II has the greater rate of nucleation at all supersaturations (Figure 1.3[2]). 
If $K_{J, I I}>K_{J, I}$ and $(1-a / c)^{3}>b$, then phase I has the higher nucleation rate but only over intermediate levels of supersaturation (Figure 1.3[3]).

Where: $\quad a=\frac{\sigma_{x}}{B_{I I}^{1 / 2}} \quad b=\left(\frac{B_{I}}{B_{I I}}\right) \quad c=\left[\frac{a}{\ln \left(K_{J, I I} / K_{J, I}\right)}\right]^{1 / 3}$

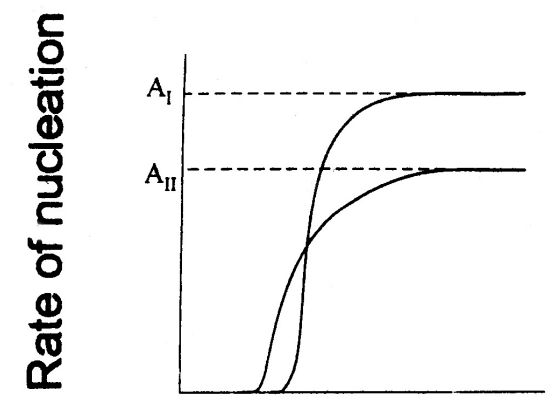

(1)

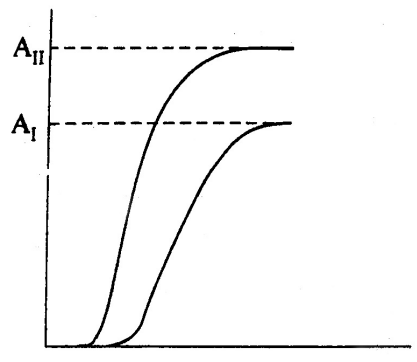

(2)

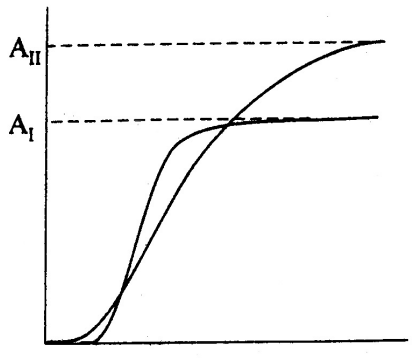

(3)

\section{Supersaturation}

Figure 1.3 Three possible nucleation rate combinations for a hypothetical dimorphic system[1].

Given that Ostwald's law implies that the nucleation rate for phase I is much greater than phase II this analysis shows that this law is only valid in specific situations[1]. The significance of this analysis to the current study lies in the understanding that there is a set of parameters $\left(K_{J}, \sigma, \gamma, v\right.$, and $\left.T\right)$ in the polymorphic calcium carbonate system where Ostwald's law does not apply, such that calcite has the fastest rate of nucleation. Within this range it is envisaged that thermodynamic influences dominate crystal nucleation and growth allowing the factors affecting the organic/inorganic interface to be more easily investigated.

\subsubsection{Heterogeneous Nucleation}

In contrast to homogeneous nucleation described above, heterogeneous nucleation involves the presence of foreign matter or 'catalytic' surfaces that induce nucleation of supersaturated solutions. The presence of an impurity typically results 
in nucleation occurring at lower supersaturation levels than normally required for homogeneous nucleation. This arises due to adsorption of crystallising material onto the impurity, which lowers $\Delta G$ of nucleation. The extent of this reduction depends on the degree to which the structure of the crystallising material is matched by the impurity. The best match and therefore the greatest reduction of $\Delta G$ possible is achieved using seed crystals[1].

\subsubsection{Crystal Growth}

Upon the formation of a stable nucleus crystal growth factors become important. Solution crystal growth is about the capture of solution ions and their integration into the crystal lattice. The ability of a crystal surface to achieve this is dependent, among other things, on the strength and number of interactions that can form between the surface and an incoming ion. This is reflected in the different growth rates observed for different crystal faces due to the inherent anisotropy of most crystal lattice structures[1].

One outcome from crystallisation theory is that the maximum number of bonds a growth unit can form when joining a crystal face is three. These have been defined as kinked ( 3 bonds), stepped ( 2 bonds) and flat (1 bond) sites (Figure 1.4). Based on the assumption that the linear growth rate $\left(v_{i}\right)$ is proportional to the total binding energy, then it is expected that $v_{\mathrm{K}}>v_{\mathrm{S}}>v_{\mathrm{F}}$ for the kinked, stepped and face bonding sites, respectively. Thus the final crystal morphology or habit is determined by the slowest growing flat faces[1].

All the kinked and stepped growth sites would be filled relatively quickly, leaving only flat surface sites. Theoretical considerations suggest that such a single binding interaction is insufficient for growth to continue on a flat surface at the crystallisation rates typically observed. Therefore such faces must have a means of creating kinked or stepped growth sites. The ease with which a surface can form multiple binding sites, and consequently the ease with which the surface can grow, is indicated by the 


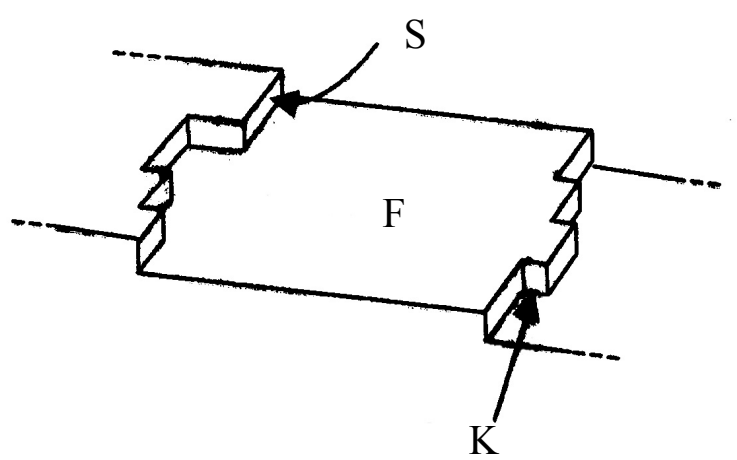

Figure 1.4 A schematic of a crystal surface showing potential growth kink (K), step (S) and face (F) sites[1].

$\alpha$-factor. The $\alpha$-factor is the ratio between the energy change in creating a multiple bonding site from a flat surface, $\Delta E$, and the thermal energy of the system on a per molecule basis:

$$
\alpha=\Delta E / k T .
$$

In terms of estimating $\alpha$ for solution growth Equation 11 becomes:

$$
\alpha=\xi\left[\left(\Delta H_{f} / R T\right)-\ln x_{e q}\right]
$$

where: $\Delta \mathrm{H}_{\mathrm{f}}=$ the heat of fusion,

$x_{\mathrm{eq}}=$ the equilibrium concentration,

$\xi=$ the crystallographic factor $\xi=\frac{E_{s l}}{E_{s s}} \approx \frac{z_{s}}{z_{t}}$,

$E_{s l}=$ the total interaction energy per molecule in the layer of the growth face,

$E_{s s}=$ the total crystallisation (or lattice) energy,

$z_{s}=$ the number of nearest neighbours per molecule in the growth face, and

$z_{t}=$ the total number of nearest neighbours in the crystal lattice.

Typical values of $\alpha$ are from 2 to 20, with different growth mechanisms being associated with different values of $\alpha$. For values of $\alpha<3$ growth is defined as continuous growth, $3<\alpha<5$ surface nucleation and for $\alpha>5$ growth proceeds via spiral growth[1]. These mechanisms will be briefly discussed below. 


\subsubsection{Continuous growth}

In continuous growth systems the energy requirement to form multiple bonded growth sites is so low that almost every growth unit arriving at the surface will find a kink or step site. Therefore the linear growth rate $(v)$ perpendicular to the surface is $v=k_{C G} \sigma$ over the entire supersaturation range[1], i.e. first order growth with a rate constant $k_{C G}$.

\subsubsection{Surface Nucleation}

In situations where the formation of growth sites is more difficult, then $v$ decreases and crystal growth proceeds via a surface nucleation mechanism. In this situation ions or molecules arriving at the surface that fail to find a growth site either return to the fluid or join other adsorbed growth units to form surface islands or nuclei. The perimeter of these islands becomes the source of the new step and kink sites, thus the islands spread laterally (Figure 1.5). Overall growth occurs perpendicular to the plane of the face, as nuclei form on the surface. The linear growth rate now becomes:

$$
v=k_{S N} \sigma^{5 / 6} \exp \left[\left(-\frac{\pi}{3 \sigma}\right)\left(\frac{\gamma_{e}}{k T}\right)^{2}\right],
$$

where $\gamma_{e}$ refers to the edge tension of a critically sized 2-D nuclei and $k_{S N}$ is the rate constant for growth via surface nucleation[1]. 


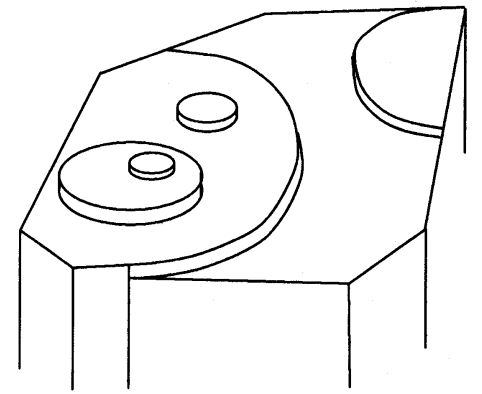

Figure 1.5 A schematic illustrating the generation of nuclei by way of surface nucleation[1].

\subsubsection{Spiral Growth}

Higher values of $\alpha$, indicating that the ability to create new growth sites is very low, are attributed to enhanced intermolecular interactions in the crystal surface resulting in a flat surface. This is seen in high $\gamma_{e}$ values, which begin to inhibit surface nucleation, particularly at low supersaturation. The consequence is that growth can only occur if a step can be created via some energetically cheap process. Built in lattice defects, such as screw dislocations, offer such a possibility. Screw dislocations have the additional advantage of extending over only part of the face, allowing growth to spiral into a hillock (Figure 1.6). For spiral growth, the linear growth rate is somewhat more complicated:

$$
v=k_{S G} \frac{\sigma^{2}}{\sigma_{1}} \tanh \left(\frac{\sigma_{1}}{\sigma}\right)
$$

where $\sigma_{1} \propto \gamma_{e} / s$ and (s) is the strength of the dislocation source, which is based on the number of interacting dislocations that make up the source. A consequence of this mechanism is that each crystal can have its own unique growth rate, as the strength of the dislocation source varies from crystal to crystal[1].

From these brief descriptions of three possible crystal growth mechanisms it is apparent that the introduction of any impurity, such as an organic molecule, would likely affect the crystal growth. It is also clear that the nature of the crystal growth process affects the final appearance of the crystal. This combination goes some way to explaining the several hundred different morphologies observed in the calcium 
carbonate system.

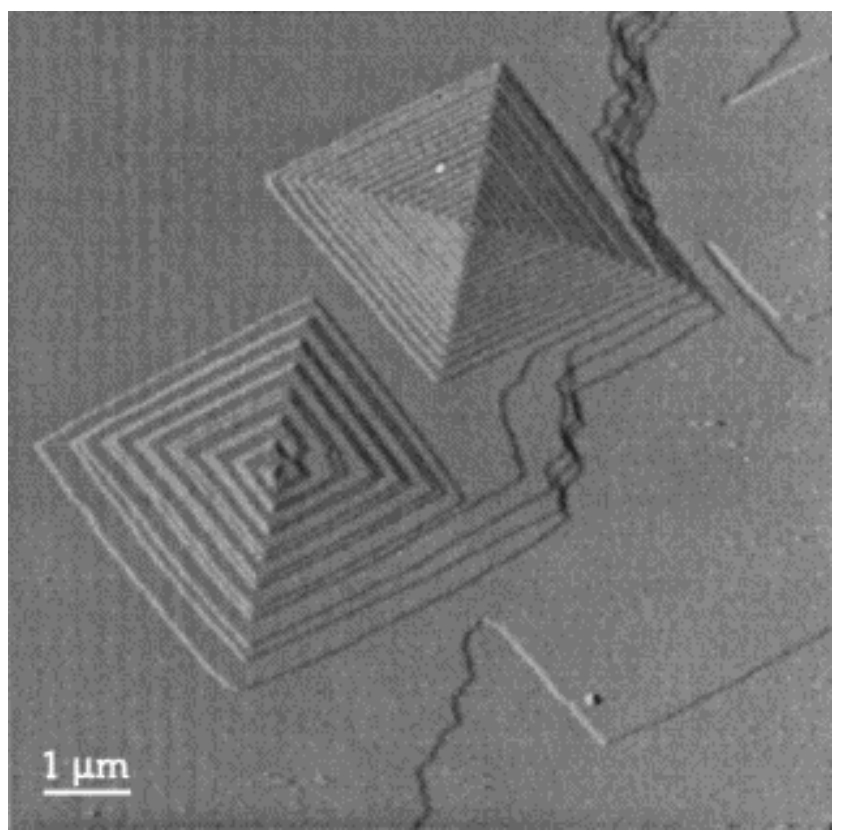

Figure 1.6 An atomic force microscope (AFM) image of two newly formed spiral growth hillocks grown on the calcite (10.4) face. (Taken from Teng et al.[6])

\subsubsection{Crystal Morphology}

The morphology of a crystal is determined by two factors, the symmetry of the internal crystal structure and the relative growth rates of the different crystal faces. The affect of the different growth rates is important, as shown above this means that the morphology can be dramatically influenced by external factors such as supersaturation, temperature, solvent and solution purity.

In the previous section (1.1.4) it was shown that the crystal growth rate is a function of supersaturation, and that in some situations this relationship is nonlinear. This means that the kinetic growth curves for different faces can intersect (Figure 1.7), such that a low supersaturation may give, for example, needle shaped crystals whereas a high supersaturation results in bipyramidal crystals.

With regards to temperature, crystal growth is a thermally activated process and therefore limited by temperature. Consequently, the rate of growth tends to increase 


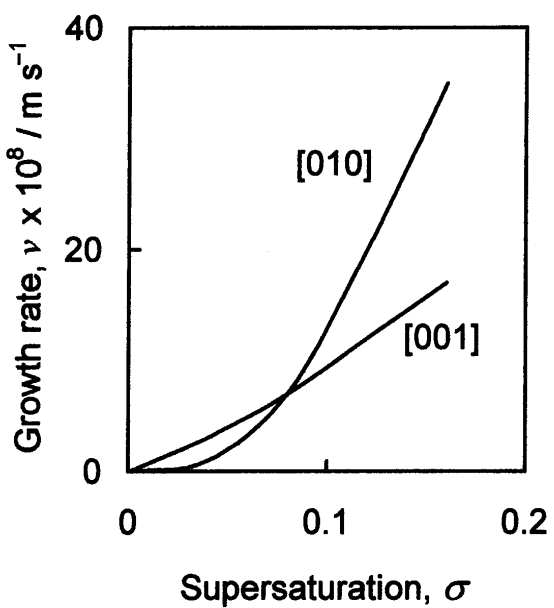

Figure 1.7 An example of the effect of supersaturation on the growth rate of particular paracetamol crystal faces[1].

with temperature, until growth on all faces becomes diffusion controlled, i.e. limited by the rates of diffusion of ions to the crystal surface from solution. Diffusion controlled growth typically leads to the formation of more isotropic crystals, as the rate of growth on all faces is the same[1].

The influence of additives and impurities is related to their ability to bond and consequently block kinked and stepped growth sites. Typically, additives adsorb onto selected faces and therefore modify the crystal morphology by blocking or restricting further grow on those faces. The effect is illustrated in Figure 1.8.

Solvents may act in a similar way to impurities. However, solvents also determine the value of $\alpha$ (see section 1.1.4) for a particular face, which subsequently alters the mechanism of crystal growth on that face. As $\alpha$ is related to solubility, changing the solvent in order to increase the solubility decreases $\alpha$ and can cause the growth rates to increase[1]. 


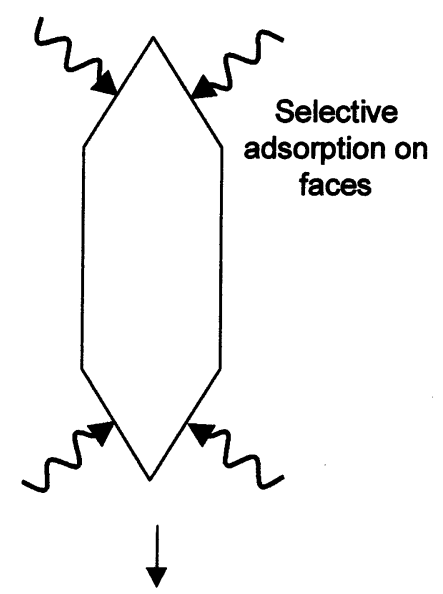

Figure 1.8 A schematic illustrating the effect of selective adsorption of impurities onto particular crystal faces and its subsequent affect on morphology[1].

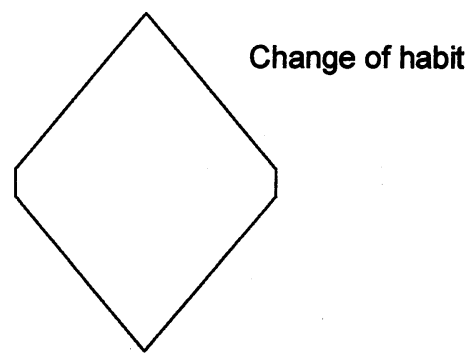

Before we move on to study how nature engineers crystal growth using organic additives, it is important to see what can be achieved by manipulating only $\mathrm{pH}$ and ionic strength. Vayssieres[7] has shown how varying the interfacial energy by altering the solution chemistry affects the properties of the resulting crystal. Through control of $\mathrm{pH}$ and ionic strength alone, Vayssieres and others[7] were able to modify the concentration of $\mathrm{H}^{+}$or $\mathrm{OH}^{-}$ions adsorbed onto metal oxide surfaces and thereby alter the interfacial energy. Control of the interfacial energy enabled the manipulation of the crystal size, shape, orientation and morphology. Working with a number of metal oxide systems Vayssieres has developed and extended this process. However, there are limitations, such as being restricted to single oxide systems. The relevance of Vayssieres' process to this study lies in highlighting the importance of interfacial energy, and in the illustration of how the interfacial energy can be manipulated without the use of organic additives, at least for oxide systems.

This brief foray into crystallisation theory has provided an understanding of the processes involved and how they can be manipulated. Both thermodynamics and 
kinetics are important, and both must be considered when trying to understand the biomineralisation process.

\subsection{Biomineralisation}

Biomineralisation can be described as the process used by organisms to grow minerals. Approximately 64 different minerals are known to be formed by organisms from many different phyla[8]. The way in which nature forms these minerals and the level of structural control achieved has attracted a significant amount of research attempting to understand the mechanism(s) involved, this research effort has been summarised in a number of reviews[9-15].

Probably the best known example of biomineralisation, outside of human bone, is nacre ('mother of pearl'). The crystallisation of $\mathrm{CaCO}_{3}$ in nacre occurs via an extracellular process involving macromolecular scaffolding. It is generally agreed that this scaffolding is pre-organised for regiospecific nucleation and the subsequent development of $\mathrm{CaCO}_{3}$ (aragonite) platelets with controlled micro-architectures[15]. Understanding how nature achieves this level of nano-structural control and incorporates it into the construction of macroscopic structures is the underlying motivation for the vast amount of research directed towards biomineralisation, particularly from a crystal engineering view point. Ultimately, if the lessons learnt from nature could be applied to other inorganic systems it would revolutionise synthetic chemistry and inorganic materials science.

Chemical deconstruction and fragment analysis, along with inference based on mechanical properties has enabled biologists, chemists, materials scientists and geologists to compile considerable information on the vast number of organic/inorganic systems found in nature. Current understanding tells us that almost all biologically controlled mineralisation processes occur in isolated environments. Although there are many different examples of biomineralisation, these can be categorised into the three types of isolated environments: extra-, inter- 
or intracellular. Extracellular mineralisation involves the production of a macromolecular matrix outside the cell, which controls nucleation and growth. Intercellular mineralisation is not widespread but involves mineralisation between groups of neighbouring cells. A small volume is isolated within the epidermal contact points of these neighbouring cells creating an isolated environment. Intracellular mineralisation involves crystallisation inside vesicles or vacuoles formed within the cell[13]. Intracellular mineralisation is a commonly adopted approach, with the formation of magnetite chains by magnetotactic bacteria being a well-known example[16]. However the focus of this study will be on synthetic approaches to understanding extracellular mineralisation.

There are many limitations to understanding the interaction between the cellular organic scaffolds, described above, and the inorganic phase. Such as the complexity of the natural system with interactions occurring on many levels. In addition, it is not always possible to extract and characterise the complete organic scaffold; essential in determining how the structure and properties of these organic molecules interact with a nucleating phase. Although surface science techniques, such as atomic force microscopy (AFM), attenuated total internal reflection infra-red spectroscopy, synchrotron-based analysis, among others, have enhanced our ability to characterise interfaces the complexity of natural systems means that in-vivo studies have proved very difficult. Although understanding the natural system is the objective, due to these limitations, the majority of studies aimed at understanding the biomineralisation process have involved simplified model organic/inorganic systems.

By combining knowledge obtained from natural systems with experimental evidence from simplified model systems Mann[17] developed a generalised scheme to describe the process of biomineralisation, Figure 1.9.

In a later paper Mann[18] went on to state that many of the processes responsible for biomineralisation are generic, of which 'four constructional processes' were 


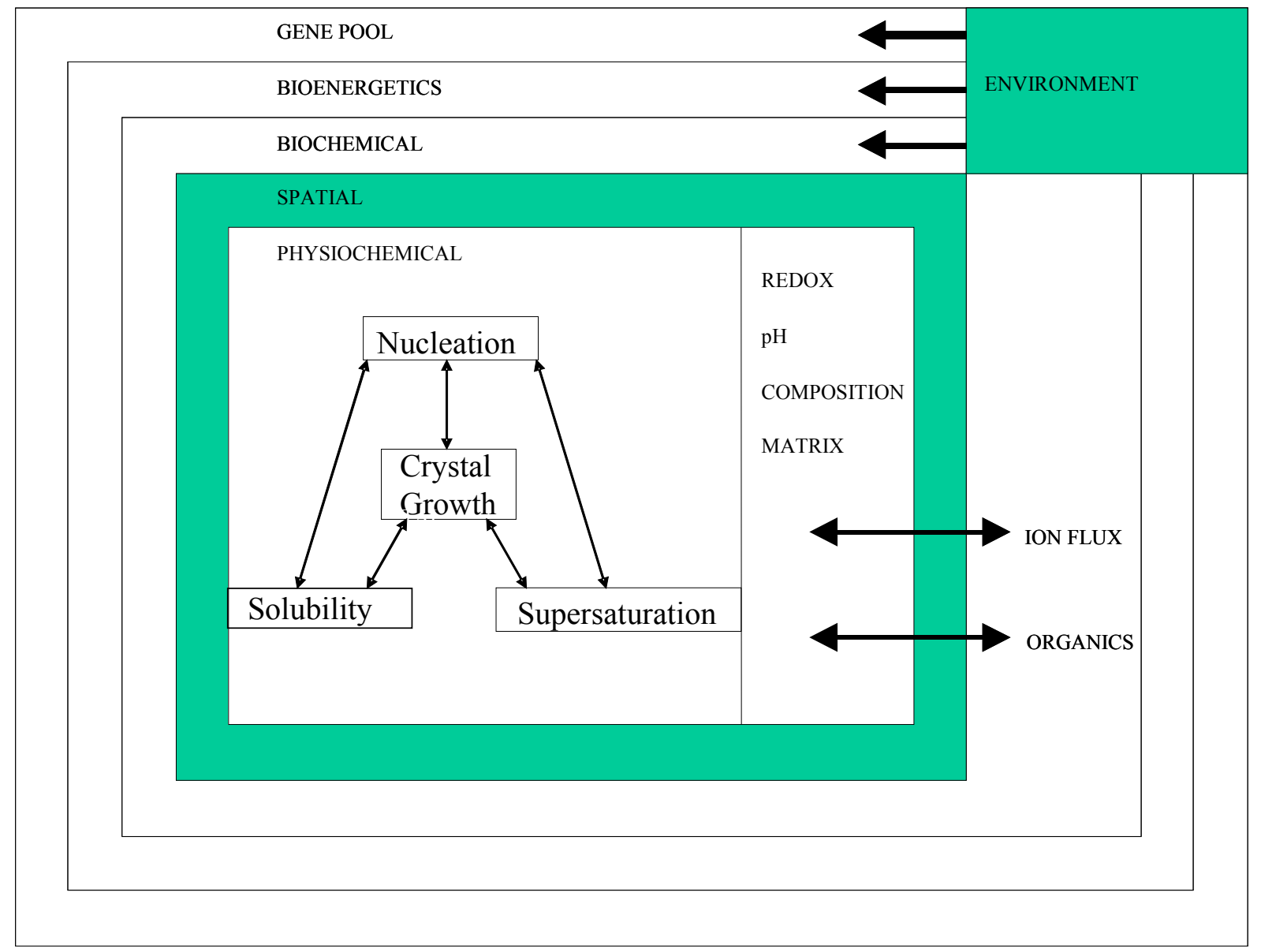

Figure 1.9 A generalised scheme of biomineralisation in organisms. The key aspect is the spatial barrier, which separates the mineralisation process from the external environment. This compartmentalisation allows the regulation of the physicochemical and biochemical properties in such a way to facilitate controlled nucleation and growth of biominerals[17].

proposed: supramolecular pre-organisation, interfacial molecular recognition, vectorial regulation and cellular processing. These processes or stages are expected to act co-operatively with feedback systems.

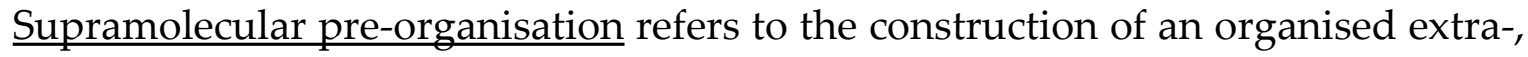
inter- or intracellular organic reaction environment before mineralisation. This typically involves the self assembly of a lipid vesicle but can involve more complex glycoprotein/carbohydrate/lipid macromolecular matrices.

Interfacial molecular recognition involves the controlled nucleation of inorganic nuclei at the organic matrix interface from the supersaturated solution. It is generally 
believed that the pre-organised architectures act as molecular templates for site directed nucleation.

Vectorial regulation is associated with the regulation of crystal growth and termination. This could be achieved through a static process involving the physical shape of the vesicle assembly or the use of specific growth inhibitors.

Cellular processing is associated with construction processes involved in the production of higher-order architectures.

In terms of understanding the interaction between the organic matrix and the nucleating inorganic phase, the interfacial molecular recognition stage is of primary interest. Elaborating on molecular recognition, Mann[19] proposed six modes of complementarity that were important in the interfacial relationship between the acidic functional groups on an organic template and the ionic crystal surface. The proposed modes of complementarity are:

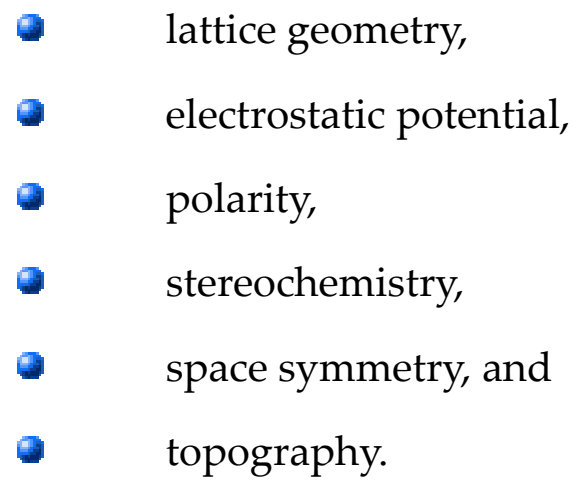

Whether with the aim of testing this model or with a view to developing the field, the last decade or so has seen a flood of studies investigating the organic templation of inorganic crystallisation. Some studies have attempted to model the natural system by using biological organic molecules such as: proteins ( $\beta$-pleated sheet proteins)[19], silicateins (silica precipitating proteins)[20,21], polypeptides[22,23], and sugars[24]. Others have used synthetic organic molecules: self-assembled monolayers (SAMs)[25-27], surfactants[28-30], block copolymers[31-33], macromolecules[34-36], dendrimers[37,38], and mono and di-carboxylic 
acids[39,40] to name a few. The vast majority of these studies have involved an empirical approach that typically involved a 'before and after' type experiment where an initial characterisation of the organic molecule was compared with the final crystal characteristics. Consequently, the nature of the biomineralisation process is inferred from correlations made between the organic molecule and the final crystal. Although far from ideal, much can be learnt from this work, for example, Aizenberg and colleagues[27,41] highlighted the importance of the choice of acidic functional group, its geometry and the effect of the parity of the alkyl chain on the face selective nucleation of $\mathrm{CaCO}_{3}$ grown on SAMs. These effects were attributed to the ability of crystal nucleation and growth to be regulated by the functionality, lattice templation and the stereochemical nature of the organic substrate. Similarly, Hunter[42] concluded that matrix-mediated nucleation is believed to occur by an epitaxial mechanism, where a lattice match between the organic matrix and the nascent crystal lowers the interfacial energy barrier to critical nucleus formation. In both these examples improved matching of the functionality, lattice geometry and stereochemistry across the organic/inorganic interface is aimed at lowering the interfacial energy barrier to nucleation and crystal growth.

The common thread between the above studies is fundamentally the aim of creating an organic 'seed' crystal. However this organic seed, potentially, has greater control than the traditional seed crystal in that it can control orientation, shape and size, enhanced by manipulation of the solution kinetics. Underlying the use of a seed is the knowledge that the presence of a foreign surface can lower the free energy barrier to nucleation and growth by forming bonds with a crystal nucleus. Provided the formation of bonds between the crystal nucleus and the substrate have a lower energy than those between the nucleus and the solvent (water), then crystallisation on the organic substrate will be favoured[3]. The influence of the organic seeding on crystal nucleation and growth is dependent on the structural and chemical match of 
the organic substrate to the nucleating crystal. This has been experimentally shown in a number of studies[15,19] where oriented crystallisation has been observed on charged carboxylate or amine monolayers, whereas neutral monolayers of octadecanol and cholesterol have inhibited crystallisation.

In terms of designing the perfect organic seed, Mann's modes of complementarity provide us with a good starting point. However there remain many questions unanswered such as, which of these six modes is most important or are they equally important? Considerable effort has gone into answering this and many of the other questions, with lattice spacing, stereochemistry, and electrostatic potential being the most widely investigated. Some of these studies have claimed that lattice matching is the primary mode[3,42], where a good atomic match between the substrate and a particular plane of the nucleating phase enhances bonding across the interface thereby reducing the enthalpic contribution to the interfacial free energy, resulting in oriented crystal nucleation. However, De Yoreo and Vekilov[3] suggested that there was currently little understanding of the geometric and stereochemical interactions between the crystal lattice and the organic modifiers. The magnitude of this interaction energy, the affect of the interaction on the interfacial energy landscape, and the impact of the change in the landscape on crystallisation are also required to obtain a complete picture of biological crystal growth. Clearly, there is much more work required before we have an understanding of the biomineralisation process.

To confound our understanding further, this discussion and Mann's model has assumed that the nucleation follows a pathway from solution to a nucleus with the ordered crystal structure of the bulk crystal. The assumption that the structure and surface energy of the embryonic nuclei will be the same as the final crystal is not necessarily the case[3]. This is because the energy barrier leading to an intermediate disordered, less stable state is less than the one leading to the most stable state. This is the basis of Ostwald's law of phases (refer section 1.1.2), which suggests that 
crystallisation will follow the pathway through all the less stable states before reaching the most stable state (Figure 1.10). This is a phenomenon that many of the early studies[17,19,28,29,43-48] did not appear to consider when relating monolayer structure (spacing, head-group chemistry) to the final crystal structure, orientation, and morphology. However more recent studies[49-51] have shown that a multi-step pathway is followed, at least in certain situations. Whether Ostwald's law holds true for all systems remains unknown due to difficulties in studying crystal nucleation. Given the nucleation theory models discussed in section 1.1.2 it is unlikely that it applies in all situations.

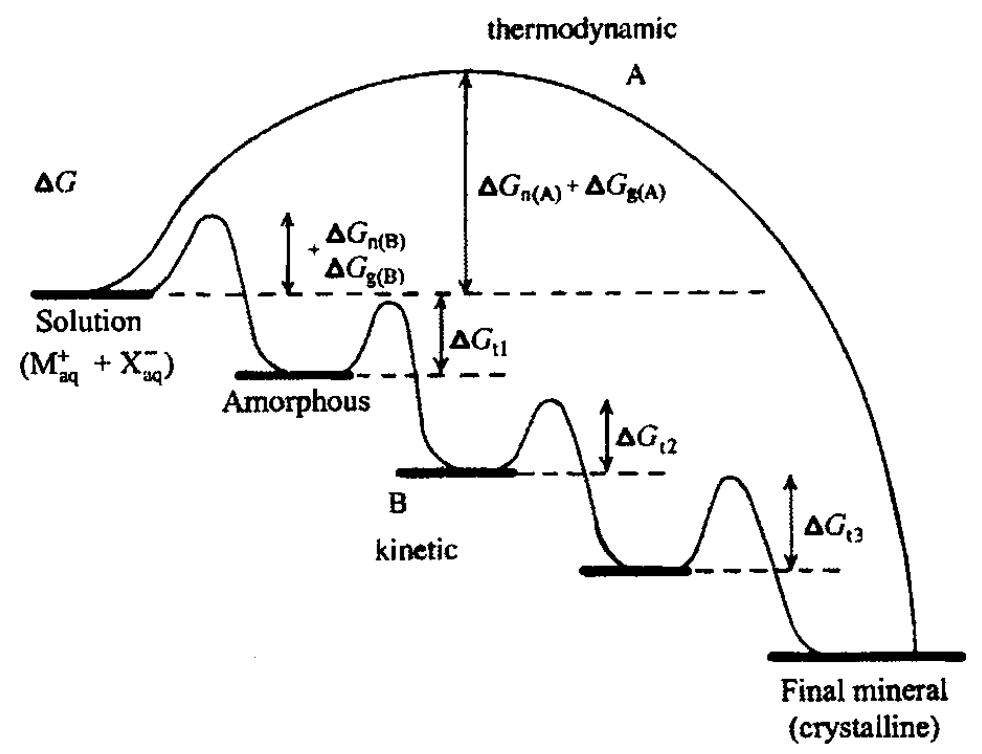

Figure 1.10 Crystallisation pathways via thermodynamic $(A)$ or kinetic $(B)$ controlled routes[52].

As indicated in Figure 1.10 the multi-step pathway is kinetically driven, in contrast to the thermodynamically driven single-step pathway. As with thermodynamics, the kinetics can be manipulated through the use of enclosed environments, such as vesicles, enabling the control of the solution chemistry (i.e. supersaturation, concentrations, $\mathrm{pH}$, etc.). Control of the degree of supersaturation can alter the critical nucleus size and therefore enable manipulation of crystal nucleation and growth. Consequently, biomineralisation in microemulsions has been 
widely investigated. $\mathrm{Li}$ and Mann[53] showed that by changing the $\left[\mathrm{H}_{2} \mathrm{O}\right] /\left[\mathrm{CaCO}_{3}\right]$ ratio in a surfactant-stabilised amorphous $\mathrm{CaCO}_{3}$ nanoparticle system, different vaterite morphologies can be observed (where vaterite is a kinetically stable but thermodynamically metastable phase). In this study alkylbenzene sulfonate stabilised amorphous calcium carbonate (ACC) was added to a sodium bis(2ethylhexyl)sulfosuccinate (NaAOT)/water-in-isooctane micro-emulsion. Stabilisation of ACC has been previously shown to be associated with the presence of polysaccharides and proteins, specifically enriched in glutamic acid, serine, and threonine, and/or inorganic ions such as $\mathrm{Mg}^{2+}$ and phosphate species[53]. Upon shaking, the combination of water, ACC and the two surfactants resulted in the crystallisation of vaterite. The different vaterite morphologies observed were attributed to changes in the $\left[\mathrm{H}_{2} \mathrm{O}\right] /\left[\mathrm{CaCO}_{3}\right]$ ratio. It is likely that these changes altered the degree of supersaturation, which in turn would have altered the surfactant conformation and changed the interfacial free energy of both the surfactant surface and the nucleating crystals.

If nucleation and growth do follow an amorphous to crystalline route then our understanding of how the final crystal structure is influenced by the organic seed is flawed. The experimentally observed preferred orientation could purely be a postnucleation adsorption effect. Further, this adsorption onto selected crystal facets would lead to the formation of different morphologies. Cölfen and Mann[52] have addressed this conundrum by developing an extended model for organic-matrix mediated nucleation, involving three different pathways (Figure 1.11). This highlights the need to have complete control over the solution chemistry in polymorphic systems when investigating thermodynamic drivers. By manipulating the solution chemistry it is possible to create a system where the nucleation rate of the most stable phase is greater than that of the metastable phases (refer section 1.1). Thus crystallisation effectively follows the thermodynamic route, A, in Figure 1.10. 


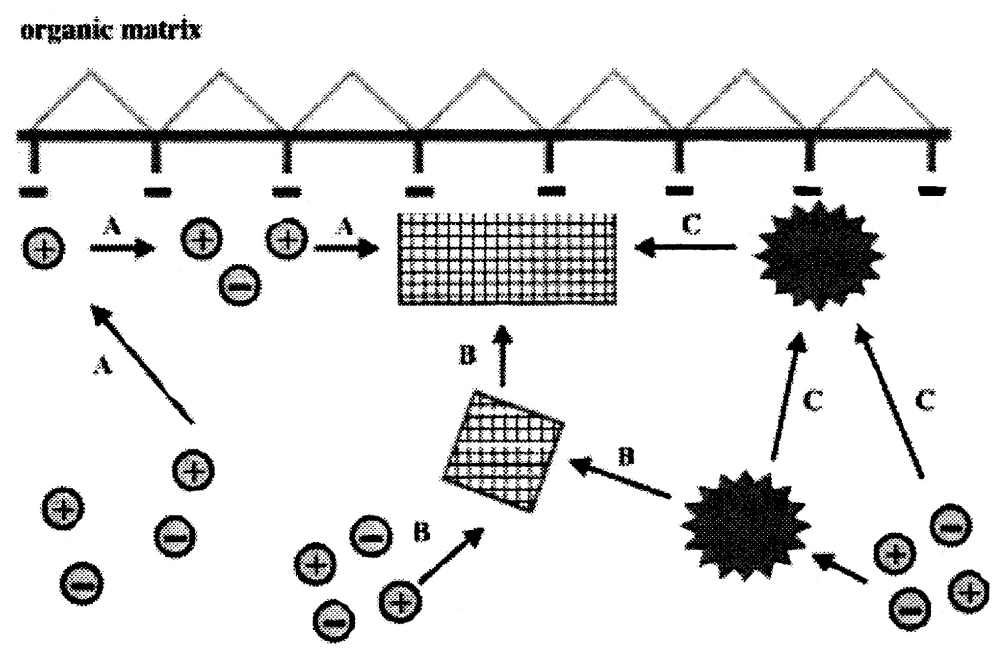

Figure 1.11 An extended three-pathway model used to describe the interaction between an organic matrix and a precipitating inorganic phase that leads to oriented crystallisation. Pathway $A$ is the more conventional view, where aqueous cations bind to the matrix, followed by counter-anions. The achievement of critical cluster size results in oriented nucleation on a specific crystallographic face. Pathway B involves the binding of solution crystallised particles on a specific crystal face. Pathway $C$ involves the formation of an intermediate amorphous phase either in solution or on the matrix interface. This amorphous primary particle then transforms via a matrix-mediated mesophase transition resulting in oriented crystallisation[52].

Once again we come back to the need to understand what is going on at the interface in situ. Unfortunately, the small number of in situ synchrotron investigations that have been performed[54,55] failed to identify the presence of a metastable phase or crystal nucleation at the interface. In addition, the availability of synchrotron-based facilities is limited thus making an indirect empirical approach more feasible. Provided that the system is optimised in favour of the single step thermodynamic route, where the nucleation rate of the stable polymorph (calcite) is greatest then the confounding effects of other crystallisation pathways should be minimised. However there is no denying the potential offered by synchrotron techniques for probing the interfacial processes.

This brief summary of the biomineralisation process, as it is currently understand, used by nature to engineer crystal structures has illustrated the complexity of natural systems. Nature uses many different organic/inorganic systems to control 
crystallisation, therefore it is not surprising that there is variability in the process. Consequently, when studying biomineralisation it is important to realise that the understanding gleaned from one organic/inorganic system may not directly cross over to other systems. The nature of a particular interface is a product of the organic and inorganic materials employed, which must be considered when attempting to understand the mechanisms involved in interfacial molecular recognition. With this in mind this review will now focus on surfactant Langmuir monolayer $/ \mathrm{CaCO}_{3}$ systems.

\subsection{Introduction to Langmuir Monolayers}

The use of Langmuir monolayers as model systems is related to the many examples of biomineralisation that involve cell membranes or intracellular vesicles. As mentioned earlier these cell membranes or vesicles typically consist of phospholipid bilayers with incorporated proteins[64]. Given that the complexity of these macromolecular cell membrane structures make the characterisation of interfacial interactions difficult, simplified surfactant structures such as microemulsions[65], reverse microemulsions[53], liposomes[66], vesicles[19], and Langmuir monolayers[43,67] have been widely studied. Consequently, the simplified planar 2-D approach, and the large body of knowledge and understanding gathered on these systems, makes surfactant monolayers a valuable substitute[15]. In addition, Langmuir monolayers created using a Langmuir-Blodgett (LB) trough enable the solution subphase below the monolayer to be controlled as it would be inside a cell.

To gain a better understanding of the interaction between the monolayer and the nucleation process, it is necessary to appreciate the complexity of the monolayer system in isolation. Extensive literature exists illustrating the extent of the phase space in monolayers and their degree of complexity. For example, the fatty acid/water phase diagram consists of up to eight phases, on varying surface pressure 
and temperature[56]. Changes to the subphase, such as $\mathrm{pH}$ and composition[57,58], alter surfactant interactions and therefore monolayer phase structure opening additional avenues for phase manipulation and determination. Owing to significant interest in Langmuir-Blodgett thin films in the early nineties, there is considerable literature on fatty acid and alcohol systems. More recently, work has focused on the monolayer-water interface[59-61] rather than purely phase structure, tilt and symmetry-based characterization[58,62]. However this effort remains limited largely due to the difficulty in probing the interface and its complexity. Hence explanations are typically limited to hydrogen bonding associated interactions[63].

\subsubsection{Chemical Structure and Varieties}

A surfactant molecule is essentially one that consists of a hydrophobic end (typically a long hydrocarbon chain) and a hydrophilic end (typically an acid and/or amine group). Therefore the number of potential molecules is vast, Figure 1.12 illustrates a few typical examples.

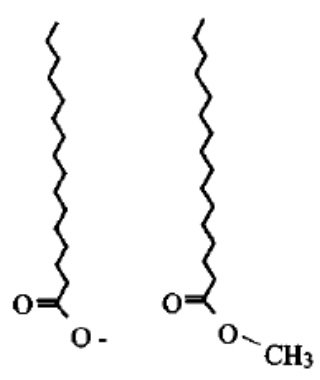

(a)

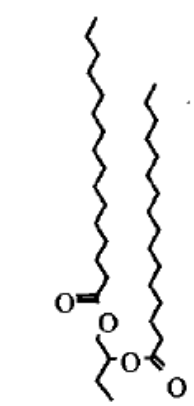

(c)

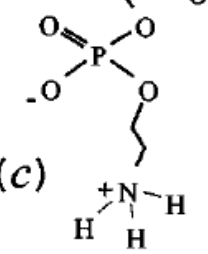

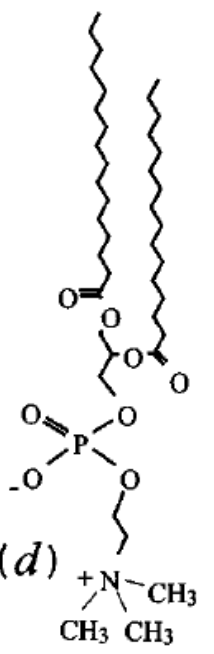

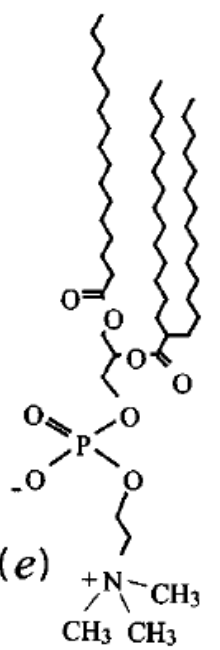

Figure 1.12 Examples of some common surfactants: (a) palmitic acid; (b) methyl palmitate; (c) diacylphosphatidylethanolamine; (d) diacylphosphatidylcholine; and (e) diacylphosphatidylcholine with an aliphatic branch.

Surfactant chemistry is extensive and complex, consequently it will not be 
covered here, for a good overview of the topic the reader is referred to Chapter 4 of 'Introduction to Soft Matter' by I. W. Hamley[64]. However in terms of crystal templation, the surfactant monolayer structure is important and will be described very briefly.

The formation of a monolayer is dependent on the chemical properties of the surfactant molecules such as: their amphiphilic nature, their solubility and concentration. The amphiphilic nature means that it will tend to self assemble such that the hydrophobic tail has little or no interaction with water and the hydrophilic head maximises its contact with water. The longer or bulkier the tail the lower the solubility in water. In addition, the length of the hydrocarbon chain determines the extent of the inter-chain van der Waal interactions, which also affects the surfactant mobility and therefore monolayer structure. Given that these two ends are joined, there are limited conformations that accommodate both the hydrophobic and hydrophilic tendencies. At medium to high concentrations (above the critical micelle concentration) the surfactant molecules tend to self assemble forming micelles, bilayers, vesicles, along with surface monolayers. However, at low concentrations there are insufficient molecules to form such elaborate structures therefore the molecules tend to adsorb to the surfaces of the container and at other interfaces, such as the air/water interface, forming monolayers. This is a very simplified description of the formation of micelles, for a more detailed account see Hamley[64]. In terms of surfactant systems this proposal will focus on monolayers formed at an air/water interface, at low surfactant concentrations.

In the absence of external pressure and at low concentrations the surfactant molecules in a monolayer will orient somewhat randomly with very little phase structure. The acid and/or amine head group will typically ionise in water within a particular $\mathrm{pH}$ range, which is an important characteristic in terms of ion binding and crystal nucleation. The presence of any packing arrangement will be due to the 
chemical structure of both the hydrocarbon chain and the head group, via steric hindrance and like-charge repulsion, respectively. Oppositely charged ions in the subphase will be attracted by the charged head groups and will go some way to neutralising the affects of like-charge repulsion. For anionic surfactants, this effect is likely to be greater for smaller cations, which can enter the interfacial layer more easily[68].

In terms of the modes of complementarity, other important properties include the geometrical arrangement of the head group, the polarity of the surfactant molecule, the valence of the head group, the presence of zwitterionic charges (i.e. contains both positively and negatively charged groups, see Figure $1.12 \mathrm{c}, \mathrm{d}$ and e), the charge density, and the packing symmetry of the molecules. Clearly, there is a great deal of versatility in the surfactant monolayer chemistry; variability that is increased through the different packing arrangements that occur on the application of an external pressure.

\subsubsection{Compression and Phases}

The application of surface pressure, by compressing the monolayer between barriers, results in an ordering of the monolayer (Figure 1.13). This ordering is the result of a rearrangement of the molecular packing in order to balance the applied pressure with the steric and electrostatic repulsion between neighbouring molecules.

For a given temperature, increasing the surface pressure can result in a phase transition (Figure 1.14a) from a liquid-like state $\left(L_{2}\right)$, where there is significant tilting in domains, to a liquid-condensed state $\left(L_{2}{ }^{\prime}\right)$ associated with close-packed head groups and then toward a condensed phase ( $S$ or $C S$ ) where there is little or no tilting and the chains are close-packed. The nature of the phase transition can take many forms depending on the chemistry of the surfactant, the temperature, the rate of application of surface pressure, and the solubility of the surfactant, among other properties. Surface pressure-area isotherms or compression isotherms are used to 
study the structure of the monolayer (Figure 1.14b). Information, such as the phase (gas, expanded or liquid, and condensed phase) and the average area per molecule (packing density) of the monolayer can be determined from the compression isotherm.

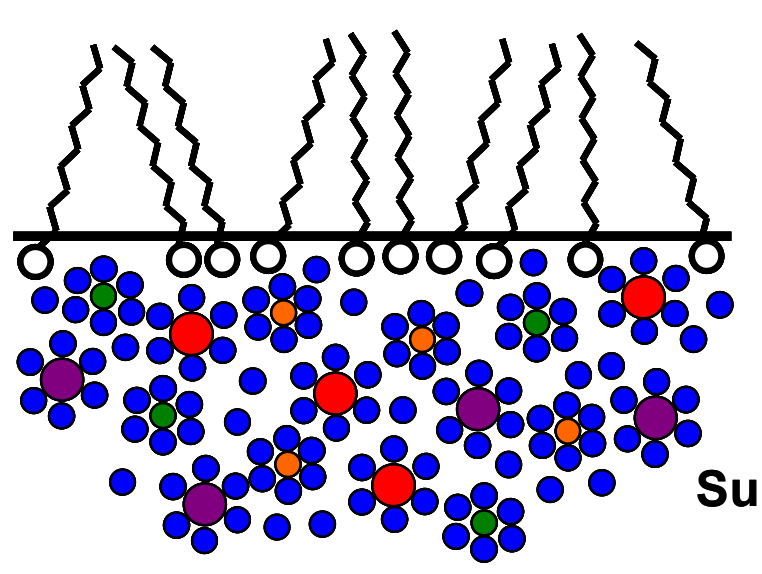

\section{Air}

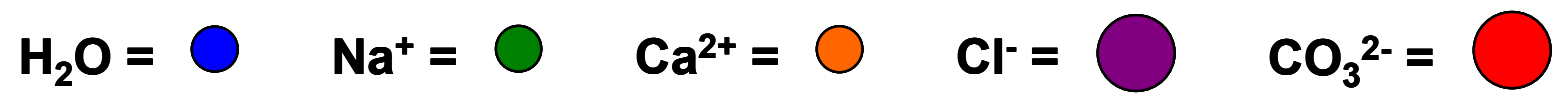

Figure 1.13 A schematic illustrating the monolayer ordering that occurs on the application of surface pressure.
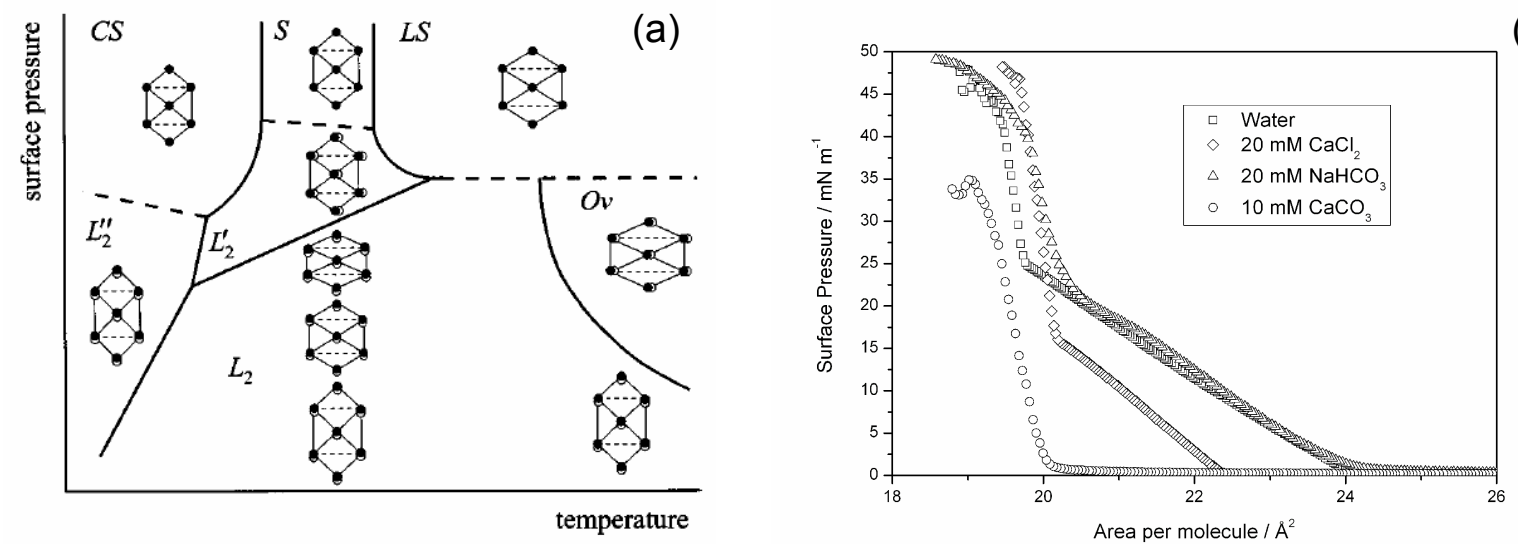

Figure 1.14 (a) A generic monolayer phase diagram[56]. (b) A pressure isotherm for a stearic acid monolayer on four different subphases. As the pressure is increased the monolayer passes through phase transitions, which can be plotted against temperature.

The packing symmetry of the surfactant molecules in a monolayer depends on the head-group size, the presence of polar groups, the number and conformation of the 
hydrocarbon chains, the chemical nature of the underlying solution, and the degree of compression being applied[64]. Due to the nature of the surfactants used in biomineralisation studies, hexagonal arrays are common[29]. However, rectangular arrays have also been observed[69].

The final structure of the monolayer and the freedom with which each molecule has to move (and thus interact with subphase cations) is determined by the nature of the phase. Consequently, knowing which monolayer phase is present, and its symmetry, is critical in understanding the organic/inorganic interface. For example the packing arrangement, and hence density, is important in terms of calculating the molecular spacing, which can then be compared to crystal lattice spacing. Likewise, the degree of monolayer compression has been shown to influence the homogeneity of crystal nucleation, with partially compressed films being optimal for controlled crystallisation[45]. The nature and mechanism of this effect was not explained but it is likely to be associated with obtaining the best lattice match between the monolayer and the nucleating crystal, thus lowering the interfacial energy of the system.

More recently X-ray techniques have been employed to provide detailed structural information on the monolayer[12-14]. To some degree this approach has supplanted surface pressure isotherm investigations as significantly more information can be obtained. A brief summary of the typical techniques and the underlying theory follows.

\subsubsection{X-ray Techniques}

X-ray methods offer the ability to non-destructively probe the monolayer structure directly at a molecular level. The monomolecular nature of the film requires the high energy flux of a synchrotron source to perform these experiments. Further, in order to avoid the significant water subphase scattering, grazing incidence X-ray diffraction (GIXD) is typically employed. Complementing the structural information obtained from GIXD is specular reflectivity (XRR). XRR 
provides information regarding the electron density perpendicular to the interface. The surface sensitive techniques of GIXD and XRR have been the subject of many reviews[12-14], therefore the following discussion is a summary of the main points.

\subsubsection{Grazing Incidence X-ray Diffraction (GIXD)}

X-ray diffraction intensity is a function of the number of scattering entities within the beam. With a wavelength of $\sim 1 \AA$, the penetration depth of an X-ray beam can be between a few microns and a few millimetres depending on the absorbing properties of the subject material. In contrast a monolayer film has a thickness of tens of Ångströms, hence to avoid saturation by the subphase scattering, and yet attain sufficient intensity, synchrotron-based GIXD is necessary. By having an incident angle $\left(\alpha_{i}\right)$ less than or equal to a critical angle $\left(\alpha_{c}\right)$, total external reflection of the X-ray radiation is achieved. The total reflection of the incident beam means that the refracted wave becomes evanescent and travels along the surface. As the amplitude of the evanescent wave decays exponentially with depth, attaining a penetration depth of $50-100 \AA$ for $\alpha_{i}<0.5 \alpha_{c}$, the crystallographic information of surface phenomena can be obtained without being dominated by subphase scattering. Combined with the larger beam 'spot', associated with small incidence angles, and the high flux of synchrotron radiation the diffracted evanescent wave is capable of providing information about the first order diffraction peaks, at least, for monomolecular films.

The critical angle $\left(\alpha_{c}\right)$ is defined by $\alpha_{c}=\cos ^{-1}(n)=(2 \delta)^{0.5} \cdot n$ is the refractive index of matter for X-rays in the $1 \AA$ wavelength range and is given by:

$$
n=1-\delta-i \beta
$$

with $\delta=2 \pi \rho r_{0} / k^{2}$, where $k=2 \pi / \lambda$ is the X-ray wave number, $\lambda$ is the wavelength, $\rho$ is the electron density and $r_{o}$ is the classical electron radius $\left(r_{o}=2.82 \times 10^{-13} \mathrm{~cm}\right) . \quad \delta$ is typically of the order of $10^{-5}$, and $\beta=-\mu / 2 k$, where $\mu$ is the linear adsorption 
coefficient. For $\lambda \approx 1 \AA$, absorption is small and $\beta<\delta$.

In 3-D crystals diffraction from a set of planes with a specific interplanar (d) spacing occurs only when the Bragg Law is satisfied. That is when (1) the scattering length vector $|Q|$ (given by $\left|k_{\mathrm{f}}-k_{\mathrm{i}}\right|=4 \pi \sin \theta / \lambda$ ) is equal to $2 \pi d_{\text {r }}$, where $d_{r}$ is the reciprocal of the interplanar spacing; and (2) the normal to the crystal plane intersects the angle between the incident and outgoing beams (Figure 1.15). For 2-D crystals, diffraction only takes place when the horizontal component of $Q$, denoted $Q_{x y}$ coincides with a vector $2 \pi\left(h \boldsymbol{a}_{r}+k \boldsymbol{b}_{r}\right)$, where $\boldsymbol{a}_{r}$ and $\boldsymbol{b}_{r}$ are the reciprocal space vectors of the unit cell parameters $\boldsymbol{a}, \boldsymbol{b}$, whereas $h$, and $k$ represent the Miller indices of the planes with spacings $d_{h k}$. In 2-D there are no restrictions on the scattering vector component normal to the film, thus Bragg scattering is manifested as rods. The finite thickness of the monolayer means that the rods are also of finite length, a length that is proportional to the thickness of the monolayer.

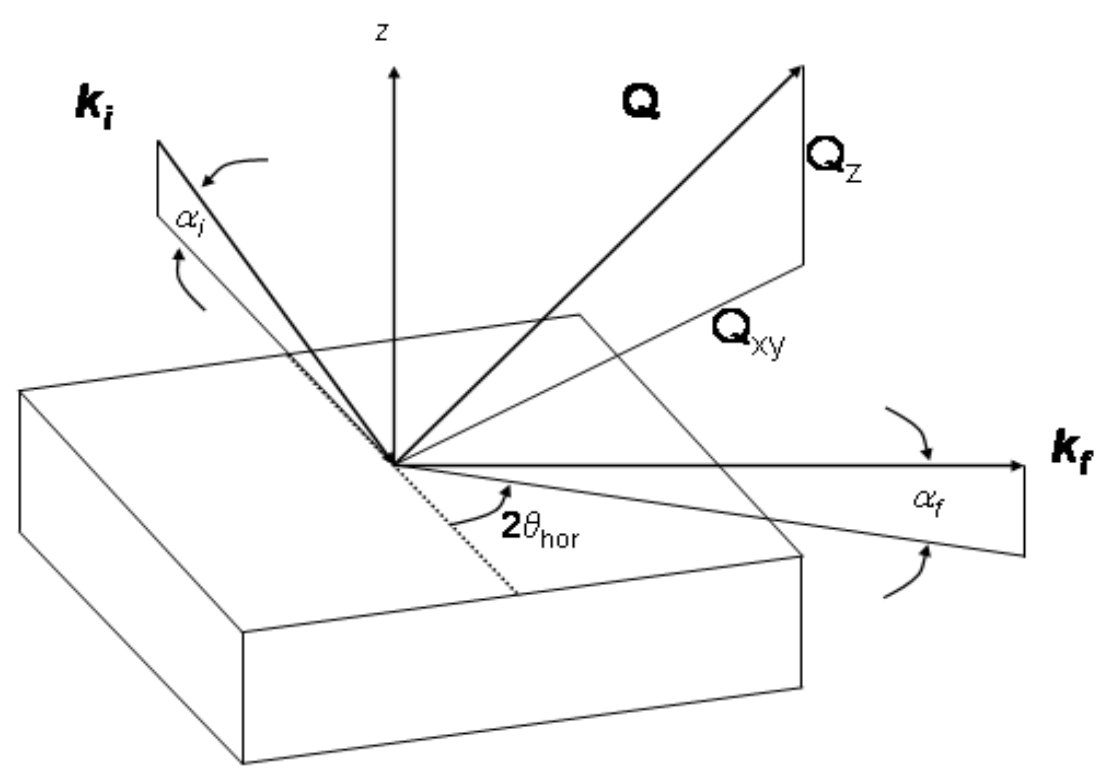

Figure 1.15 A schematic of the GIXD and specular reflectivity geometry from a horizontal surface. $K_{i}$ and $K_{f}$ are the incident and reflected wave vectors, with angles $\alpha_{i}$ and $\alpha_{f}$ between the beam and the surface, respectively. $Q$ is the diffracted wave vector with components $Q_{z}$ and $Q_{x y}$. 


\subsubsection{X-ray Reflectometry (XRR)}

Specular X-ray reflectivity enables information about the electron density variation in a monolayer to be probed in the direction normal to the interface. The specular nature of the technique refers to the measurement of the reflected ray in plane with the incident wave vector $k_{i}$ and the vector normal to the surface at an angle equal to the incident angle $\left(\alpha_{i}\right)$, Figure 1.15. For an ideal surface the specular reflectivity is given by the Fresnel law of optics, which within the limit of small incidence angle is:

$$
R_{F}\left(q_{z}\right)=\left[\frac{\left\{q_{z}-\left(q_{z}^{2}-q_{c}^{2}\right)^{1 / 2}\right\}}{\left\{q_{z}+\left(q_{z}^{2}-q_{c}^{2}\right)\right\}^{1 / 2}}\right]^{2}
$$

where $q_{z}=\left(\frac{4 \pi}{\lambda}\right) \sin \alpha_{i}$ and, $q_{c}=\left(\frac{4 \pi}{\lambda}\right) \sin \alpha_{c}$ is the critical value of $q_{z}$ for total external reflection[13]. When $q_{z}<q_{c}$ then $R_{F}=1$, however as $q_{z}$ exceeds $q_{c}, R_{F}$ decreases significantly placing importance on the high flux capacity of synchrotron sources. The validity of Equation 16 lies in the assumption of ideally flat surfaces where the electron density varies in a step-like fashion between two constant values. Hence when $\rho(z)$ varies continuously the reflectivity is changed to:

$$
R\left(q_{z}\right)=R_{F}\left(q_{z}\right)\left|\phi\left(q_{z}\right)\right|^{2}
$$

where

$$
\phi\left(q_{z}\right)=\left(\frac{1}{\rho_{\infty}}\right) \int\left[\frac{d \rho(z)}{d z}\right] \exp \left(i q_{z} z\right) d z
$$

and $\rho_{\infty}$ is the bulk subphase electron density. Unfortunately Equation 17 is very complex and only its modulus (not the phase) can be solved from the measured reflectivity. Consequently, the specular reflectivity data is analysed by fitting a parametrised model density profile to the measured data using the above equations.

\subsubsection{Effects of Environment}

A Langmuir monolayer is a dynamic structure with individual surfactant molecules diffusing in and out of the monolayer, at some temperature dependant 
rate. The structure is also known to change in response to $\mathrm{pH}$ and the presence of subphase counter-ions, consequently the actual structure is very variable and incredibly susceptible to changes in environment. What must also be realised is that a monolayer is not a uniform arrangement of surfactant molecules and that the phase structures discussed above, are in fact averages. Brewster Angle Microscopy (BAM) provides a more accurate picture of the phase structure within a monolayer. Figure 1.16 illustrates the formation of domains of different phases (indicated by light and dark regions). Depending on the mobility and solubility of the surfactant molecules a homogeneous monolayer may form, given sufficient time to reach equilibrium.

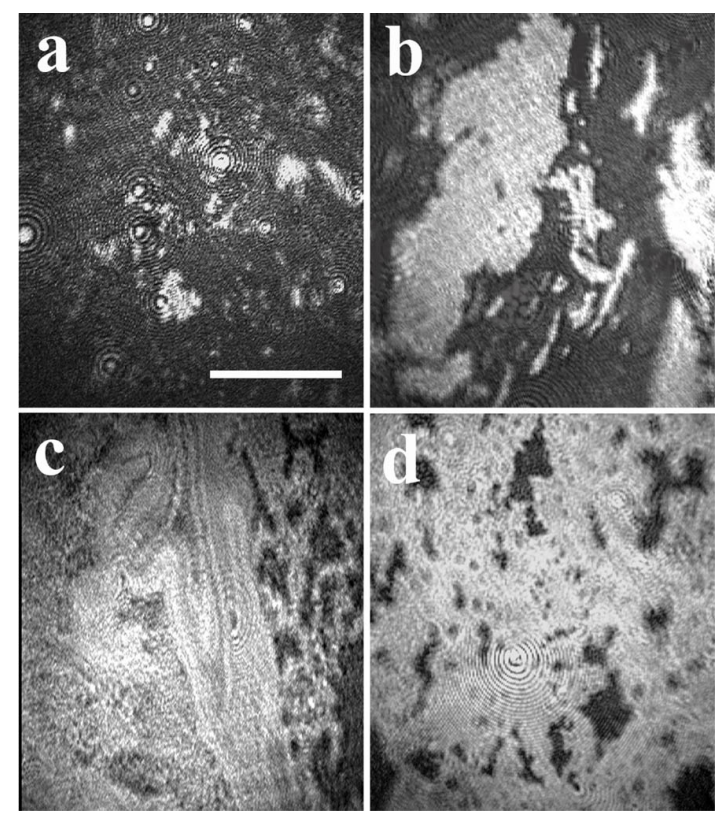

Figure 1.16 BAM images of uncompressed monolayers $\left(\Pi=0 \mathrm{mN} \mathrm{m}{ }^{-1}\right)$ of (a) palmitic acid; (b) lignoceric acid; and compressed monolayers of (c) lignoceric acid at $\Pi=29 \mathrm{mN} \mathrm{m}^{-1}$ and (d) triacontanoic acid at a pressure of $27 \mathrm{mN} \mathrm{m}^{-1}$. Scale bar $=0.5 \mathrm{~mm}[70]$.

The influence of $\mathrm{pH}$ is associated with the ionisation of the head group. Depending on the chemical nature of the surfactant, changes in the subphase $\mathrm{pH}$ will result in different degrees of ionisation. As mentioned earlier, ionisation results in like-charge repulsion, thus affecting the packing. The presence of subphase counterions (such as $\mathrm{Ca}^{2+}$ ) is known to initiate solid-like monolayer structuring. If the surface pressure is low then the presence of counter-ions will result in domains of 
solid-like phase behaviour separated by regions of gas-like phase behaviour, i.e. a heterogeneous monolayer. Impurities are also known to alter the structure of the monolayer, with the magnitude of the effect very much dependant on the nature of the impurity. The ionic strength of the subphase will also indirectly influence monolayer structure.

Having briefly described the Langmuir monolayer system, it is necessary to understand calcium carbonate chemistry before confronting the nature of the organic/inorganic interface.

\subsection{Calcium Carbonate}

The choice of calcium carbonate from the many inorganic materials that have been investigated, for example hydroxyapatite[71], calcium phosphate[50] and barium sulfate[43], is based on 1) it being a common biomineral, 2) the large body of knowledge available regarding its crystallisation and 3) the versatility offered by the $\mathrm{CaCO}_{3}$ polymorphs.

There are known to be eight polymorphs of calcium carbonate, three common anhydrous crystalline forms: calcite, aragonite and vaterite, two hydrated crystalline forms: calcium carbonate hexahydrate $\left(\mathrm{CaCO}_{3} \cdot 6 \mathrm{H}_{2} \mathrm{O}\right)$ and calcium carbonate monohydrate $\left(\mathrm{CaCO}_{3} \cdot \mathrm{H}_{2} \mathrm{O}\right)$, two high temperature forms: calcite II and calcite III, and an amorphous phase. Calcite, aragonite and amorphous $\mathrm{CaCO}_{3}$ are common in biological systems. Vaterite is a metastable phase and is rarely formed in nature. Calcite is the most abundant polymorph of calcium carbonate, a consequence of being the most thermodynamically stable phase.

Table 1 lists the crystal structure details for calcite, aragonite and vaterite. In all three structures the $\mathrm{Ca}^{2+}$ and $\mathrm{CO}_{3}{ }^{2-}$ ions are arranged in alternate layers perpendicular to the $c$ axis. However in calcite and aragonite the planar $\mathrm{CO}_{3}{ }^{2-}$ ions are oriented perpendicular to the $c$ axis, which can be seen in Figure 1.17. In vaterite the $\mathrm{CO}_{3}{ }^{2-}$ ions are oriented parallel to the $c$ axis[10]. Interestingly, the orientation of 
the carbonate ions in the plane parallel to the $c$ axis is believed to be randomly distributed among three or more different positions[72]. This random rotation around the carbon atom makes structure determination difficult.

The similarities between calcite and aragonite do not end with the orientation of the carbonates, the inter-ion distances and angles within the basal faces are also very similar. However the important difference between calcite and aragonite lies in the layering of the carbonate anions. In calcite, the $\mathrm{CO}_{3}{ }^{2-}$ ions lie in a single plane midway between each pair of $\mathrm{Ca}^{2+}$ planes. In contrast, the $\mathrm{CO}_{3}{ }^{2-}$ ions are staggered in two layers between each $\mathrm{Ca}^{2+}$ layer with alternate $\mathrm{CO}_{3}{ }^{2-}$ groups rotated $\pm 30^{\circ}$ in aragonite[73]. 
Table 1 Crystallographic characteristics of the three crystalline phases of calcium carbonate found in nature.

\begin{tabular}{|c|c|c|c|}
\hline & Calcite $^{\#}$ & Aragonite & Vaterite \\
\hline JCPDS File & $47-1743$ & $41-1475$ & $33-0268$ \\
\hline$a(\AA)$ & 4.9896 & 4.9623 & 7.1473 \\
\hline$b(\AA)$ & - & 7.968 & - \\
\hline$c(\AA)$ & 17.061 & 5.7439 & 16.917 \\
\hline Volume $[C D]\left(\AA^{3}\right)$ & 367.85 & 227.11 & 748.41 \\
\hline Z & 6 & 4 & 12 \\
\hline Symmetry & $\begin{array}{c}\text { Rhombohedral } \\
\text { (pseudo-hexagonal } \\
\text { cell) }\end{array}$ & Orthorhombic & Hexagonal \\
\hline Space Group & $\mathrm{R} \overline{3} \mathrm{c}(167)$ & Pmcn (62) & $\mathrm{P}_{63} / \mathrm{mmc}(194)$ \\
\hline Ca Coordination & 6 & 9 & $8^{*}$ \\
\hline Density $\left(\mathrm{g} / \mathrm{cm}^{3}\right)$ & 2.711 & 2.927 & 2.665 \\
\hline Solubility $\left(@ 25^{\circ} \mathrm{C}\right)[5]$ & $3.31 \times 10^{-9}$ & $4.57 \times 10^{-9}$ & $1.23 \times 10^{-9}$ \\
\hline Cleavage Planes & $\{10.1\}$ perfect & $\begin{array}{c}\{010\} \text { imperfect }, \\
\{110\} \text { poor }\end{array}$ & \\
\hline
\end{tabular}

\# The details for calcite refer to the hexagonal pseudo-cell structure, which is more commonly used, however it actually has a rhombohedral structure as indicated.

* In vaterite each calcium atom is coordinated to six carbonate oxygen atoms at a distance of $\approx 2.4 \AA$ but there are a further two oxygen atoms at $\approx 2.9 \AA$ distance, sufficiently close to be considered to be bound to the calcium atom[72].

In terms of providing a comparison for the influence of the Langmuir monolayer on crystallisation, in the absence of any templation the most commonly observed nucleation face of calcite is the (10.4) plane. This is attributed to the electrostatic potential of this face, with both $\mathrm{Ca}^{2+}$ and $\mathrm{CO}_{3}{ }^{2-}$ ions present the charge on this particular face is neutral[74]. As for aragonite, crystallised in the presence of $\mathrm{Mg}^{2+}$, the $\{110\}$ face is most likely to be observed in the absence of any templating[73].

\subsection{Monolayer/Crystal Interactions}

This section will examine the combined current knowledge and understanding of 

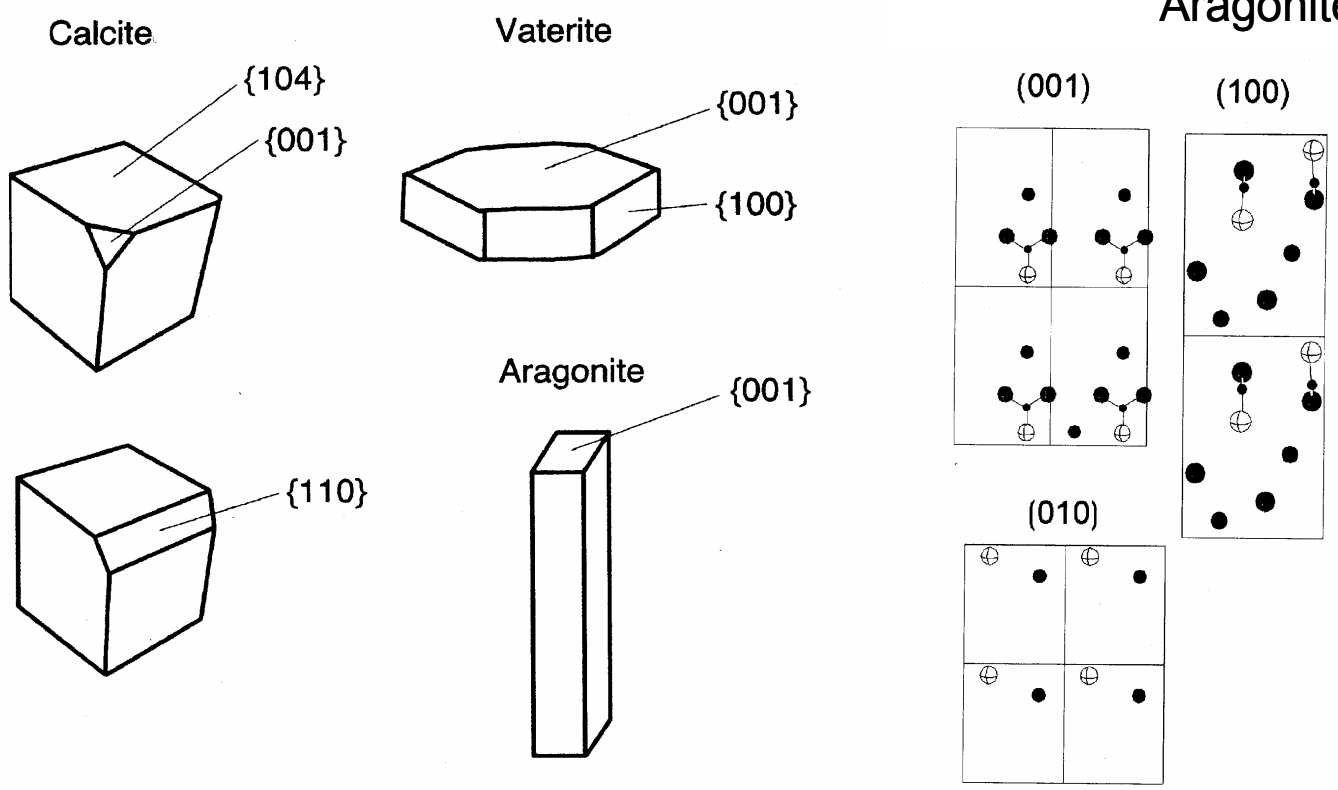

(001)
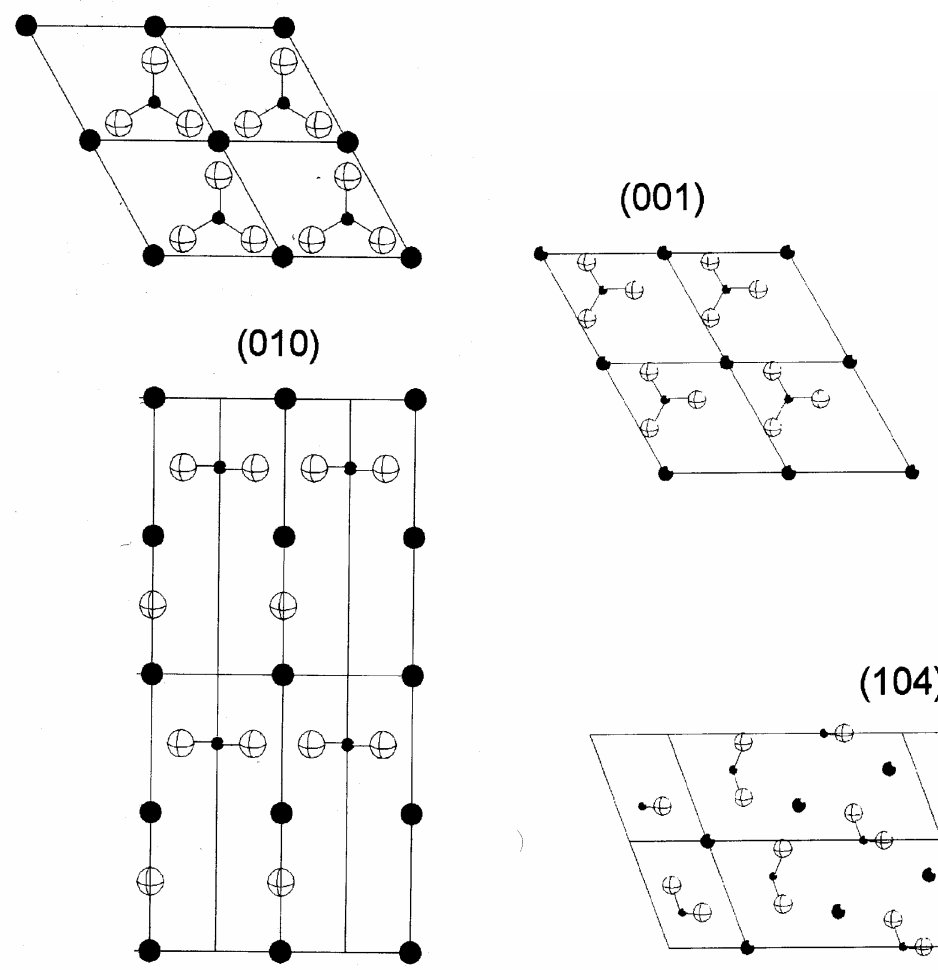

(110)

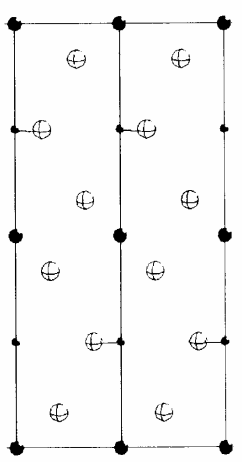

(104)

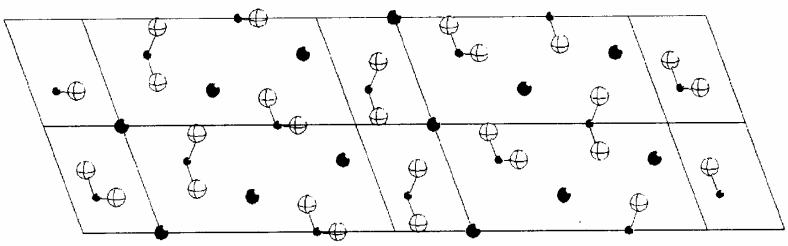

Vaterite

\section{Calcite}

Figure 1.17 The typical crystal habits of calcium carbonate. The important faces are labelled and the crystal lattice arrangement is shown[75]. In the calcite (001) schematic the carbonate groups are actually displaced into the page relative to the calcium ions. All the atoms in the calcite (110) and (104) planes are in the plane of the page. In the aragonite schematic the two different oxygen atoms are represented as filled and unfilled spheres. 
biomineralisation, Mann's six modes of complementarity, Langmuir monolayers and calcium carbonate with the aim of understanding what is important, and why, within the context of crystal engineering. The following discussion will consider an example system consisting of a stearic acid monolayer spread over a supersaturated aqueous $\mathrm{CaCO}_{3}$ subphase with $\mathrm{Na}^{+}, \mathrm{Cl}^{-}, \mathrm{OH}^{-}$ions added for $\mathrm{pH}$ and ionic strength adjustment.

\subsubsection{Stearic acid/Ca ${ }^{2+}$ Bonding}

For bonding between subphase ions and monolayer head groups to occur, previous studies have shown that the head group has to be ionised. This is inferred by the lack of interaction between the cations and the monolayer at low $\mathrm{pH}$, when there is little or no dissociation[76]. However, as the $\mathrm{pH}$ is increased the surfactant becomes increasingly deprotonated and converted to a salt. The intrinsic $\mathrm{pKa}$ indicates the point of $50 \%$ dissociation and for a typical long chain fatty acid this is in the $\mathrm{pH}$ range of 5-6. However the particular $\mathrm{pH}$ range that the acid to salt conversion occurs over is specific to the individual cation species present. This dependence is generally attributed to the competition for the carboxylate group between the metal ion and the proton[76].

In terms of the extent of the reaction, divalent cations are generally found to be the most effective and the salts formed are traditionally known as "soaps"[76]. In a previous Langmuir-Blodgett study, Sobotka[77] determined that the percentage of soap, for a stearic acid/ $\mathrm{Ca}^{2+}$ system, increased from $30 \%$ at $\mathrm{pH}=6$ to $100 \%$ at $\mathrm{pH}=8$. In comparison, in a similar study, Kobayashi et al.[78] found the percentage of soap for an arachidic acid/ $\mathrm{Ca}^{2+}$ system to vary from $0 \%$ to $100 \%$ over the $\mathrm{pH}$ range of 4.6 to 7.5 .

The studies above have focussed on electrostatic bonding between the $\mathrm{Ca}^{2+}$ ion and the charged head group yet there are four main types of metal-carboxylate interactions that could apply to the bonding between stearic acid and $\mathrm{Ca}^{2+}$ ions 
(Figure 1.18). As described above, the carboxylate group can act as an uncoordinated anion (Figure 1.18a), alternatively it could form some type of metal-ligand coordination structures, such as: a monodentate ligand (Figure 1.18b), a bidentate chelate (Figure 1.18c) or a bridging bidentate (Figure 1.18d)[79]. Gericke and Hühnerfuss[79] found, using infrared reflection-absorption spectrometry, that for calcium octadecanoate the IR bands corresponded primarily to an ionic carboxylatemetal interaction. However, there was a weak band corresponding to a small amount of covalent bonding. This mixed bonding behaviour has been seen in other calcium carboxylates, e.g. calcium acetate[79]. A comparative study investigating the binding of alkaline earth ions $\left(\mathrm{Mg}^{2+}, \mathrm{Ca}^{2+}\right.$ and $\left.\mathrm{Ba}^{2+}\right)$ and transition metal ions $\left(\mathrm{Co}^{2+}, \mathrm{Cd}_{2}{ }^{+}\right.$and $\mathrm{Pb}^{2+}$ ) to fatty acid LB films, found that the latter group has a tendency to form more covalent bonds. The presence of $d$ and $f$ orbitals means that the transition metal ions tend to form coordination complexes with substantial covalent character. Surface potential measurements also showed alkaline earth metal ions interact electrostatically[58].

(a)

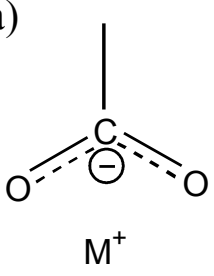

(b)

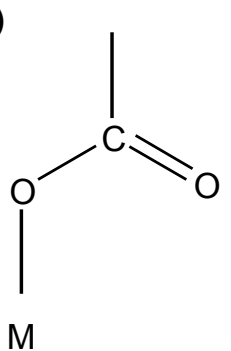

(c)

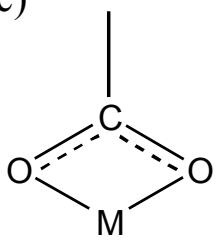

(d)

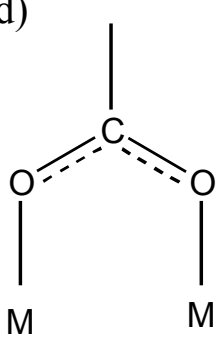

Figure 1.18 The four main types of metal ion/carboxylate interactions observed: (a) an uncoordinated anion, (b) a monodentate ligand, (c) a bidentate chelate and (d) a bridging bidentate interaction.

Interestingly, a study investigating the influence of di-carboxylic acids on calcite crystallisation found that the crystal/di-acid interaction is via bidentate binding. This cooperative binding of both carboxylates is strongest for malonic acid, however in the longer-chain di-carboxylates the acid groups behave independently[39]. Given that there is little difference in the $\mathrm{Ca}^{2+}$-binding stability constants then this difference was attributed to loss of conformation entropy in the longer chain derivatives. 
A more recent study[40] into the adsorption of small di- and tri-carboxylic acids from water onto calcite found the mechanism of adsorption to be a complexation of the $-\mathrm{Ca}^{+}$surface site by the two carboxylates, a mechanism similar to that observed in the solution complexation of $\mathrm{Ca}^{2+}$ ions. Geffroy et al.[40] also found that the surface complexation resulted in a ring formation (Figure 1.19), which involves the binding of the two carboxylates to the $-\mathrm{Ca}^{+}$surface site, displacing water in the process.

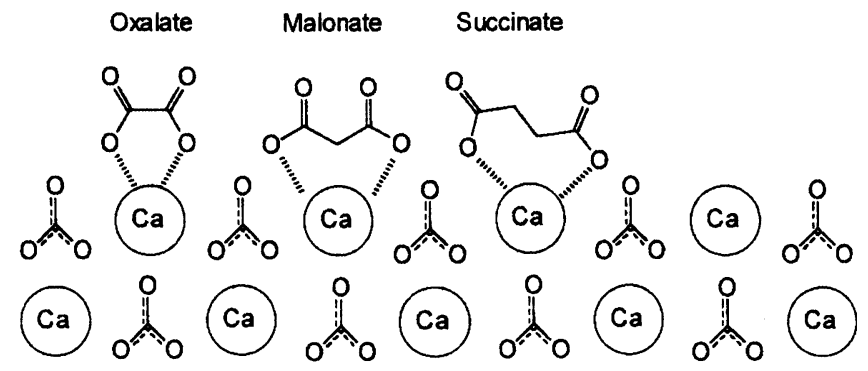

Figure 1.19 Suggested mechanism of surface complexation of dicarboxylates on a calcite surface[40].

A comparison of different aliphatic dicarboxylates found, in line with the general view, that five-membered chelate rings are the most stable and that the complexation strength increased with the number of $\mathrm{CH}_{2}$ groups: oxalate > malonate > succinate. When comparing the strength of the surface complexation of the dicarboxylates with aliphatic diols and catechol, Geffroy et al.[40] also found a trend reflecting the relative electron donor abilities of the oxygen that chelates the $-\mathrm{Ca}^{+}$surface site: enolate > carboxylate > hydroxyl. Figure 1.20 illustrates the suggested complexation mechanism for 2-hydroxy carboxylates, which display the strongest surface complexes.

Another study, this time using in situ synchrotron X-ray scattering, investigating fatty acid Langmuir monolayers on supersaturated calcium bicarbonate subphases found the cation binding ratio to be 1:4-8 surfactant molecules. This was at an estimated $50 \%$ deprotonation level within a $\mathrm{pH}$ range of 6.3-7.4. This was contrary to the $2: 1$ or $1: 1$ ratios expected for bidentate or epitaxial adsorption[69]. However this may reflect a 50\% deprotonation and a relatively low subphase ionic strength, as 
studies have shown that increased subphase concentration (ionic strength) can lead to increased soap percentage for a given $\mathrm{pH}[76]$.
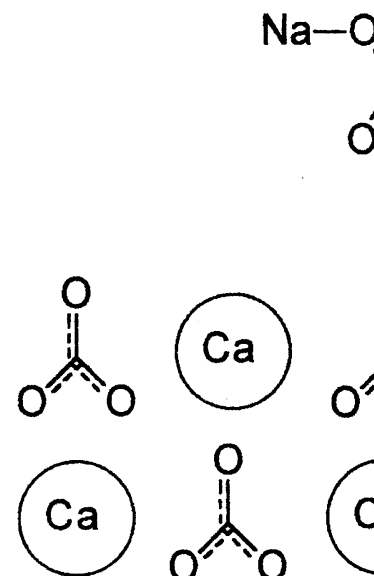<smiles>[O]C(=O)O</smiles>
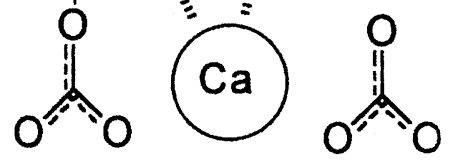

Figure 1.20 Suggested mechanism of surface complexation of $\alpha$-hydroxy carboxylates on a calcite surface[40].

These studies show no conclusive findings regarding the nature of the calciumcarboxylate (particularly stearic acid) interaction. However, given that mixed electrostatic and covalent binding has been observed in calcium carboxylates then it is possible that the nature of the binding changes depending on the chemical environment, which is where the modes of complementarity may play a role.

\subsubsection{Modes of Complementarity}

This section presents a brief description of the six modes of complementarity (lattice geometry, symmetry, electrostatics, stereochemistry, polarity and topography) proposed by Mann[19], with a particular focus on how they might be considered in a $\mathrm{Ca}^{2+} /$ stearic acid monolayer system.

\subsubsection{Lattice Geometry and Symmetry}

The lattice matching and symmetry modes are closely related, the aim is to match the crystal lattice spacing and symmetry of the organic seed to that of the crystal being precipitated. In principle this is relatively simple, however achieving this 
experimentally in a monolayer is more difficult. Figure 1.21 depicts models of four calcite faces, clearly showing the spacing and symmetry of the $\mathrm{Ca}^{2+}$ and $\mathrm{CO}_{3}{ }^{2-}$ ions. The aim of creating a stearic acid monolayer seed is to recreate this spacing and symmetry by altering the surface compression of the monolayer and the chemistry of the surfactant molecule.

\subsubsection{Electrostatic Matching}

In terms of electrostatic matching the focus is on charge (positive or negative), and charge density. A comparison of the (10.4), (10.0), (00.1) and (01.2) faces shows that the (10.4) and (10.0) are neutral whereas the (00.1) and (01.2) are positive. Therefore in terms of electrostatic charge matching, a negative stearic acid monolayer provides a better match for the latter two faces. The other consideration is charge density, the best match will depend on the valance, spacing and degree of ionisation of the monolayer.

\subsubsection{Stereochemical Matching}

Stereochemical matching is commonly considered to refer to matching of the carboxylate head-group orientation with the carbonate anions in the crystal lattice. As such, the term stereochemistry is incorrectly used in the literature as it does not refer to a chiral centre. For the remainder of this discussion the term stereochemistry will be used for consistency with the literature, however in the remainder of this thesis the term spatial geometry matching will be used. If the monolayer is fully compressed and the carboxylate is perpendicular to the interface then a stereochemical match is only possible with the $\{10.0\}$ face, as it is the only face where the carbonate anions are perpendicular to the nucleating face, as shown in Figure 1.21. However, for a partially compressed monolayer, where the surfactant molecules are tilted, there is a potential stereochemical match for both the (10.4) and (01.2) faces. The parallel configuration of the carbonate anions to the (00.1) face 
means the occurrence of stereochemical matching is very unlikely.
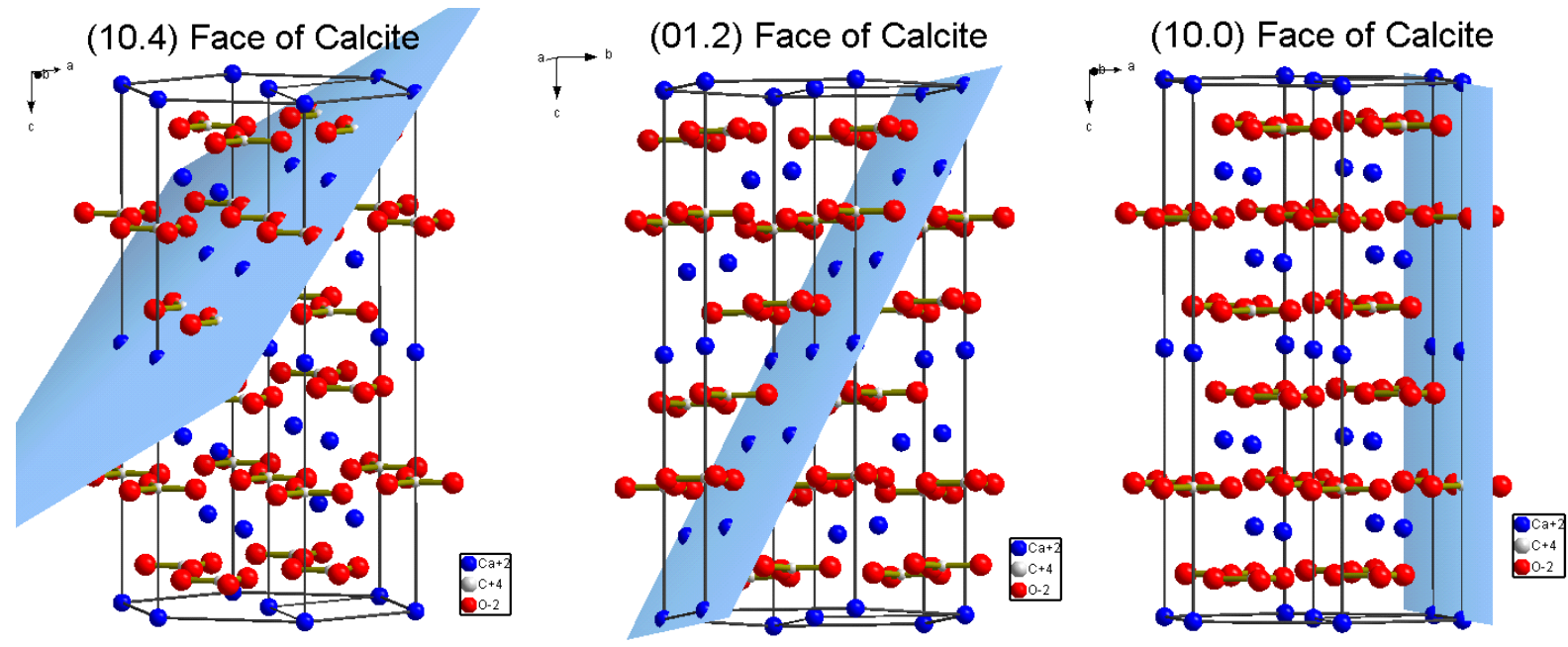

(10.4) Face of Calcite

(01.2) Face of Calcite
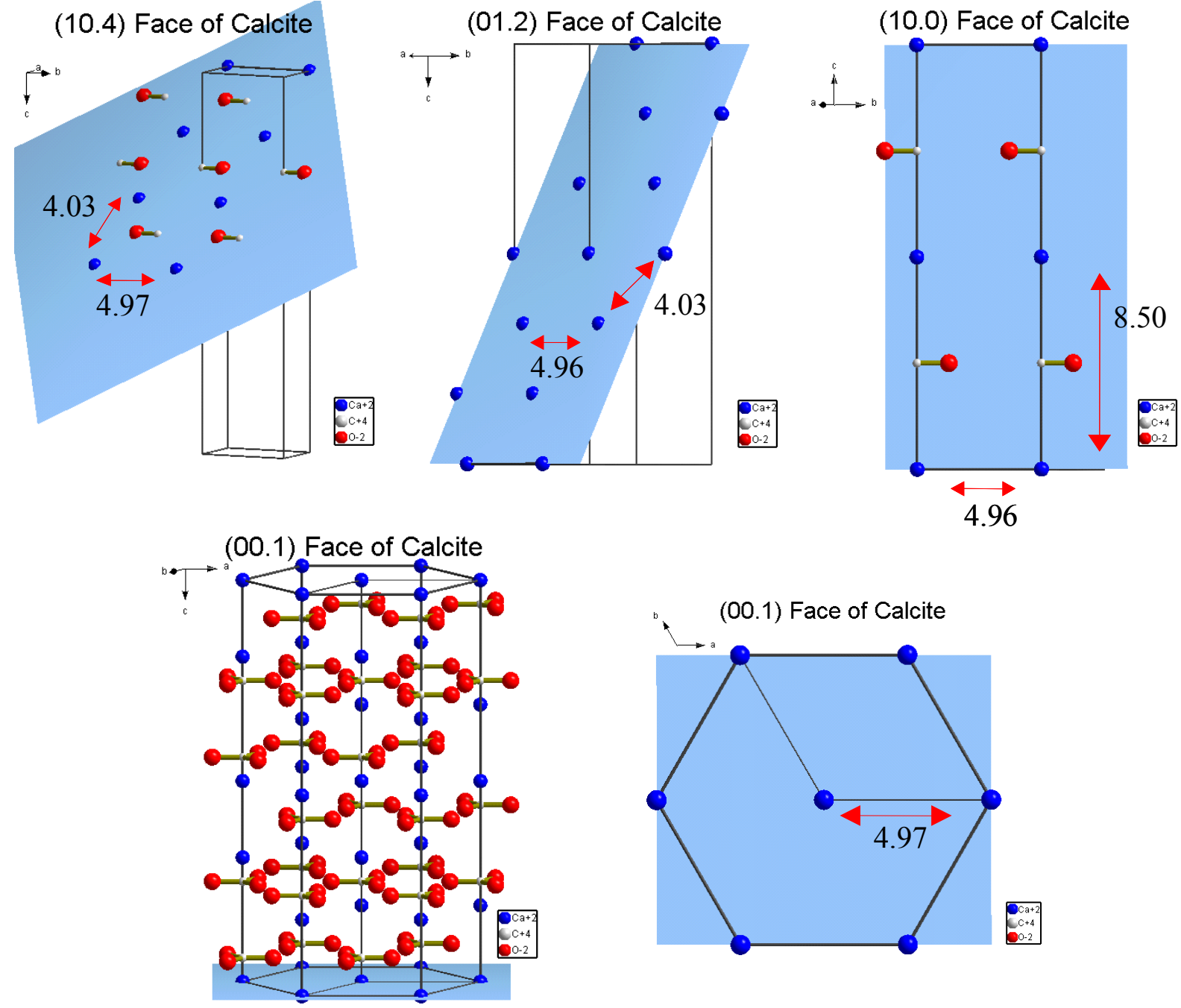

Figure 1.21 Four crystal faces of calcite, showing the, symmetry, spacing and composition of the face. 


\subsubsection{Topography}

The importance of topography lies in the way it influences the spatial charge distribution, confines and controls solution chemistry, and restricts crystal growth. The latter two are concerned with enclosed environments, such as vesicles. In terms of spatial charge distribution and controlled crystal growth the aim is to create an organised array of charge sites, which match the crystallography of the incipient nucleus. According to Mann[19], the simplest way to achieve this is through curvature of the substrate surface as shown in Figure 1.22. Thus the surface curvature or the topography plays an important role in controlling crystallisation.

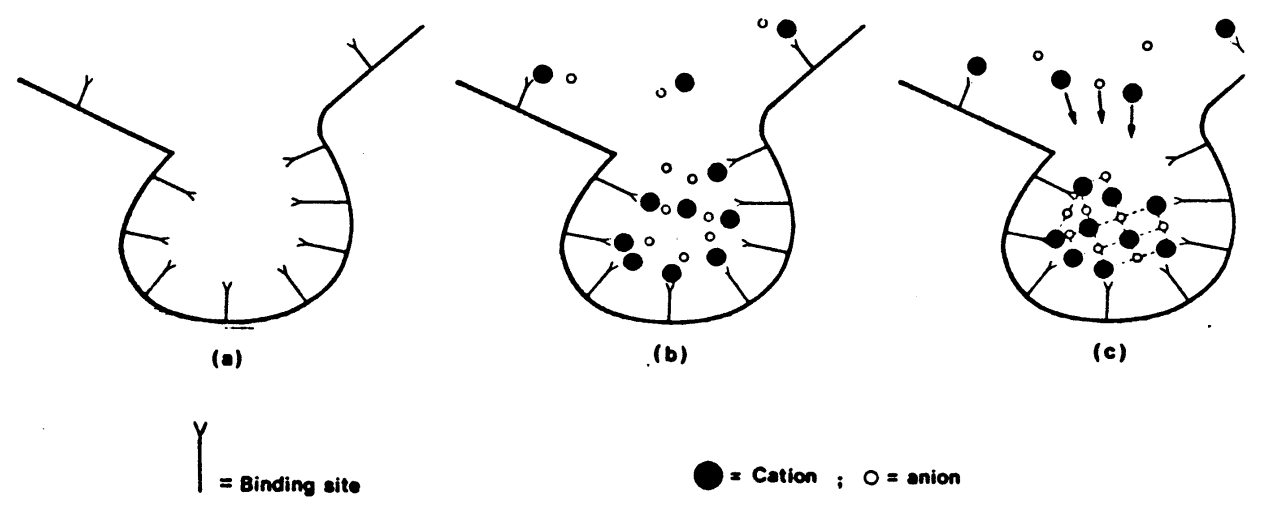

Figure 1.22 A schematic of the influence of surface topography on the spatial charge distribution and therefore controlled crystallisation[19].

Unfortunately, in the Langmuir monolayer system used in this study there is no scope for this type of topographic influence on crystallisation. Having said that, based on a GIXD study[80], it was suggested the possible occurrence of periodic buckling of the monolayer in the presence of a precipitated inorganic film. Kmetko[80] observed small secondary maxima (oscillations) in GIXD Bragg rod scans of $\mathrm{MgCl}_{2}$ and $\mathrm{MnCl}_{2}$ films grown under a heneicosanoic acid Langmuir monolayer. It was proposed that these oscillations arose due to periodic buckling or protrusion of the aliphatic chain (Figure 1.23). This phenomenon has been observed in an arachidic acid monolayer with cadmium salt, also on a solid substrate in Langmuir- 
Blodgett films. The possibility of a textured monolayer surface adds another level of complexity to the organic/inorganic interfacial interaction and it is likely that this rearrangement of the monolayer would occur during the nucleation of the crystal and only in particular circumstances.

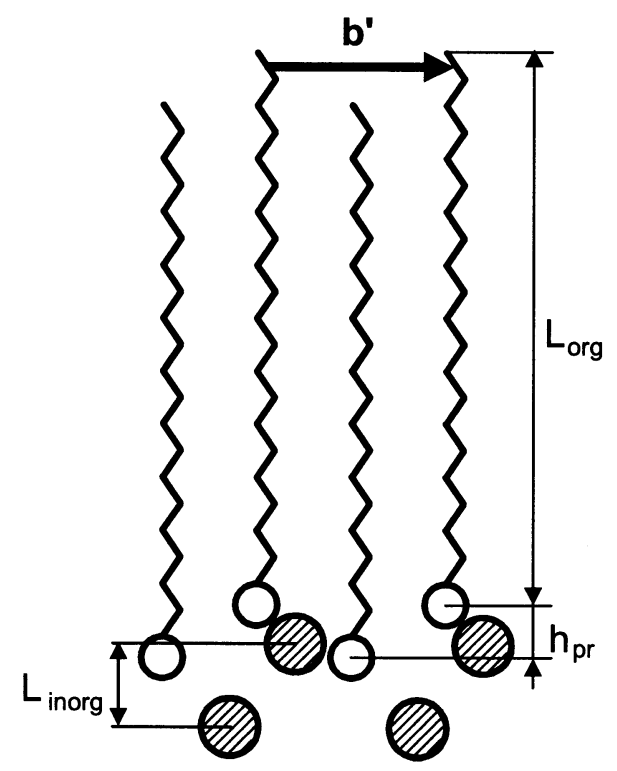

Figure 1.23 A schematic of the monolayer buckling or protrusions postulated by Kmetko[80] to explain small secondary oscillations in grazing incidence X-ray diffraction Bragg rod studies of $\mathrm{MnCl}_{2}$ and $\mathrm{MgCl}_{2}$ films precipitated under heneicosanoic acid.

The mechanism for such a process is unknown but Kmetko postulated that it occurs due to the subphase ions compressing the head-group packing to the point where out-of-plane protrusions occur to relieve in-plane packing stresses. Ordinarily the small ions would form weaker ionic bonds as individuals, therefore to create such an effect it is suggested that they must interact as large aqueous species as a consequence of hydration and hydrolysis before binding[80].

\subsubsection{Polarity}

The influence of polarity on crystal growth has received very little attention, this is likely to be due to the difficulty in separating the effect from electrostatic factors. The importance of the dipole strength of the surfactant molecule, and therefore the 
monolayer, lies in its role, along with the surface charge density, in determining the nature of the short-range interactions[81]. The interaction between a deprotonated surfactant molecule and a cation in the subphase has ion-ion and ion-dipole components. The relative importance of the ion-dipole interaction will be dependant on the chemistry of the surfactant molecule, the properties of the counter-ion, and the dielectric properties of the solvent. For example, given an equal charge, a cation will generally interact more strongly with dipoles than anions would, because the charge is more concentrated on the usually smaller cation.

This dependence on the surfactant chemistry enables the dipole moment, and therefore the ion-dipole interaction, to be manipulated. By placing an electron withdrawing group elsewhere in the chain, the electron density at the head group will be reduced changing the dipole moment of the molecule. Consequently, the iondipole interaction would be reduced, but it is unknown how will this affect the overall interaction. For example, knowing how a change in the dipole moment would affect the mixed ionic/covalent bonding in the $\mathrm{Ca}^{2+}$-stearic acid system, as described in section 1.5.1, could be extremely important in determining the nature of the bonding.

To obtain an indication of the strength of the ion-ion and ion-dipole interactions and to investigate how they can be manipulated we turn to Equations 19 and 20. For a charge-charge interaction the interaction energy $w(r)$ is:

$$
w(r)=\frac{Q_{1} Q_{2}}{4 \pi \varepsilon \varepsilon_{\mathrm{o}} r}(\text { coulomb energy }) .
$$

Similarly, for a charge-fixed dipole interaction the interaction energy $w(r)$ is:

$$
w(r)=\frac{-Q u \cos \theta}{4 \pi \varepsilon \varepsilon_{\mathrm{o}} r^{2}}
$$

where $w(r)$ is in $J$, the electric charge $(Q)$ is in $C$, the electric dipole moment $(u)$ is in $(\mathrm{C} \mathrm{m}), r$ is the distance between the interacting molecules, $\varepsilon_{\mathrm{o}}$ is the dielectric permittivity of free space $\left(8.854 \times 10^{-12} \mathrm{C}^{2} \mathrm{~J}^{-1} \mathrm{~m}^{-1}\right)$ and $\varepsilon$ is the dielectric strength of the 
medium[82]. The $(\cos \theta)$ term in Equation 20 means that the magnitude of the charge-dipole interaction will be affected by the degree of surfactant tilt. At low surface compression the molecules are likely to have significant tilt therefore the interaction will be reduced compared to that of an erect compressed 'solid-like' phase. The $r^{2}$ term in the denominator shows that the charge-dipole interaction energy will decay quadratically in contrast to the linear decay of the charge-charge interaction energy. Therefore, the importance of the ion-dipole interaction increases as $r$ decreases.

Equations 19 and 20 show an increase in interaction energy can be achieved by either increasing the charge density or reducing the local dielectric constant. Vogel and Möbius[83] showed how the latter can be achieved by the introduction of alkyl groups in the vicinity of a surfactant head group. For example, methylation was found to increase the head group hydration shell distance, thereby increasing the effective dipole moment. This increase reflects a reduction of the high dielectric constant of water from $\varepsilon=78$ to $\varepsilon=5$ in the vicinity of polar interfaces, which was attributed to changes in the water structure[83]. This manipulation of the local dielectric constant highlights the importance of the subphase to interfacial interactions.

\subsubsection{Solvation Implications}

The structuring of water molecules around ions, molecules, particles, and surfaces is also important in terms of crystallisation. For ions to leave the solution to join a growing crystal then either the bound water has to be removed (dehydration) or the water has to be incorporated into the crystal structure. The release or incorporation of bound water has significant thermodynamic implications in terms of the process of crystallisation. The different strengths of the ion-water bonds and ion-crystal bonds have an enthalpic effect, which will vary from system to system. In contrast, according to De Yoreo and Vekilov[3], the entropic effects appear to be consistent 
( $20 \mathrm{~J} \mathrm{~K}^{-1}$ per mole of water released or trapped); positive on the release of water and negative for trapped water. Therefore the tendency of the subphase and monolayer ions to be hydrated must be considered when investigating the nature of the interfacial interaction.

This overview of the factors that are believed to be important in determining the nature of the organic/inorganic interface highlights the complexity of the situation. In the next section the research activities pertaining to crystallisation under Langmuir monolayers is summarised, providing experimental evidence for much of the theory that has been discussed above.

\subsubsection{Current Understanding}

One of the earliest studies investigating calcium carbonate growth on surfactant monolayers was by Mann et al.[45]. This study involved the growth of vaterite under stearic acid monolayers, in which it was found that the vaterite crystals exhibited preferred orientation in the [00.1] direction. This suggested that nucleation occurred on the (00.1) face, with the $c$ and $a$ axes oriented perpendicular and parallel to the monolayer, respectively. These results were attributed to electrostatic and stereochemical interactions at the interface. In terms of electrostatics, the formation of a Stern layer of $\mathrm{Ca}^{2+}$ counter-ions favoured the nucleation of faces containing only $\mathrm{Ca}^{2+}$ ions, such as the (00.1) face of vaterite. However, charge accumulation cannot account for vaterite formation alone, as the (00.1) face of calcite also consists solely of $\mathrm{Ca}^{2+}$ ions. Mann et al. attributed the crystallisation of vaterite (as opposed to calcite) to stereochemical matching, stating that the carbonate anions are arranged parallel to the (00.1) face in calcite, in contrast to the perpendicular arrangement in the (00.1) face of vaterite. The perpendicular conformation is believed to match the orientation of the carboxylate groups on the monolayer. Mann et al. went on to state that there was no evidence for a match in the lattice geometry and concluded that electrostatic and stereochemical matching overrides lattice mismatch. Further, if lattice matching 
were important then calcite would be expected rather than vaterite due to a better lattice spacing match with the partially compressed monolayer. Although the preferred alignment and the resultant polymorph have been accounted for, the influence of kinetics on the crystallisation of vaterite in place of calcite was not discussed.

In contrast to the above study, matching of the crystal lattice geometry with the spacing of the monolayer head groups is the most common explanation given for oriented crystallisation[84]. For example, the influence of lattice geometry was observed in a study[29] investigating the nucleation and growth of calcium carbonate under stearic acid monolayers. Under a compressed monolayer $\left(\Pi=45 \mathrm{mM} \mathrm{m}^{-1}\right)$ calcite crystals were nucleated with the $\{1 \overline{1} .0\}$ face oriented parallel to the monolayer/subphase interface. Unlike the Mann et al.[45] example above where a uni-polar (00.1) face was nucleated at the interface, the $\{1 \overline{1} .0\}$ face is neutral, containing both $\mathrm{Ca}^{2+}$ and $\mathrm{CO}_{3}{ }^{2-}$ ions. Given the electrostatic mismatch of the negatively charged monolayer and the neutral $\{1 \overline{1} .0\}$ face, the preferred orientation was attributed to matching of the lattice geometry. For this to be the case, it would be necessary to have a good spatial fit between the monolayer structure and the crystal lattice spacing. When compressed on an aqueous subphase, simple fatty acid monolayers have been found to form hexagonal or pseudo-hexagonal structures with a head-group spacing of approximately $5 \AA$ [56]. In terms of the interaction between divalent cations $\left(\mathrm{M}=\mathrm{Ca}^{2+}\right)$ and stearic acid, in X-ray reflectivity studies completed by DiMasi et al.[85] it was suggested that the general stoichiometry is $\mathrm{MSt}_{\mathrm{x}}$ (where $\mathrm{x}=2$ 4 and $\mathrm{St}=$ stearate). Given the monolayer structure and spacing, the nature of the $\mathrm{Ca}^{2+} /$ stearic acid stoichiometry, and the lattice spacing of the $\{1 \overline{1} .0\}$ face, there is a reasonably close match.

However, if a geometric match was all that was required then the (00.1) face of calcite should also have been observed as it provides an exact match for the 
hexagonal monolayer structure. This was not the case. The difference between the $\{1$ $\overline{1} .0\}$ and (00.1) faces of calcite lies in the orientation of the carbonate anions, which are perpendicular to the $\{1 \overline{1} .0\}$ surface and parallel to the (00.1) face. Thus the stereochemistry of the carboxylate head group matches the $\{1 \overline{1} .0\}$ faces but not the (00.1) face[29]. Therefore the matching of lattice geometry is important but not sufficient to be the sole determinant of preferred orientation, at least in some systems.

Interestingly, the above study shows lattice geometry/stereochemical matching of the $\{1 \overline{1} .0\}$ face dominating the lattice geometry/electrostatic matching of the Ca(00.1) face. This could be accounted for by considering the degree of deprotonation. These experiments were performed in the $\mathrm{pH}$ range of 5.8-6.0, thus with a $\mathrm{pKa}$ of 5.1 for stearic acid, the monolayer would be approximately $60 \%$ deprotonated. Assuming ordered deprotonation, then one could imagine $\mathrm{HCO}_{3}{ }^{-}$or $\mathrm{CO}_{3}{ }^{2-}$ ions sitting on protonated acid sites at the interface, offsetting the $\mathrm{Ca}^{2+}$ charge. This would also account for the $\mathrm{MSt}_{2}$ stoichiometry observed in X-ray reflectivity studies. Further, Duffy and Harding[74] showed that vacancies or distortions in the lattice structure could accommodate electrostatic mismatch. Another consideration is the nature of the $\mathrm{Ca}^{2+}$ /stearic acid interaction. Referring back to section 1.5.1, regarding stearic acid/ $\mathrm{Ca}^{2+}$ bonding, and considering the degree of deprotonation, it is possible that there is a significant amount of covalent bidentate binding as that found by Mann, Heywood et al.[39] for $\mathrm{Ca}^{2+} /$ dicarboxylates.

Another study by Heywood and Mann et al.[28] supports this notion that ionic binding and electrostatic matching are not necessary for oriented growth. In this study calcium carbonate was crystallised under stearic acid, octadecylamine and octadecanol monolayers. At low $\mathrm{Ca}^{2+}$ levels, oriented vaterite was observed under both positively (stearic acid) and negatively (octadecylamine) charged monolayers, whereas octadecanol was found to inhibit nucleation. The crystals under both 
charged monolayers displayed a preferred orientation on the (00.1) face. The preferred orientation in the stearic acid monolayer experiment could be related to an electrostatic match between the negative monolayer and the uni-polar $\mathrm{Ca}^{2+}-(00.1)$ face. In addition, a stereochemical match between the perpendicular alignment of the carbonate groups with the carboxylate head-groups would have favoured the (00.1) face. However, this does not explain the preferred orientation observed for the octadecylamine monolayer. This result shows that $\mathrm{Ca}^{2+}$ binding is not critical for the formation of vaterite on the uni-charged (00.1) face as the amine monolayer surface is also positively charged[29]. This conclusion appears to overlook the occurrence of a uni-charged $\mathrm{CO}_{3}{ }^{2-}$ (00.1) face being nucleated at the positive octadecylamine monolayer. This scenario would suggest that electrostatics are playing a role.

Donners et al.[86] provides another example of the importance of stereochemical recognition at the expense of lattice geometry. In this study the nucleation and growth of $\mathrm{CaCO}_{3}$ in the presence of poly(L-isocyanoalanyl-D-alanine), both in solution and on a coated glass surface was investigated. It was suggested that a stereochemical match between the carbonate alignment and the orientation of the carboxylates in the polymer led to the unusual preference for the (01.1) face. Molecular modelling showed the lack of a match between the lattice spacing of the carbonate ions and the carboxylate end groups, hence lattice geometry does not appear to be important. However, there was no mention made of the electrostatic interactions.

With a view to exploring the importance of stereochemical effects further, studies probing the affect of different head group chemistry is instructive. A comparison of monolayers with carboxylic acid, sulfate and phosphonate head groups showed that stereochemical matching between the head groups and the nucleation face does affect crystal orientation[73]. It was found that for eicosanoic acid monolayers, calcite is crystallised on the (1 $\overline{1} .0)$ plane in contrast to the (001) face observed for $n$ - 
eicosyl sulfate and $n$-eicosyl phosphonate. The observation of the $(1 \overline{1} .0)$ plane for eicosanoic acid is in accordance with earlier studies and can be attributed to the complementary alignment of the monolayer carboxylates and the crystal carbonates. However, the tridentate oxygen motif of the sulfate and phosphonate do not provide the same stereochemical match. The occurrence of the (00.1) face was attributed to a better match of lattice geometry. It was stated that the different packing of the tridentate sulfate and phosphonate head groups improved the lattice match. It is likely that the loss of the stereochemical matching meant that the lattice matching and electrostatic matching became more important.

As part of the same study, similar behaviour was observed for aragonite crystallisation, which is stabilised through the addition of $\mathrm{Mg}^{2+}$. Nucleation under the eicosanoic acid monolayer is oriented with the (100) aragonite face parallel to the monolayer. This was attributed to potential geometric and stereochemical interactions. In contrast the (001) face was observed for $n$-eicosyl sulfate and $n$ eicosyl phosphonate. The occurrence of preferred orientation in aragonite is interesting considering that Kuther et al.[75] found that aragonite would not grow epitaxially on ordered substrates, such as SAMs. This was accounted for by the combination of an orthorhombic space group and the lack of 3-fold symmetry. Heywood et al.[73] attributed the presence of the (001) face to the same geometric arrangement that led to the calcite (00.1) face being observed.

In terms of ascertaining the importance of geometric matching and in general elucidating the nature of the molecular recognition that occurs at the interface during nucleation in situ FTIR has been employed. One such study[87] investigated calcite crystallisation under stearic acid, octadecyl sulfate and polymerised 10,12pentacosadiynoic acid monolayers showing preferred orientation of calcite on the (01.0), (00.1), and (01.2) planes, respectively. The surfactant monolayers were shown to uniquely rearrange in order to optimise the geometric and stereochemical fit to the 
growing calcite crystals. For stearic acid mineralisation results, increased tilting of the molecules away from the surface normal to accommodate the geometry of the (01.0) plane was observed. In octadecyl sulfate monolayers, the hydrocarbon chain becomes more disordered during mineralisation expanding the monolayer in order to fit the carbonate spacing in the calcite $\mathrm{CO}_{3}{ }^{2-}-(00.1)$ plane. As for acidic polydiacetylene, the (01.2) calcite face is aligned with the direction of the polymer backbone. From the FTIR spectra it is indicated that the alkyl side chains reorganise to optimise the stereochemical fit of the (01.2) calcite plane. Symmetry reduction and stereochemical and lattice matching all appear to be important. This reorganisation of the monolayer is likely to be a reflection of the extra degree of freedom imparted by the relatively low level of compression, $\left(\sim 10 \mathrm{mN} \mathrm{m}^{-1}\right)$.

Berman and Charych[88] also investigated the growth of calcite on a polymerised 10,12-pentacosadiynoic acid ( $\mathrm{p}$-PDA) monolayer. The 25-carbon chain acid was polymerised in situ with the aim of increasing the rigidity of the monolayer. Preferred orientation was observed in calcite, with a precise lattice match between the $a$ axis and the monolayer periodicity. Further, it was found that there was a stereochemical fit between the tilted monolayer and the inclined carbonate on the (01.2) calcite face. This combination was found to induce total control over nucleation. The level of monolayer compression is not stated in this study but it appears that polymerisation of the hydrocarbon backbone is not sufficient to ensure complete rigidity, as the molecules are still able to tilt. However, lattice matching does appear to be assured with polymerisation, assuming the correct head group spacing is incorporated into the polymer structure.

In contrast to the many studies discussed above promoting the importance of stereochemical and lattice matching, recent computer modelling data highlights the importance of electrostatics. One study in which the crystallisation of calcite under stearic acid monolayers was modelled, Duffy and Harding[84] showed that 
nucleation is influenced by the degree of ionisation and competition between the crystal/substrate and water/substrate interactions. The degree of ionisation influences the strength of the crystal/substrate adhesion. Fully ionised substrates have a stronger adhesion than neutral substrates and are therefore better at promoting nucleation. Consequently, $\mathrm{pH}$ is important as it controls the degree of ionisation. The calculations showed that the crystallisation of neutral faces (such as the calcite (10.4) face) on ionised monolayers is unfavourable. Unless the density of the surface carbonates (for anionic monolayers) is low enough to allow substitution of the ions with the monolayer head-groups, electrostatic neutrality cannot be achieved. Duffy and Harding[84] claim that it is only after all these conditions are satisfied that stereochemical matching becomes important. Since the organic template is not a rigid structure, stereochemical interactions can only be a final consideration as thermal fluctuations and entanglement may disrupt the substrate. They go on to suggest that if the substrate was cross-linked and rigid then stereochemical interactions would play a more important role in biomineralisation. It was also proposed that electrostatics plays an important role in determining the final crystal morphology as ionised and neutral monolayers tend to stabilise different surfaces, (10.4) for neutral monolayers and (10.0) or (00.1) for the ionised monolayers.

Duffy and Harding[84] went on to suggest that many of the comparisons made supporting lattice geometry matching, as a means of generating preferred crystal orientation, are incorrectly based on perfect un-relaxed crystal structures and an idealised arrangement of functional groups on the organic matrix. Considering that these functional groups are in water, a very dynamic system, the actual structural arrangement could be far from ideal[84]. Making a comparison between the unrelaxed crystal structure and an idealised monolayer phase arrangement is likely to be flawed, but because the monolayer has the freedom to rearrange itself, the likelihood of lattice geometry matching across the interface remains a possibility. 
This is reflected in Mann's observation that partially compressed monolayers are the best for controlled crystallisation[45]. All this really tells us is that the monolayer is not a true template in the sense that it is preformed waiting for nucleation to commence. Rather the ordered interface is self-assembled as part of the nucleation and growth processes[74].

Although the monolayer cannot be thought of as a rigid template, the extent to which it can rearrange during nucleation is variable. This is illustrated by Buijnsters et al.[89], who observed preferred orientation of calcite on the (10.0) plane using an amide-containing phospholipid monolayer. What is interesting in this study is the use of hydrogen bonding between the phospholipid molecules to increase the monolayer rigidity and to force the phosphate head groups to adopt a bidentate orientation towards the aqueous solution. When this ability to form hydrogen bonds is removed lateral pressure is required to observe a similar monolayer conformation and to achieve preferred orientation of the crystallised calcite.

It has been shown above that the physical packing and the chemical nature of the monolayer are important in terms of influencing the degree of subphase ion/monolayer matching. The crystal uniformity and nucleation density has also been found to change with the degree of freedom of the monolayer. For both stearic acid and octadecylamine monolayers the nucleation density was higher for solid-like monolayers than for liquid-like[28]. Mann[45] observed a similar effect with the uniformity of vaterite nucleation and stated that partial monolayer compression was optimal for controlled crystallisation. These observations fit with BAM studies[70], which showed preferential nucleation at domain boundaries where there is probably more scope for rearrangement of the monolayer structure to match the crystal lattice thus lowering the interfacial energy of the system.

In a similar situation, the nucleation density of aragonite crystallised under eicosanoic acid, n-eicosyl sulfate and n-eicosyl phosphonate monolayers was 
repeatedly less than that observed for calcite. This was attributed to $\mathrm{Mg}^{2+}$ substitution of $\mathrm{Ca}^{2+}$ at the monolayer, which was added (at the high $\mathrm{Ca}^{2+} / \mathrm{Mg}^{2+}$ ratio of 1:6) to ensure aragonite crystallisation. Alternatively, the decrease may reflect the reduced number of stereochemically equivalent carbonates per unit area, as all the carbonates in the calcite ( $1 \overline{1} .0)$ plane are the same, however in the aragonite $(100)$ face there are two different carbonate orientations[73].

The monolayer variability discussed above can, at least in part, be attributed to differences in the ion concentration, ionic strength, $\mathrm{pH}$ of the subphase and temperature, which are known to affect the structure of surfactant assemblies. Therefore it is important to monitor both template and mineral during nucleation and growth, to correctly distinguish between epitaxial, stereochemical and kinetic control and when making comparisons between different studies. This variability in the system is displayed in a surface X-ray scattering study[69] that was attempting to repeat earlier studies, and found that vaterite crystals nucleated under stearic or arachidic acid films were not oriented relative to the monolayer. This polymorph selectivity was attributed to solution kinetics, particularly the $\mathrm{CO}_{2}$ escape rate, with vaterite being favoured over calcite when mineralisation occurs more slowly. Also based on the lack of preferred orientation observed and evidence of macroscopic reorientation in the largest crystals, due to surface tension effects, it is suggested that previous studies were incorrect about the presence of preferred orientation[69]. This is a rather bold statement given the body of work that supports the idea of preferred orientation and controlled crystallisation. However, this lack of preferred orientation is supported by a similar study using grazing incidence X-ray diffraction[80], which found that although $\mathrm{BaF}_{2}$ and $\mathrm{SrF}_{2}$ displayed epitaxially oriented crystal growth under heneicosanoic acid monolayers, $\mathrm{CaCO}_{3}$ did not. Kmetko[80], in this latter study, was not so bold as to condemn previous ex situ studies but he could not explain the discrepancy. In a more recent study[90] involving synchrotron X-ray 
scattering studies of the growth of magnesium calcites on SAMs the occurrence of preferred orientation has been shown. This suggests that the issue may lie with the technique or experimental approach rather than the absence of oriented crystallisation but we will have to wait for further studies to confirm this.

Variability between similar studies could also be attributed to a failure to account for all the influences on the organic/inorganic interface. One such influence that has been widely overlooked is polarity (another of the modes of complementarity). The author has failed to find any experimental study investigating the influence of the dipole moment on the crystallisation of an inorganic phase under a Langmuir monolayer. The only study found was a computational study by Duffy and Harding[74]. Calculations investigating crystallisation of the (01.2) calcite face showed that a well-defined substrate charge density was required. This was due to the requirement to quench the surface dipole moment. However this is inconsistent with experimental evidence, which shows that nucleation of the polar (01.2) calcite face occurs on a range of different organic substrates. It is proposed that the different organic substrates counter the dipole moment by modifying the charge density of the interfacial cation plane. This is achieved by introducing vacancies, surface reconstruction, ionising the organic acid groups or by adsorbing charged ions from the solution onto the crystal surface. The first two often result in high-energy surfaces. Removal of surface $\mathrm{Ca}^{2+}$ ions also serves to accommodate any lattice mismatch as well as quench the dipole moment. The use of vacancies also has morphological implications, as it would be expected to find a reduced crystal growth rate in the direction of poor matching due to the presence of defects. Calculations show that the (00.1) surface has a lower interfacial energy than the (01.2). Therefore the presence of the (01.2) surface over the (00.1) surface is surprising. Two reasons were proposed for this observation, the first suggested that small nuclei (in the order of a few $\mathrm{CaCO}_{3}$ units wide) oriented in the (01.2) direction are more stable than 
nuclei oriented in the (00.1) direction. Secondly, simulations show the formation of a bilayer structure consisting of $\mathrm{Ca}^{2+}$ and $\mathrm{HCO}_{3}{ }^{-}$ions above the charged monolayer and it is the stereochemical matching of these $\mathrm{HCO}_{3}{ }^{-}$ions to the $\mathrm{CO}_{3}{ }^{2-}$ ions in the $(01.2)$ surface that lead to the preferred orientation. Duffy and Harding[74] suggest that if such a bilayer should form, then kinetically the (01.2) surface would be favoured. However, they do not explain the reasons for the formation of this bilayer, but it is likely to be a counter-ion effect. Neither do they explain the $\mathrm{HCO}_{3}{ }^{-}$orientation, which could be explained by way of a stereochemical match with the carboxylate head group of the stearic acid monolayer. Duffy and Harding[74] conclude by stating that for a crystal to grow in a particular direction that involves a polar face then the local geometric matching and the global electrostatics (i.e. the quenching of macroscopic dipoles) must be met.

Many of the above studies have attempted to account for preferred orientation and polymorph selection in terms of matching of lattice geometry, stereochemistry, symmetry, and electrostatics across the monolayer/crystal interface. The result is a number of conflicting and variable results. This can be partly attributed to the failure to account for kinetic influences. Loste et al.[70] illustrate a good example of how kinetics can influence crystallisation. Investigating how the monolayer chain length influences crystal polymorph and morphology, Loste et al. found the rate of $\mathrm{CO}_{2}$ diffusion through the monolayer, and thus the kinetics of precipitation, decreased with increasing chain length. Consequently, the influence of chain length and monolayer domain structure on morphology and polymorph control is confounded with the influence of the kinetics. However it is interesting to note that, although compressed to $30 \mathrm{mN} \mathrm{m}^{-1}$, the shortest surfactant investigated, palmitic acid $\left(\mathrm{CH}_{3}\left(\mathrm{CH}_{2}\right)_{14} \mathrm{COOH}\right)$, showed considerable movement. In contrast, stearic acid $\left(\mathrm{CH}_{3}\left(\mathrm{CH}_{2}\right)_{16} \mathrm{COOH}\right)$ and the other longer surfactants exhibited little macroscopic movement. This is consistent with the increased van der Waal interaction expected 
between the longer chain molecules within the domains. Brewster Angle Microscopy showed that crystals preferentially grew under compressed monolayer domains and at domain boundaries. The shorter chain length monolayers, palmitic and stearic acid, were found to give greater amounts of aragonite, vaterite and un-oriented calcite, whereas the longer chains gave oriented calcite. It is likely that this polymorph selectivity is due to changes in the kinetics rather than any direct affect of chain length.

Another example of the influence of kinetics was illustrated in a two part study by Heywood et al.[28,29]. Vaterite crystallised under a stearic acid monolayer at low $\mathrm{Ca}^{2+}$ ion concentration $\left(\left[\mathrm{Ca}^{2+}\right] \approx 4.5 \mathrm{mmol} \mathrm{L}{ }^{-1}\right)$ whereas calcite crystallised at high $\mathrm{Ca}^{2+}$ concentrations $\left(\left[\mathrm{Ca}^{2+}\right] \approx 9 \mathrm{mmol} \mathrm{L}^{-1}\right)$. This was attributed to an earlier observation by Turnball[91] who suggested that vaterite formation was favoured under conditions of high $\mathrm{HCO}_{3}^{-} / \mathrm{Ca}^{2+}$ ratios whereas calcite formation tends to occur at stoichiometric proportions. A second explanation was based on the belief that the more open structure of vaterite has a greater tolerance to disorientation of the carbonate ions thus the formation of vaterite nuclei is kinetically favoured through the stabilisation of disordered clusters[28]. It was concluded that further work was required to validate these explanations[29]. An alternative explanation for the apparent phase selection could simply be the different levels of supersaturation. Changes in supersaturation are known to affect the relative rates of nucleation of the two phases, as discussed in section 1.1.2. It is possible that the solution conditions correspond to scenario (3) where $K_{J, I I}>K_{J, I}$ and $(1-a / c)^{3}>b$, meaning that phase I (vaterite) has the higher nucleation rate but only over intermediate levels of supersaturation (Figure 1.3[1]).

In this section an overview of research completed with the aim of understanding crystallisation under Langmuir monolayers has been provided. Lattice geometry 
(and symmetry), stereochemistry and electrostatics have been widely used to explain the preferred orientation of the crystal phase. The particular importance of these different influences remains to be determined, and in fact it is likely to vary depending on other influences such as the $\mathrm{pH}$ and kinetics. There is a lack of information regarding the importance of polarity and topography on the nature of the interfacial interaction. Further, a clear picture is lacking of how the kinetic influences, such as $\mathrm{pH}$ and ionic strength, influence the thermodynamic influences, such as lattice geometry and electrostatics. It is envisaged, perhaps naively, that a complete understanding of the organic/inorganic interfacial interaction will enable comparisons between systems to be readily made and synthetic systems to be designed with a view to achieving complete control over crystal nucleation and growth.

\subsection{Summary}

This chapter has reviewed the current understanding of matrix mediated calcium carbonate crystal nucleation and growth under surfactant monolayers. The attraction for this field is great, given that an understanding of the molecular-specific interactions between organic molecules and selected crystal faces offers the potential to design templates and additives that enable specific morphological control of crystal growth.

It is clear that Mann's modes of complementarity are important, however the questions of how, why, where, when and to what extent remains debated. For a large part, this debate is due to the large number of different systems found in nature but is also a reflection of how far we have yet to go before we completely understanding the biomineralisation processes.

It is also clear that many of the studies attempting to understand biomineralisation have over-simplified the situation. By ignoring the role of kinetics or by focussing on only one or two of the modes these studies have possibly erred in 
their conclusions. In the researchers defence, much of the early work in this field has been restricted by the lack of experimental techniques able to provide high-resolution structural details. Therefore there is only circumstantial evidence to suggest that molecular recognition is an important factor in controlling inorganic crystallisation. The use of synchrotron techniques offers great potential, the ability to achieve highresolution structural detail in situ shows promise for elucidating the mechanisms of organic matrix-mediated crystal nucleation and growth. That is, once the experimental discrepancies can be eliminated.

To advance this area of crystal engineering this review has highlighted a number of areas that require further study. These include:

- an understanding of how polarity or the surfactant dipole moment affects the ion/head group binding;

- a clear picture of the effects of lattice geometry, polarity, electrostatics etc. in a system where the influence of kinetics is minimised;

- a study that monitors the influence of all the modes simultaneously, in this way a clear picture of the interaction between the modes can be obtained;

- the nature of the ion/head group binding, and how it changes with different chemistries; and

- knowledge of the different binding strengths of a surfactant to the different crystal faces is key to predicting crystal orientation.

To further complicate matters a growing number of studies in the last five years have suggested that oriented crystal nucleation and growth may proceed via an amorphous calcium carbonate (ACC) pathway (refer Figure 1.11). The explanation for the lack of evidence of ACC in monolayer systems is attributed to the inability to detect ACC before it crystallises. It is likely that this only occurs in selected systems based on crystal nucleation rates discussed in section 1.1.2 however, this needs to be confirmed. 
This list of outstanding issues in the field of biomineralisation is not exhaustive, and this thesis only attempts to address a subset of them. Broadly speaking the aim of this study is to further our understanding towards the ultimate goal of synthetic crystal engineering that rivals nature.

\subsection{References}

[1] R. Davey and J. Garside, From Molecules to Crystallizers, Oxford University Press, 2001.

[2] A. Adamson and A. Gast, Physical Chemistry of Surfaces, New York: WileyInterscience, 1997.

[3] J. De Yoreo and P. Vekilov, "Principles of Crystal Nucleation and Growth," Reviews in Mineralogy and Geochemistry vol. 54, 2003, pp. 57-93.

[4] J. Mullin, Crystallization, Butterworth-Heinemann, Oxford., 2001.

[5] K. Sawada, "The Mechanisms of Crystallization and Transformation of Calcium Carbonates," Pure E Applied Chemistry, vol. 69, 1997, pp. 921-928.

[6] H. Teng, P. Dove, and J. De Yoreo, "Reversed Calcite Morphologies Induced by Microscopic Growth Kinetics: Insight into Biomineralization," Geochimica et Cosmochimica Acta vol. 63, 1999, pp. 2507-2512.

[7] L. Vayssieres, "On the Design of Advanced Metal Oxide Nanomaterials," Int. J. Nanotechnology vol. 1, 2004, pp. 1-41.

[8] S. Weiner and P. Dove, "An Overview of Biomineralization Processes and the Problem of the Vital Effect," Reviews in Mineralogy and Geochemistry vol. 54, 2003, pp. 1-29.

[9] S. Mann, "The Chemistry of Form," Angew. Chem. Int. Ed. vol. 39, 2000, pp. 33923406.

[10] F. Meldrum, "Calcium Carbonate in Biomineralisation and Biomimetic Chemistry," International Materials Reviews vol. 48, 2003, pp. 187-224.

[11] E. Dujardin and S. Mann, "Bio-Inspired Materials Chemistry," Advanced Materials, vol. 14, 2002, pp. 775-788.

[12] L. Addadi and S. Weiner, "Control and Design Principles in Biological Mineralization," Angew. Chem. Int. Ed. vol. 31, 1992, pp. 153-169.

[13] S. Weiner and L. Addadi, “Design Strategies in Mineralized Biological Materials," J. Mater. Chem., vol. 7, 1997, pp. 689-702.

[14] S. Mann, "Biomineralization: the Form(id)able Part of Bioinorganic Chemistry!," J. Chem. Soc. Dalton Trans., 1997, pp. 3953-3961.

[15] K. Naka and Y. Chujo, "Control of Crystal Nucleation and Growth of Calcium Carbonate by Synthetic Substrates," Chemistry of Materials, vol. 13, 2001, pp. 3245-3259. 
[16] A. Komeili, H. Vali, T. Beveridge, and D. Newman, "Magnetosome Vesicles are Present Before Magnetite Formation, and MamA is Required for their Activation," Proceedings of the National Academy of Sciences vol. 101, 2004, pp. 3839-3844.

[17] S. Mann, "Molecular Recognition in Biomineralization," Nature vol. 332, 1988, pp. 119-124.

[18] S. Mann, "Biomineralization and Biomimetic Materials Chemistry," J. Mater. Chem., vol. 5, 1995, pp. 935-946.

[19] S. Mann, B. Heywood, J. Didymus, S. Rajam, V. Wade, J. Walker, P. Calvert, and M. Alper, "Biomineralization: New Routes to Crystal Engineering," Materials Synthesis Utilizing Biological Processes, Boston, Massachusetts, USA. Materials Research Society, 1989, pp. 25-37.

[20] S. Patwardhan and S. Clarson, "Silicification and Biosilicification: Part 5 An Investigation of the Silica Structures Formed at Weekly Acidic $\mathrm{pH}$ and Neutral $\mathrm{pH}$ as Facilitated by Cationically Charged Macromolecules," Materials Science and Engineering C vol. 23, 2003, pp. 495-499.

[21] S. Patwardhan and S. Clarson, "Silicification and Biosilicification: Part 6 Poly-LHistidine Mediated Synthesis of Silica at Neutral $\mathrm{pH}$, " J.Inorganic and Organometallic Polymers vol. 13, 2003, pp. 49-53.

[22] D. Walsh, E. Boanini, J. Tanaka, and S. Mann, "Synthesis of tri-Calcium Phosphate Sponges by Interfacial Deposition and Thermal Transformation of Self-Supporting calcium Phosphate Films," J.Mater.Chem., vol. 15, 2005, pp. 10431048.

[23] T. Watanabe, I. Fukuda, K. China, and Y. Isa, "Molecular Analysis of Protein Components of the Organic Matrix in the Exoskeleton of Two Scleractinian Coral Species," Comparative Biochemistry and Physiology Part B vol. 136, 2003, pp. 767-774.

[24] C. Chan, G. De Stasio, S. Welch, M. Girasole, B. Frazer, M. Nesterova, S. Fakra, and J. Banfield, "Microbial Polysaccharides Template Assembly of Nanocrystal Fibers," Science vol. 303, 2004, pp. 1656-1658.

[25] A. Travaille, L. Kaptijn, P. Verwer, B. Hulsken, J. Elemans, R. Nolte, and H. van Kempen, "Highly Oriented Self-Assembled Monolayers as Templates for Epitaxial Calcite Growth," Journal of the American Chemical Society, vol. 125, 2003, pp. 11571-11577.

[26] A. Travaille, E. Steijven, H. Meekes, and H. vanKempen, "Thermodynamics of Epitaxial Calcite Nucleation on Self-Assembled Monolayers," Journal of Physical Chemistry B, vol. 109, Mar. 2005, pp. 5618-5626.

[27] J. Aizenberg, A. Black, and G. Whitesides, “Oriented Growth of Calcite Controlled by Self-Assembled Monolayers of Functionalized Alkanethiols Supported on Gold and Silver," Journal of the American Chemical Society, vol. 121, 1999, pp. 4500-4509. 
[28] S. Rajam, B. Heywood, J. Walker, S. Mann, R. Davey, and J. Birchall, “Oriented Crystallization of $\mathrm{CaCO}_{3}$ Under Compressed Monolayers Part 1. Morphological Studies of Mature Crystals," J. Chem. Soc. Faraday Trans. vol. 87, 1991, pp. 727734.

[29] B. Heywood, S. Rajam, and S. Mann, “Oriented Crystallization of $\mathrm{CaCO}_{3}$ Under Compressed Monolayers Part 2. Morphology, Structure and Growth of Immature Crystals," J. Chem. Soc. Faraday Trans. vol. 87, 1991, pp. 735-743.

[30] C. Ma, H. Lu, R. Wang, L. Zhou, F. Cui, and F. Qian, "Comparison of Controlled Crystallization of Calcium Phosphates under Three Kinds of Monolayers," J.Crystal.Growth, vol. 173, 1997, pp. 141-149.

[31] P. Simon, R. Ulrich, H. Spiess, and U. Wiesner, "Block Copolymer-Ceramic Hybrid Materials from Organically Modified Ceramic Precursors," Chemistry of Materials, vol. 13, 2001, pp. 3464-3486.

[32] C. Park, J. Yoon, and E. Thomas, "Enabling Nanotechnology with Self Assembled Block Copolymer Patterns," Polymer vol. 44, 2003, pp. 6725-6760.

[33] G. Soler-Illia, E. Crepaldi, D. Grosso, and C. Sanchez, "Block CopolymerTemplated Mesoporous Oxides," Current Opinion in Colloid and Interface Science vol. 8, 2003, pp. 109-126.

[34] D. Volkmer, M. Fricke, C. Agena, and J. Mattay, "Interfacial Electrostatics Guiding the Crystallization of $\mathrm{CaCO}_{3}$ Underneath Monolayers of Calixarenes and Resorcarenes," J.Mater.Chem., 2004, pp. 2249-2259.

[35] L. Zhang, H. Liu, R. Zhang, L. Zhang, D. Qian, Y. Mu, X. Yu, and X. Feng, “The Mineralization Process of Calcium Phosphate Induced by the LB Films of Porphyrin," Colloids and Surfaces A: Physicochemical and Engineering Aspects vol. 257-258, 2005, pp. 307-312.

[36] S. Champ, J. Dickinson, P. Fallon, B. Heywood, and M. Mascal, "HydrogenBonded Molecular Ribbons as Templates for the Synthesis of Modified Mineral Phases," Angew. Chem. Int. Ed. vol. 39, 2000, pp. 2716-2719.

[37] L. Estroff and A. Hamilton, "At the Interface of Organic and Inorganic Chemistry: Bioinspired Synthesis of Composite Materials," Chemistry of Materials, vol. 13, 2001, pp. 3227-3235.

[38] K. Naka and Y. Chujo, "Effect of Anionic Dendrimers on the Crystallization of Calcium Carbonate in Aqueous Solution," C.R. Chimie vol. 6, 2003, pp. 11931200.

[39] S. Mann, J. Didymus, N. Sanderson, B. Heywood, and E. Aso Samper, "Morphological Influence of Functionalized and Non-Functionalized $\omega$ Dicarboxylates on Calcite Crystallization," J. Chem. Soc. Faraday Trans. vol. 86 , 1990, pp. 1873-1880.

[40] C. Geffroy, A. Foissy, J. Persello, and B. Cabane, "Surface Complexation of Calcite by Carboxylates in Water," J.Colloid and Interface Sci., vol. 211, 1999, pp. 45-53. 
[41] Y. Han and J. Aizenberg, "Face-Selective Nucleation of Calcite on SelfAssembled Monolayers of Alkanethiols: Effect of the Parity of the Alkyl Chain," J. Am. Chem. Soc. vol. 42, 2003, pp. 3668-3670.

[42] G. Hunter, "Interfacial Aspects of Biomineralization," Current Opinion in Solid State $\mathcal{E}$ Materials Science vol. 1, 1996, pp. 430-435.

[43] B. Heywood and S. Mann, "Template-Directed Inorganic Crystallization: Oriented Nucleation of Barium Sulfate under Langmuir Monolayers of an Aliphatic Long chain Phosphonate," Advanced Materials vol. 8, 1992, pp. 14921498.

[44] B. Heywood and S. Mann, "Crystal Recognition at Inorganic-Organic Interfaces: Nucleation and Growth of Oriented $\mathrm{BaSO}_{4}$ under Compressed Langmuir Monolayers," Advanced Materials, vol. 4, 1992, pp. 278-282.

[45] S. Mann, B. Heywood, S. Rajam, and J. Birchall, "Controlled Crystallization of $\mathrm{CaCO}_{3}$ Under Stearic Acid Monolayers ," Nature vol. 334, 1988, pp. 692-695.

[46] S. Mann, B. Heywood, S. Rajam, and J. Birchall, "Interfacial Control of Nucleation of Calcium Carbonate Under Organized Stearic Acid Monolayers," Proc. R. Soc. London vol. A423, 1989, pp. 457-471.

[47] B. Heywood and S. Mann, "Organic Template-Directed Inorganic Crystallization: Oriented Nucleation of $\mathrm{BaSO}_{4}$ Under Compressed Langmuir Monolayers," Journal of the American Chemical Society, vol. 114, 1992, pp. 46814686.

[48] B. Heywood and S. Mann, "Template-Directed Nucleation and Growth of Inorganic Materials," Advanced Materials, vol. 6, 1994, pp. 9-20.

[49] L. Addadi, S. Raz, and S. Weiner, "Taking Advantage of Disorder: Amorphous Calcium Carbonate and Its Role in Biomineralization," Advanced Materials, vol. 15, 2003, pp. 959-970.

[50] L. Zhang, H. Liu, X. Feng, R. Zhang, L. Zhang, Y. Mu, J. Hao, D. Qian, and Y. Lou, "Mineralization Mechanism of Calcium Phosphates under Three Kinds of Langmuir Monolayers," Langmuir vol. 20, 2004, pp. 2243-2249.

[51] X. Xu, J. Han, and K. Cho, "Formation of Amorphous Calcium Carbonate Thin Films and Their Role in Biomineralization," Chemistry of Materials, vol. 16, 2004, pp. 1740-1746.

[52] H. Cölfen and S. Mann, "Higher-Order Organization by Mesoscale SelfAssembly and Transformation of Hybrid nanostructures," Angew.Chem.Int.Ed. vol. 42, 2003, pp. 2350-2365.

[53] M. Li and S. Mann, "Emergent Nanostructures: Water-Induced Mesoscale Transformation of Surfactant-Stabilized Amorphous Calcium Carbonate Nanoparticles in Reverse Microemulsions," Advanced Functional Materials, vol. 12, 2002, pp. 773-779.

[54] E. DiMasi and L. Gower, "Synchrotron X-ray Observations of a Monolayer Template for Mineralization," Langmuir 2002, pp. 301-306. 
[55] J. Kmetko, A. Datta, G. Evmenenko, and P. Dutta, “The Effects of Divalent Ions on Langmuir Monolayer and Subphase Structure: A Grazing-Incidence Diffraction and Bragg Rod Study," Journal of Physical Chemistry B, vol. 105, Nov. 2001, pp. 10818-10825.

[56] V. Kaganer, H. Möhwald, and P. Dutta, "Structure and Phase Transitions in Langmuir Monolayers," Reviews of Modern Physics, vol. 71, 1999, pp. 779-819.

[57] R. Johann, D. Vollhardt, and H. Möhwald, "Shifting of Fatty Acid Monolayer Phases Due to Ionization of the Headgroups," Langmuir vol. 17, 2001, pp. 45694580 .

[58] M. Yazdanian, H. Yu, and G. Zografi, "Ionic Interactions of Fatty Acid Monolayers at the Air/Water Interface," Langmuir vol. 6, 1990, pp. 1093-1098.

[59] P. Miranda, Q. Du, and Y. Shen, "Interaction of Water with a Fatty Acid Langmuir Film," Chemical Physics Letters, vol. 286, 1998, pp. 1-8.

[60] D. Nutt and A. Stone, "Theoretical Studies of the Interface Between Water and Langmuir Films of Aliphatic Alcohols," J.Chem.Phys., vol. 119, 2003, pp. 56705679 .

[61] E. Le Calvez, D. Blaudez, T. Buffeteau, and B. Desbat, "Effect of Cations on the Dissociation of Arachidic Acid Monolayers on Water Studied by PolarizationModulated Infrared Reflection-Absorption Spectroscopy," Langmuir vol. 17, 2001, pp. 670-674.

[62] T. Bohanon, B. Lin, M. Shih, G. Ice, and P. Dutta, "Determination of Lattice Structure and Calculation of Molecular Tilt in Lipid Monolayers on Water Using X-ray Diffraction," Phys.Rev.B, vol. 41 , 1990, pp. 4846-4849.

[63] Y. Wang, X. Du, and H. Liu, "Chain Orientation and Headgroup Structure in Langmuir Monolayers of Stearic Acid and Metal Stearate (Ag, Co, Zn, and Pb) Studied by Infrared Reflection-Absorption Spectroscopy," J.Chem.Phys., vol. 124 , 2006, pp. 134706-1-134706-9.

[64] I. Hamley, Introduction to Soft Matter Polymers, Colloids, Amphiphiles and Liquid Crystals, John Wiley \& Sons, Ltd, 2000.

[65] D. Kuang, A. Xu, Y. Fang, H. Ou, and H. Liu, "Preparation of Inorganic Salts $\left(\mathrm{CaCO}_{3}, \mathrm{BaCO}_{3}, \mathrm{CaSO}_{4}\right)$ Nanowires in the Triton X-100/Cyclohexane/Water Reverse Micelles," J.Crystal.Growth, vol. 244, 2002, pp. 379-383.

[66] E. Eanes, P. Calvert, and M. Alper, "Calcium Phosphate Precipitation in Liposomal Suspensions," Materials Synthesis Utilizing Biological Processes, Boston, Massachusetts, USA. Materials Research Society, 1989, pp. 15-24.

[67] S. Mann, B. Heywood, S. Rajam, J. Walker, R. Davey, and J. Birchall, "Crystal Synthesis Under Langmuir Monolayers," Advanced Materials, vol. 2, 1990, pp. 257-261.

[68] Y. Li, P. Zhang, F. Dong, X. Cao, X. Song, and X. Cui, “The Array and Interfacial Activity of Sodium Dodecyl Benzene Sulfonate and Sodium Oleate at the Oil/Water Interface," J.Colloid and Interface Sci., vol. 290, 2005, pp. 275-280. 
[69] E. DiMasi, M. Olszta, V. Patel, and L. Gower, "When is Template Directed Mineralization Really Template Directed?," CrystEngComm, vol. 5, 2003, pp. 346350.

[70] E. Loste, E. Diaz-Marti, A. Zarbakhsh, and F. Meldrum, "Study of Calcium Carbonate Precipitation under a Series of Fatty Acid Langmuir Monolayers Using Brewster Angle Microscopy," Langmuir vol. 19, 2003, pp. 2830-2837.

[71] A. Milev, G. Kannangara, and M. Wilson, "Template-Directed Synthesis of Hydroxyapatite from a Lamellar Phosphonate Precursor," Langmuir vol. 20, 2004, pp. 1888-1894.

[72] S. Kamhi, "On the Structure of Vaterite, $\mathrm{CaCO}_{3}$ " Acta Cryst., vol. 16, 1963, pp. 770-772.

[73] B. Heywood and S. Mann, "Molecular Construction of Oriented Inorganic Materials: Controlled Nucleation of Calcite and Aragonite under Compressed Langmuir Monolayers," Chemistry of Materials, vol. 6, 1994, pp. 311-318.

[74] D. Duffy and J. Harding, "Growth of Polar Crystal Surfaces on Ionized Organic Substrates," Journal of Physical Chemistry B vol. 20, 2004, pp. 7637-7642.

[75] Kuther, J.; Seshadri, R.; Knoll, W.; Tremel, W. “Templated Growth of Calcite, Vaterite and Aragonite Crystals on Self-Assembled Monolayers of Substituted Alkylthiols on Gold." J. Mater. Chem. 1998, 8, 641-650.

[76] Schwartz, D. K. “Langmuir-Blodgett Film Structure.” Surface Science Reports, 27, 1997; pp 241-334.

[77] Sobotka, H.; Demeny, M.; Chanley, J. D. "Radioactive tracer studies of monolayers : II. Comparison of floating and built-up monolayers." Journal of Colloid Science 1958, 13 (6), 565-568.

[78] Kobayashi, K.; Takaoka, K.; Ochiai, S. "Application of X-ray photoelectron spectroscopy and fourier transform IR-reflection absorption spectroscopy to studies of the composition of Langmuir-Blodgett films." Thin Solid Films 1988, 159 (1-2), 267-273.

[79] Gericke, A.; Hühnerfuss, H. "The Effect of Cations on the Order of the Saturated Fatty Acid Monolayers at the Air-Water Interface as Determined by Infrared Reflection-Absorption Spectrometry." Thin Solid Films. 245, 1994; pp 74-82.

[80] Kmetko, J. "Effects of Divalent Ions on Langmuir Monolayers: Synchrotron X-ray Scattering Studies." PhD Thesis, Northwestern University, Evanston, Illinois, USA., 2002.

[81] Vogel, V.; Möbius, D. "Local Surface Potentials and Electric Dipole Moments of Lipid Monolayers: Contributions of the Water/Lipid and the Lipid/Air Interfaces." Journal of Colloid and Interface Science 1988, 126 (2), 408-420.

[82] Israelachvili, J. N. "Intermolecular and Surface Forces;" 2nd Ed. ed.; Academic Press: 1992.

[83] Vogel, V.; Möbius, D. "Hydrated Polar Groups in Lipid Monolayers: Effective Local Dipole Moments and Dielectric Properties." Thin Solid Films 1988, 159, 73- 
81.

[84] Duffy, D. M.; Harding, J. H. "Simulation of Organic Monolayers as Templates for the Nucleation of Calcite Crystals." Langmuir 2004, 20, 7630-7636.

[85] DiMasi, E.; Patel, V. M.; Munisamy, S.; Olszta, M.; Gower, L. B. "Synthetic Seashells: Biomimetic Mineral Nucleation at a Langmuir Monolayer." NSLS Activity Report 2001, 2-68-2-72.

[86] Donners, J. J. J. M.; Nolte, R. J. M.; Sommerdijk, N. A. J. M. “A Shape-Persistent Polymeric Crystallization Template for $\mathrm{CaCO}_{3}$." J. Am. Chem. Soc. 2002, 124, 9700-9701.

[87] Ahn, D. J.; Berman, A.; Charych, D. "Probing the Dynamics of TemplateDirected Calcite Crystallization with in Situ FTIR." J. Phys. Chem. 1996, 100, 12455-12461.

[88] Berman, A.; Charych, D. "Oriented Nucleation of Inorganic Salts on Polymeric Long Chain Acid Monolayers." J. Crystal. Growth 1999, 198/199, 796-801.

[89] Buijnsters, P. J. J.; Donners, J. J. J. M.; Hill, S. J.; Heywood, B. R.; Nolte, R. J. M.; Zwanenburg, B.; Sommerdijk, N. A. J. M. “Oriented Crystallization of Calcium Carbonate under Self-Organized Monolayers of Amide-Containing Phospholipids." Langmuir 2001, 17, 3623-3628.

[90] Kwak, S.-Y.; DiMasi, E.; Han, Y. J.; Aizenberg, J.; Kuzmenko, I. “Orientation and $\mathrm{Mg}$ Incorporation of Calcite Grown on Functionalized Self-Assembled Monolayers: A Synchrotron X-ray Study." Crystal Growth E Design, 5, 2005; pp 2139-2145.

[91] Turnbull, A. G. "A thermochemical study of vaterite." Geochimica et Cosmochimica Acta 1973, 37 (6), 1593-1601. 


\section{Chapter 2: Experimental}

\subsection{Materials and Methods}

The surfactants investigated in this study: octadecanoic acid (ODA, >99\%, Merck), octadecanol (ODOH, 299\%, Fluka), DL-2-hydroxyoctadecanoic acid (2-HSA, >99\%, Sigma), 2-methyloctadecanoic acid (2-MODA, 97\%, Aldrich), 2-bromooctadecanoic acid (2-BODA, Sigma), and 3-hydroxyoctadeacnoic acid (3-HSA, 97\%, Indofine Chemicals Inc.) were used as supplied, without further purification. The seventh surfactant ODMA was synthesised in-house using the methodology described below. Analytical grade chloroform (Labscan AR) was used as the spreading solvent.

\subsection{ODMA Synthesis}

There is a number of synthetic routes for alkylated alkanedioic acids, which have been outlined by Diaper and Kuksis[1]. The particular process chosen was used by Sharma and Biswas[2] in the synthesis of a series of $n$-alkyl malonic acids, and involves relatively uncomplicated chemistry (Figure 2.1).

The methodology first required $\sim 40 \mathrm{~mL}$ of sodium ethoxide, this was produced fresh from dry ethanol $(\mathrm{EtOH})$ and sodium metal under flowing nitrogen. Upon completion of the reaction, $9 \mathrm{~mL}$ of diethylmalonate $(>99 \%$, Fluka) was added. This was followed by the slow addition of octadecylbromide (98\%, Sigma). The solution was left gently refluxing until the $\mathrm{pH}$ of the solution became neutral which was typically achieved in $\sim 30$ hours. The NaBr precipitate was filtered off and washed with EtOH. The filtrate was rotary-evaporated to remove the EtOH. The $\mathrm{NaBr}$ precipitate was redissolved in water and combined with the distilled product. The mixture was shaken and allowed to separate. The lower aqueous layer was extracted 
twice with ethyl acetate. The ester/product mix was dried with magnesium sulfate (>99\%, Pure Science) overnight and then filtered. After two recrystallisation cycles the ethyl acetate was then removed, before the deprotection using $2 \mathrm{M} \mathrm{KOH}$. Further purification of the potassium salt was performed before the final acidification step.

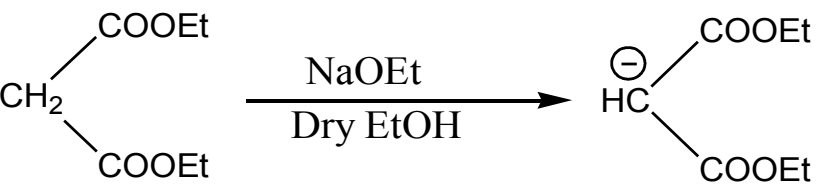

$+$

$\mathrm{CH}_{3}\left(\mathrm{CH}_{2}\right)_{17} \mathrm{Br}$
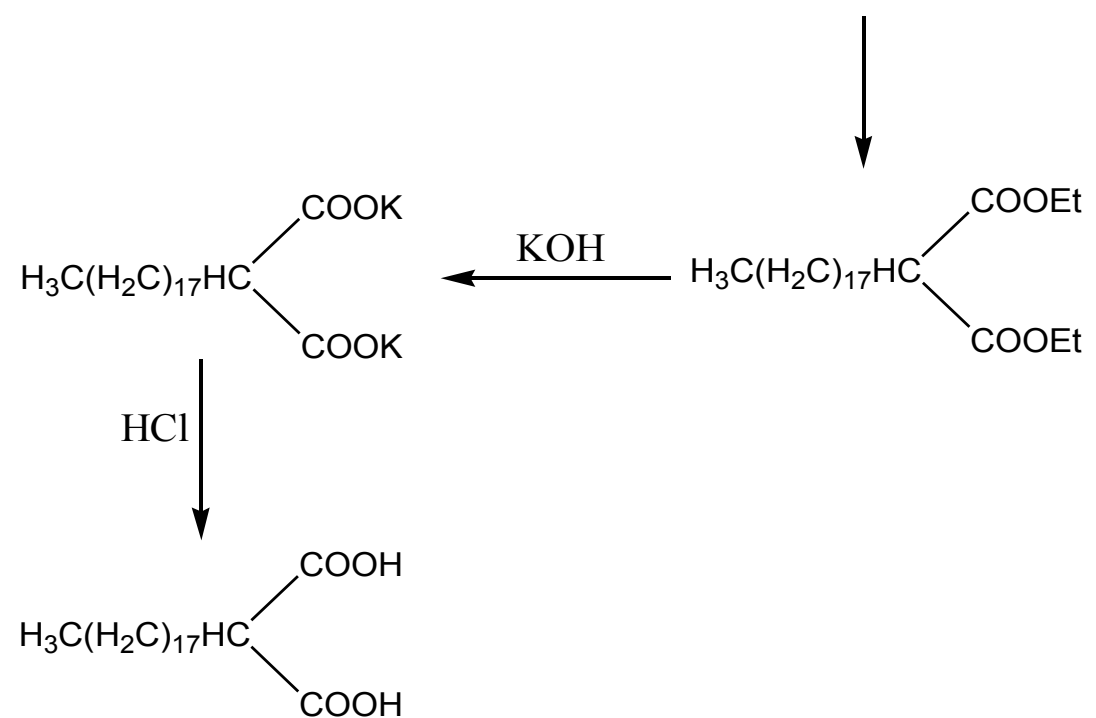

Figure 2.1 A scheme for the synthesis of octadecylmalonic acid (ODMA).

- Elemental analysis: Calculated for $\mathrm{C}_{21} \mathrm{H}_{40} \mathrm{O}_{4}$ : $\mathrm{C} 70.74 \%, \mathrm{H} 11.31 \%$, and $\mathrm{O}$ 17.95\%. Measured: C 71.01\%, H 11.25\%, and O 17.74\%.

- NMR analysis: Proton $\mathrm{C}_{3}-\left(\mathrm{CH}_{2}\right)_{17} 0.86 \delta ; \mathrm{CH}_{3}-\left(\mathrm{C}_{2}\right)_{16} 1.28 \delta ;\left(\mathrm{CH}_{2}\right)_{16}-\underline{\mathrm{CH}}_{2}-\mathrm{CH}$

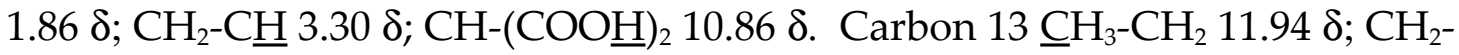

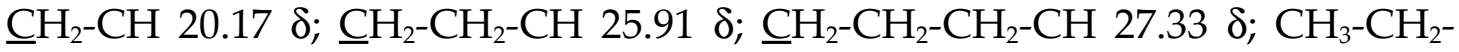
$\mathrm{CH}_{2}-\left(\mathrm{CH}_{2}\right)_{12}-\mathrm{CH}_{2} 27.80 \delta$; $\left(\underline{\mathrm{CH}}_{2}\right)_{12}-\mathrm{CH}_{2}-\mathrm{CH}_{2}-\mathrm{CH}_{2}-\mathrm{CH} 28.16 \delta ; \mathrm{CH}_{3}-\mathrm{CH}_{2}-\mathrm{CH}_{2}-$ $\left(\mathrm{CH}_{2}\right)_{12} 30.39 \delta ; \mathrm{CH}_{2}-\mathrm{CH}_{2}-\underline{\mathrm{CH}}-(\mathrm{COOH})_{2} 49.73 \delta ; \mathrm{CH}_{2}-\mathrm{CH}-(\underline{\mathrm{COOH}})_{2} 168.55 \delta$. Note due to solubility issues this NMR was obtained using tetrahydrofuran- $\mathrm{d}_{8}$. The full patterns can be found in Appendix 1. 


\subsection{Aqueous Subphases}

The aqueous subphase solutions were made from calcium chloride dihydrate ( $>99 \%$, Sigma Aldrich), sodium bicarbonate ( $>99 \%$, Romil), and ultrapure deionised water (double-distillation fed Millipore purification unit, resistivity of $18.2 \mathrm{M} \Omega \mathrm{cm}$ ). The $\mathrm{pH}$ of the $20 \mathrm{mM} \mathrm{NaHCO}_{3}$ subphase ( $\mathrm{pH} \sim 8.5$ ) was reduced by bubbling carbon dioxide, generating a $\mathrm{CO}_{2}$ supersaturated solution with a $\mathrm{pH} \sim 6.0$. The $10 \mathrm{mM}$ calcium carbonate subphase $(\mathrm{pH} \quad$ 5.8 ) was obtained by combining equal concentration volumes of $20 \mathrm{mM} \mathrm{CaCl}_{2}$ and $20 \mathrm{mM} \mathrm{NaHCO}_{3}$ solutions, presupersaturated in $\mathrm{CO}_{2}(\mathrm{~g})$. In comparison the bulk $\mathrm{pH}$ value for the water and $20 \mathrm{mM}$ $\mathrm{CaCl}_{2}$ subphases were 5.6 and 5.5, respectively.

When making comparisons across different electrolytes it is common to use concentrations that result in a standard concentration, activity, ionic strength or $\mathrm{pH}$. However, because this study is focussed on the interfacial region, comparisons of bulk concentrations etc. is of limited value. Ideally, surface-based values would be used, however there is no definitive universal approach to calculating surface based quantities. The selection of $10 \mathrm{mM}$ for the CCCS was based on common practise, where the Kitano method[3], which achieves a concentration of $\sim 9 \mathrm{mM}$ is the benchmark for many calcium carbonate crystallisation investigations, in particular, those utilising a Langmuir monolayer to induce crystallisation[4]. Hence the selection was based on literature precedence.

Experiments investigating the effect of concentration for both the pure acid and pure alcohol on 10, 20 and $40 \mathrm{mM} \mathrm{CaCl}_{2}$ showed no variation, within experimental error, under our experimental conditions. Similar experiments with 100 mol.\% ODA on 5, 10, 15 and $20 \mathrm{mM} \mathrm{NaHCO}_{3}$ again showed little variation with concentration. Further the monolayers on 5 and $10 \mathrm{mM} \mathrm{NaHCO}_{3}$ subphases were found to be very unstable, collapsing at relatively low pressures. This instability was likely due to the higher levels of $\mathrm{CO}_{2}$ gas that were able to be achieved at the lower $\mathrm{HCO}_{3}^{-}$ 
concentrations leading to excessive bubbling which disrupted the monolayer. In contrast, bubbling was unable to lower the $\mathrm{pH}$ sufficiently in solutions with concentrations greater than $20 \mathrm{mM} \mathrm{NaHCO}$.

Given this lack of monolayer response to subphase concentration variation and that the surface concentration, of which we are ultimately interested, is not reflected in the bulk measurements it was deemed adequate to use the $20 \mathrm{mM} \mathrm{CaCl}_{2}$ and $\mathrm{NaHCO}_{3}$ solutions generated to formulate the saturated $\mathrm{CaCO}_{3}$ crystallising subphase. Further the aim of the subphase analysis was not to provide a direct comparison of the monolayer behaviour on the different subphases but rather a deconstruction of the CCCS in order to elucidate the nature of the specific ion interactions.

A similar view was held for the control of subphase $\mathrm{pH}$; where, across the four subphases the variation in $\mathrm{pH}$ was $\sim 0.5 \mathrm{pH}$ units. The $\mathrm{NaHCO}_{3}$ and $\mathrm{CCCS}$ subphases were found to be limited in terms of the obtainable $\mathrm{pH}$ by the buffering effect of the $\mathrm{H}_{2} \mathrm{CO}_{3} / \mathrm{HCO}_{3}{ }^{-} / \mathrm{CO}_{3}{ }^{2-}$ equilibrium, hence a uniform $\mathrm{pH}$ of 5.8 to 6.0 would have been required across all the subphases. However, adjusting the $\mathrm{pH}$ of the water subphase to $\mathrm{pH} 6$ would have required the addition of potential determining ions, which would have confounded the investigation into the role of water alone.

Experimentation into the effect of $\mathrm{pH}$ for the pure acid on $20 \mathrm{mM} \mathrm{CaCl}_{2}$ did show some variation across the $5.5-6.0 \mathrm{pH}$ range. $A_{m}$ at onset and at $\Pi=10 \mathrm{mNm}^{-1}$ showed negligible change but the $\Pi$ at transition and $\Delta \mathrm{V}_{\max }$ values did reflect some changes in the $\mathrm{pH}$. Despite this it was deemed sufficient to us the unaltered systems as the main focus was qualitative trend analysis rather direct quantitative comparison of individual systems.

\subsection{1 pH Measurements}

$\mathrm{pH}$ measurements were performed using a Mettler Toledo Seven Easy pH meter with a flat electrode for use in the Langmuir trough. 


\subsection{Surface Pressure-Area Isotherms}

Surface pressure and potential isotherms were measured at room temperature $\left(\sim 20^{\circ} \mathrm{C}\right)$ using a NIMA 702BAM PTFE trough (area $\left.\sim 700 \mathrm{~cm}^{2}\right)$. Prior to each experiment the trough and barriers were cleaned thoroughly with AR grade chloroform, and subsequently rinsed with ultra pure water. Whatman chromatography paper (Char 1) was used for the Wilhelmy plate. With the barriers fully open, $50-100 \mu \mathrm{L}$ of $\sim 0.5-1.5 \mathrm{mg} \mathrm{mL}^{-1}$ solution (chloroform solvent) was spread onto the surface of the water using a Hamilton microsyringe. After 10 minutes it was assumed that the solvent had evaporated and only a layer of solute remained at the subphase/air interface. The barriers were compressed at a speed of $100 \mathrm{~cm}^{2} \mathrm{~min}^{-1}$. This relatively high speed was adopted for two reasons 1) to ensure non-equilibrium conditions and 2) to minimise calcium carbonate nucleation. The higher compression speeds also have the advantage of avoiding inherent drift often observed in surface potential measurements.

\subsubsection{Surface Potential}

Surface potential measurements were made using a Trek Electrostatic Voltmeter (320C) and a 3250 high-sensitivity vibrating-plate probe from Trek INC, Medina NY, USA (Figure 2.2). In all cases the null voltage was established on the bare subphase, and the measurements were logged in conjunction with the surface pressure and area by the NIMA software (Version 5.16).

The surface potential sensor consists of a stationary stainless steel electrode in the subphase, and a second vibrating electrode $\sim 2 \mathrm{~mm}$ above the air/water interface. The vibration of the air-based electrode creates periodic variations in the capacitance, and the resulting change in the current is compensated for by a change in the potential. The presence, and conformation of the monolayer, in conjunction with the any induced subphase restructuring alters the capacitance between the electrodes. Having established a null voltage prior to adding the surfactant, the potential $(\Delta V)$ 
associated with the monolayer can be measured.
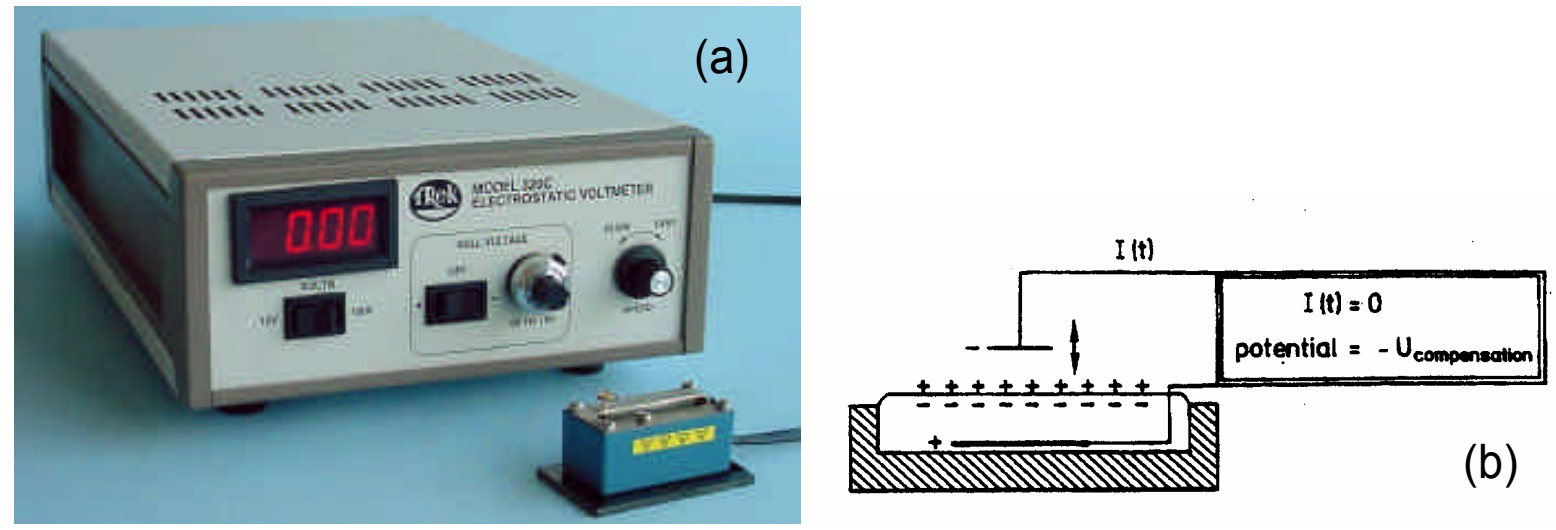

Figure 2.2 (a) A picture of the Trek 320-H-CE electrostatic voltmeter with a 3250 vibrating plate probe used in experimentation. (b) A schematic of the likely experimental set up, based on the work completed by Vogel and Möbius $\{5,6\}$.

The change in surface potential by the formation of the monolayer, $\Delta V$, is defined by the Helmholtz equation[5,6]:

$$
\Delta V=12 \pi n \mu_{\perp}
$$

where $n$ is the number of molecules and is given by $1 / A_{0}$, the mean area per molecule $\left(A_{0}\right)$ and $\mu \perp$ is the effective dipole moment normal to the interface. For charged monolayers $\Delta V$ includes the potential $\Psi$ of the diffuse double layer, thus Equation 21 becomes:

$$
\Delta V=12 \pi \frac{\mu_{\perp}}{A_{0}}+\psi
$$

Although the underlying assumptions made in the derivation of this equation mean that the equation is only valid when the contribution of the dipoles to $\Delta V$ of a monolayer are independent of $\Psi_{0}$, it does provide a practical approximation.

\subsubsection{Analysis of the Isotherm Data}

Five key values were extracted from the data and were used in conjunction with surface potential measurements for trend comparison.

1. The area per molecule at the onset transition, that is from the liquid expanded 
or condensed/liquid expanded coexistence phase to the tilted condensed phase ( $L_{2}$ for fatty acids or $L_{2}^{\prime}$ for fatty alcohols).

2. The area per molecule at a constant pressure of $10 \pm 0.1 \mathrm{mN} \mathrm{m}^{-1}$.

3. The area per molecule at the transition from the tilted $L_{2}$ phase for fatty acids or $L_{2}^{\prime}$ for fatty alcohols to the untilted $L S$ phase.

4. The surface pressure at the tilted $L_{2} / L_{2}$ ' to the untilted LS phase transition.

5. The area per molecule at a constant pressure of $25 \pm 0.1 \mathrm{mN} \mathrm{m}^{-1}$.

The two fixed pressures $\left(\Pi=10\right.$ and $25 \mathrm{mN} \mathrm{m}^{-1}$ ) were chosen as they lie in the tilted and untilted phases respectively for the majority of systems investigated. In all cases the transition areas and pressures were determined by finding the intersection of the linear regions that proceed and follow the transition.

The surface potential results presented are the maximum value reached during the isotherm, this approach is consistent with the methodology employed in the literature[7]. The observed trend is independent of alignment of the $\Delta \mathrm{V}_{\max }$ collection point with any one of the surface pressure parameters, as discussed above.

\subsubsection{Reproducibility}

Weak molecular interactions between the surfactant molecules that constitute the monolayer means that the system is dynamic. Further, high compression speeds, external vibrations, the method of film application, the time allowed for equilibration, thermal fluctuations, impurities and the polydispersity of the film all contribute to variable monolayer behaviour.

Consequently for all systems a minimum of four isotherms were performed, with as many as 20 replications for the less reproducible systems. Reproducibility was adjudged by visual comparison of the isotherms and by statistical analysis of the variation. For each isotherm the five key values (described above) were determined, these were then averaged and a standard deviation generated. Using these sample 
averages $(\bar{x})$ and standard deviations $(\sigma)$, a test ratio (TR) was determined (Equation 23), which is a measure of how each isotherm represents the overall sample monolayer behaviour. The isotherm with the lowest TR was then selected as a representative isotherm and used in images. Any comparative analysis of different systems was based on the calculated averages.

$$
T R=\left(\frac{x-\bar{x}}{\sigma}\right)^{2}
$$

\subsubsection{Brewster Angle Microscopy}

Interfacial reflectivity depends on the polarisation of the incident light and the incidence angle. For an interface where the change in refractive index from $n_{1}$ to $n_{2}$ is sharp (a Fresnel interface), and the light is P-polarised, the reflectivity vanishes when

the incident angle equals the Brewster angle $\left(\theta_{B}\right)$, defined as $\tan \theta_{B}=\frac{n_{2}}{n_{1}}$. In reality, rather than vanishing completely at the Brewster angle the reflected light intensity is reduced to a minimum. This minimum reflected light intensity is strongly dependent on the properties of the interface, where the interfacial reflectivity at the Brewster angle for P-polarised light has three contributing factors:

1) the thickness of the interface, where a dense monolayer of surfactant introduces a variation of refractive index over a thickness of $20-25 \AA$. This thickness is dependent on the phase domain of the monolayer.

2) The roughness of the interface, originating from thermal fluctuations. Ordinarily with the surface tension of water being so high, these effects are negligible, producing only a small error in the film thickness $(\sim 3 \AA)$.

3) The anisotropy of the monolayers. Some phases are optically isotropic, which can greatly increase the reflected intensity[8].

Images and video footage were collected of the monolayer isotherms using a MicroBAM2 from Nanofilm Technologie GmbH. With a fixed angle of incidence 
$53 \pm 2^{\circ}$ (the Brewster angle for an air water interface) and parallel polarised light, the reflectivity differences associated with variation in the refractive index created by the monolayer were imaged. The light source was a class B laser diode light of $659 \mathrm{~nm}$ and $>20 \mathrm{~mW}$, with a maximum optical power of $30 \mathrm{~mW}$ at the aperture of the instrument. The beam is collimated with a diameter of approximately $6 \mathrm{~mm}$.

\subsection{Crystallisation}

Crystal growth experiments were performed in a NIMA 102 PTFE trough (Figure 2.3). Solutions of $20 \mathrm{mM} \mathrm{CaCl}$ and $20 \mathrm{mM} \mathrm{NaHCO}_{3}$ were bubbled with carbon dioxide at a rate of $\sim 10 \mathrm{~mL} / \mathrm{min}$ for at least 30 minutes. Equi-volumes of the two solutions were then combined and added to the trough. $15 \mu \mathrm{L}$ of approximately 1.2 $\mathrm{mg} / \mathrm{mL}$ surfactant solution was applied to the air/water interface using a $10 \mu \mathrm{L}$ Hamilton glass syringe, and left for 10 minutes to allow solvent evaporation and monolayer equilibration. Pressure was subsequently applied and maintained at the required level using NIMA software. The pressure was applied at a rate of $10 \mathrm{~cm}^{2} \mathrm{~min}^{-1}$ until the desired pressure of either 10 or $25 \mathrm{mN} \mathrm{m}^{-1}$ was obtained. The trough was temperature controlled at $20 \pm 1.0^{\circ} \mathrm{C}$ using a water bath/chiller unit combination. In order to minimise evaporation from the trough over the extended growth period, water reservoirs and thermostatically controlled heat lamps were placed inside the trough cabinet as a means of maintaining a constant humidity and air temperature.

Crystals were harvested after approximately 16 hours of growth using $12 \mathrm{~mm}$ diameter glass cover slips via the horizontal Langmuir-Schaefer method[9]. In order to maximise the crystal yield the glass cover slips were coated with a hydrophobic hexamethyldisilazane (HDMS) layer via overnight exposure to HDMS vapour in a glass desiccator. Immediately after harvesting, the crystals were carefully washed with water, dabbed dry, mounted on an SEM stub and oven dried at $\sim 50^{\circ} \mathrm{C}$ overnight. In all cases three collections were made from different areas in the trough, and each 


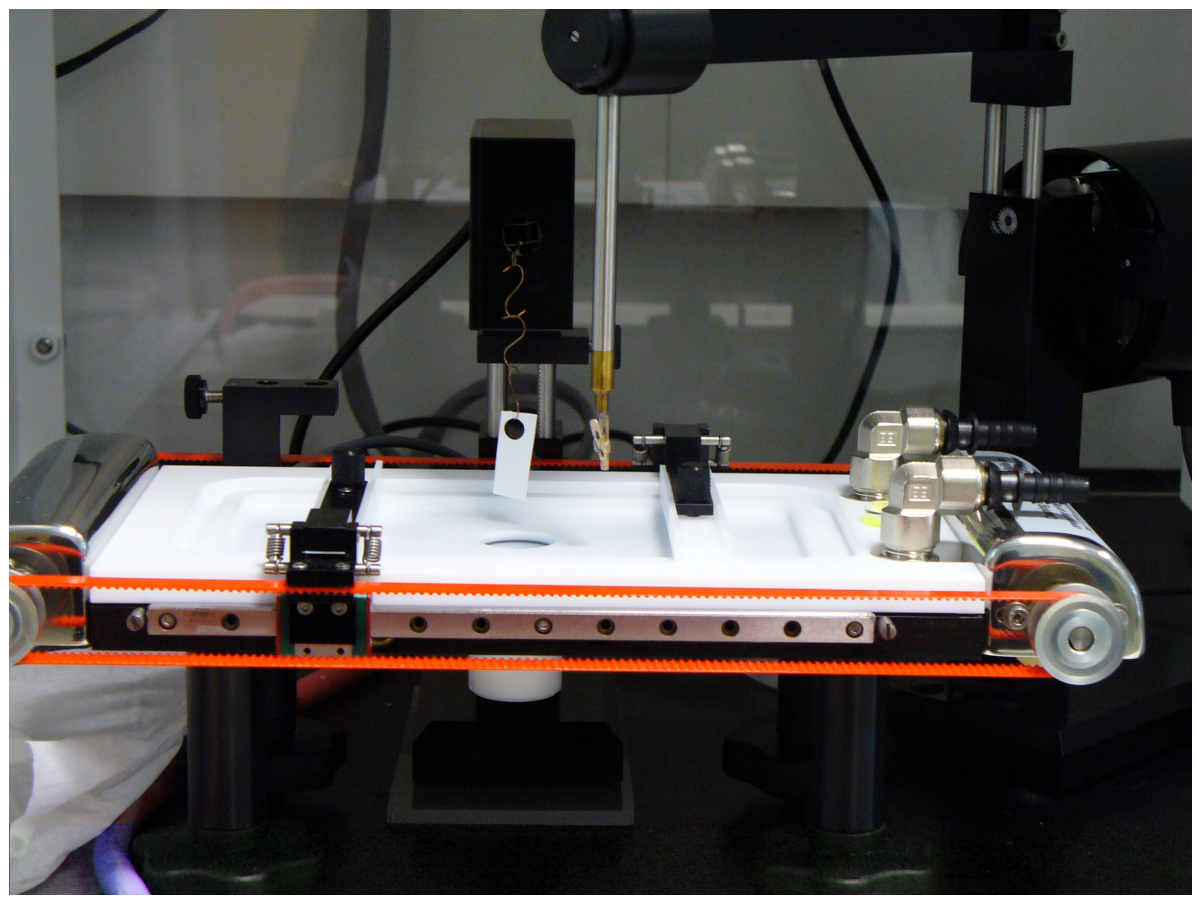

Figure 2.3 A photograph of the NIMA 102 Langmuir-Blodgett trough that was used for crystallisation experiments. In the centre of the picture is the Wilhelmy plate based surface pressure sensor.

growth system was repeated. Imaging involved a JEOL 5300 LVM SEM after sputter coating with $4 \mathrm{~nm}$ of gold. In order to minimise charging effects, images were commonly collected using the back-scattered mode. A minimum of 30 crystal images were collected for each system, from which a representative average was obtained.

Analysis of the crystals involved the measurement of inter-edge angles in order to ascertain the nucleation face, as described by Archibald et al.[10]. Using a digitised image of the crystals, the inter-edge angles were measured using SemAfore software (Version 5.0, JEOL (Skandinaviska) AB, Hammarbacken 6 A, Sweden). Nucleation face assignment was based on a comparison of these angle measurements with computer-generated idealised rhombohedral models of calcite with a known orientation, using SHAPE for Windows (Version 7.2.2, Shape Software, Kingsport, TN 37663 USA). The protocol for nucleation face assignment involved the modelling of 48 different faces of calcite[11,12]. See Appendix 4 for images of the 48 models.

Crystallographically there are three types of preferred orientation, with crystals 
oriented with a definite zone axis but somewhat randomly about this direction being the most common form. A zone axis is defined as a vector parallel to a set of faces or planes within a crystal, and thus meets the Weiss' Zone Law: uh $+v k+w l=0[13]$. Assigned crystal nucleation faces were grouped according to common zone axes in order to probe the nature of the preferential crystal orientation.

\subsection{X-ray Techniques}

GIXD and XRR measurements were performed at the Argonne National Laboratory, Advanced Photon Source (APS) on ChemMatCARS beam line 15 ID-C. The X-ray wavelength was $1.2244 \AA(10.126 \mathrm{keV})$. The beam line was fitted with a PTFE Langmuir trough for in situ X-ray scattering from the liquid surface. Surface pressure was determined using a Wilhelmy plate and maintained using the NIMA V5.16 software. The experimental subphase temperature was maintained at $20^{\circ} \mathrm{C}$, under an atmosphere of helium. The beam line and trough set up has been described previously[14].

To avoid 'beam damage' the trough was regularly moved to expose new areas of the monolayer to the beam footprint, this occurred between GIXD and during XRR experiments, as is common practised for studies of this kind. Throughout experimentation data was checked for peak shape irregularities and XRR patching errors as indicators for beam damage.

\subsubsection{GIXD Measurement and Analysis}

GIXD experimental data were collected using a Pilatus 100K detector in either a pinhole geometry with a resolution of $5 \mathrm{mrad}$, or line scans taken in a two-slit geometry with a resolution of $2 \mathrm{mrad}$. The incident angle $(\alpha)$ was $0.1^{\circ}$, and scans of the reflected angle $(\beta)$ involved values of $0.1,4$ and $8^{\circ}$. Standard GIXD scans were performed through a $2 \theta$ range of $13-19.5^{\circ}$ and the pinhole scans were performed through a range of $16.3-19.5^{\circ}$. 
In order to assess whether the pinhole geometry introduced spatial or angular irregularities into diffraction images, due to the large beam 'footprint', duplicate parallel GIXD and pinhole scans were performed at the beginning of each crystallisation experiment. A comparison of these scans found no evidence of irregular peak shapes or other irregularities in moving between geometries.

\subsubsection{Symmetry and Peak (hk) Assignment}

In GIXD the scattered intensity is monitored as a function of: (1) the angle between the incident and scattered beams in the plane of the subphase; and (2) the angle between the scattered beam and the subphase surface (Figure 1.15). As the monolayer consists of many independently oriented domains the diffraction pattern is a 2-D powder. Thus peaks in the diffraction pattern reflect an averaging of the periodicity or crystallinity of the monolayer domains. This averaging and the 2-D nature of monolayers means that of the three components $\left(Q_{x}, Q_{y}\right.$, and $\left.Q_{z}\right)$ of the momentum transfer vector $Q$, only the vertical $Q_{z}$ component can be individually determined. In terms of $Q_{x}$ and $Q_{y}$, only the combined $Q_{x y}$ value $\left(Q_{x y}^{2}=Q_{x}^{2}+Q_{y}^{2}\right)$ can be measured.

In reciprocal space the scattering pattern is given by the product of two terms: the form factor, and the structure factor. The form factor of the individual rod-like molecules in reciprocal space is a disk on a plane normal to the long axis of the molecule. The structure factor reflects the translational order of the molecular centres in the plane of the molecules and for a 2-D lattice leads to 'Bragg rods' normal to the monolayer plane. The relevance of this lies in the observation of peaks, where the intersection of the reciprocal disk with the Bragg rods gives rise to six first-order diffraction maxima. Of these six maxima only three can be observed, as peaks below the water plane cannot be measured.

Differences in the symmetry brought about by packing and tilt are reflected in the intersection of the reciprocal disk and the Bragg rods. Consequently, examination of 
the peak positions gives insight into the lattice packing and occurrence of tilt. The observation of a single in-plane peak represents an untilted hexagonal phase, where it is implied that the single diffraction peak is in fact triply degenerate peaks (Figure 2.4). Two peaks point to a rectangular lattice (also referred to as a distorted hexagonal lattice) with the respective $Q_{z}$ values indicating the magnitude and direction of tilt. An untilted centred-rectangular lattice is indicated by two in-plane peaks, in contrast to a nearest neighbour (NN) tilted phase which exhibits one inplane and one out-of-plane peak. Two out-of-plane peaks are indicative of a next nearest neighbour (NNN) tilted centred rectangular phase. For centred rectangular phases, symmetry dictates that one peak is doubly degenerate and the other nondegenerate, hence the ratio of peak intensities should be approximately 2:1. The centred rectangular cell is brought about by an elongation in one direction, such that if the unit cell stretches (i.e. due to tilting) in the direction of the nearest neighbour molecule then $\left|Q_{n}\right|>\left|Q_{d}\right|$ (subscripts $n$ and $d$ denote nondegenerate and degenerate peaks); the opposite inequality indicates shrinkage in that direction. The observation of three out-of-plane peaks is indicative of intermediate (between NN and NNN) or oblique tilt.

Following the assignment of symmetry and associated degeneracy, peak indexing can be performed. This is most readily achieved for the hexagonal lattice, where the $(1,0),(0,1)$ and $(1, \overline{1})$ reflections are all assigned to the one triply degenerate peak. For the intermediate tilted systems the three distinct peaks are assigned $(1,0),(0,1)$ and $(1, \overline{1})$ reflections. Further the relationship between the peaks has been shown to be $Q_{z}^{a}=Q_{z}^{b}+Q_{z}^{a b}$ where peak $a$ has the largest $Q_{z}[15,16]$. From this more general equation comes the distinction between degenerate and nondegenerate peaks such that the ratio $Q_{n z}: Q_{d z}$ can only be $0: 1$ or 2:1 as applied to NN and NNN tilted phases. The degenerate peak in NN or NNN tilted phases is assigned the $(1,1)$ and $(1, \overline{1})$ 

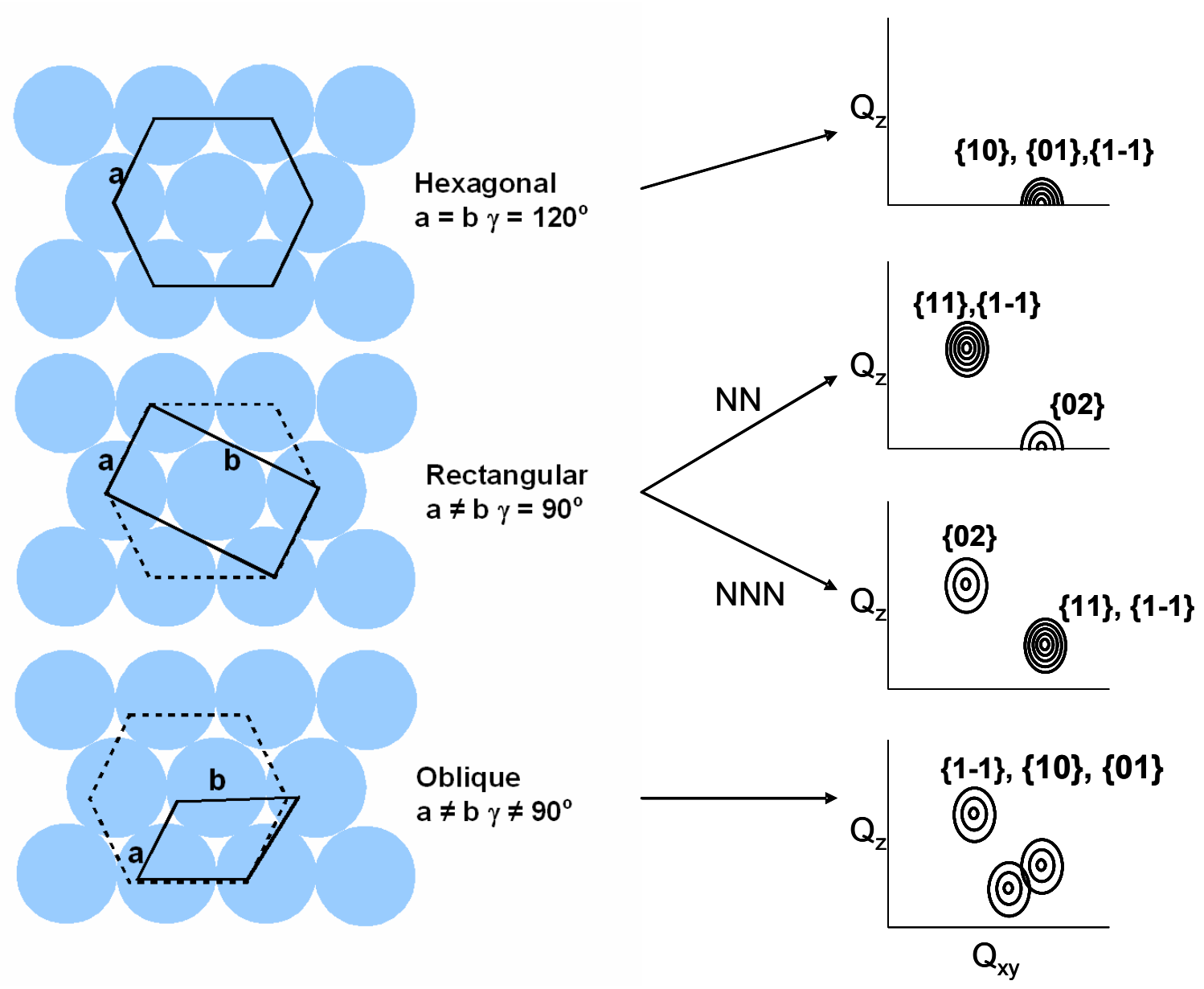

Figure 2.4 An illustration of the three different monolayer packing symmetries. In the hexagonal arrangement the monolayers are untilted, and equally spaced. The centred rectangular lattice is a distorted hexagonal arrangement, due to tilt along the bonding direction. The rectangular lattice can be manifested as either a NN or NNN tilted phase. Finally, the oblique lattice reflects a further distortion from the hexagonal lattice, due to an intermediate tilting direction.

reflections, given that for the rectangular unit cell the $(1,0)$ and $(0,1)$ reflections are forbidden, as are all reflections where $h+k$ is odd. Automatically then, the nondegenerate peak is assigned as the $(0,2)$ reflection.

\subsubsection{Lattice Parameter Determination}

Determination of symmetry and assignment of $h k$ reflections enables the calculation of the lattice parameters. The lattice spacings are obtained from the inplane diffraction data, such that: 


$$
d_{h k}=\frac{2 \pi}{Q_{x y}^{h k}}
$$

from which the lattice parameters can be calculated. For hexagonal symmetry and rearranging for $a$ gives:

$$
a=\frac{2 \pi}{Q_{x y} \sqrt{\left[\frac{4}{3}\left(h^{2}+h k+k^{2}\right)\right]}} .
$$

To aid the comparison of systems the hexagonal lattice is converted to a rectangular cell. For the hexagonal cell this is straight-forward as the hexagonal cell can be considered a special case of the rectangular cell, where $a_{\text {rect }}=a_{\text {hex }}$ and $b_{\text {rect }}=\sqrt{3} \cdot a_{\text {hex }}$ and the angle between $a$ and $b$ changes from $120^{\circ}$ to $90^{\circ}$ (Figure 2.3).

For a centred rectangular cell where two peaks are observed, one degenerate (subscript $d$ ) and the other nondegenerate (subscript $n$ ), the following equations are employed to calculate the lattice parameters[17]:

$$
\begin{gathered}
a=\frac{4 \pi}{\sqrt{4 Q_{d x y}^{2}-Q_{n x y}^{2}}}(\AA) \\
b=\frac{4 \pi}{Q_{n x y}}(\AA) .
\end{gathered}
$$

The distortion $(\delta)$ of the rectangular cell from hexagonal is:

$$
\delta=\sqrt{3}-\frac{b}{a} .
$$

For intermediate tilt or an oblique lattice the reduction in symmetry complicates matters. As in the previous hexagonal case the classic 3-D crystal expression for calculating lattice planes is modified for the 2-D crystal, such that:

$$
d_{h k}=\frac{\sin \gamma}{\sqrt{\left(\frac{h}{a}\right)^{2}+\left(\frac{k}{b}\right)^{2}-\frac{2 h \mathrm{k}}{a \cdot b} \cos \gamma}}
$$

where $d_{h k}=\frac{2 \pi}{Q_{x y}^{h k}}$, and the lattice parameters $(a, b$ and $\gamma)$ are deduced by way of least squares minimisation for the three pairs of $h k$ values. The result is values expressed in terms of the primitive (oblique) lattice cell. 


\subsubsection{Tilt and Azimuth}

The tilt angle $(\theta)$ is defined as the angle from the surface normal to the molecular chain (Figure 2.5). The tilt azimuth $(\psi)$ is the in-plane direction of tilt, with NN defined as $\psi=0^{\circ}$ and for NNN $\psi=90^{\circ}$. Generically the tilt angle is determined using Equation 25, where the three equations (one for each hk) are solved simultaneously to yield the tilt angle and azimuth.

$$
\cos \psi_{\mathrm{r}}^{\mathrm{hk}}=\frac{\mathrm{Q}_{\mathrm{z}}^{\mathrm{hk}}}{\mathrm{Q}_{\mathrm{xy}}^{\mathrm{hk}} \tan \theta}
$$

where $\theta$ is the tilt angle, $Q_{z}^{h k}$ and $Q_{x y}^{h k}$ correspond to the peak positions, and $\psi_{r}^{h k}$ is the tilt azimuth between the tilt direction and the reciprocal space lattice vector. For NN and NNN oriented tilt angles, where the tilt azimuth is already defined, Equation 25 simplifies to:

$$
\tan \theta=\frac{Q_{d z}}{\sqrt{Q_{d x y}^{2}-\left(\frac{Q_{n x y}}{2}\right)^{2}}} \text { for NN. }
$$

and

$$
\tan \theta=\frac{Q_{n z}}{Q_{n x y}} \text { for NNN. }
$$

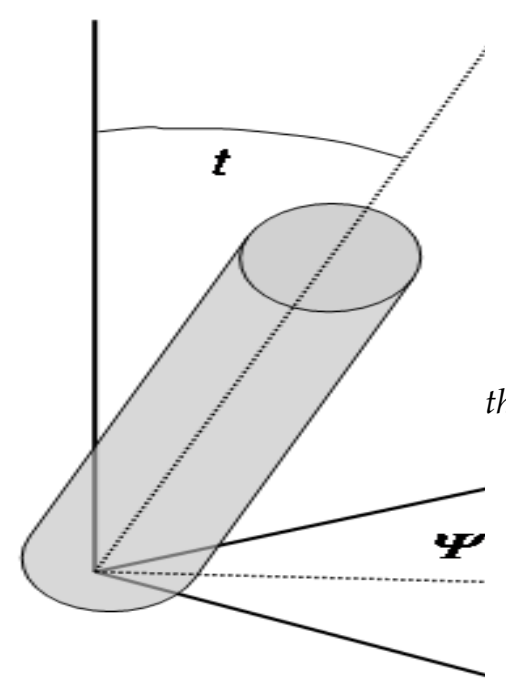

Figure 2.5 A schematic defining the polar tilt angle $(t)$ and the tilt direction or azimuth $(\Psi)$. 
However for intermediate tilt, calculating the tilt angle and azimuth becomes considerably more complex. Provided the condition $Q_{z}^{a}=Q_{z}^{b}+Q_{z}^{a b}$ is met then the derivation of Wiegart et al.[16] can be employed to calculate the tilt angle for intermediate or oblique tilt azimuths as follows:

$$
\theta=\tan ^{-1} \frac{Q_{z}^{i}}{Q_{x y}^{i} \cos \psi_{r}^{i}}
$$

where $i=a, b, a b$ (denoting the three hk pairs), and $\psi_{r}^{i}$ is determined as:

$$
\begin{gathered}
\psi_{r}^{a}=\psi_{r}^{b}+\gamma_{r} \\
\psi_{r}^{b}=\tan ^{-1}\left(\frac{1}{\sin \gamma_{D}}\left[\cos \gamma_{D}-\frac{Q_{z}^{a} Q_{x y}^{b}}{Q_{z}^{b} Q_{x y}^{a}}\right]\right) \\
\psi_{r}^{a b}=\cos ^{-1}\left(\frac{Q_{z}^{a b} Q_{x y}^{b} \cos \psi_{r}^{b}}{Q_{z}^{b} Q_{x y}^{a b}}\right)
\end{gathered}
$$

$\gamma_{r}=\pi-\gamma$ and coincides with $\gamma_{D}$, the angle of the 2-D unit cell in real space.

\subsubsection{Area Calculations}

Having calculated the lattice parameters and tilt angle the areas per molecule can be determined, of which there are two:

$$
A_{x y}=a \cdot b \sin (\gamma) \quad\left(\AA^{2}\right),
$$

defines the area per unit cell, and

$$
A_{o}=A_{x y} \cos (\theta) \quad\left(\AA^{2}\right),
$$

which defines the cross-sectional area of the chain. 


\subsubsection{Long Range Order}

To obtain a measure of the extent of the long range order, the coherence length is calculated, which involves deconvoluting the real variation from that generated by the experimental geometry $\left(F W H M_{\text {meas }}-F W H M_{\text {res }}\right)$, assuming the deconvolution of two Lorentzians[15]. $F W H M_{r e s}=\frac{4 \pi}{\lambda} \sin \left(\frac{2 \theta}{2}\right)$ with $\theta=0.1234$ rad for the standard position sensitive detector set up or $0.086 \mathrm{rad}$ for the pinhole detector. Thus the domain coherence length $(l)$ is:

$$
l=\frac{2}{\left(F W H M_{\text {meas }}-F W H M_{\text {res }}\right)}
$$

\subsubsection{GIXD Calculations}

Analysis of the GIXD data involved the summation of the 2-D data in the $Q_{x y}$ and $Q_{z}$ planes. The resulting profiles were peak fitted, a Lorentzian curve fit was performed for the $Q_{x y}$ profiles and a Gaussian fit for the $Q_{z}$ profiles. In both cases the standard equations were modified to account for any linearly sloping background. From the fits, peak position, FWHM and integrated intensity were obtained, which were subsequently fed into the equations outlined above (Equations 24 to 22). The data was also inputted into a 2-D contour plot using a random matrix generated using OriginPro 7.5 (OriginLab Corporation, USA).

\subsubsection{XRR Measurements and Calculations}

Reflectivity measurements were performed using an Oxford Cyberstar 1000 scintillation photomultiplier and the Pilatus $100 \mathrm{~K}$ detector. Measurements were made for $Q_{z}$ values up to $0.8 \mathrm{~A}^{-1}\left(\sim 37 \times \alpha_{c}\right)$, with a resolution of $3 \mathrm{mrad}$.

\subsubsection{XRR Analysis}

XRR data was analysed by fitting parametrised model density profiles to the measured data (Figure 2.6). The most common approach (and the one used here) 
used to generate a $\rho(z)$ profile involves representing the monolayer as a stack of boxes. Each box has a constant density $\left(\rho_{i}\right)$ and thickness or length $\left(l_{i}\right)$. The model also accounts for the electron density of the semi-infinite subphase and the vertical roughness $(\sigma)$ of the interface. The box model is iteratively altered such as to perfect the fit between the calculated and measured reflectivity. Refinement of the reflectivity model utilised the Stochfit software[18]. Model selection was based on the $\chi^{2}$ value for the model fit, a visual assessment of the fit, the calculated errors and the appropriateness of the calculated electron density profile, not necessarily in this order.

Using the fitted electron density profiles, information about the monolayer and interfacial subphase was obtained by assigning each box to a fragment of the molecule. This was achieved by calculating the total number of electrons $(\mathrm{N})$, where $\mathrm{N}=\rho \mathrm{AL}_{\mathrm{T}}$ and assigning them to each of the boxes based on the proportion of the total electron density found in each box, where $\mathrm{A}$ is the area per molecule, $\mathrm{L}_{\mathrm{T}}$ is the total length of the boxes and $\rho=\mathrm{L}_{i} \rho_{i} \mathrm{~S},\left(\mathrm{~L}_{i}\right.$ is the box width, $\rho_{i}$ is the box height and $\mathrm{S}$ is the scattering length density for bulk water $\left(0.334\right.$ e $\left.\left.\AA^{-3}\right)\right)$. Having assigned the number of electrons in each box, molecular fragments correlating to the number of electrons was determined, beginning from the tail. The excess in the head group was attributed to water or subphase ions. 
(a)

Reflectivity

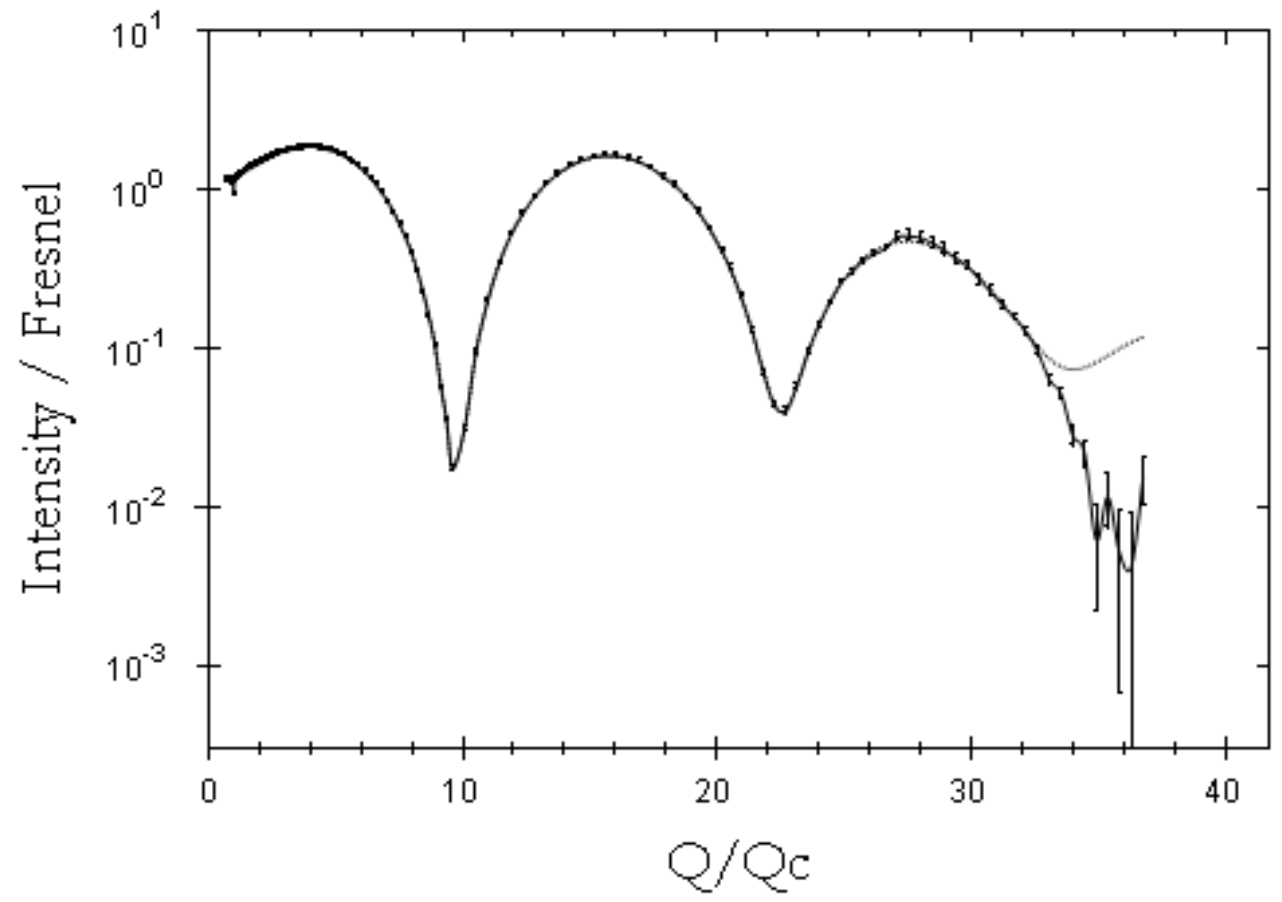

(b)

Electron Density Profile

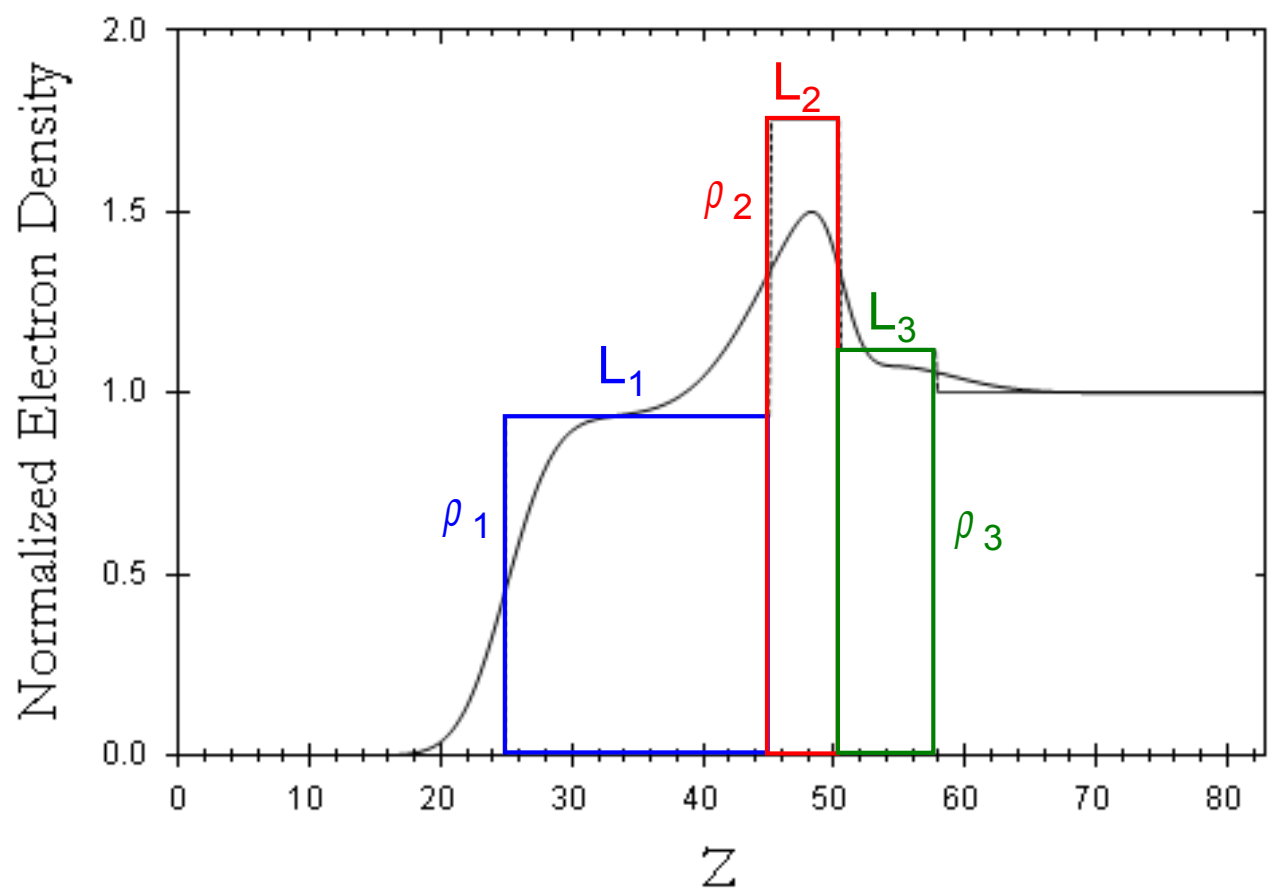

Figure 2.6 XRR and electron density profiles for 2-HSA on $\mathrm{CaCl}_{2}$ at $\Pi=25 \mathrm{mN} \mathrm{m}^{-1}$. (a) In the reflectivity profile the solid line reflects the model fit of the experimentally determined data (points). (b) The electron density profile the dashed line represents the model boxes and the solid line is the smoothed box model. Highlighted in colour is the three box model with the model parameters labelled. 
[1] D. Diaper and A. Kuksis, "Synthesis of Alklyated Alkanedioic Acids," Chemical Reviews, vol. 59 , 1959, pp. 89-178.

[2] B. Sharma and A. Biswas, "X-Ray Diffraction Study if n-Alkyl Malonic Acids," Analytical Chemistry, vol. 30, 1958, pp. 1356-1361.

[3] Y. Kitano, K. Park and D.W. Hood, J. Geophys. Res. 67 (1962) (12), pp. 4873-4877.

[4] S. Rajam, B. Heywood, J. Walker, S. Mann, R. Davey, and J. Birchall, "Oriented Crystallization of $\mathrm{CaCO}_{3}$ Under Compressed Monolayers Part 1. Morphological Studies of Mature Crystals," J.Chem.Soc.Faraday Trans. vol. 87, 1991, pp. 727-734.

[5] V. Vogel and D. Möbius, "Local Surface Potentials and Electric Dipole Moments of Lipid Monolayers: Contributions of the Water/Lipid and the Lipid/Air Interfaces," J.Colloid and Interface Sci., vol. 126 , 1988, pp. 408-420.

[6] V. Vogel and D. Möbius, "Hydrated Polar Groups in Lipid Monolayers: Effective Local Dipole Moments and Dielectric Properties," Thin Solid Films vol. 159, 1988, pp. 73-81.

[7] O. Oliveira Jr and C. Bonardi, "The Surface Potential of Langmuir Monolayers Revisited," Langmuir vol. 13, 1997, pp. 5920-5924.

[8] S. Henon and J. Meunier, "Microscope at the Brewster angle: Direct observation of first-order phase transitions in monolayers," Review of Scientific Instruments, vol. 62, Apr. 1991, pp. 936-939.

[9] I. Langmuir and V.J. Schaefer, "Activities of Urease and Pepsin Monolayers," Journal of the American Chemical Society, vol. 60, Jun. 1938, pp. 1351-1360.

[10] D. Archibald, S. Qadri, and B. Gaber, "Modified Calcite Deposition Due to Ultrathin Organic Films on Silicon Substrates," Langmuir vol. 12, Jan. 1996, pp. 538-546.

[11] “PDF Calcite 5-586," ICDD Database, 2008.

[12] "http://database.iem.ac.ru/mincryst/s_carta.php?CALCITE+706," (H.Effenberger, K.Mereiter, I.Zemann (1981);* Z.Kristallogr., 156, 233-243), 2008.

[13] Physics in Industry: X-ray Diffraction by Polycrystalline Materials, London: Chapman \& Hall Limited, 1960.

[14] B. Lin, M. Meron, J. Gebhardt, T. Graber, M.L. Schlossman, and P.J. Viccaro, "The liquid surface/interface spectrometer at ChemMatCARS synchrotron facility at the Advanced Photon Source," Physica B: Condensed Matter, vol. 336, Aug. 2003, pp. $75-80$.

[15] V. Kaganer, H. Möhwald, and P. Dutta, "Structure and Phase Transitions in Langmuir Monolayers," Reviews of Modern Physics, vol. 71, 1999, pp. 779-819.

[16] L. Wiegart and B. Struth, "Geometric boundary condition for the chain alignment in lipid monolayers," Physica B: Condensed Matter, vol. 357, Feb. 2005, pp. 126-129.

[17] L. Cristofolini, M. Fontana, C. Boga, and O. Konovalov, “Microscopic Structure of Crystalline Langmuir Monolayers of Hydroxysteric Acids by X-ray Reflectivity and GID: OH Group Position and Dimensionality Effect," Langmuir 
vol. 21, 2005, pp. 11213-11219.

[18] S.M. Danauskas, D. Li, M. Meron, B. Lin, and K.Y.C. Lee, "Stochastic fitting of specular X-ray reflectivity data using \it StochFit," Journal of Applied Crystallography, vol. 41, Dec. 2008, pp. 1187-1193. 


\section{Chapter 3: Mixed Octadecanoic Acid/ Octadecanol Monolayers}

\subsection{Introduction}

In terms of monolayer templated crystallisation, accepted wisdom has recently shifted to charge density and non-specific electrostatic interactions as being the dominating forces[1]. However, such non-specificity does not explain the widely observed phenomena of face-selective nucleation. It is generally accepted that growth of calcite under a charged anionic monolayer leads to oriented nucleation on a charged crystal face (00.1) or (01.2), in contrast to the neutral (10.4) face expressed in the presence of a neutral monolayer. Although charge density and ion-ion electrostatics are likely promoters of nucleation, the selection of specific faces requires interactions with some degree of directionality. This points towards spatial geometrical influences[2], which encompass directional electrostatic dipole interactions (e.g. hydrogen bonding).

In order to gain a better understanding of the interrelationship between the monolayer and subphase, in this chapter we focus on the monolayer behaviour of fatty acid/long chain alcohol mixtures. Mixed systems have the benefit of providing a more accurate model for biological cell membranes, which contain a combination of lipid molecules in conjunction with proteins and other molecules[3]. Hence there is considerable prior work on pure and mixed fatty acid and alcohol monolayers, including investigations on calcium-based and pH-adjusted subphases[4-6]. Predominantly these studies[7,8] have focused, however, on investigating interactions within the monolayer rather than monolayer/subphase interactions. 
Additionally they are invariably performed at low $\mathrm{pH}(\sim 2)$ to ensure a thermodynamically ideal neutral monolayer. However, in this study we look to investigate the interfacial interaction in partially charged and non-equilibrium conditions in order to more closely mimic the natural conditions of biomineralisation at a membrane. As such the attraction of mixed acid/alcohol systems lies in the ability to systematically modify the surface charge, thus altering the hydrogenbonding capacity of the monolayer and the interfacial water.

In addition to the use of mixed monolayers, the crystallising subphase is broken up into its constituents in true reductionistic style, as such the monolayer-subphase interactions were studied on all four subphases (water, calcium chloride, sodium bicarbonate and CCCS). It is envisaged that the systematic deconstruction and reconstruction of the crystallising subphase will provide an improved understanding of the molecular recognition and self-assembly processes.

Experimental findings were supported by numerical calculations aimed at fitting the surface potential data. This involved a combined Demchak Fort/PoissonBoltzmann based model, which is described in detail in the next section.

\subsubsection{Theoretical Calculations}

The experimentally determined surface potential is the summation of all charge separation residing between the two electrodes, thus it includes contributions from the air, monolayer, subphase and all the associated interfaces. A common approach to modelling such a complex system involves either eliminating the electric double layer contribution from the subphase by using non-ionised monolayers $[9,10]$ or by assuming that the monolayer component is constant between the systems examined[11]. Unfortunately neither of these simplifications are appropriate for this study, hence a combined approach was employed. This involves an amalgamation of the three-layer capacitor model, developed by Demchak and Fort[12], with the GouyChapman-Stern (GCS) model employed by Lochhead et al.[13] (Figure 3.1). The 
former was developed to describe the surface potential associated with the monolayer, whereas the latter describes the double layer contribution.

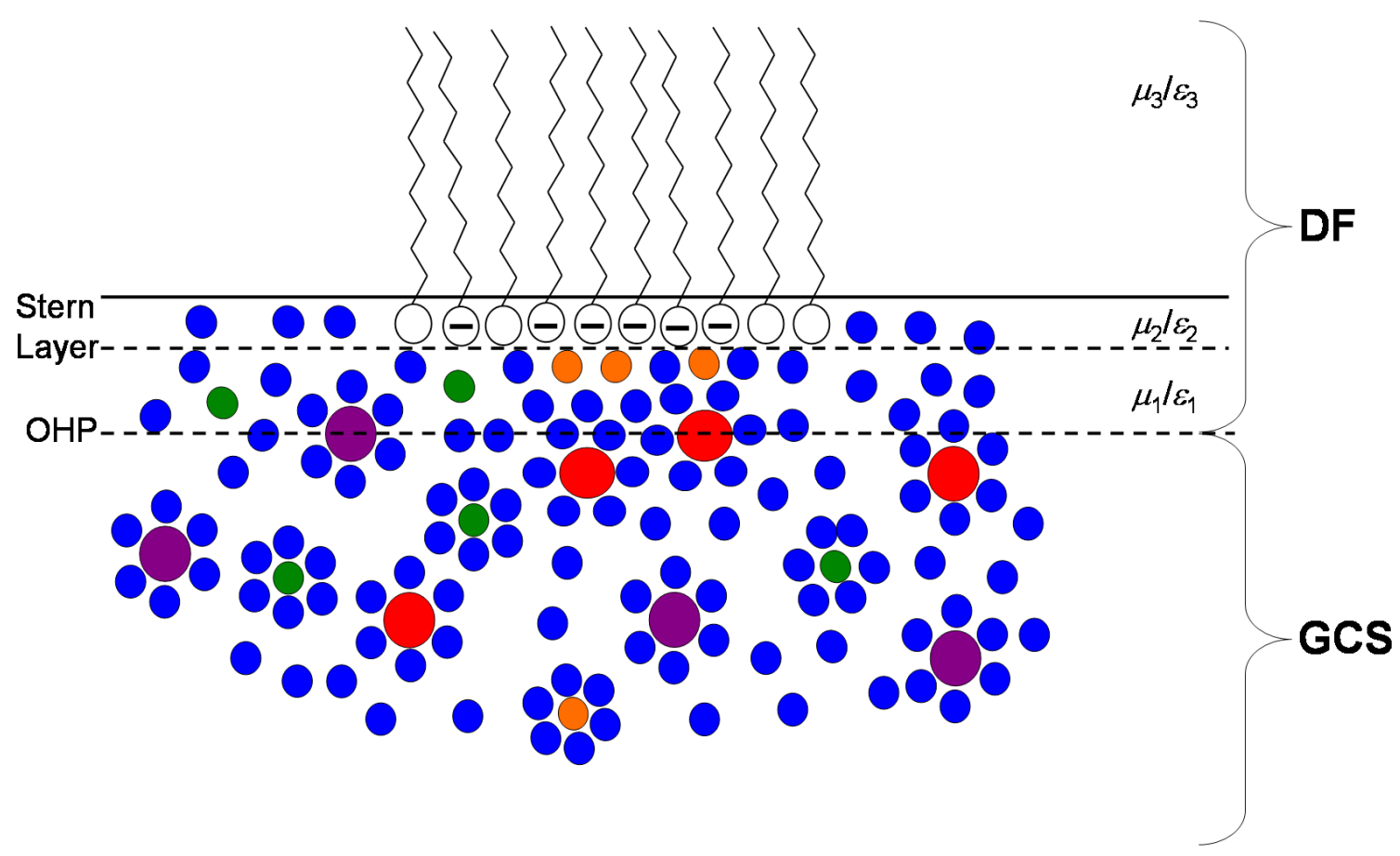

Figure 3.1 A schematic of the combined Demchak-Fort/Gouy Chapman Stern numerical model employed to elucidate the experimental surface potential data.

The three-layer capacitor (DF) model is a refinement of the Helmholtz model, where it is assumed that the monolayer can be divided into three layers that contribute to the surface potential. Thus

$$
\Delta \mathrm{V}=\frac{1}{\mathrm{~A}_{\mathrm{m}} \varepsilon_{\mathrm{o}}}\left(\frac{\frac{\mu_{1}}{\varepsilon_{1}}+\frac{\mu_{2}}{\varepsilon_{2}}+\frac{\mu_{3}}{\varepsilon_{3}}}{)}\right)
$$

where $\mu_{\mathrm{i}}$ is the average dipole moment per molecule and $\varepsilon_{\mathrm{i}}$ is the local dielectric constant for each of the three layers, $\mathrm{A}_{\mathrm{m}}$ is the average area per molecule and $\varepsilon_{\mathrm{o}}$ is the permittivity of free space. The three components are conventionally defined as the immediate interfacial subphase component $\left(\mu_{1} / \varepsilon_{1}\right)$, the monolayer head groups $\left(\mu_{2} / \varepsilon_{2}\right)$ and the hydrocarbon tails $\left(\mu_{3} / \varepsilon_{3}\right)$. 
The GCS model is a modification of the Gouy-Chapman (GC) solution to the Poisson-Boltzmann (PB) equation for planar diffuse double layers, where the PB equation details the relationship between an electrical potential and the distribution of charged species. The standard GC approach, using the Grahame equation that relates surface potential $(\psi(0))$ to surface charge $(\sigma)$ (Equation 41), tends to overestimate the surface potential. This discrepancy is due to a failure to account for the loss of bound ions from the double layer and the associated change in the surface charge,

$$
\sigma=-\left[2 \varepsilon \varepsilon_{o} k \mathrm{~T} \sum_{i}^{m} \mathrm{n}_{\mathrm{i}, \mathrm{b}}\left[\exp \left(\frac{-z_{i} e \Psi(0)}{k \mathrm{~T}}\right)-1\right]\right]^{1 / 2} .
$$

The Stern modification corrects for this overestimation by accounting for the adsorption of charge determining species (e.g. counter-ion binding). In this study, the McLaughlin methodology[14] was employed to make the correction. Based on the Langmuir adsorption isotherm equation (Equation 42) the propensity for ions to bind to the surface is determined, from which the surface charge is adjusted.

$$
K_{\text {int }}=\frac{\left\{\mathrm{COO}^{-} \mathrm{M}^{+}\right\}}{\left\{\mathrm{COO}^{-}\right\}\left[\mathrm{M}^{+}\right]_{s}}
$$

where $K_{\text {int }}$ is the intrinsic binding constant. Note that \{\} indicates the surface concentration of the species whereas []$_{s}$ represents the normal volume-based concentration adjacent to the surface. For simplification, the charge of the species is dropped in our notation for the binding constants, i.e. $K_{\mathrm{Ca}}$ represents the binding constant for the $\mathrm{Ca}^{2+}$ ion.

Using the McLaughlin methodology, the correction term employed by Lochhead et al.[13] was extended to incorporate four new terms. The aim was to explore the likelihood of anion binding via hydrogen bonding to alcohol and/or protonated acid head groups, where a positive binding constant might indicate a mechanism to explain how the bicarbonate ion affects monolayer behaviour. It should be noted that the McLaughlin methodology assumes 1:1 binding for all ions, irrespective of 
valence. The following details the extension of the methodology.

Surface pressure isotherms provide information regarding the average area per molecule $\left(A_{m}\right)$ of the monolayer leading to the following expression for the surface charge density:

$$
\sigma=\frac{-e}{A_{m}} .
$$

$1 / A_{m}$ equates simply to the total number of possible head group binding sites. The surface charge is defined by the total surface concentration of charged surfactant and surfactant-ion pairs for both the acid and alcohol molecules, such that:

$$
\begin{aligned}
-\sigma / e=\left\{\mathrm{COO}^{-}\right\} & +\left\{\mathrm{COOH} \cdots \mathrm{OH}^{-}\right\}+\left\{\mathrm{COOH}^{-} \cdot \mathrm{HCO}_{3}^{-}\right\}+\left\{\mathrm{H}_{2} \mathrm{COH} \cdots \mathrm{OH}^{-}\right\} \\
& +\left\{\mathrm{H}_{2} \mathrm{COH} \cdots \mathrm{HCO}_{3}^{-}\right\}-\left\{\mathrm{COO}^{-} \mathrm{Ca}^{2+}\right\}
\end{aligned}
$$

where $\left\{\mathrm{COOH} \bullet . \mathrm{OH}^{-}\right\}$denotes a hydrogen-bonded complex between, in this case, the protonated acid head group and a hydroxide counter-ion Note that $\mathrm{COO}^{-} \mathrm{Na}^{+}$, for example, does not add to the total surface charge and so is not included in Equation 44. Rearranging Equation 44 and substituting in the relevant Langmuir adsorption expressions yields:

$$
\begin{aligned}
-\sigma / e=\left\{\mathrm{COO}^{-}\right\} & +K_{\mathrm{OH}}\{\mathrm{COOH}\}\left[\mathrm{OH}^{-}\right]_{s}+K_{\mathrm{HCO}_{3}}\{\mathrm{COOH}\}\left[\mathrm{HCO}_{3}^{-}\right]_{s}+K_{\mathrm{OHOH}_{2}}\left\{\mathrm{H}_{2} \mathrm{COH}\right\}\left[\mathrm{OH}^{-}\right]_{s} \\
& +K_{\mathrm{OHHCO}_{3}}\left\{\mathrm{H}_{2} \mathrm{COH}\right\}\left[\mathrm{HCO}_{3}^{-}\right]_{s}-K_{\mathrm{Ca}}\left\{\mathrm{COO}^{-}\right\}\left[\mathrm{Ca}^{2+}\right]_{s}
\end{aligned}
$$

and since $\{\mathrm{COOH}\}=K_{\mathrm{H}}\left\{\mathrm{COO}^{-}\right\}\left[\mathrm{H}^{+}\right]_{\mathrm{s}}$

$$
\begin{gathered}
-\sigma / e=\left\{\mathrm{COO}^{-}\right\}\left(1-K_{\mathrm{Ca}}\left[\mathrm{Ca}^{2+}\right]_{s}+K_{\mathrm{H}}\left[\mathrm{H}^{+}\right]_{s} K_{\mathrm{OH}}\left[\mathrm{OH}^{-}\right]_{s}+K_{\mathrm{H}}\left[\mathrm{H}^{+}\right]_{s} K_{\mathrm{HCO}_{3}}\left[\mathrm{HCO}_{3}^{-}\right]_{s}\right) \\
+\left\{\mathrm{H}_{2} \mathrm{COH}\right\}\left(K_{\mathrm{OHOH}}\left[\mathrm{OH}^{-}\right]_{s}+K_{\mathrm{OHHCO}_{3}}\left[\mathrm{HCO}_{3}^{-}\right]_{s}\right)
\end{gathered}
$$

$\left\{\mathrm{COO}^{-}\right\}$can be defined by considering the total surface concentration of acid molecules, including both free and bound groups. Such that:

$$
\left\{\mathrm{COO}^{-}\right\}^{\mathrm{Tot}}=\left\{\mathrm{COO}^{-}\right\}+\left\{\mathrm{COO}^{-} \mathrm{H}^{+}\right\}+\left\{\mathrm{COO}^{-} \mathrm{Na}^{+}\right\}+\left\{\mathrm{COO}^{-} \mathrm{Ca}^{2+}\right\} .
$$

Rearrangement and substitution of the appropriate Langmuir adsorption expressions gives a value for $\left\{\mathrm{COO}^{-}\right\}$: 


$$
\left\{\mathrm{COO}^{-}\right\}=\frac{\left\{\mathrm{COO}^{-}\right\}^{\text {Tot }}}{\left(1+K_{\mathrm{H}}\left[\mathrm{H}^{+}\right]_{s}+K_{\mathrm{Na}}\left[\mathrm{Na}^{+}\right]+K_{\mathrm{Ca}}\left[\mathrm{Ca}^{2+}\right]_{s}\right)} .
$$

Substituting Equation 46 into Equation 45 and adding a term for the alcohol mole fraction (f) yields:

$$
\begin{gathered}
\sigma=\frac{-e(1-f)\left\{\mathrm{COO}^{-}\right\}^{t o t}\left[1-K_{\mathrm{Ca}}\left[\mathrm{Ca}^{2+}\right]_{s}+K_{\mathrm{H}}\left[\mathrm{H}^{+}\right]_{s} K_{\mathrm{OH}}\left[\mathrm{OH}^{-}\right]_{s}+K_{\mathrm{H}}\left[\mathrm{H}^{+}\right]_{s} K_{\mathrm{HCO}_{3}}\left[\mathrm{HCO}_{3}{ }^{-}\right]_{s}\right]}{\left[1+K_{\mathrm{H}}\left[\mathrm{H}^{+}\right]_{s}+K_{\mathrm{Na}}\left[\mathrm{Na}^{+}\right]_{s}+K_{\mathrm{Ca}}\left[\mathrm{Ca}^{2+}\right]_{s}\right]} \\
-e f\left\{\mathrm{H}_{2} \mathrm{COH}\right\}\left[K_{\mathrm{OHOH}}\left[\mathrm{OH}^{-}\right]_{s}+K_{\mathrm{OHHCO}_{3}}\left[\mathrm{HCO}_{3}^{-}\right]_{s}\right]
\end{gathered}
$$

The initial values for $K_{\mathrm{int}}$ were taken from Lochhead et al.[13]: $\mathrm{p} K_{\mathrm{H}}=5.4, \mathrm{p} K_{\mathrm{Na}}=-0.771$ and $\mathrm{p} K_{\mathrm{Ca}}=0.51$; the new terms $\mathrm{p} K_{\mathrm{OH}}, \mathrm{p} K_{\mathrm{OHOH}}, \mathrm{p} K_{\mathrm{HCO}_{3}}$ and $\mathrm{p} K_{\mathrm{OHHCO}_{3}}$ were initially set to -10 . Equations 41 and 47 were solved simultaneously using a least squares approach to obtain a value for $\psi(0)$, with $\mathrm{p} K_{\mathrm{H},} \mathrm{p} K_{\mathrm{Na},} \mathrm{p} K_{\mathrm{Ca}} \mathrm{p} K_{\mathrm{OH}}, \mathrm{p} K_{\mathrm{OHOH}}, \mathrm{p} K_{\mathrm{HCO}_{3}}$, $\mathrm{p} K_{\mathrm{OHHCO}_{3}}, \sigma$, and $\psi(0)$ as adjustable parameters. This value of $\psi(0)$ is then combined with the monolayer contribution (Equation 40) to fit the measured surface potential:

$$
\Delta \mathrm{V}=\frac{1}{\mathrm{~A}_{\mathrm{m}} \varepsilon_{\mathrm{o}}}\left(\frac{\mu_{1}}{\varepsilon_{1}}+\frac{\mu_{2}}{\varepsilon_{2}}+\frac{\mu_{3}}{\varepsilon_{3}}\right)+\psi(0)
$$

The adjustable parameters in this process were the high and low pressure values for $\mu_{1} / \varepsilon_{1}, \varepsilon_{2}$, and $\varepsilon_{3}$. Limits for these parameters were based on literature values along with the following justifications (parameter ranges are summarised in Table 2).

- The dipole moment for the tails $\left(\mu_{3}\right)$ was not fitted, the literature value of 330 $\mathrm{mD}$ was used[15].

- The dipole moment for the head groups $\left(\mu_{2}\right)$ was not adjusted from the literature values of $1000 \mathrm{mD}$ and $990 \mathrm{mD}[15]$, for the alcohol and acid, respectively, mixed systems were calculated as a weighted average.

- $\varepsilon_{3}$ at high pressure - restricted to be below 10 (based on a value for a stearic acid film of 2.7[16]). Its lower bound is expected to be greater than its value at low pressure as discussed by Oliveira Jr et al.[15]. 
- $\varepsilon_{3}$ at low pressure - restricted to between 1.1 and 5. The lower limit is based on the values for dry air (1.0005364) and steam (1.00587)[17]. The upper limit encompasses the idea that at lower pressures the increased tilt and loss of tailtail interaction would decrease the polarizability. Literature values for $\varepsilon_{3}$ range from 2.1 to $5.3[18]$.

- $\varepsilon_{2}$ at high pressure was limited to below 15 but greater than the value determined at low pressure. Values of 6-8 have been suggested in the literature[19], but given the mixed nature of the monolayer used here and the expected enhanced intramonolayer hydrogen bonding as a direct consequence of this, then the dielectric constant may be increased by the already polarized state of the head groups.

- $\varepsilon_{2}$ at low pressure was bound between the estimated high pressure value and the permittivity of bulk water (78.5), in accordance with literature[15]. However rather than utilising a step change between the high and low pressure values of $\varepsilon_{2}$, employed by Oliveira Jr et al.[15] we have applied a Boltzmann growth model to provide a better fit with our experimental data, Equation 43 , where $\mathrm{A}_{1}=\varepsilon_{2}$ at high pressure, $\mathrm{A}_{2}=\varepsilon_{2}$ at low pressure, $x_{0}$ is a mid point constant approximating $A_{c}$, where $A_{c}$ is the critical area per molecule at which the surface potential begins to rise and $\tau$ is a fitting parameter equating to area per molecule on compression,

$$
\varepsilon_{2}=\frac{\mathrm{A}_{1}-\mathrm{A}_{2}}{\left(1+e^{\left(x-x_{0}\right) / \tau}\right)}+\mathrm{A}_{2} .
$$

- $\mu_{1} / \varepsilon_{1}$ was fitted as a single variable. However as with the previous parameters it was assumed to have both a low and high pressure value. High and low pressure values were based around $A_{c}$ the point of intersection. The value of $\mu_{1} / \varepsilon_{1}$ at high pressure was free, whereas at low pressure it was restricted to between -15 and 15 . Limits were chosen to represent a broad range of 
possible low pressure monolayer conformations, as are expected for different subphases, being more realistic than assuming gaseous behaviour for all systems.

Table 2 Fitting parameter ranges for monolayer potential in the Demchak Fort/Gouy Chapman Stern model.

\begin{tabular}{|c|}
\hline$\varepsilon_{3}$ at low pressure $<\varepsilon_{3}$ at high pressure $<10$ \\
\hline $1.1<\varepsilon_{3}$ at low pressure $<5$ \\
\hline $\mathrm{A}_{1}$ component of $\varepsilon_{2}<15$ \\
\hline $\mathrm{A}_{1}$ component of $\varepsilon_{2}<\mathrm{A}_{2}$ component of $\varepsilon_{2}$ \\
\hline $\mathrm{A}_{2}$ component of $\varepsilon_{2}<78.50$ \\
\hline$\tau$ component of $\varepsilon_{2}>0.1$ \\
\hline$-15<\mu_{1} / \varepsilon_{1}$ at low pressure $<15$ \\
\hline
\end{tabular}

Underlying this model is a number of simplifications and assumptions. Firstly, the DF model incorporates mutual polarization of the three contributions into the dielectric constant terms. This can lead to an overestimation of the dielectric terms[9]. In addition, when fitting the dielectric constant terms care needs to be taken to consider the nature of the monolayer material, in order to avoid spurious values. The electric double layer is a complex phenomenon that involves both chemical and electrical effects. For example, its formation is driven by the chemical affinity of charge determining ions for the surface[20], at the same time like-charge repulsion and electric potential are mediating the ion distribution. Hence the GCS model, which only considers electrical effects is already a simplification. In additional to this there is a number of well known limitations associated with the ability of the PB equation to model these electrical considerations, beginning with it being a mean field theory. Considering that the monolayers in this study are not only partially dissociated but also a mixture of ionic and non-ionic surfactants, the assumption that the surface charge is uniformly distributed and constant is simplistic. Similarly, to assume that the electrolyte consists of point charges dispersed in a structure-less solvent having a uniform dielectric constant is also 
rudimentary. More recently there have been several attempts to improve PB-based models by incorporating additional interactions such as: image forces, dispersion forces, specific ion and ion hydration effects[21]. However, often these approaches introduce additional fitted parameters to an already underspecified problem. Finally, the additive approach to combining these models (DF + GCS) assumes the molecular dipole is independent of the double layer, which is again an oversimplification.

Despite its flaws this model was employed to aid the analysis of experimental results and ultimately elucidate the monolayer/subphase interactions. The model was chosen for its simplicity, which enabled it to be modified in order to incorporate anion binding, thought to be a contributing influence in the monolayer behaviour. Further, the model is well established with significant literature studies from which to compare computed parameter values, in order to validate the model.

\subsection{Results and Discussion}

Analysis of monolayer behaviour is based on a comparison of experimental results with the rule of mixtures (ROM), which represents the absence of any interaction between the acid and alcohol surfactant molecules. That is, when the components of a mixed system are immiscible, the area per molecule $\left(A_{m}\right)$ of the mixture is simply the weighted average of the $A_{m}$ of the pure components:

$$
\mathrm{A}_{12}=\mathrm{x}_{1} \mathrm{~A}_{1}+\mathrm{x}_{2} \mathrm{~A}_{2} \text {. }
$$

In this way inferences can be made with regard to possible interactions within the system that manipulate the monolayer behaviour; whether entropic or enthalpic in nature and within the monolayer itself or between the monolayer and subphase.

Upon comparing the behaviour of the five key values, outlined earlier (Chapter 2), on the four different subphases to the ROM, four general trends emerged for the mixed-monolayer behaviour. (The reader is referred to Appendix A2 for full graphical illustration of the monolayer trends, including the model fits). Figure 3.2 illustrates these trends in a comparison of the $A_{m}$ at the onset phase transition for 
each of the subphases. Condensation of the monolayer manifests itself as a negative deviation of the $A_{m}$ from the ROM line, (Figure 3.2a, for water). Figure 3.2b displays the same phase transition for a $20 \mathrm{mM} \mathrm{CaCl}_{2}$ subphase, which in contrast, shows ROM behaviour. For $20 \mathrm{mM} \mathrm{NaHCO}_{3}$ (Figure 3.2c) we observe what we have termed alcohol-like behaviour, where the $A_{m}$ of the mixtures is similar to that of the pure alcohol. Finally, Figure 3.2d shows acid-like behaviour for a $10 \mathrm{mM}$ CCCS. The description and discussion of results will revolve around the assignation of one of these four trends (condensation, ROM, alcohol-like or acid-like behaviour) and an interpretation of why the specific behaviour presents itself for a given system.
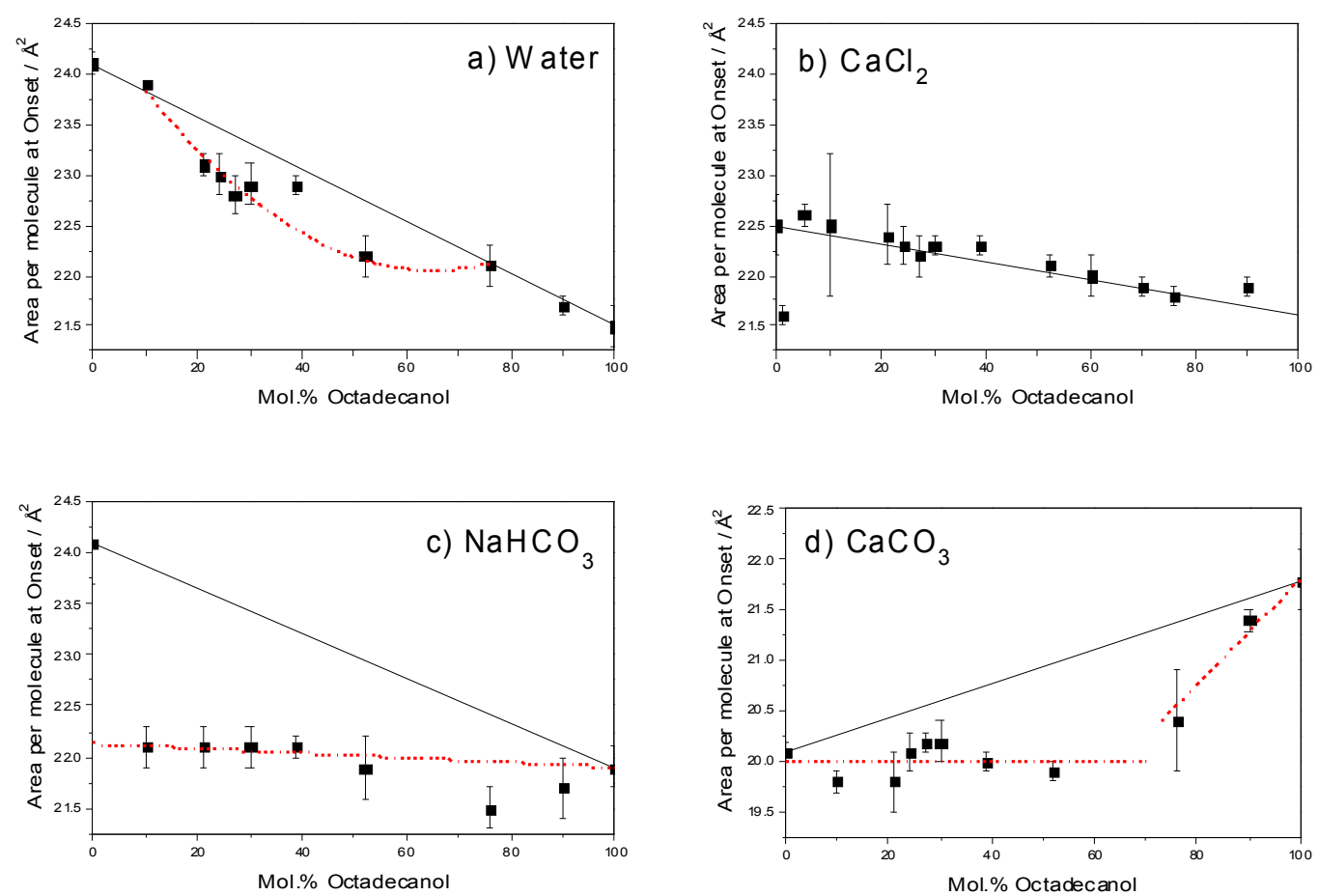

Figure 3.2 Mean area per molecule of the mixed $\mathrm{ODA}$ and $\mathrm{ODOH}$ system as a function of the $\mathrm{ODOH}$ added on four different subphases at $\sim 20^{\circ} \mathrm{C}$. Depicted are examples of the four general trends utilized to describe the mixed monolayer behaviour. a) Condensation behaviour on water at $p H \sim 5.6, b)$ ROM behaviour: $20 \mathrm{mM}$ $\mathrm{CaCl}_{2}$ at $\left.\mathrm{pH} \sim 5.5, \mathrm{c}\right)$ alcohol-like behaviour: $20 \mathrm{mM} \mathrm{NaHCO}$ slus $\mathrm{CO}_{2(g)}$ at $\mathrm{pH} \sim 6.0$ and d) acid-like behaviour: $10 \mathrm{mM} \mathrm{CCCS}$ plus $\mathrm{CO}_{2(g)}$ at $p H \sim 5.8$. In all graphs the solid line represents the rule of mixtures trend and the dashed line is drawn to guide the eye to the assigned monolayer behaviour. 
Figure 3.3 shows representative surface pressure isotherms for the pure acid on the four subphases investigated. On water and $\mathrm{CaCl}_{2}$ the isotherms illustrate typical fatty acid behaviour at room temperature with a well-defined tilted $L_{2}$ to untilted $L S$ transition. The isotherms obtained for $\mathrm{NaHCO}_{3}$ have similar form but with less welldefined transitions. The presence of a CCCS results in the loss of the tilted phase(s) and the isotherm consists of the single untilted LS phase on the application of pressure. In contrast the pure alcohol surface pressure isotherms have identical form on all four subphases, displaying characteristic fatty-alcohol behaviour. The key feature of the pure alcohol isotherm is the tilted $L_{2}{ }^{\prime}$ to untilted $L S$ transition.

In general the phase diagrams for pure fatty-acid and alcohol monolayers are similar with the key difference being the observation of a nearest neighbour (NN) tilted phase $\left(L_{2}\right)$ in fatty-acid systems, absent in fatty-alcohol systems. (For a full phase diagram and a description of these phases the reader is referred to Kaganer et al.[5].)

What follows is a description of the phase behaviour of the mixed monolayers on each of the four subphases. 


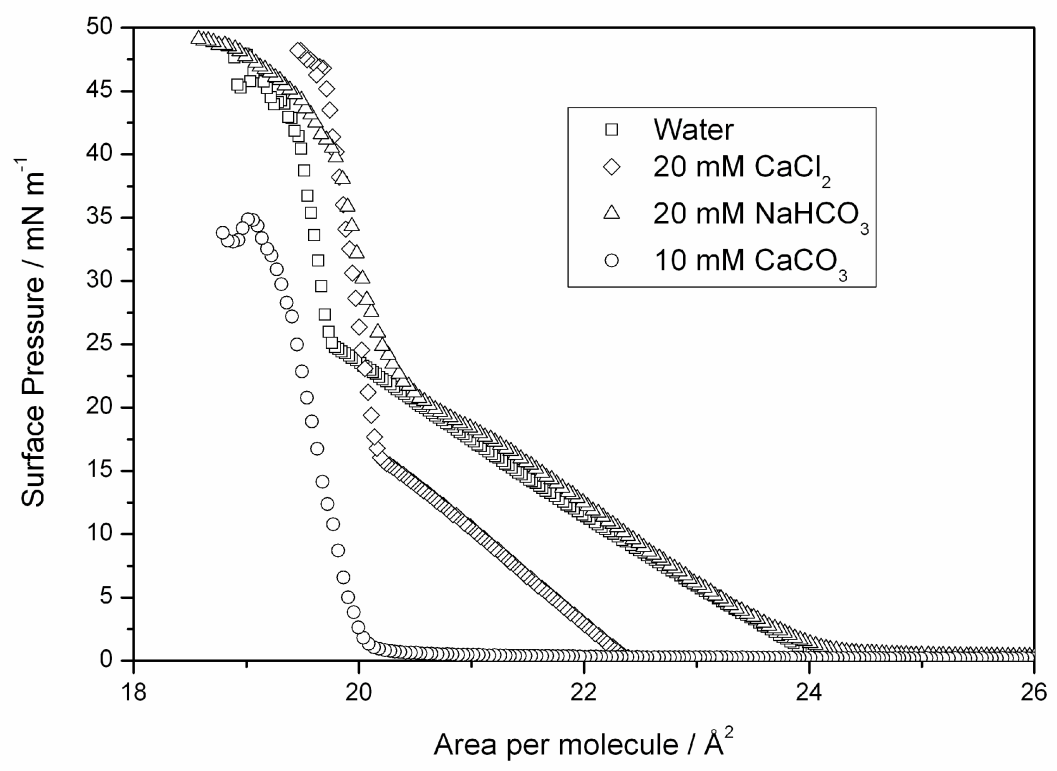

Figure 3.3 Representative surface pressure isotherms of pure octadecanoic acid on the different subphases investigated. The inflection point in the water, $\mathrm{CaCl}_{2}$, and $\mathrm{NaHCO}_{3}$ isotherms is associated with a tilted $\mathrm{L}_{2}$ to untilted LS transition. This transition is absent for the CCCS where the monolayer is untilted at all pressures.

\subsubsection{Ultrapure Water}

Pure octadecanoic acid exhibits typical fatty-acid behaviour as reported in the literature for a water subphase at $\mathrm{pH} 5.6$ and a temperature of $20 \pm 1^{\circ} \mathrm{C}[22-24]$. The onset to $L_{2}$ transition occurs at $24.1 \pm 0.1 \AA^{2} /$ molecule (Figure 3.2a) and the $L_{2}$ to $L S$ transition at $19.8 \pm 0.1 \AA^{2} /$ molecule. Likewise the transitions for pure octadecanol (Figure 3.2a) occur at $21.5 \pm 0.2 \AA^{2} /$ molecule (onset to $L_{2}^{\prime}$ transition) and $20.0 \pm 0.2$ $\AA^{2} /$ molecule ( $L_{2}{ }^{\prime}$ to $L S$ transition) in agreement with literature values[25].

The maximum surface potential $\left(\Delta \mathrm{V}_{\max }\right)$ measured for the pure acid is $263 \pm 7 \mathrm{mV}$ in accordance with literature values for stearic acid monolayers under similar conditions[15,26]. This compares with $420 \pm 20 \mathrm{mV}$ for the pure alcohol, which is also consistent with literature[4]. Similarities in the chemistry and therefore the molecular dipoles means that the surface potential values for pure alcohol and pure protonated acid monolayers are comparable[12]. Literature values for protonated $(\mathrm{pH} 2)$ stearic acid monolayers are of the order of $400 \mathrm{mV}[4,27]$. However, partial 
dissociation at pH 5.6 and the subsequent double layer formation leads to a reduction in $\Delta \mathrm{V}_{\max }$ for the pure acid system.

The $\Delta \mathrm{V}_{\max }$ data approximates acid-like behaviour (Figure 3.4). As expected the pure alcohol monolayer has the largest value of $\Delta \mathrm{V}_{\max }$, reflecting the absence of a double layer to counter the molecular dipole moment.

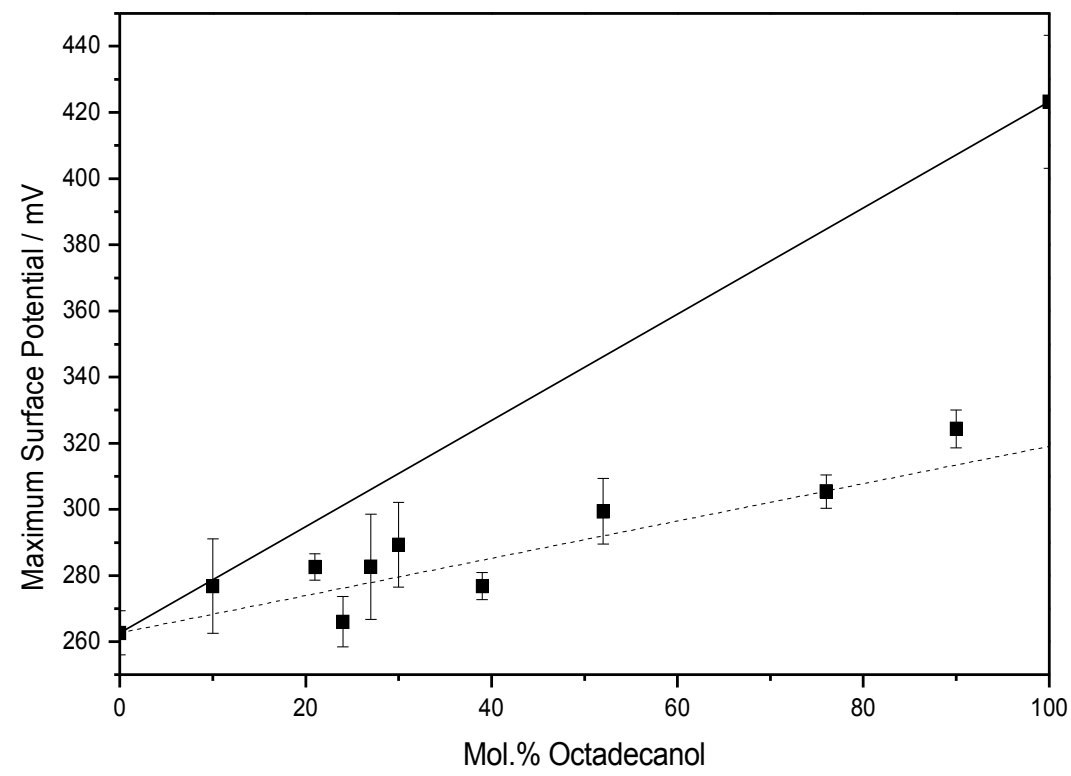

Figure 3.4 Surface potential measurements for the mixed monolayers on water. The solid line represents ROM behaviour and the dashed line is added to highlight the acid-like behaviour of the data.

For the mixed systems, $\Delta \mathrm{V}_{\max }$ would be expected to lie between the two pure systems reflecting the gradual weakening of the double layer. This is only partially true, and is evident in the gradual rise in $\Delta \mathrm{V}_{\max }$. However, the mixed systems exhibit a strong acid-like trend. The substitution of acid with alcohol molecules should lead to a decrease in the absolute number of dissociated acid molecules in the monolayer, subsequently decreasing the double layer potential and $\Delta \mathrm{V}_{\max }$ should increase in accordance with the ROM trend. The suppression of $\Delta \mathrm{V}_{\max }$ is indicative of increasing acid dissociation (or higher surface $\mathrm{pH}$ ), suggesting a shift in the apparent $\mathrm{p} K_{\mathrm{a}}$ of the acid such that, as the number of acid molecules is reduced, the degree of dissociation 
of the remaining head groups is increased and a near-constant monolayer surface charge density is maintained. It is generally accepted that the $\mathrm{pH}$ at the surface $\left(\mathrm{pH}_{\mathrm{s}}\right)$ is different from that in the bulk, however literature evidence points to a raising of the $\mathrm{pK}_{\mathrm{a}}$ such that $\mathrm{pH}_{\mathrm{s}}$ is lower than that in the bulk[28,29]. Hence some other explanation is required that considers the interplay of interactions and forces at the interface, both within the monolayer, and between the monolayer and the subphase.

The presence of hydrogen bonding has been reported in partially dissociated pure fatty acid monolayers[30,31]. In addition, the substitution of acid for alcohol molecules in mixed systems could potentially enhance the propensity for such interand intramonolayer interactions, due to the closer head-group packing. The introduction of alcohol molecules would also lead to a restructuring of the localised water structure, as alcohol monolayers are known to promote the formation of highly hydrogen bonded ice-like structures in the subphase[32]. The redistribution of electron density within the head groups associated with hydrogen bonding could lead to an enhanced dissociation of the remaining acid groups, resulting in a negative deviation from ROM in the surface potential measurements.

If the maintenance of $\Delta \mathrm{V}_{\max }$, with increasing alcohol content, is the result of an enhancement of intramonolayer hydrogen bonding, the $A_{m}$ data should also reflect this. The results show condensation behaviour for the $\mathrm{A}_{\mathrm{m}}$ at both the onset to $L_{2} / L_{2}{ }^{\prime}$ transition (Figure 3.2a) and at a fixed surface pressure within the $L_{2} / L_{2}^{\prime}$ phase domain $\left(\Pi=10 \mathrm{mN} \mathrm{m}^{-1}\right)$. The reduction in $A_{m}$ is particularly evident between the compositions of $10 \mathrm{~mol} . \% \mathrm{ODOH}$ and $77 \mathrm{~mol} . \% \mathrm{ODOH}$. At the higher pressure of 25 $\mathrm{mN} \mathrm{m}{ }^{-1} \mathrm{ROM}$ behaviour is followed.

To a first approximation, the introduction of alcohol would reduce the overall charge density and more specifically like-charge repulsion. This combined with the smaller head group size of the alcohol would act to reduce the $A_{m}$ in proportion to the alcohol content leading to ROM-type behaviour. However, from the surface 
potential data discussed above, the monolayer-surface charge density is almost maintained upon substitution of alcohol due to the enhanced hydrogen bonding. Therefore like-charge repulsion remains significant and ROM would not be expected. Deviation from ROM (whether condensation or acid-like) would then depend on the extent of like-charge repulsion coupled with the formation of a hydrogen-bonded network. On a monolayer domain scale, the redistribution of charge associated with the hydrogen-bonded network would mitigate like-charge repulsion. Therefore acidlike behaviour would not be expected and one would predict condensation, as is observed.

To a point, the substitution of acid by alcohol would lead to a reduction in likecharge repulsion and steric constraints, potentially shortening the hydrogen bonds, the associated increase in bond strength may also mitigate increased deprotonation. Similarly, the relative rotational freedom of the alcohol (as the head group diameter is smaller than the tail) allows optimization of the relative orientations of the alcohol/acid/carboxylate head groups, increasing the number of hydrogen bonds by mitigating steric limitations. This rotational freedom could also facilitate reorientation of the acid head groups to create favourable carboxyl oxygen lone pair interactions, which was shown by Rebel et al.[33] to affect the acid $\mathrm{p} K_{\mathrm{a}}$ value. Here a shift in $\mathrm{p} K_{\mathrm{a}}$ was observed for different arrangements of the carboxylic acid functional groups in di-acid molecules.

Together, these effects would produce the observed condensation behaviour at pressures where head-group chemistry and symmetry define phase behaviour (here at onset and $\Pi=10 \mathrm{mN} \mathrm{m}^{-1}$ ). Moreover, consistent with this argument, the condensation is symmetric around the point of equal numbers of acid and alcohol molecules. The 1:1 ratio of acid to alcohol would maximise the carbonyl-oxygen to hydroxyl-hydrogen bonding, reported to be the longest lived[34]. This is mirrored in the condensation behaviour of the $L_{2} / L_{2}$ ' to $L S$ transition pressure data. As the $\mathrm{A}_{\mathrm{m}}$ is 
already reduced within this condensed region the pressure required to achieve the critical $\mathrm{A}_{\mathrm{m}}$ and induce a phase transition is also reduced. The $L_{2} / L_{2}{ }^{\prime}$ (tilted) to $L S$ (untilted) transition is principally dependent on the $A_{m}[35]$, and is a balance between the reduction in lattice spacing and the desire to maximise interactions between the tails[5]. Beyond this transition, it is generally accepted that the phase behaviour of fatty acid and alcohol monolayers is very similar[5], reflecting the dominance of the untilted hydrocarbon tail interactions. Consequently, the $\mathrm{A}_{\mathrm{m}}$ at a surface pressure of $25 \mathrm{mN} \mathrm{m}^{-1}$ displays ROM behaviour across the full range of acid/alcohol mixtures.

While this has been discussed purely from the perspective of intra- and intermolecular interactions, consideration of combinatorial entropic effects and their role in modifying these interactions should not be ignored. These entropic effects might play a considerable role in defining the phase behaviour of the mixed monolayer, modifying monolayer-subphase interactions and solvation of subphase ions. This is true for all subphases. In the case of water, formation of a hydrogenbonded network, the main driver in defining the state of the monolayer, is supported by entropically-driven mixing of the monolayer, both in terms of the head group interactions and local water structuring.

The formation of an intramonolayer hydrogen-bonding network upon substitution of alcohol explains the observed mixed fatty acid/alcohol monolayers behaviour on a water subphase. This hypothesis is supported by numerical calculations. Values for surface charge density and surface $\mathrm{pH}\left(\mathrm{pH}_{\mathrm{s}}\right)$ were found to remain approximately constant with increasing alcohol content, consistent with increased deprotonation of the remaining acid head groups. There was no evidence that this was achieved through anion binding.

\subsection{2 $20 \mathrm{mM}$ Calcium Chloride}

An enhanced double layer and a significant calcium-ion effect are evident for 
calcium chloride subphases. The presence of calcium ions mitigates like-charge repulsion, alters the localised $\left[\mathrm{H}^{+}\right]$or $\mathrm{pH}_{\mathrm{s}}[13,36]$, and has the capacity to disrupt hydrogen bonding between the head groups, between head groups and water and the hydrogen-bonded water network. The reduction in like-charge repulsion is seen most dramatically in the decreased $A_{m}$ of the pure acid at onset, from $24.1 \pm 0.1$ $\AA^{2} /$ molecule on water to $22.5 \pm 0.3 \AA^{2} /$ molecule for $\mathrm{CaCl}_{2}$ (Figures $3.2 \mathrm{a}$ and $3.2 \mathrm{~b}$ ). Such a reduction is not observed in the pure alcohol system, where the $A_{m}$ remains unchanged between the two subphases $\left(21.6 \pm 0.1\right.$ compared to $21.5 \pm 0.2 \AA^{2} /$ molecule on water). The different response of the acid monolayer is consistent with partial dissociation at $\mathrm{pH} \sim 5.5$.

The overall trend of the mixed systems for the $\mathrm{CaCl}_{2}$ subphase is to decrease $\mathrm{A}_{\mathrm{m}}$ upon increasing alcohol content, reflecting the smaller head group, as was the case on water. However, a closer comparison shows two distinct behaviours emerge: at low and high-alcohol content. These two behaviours correspond to the dominance of monolayer/subphase electrostatic interactions and intramonolayer hydrogen bonding, respectively.

\subsubsection{Low-alcohol content}

At low-alcohol content (up to $\sim 40 \mathrm{~mol}$ \%) all data (area per molecule, pressure and surface potential) follow the ROM (for example, Figure 3.2b). Here the presence of the calcium ions disrupt the hydrogen-bonding network and associated increase in acid dissociation. The latter is supported by an increase in the $\mathrm{pH}_{\mathrm{s}}$ from $\sim 3$ for water to $\sim 5$ for $\mathrm{CaCl}_{2}$ in numerical calculations. In addition, the $\mathrm{Ca}^{2+}$ and $\mathrm{Cl}^{-}$ions significantly increase the ionic strength, decreasing the surface potential and Debye length compared to that for water. However, in terms of monolayer behaviour, the dominant effect is $\mathrm{Ca}^{2+} / \mathrm{COO}^{-}$binding which effectively negates any like-charge repulsion resulting in a significant decrease in $\mathrm{A}_{\mathrm{m}}$. In addition, binding restricts head group reorientation reducing the capacity for intramonolayer hydrogen bonding. 
Clearly there is a strong electrostatic interaction between the calcium ions and the acid head groups, which is seen none more readily than in the surface potential data (Figure 3.5). On $20 \mathrm{mM} \mathrm{CaCl}_{2}$ the measured surface potential of the pure acid system is $\sim 70 \mathrm{mV}$ higher than that on pure water (Figure 3.4), reflecting significant changes in the interfacial interaction.

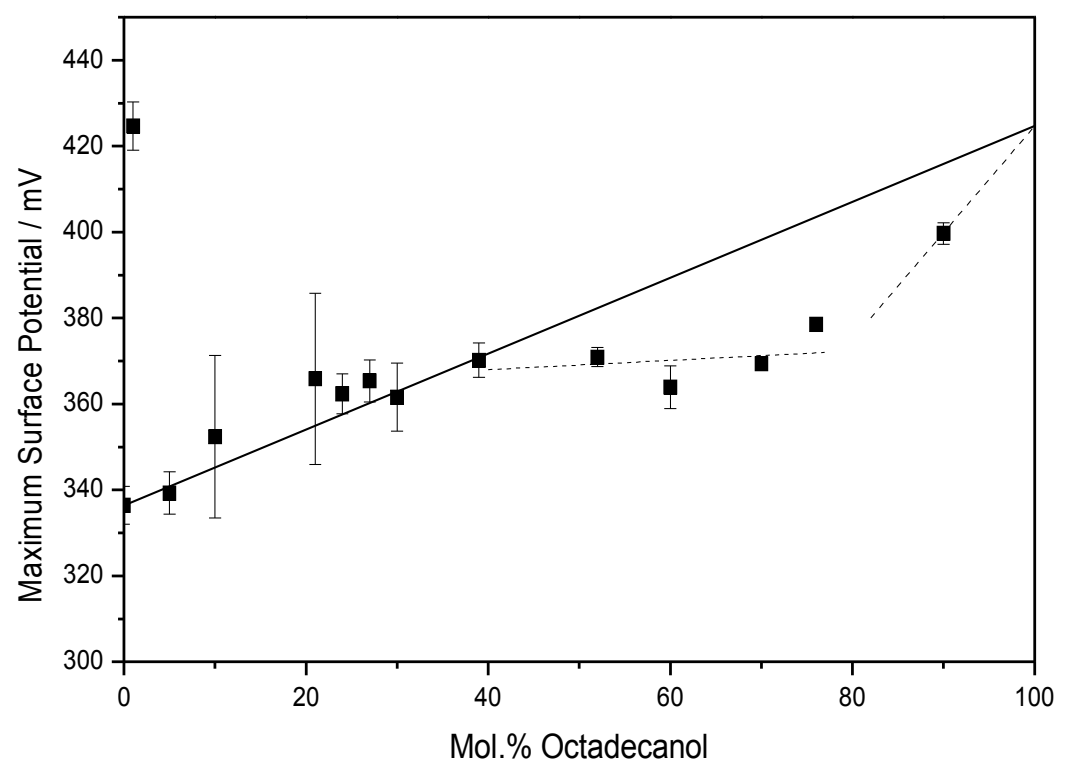

Figure 3.5 Surface potential plot for the mixed systems for a $20 \mathrm{mM} \mathrm{CaCl}$ subphase. The solid line reflects ROM behaviour, which is followed in the low-alcohol content monolayer (up to 40 mol.\%). The data shows significant negative deviation away from ROM for higher alcohol content, highlighted by the dashed line added to guide the eye.

As the monolayer charge density is decreased, upon alcohol substitution, the potential associated with the double layer $(\psi(0))$ would be expected to also reduce, increasing the measured surface potential. However, calculations show that $\psi(0)$ is approximately constant, with the exception of the pure alcohol. This disparity can be accounted for by considering the interfacial water, $\mu_{1} / \varepsilon_{1}$. The reduced monolayer charge density will alter the average head-group hydration-shell distance, which has been shown to significantly reduce the dielectric constant of the surface water $\left(\varepsilon_{1}\right)$ [37]. Similarly, the decreased cation concentration at the surface will reduce the local 
water concentration (reflecting the loss of water of solvation), also reducing $\varepsilon_{1}$. A reduction in $\varepsilon_{1}$ will lead to a smaller Debye length, despite the small decrease in the ionic strength (with a reduction in monolayer ionisation), diminishing the charge separation and therefore reducing the double layer component of the measured surface potential further. As this reduction, in both the amount and separation of charge, is associated with the substitution of the alcohol it follows that ROM behaviour is observed. Numerical calculations support this in returning significantly elevated negative values for $\mu_{1} / \varepsilon_{1}$ consistent with a reduced dielectric constant at the interface. Hence in terms of the model, the increasing $\Delta \mathrm{V}_{\max }$ with alcohol content is accounted for by the $\mu_{1} / \varepsilon_{1}$ term rather than $\psi(0)$. In reality, changes in both would be expected. The lack of change in $\psi(0)$ is likely due to the inability of the GCS theory to account for chemical interactions and the inherent error associated with the displacement of $\psi(0)$ and $\sigma$ to the outer Helmholtz plane.

The pressure at the $L_{2}$ to $L S$ transition, for the pure acid, is significantly reduced compared to that measured on water $\left(17.0 \pm 0.1\right.$ compared to $25.7 \pm 0.4 \mathrm{mN} \mathrm{m}^{-1}$ on water), while for pure alcohol there is negligible change $(13.1 \pm 0.4$ compared to $13.6 \pm$ $0.5 \mathrm{mN} \mathrm{m}^{-1}$ on water), within measurement error. Again, this indicates that the presence of $\mathrm{Ca}^{2+}$ ions reduces like-charge repulsion and induces the formation of bidentate complexes leading to a decrease in the pressure required to achieve the phase transition.

Together these data reveal that electrostatic interactions between the monolayer and the subphase dominate monolayer behaviour, effectively eliminating intramonolayer hydrogen bonding. This, in conjunction with the factors discussed above, leads to large negative values for the surface potential contribution originating from the interfacial subphase $\left(\mu_{1} / \varepsilon_{1}\right)$ and causes a reduction in the overall surface potential for low-alcohol content monolayers. 


\subsubsection{High-alcohol content}

A switch in behaviour is observed at $~ 50 \mathrm{~mol} . \%$ alcohol. This is most evident in the surface potential (Figure 3.5) and surface pressure at the $L_{2}$ to $L S$ phase transition data, both of which show a dramatic negative deviation away from ROM behaviour. This reflects a loss of monolayer/cation interactions corresponding to a reduction in the monolayer charge density. With the reduced $\mathrm{Ca}^{2+} /$ carboxylate interaction there is an increase in hydrogen bonding between monolayer head groups. Increased hydrogen bonding, as evidenced and discussed for the water subphase data, leads to higher degrees of dissociation facilitating a constant double-layer potential. That is, when intramonolayer hydrogen bonding dominates, the monolayer acts to maintain a constant charge density by inducing further dissociation of the remaining acid head groups. These findings are supported by the constant $\mathrm{pH}_{\mathrm{s}}$ values calculated numerically.

For high-alcohol content monolayers the system is dominated by hydrogenbonding interactions and the data more directly correlates with that observed on water. Indeed inspection of the surface potential and surface pressure at the $L_{2}$ to $L S$ phase transition data shows that the behaviour directly correlate with that of the water system, taking into consideration the already shifted absolute values as discussed above. The area per molecule data however requires further consideration.

On comparing the water and $\mathrm{CaCl}_{2}$ subphase data, the expected condensation behaviour, associated with increased hydrogen bonding, is not apparent for the $\mathrm{CaCl}_{2} \mathrm{~A}_{\mathrm{m}}$ plots (for example, Figure 3.2b). The apparent absence of any condensation behaviour for the $\mathrm{CaCl}_{2}$ subphase reflects the already condensed $\mathrm{A}_{\mathrm{m}}$, as attributed to reduced like-charge repulsion. Unlike the water subphase where the reduction of like-charge repulsion is brought about by the introduction of alcohol, for $\mathrm{CaCl}_{2}$ this role has already been partially fulfilled by the presence of the calcium ions. As such, the data manifests itself as more closely following ROM for all pressures, but this is 
only strictly the case for the high-pressure data. At lower pressures the precondensed monolayer masks the hydrogen-bonding effect.

The behaviour of the mixed monolayers for the $\mathrm{CaCl}_{2}$ subphase is complex, with evidence of a switch from electrostatic dominance at low-alcohol contents (i.e. monolayer/subphase interactions prevailing), to behaviour more consistent with hydrogen bonding determining the monolayer response (i.e. intramonolayer interactions dominating), similar to that observed in the pure water systems.

\subsection{3 $20 \mathrm{mM}$ Sodium Bicarbonate}

Moving to a sodium bicarbonate subphase introduces additional complexity. The bicarbonate anion concentration in an open, non-equilibrium system is very dynamic due to its participation in the $\mathrm{H}_{2} \mathrm{CO}_{3} / \mathrm{HCO}_{3}{ }^{-} / \mathrm{CO}_{3}{ }^{2-}$ acid/base equilibria, despite being the dominant ion between $\mathrm{pH} 6$ and 8. In addition, this subphase is supersaturated in carbon dioxide gas in order to lower the $\mathrm{pH}$ to $\sim 6$, which significantly perturbs the bicarbonate concentration.

Figure 3.6 shows the surface potential of the mixed systems for the sodium bicarbonate subphase. The pure acid monolayer has a maximum surface potential of $240 \pm 11 \mathrm{mV}$ in comparison to $437 \pm 3 \mathrm{mV}$ for the pure alcohol monolayer. These values correspond well with those measured on pure water (Figure 3.4). In contrast, the surface potential of the pure acid monolayer for the $\mathrm{CaCl}_{2}$ subphase is considerably higher (Figure 3.5). This is due to the strong interaction between the calcium ions and the acid head groups leading to a reduced ability for the double layer to counter the molecular dipole. The similarity of the sodium bicarbonate and water data suggests that electrostatic interactions between the monolayer and sodium ions, alone, do not determine the monolayer behaviour. This is consistent with the negative binding constant for sodium to carboxylates. In fact, unlike the calcium ions, the weakly kosmotropic nature (small highly charged ions that are strongly hydrated), and relatively poor binding affinity of $\mathrm{Na}^{+}$ions (to $\mathrm{COO}^{-}$) means 
that there is a tendency for these ions to be found preferentially in the bulk.

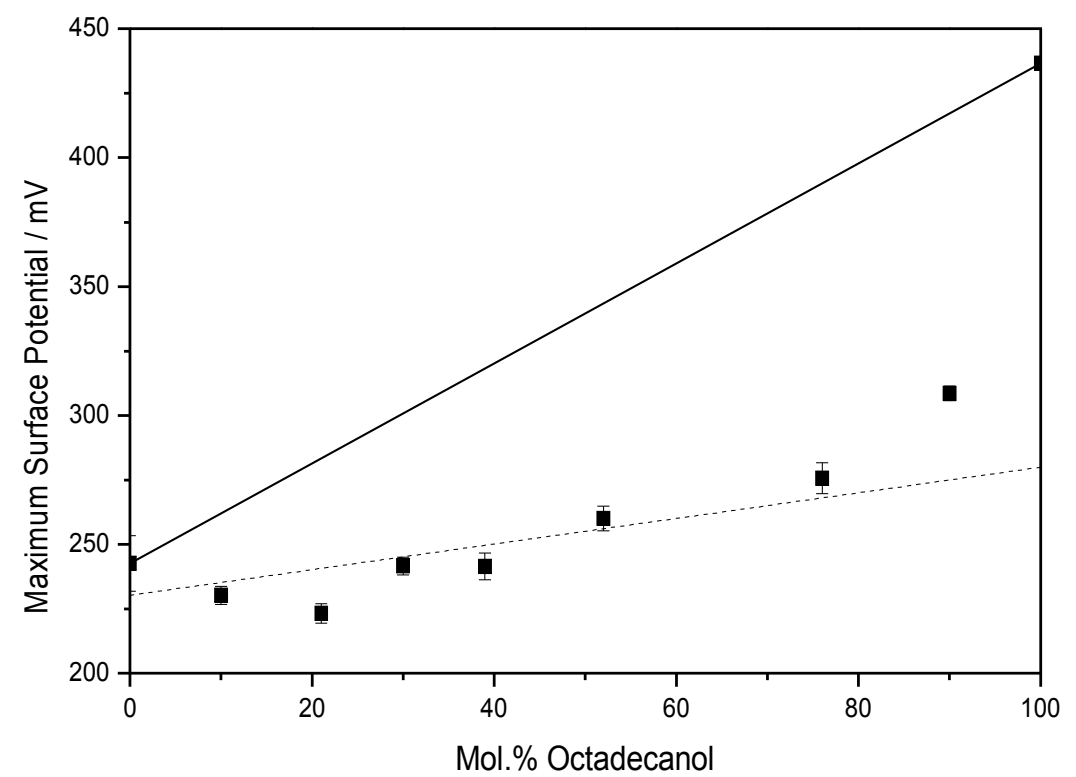

Figure 3.6 Variation of surface potential as a function of alcohol substitution for the $20 \mathrm{mM} \mathrm{NaHCO}$ subphase. The solid line represents the rule of mixtures trend and the dashed line guides the eye to the acid-like behaviour of the experimental results.

However, a closer comparison of the trends on water and sodium bicarbonate reveal several differences between the two subphases despite the lack of sodium interaction. On sodium bicarbonate, depressed surface potential values (Figure 3.6 c.f. Figure 3.4), a more pronounced condensation behaviour for the pressure at transition, smaller absolute $A_{m}$ values and the overall $A_{m}$ trends (compare Figures 3.2a and 3.2c), all indicate significant deviation from the water system. In fact, with the exception of the pure acid, the sodium bicarbonate data (for example Figure 3.2c) more closely match the behaviour of the calcium chloride data (for example Figure $3.2 b)$.

Combined, this data conclusively points to a strong interaction between the sodium bicarbonate subphase and the monolayer. The weak binding affinity of sodium ions implies that the bicarbonate anion is key in establishing an interface that incorporates aspects of the hydrogen-bonding dominated water system and the 
electrostatics-dominated calcium chloride system. More specifically the role of the $\mathrm{HCO}_{3}{ }^{-}$ion may include: 1) participation in the hydrogen-bonding network, invoking enhanced condensation, 2) ion-pair formation to mitigate like-charge repulsion, and 3) Hofmeister related $\mathrm{HCO}_{3}^{-} /$water interactions.

\subsubsection{A Hydrogen-Bonded Network}

The formation of a hydrogen-bonding network was shown in the water system to induce a greater degree of dissociation of the acid head-groups while also mitigating like-charge repulsion. Both of these effects are seen to a greater degree when bicarbonate ions are present in the subphase. This suggests that the bicarbonate ion actively manipulates the hydrogen-bonding network, increasing the capacity to hydrogen bond by influencing the orientational restructuring within the monolayer, via ion/dipole interactions or by directly participating in the network. The latter is the most likely, contributing both to the maintenance of the negatively charged interfacial region and the condensation of the monolayer through a more extensive hydrogen-bonding network. It should be noted that unlike the bicarbonate ion, the chloride ion cannot participate in hydrogen bonding and hence is unable to manipulate the hydrogen-bonding network in this way.

\subsubsection{Complexation}

Complexation provides a means by which the negative $\mathrm{HCO}_{3}^{-}$ion can approach and thereby influence the negative monolayer[13]. The neutralisation or reversal of charge with the formation of a complex is suggested to facilitate the approach of the co-ion to the monolayer. X-ray reflectivity studies[38] have shown, at least for the counter-ions, that the complexes are able to form chemisorption-like coordinate covalent bonds with the carboxylate head groups, rather than electrostatic interactions that would be weakened by ion solvation. Calculations by Lochhead et al.[13] showed that the surface concentration of the neutral and positively charged 
complexes was higher than that of the un-complexed co-ions, suggesting that complexes containing the bicarbonate ion (i.e. $\left[\mathrm{NaHCO}_{3}\right]^{0},\left[\mathrm{Na}_{2} \mathrm{HCO}_{3}\right]^{+},\left[\mathrm{H}_{2} \mathrm{CO}_{3}\right]^{0}$, etc.) could approach the monolayer surface and influence the monolayer behaviour. However, quantitative assessment of such speciation is very difficult, as such, this mechanism remains conjecture at this stage.

The existence of a neutral complex participating in the hydrogen-bonding network may be more readily rationalized than the negatively charged bicarbonate ion itself, but as will be discussed below the chaotropic nature (large weakly charged ions that are readily polarizable) of the bicarbonate ion may be sufficient to counter act its co-ion status.

\subsubsection{Hofmeister Related Interactions}

The propensity for an ion to complex is in part related to its charge density, with small highly charged ions forming complexes more readily. Similarly, Hofmeisterrelated ion-water interactions are associated with ionic charge density[39,40], where, for example, the way each individual species interacts with water influences its localised distribution. Studies investigating the Hofmeister series[41] and the surface concentration of ions in solution[42] show that chaotropes tend to residue at the surface where the polarizability of the ions is enhanced by the asymmetric solvent distribution, which strengthens the ion-water interactions[43]. Correspondingly, kosmotropes are strongly hydrated, having a stronger interaction with water than water-water interactions. Hence such ions tend to gravitate towards the bulk in order to maximise their water interactions.

The introduction of a surfactant monolayer however alters the density of the water in the vicinity of the monolayer via ion-dipole or dipole-dipole interactions. This is evident in studies which show the ice-like packing of water molecules below monolayers of fatty alcohols[32,44]. The nature of these interactions can lead to the creation of zones of high or low density water, which then favour particular ions, 
kosmotropes in high density water and chaotropes in low density water. Thus attraction of ions to the surface involves balancing ion-ion and ion-dipole interactions with hydration effects. For example, Cheng et al.[41] have shown that surfactants can selectively modify the anion affinity for the surface. Thus the propensity for particular ions to be attracted to or repelled from the interface is specific to the ion/surfactant combination.

With the view to defining the interfacial structure it is apparent that aspects of all three of these factors plays a role, especially when the crystal structure of sodium bicarbonate is considered. Crystalline sodium bicarbonate has a unique chain-like structure[45,46] (Figure 3.7), which incorporates charge compensation and extensive hydrogen bonding. It is hypothesised that a similar less ordered and hydrated structure forms at the monolayer subphase interface, where the incorporation of the sodium ion facilitates cation-mediated homoionic hydrogen bonding $[47,48]$, thus generating an extensively hydrogen-bonded network. Ordinarily the sodium concentration at the surface would be low, as indicated by the negative binding constant, however upon the formation of an ion-pair with the bicarbonate anion the propensity for surface activity increases. Likewise, like-charge repulsion would tend to restrict the opportunity for bicarbonate, and bicarbonate-carboxylate hydrogen bonding but as discussed by Braga et al. $[47,48]\}$ the presence of the cation leads to significant charge compression mitigating like-charge repulsion.

Numerical calculations support this model, with a significant increase in the sodium binding constant term, $\mathrm{p} K_{\mathrm{Na}}$. However, there was no subsequent increase in the bicarbonate binding constant. This is attributed to the hydrated anion being located in the 'second' layer of the chain complex (Figure 3.7), and therefore it is not considered part of the Stern layer per se. 


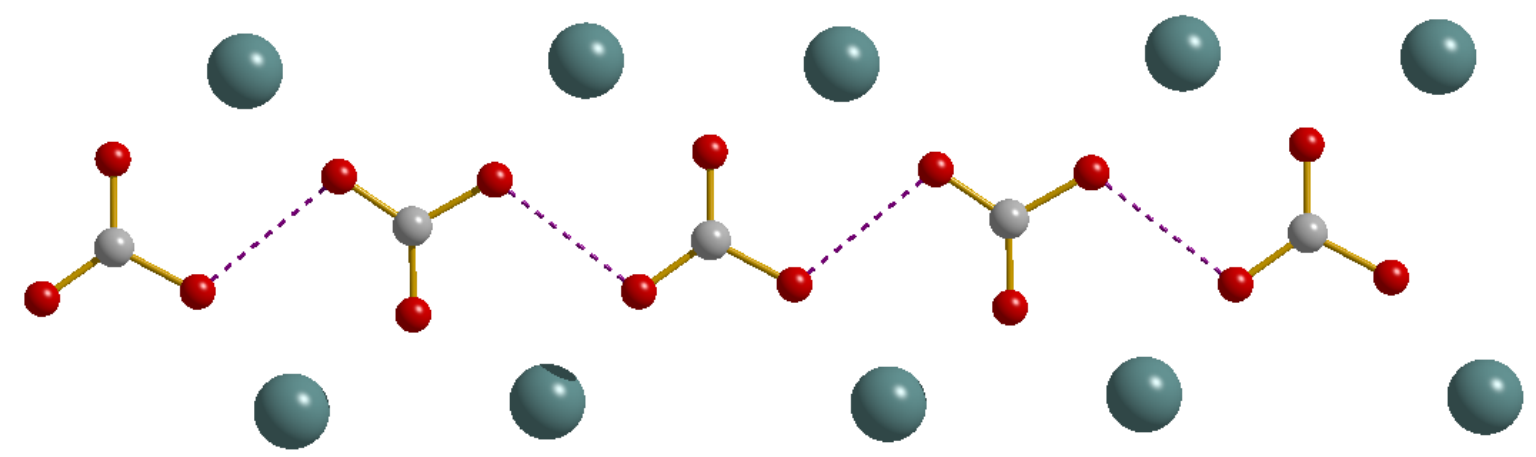

Figure 3.7 A 2-dimensional representation of the chain structure of crystalline sodium bicarbonate, viewed down the $a$ and $b$ axes. The large spheres represent the sodium ions, with the bicarbonate layer in between. The dashed lines depict the O-H‥O hydrogen bond between adjacent bicarbonate molecules.

Hence, a hydrated sodium bicarbonate hydrogen bonded chain-like structure associated with the monolayer is envisaged; oriented such that the sodium ions are in closest proximity with the monolayer. The calculated $\mathrm{pH}_{\mathrm{s}}$ is of the order observed for $\mathrm{CaCl}_{2}$, yet the potential and $\mu_{1} / \varepsilon_{1}$ fall between those of water and $\mathrm{CaCl}_{2}$, reflecting the hydrogen bonding/electrostatics combination. Similarly, with changes to the Hofmeister-related water-structuring brought about by network formation, it is conceivable that the formation of such a structure would alter ion hydration, the local dipole moment, dielectric constant, and surface potential thus explaining the mixed $\mathrm{A}_{\mathrm{m}}$ data[39,40].

Entropically, the increased order associated with a hydrogen-bonded network would be unfavourable, however we suggest that network formation is enthalpically driven by the formation of stronger interactions. The increased order of the network is offset by the release of water.

The concept of forming such a hydrogen-bonded sodium bicarbonate network is supported by the complexation phenomena proposed by Lochhead et al.[13], although such an extended structure was not considered. Additionally, the 2dimensional nature of the interface complements the linear nature of a $\mathrm{NaHCO}_{3}$ chain structure. In contrast, it is common for $\mathrm{NaCl}$ to be employed as a non- 
interacting electrolyte in surfactant systems, as the $\mathrm{Cl}^{-}$ion is unable to hydrogen bond. Thus the bicarbonate co-ion results in a unique structure, which imparts aspects of both hydrogen bonding and electrostatic interactions into the system, thus defining the interfacial structure.

In summary, cation mediated hydrogen bonding is believed to create a chain-like structure under mixed octadecanoic acid/octadecanol monolayers, similar to that observed for crystalline sodium bicarbonate. Such a structure incorporates aspects of electrostatic and hydrogen bonding based interactions that account for the observed surface pressure and surface potential phenomena. The bicarbonate anion is the principal factor in the establishment of the proposed interfacial structure. In comparison, the inability for the $\mathrm{Cl}^{-}$ion to hydrogen bond and its inactivity as a potential determining ion renders sodium chloride a non-interacting electrolyte for surfactant systems.

\subsubsection{0 mM Calcium Carbonate Crystallising Subphase}

The aim of this study was to improve our understanding of the interfacial interaction in a calcium carbonate crystallising system, by reducing the system to its components. Having formulated mechanisms for the interfacial interaction on water, $\mathrm{CaCl}_{2}$ and $\mathrm{NaHCO}_{3}$ subphases, in this section we look to use this information to enhance our understanding of $\mathrm{CaCO}_{3}$ nucleation at a monolayer. The main observations upon moving to a CCCS are a large reduction in $\mathrm{A}_{\mathrm{m}}$ (for example, Figure 3.2d) and ROM behaviour for $\Delta \mathrm{V}_{\max }$ (Figure 3.8).

For the first time the $A_{m}$ for the pure acid is smaller than that for the pure alcohol (for example at onset the $A_{m}$ are $20.1 \pm 0.1$ and $21.8 \pm 0.3 \AA^{2} /$ molecule, respectively, Figure $3.2 \mathrm{~d}$ ). The effect of this degree of condensation is the loss of the tilted phase at alcohol contents below $77 \mathrm{~mol} \%$ (at higher alcohol content the isotherm again correlates to that of the other subphases). On increasing alcohol content the $A_{m}$ at onset (Figure 3.2d) and $\Pi=10 \mathrm{mN} \mathrm{m}^{-1}$ (although weakly for the latter) display acid- 


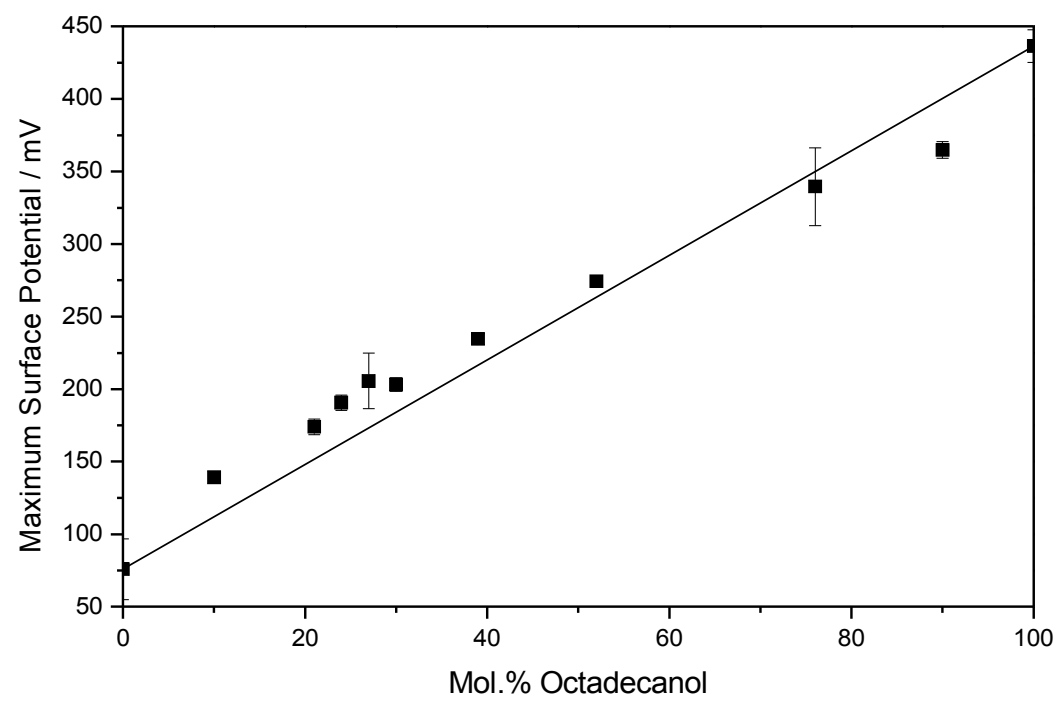

Figure 3.8 Surface potential plot for the mixed systems for the CCCS. The solid line reflects ROM behaviour.

like behaviour, again dissimilar to the condensation, ROM and condensation, and alcohol-like behaviour observed for the water, $\mathrm{CaCl}_{2}$ and $\mathrm{NaHCO}_{3}$ subphases, respectively. However the $A_{m}$ at $\Pi=25 \mathrm{mN} \mathrm{m}^{-1}$ exhibits ROM behaviour, as expected, reflecting the dominance of the tail interactions in defining the monolayer behaviour.

The surface potential data display a ROM trend for the full extent of the mixed systems (Figure 3.8). However the difference in $\Delta \mathrm{V}_{\max }$ from the pure acid $(76 \pm 21 \mathrm{mV})$ to the pure alcohol $(437 \pm 11 \mathrm{mV})$ was significantly larger for the CCCS than for any other subphase. Similarly, the absolute potential values for the acid-rich monolayers were also significantly lower than those observed for the other subphases. This points towards a significant double layer potential, that steadily declines in step with the substitution of alcohol molecules.

In order to achieve concurrently, a large decrease in surface potential and area per molecule, the formation of a hydrogen-bonded soap complex is hypothesised, involving the acid heads, $\mathrm{Ca}^{2+}$ ions and $\mathrm{HCO}_{3}^{-}$ions. Although similarly postulated 
for the $\mathrm{NaHCO}_{3}$ subphase, the strong $\mathrm{Ca}^{2+}$ binding leads to a more condensed and ordered soap network, with less solvation. Keeping in mind that this structure would be a precursor to a likely amorphous $\mathrm{CaCO}_{3}$ phase, the extent of ordering is small and localised at best. The necessity for charge compensation and bicarbonate hydrogen bonding means that the soap network is loosely ordered into alternating charged layers, thus creating a sizeable dipole, and the reduction in $\Delta \mathrm{V}_{\max }$.

The strong ROM behaviour of $\Delta \mathrm{V}_{\max }$ for all alcohol contents can be attributed to significant cation binding and the presence of a hydrogen-bonded network. Steric implications associated with the high degree of compression, brought about by the mitigation of like-charge repulsion and the hydrogen-bonded network, reduce the capacity for intramonolayer hydrogen bonding. Therefore unlike $\mathrm{CaCl}_{2}$ there is no apparent enhancement in the degree of acid dissociation that might lead to a deviation from ROM behaviour.

The numerical model provides tentative support for the proposed interface structure, with values consistent with the presence of electrostatic and hydrogen bonding based interactions. The monolayer dipole components, $\mu_{1} / \varepsilon_{1}$, $\varepsilon_{2}$, and $\varepsilon_{3}$ all show trends consistent with the ROM behaviour of $\Delta \mathrm{V}_{\max }$. At low alcohol contents the dielectric constant terms are all elevated reflecting the strong interfacial interaction and the highly compressed monolayer state. However, on increasing alcohol content these values reduce to the levels observed for $\mathrm{CaCl}_{2}$, following a ROM trend. While $\psi(0), \sigma$ and $\mathrm{pH}_{\mathrm{s}}$ display acid-like trends reflecting the influence of the strong cation binding evident in the $\mathrm{A}_{\mathrm{m}}$ results. Unsurprisingly, the model does not provide conclusive evidence for the proposed structure as it fails to account for the formation of complex structures as described above. Despite this we can obtain a good fit for the experimental $\Delta \mathrm{V}_{\max }$ results and qualitative trends that support our hypothesis. 


\subsection{Conclusions}

To advance our understanding of the monolayer/subphase interactions that define and control monolayer structure and subsequent crystallisation at soft interfaces, we have investigated the behaviour of mixed octadecanoic acid/octadecanol systems on four different subphases: ultrapure water, calcium chloride, sodium bicarbonate and a CCCS.

Analysis of our results have led us to propose the following conclusions for the non-equilibrium, mixed monolayer, nucleating subphase systems:

1. On increasing alcohol content, the degree of deprotonation of the acid molecules can be enhanced via hydrogen bonding, thus maintaining a constant surface charge. However this phenomenon is readily cancelled by dominant electrostatic interactions such as the presence of a divalent cation.

2. The presence of the bicarbonate anion can lead to the formation of a cationmediated hydrogen-bonded network.

3. The combination of a strongly binding counter-ion $\left(\mathrm{Ca}^{2+}\right)$ and the bicarbonate anion leads to a hydrogen-bonded soap-like network that acts as a driver for extensive compression of the monolayer, beyond that achieved through electrostatic interactions alone.

The bicarbonate anion is highly influential in terms of interfacial structure, and when present, should always be considered in any analysis of interfacial dynamics. This extends to the processes of templated crystal nucleation, where directionality, of some form, is required to achieve preferential orientation. We suggest that the formation of such a hydrogen-bonded network may introduce the required degree of directionality into the interfacial interaction. Crystallisation studies to explore this further are currently underway.

The combined DF and GCS model was found to readily fit the experimental results. However, this was not necessarily an indication of a good model but rather a 
reflection of the number of fitted parameters. The difficulty in fitting the data lay in obtaining a fit with values that were feasible and realistic. For example, it was found that in some cases that the dielectric constant terms tended to be large, likely compensation for the inability of the GCS theory to account for chemical effects, such as hydrogen bonding, Hofmeister effects, ion-specific effects among other subtle contributing influences. This is a reflection of the technique itself which measures a bulk averaged property.

\subsection{References}

[1] M. Fricke and D. Volkmer, "Crystallization of Calcium Carbonate Beneath Insoluble Monolayers: Suitable Models of Mineral-Matrix Interactions in Biomineralization?," Topics in Current Chemistry, vol. 270, 2007, pp. 1-41.

[2] P. Calvert and S. Mann, "The negative side of crystal growth," Nature, vol. 386, 1997, pp. 127-128.

[3] W. Harkins and T. Anderson, "I. A Simple Accurate Film Balance of the Vertical Type for Biological and Chemical Work, and a Theoretical and Experimental Comparison with the Horizontal Type. II. Tight Packing of a Monolayer by Ions.," Journal of the American Chemical Society, vol. 59, 1937, pp. 2189-2197.

[4] G. Gaines Jnr., Insoluble Monolayers at Liquid-Gas Interfaces, John Wiley \& Sons , 1966.

[5] V. Kaganer, H. Möhwald, and P. Dutta, "Structure and Phase Transitions in Langmuir Monolayers," Reviews of Modern Physics, vol. 71, 1999, pp. 779-819.

[6] T. Kim and D. Ahn, "In-situ FTIR Investigation of Heterogeneous Langmuir Monolayers at the Air/Water Interface," Materials Science and Engineering $C$, vol. 24,2004 , pp. 205-208.

[7] M. Shih, M. Durbin, A. Malik, P. Zschack, and P. Dutta, "Lattice Structures and Molecular Tilts in Langmuir Monolayers of Saturated Fatty Acid-Alcohol Mixtures," J.Chem.Phys., vol. 101, 1994, pp. 9132-9136.

[8] B. Fischer, E. Teer, and C. Knobler, "Optical Measurement of the Phase Diagram of Langmuir Monolayers of Fatty Acid-Alcohol Mixtures," J.Chem.Phys., vol. 103, 1995, pp. 2365-2368.

[9] D. Taylor, O. Oliveira Jr, and H. Morgan, "Models for interpreting surface potential measurements and their application to phospholipid monolayers," J.Colloid and Interface Sci., vol. 139, Oct. 1990, pp. 508-518.

[10] D. Taylor and G. Bayes, "Calculating the Surface Potential of Unionized Monolayers," Physical Review E, vol. 49, 1994, pp. 1439-1449.

[11] V. Shapovalov and G. Brezesinski, "Breakdown of the Gouy-Chapman Model 
for Highly Charged Langmuir Monolayers: Counterion Size Effect," Journal of Physical Chemistry B, vol. 110, May. 2006, pp. 10032-10040.

[12] R. Demchak and T. Fort, "Surface Dipole Moments of Close-Packed Un-ionized Monolayers at the Air-Water Interface," J.Colloid and Interface Sci., vol. 42 , 1974, pp. 191-202.

[13] M. Lochhead, S. Letellier, and V. Vogel, "Assessing the Role of Interfacial Electrostatics in Oriented Mineral Nucleation at Charged Organic Monolayers," Journal of Physical Chemistry B, vol. 101, 1997, pp. 10821-10827.

[14] S. McLaughlin, N. Mulrine, T. Gresalfi, G. Vaio, and A. McLaughlin, "Adsorption of divalent cations to bilayer membranes containing phosphatidylserine," The Journal of General Physiology, vol. 77, Apr. 1981, pp. 445-473.

[15] O. Oliveira Jr, D. Taylor, and H. Morgan, "Modelling the Surface Potential-Area Dependence of a Stearic Acid Monolayer," Thin Solid Films, vol. 210/211, 1992, pp. 76-78.

[16] V. Agarwal and B. Ichijo, "Determination of Dielectric Constant of Stearic Acid Films Using Varying Gap Immersion Method," Electrocomponent Science and Technology, vol. 4 , 1977, pp. 23-28.

[17] CRC Handbook of Chemistry and Physics, The Chemical Rubber Co., 2005.

[18] V. Tsukanova, D. Grainger, and C. Salesse, "Monolayer Behavior of NBDLabeled Phospholipids at the Air/Water Interface," Langmuir, vol. 18, Jul. 2002, pp. 5539-5550.

[19] P. Dynarowicz-Latka, A. Cavalli, D.A. Silva Filho, M. dos Santos, and O.N. Oliveira, "Dipole moments in Langmuir monolayers from aromatic carboxylic acids," Chemical Physics Letters, vol. 326, 2000, pp. 39-44.

[20] G. Lamm, "The Poisson-Boltzmann Equation," Reviews in Computational Chemistry, vol. 019, John Wiley \& Sons, 2003, pp. 147-367.

[21] M. Manciu and E. Ruckenstein, "Specific ion effects via ion hydration: I. Surface tension," Advances in colloid and interface science, vol. 105, 2003, pp. 63-101.

[22] J. Simon-Kutscher, A. Gericke, and H. Huhnerfuss, "Effect of Bivalent $\mathrm{Ba}, \mathrm{Cu}, \mathrm{Ni}$, and $\mathrm{Zn}$ Cations on the Structure of Octadecanoic Acid Monolayers at the AirWater Interface As Determined by External Infrared Reflection-Absorption Spectroscopy," Langmuir, vol. 12, Feb. 1996, pp. 1027-1034.

[23] A. Bibo and I. Peterson, "Phase Diagrams of Monolayers of Long Chain Fatty Acids," Advanced Materials, vol. 2, 1990, pp. 309-311.

[24] Y. Lee, Y. Yang, and Y. Shen, "Monolayer Characteristics of Mixed Octadecylamine and Stearic Acid at the Air/Water Interface," Journal of Physical Chemistry B, vol. 109 , 2005, pp. 4662-4667.

[25] G. Lawrie and G. Barnes, “Octadecanol Monolayers: The Phase Diagram," J.Colloid and Interface Sci., vol. 162, Jan. 1994, pp. 36-44.

[26] V. Vogel and D. Möbius, "Local Surface Potentials and Electric Dipole Moments of Lipid Monolayers: Contributions of the Water/Lipid and the Lipid/Air 
Interfaces," J.Colloid and Interface Sci., vol. 126 , 1988, pp. 408-420.

[27] O. Oliveira Jr and C. Bonardi, "The Surface Potential of Langmuir Monolayers Revisited," Langmuir, vol. 13, 1997, pp. 5920-5924.

[28] J.R. Kanicky, A. Poniatowski, N. Mehta, and D. Shah, "Cooperativity among Molecules at Interfaces in Relation to Various Technological Processes: Effect of Chain Length on the pKa of Fatty Acid Salt Solutions ," Langmuir, vol. 16, 2000, pp. 172-177.

[29] J.R. Kanicky and D.O. Shah, "Effect of Degree, Type, and Position of Unsaturation on the pKa of Long-Chain Fatty Acids," J.Colloid and Interface Sci., vol. 256, Dec. 2002, pp. 201-207.

[30] A. Gericke and H. Huhnerfuss, "In Situ Investigation of Saturated Long-Chain Fatty Acids at the Air/Water Interface by External Infrared ReflectionAbsorption Spectrometry," Journal of Physical Chemistry, vol. 97, 1993, pp. 1289912908.

[31] R. Johann, D. Vollhardt, and H. Möhwald, "Study of the pH Dependence of Head Group Bonding in Arachidic Acid Monolayers by Polarization Modulation Infrared Reflection Absorption Spectroscopy," Colloids and Surfaces A: Physicochemical and Engineering Aspects, vol. 182, 2001, pp. 311-320.

[32] I. Kuzmenko, H. Rapaport, K. Kjaer, J. Als-Nielsen, I. Weissbuch, M. Lahav, and L. Leiserowitz, "Design and Characterization of Crystalline Thin Film Architectures at the Air-Liquid Interface: Simplicity to Complexity," Chemical Reviews, vol. 101, 2001, pp. 1659-1696.

[33] J. Rebel, R. Duff, W. Gordon, and K. Parris, "Convergent Functional Groups Provide a Measure of Stereoelectronic Effects at Carboxyl Oxygen," Journal of the American Chemical Society, vol. 108 , 1986, pp. 6068-6069.

[34] N. Winter, J. Vieceli, and I. Benjamin, "Hydrogen-Bond Structure and Dynamics at the Interface Between Water and Carboxylic Acid-Functionalized SelfAssembled Monolayers," Journal of Physical Chemistry B, vol. 112 , 2008, pp. 227231.

[35] E. Teer, C. Knobler, C. Lautz, S. Wurlitzer, J. Kildae, and T. Fischer, "Optical Measurements of the Phase Diagrams of Langmuir Monolayers of Fatty Acid, Ester, and Alcohol Mixtures by Brewster-Angle Microscopy," J.Chem.Phys., vol. 106,1997, pp. 1913-1920.

[36] E. Le Calvez, D. Blaudez, T. Buffeteau, and B. Desbat, "Effect of Cations on the Dissociation of Arachidic Acid Monolayers on Water Studied by PolarizationModulated Infrared Reflection-Absorption Spectroscopy," Langmuir, vol. 17, 2001, pp. 670-674.

[37] V. Vogel and D. Möbius, "Hydrated Polar Groups in Lipid Monolayers: Effective Local Dipole Moments and Dielectric Properties," Thin Solid Films, vol. 159, 1988, pp. 73-81.

[38] J. Li, K. Liang, G. Scoles, and A. Ulman, “Counterion Overlayers at the Interface 
between an Electrolyte and an .omega.-Functionalized Monolayer SelfAssembled on Gold. An X-ray Reflectivity Study," Langmuir, vol. 11, Nov. 1995, pp. 4418-4427.

[39] K. Collins and M. Washabaugh, "The Hofmeister Effect and the Behaviour of Water at Interfaces," Quarterly Reviews of Biophysics, vol. 18, 1985, pp. 323-422.

[40] M. CACACE, E. Landau, and J. RAMSDEN, "The Hofmeister series: salt and solvent effects on interfacial phenomena," Quarterly Reviews of Biophysics, vol. 30, 2000, pp. 241-277.

[41] J. Cheng, C. Vecitis, M. Hoffmann, and A. Colussi, "Experimental Anion Affinities for the Air/Water Interface," Journal of Physical Chemistry B, vol. 110, Dec. 2006, pp. 25598-25602.

[42] B. Garrett, "Ions at the Air/Water Interface," Science, vol. 303, 2004, pp. 11461147.

[43] G. Archontis and E. Leontidis, "Dissecting the stabilization of iodide at the airwater interface into components: A free energy analysis," Chemical Physics Letters, vol. 420, Mar. 2006, pp. 199-203.

[44] P. Miranda, Q. Du, and Y. Shen, "Interaction of Water with a Fatty Acid Langmuir Film," Chemical Physics Letters, vol. 286, 1998, pp. 1-8.

[45] R. Sass and R. Scheuerman, "The crystal structure of sodium bicarbonate," Acta Cryst., vol. 15, 1962, pp. 77-81.

[46] B. Sharma, "Sodium bicarbonate and its hydrogen atom," Acta Cryst. vol. 18, 1965, pp. 818-819.

[47] D. Braga, L. Maini, F. Grepioni, F. Mota, C. Rovira, and J. Novoa, "Interanionic (-)O-H...O(-) Interactions: A Solid-State and Computational Study of the Ring and Chain Motifs," Chemistry - A European Journal, vol. 6, 2000, pp. 4536-4551.

[48] D. Braga, E. D'Oria, F. Grepioni, F. Mota, J. Novoa, and C. Rovira, "O-H...O Interactions Involving Doubly Charged Anions: Charge Compression in Carbonate-Bicarbonate Crystals," Chemistry - A European Journal, vol. 8, 2002, pp. 1173-1180. 


\section{Chapter 4: Morphology and Orientation for Mixed Monolayer Systems}

\subsection{Introduction}

Exploration of the intrinsic monolayer properties, detailed in the previous chapter, has allowed us to identify a potential mechanism for information transfer from the monolayer to a growing calcium carbonate crystal. In this chapter we analyse the subsequent calcium carbonate crystallisation (particularly calcite) under these same mixed monolayers in order to validate and develop the hypothesis.

Crystallisation, both homogeneous and heterogeneous, was described in Chapter 1 , therefore the following is only a brief reminder of the relevant points. Solutionbased calcium carbonate crystallisation involves two main stages: nucleation and growth. Nucleation involves the complexation, agglomeration, deprotonation $\left(\mathrm{HCO}_{3}^{-}\right.$to $\left.\mathrm{CO}_{3}^{2-}\right)$, ordering, and dehydration of ions (not necessarily in this order). For nuclei of sufficient size, this is followed by growth or ripening.

Heterogeneous crystal growth under a 2-dimensional insoluble Langmuir monolayer is no different, except that the monolayer can thermodynamically promote crystallisation, where the monolayer functions as a seed crystal, lowering the barrier to nucleation. Additionally, ionised monolayers can act to concentrate ions at the interface, modifying the kinetics of crystallisation. The degree of complementarity between the monolayer and the nucleating crystal influences the magnitude of these effects. Properties such as surface charge, geometry, symmetry, polarity and lattice spacing all contribute to the promotion and stabilisation of crystal nuclei. The insolubility of the surfactant molecules limits the manipulation of crystal 
nucleation and growth by the monolayer to orientational and kinetic effects. As such, in this chapter we will deal with the presence or absence of face-selective nucleation, and morphological modifications brought about by monolayer-facilitated crystal growth.

Much has been made of the path from nuclei to crystalline calcite[1], whether calcite nucleates directly or forms via (following Ostwald's rule of phases) metastable amorphous calcium carbonate and vaterite phases. Intuitively, the mechanism will likely depend on the extent of complementarity between the monolayer and the thermodynamically stable calcite phase, and the surface charge of the monolayer. Either way, the occurrence of face-selective nucleation implies some level of information transfer between the monolayer and the calcite crystal whether it is at the nuclei level or the vaterite to calcite transformation.

In this chapter we probe the crystallisation of calcite under a range of octadecanoic acid/octadecanol mixed monolayers with the aim of correlating monolayer properties with those of the resulting crystals. This involves the comparison of morphological and orientation-based properties of crystals grown under monolayers of increasing octadecanol substitution. Crystal orientation is qualitatively determined for each system using the inter-edge angle method[2]. Finally the trends in morphology and orientation are correlated with the hypothesis developed in the previous chapter for defining the assembly of ions at the monolayer-subphase interface. In addition this discussion will address the roles that charge density, lattice matching and symmetry have on face-selective nucleation.

\subsection{Results}

Mineralisation at the air/water interface in the absence of a monolayer is illustrated in Figure 4.1. Nucleation occurs preferentially at the interface where the surface energy is high, however the induction times are typically longer than those experienced in the presence of a monolayer. Crystal morphology and orientation are 
non-specific with regions of intergrowth, consistent with that observed by Loste et al. [3] under similar conditions.

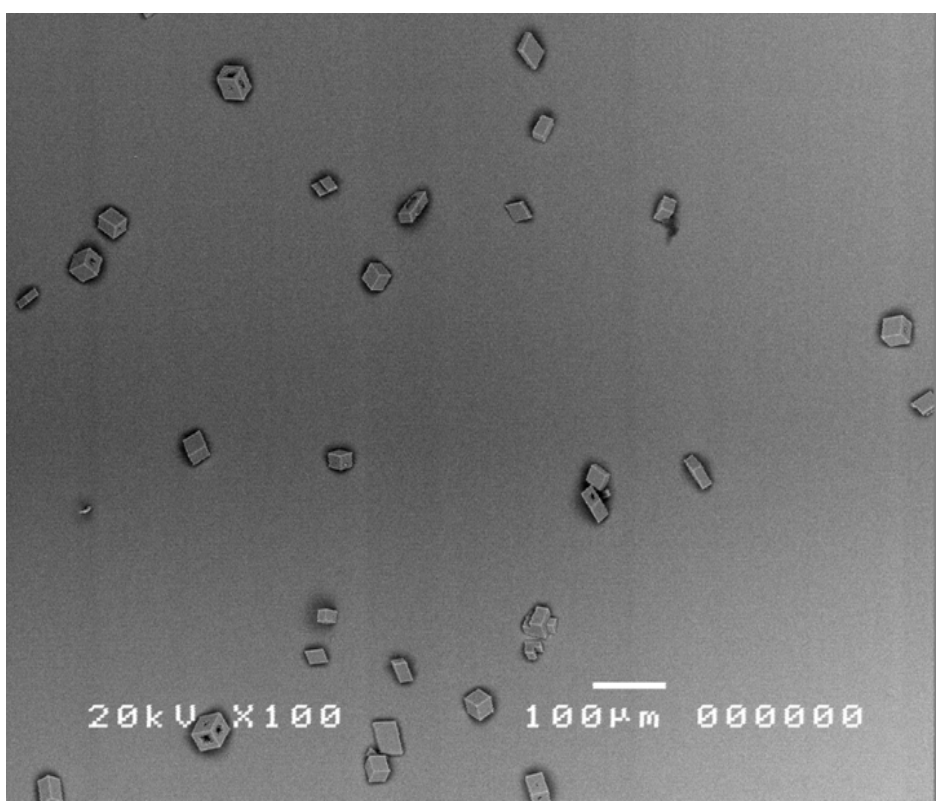

Figure 4.1 Nucleation at the air/water interface in the absence of a monolayer. The product is randomly oriented rhombohedral calcite crystals with very little elongation.

Growth under monolayers has been well characterised[4-8], particularly for the more common surfactant systems of pure octadecanol and octadecanoic acid. Consequently the evaluation of growth under mixed monolayers will employ the well established crystal descriptors for calcite: type I, II, and III, in addition to the classic rhombohedral growth (Figure 4.2)[3,9-12]. It is important to note that as discussed by Loste et al.[3] type III crystals are in fact inverted in the harvesting process and therefore are an extension of the truncated rhombohedral crystal. However, before assessing crystal morphology and orientation it is pertinent to revisit and elaborate on the monolayer behaviour before and during crystallisation. 

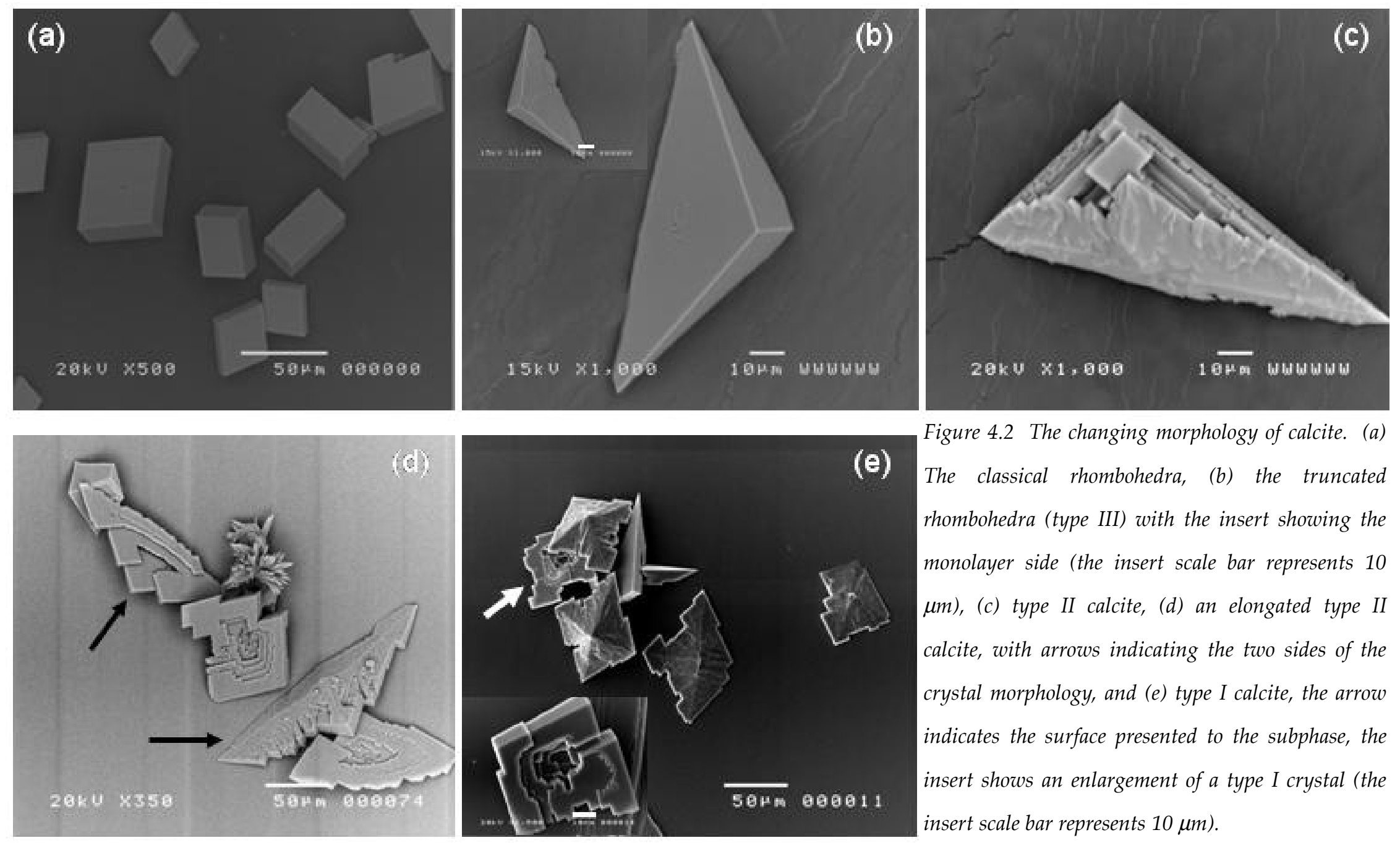

Figure 4.2 The changing morphology of calcite. (a)

The classical rhombohedra, (b) the truncated

rhombohedra (type III) with the insert showing the monolayer side (the insert scale bar represents 10 $\mu \mathrm{m})$, (c) type II calcite, (d) an elongated type II calcite, with arrows indicating the two sides of the crystal morphology, and (e) type I calcite, the arrow indicates the surface presented to the subphase, the insert shows an enlargement of a type I crystal (the insert scale bar represents $10 \mu \mathrm{m}$ ). 


\subsubsection{Monolayer Behaviour}

In the previous chapter we illustrated the significant reduction in $A_{m}$, the loss of a tilting transition, and the rule of mixtures behaviour of the surface potential, associated with a CCCS. We attributed these effects to the interfacial interaction, or more specifically the formation of a hydrogen-bonded soap network. Although somewhat disordered and hydrated, such a network forms the precursor to crystal nucleation. Further, the nature of the cation-mediated hydrogen-bonded network provides a mechanism for achieving face-selective nucleation.

An exception to the general loss of tilt on CCCS occurs at high alcohol contents (>70 mol.\%, Figure 4.3). The tilting transition occurs at $\sim 6 \mathrm{mN} \mathrm{m}^{-1}$ for the $75 \mathrm{~mol} . \%$ ODOH monolayer, meaning that at the lowest crystal growth pressure $\left(10 \mathrm{mN} \mathrm{m}^{-1}\right)$ there is no tilt. Consequently only crystals grown under pure octadecanol at $\Pi=10 \mathrm{mN} \mathrm{m}^{-1}$ experience a tilted monolayer. Despite this, there remains the possibility that the presence of a tilted phase during the monolayer equilibration time (not observed for lower alcohol contents) could impart a subtly altered packing structure in the final monolayer. An evaluation of crystals grown under $100 \%$ ODOH at $\Pi=10$ and $25 \mathrm{mN} \mathrm{m}^{-1}$ shows no significant difference, suggesting that, in terms of manipulating crystal morphology, the influence of historical packing conformation is negligible. 


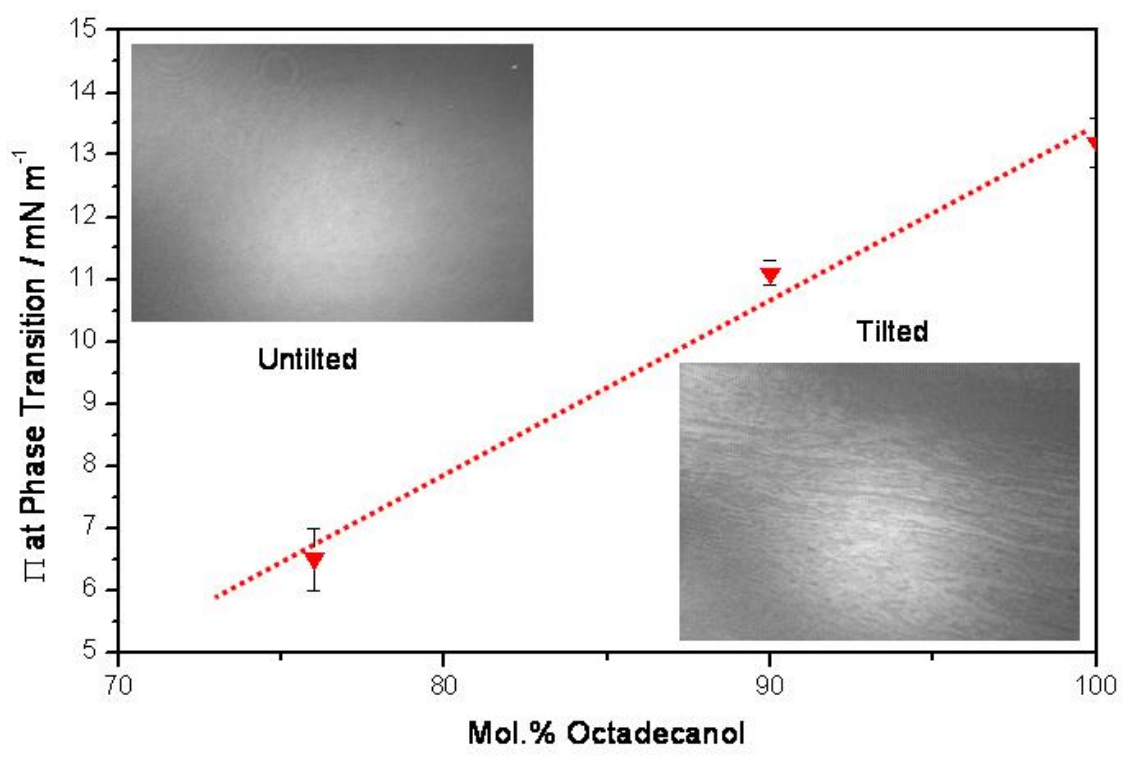

Figure 4.3 Evidence for the absence and presence of tilt in the mixed monolayers on CCCS. The red dashed line guides the eye to the tilting transition from the tilted $L_{2}$ phase to the untilted LS phase for the mixed monolayer systems. Brewster angle microscopy images are included as inserts to illustrate the absence and presence of tilt. The image of the untilted LS phase (upper left) for the CCCS at low alcohol contents lacks contrast due to the absence of tilt. In contrast, the presence of the tilted $L_{2}$ phase is reflected in the contrasting domains (lower right).

Another feature of these monolayers is their propensity for 'slow collapse' during the crystallisation experiments (Figure 4.4), where the concept of slow collapse refers to the time-dependent degradation of the monolayer at pressures greater than the equilibrium spreading pressure (ESP) but below the traditional (fast) collapse pressure. The latter is characterised by a fracture or catastrophic mechanism as opposed to the gradual degradation observed in slow collapse[13]. However both modes of collapse involve expulsion of molecules from the monolayer associated with the nucleation and growth of 3-D phases. Ybert et al.[14] found three distinct mechanisms for collapse, dependent on the surface pressure and the rate of compression, of which slow collapse was associated with the formation of multilayer islands. Higher pressures led to folding, with 'giant folds' forming with low 
compression speeds. For phospholipid monolayers these giant folds are reported to correlate to the formation of vesicles via a first order transition[15].

Examination of the mixed monolayer's slow collapse behaviour during crystallisation experiments indicates a trend of increasing monolayer stability (where stability is defined by the absence of slow collapse) with increasing alcohol content. Further comparison of the low and high pressure behaviour (Figure 4.4) shows that the monolayer is generally more stable at low pressures. These observations are consistent with the pure alcohol having a higher ESP $\left(\sim 35 \mathrm{mN} \mathrm{m}^{-1}[16]\right)$ than the pure acid monolayer $\left(\mathrm{ESP}=2-7.3 \mathrm{mN} \mathrm{m}^{-1}[16-18]\right)$.

(a)

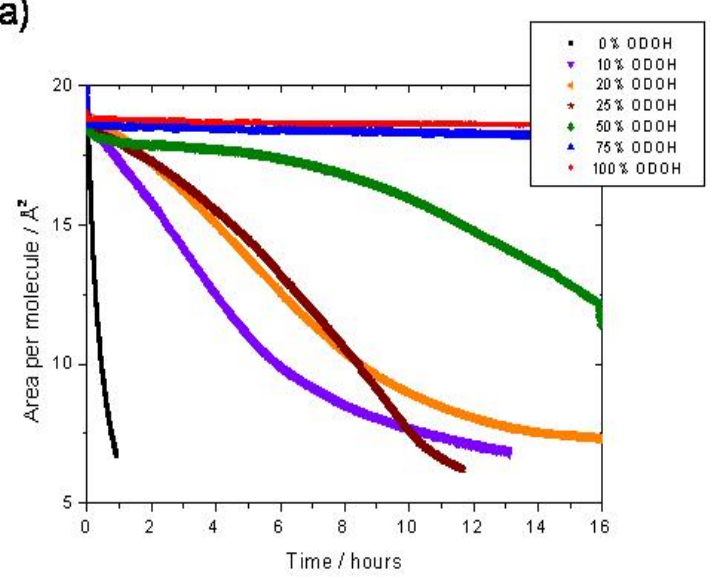

(b)

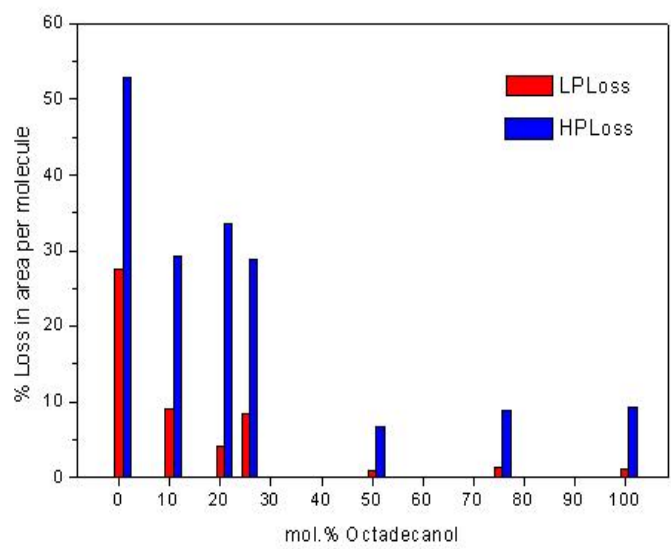

Figure 4.4 (a) The change in area per molecule with time (monolayer stability) during the crystal growth experiments at a constant surface pressure of $25 \mathrm{mN} \mathrm{m}^{-1}$. (b) A comparison of the loss in area after 4 hours of crystal growth for the two pressures $\left(L P=10 \mathrm{mN} \mathrm{m}^{-1}\right.$ and $\left.H P=25 \mathrm{mN} \mathrm{m}^{-1}\right)$.

In order to explore this further, the long term stability of monolayers of $10 \mathrm{~mol} \%$ $\mathrm{ODOH}$ on water and carbon dioxide bubbled $\mathrm{CaCl}_{2}$ subphases at $\Pi=25 \mathrm{mN} \mathrm{m}^{-1}$ was examined. The relative instability on water (Figure $4.5, \sim 30 \%$ reduction in $\mathrm{A}_{\mathrm{m}}$ ) reflects the low ESP resulting in slow collapse probably via a folding mechanism given the high pressure. In contrast the presence of $\mathrm{Ca}^{2+}$ ions in the subphase leads to significant stabilisation $\left(\sim 5 \%\right.$ reduction in $\left.\mathrm{A}_{\mathrm{m}}\right)$. This is consistent with the use of divalent cations to improve the properties of Langmuir-Blodgett films[19]. In the 
case of the CCCS-based system, there is a large increase in monolayer instability $\left(\sim 70 \%\right.$ reduction in $\left.A_{m}\right)$. This instability is most likely brought about by the increased solubility and/or the presence of defects (calcium carbonate nuclei) promoting nucleation and growth of a 3-D surfactant phase, whether it be folds or vesicles. The different profile shapes of water and CCCS in Figure 4.5 reflect a different nucleation and growth mechanism for the 3-D surfactant phase[20]. Whether instantaneous or progressive nucleation, whether folding or vesicular, the actual mechanism for collapse is dependent on temperature, compression speed, ESP, experimental pressure, defects, surfactant and subphase chemistry, resulting in very unpredictable behaviour. This dynamism and heterogeneity is a significant difference when comparing these results for Langmuir monolayers with SAM-based growth studies[21,22].

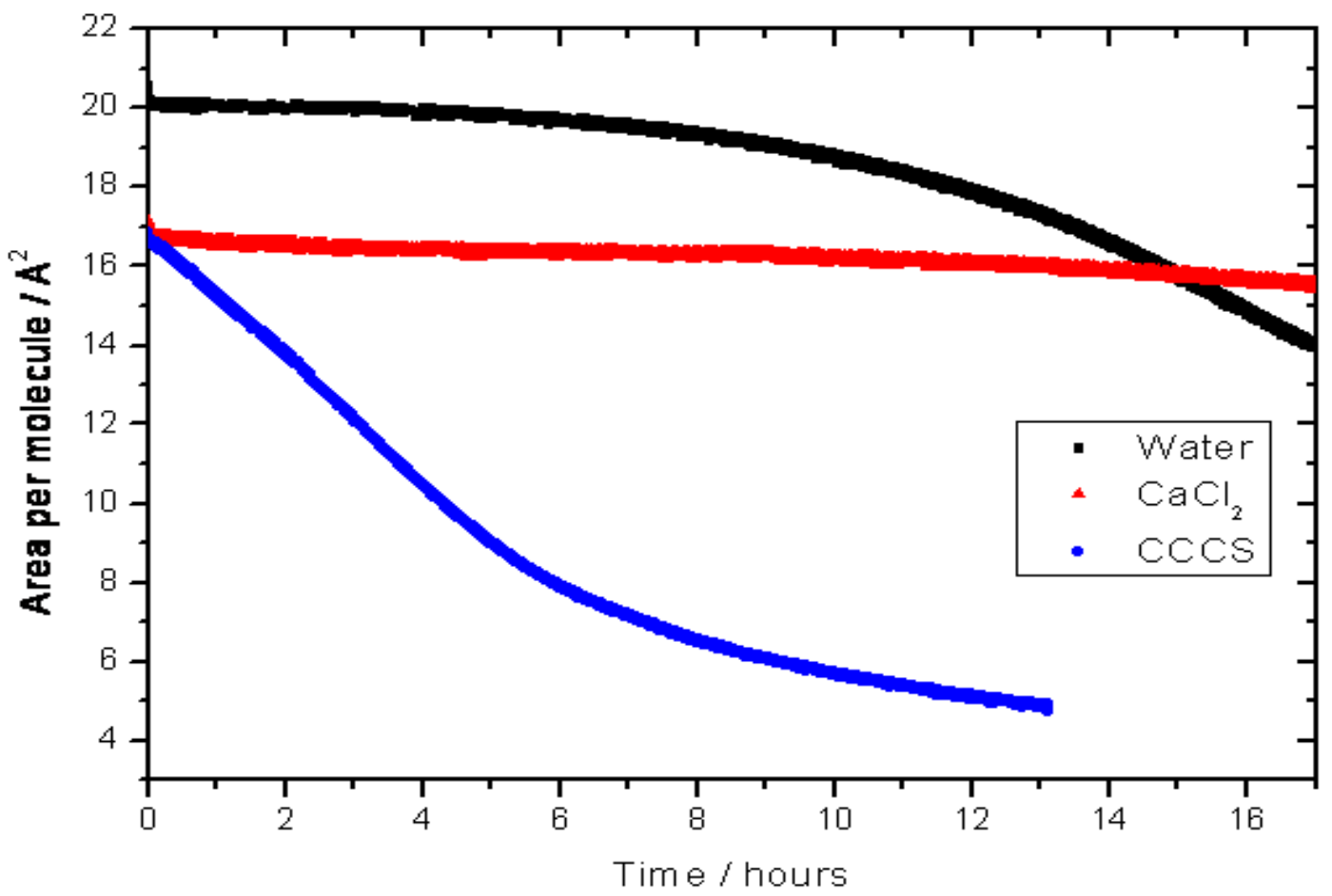

Figure $4.510 \mathrm{~mol} . \% \mathrm{ODOH}$ on water, bubbled calcium chloride and calcium carbonate crystallising subphases at a pressure of $25 \mathrm{mN} \mathrm{m} \mathrm{m}^{-1}$. The best stability being achieved on the carbon dioxide bubbled calcium chloride subphase. 
Finally, in the previous chapter we showed the surface potential of the mixed monolayer/CCCS system tracks with increasing alcohol content. This was attributed to significant cation binding and the formation of a hydrogen-bonded network. At high charge densities, binding and network formation result in a degree of layering of charge parallel to the interface. Along with an altered subphase dielectric constant, this ordering of charge leads to a large decrease in the measured surface potential. The extent of this effect is reduced with increasing alcohol content, as the reduced charge density leads to a reorientation of the charged layers so as to present a more neutral arrangement of ions.

\subsubsection{Low Pressure $\left(\Pi=10 \mathrm{mN} \mathrm{m}^{-1}\right)$}

A comparison of the crystals grown under mixed monolayers at a fixed surface pressure of $10 \mathrm{mN} \mathrm{m}^{-1}$ is shown in Figure 4.6. The pure alcohol system (100 mol.\% ODOH) displays the classic rhombohedral calcite, thus oriented on $\{10.4\}$ faces (Figure 4.6a). There are very few crystal defects and minimal elongation. The situation is very similar for the $75 \mathrm{~mol}$ \% ODOH system (Figure 4.6b) with the exception of a greater number of crystals and the introduction of limited rhombohedral truncation (Type III). Conversely, the step to $50 \mathrm{~mol}$ \% ODOH (Figure 4.6c) results in a significant increase in the propensity for truncation. Very few $\{10.4\}$ oriented rhombohedral crystals are seen. Rather there is now a prevalence for type III truncated rhombohedra with smooth $\{10.4\}$ side faces. The triangular type III crystals show few defects and are inter-dispersed with a small amount of type II crystals. There is no evidence of the elongated irregular type II crystals. Decreasing the alcohol content further $(25 \mathrm{~mol} . \% \mathrm{ODOH}$, Figure $4.6 \mathrm{~d})$ leads to a prevalence of the irregular type II crystals. Like the $50 \mathrm{~mol} \% \mathrm{ODOH}$ system there is an absence of type I crystals. Possibly as a result of the increased elongation and irregularity, a substantial number of crystals are inverted. This inversion permits a view of the crystal surface in contact with the monolayer, which shows considerable topography. 
A small proportion of the type II crystals display the beginnings of a central depression as described by Rajam et al.[9]; attributed to diffusion-limited growth. Decreasing alcohol content further $(20 \mathrm{~mol}$ \% ODOH) leads to the domination of type II-like crystals and increased numbers of type I, with very little type III. Associated with the type II domination is significant elongation in the majority of crystals. A further reduction of alcohol to $10 \mathrm{~mol}$ \% (Figure 4.6e) shifts the numbers towards type I crystals with reduced type II and no type III. Crystal density remains high, in contrast to the pure acid system $(0 \mathrm{~mol} \% \mathrm{ODOH}$, Figure $4.6 \mathrm{f})$ where there is a significant reduction in the number of crystals successfully harvested. Surprisingly, the pure acid system shows very few type I crystals possibly due to size and irregularity contributing to the crystals being dislodged during harvesting and washing. Instead there is a predominance of type II with a small amount of defectridden type III.

At low pressure there is a clear transition on decreasing the alcohol content in mixed monolayers from the classical rhombohedral morphology to an elongated irregular truncated calcite. This transition reflects the variation in binding capacity and double layer potential of the different monolayer compositions. Morphologically there appear to be two steps, from $25 \%$ to $50 \%$ and $50 \%$ to $75 \%$. The former goes from the classical rhombohedral calcite to the truncated triangular calcite (type III) associated with nucleation on a face other than $\{10.4\}$. The second jump reflects a switch from oriented but regular rhombohedral calcite (type III) to very irregular elongated calcite of types I and II.

\subsubsection{High Pressure $\left(\Pi=25 \mathrm{mN} \mathrm{m}^{-1}\right)$}

Increasing the pressure did not alter the morphology under pure alcohol monolayers (Figure 4.7a). The crystals exhibited a defect-free rhombohedral morphology with some elongation. Similarly crystals grown under the $75 \mathrm{~mol} \%$ and 50 mol.\% ODOH monolayers at high pressure (Figure $4.7 \mathrm{~b}$ and c, respectively) were 
effectively the same as those observed at low pressure. At $25 \mathrm{~mol} \%$ ODOH (Figure 4.7d) the crystal morphology at high pressure differs significantly from that observed at low pressure. Rather than crystal size increasing and elongation becoming more pronounced, moving towards type II crystals, at high pressure the morphology remains very similar to that under $50 \mathrm{~mol}$ \% ODOH. The morphology is dominated by type III crystals that exhibit few defects and little elongation. Further there remained a significant number of rhombohedral crystals which at low pressures are effectively absent. Decreasing the alcohol content further (to 20 mol.\%) leads to increased type I and II numbers, however type III still dominates with little elongation and only a small increase in the frequency of defects. At 10 mol.\% ODOH (Figure 4.7e) the degree of elongation, irregularity and frequency of defects is increased from the $20 \mathrm{~mol} \% \mathrm{ODOH}$ system. However, in comparison to the low pressure system, morphology continues to be dominated by types II and III truncated rhombohedra. Crystallisation under pure acid (0 mol.\% $\mathrm{ODOH}$, Figure 4.7f) monolayers at high pressures is characterised by a decrease in nucleation density, increased elongation and truncation but there remain minimal type I crystals. In comparison to the low pressure system, there is a general trend of reduced elongation and irregularity in the crystals grown at high pressure. 

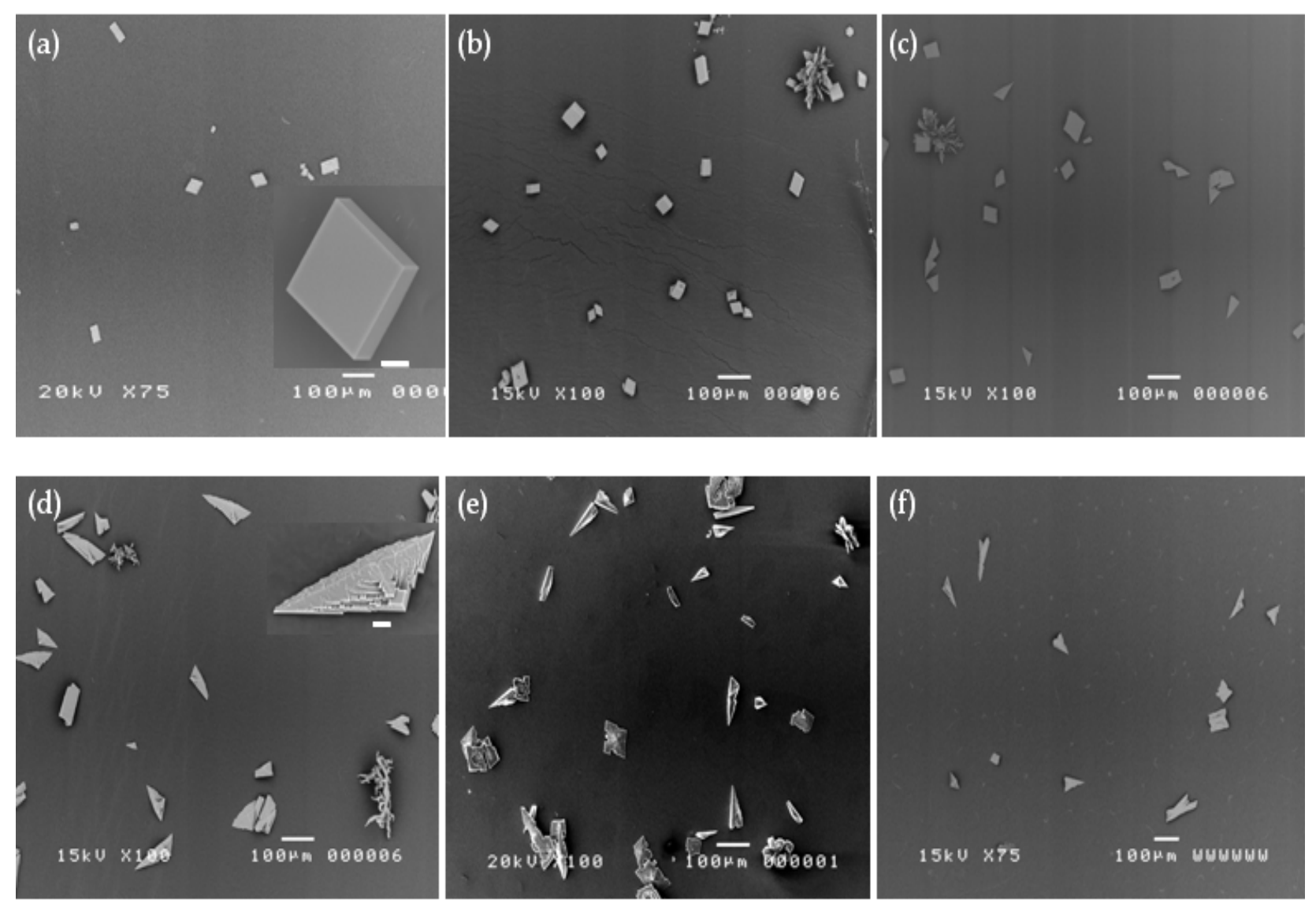

Figure 4.6 Overviews of the changes in crystal morphology as the octadecanol content of the monolayer is increased at a constant pressure of $10 \mathrm{mN} \mathrm{m}{ }^{-1}$. (a) $100 \mathrm{~mol} \% \mathrm{ODOH}$, (b) $75 \mathrm{~mol} . \% \mathrm{ODOH}$, (c) $50 \mathrm{~mol} . \% \mathrm{ODOH}$, (d) $25 \mathrm{~mol} . \% \mathrm{ODOH}$, (e) $10 \mathrm{~mol} . \% \mathrm{ODOH}$, and (f) 0 mol.\% ODOH. The inserts show enlarged images of a typical crystal (the scale bar $=10 \mu \mathrm{m}$ ). 

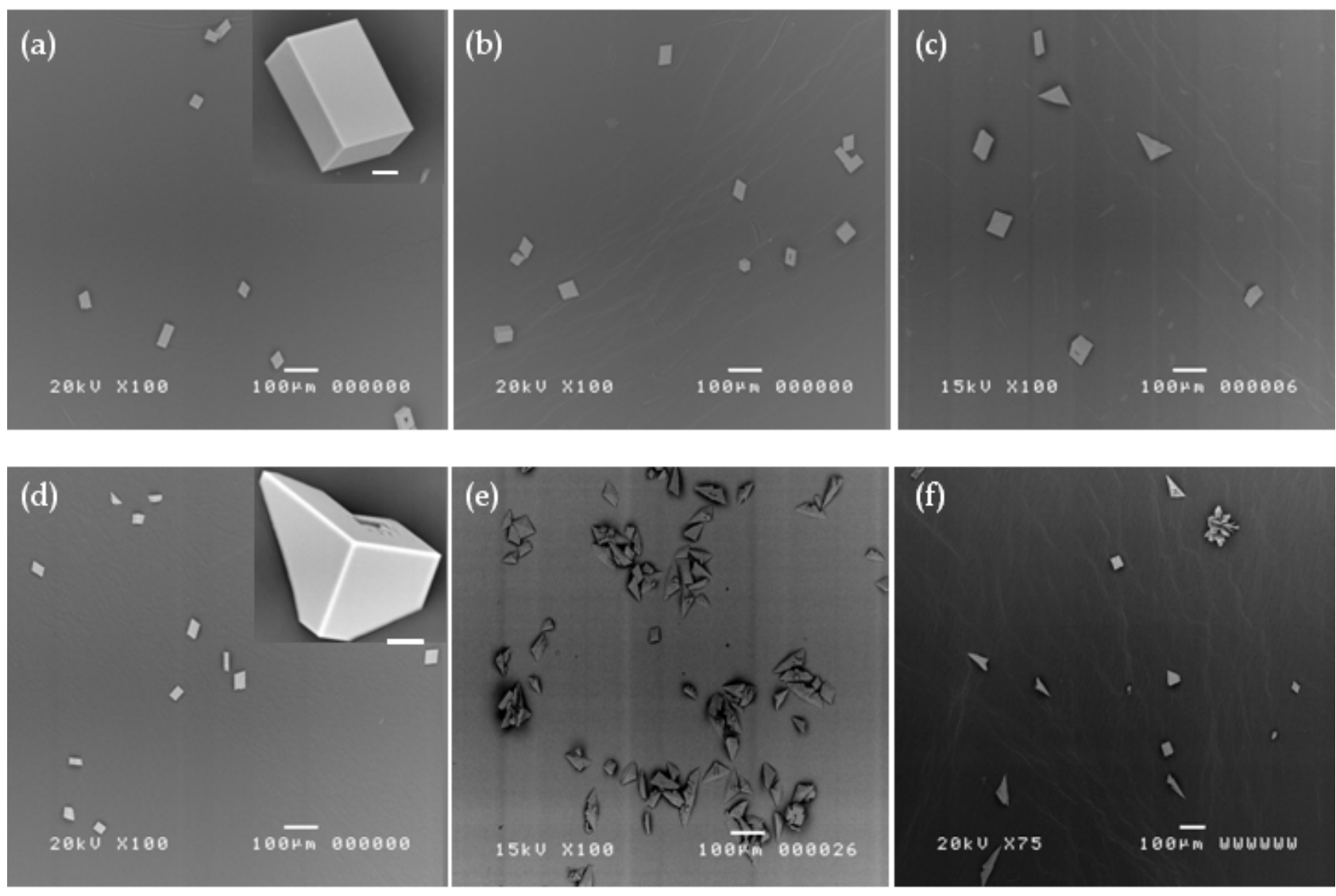

Figure 4.7 Overviews of the changes in crystal morphology as the octadecanol content of the monolayer is increased at a constant pressure of $25 \mathrm{mN} \mathrm{m}^{-1}$. (a) $100 \mathrm{~mol} \%$ $\mathrm{ODOH},(b) 75 \mathrm{~mol} \% \mathrm{ODOH}$, (c) $50 \mathrm{~mol} . \% \mathrm{ODOH}$, (d) $25 \mathrm{~mol} \% \mathrm{ODOH}$, (e) $10 \mathrm{~mol} \% \mathrm{ODOH}$, and (f) $0 \mathrm{~mol} \% \mathrm{ODOH}$. The inserts show enlarged images of a typical crystal (the scale bar $=10 \mu \mathrm{m}$ ). 
Again, as in the low pressure situation, there is a transition on decreasing alcohol content in mixed monolayers from the classical rhombohedral morphology to an elongated irregular truncated calcite. However in contrast to the low pressure crystal morphology, the transition is delayed. Taken from the perspective of the degree of truncation, elongation and irregularity, the $0 \mathrm{~mol} \% \mathrm{ODOH}$ system at high pressure is equivalent to the 50 to $25 \mathrm{~mol}$ \% ODOH system at low pressure.

\subsubsection{Crystal Orientation}

In order to obtain an idea of the nucleation face or the orientation of the nucleated crystals, the inter-edge angle methodology popularised by Archibald et al.[2] was employed. A caveat must be placed over the results in that the technique assumes perfect alignment of crystals on the SEM stub, as it was under the monolayer. Given surface tension issues when harvesting and perturbation during washing and mounting on the stub there is significant scope for miss-alignment. Further, analysis of the nucleation face topography suggests that during growth there is some realignment, as indicated by edge-like artefacts (Figure 4.13), likely due to gravitational effects associated with the size of the growing crystal. Thus the technique does not result in a categorical assignment of the nucleation face, but is a good method for giving qualitative support for a preferred orientation.
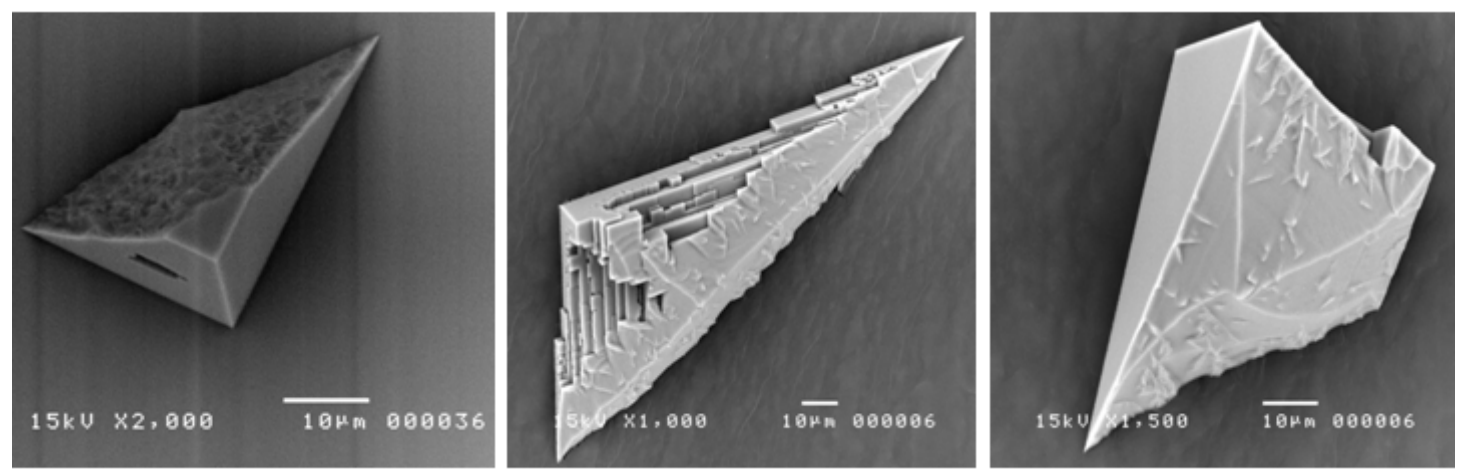

Figure 4.8 SEM images illustrating the textured upper crystal surface, the surface in contact with the monolayer. 
Figure 4.12 summarises the results for low and high pressure. The relatively low percentage returns for any one face is consistent with literature[2] and is a reflection of errors in the technique and the dynamic nature of Langmuir monolayers. However the consistent dominance of the $\{11.15\}$ and $\{10.4\}$ faces at both pressures supports the qualitative value of the technique.

Taking into account the difficulty, and in some cases an inability, in determining the orientation of many of the irregular crystals, the results show that the $\{11.15\}$ face is consistently the most common at low alcohol contents for both pressures investigated. At high alcohol contents, there is a switch to the classic rhombohedral $\{10.4\}$ face. Interestingly the transition between these two faces differs for the two pressures investigated. At high pressure an abrupt change from $\{11.15\}$ at $50 \mathrm{~mol} \%$ $\mathrm{ODOH}$ to $\{10.4\}$ at $75 \mathrm{~mol} \% \mathrm{ODOH}$ occurs. In contrast, at low pressure this transition is more gradual, involving an intermediate orientation: $\{11.15\}$ at $25 \mathrm{~mol} . \%$ ODOH to $\{10.16\}$ at $50 \mathrm{~mol} . \%$ to $\{10.4\}$ at $75 \mathrm{~mol} . \%$.

Reviewing the secondary faces (the second and third most common faces) there appears to be a common zone axis. At low alcohol contents the faces (11.12) and (11.9) have the greatest representation after $\{11.15\}$, with all three faces belonging to the $[1 \overline{1} .0]$ zone axis. This is in contrast to the [01.0] zone axis for the $\{10.4\}$ and (10.16) and (10.10) faces.

In summary, there appears to be two important transition events as the alcohol content is decreased in crystals grown under mixed octadecanoic acid/octadecanol monolayers:

1. a nucleation face or orientation change around the $50-75$ mol.\% ODOH level; and

2. a morphological change from truncated rhombohedra to type II and I calcite at $\sim 75$ - 90 mol.\%.

The specific composition for these transitions is, at least in part, pressure dependent. 


\subsection{Discussion}

There is substantial evidence that monolayer chemistry and symmetry exert an influence on crystal nucleation and growth. Precisely how this comes about remains an enigma. The aims of this chapter were to:

1. investigate the influence of mixed monolayer compositions on calcite crystallisation;

2. elucidate the mechanism of the monolayer/crystal interaction; and

3. assess whether there is any correlation between this mechanism and the previously proposed hypothesis of a hydrogen-bonded soap network functioning as nuclei precursor.

This was achieved by correlating the monolayer behaviour with two key aspects of the resulting calcium carbonate crystal properties: face selective nucleation (or preferential orientation) and morphology. 


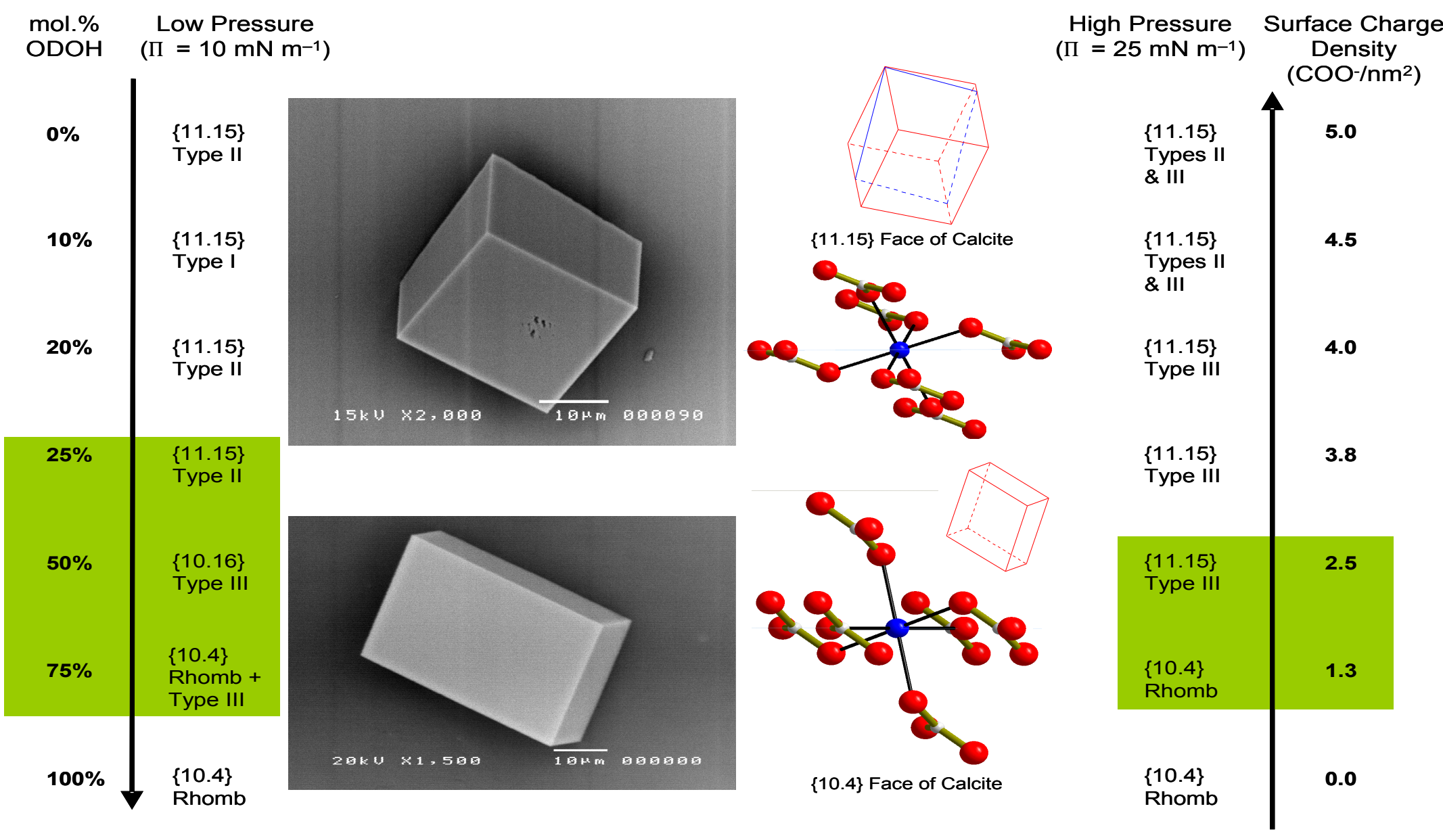

Figure 4.9 Overview of the low and high pressure nucleation face assignment data, showing the most prominent nucleation faces for the different mixed monolayer systems. Charge density is based upon a full ionised monolayer (given a surface $p H$ of 7 at the point of crystallisation) and an average $A_{m}$ of $20 \AA^{2} / m o l e c u l e$. 


\subsubsection{Face selective nucleation}

Generically, nucleation is driven by the metastability of the solution and the desire to minimise energy. It is for this reason that heterogeneous nucleation is ubiquitous, with nuclei forming at interfaces. In the absence of a monolayer, crystal orientation is random but almost exclusively involves one of the faces of the form $\{10.4\}$. This is due to the $\{10.4\}$ faces having the lowest surface energy (especially when hydrated)[23], which is in part due to the high atomic density. The introduction of a monolayer introduces the propensity for enhanced matching at the interface leading to the expression of higher energy crystal faces. Consequently, the expression of $\{10.4\}$ faces under the high alcohol monolayers is indicative of little or no templation. Conversely the expression of $\{11.15\}$ faces under low alcohol monolayers implies a stronger interaction across the interface and a monolayer structure that has properties commensurate with that particular crystal face.

The domination of the $\{11.15\}$ faces through such a large compositional range (0 $25 \%$ and $0-50 \%$ alcohol for the low and high pressure systems, respectively) reflects a considerable versatility and ability to accommodate defects by both the monolayer and the crystal nuclei. Regardless, the result indicates some degree of face-selective nucleation, which is supported by the assignment of zone axes.

The grouping of these dominant and secondary nucleation faces to zones, [01.0] and $[1 \overline{1} .0]$, is further indication of preferential orientation. The nature of the preferential orientation may not be of the order typically observed for SAM-based crystallisation studies due to the heterogeneity and dynamism of the monolayer, however there is a degree of FSN. Closer examination of the properties of the faces assigned to the two zones leads to a number of correlations (Table 3).

- The faces nucleated under high alcohol content monolayers belong to the [01.0] zone in contrast to the [1 $\overline{1} .0]$ zone that accounts for the low alcohol derived crystal faces. 
- All faces in the [01.0] zone have one $\mathrm{Ca}^{2+}$ lattice dimensions of length $4.97 \AA$, whereas the $\left[\begin{array}{l}1 \\ 1\end{array} .0\right]$ zone faces have one dimension of $8.61 \AA$.

- Based on the 2D Ca ${ }^{2+}$ lattice area, on average the [01.0] zone faces have a higher density than the $[1 \overline{1} .0]$ zone faces.

Table 3. Selected properties of the dominant nucleation faces as determined by inter-edge angle measurements.

\begin{tabular}{|c|c|c|c|c|}
\hline Face & Zone Axis & Angle to (00.1) & $\begin{array}{c}\text { Ca }{ }^{2+} \text { Lattice } \\
\text { spacing }(\AA)\end{array}$ & $\begin{array}{c}\mathrm{Ca}^{2+} \text { Lattice } \\
\text { Area }\left(\mathrm{nm}^{2}\right)\end{array}$ \\
\hline$(10.4)$ & {$[01.0]$} & 44.63 & $\begin{array}{l}a=4.0320 \\
b=4.9688\end{array}$ & 0.20 \\
\hline$(10.16)$ & {$[01.0]$} & 13.86 & $\begin{array}{l}a=4.9688 \\
b=11.8195\end{array}$ & 0.59 \\
\hline$(10.10)$ & {$[01.0]$} & 21.54 & $\begin{array}{c}a=4.9687 \\
b=8.1015\end{array}$ & 0.40 \\
\hline$(11.15)$ & {$[1 \overline{1} .0]$} & 24.51 & $\begin{array}{c}a=8.606 \\
b=13.7285\end{array}$ & 1.18 \\
\hline$(11.12)$ & {$[1 \overline{1} .0]$} & 29.68 & $\begin{array}{l}a=6.3989 \\
b=8.6061\end{array}$ & 0.55 \\
\hline$(11.9)$ & {$[1 \overline{1} .0]$} & 37.23 & $\begin{array}{l}a=8.6061 \\
b=9.4719\end{array}$ & 0.82 \\
\hline
\end{tabular}

In terms of describing how FSN arises we return to the idea of a cation-mediated hydrogen-bonded network. Nucleation begins with the agglomeration of ions, a process facilitated by the monolayer. Interactions (such as charge neutralisation, dissociation, like-charge repulsion, hydrogen bonding, dipole interactions, dispersion forces among others) between the monolayer head groups, the head groups and hydrated ions, and between the ions themselves leads to some distribution of ions at the interface. The primary rule for the arrangement of these ions is energy minimisation and may involve bond formation. In the previous chapter a linear chain-like structure loosely styled on the cation-mediated hydrogenbonded network of crystalline $\mathrm{NaHCO}_{3}$ was proposed as a potential structure at the interface. Such a structure provided the mechanism for separating similarly charged faces that, on a pure electrostatic basis, would be inseparable. The possible inclusion 
of the surfactant head groups in the network facilitates complementarity between the monolayer carboxylates and the calcite crystal carbonates leading to a particular orientation. The heterogeneity and dynamic nature of the monolayer and the variable subphase chemistry, on a molecular length scale, means that the interfacial interaction is more a case of synergy rather than rigid templation.

Historically FSN has been attributed to lattice matching[10], spatial geometry matching (often incorrectly termed stereochemistry)[24] and more recently nonspecific electrostatics[25] as the source of the preferential orientation. However the following discussion will illustrate how the idea of a single dominant effect is overly simplistic.

\subsubsection{Lattice Matching}

Lattice matching is based upon identifying equivalent lattice parameters and symmetry between the monolayer and specific crystal faces. The pure octadecanoic acid monolayer at a pressure of $c a .25 \mathrm{mN} \mathrm{m}^{-1}$ has been shown to have an $\mathrm{A}_{\mathrm{m}}$ of 19.2 $\AA^{2}$ and a hexagonal lattice cell $\left(a=4.71 \AA\right.$ )[26]. Therefore given the slightly larger $\mathrm{A}_{\mathrm{m}}$ of $19.8-20.5 \AA^{2}$ at low pressure and $19.3-19.7 \AA^{2}$ at high pressure for the mixed systems on the CCCS, and assuming a similar symmetry, nucleation on the hexagonal (00.1) face of calcite $(a=4.97 \AA$ ) might be expected.

However this approach ignores the capacity for the monolayer to change with both time, for example the slow collapse as discussed earlier, and the nucleation event itself. In Chapter 6 evidence for the latter will be presented. The likelihood of such a rearrangement is great considering the dynamic nature of the monolayer. However the extent of monolayer restructuring depends on the strength of the overall interfacial interaction and steric considerations (which is dependent on the surface pressure and chemical functionality of the surfactant). Although monolayer restructuring complicates the design process it offers improved matching facilitated by the ability (even if limited) for the monolayer and nuclei to find the most 
energetically favourable conformation.

Assessing the level of lattice matching in this study we find a significant mismatch between the monolayer $\left(\mathrm{A}_{\mathrm{m}} \sim 20 \AA^{2}\right)$ and the $\{11.15\}$ faces $\left(\mathrm{Ca}^{2+}\right.$ lattice area $\sim 118 \AA^{2}$ ). In addition, from the literature we know that on a room temperature CCCS the monolayer would have a hexagonal lattice[26] which is inconsistent with the rectangular symmetry of the $\{11.15\}$ faces. The large crystal lattice may originate from the necessity of finding a common multiple for the two sets of lattice dimensions as described by Kewalramani et al.[27]. Alternatively this disparity could reflect a low surface charge in the mixed monolayer systems, brought about by the presence of alcohol and/or significant protonation of the acid molecules. Therefore considering simultaneously, the initial interfacial $\mathrm{pH}$, the dynamics of the monolayer, the ability of the crystal nuclei to accommodate defects, and the hydrated and likely, amorphous nuclei then there is reasonable scope for a good interfacial fit. However there is insufficient evidence to state that lattice matching is the controlling interaction and any claim to this extent is too simplistic.

\subsubsection{Spatial Geometry Matching}

A comparison of the inter-plane angles for the different nucleation faces enables an assessment of the spatial geometry matching between carbonate anions and the carboxylate head groups. In calcite the $\mathrm{CO}_{3}{ }^{2-}$ ions are aligned perpendicular to the $c$ axis, and therefore the angle of the nucleation face to the (00.1) face provides a measure of the angle of the planar carbonates to the nucleation plane. Table 3 shows these angles for the various nucleation faces. Given that the monolayer is untilted, and assuming that the chain is in an all-trans configuration with herring-bone packing (which is likely due to the reduced area), then the theoretical carboxylate orientation would be at an angle of $45-90^{\circ} \mathrm{C}$ to the interface and nucleation plane. However the combination of low surface pressure, reduced like-charge repulsion and the smaller alcohol head group allows greater freedom for the carboxylate to re- 
orientate.

Molecular modelling performed by D. Duffy and J. Harding of University College London, U.K. in conjunction with A. M. Travaille[28] shows that there may in fact be a considerable range of orientations present. The system modelled was a SAM of 25 16-mercaptohexadecanoic acid molecules in a vacuum at $0 \mathrm{~K}$. The angle of the final $\mathrm{H}_{2} \mathrm{C}-\mathrm{COOH}$ bond was $\sim 45^{\circ}$ to the substrate. In contrast, the addition of water, $\mathrm{Ca}^{2+}$ and $\mathrm{HCO}_{3}{ }^{-}$ions at $300 \mathrm{~K}$ led to a very broad bimodal peak spanning a $\mathrm{H}_{2} \mathrm{C}-\mathrm{COOH}$ bond angle of $0.5-75^{\circ}$. The most intense peak (frequency $\sim 3.0$ molecules) was located at $\sim 25^{\circ}$ and a second weaker peak (frequency $\sim 1.5$ molecules) was broad and centred at $\sim 54^{\circ}$. The suggestion in this study was that the two peaks correlated with the $\{01.2\}\left(27^{\circ}\right)$ and $\{01.5\}\left(52^{\circ}\right)$ faces rather than the $\{00.1\}\left(90^{\circ}\right)$ hexagonal face, which based on symmetry provided a better match to the hexagonal SAM.

Again this view is perhaps too simplistic. Based on a SAM of only 25 molecules combined with the broad distribution of $\mathrm{H}_{2} \mathrm{C}-\mathrm{COOH}$ bond angles, and an additional peak intensity of only 3.0 molecules, the result is not conclusive and there remains scope for reorientation driven by other interactions. Furthermore this SAM model involves tilted molecules (at an angle of $30^{\circ}$ ) and therefore the molecules are well spaced, providing the carboxylate head groups significant rotational freedom. Thus the study is not representative of the untilted Langmuir monolayers on the CCCS. Despite this, the modelling does suggest that an angle of $14^{\circ}$ for the (10.16) face is at least possible.

Again there is insufficient evidence to suggest that spatial geometry matching of the carboxylate and carbonate groups directs FSN. However the importance of spatial geometry matching in FSN is dependent on steric implications, therefore its role will vary with surface pressure and surfactant chemistry.

\subsubsection{Electrostatics}

Electrostatics is the most commonly employed explanation for FSN. 
Unfortunately the term 'electrostatics' is loosely used and therefore an understanding of precisely what is meant is not always clear. Typically discussion of electrostatics is based around charge density and therefore focuses on ion-ion interactions, neglecting the important dipole interactions which can impart directionality. Ion-ion interactions are important and in cases of high charge density can account for FSN, where there are few highly charged crystal faces, (in calcite these are the $\{00.1\},\{01.2\}$, and $\{01.5\}$ faces $)$. Additionally the high charge densities also influence the nucleation kinetics, potentially limiting the options for FSN. However at medium to low charge densities, dipole, symmetry and lattice interactions play an increasingly important role.

Further analysis of the results highlights a number of contradictory and anomalous trends that include:

- two faces of relatively fixed surface termination, (11.15) and (10.4), dominate relatively large ranges of monolayer surface charge;

- the transition between these two faces differs for low and high pressures yet the mean monolayer surface charge varies negligibly (Figure 4.12); and

- the transition at high pressure occurs relatively abruptly (between 50 $75 \mathrm{~mol} . \% \mathrm{ODOH})$, which is inconsistent with the rule of mixtures behaviour of the maximum surface potential (Chapter 3).

The evidence supporting the importance of electrostatics in FSN is strong but the inconsistencies highlighted above suggest that other effects need to be considered.

Individually lattice, spatial geometry and electrostatic matching fails to explain the observed results. However the concept of a cation-mediated hydrogen-bonded network incorporates all these aspects and adequately accounts for the results.

\subsubsection{Morphology}

Under standard conditions the equilibrium crystal shape for calcium carbonate is the classic rhombohedron, where morphology is determined by surface energies, 
such is the case with the expression of the lowest energy $\{10.4\}$ faces. In nonequilibrium cases, crystal morphology is also a function of FSN and the growth process. Morphologically, the results shown here exhibit a continuum of crystal habit modifications, from classic rhombohedra (equilibrium growth) to truncated rhombohedra (type III calcite), to type II and finally to type I calcite. The latter morphologies displaying significant rhombohedon truncation, elongation and irregularity.

Truncation can be attributed to FSN, with the area of the truncated face defined by the position $(m)$ of the nucleation face relative the centre of the rhombohedron (Figure 4.10). The value of $m$ is related to the growth rate which in turn is a function of the interfacial match and the solution kinetics.

Elongation reflects anisotropic growth of the crystal, ultimately due to differing growth rates for the exposed facets. Given that classic or truncated rhombohedra have $\{10.4\}$ faces exposed to the subphase it is unlikely that elongation is caused by face-specific surface energies. A more plausible explanation was put forward by Pokroy and Aizenberg[29] who correlated the direction of asymmetric lateral growth of the crystals to the direction(s) of greatest lattice match between the SAM and the crystal.

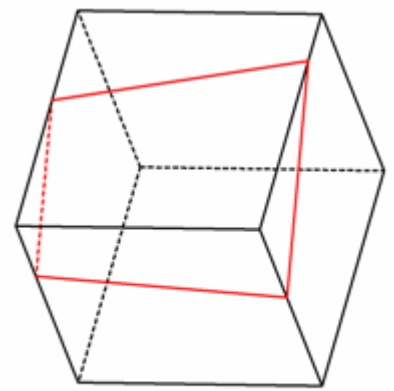

$m=1$

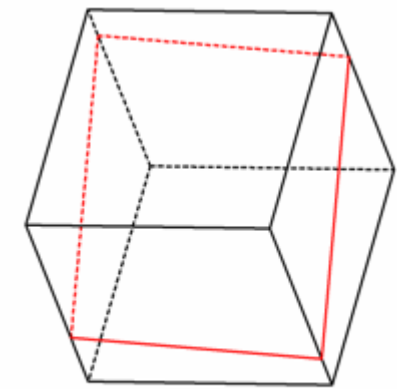

$\mathrm{m}=\mathbf{0}$

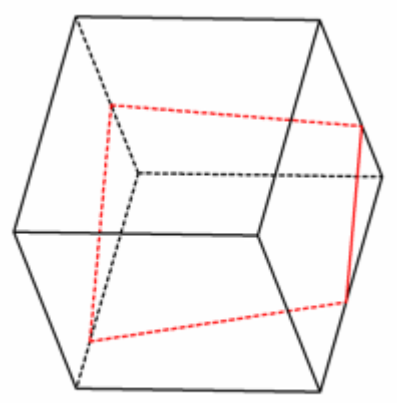

$m=-1$

Figure 4.10 Computer generated (SHAPE for Window V7.2.2) calcite rhombohedrons oriented with the (11.15) face in the plane of the page. The red polygon represents the nucleation face for different distances from the centre of the crystal ( $m$ ), highlighting the range of morphologies that result. 
The irregularity expressed in the larger type I and II crystals appears to result from the culmination of four different effects: truncation, elongation, agglomeration (crystal nuclei intergrowth) and diffusion limited growth.

With the aforementioned effects in mind, analysis of the results shows four trends:

- crystal truncation, irregularity and elongation are significantly more prevalent at low alcohol contents, with no evidence of significant rhombohedral distortion at high alcohol contents;

- a comparison of the high and low pressure calcite crystals shows that the high pressure systems (and therefore high charge density) generally result in less irregularity and elongation;

- monolayer stability (as indicated by the constant pressure plots, Figure 4.4) for each of the growth experiments appears to influence crystal morphology;

- and finally, the monolayer stability is dependent on surface pressure and the alcohol content.

The interrelationship between these four observations is represented in Figure 4.11. The prominence of monolayer stability is somewhat surprising given the relative lack of its discussion in the literature. Using the pure acid system as an example we can correlate monolayer stability with crystal morphology independently of the surface pressure and therefore the charge density. (Note that for a given ensemble average surface pressure e.g. $\Pi=10 \mathrm{mN} \mathrm{m}^{-1}$, monolayer stability was observed to vary considerably between repeat experiments). In Figure 4.8 four pure acid experiments are shown (two repeats for each of 10 and $25 \mathrm{mN} \mathrm{m}^{-1}$ ), where crystal irregularity increases with enhanced monolayer stability. This is a general result for all monolayers investigated. 


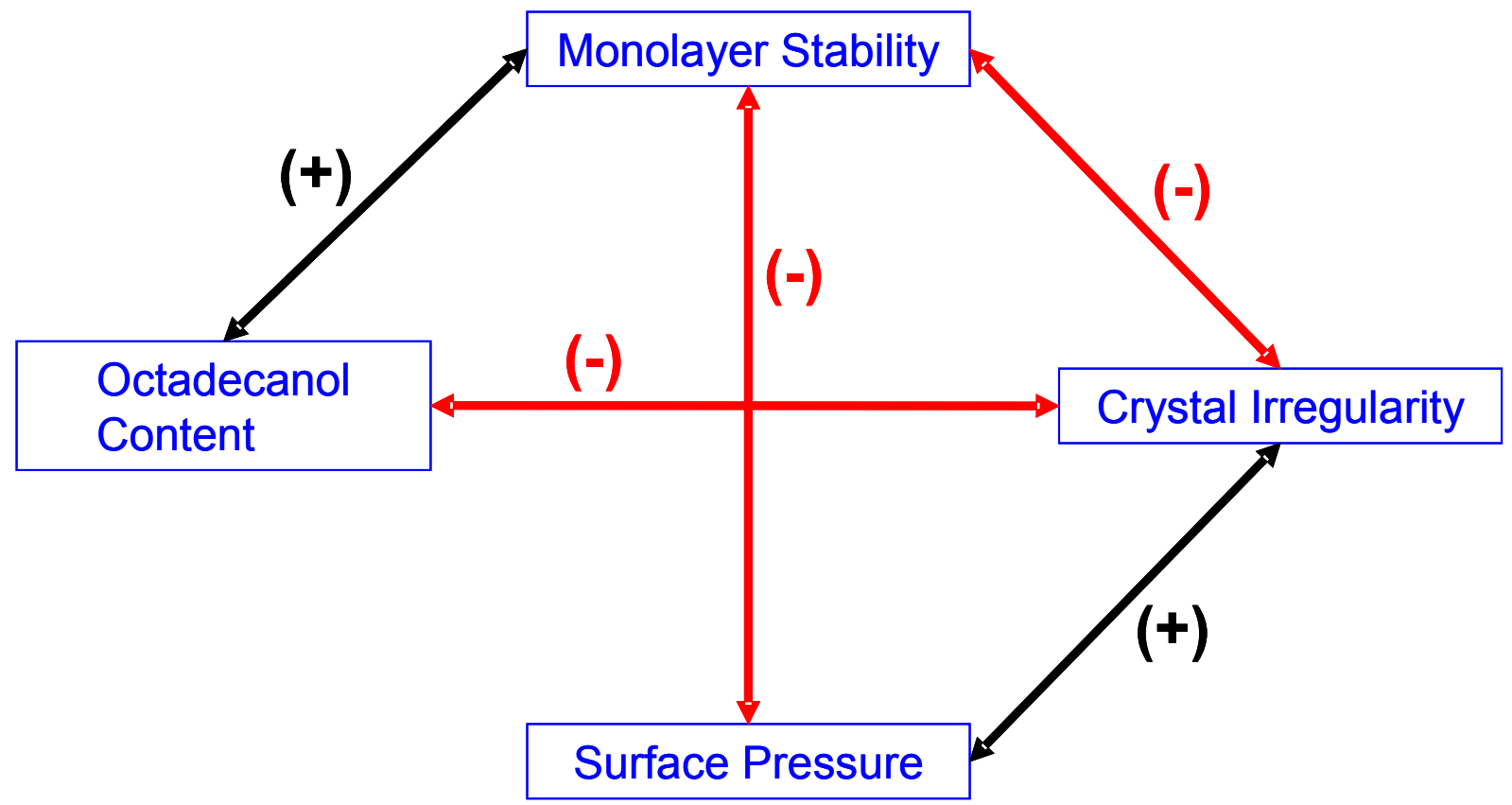

Figure 4.11 A schematic illustrating the interrelationships occurring between monolayer stability and crystal morphology. The (-) or red lines depict an inverse relationship whereas the (+) or black lines depict a positive relationship.

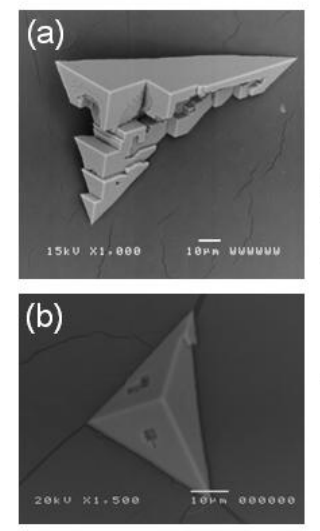

$\Pi=10 \mathrm{mN} \mathrm{m}^{-1}$

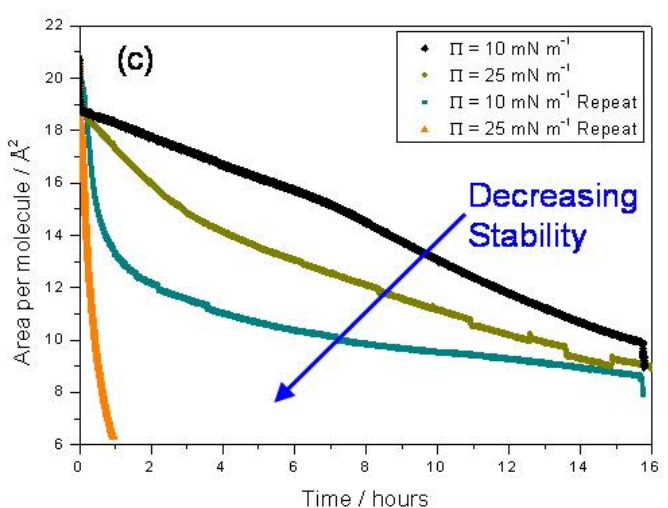

Figure 4.12 The correlation between monolayer stability and morphology. In order of decreasing monolayer stability $(a)>(d)>(b)>(e)$, correlating to the black, olive, teal and orange lines as shown in (c). The crystals (a) and $(b)$ are grown at $\Pi=10 \mathrm{mN} \mathrm{m} \mathrm{m}^{-1}$ whereas $(d)$ and (e) were grown at $\Pi=25 \mathrm{mN} \mathrm{m} \mathrm{m}^{-1}$.

\subsubsection{Monolayer Stability}

In order to understand these observations, identifying the origin of monolayer stability or instability is paramount. Monolayer stability is inherently associated with the ESP and slow collapse. As such it is of no surprise that stability is improved 
with increasing $\mathrm{ODOH}$ content given that the ESP of the alcohol is significantly higher than that for the acid. Consequently slow collapse for the high $\mathrm{ODOH}$ monolayers is absent with the small loss in $A_{m}$ due to other effects. In contrast, at low levels of ODOH the monolayer instability is great. The ESP of a monolayer is purely a measure of combined influence of the many drivers at play. That is hydrophobicity, hydrophilicity, entropy, solubility, like-charge repulsion, dipole and van der Waal interactions, hydration effects, surface energies and chemical free energy of the surfactant phases among other effects, all contribute to the propensity for a surfactant to form a stable monolayer. Similarly the formation of a cationmediated hydrogen-bonded network would impact on the stability of the monolayer.

In terms of the affect of surface pressure on monolayer stability, this is again tied to the ESP. Both pressures examined $\left(10\right.$ and $\left.25 \mathrm{mN} \mathrm{m}^{-1}\right)$ are below the ESP for pure ODOH monolayers and above the ESP for pure ODA monolayers hence the associated stability or lack of it. At higher pressures there is likely to be a larger contribution to the ESP from the driving force for phase change to a more stable 3-D phase (a vesicle or solid) due to the metastability associated with the higher concentrations. Consequently the monolayer is more unstable at higher pressures.

In addition to slow collapse, brought about by pressures above the ESP, perturbation associated with the release of $\mathrm{CO}_{2}$ and nucleation events may also reduce the monolayer stability.

\subsubsection{Perturbation}

The release of carbon dioxide from the subphase during crystallisation experiments is associated with the formation of millimetre-sized bubbles that rise to and break at the subphase surface. Perturbation of the wilhelmy plate by breaking bubbles has been observed, supporting this idea of perturbation-derived instability. However, Figure 4.5 shows a 10 mol.\% ODOH monolayer on carbon dioxide bubbled $\mathrm{CaCl}_{2}$ subphase to be more stable than a water or CCCS subphase. Any disruption of 
the monolayer by evolving gases is offset by the stabilisation of the calcium ions, casting doubt over the significance of $\mathrm{CO}_{2}$-related perturbation.

The significant difference in monolayer stability between the $\mathrm{CaCl}_{2}$ and CCCS subphases intimates that the nucleation event(s) could constitute an alternative source of perturbation. However the influence of nucleation events on monolayer stability is very difficult to decouple from the physicochemical environment from which the nuclei precipitate. One possible pointer to the influence of crystallisation is observation of undulations and ridges on the crystal nucleation surfaces (Figure 4.13). This surface topography increases with increasing monolayer surface charge, however there is negligible difference between high and low pressures. Assuming that the crystal topography is representative of the monolayer topography then three things can be deduced:

1. there is a strong interaction between crystal and monolayer;

2. the monolayer does present folds associated with slow collapse; and

3. the strength of the interfacial interaction results in slow collapse via a modification of the monolayer ESP upon nucleation.

The rough crystal nucleation surfaces also present evidence for crystal rearrangement during growth (edge-like ridges on the surfaces defining several roughened facets, Figure 4.13). Such a phenomena would exacerbate the slow collapse, thus the combination of folding and crystal rearrangement could explain the $\sim 70 \%$ loss in the area per molecule for the most unstable pure acid system.

In summary, monolayer stability or more precisely the equilibrium spreading pressure of the monolayer is intimately linked to the crystal morphology, in terms of elongation and irregularity. This relationship is based upon a strong interaction between the monolayer and the subphase. A weak interaction, at high $\mathrm{ODOH}$ content and high monolayer surface pressures, leads to the expression of regular smooth $\{10.4\}$ faces with little elongation. 
At low monolayer surface pressures the relative rotational freedom of the individual surfactant molecules is likely to result in larger domains and generally greater monolayer stability. This flexibility would facilitate an improved interaction with the hypothesised hydrogen-bonded soap network thus stabilising the monolayer further, just as divalent cations (which result in cation/surfactant-dimer pairs) stabilise monolayers for creating Langmuir-Blodgett films. Hence crystals derived from low pressure monolayers exhibit greater elongation and irregularity.

Conversely, at higher pressures steric limitations restrict the monolayer's ability to facilitate network formation through spacing and symmetry rearrangements, the network domain size is small and therefore more soluble. Coupled with the affect of the increased pressure and nucleation-related buckling, the monolayer is commensurately more unstable. The smaller network domains also result in less lateral growth in subsequent crystallisation. The suggestion of pressure-dependent domain size is supported by increased nucleation density (Figure 4.1), most evident in the early stages of growth before ripening processes begin to dominate, where the high energy domain boundary sites readily promote nucleation.
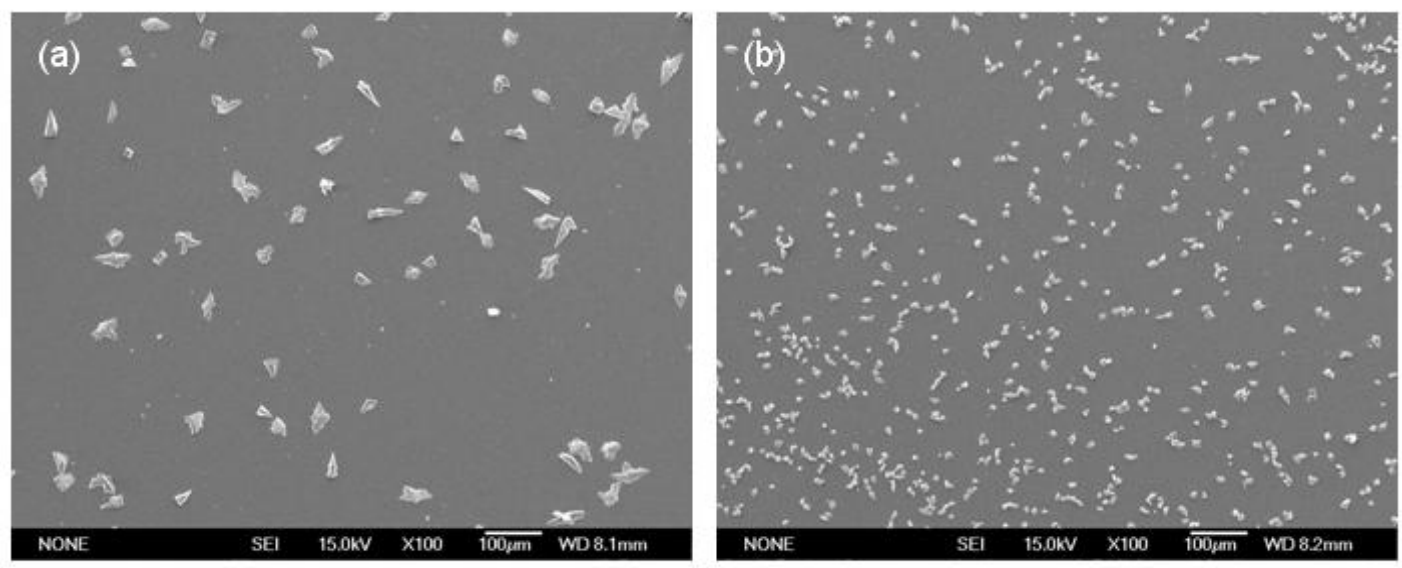

Figure 4.13 SEM images of the variation in nucleation density with surface pressure of pure octadecanoic acid monolayers after 1 hour of growth, (a) $\Pi=10 \mathrm{mN} \mathrm{m}^{-1}$, and (b) $\Pi=30 \mathrm{mN} \mathrm{m}^{-1}$.

In summary, monolayer stability is not the cause of crystal morphology modifications rather it is an expression of the strong interfacial interaction that leads 
to these morphological variations in crystal shape.

\subsection{Conclusions}

This study investigated the properties of calcite nucleation and growth under a range of mixed octadecanoic acid/octadecanol monolayers at two pressures. There is evidence to suggest that the morphological descriptors of Rajam et al.[9] are in fact stages in a morphological spectrum from classic rhombohedra to irregular plate-like (type I) crystals. Further, this continuum reflects increasing modification brought about by the thermodynamic and kinetic implications of higher charge density monolayers.

Evaluation and discussion of preferential orientation leads to a number of conclusions regarding the role of charge density, lattice matching, symmetry and spatial geometry matching in face-selective nucleation. These include:

- the requirement of significant charge density to observe oriented nucleation;

- the domination of face selectivity by electrostatics at high charge densities; but

- more often face selectivity is achieved through some combination of spatial geometry, lattice matching, and electrostatics, in which case the concept of a cation-mediated hydrogen-bonded network may provide an improved understanding.

In terms of morphology, analysis has shown that truncation, elongation, agglomeration and diffusion limited growth all contribute to the gross crystal shape. In addition, the following points are of note:

- morphological irregularity and elongation are signs of a strong interfacial interaction, involving enhanced matching across the interface; and

- the strength of the interfacial interaction is also expressed in terms of monolayer stability.

Ultimately crystal templation beneath Langmuir monolayers is a complex interrelationship of many factors and to suggest that one factor (such as charge 
density) dominates is often too simplistic.

\subsection{References}

[1] H. Cölfen and S. Mann, "Higher-Order Organization by Mesoscale SelfAssembly and Transformation of Hybrid nanostructures," Angew. Chem. Int. Ed., vol. 42, 2003, pp. 2350-2365.

[2] D. Archibald, S. Qadri, and B. Gaber, "Modified Calcite Deposition Due to Ultrathin Organic Films on Silicon Substrates," Langmuir, vol. 12, Jan. 1996, pp. 538-546.

[3] E. Loste, E. Diaz-Marti, A. Zarbakhsh, and F. Meldrum, "Study of Calcium Carbonate Precipitation under a Series of Fatty Acid Langmuir Monolayers Using Brewster Angle Microscopy," Langmuir, vol. 19, 2003, pp. 2830-2837.

[4] B. Heywood and S. Mann, "Organic Template-Directed Inorganic Crystallization: Oriented Nucleation of $\mathrm{BaSO}_{4}$ Under Compressed Langmuir Monolayers," Journal of the American Chemical Society, vol. 114, 1992, pp. 46814686.

[5] S. Mann, B. Heywood, S. Rajam, and J. Birchall, "Controlled Crystallization of $\mathrm{CaCO}_{3}$ Under Stearic Acid Monolayers ," Nature, vol. 334, 1988, pp. 692-695.

[6] E. DiMasi, V. Patel, S. Munisamy, M. Olszta, and L. Gower, "Synthetic Seashells: Biomimetic Mineral Nucleation at a Langmuir Monolayer," NSLS Activity Report, 2001, pp. 2-68-2-72.

[7] E. Landau, S. Grayer Wolf, M. Levanon, L. Leiserowitz, M. Lahav, and J. Sagiv, "Stereochemical Studies in Crystal Nucleation. Oriented Crystal Growth of Glycine at Interfaces Covered with Langmuir and Langmuir-Blodgett Films of Resolved $\alpha$-Amino Acids," Journal of the American Chemical Society, vol. 111, 1989, pp. 1436-1445.

[8] D. Duffy and J. Harding, "Modelling the Interfaces Between Calcite Crystals and Langmuir Monolayers," J.Mater.Chem., vol. 12, 2002, pp. 3419-3425.

[9] S. Rajam, B. Heywood, J. Walker, S. Mann, R. Davey, and J. Birchall, "Oriented Crystallization of $\mathrm{CaCO}_{3}$ Under Compressed Monolayers Part 1. Morphological Studies of Mature Crystals," J. Chem. Soc. Faraday Trans., vol. 87, 1991, pp. 727734.

[10] B. Heywood, S. Rajam, and S. Mann, “Oriented Crystallization of $\mathrm{CaCO}_{3}$ Under Compressed Monolayers Part 2. Morphology, Structure and Growth of Immature Crystals," J. Chem. Soc. Faraday Trans., vol. 87, 1991, pp. 735-743.

[11] S. Mann, B. Heywood, S. Rajam, and J. Walker, "Structural and stereochemical relationships between Langmuir monolayers and calcium carbonate nucleation," Journal of Physics D: Applied Physics, 1991, pp. 154-164.

[12] B. Heywood and S. Mann, "Template-Directed Nucleation and Growth of Inorganic Materials," Advanced Materials, vol. 6, 1994, pp. 9-20. 
[13] M. Weis, "Kinetics of slow collapse process: Thermodynamic description of rate constants," Applied Surface Science, vol. 253, Nov. 2006, pp. 1469-1472.

[14] C. Ybert, W. Lu, G. Moller, and C.M. Knobler, "Collapse of a Monolayer by Three Mechanisms," The Journal of Physical Chemistry B, vol. 106, Feb. 2002, pp. 2004-2008.

[15] A. Gopal and K.Y. Lee, "Morphology and Collapse Transitions in Binary Phospholipid Monolayers," The Journal of Physical Chemistry B, vol. 105, Oct. 2001, pp. 10348-10354.

[16] G. Barnes, "The potential for monolayers to reduce the evaporation of water from large water storages," Agricultural Water Management, vol. 95, Apr. 2008, pp. 339-353.

[17] G. Gaines Jnr., Insoluble Monolayers at Liquid-Gas Interfaces, John Wiley \& Sons , 1966.

[18] R. Heikkila, C. Kwong, and D. Cornwell, "Stability of Fatty Acid Monolayers and the Relationship Between Equilibrium Spreading Pressure, Phase Transformations, and Polymorphic Crystal Forms," Journal of Lipid Research, vol. 11, 1970, pp. 190-194.

[19] M. Petty, Langmuir-Blodgett Films: An Introduction, Cambridge University Press, 1996.

[20] D. Vollhardt and U. Retter, "Nucleation in insoluble monolayers. 1. Nucleation and growth model for relaxation of metastable monolayers," The Journal of Physical Chemistry, vol. 95, May. 1991, pp. 3723-3727.

[21] J. Aizenberg, A. Black, and G. Whitesides, “Oriented Growth of Calcite Controlled by Self-Assembled Monolayers of Functionalized Alkanethiols Supported on Gold and Silver," Journal of the American Chemical Society, vol. 121, 1999, pp. 4500-4509.

[22] A. Travaille, L. Kaptijn, P. Verwer, B. Hulsken, J. Elemans, R. Nolte, and H. van Kempen, "Highly Oriented Self-Assembled Monolayers as Templates for Epitaxial Calcite Growth," Journal of the American Chemical Society, vol. 125, 2003, pp. 11571-11577.

[23] N. de Leeuw and S. Parker, "Surface Structure and Morphology of Calcium Carbonate Polymorphs Calcite, Aragonite and Vaterite: An Atomistic Approach," Journal of Physical Chemistry B, vol. 102, 1998, pp. 2914-2922.

[24] J. Donners, R. Nolte, and N. Sommerdijk, "A Shape-Persistent Polymeric Crystallization Template for $\mathrm{CaCO}_{3}$," Journal of the American Chemical Society, vol. 124, 2002, pp. 9700-9701.

[25] M. Fricke and D. Volkmer, "Crystallization of Calcium Carbonate Beneath Insoluble Monolayers: Suitable Models of Mineral-Matrix Interactions in Biomineralization?," Topics in Current Chemistry, vol. 270, 2007, pp. 1-41.

[26] E. DiMasi, M. Olszta, V. Patel, and L. Gower, "When is Template Directed Mineralization Really Template Directed?," CrystEngComm, vol. 5, 2003, pp. 346- 
350.

[27] S. Kewalramani, G. Dommett, K. Kim, G. Evmenenko, H. Mo, B. Stripe, and P. Dutta, "Aggregation-governed oriented growth of inorganic crystals at an organic template," The Journal of Chemical Physics, vol. 125, Dec. 2006, pp. 224713224717.

[28] A. Travaille, "Soft Interactions, Solid Results Nucleation of Calcite on Organic Monolayers and Knowledge Creation in a University Research Institute," PhD Thesis, Radboud University Nijmegen, 2005.

[29] B. Pokroy and J. Aizenberg, "Calcite shape modulation through the lattice mismatch between the self-assembled monolayer template and the nucleated crystal face," CrystEngComm, vol. 9, 2007, pp. 1219-1225. 


\section{Chapter 5: 2-Hydroxyoctadecanoic Acid}

\section{MONOLAYERS}

\subsection{Introduction}

Chapters 3 and 4 highlighted the role of the acid and alcohol functional groups play in influencing monolayer behaviour and calcite crystallisation. In this chapter these the influence of these functional groups is explored in the guise of a single molecule, DL-2-hydroxyoctadecanoic acid (Figure 5.1, 2-HSA). 2-HSA combines the electrostatic acid functionality with the alcohol or hydroxyl functional group in the one head group. Unlike the mixed monolayer systems, where the acid and alcohol molecules were free to order based on energetics, in 2-HSA the two functional groups are covalently linked.

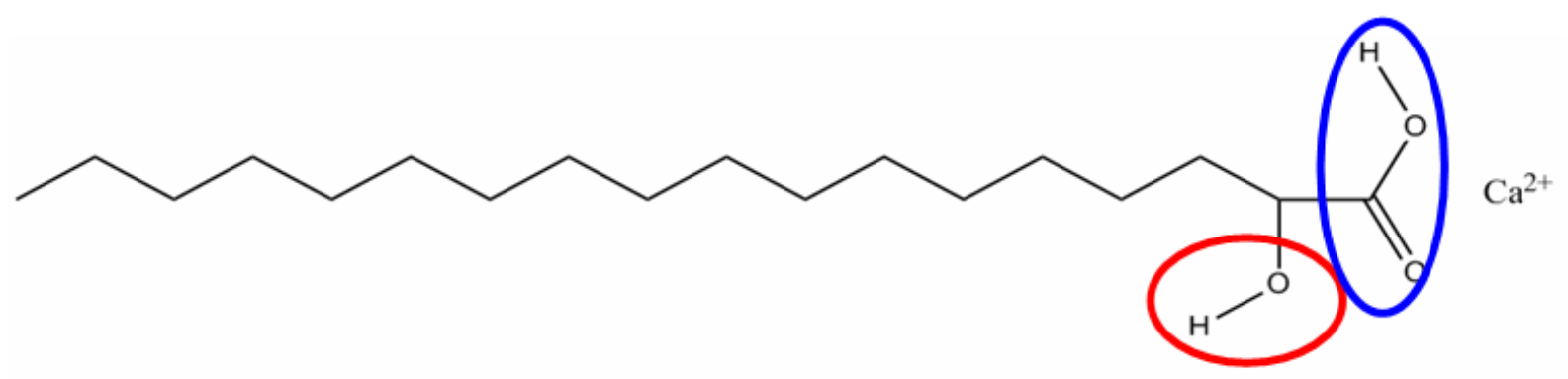

Figure 5.1 A schematic of DL-2-hydroxyoctadecanoic acid molecule with the hydroxyl (red) and acid (blue) functional groups highlighted.

2-HSA is used in therapeutic and cosmetic applications to facilitate skin care and disease treatment, where its role has been linked to strong chelation of calcium ions[1]. However, its selection in this study lies primarily in the subtle modification of the head group electron density. The hydroxyl group draws charge away from the carboxyl group, creating a single large polar head group. This is in contrast to the 
bipolar nature of 7- 9- and 12-HSA[2-10]. Unfortunately, the size, shape (steric implications), the propensity for hydrogen bonding, and the binding capacity of the head group are also modified with the addition of a hydroxyl group. Consequently, a relatively small modification leads to significant changes in the interfacial interaction.

Previous investigations of the behaviour of hydroxy-fatty acids has shown that the location of the hydroxyl group at the 2- or 3- position, as opposed to further up the chain, has significant consequences. For example, Kellner and Cadenhead[2] showed that for 2-hydroxyhexadecanoic acids (2-HHA) the monolayer was condensed at all pressures and the film was very rigid such that it displaced the Wilhelmy plate from vertical. The rigidity of the monolayer was attributed to hydrogen bonding between the hydroxyl group and the neighbouring carbonyl oxygen, resulting in an increased equilibrium spreading pressure (ESP) and melting point for 2-HHA compared to hexadecanoic acid, $\left(17.8 \pm 0.2 \mathrm{mN} \mathrm{m}^{-1}\right.$ and $87^{\circ} \mathrm{C}$ c.f. 10.7 $\pm 0.8 \mathrm{mN} \mathrm{m}^{-1}$ and $\left.63^{\circ} \mathrm{C}\right)$. The increased monolayer stability brought about by intermolecular hydrogen bonding between neighbouring molecules has also been associated with higher $\mathrm{pKa}$ values for 2-HSA[7].

In terms of the $\Pi$-A isotherm behaviour, 2-HSA behaves more akin to nonsubstituted octadecanoic acid with the addition of a two-phase coexistence region at zero pressure. This is quite unlike the bipolar mid-chain substituted hydroxy acids which present the two phase coexistence region (represented as a plateau in a П-A isotherm) at higher pressures[4,8]. The difference lies in the creation of a single large mono-polar head group. A geometric comparison of the head group, perpendicular to the tilt direction, and alkyl chain lattices shows that the chain packing becomes disordered in order to accommodate the size mismatch. Consequently 2-HSA does not form well-shaped condensed phase domains with a highly crystalline structure.

The packing implications for increased head group size is illustrated in a grazing 
incidence X-ray diffraction (GIXD) study of 2-hydroxypalmitic acid monolayers. Weidemann et al.[6] reported a distribution of diffraction intensity along a characteristic arc in reciprocal space. The diffuse diffraction intensity was attributed to a superposition of different lattices, ascribed to variations of tilt azimuth, representing the disordered packing of the alkyl chains. Disorder was attributed to a mismatch between the enlarged head groups and the chains[3]. In comparison to acids with hydroxyl groups substituted in the mid-chain position, the alkyl chains of 2-HSA have greater configurational freedom, hence the disordered state[9].

Another consequence of the addition of the hydroxy group at the second carbon is chirality. Investigating the differences between the two enantiomeric forms of 2-HHA, Neumann et al.[5] found that the presence of divalent cations, such as $\mathrm{Ca}^{2+}$, result in considerable condensation and potential for increased chiral discrimination. A comparison of $\mathrm{Ca}^{2+}, \mathrm{Pb}^{2+}$ and $\mathrm{Zn}^{2+}$ subphase ions showed $\mathrm{Ca}^{2+}$ to incite the greatest condensation effect. This contrasts with the study by Yazdanain et al.[11] that showed the opposite trend for octadecanoic acid monolayers. In terms of chiral discrimination and therefore the potential for phase separation, electrostaticallybased surfactant-cation binding is generally thought to be homochiral in nature. In line with this theory, $\mathrm{Ca}^{2+}$ was found to show relatively (compared to $\mathrm{Pb}^{2+}$ and $\mathrm{Zn}^{2+}$ ) poor chiral discrimination.

Clearly, the attempt to explore the effect a subtle change in head group chemistry has on monolayer behaviour and subsequent calcium carbonate nucleation is not straight-forward. With the literature focussing on more theoretical-based studies employing low $\mathrm{pH}$ water subphases, a greater understanding of the interfacial interaction is required before the impact of the hydroxyl substitution on mineral nucleation can be elucidated. To this end, described in this chapter is an investigation of the monolayer behaviour and the interfacial interaction with the subphase. As in Chapter 3, this involves the characterisation of 2-HSA monolayers 
on four subphases: water, calcium chloride, sodium bicarbonate and a calcium carbonate crystallising subphase. Experimentation involved surface pressure and potential measurements complemented by Brewster angle microscopy imaging. Further due to the strength of the interaction with the calcium containing subphases this system was selected for synchrotron-based grazing incidence X-ray diffraction (GIXD), and X-ray reflectivity.

\subsection{Results \& Discussion}

Figure 5.2 illustrates the typical behaviour of 2-HSA monolayers on subphases of water, calcium chloride, sodium bicarbonate and a calcium carbonate crystallising subphase. With the exception of the water subphase, the general behaviour of the 2HSA monolayers is significantly different from that observed for ODA and ODOH mixtures. The uneven and curvilinear profiles are indicative of a reduction in crystallinity in comparison to the ODA/ODOH mixtures. This is consistent with the previous literature that attributed the disorder to the large head group size[3]. This increased head group size is also reflected in an increased $A_{m}$ at onset, compared to the mixed ODA/ODOH systems discussed in Chapter 3. Additionally the introduction of subphase ions, particularly $\mathrm{Ca}^{2+}$, leads to a significant expansion of the $A_{m}$ at onset (Figure 5.3). Again this is at odds with the condensation behaviour observed for the mixed systems. Further, the presence of calcium ions results in the formation of a very rigid film that physically moves the Wilhelmy plate, creating perturbations in the isotherm profile. Greatest perturbation occurs at lower pressures when the monolayer experiences the largest movement. 


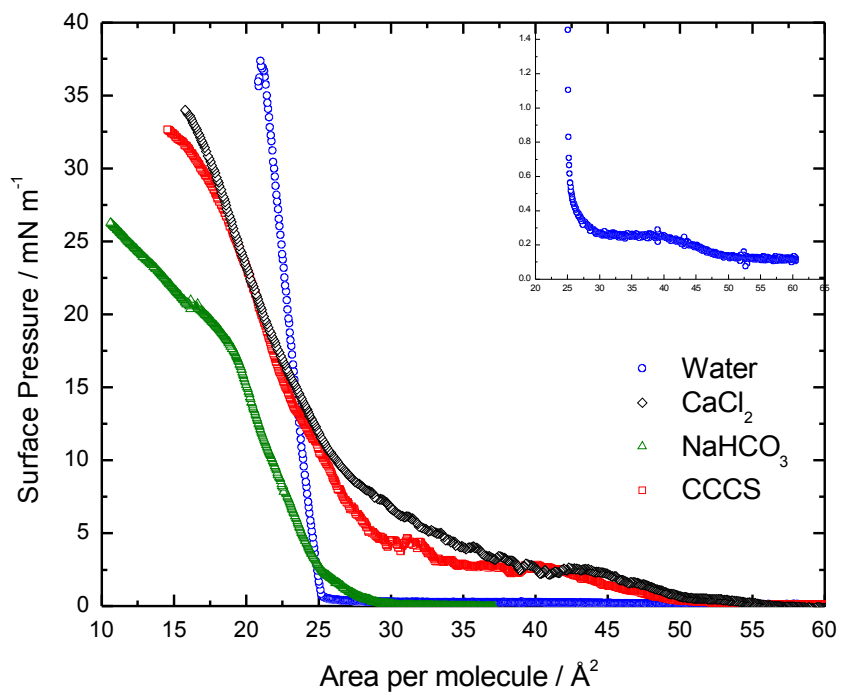

Figure 5.2 Surface pressure isotherms for 2-hydroxyoctadecanoic acid on different subphases: water at pH 5.6, $\mathrm{CaCl}_{2}$ at $\mathrm{pH} 5.5, \mathrm{NaHCO}_{3}$ at $\mathrm{pH} 6.0$ and a calcium carbonate crystallising subphase at $\mathrm{pH} 5.8$.

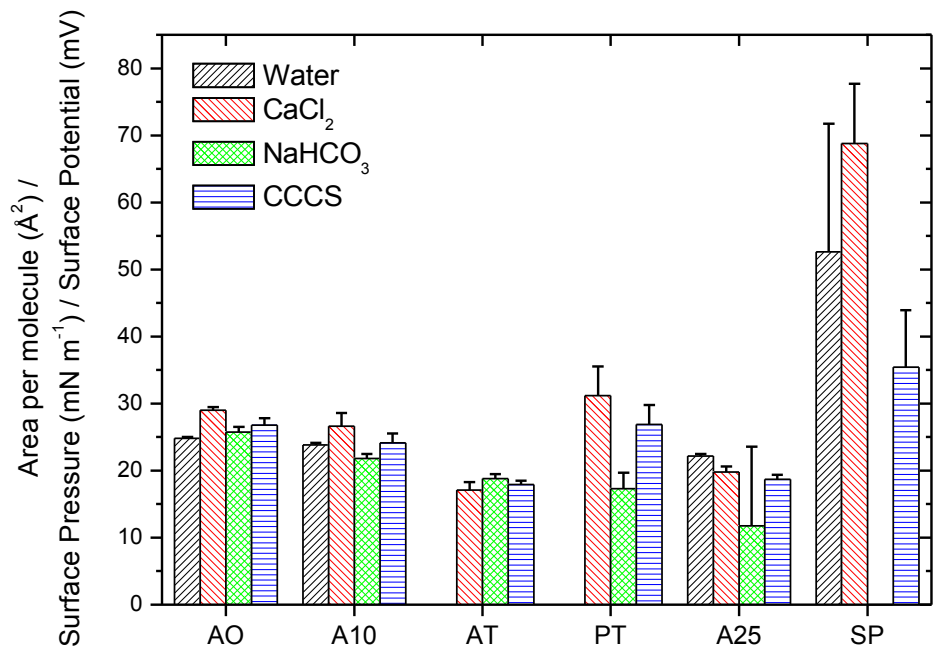

Figure 5.3 The overall trends in the surface pressure and potential isotherms for the different subphases. Note, there was no transition apparent on the water subphase hence the absence of AT and PT columns, also the maximum surface potential for the $\mathrm{NaHCO}_{3}$ subphase was $-30.6 \pm 12.0 \mathrm{mV}$ and therefore is not shown. (Key: $A O=$ the $A_{m}$ at onset, $A_{10}=$ the $A_{m}$ at $\Pi=10 \mathrm{mN} \mathrm{m}^{-1}, A T=$ the $A_{m}$ at the phase transition, $P T=\Pi$ at the phase transition, $A_{25}=$ the $A_{m}$ at $\Pi=25 \mathrm{mN} \mathrm{m}^{-1}$, and $S P=$ the maximum surface potential). 
Figure 5.3 shows the key characteristics of the monolayers: $A_{m}$ at onset, $A_{m}$ at $\Pi=10 \mathrm{mN} \mathrm{m}^{-1}, A_{m}$ at the phase transition, $\Pi$ at the phase transition (N.B. the isotherm for 2-HSA on water does not display a phase transition), the $A_{m}$ at $\Pi=25 \mathrm{mN} \mathrm{m}^{-1}$ and the maximum surface potential. In the following section the results will be attributed to the nature of the interfacial interaction that is unique to the subphase, with similarities and differences to the mixed systems noted. Initially at least, the addition of an active or participatory functional group leads to a stronger expression of the influence of the different subphase conditions. The specifics of these interactions will now be discussed more fully on an individual subphase basis.

\subsubsection{Water}

2-HSA on water at a $\mathrm{pH}$ of 5.6 behaves in a manner consistent with literature studies performed at $\mathrm{pH} 3[10]$. Immediately, from evaporation of the spreading solvent, the monolayer exists as two coexisting phases. The application of pressure results in a comparatively sharp transition that leads directly to collapse without any apparent intermediate transition to an untilted phase. At the early stages of compression, $\Pi<0.5 \mathrm{mN} \mathrm{m}^{-1}$, a plateau-like transition was observed. Brewster angle microscopy suggested no change in phase, only the merging of large uniform (in terms of contrast) islands of surfactant (Figure 5.4). Exploring this phenomena further, it was found that this plateau was dependent on compression speed and was concluded an artefact of these higher rates of compression.

Assuming a tilted monolayer, the lack of BAM contrast in conjunction with the lack of an observable phase transition, points to an universally heterogeneous or disordered film. This heterogeneity is confirmed by both literature[3,6], where it is attributed to a varying tilt azimuth, and GIXD. At all pressures investigated the presence of variable tilt was confirmed, manifested as a distribution of scattering intensity that was dominated by two or three peaks, consistent with a mix of NN and NNN tilted phases (Figure 5.5). Figure 5.6 shows the change in the diffraction 


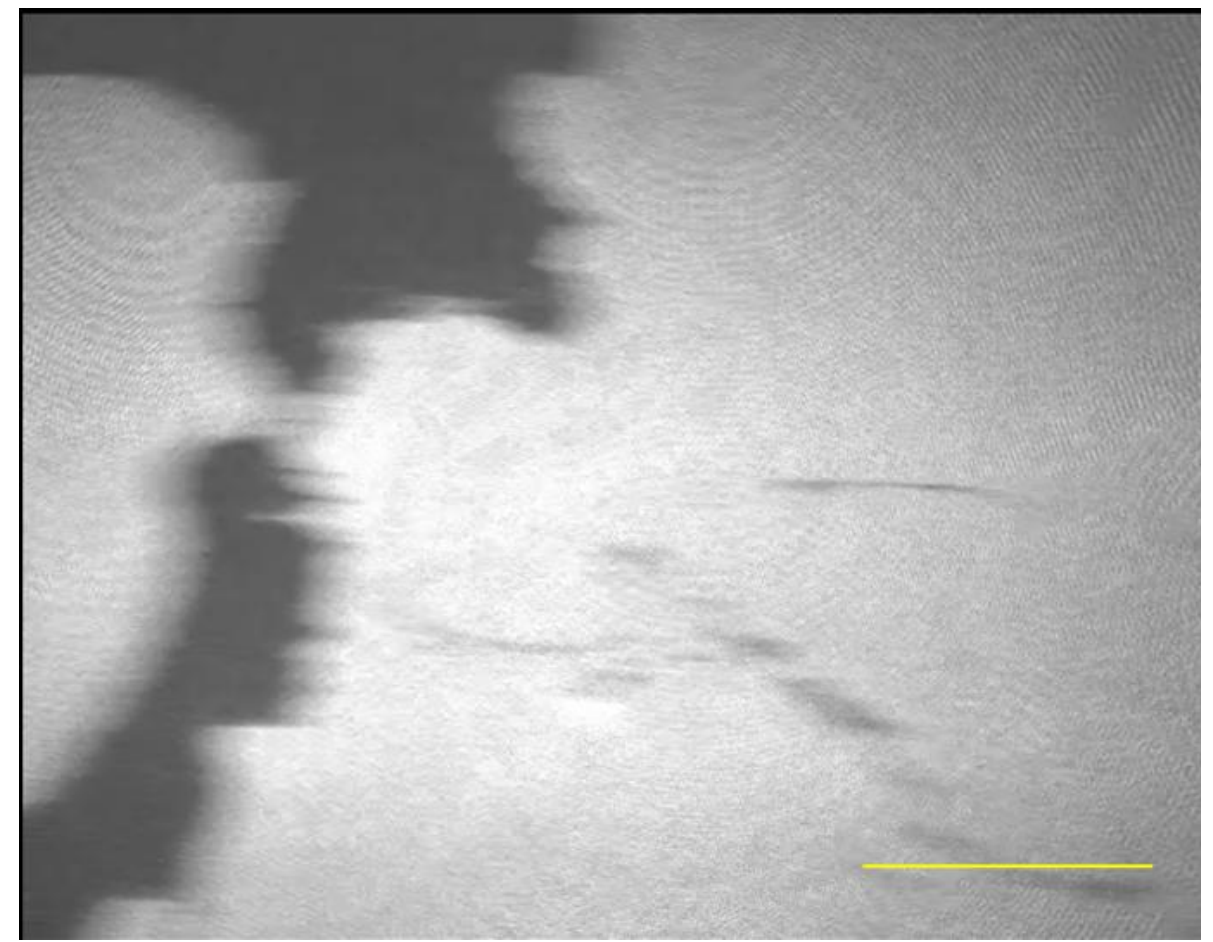

Figure 5.4 A BAM image showing the large islands present on water in the absence of pressure. Apparent is the lack of contrast pointing to a homogeneous, but as it turns out disordered monolayer. The scale bar $=1$ $\mathrm{mm}$. (Each of the BAM images is of an area of monolayer 4.128 by $3.616 \mathrm{~mm}$, based on a $640 \times 480$ image and a $x$-scale of 5.65 microns/pixel and a $y$-scale of 8.60 microns/pixel.)

pattern with increasing pressure. The zero pressure contour plot (Figure 5.5a) only shows two clear peaks at $Q_{x y}=1.275$ and $1.437 \AA^{-1}$, however on summing the data a third peak at $Q_{x y}=1.379 \AA^{-1}$ becomes apparent, as shown in the fitted profiles (Figure 5.5c). Three peaks is normally associated with an intermediate or oblique tilted lattice, however for this to be the case the condition $Q_{z}^{a}=Q_{z}^{b}+Q_{z}^{a b}$ must be met and the peak integrated intensities should be approximately equal[12]. With $Q_{z}$ values of $0.844,0.525$, and $0.00 \AA^{-1}$ this is clearly not the case, hence the three peaks must arise from a combination of NN, and NNN phases. Similarly disordered systems have been observed for other hydroxy-fatty acids[3].

Attributing the highest $Q_{z}$ peak to a combination of NN and NNN phases, the middle peak to NNN tilt and the in-plane peak to NN and untilted (U) phases we can approximate the phase composition. Correlating the integrated peak intensities 
(908 : $585: 242$, for $\Pi=0 \mathrm{mN} \mathrm{m}^{-1}$ ) with a ratio of $\mathrm{NN}: \mathrm{NNN}: \mathrm{U}$ phases based on the expected intensities associated with degeneracy gave a changing ratio shown in Figure 5.7a. These ratios show that the proportion of NNN phase remains relatively constant, whereas the initial NN phase is lost in favour of $U$ phase up to $\sim 10 \mathrm{mN} \mathrm{m}^{-1}$. Beyond $\Pi=10 \mathrm{mN} \mathrm{m}^{-1}$ this trend is reversed. At $\Pi=30 \mathrm{mN} \mathrm{m}^{-1}$ there is a suggestion that the NNN phase content is reduced in favour of the untilted phase. The explanation for such behaviour is unclear and further studies are required to: verify the phenomena, to provide a more accurate measurement of phase composition, and to assess whether the ratios observed here are characteristic of the monolayer or are random.

In terms of the lattice parameters, Figure $5.7 \mathrm{~b}$ shows how the different lattice dimensions change with pressure. The lattice parameters complement the phase composition in that the largest movement with pressure occurs in the NN and NNN phases, with the $U$ phase remaining relatively stable. Also of interest is the correlation between the NNN and NN lattice parameters and the stable U phase, such that, $a_{\mathrm{NNN}} \approx a_{U}$ and $b_{\mathrm{NN}} \approx b_{\mathrm{U}}$ and all four lattice parameters remain relatively stable with pressure, while $a_{N N}$ and $b_{\mathrm{NNN}}$ decrease with pressure. Thus the reduction in lattice parameters correlates with the reduction in tilt, such that the direction of shrinkage is consistent with the tilt direction.

This heterogeneity in the tilt direction of the monolayer has been attributed to a size mismatch between the large mono-polar head group and the diameter of the tails. The molecular spacing in the monolayer is dominated by the large head groups, limiting the tail-tail interactions. Alteration of the tilt angle per se is energetically expensive therefore the tails adopt a variation in the tilt direction to reduce the overall energetics of the monolayer. On a monolayer scale, the head/tail group mismatch leads to an entropically driven disordering of the monolayer tilt characteristics. However, with the application of pressure the degree of monolayer 
disorder is reduced with the reduction in tilt (which is observed for both the NN and NNN phases), as indicated by the movement of the peaks to smaller $Q_{z}$, and larger $Q_{x y}$ or smaller lattice values with increasing pressure. Perhaps coincidently, the rate of this gradual change is similar to that observed for monolayers consisting of a 60/40 mix of 2-hydroxypalmitic acid (HPA) and palmitic acid (PA)[3]. This investigation of mixed systems was instigated to confirm the large head group size as the source of the heterogeneity of tilt azimuth. The similarity between the pure 2-HSA and the 60/40 mixed HPA/PA system may reflect the longer tail of 2-HSA, such that a reduction in tilt associated with longer tails is equivalent to a reduction in head group size. 


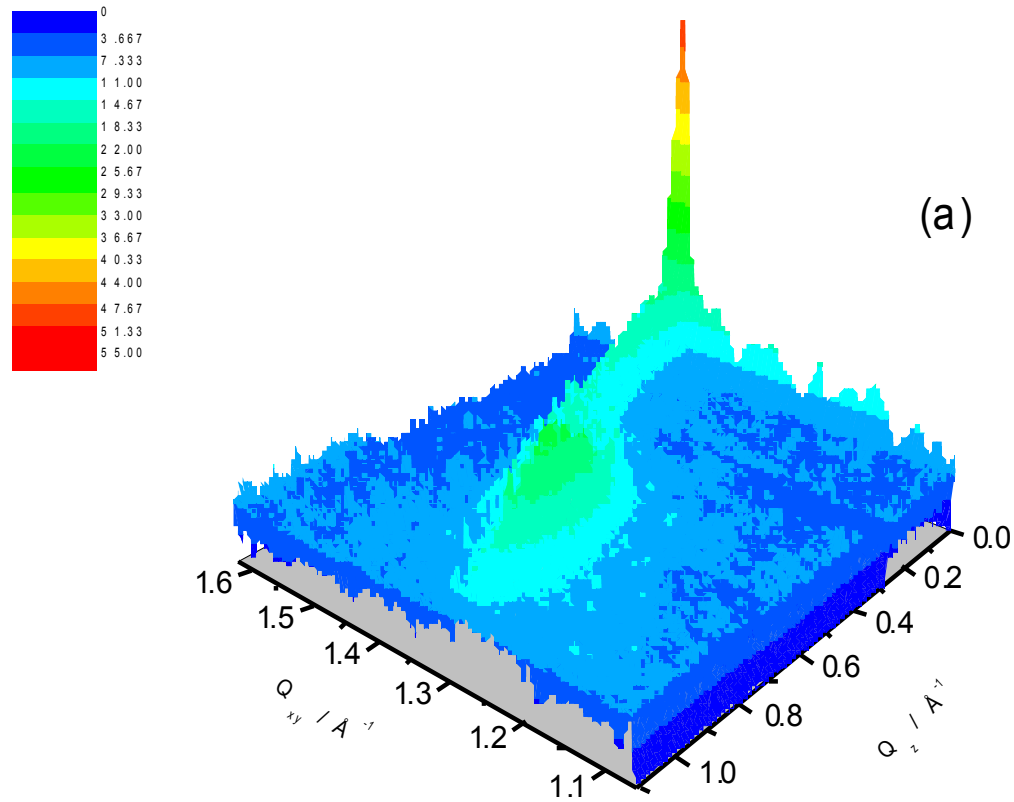

(b)

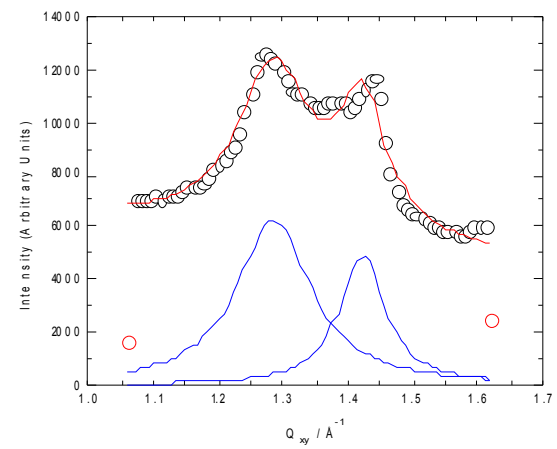

(c)

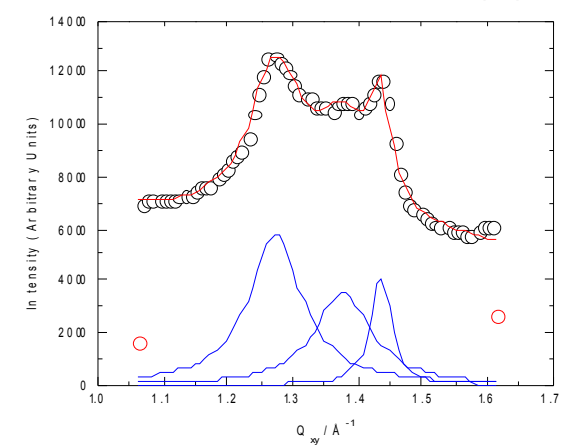

(d)

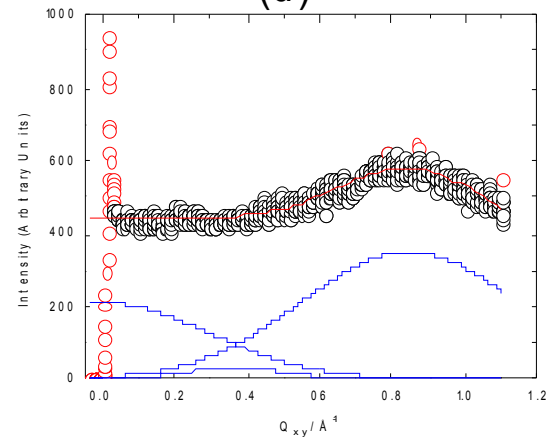

Figure 5.5 GIXD results for 2-HSA on water at $\Pi=0 \mathrm{mN} \mathrm{m} \mathrm{m}^{-1}$. (a) A 3-D contour plot illustrating the diffraction arc. (b) and (c) The fitting of two and three peak models, respectively, to the summed $Q_{x y}$ profile, with the three-peak model providing the best match. (d) The summed $Q_{z}$ profile and fit. 

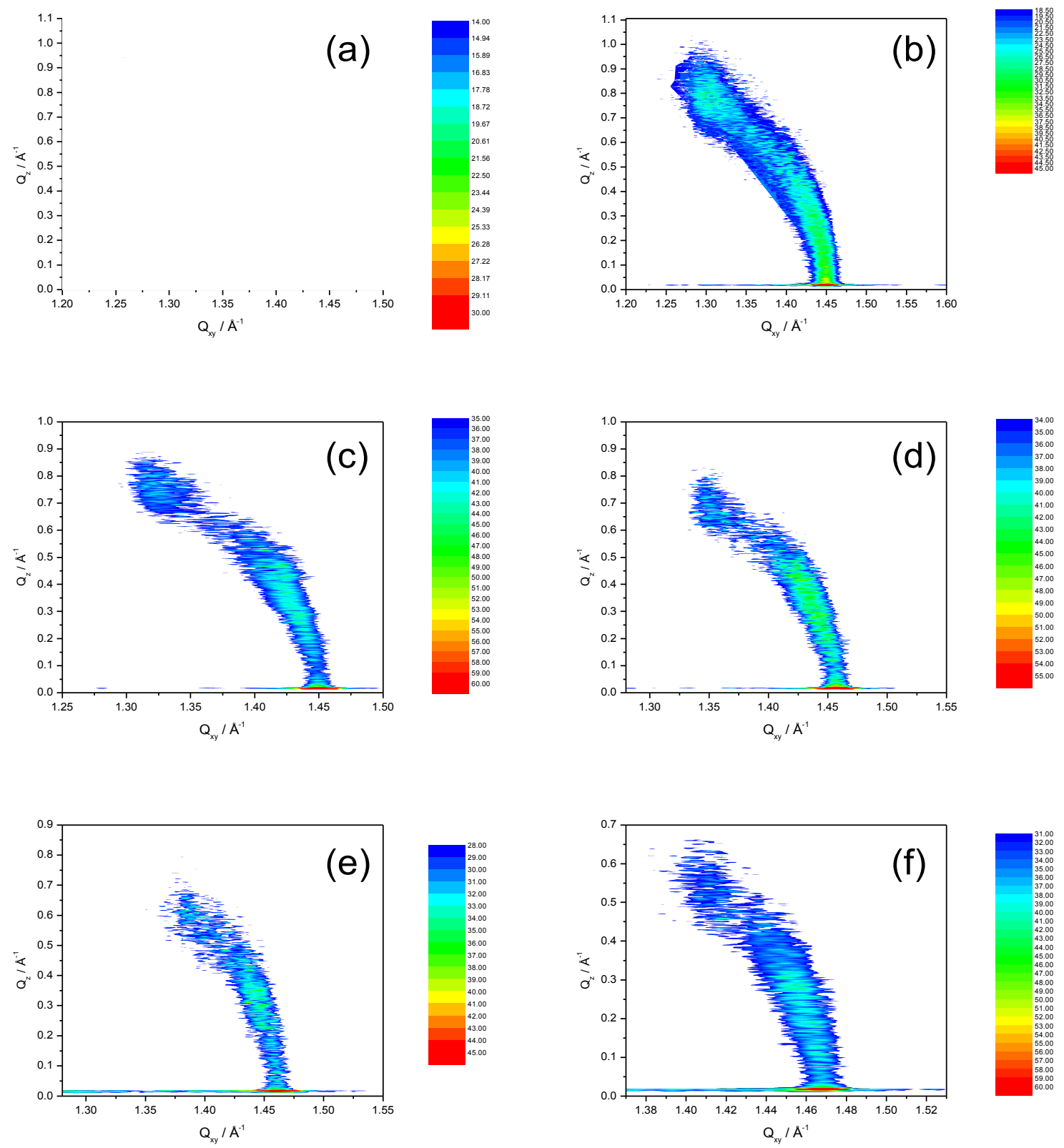

Figure 5.6 2-D contour plots of the behaviour of 2-HSA on water with increasing surface pressure. (a) 0 , (b) 5, (c) 10, (d) 17, (e) 25, and (f) $30 \mathrm{mN} \mathrm{m}$. 
(a)

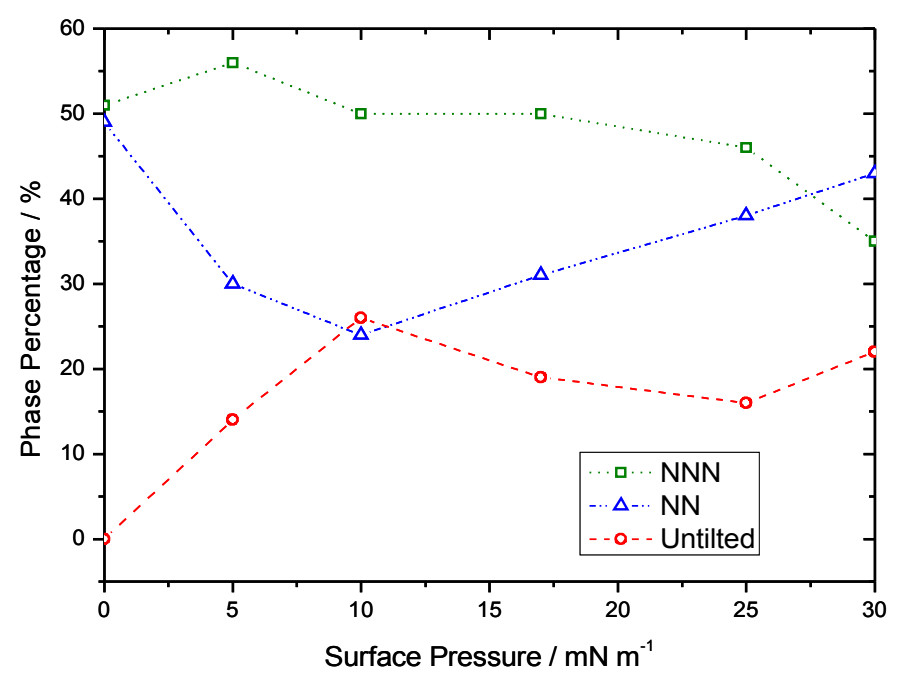

(b)

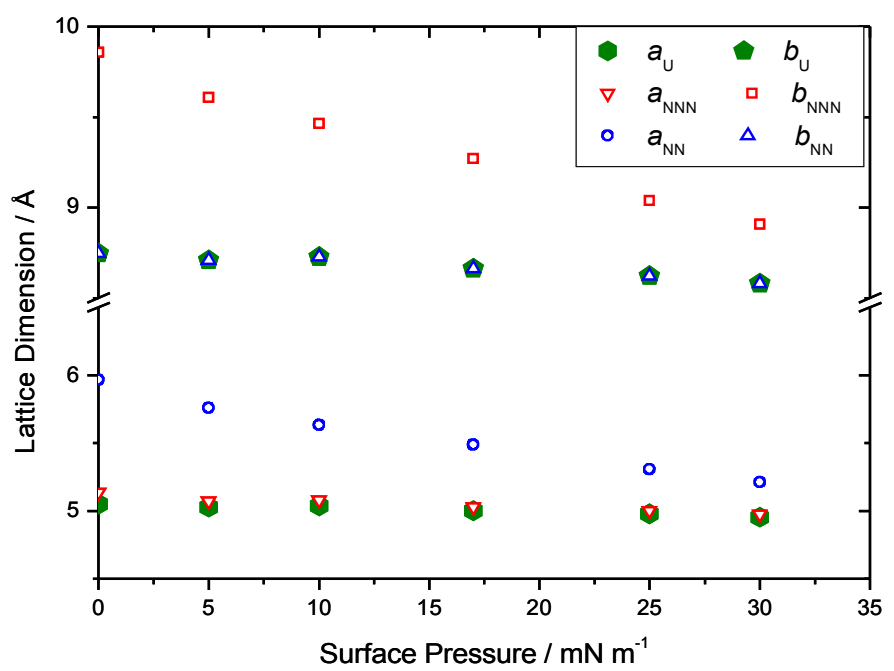

Figure 5.7 GIXD analysis of 2-HSA on water at $\Pi=0 \mathrm{mN} \mathrm{m}^{-1}$. (a) Variation of the phase composition of the monolayer with pressure, lines drawn to guide the eye. (b) Changes in the lattice parameters $a$, and $b$ with pressure for the three phases.

At a pressure of $35 \mathrm{mN} \mathrm{m}^{-1}$ Wediemann et al.[3] showed this transition to culminate in a single, in-plane peak. However this was not observed for 2-HSA, likely due to the fast compression speeds leading to a somewhat premature collapse. Although not directly observed, collapse was consistent with brittle-fracture (shown for the $\mathrm{NaHCO}_{3}$ subphase in Figure 5.17), characterised by a sudden and jerky movement of the monolayer. The brittleness of the monolayer reflects the extensive 
hydrogen bonding that the hydroxyl group adds to the system[2].

In terms of the surface potential behaviour it is helpful to recall the typical potential profile for the mixed systems, which consists of a plateau at pre-onset pressures, followed by a small and gradual increase coinciding with onset. The slight increase attains a maximum around the transition to an untilted phase before decreasing. This profile being consistent with the loss of tilt leading to an increase in the molecular dipole associated with increased vertical charge separation. The subsequent decline coincides with the initiation of collapse, where the formation of 3D structures results in a countering of the molecular dipole. The nature of this decrease in potential is specific to the mechanism of collapse.

For 2-HSA on water, the surface potential profile (Figure 5.8) begins with a plateau for the expanded film, in accordance with the mixed monolayer systems. However, unlike the mixed systems there is no increase in potential associated with onset, rather the potential displays a gradual decline. This response to onset is attributed to the lack of a tilted-to-untilted transition and the early stages of collapse. The maximum surface potential on water is $\sim 52.6 \mathrm{mV}$, which is considerably lower than the $\sim 260 \mathrm{mV}$ and $\sim 420 \mathrm{mV}$ recorded for pure ODA and ODOH, respectively. Such a significant drop in the potential is difficult to account for; however, at least in part, this can be attributed to the increased propensity for intramolecular hydrogen bonding. Greater hydrogen bonding has two affects: (1) it will result in a significant change in the water structuring at the interface; and (2) as described in Chapter 3, it may increase the countering double layer potential by increasing the degree of dissociation. Ultimately further work is required to elucidate this drop in the measured surface potential.

The XRR data for 2-HSA on water was measured at two pressures $\Pi=10$ and $25 \mathrm{mN} \mathrm{m}^{-1}$. Given the three-phase composition it is assumed that the XRR data is a weighted average of the three phases. For example at $\Pi=10 \mathrm{mN} \mathrm{m}^{-1}, \mathrm{~A}_{x y}$ is $24.0 \AA^{2}$ 


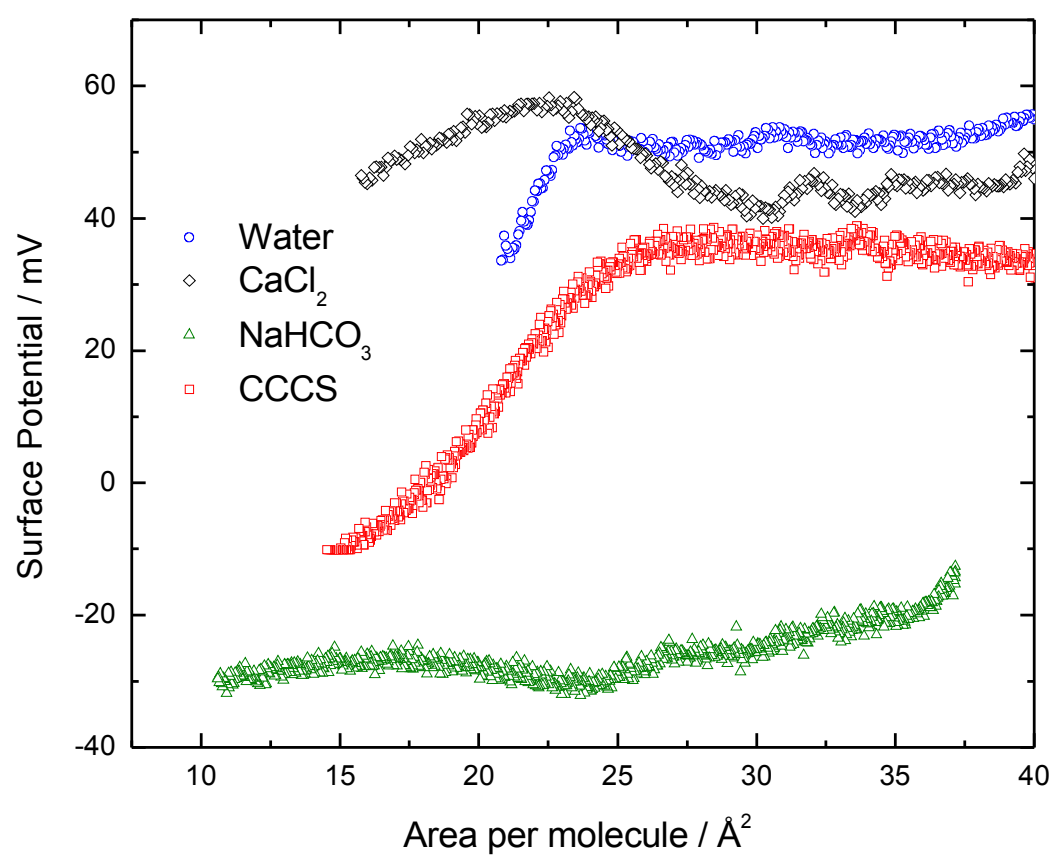

Figure 5.8 Surface potential profiles for 2-HSA on subphases of water, calcium chloride, sodium bicarbonate and CCCS.

for NNN, $24.6 \AA^{2}$ for NN and $22.0 \AA^{2}$ for $U$, which gives an average of $23.6 \AA^{2}$, for a phase ratio of $0.5: 0.24: 0.26$. This compares with an isotherm based $A_{m}$ of $23.8 \AA^{2}$.

At the lower pressure two models are difficult to separate, in terms of reflectivity fit, both are detailed in Table 4. The first, a two-box model, has the best statistics but overall the fit visually is not as good as the second model. The second model, a three-box model, has reasonable statistics and a better visual fit but the subphase roughness is unusual. The third box in this model represents some subphase ordering, which increases the localised electron density above bulk water. Therefore there is a suggestion that there is some degree of water restructuring at the interface but the irregular subphase roughness suggests that a third separate box does not quite capture this.

Using the weighted average $A_{m}$ for the GIXD data and the surface pressure isotherms, the total electron count was calculated. Based on the electron density ratio 
of the boxes these electrons were assigned to the boxes and then attributed to molecular groups. Thus the two-box model consists of a $\left[\mathrm{CH}_{3}\left(\mathrm{CH}_{2}\right)_{13}\right]$ box and a $\left[\left(\mathrm{CH}_{2}\right)_{2} \mathrm{CHOHCOOH}+2 \times \mathrm{H}_{2} \mathrm{O}\right]$. In comparison, the three-box model yields boxes: $\left[\mathrm{CH}_{3}\left(\mathrm{CH}_{2}\right)_{14}\right],\left[\mathrm{CH}_{2} \mathrm{CHOHCOOH}+3 \times \mathrm{H}_{2} \mathrm{O}\right]$ and $\left[10 \times \mathrm{H}_{2} \mathrm{O}\right]$. The differences being an extra $\mathrm{CH}_{2}$ group in the head group box of the two-box model, which is replaced by a water in the three-box model. Overall the best fit is with the three-box model given the better reflectivity fit and the lower head group alkyl content suggested by the model. However, the improved fit of three box model, in comparison to the two box model, of the data is small. Given the averaging that occurs, due to the 3 hour measurement time, and the similarity of the fits, it is difficult to exclude either model.

Table 4 The best fitting models for 2-HSA on water at $\Pi=10$ and $25 \mathrm{mN} \mathrm{m}^{-1}$.

\begin{tabular}{|c|c|c|c|c|c|c|c|c|c|}
\hline $\begin{array}{c}\Pi \\
\left(\mathrm{mN} \mathrm{m}^{-1}\right)\end{array}$ & $\begin{array}{c}\text { No. of } \\
\text { Boxes }\end{array}$ & $\rho_{1} / \rho_{\mathrm{w}}$ & $\begin{array}{c}\mathrm{L}_{1} \\
(\AA)\end{array}$ & $\rho_{2} / \rho_{\mathrm{w}}$ & $\begin{array}{c}\mathrm{L}_{2} \\
(\AA)\end{array}$ & $\rho_{3} / \rho_{\mathrm{w}}$ & $\begin{array}{c}\mathrm{L}_{3} \\
(\AA)\end{array}$ & $\begin{array}{c}\mathrm{L}_{\mathrm{T}} \\
(\AA)\end{array}$ & $\begin{array}{c}\sigma \\
(\AA)\end{array}$ \\
\hline 10 & 2 & 0.97 & 15.13 & 1.24 & 7.88 & & & 23.01 & 1.75 \\
\hline 10 & 3 & 0.99 & 15.1 & 1.26 & 7.83 & 1.03 & 12.82 & 35.75 & 2.31 \\
\hline 25 & 2 & 0.97 & 17.33 & 1.41 & 4.83 & & & 22.16 & 3.14 \\
\hline
\end{tabular}

$\left(\rho_{1} / \rho_{w}\right.$ is the linear electron density of the box relative to the subphase, $S L D=0.334 \mathrm{e} / \AA^{3}, L_{i}$ is the box length, $L_{T}$ is the total box length and $\sigma$ is the subphase roughness).

At $\Pi=25 \mathrm{mN} \mathrm{m}^{-1}$ the two-box model provides the best fit of the experimental reflectivity. With an isotherm based $A_{m}$ of $22.2 \AA^{2}$ and a GIXD based $A_{x y}$ of $22.54 \AA^{2}$, the first box in the model accounts for the 16 tail-carbons. The head group box has sufficient electron density for the remaining [CHOHCOOH] plus one water molecule. This arrangement reflects the domination of steric affects at higher pressures, where the penetration of the head group into the subphase, and the degree of hydration is reduced. The total length is smaller than that at $\Pi=10 \mathrm{mN} \mathrm{m}^{-1}$, which is consistent with the slight reduction in the amount of untilted phase present (Figure 5.7a). Hence the small rearrangement of the head group reflects an unexpected small 
shift to a more erect NN phase rather than an increase in the untilted phase content.

Under similar conditions (subphase $\mathrm{pH}$ unspecified), Cristofolini et al.[7] fitted a two-box model with quite different parameters $\left(\rho_{1} / \rho_{\mathrm{w}}=0.31 \mathrm{e} / \AA^{3}, \mathrm{~L}_{1}=15.6 \AA, \rho_{2} / \rho_{\mathrm{w}}=\right.$ $0.42 \mathrm{e} / \AA^{3}$, and $\mathrm{L}_{2}=9.36 \AA$ ). From these values it was deduced, from the large head group box, that six carbons were submerged in the subphase and the remaining 12 contributed to the tail box. This differs from our calculations as Cristofolini et al. [7] ignored the possibility of any water in the head-group box. Given the highly polar nature of the head group it is very likely that there would be significant hydrogen bonding between the subphase water and the head group. Therefore this approach by Cristofolini et al.[7] is overly simplistic.

In summary, 2-HSA monolayers on water present a three-phase coexistence of NNN, NN, and $U$ phases up to $\Pi=30 \mathrm{mN} \mathrm{m}^{-1}$. There is evidence that, with increasing pressure, the NN and $\mathrm{U}$ compositions fluctuate as the tilt angle of the NN phase reduces. In contrast, the level of NNN phase remains relatively constant as tilt is reduced.

\subsubsection{Calcium Chloride}

The introduction of calcium ions to the subphase has a dramatic effect on the monolayer behaviour as shown in Figure 5.2. A comparison with water shows the isotherm to have a larger $A_{m}$ at both onset and $\Pi=10 \mathrm{mN} \mathrm{m}^{-1}$, a trend reversed at higher pressures (Figure 5.3). However, analysis of GIXD data shows that, in fact, the monolayer exhibits untilted, hexagonal symmetry with an $\mathrm{A}_{\mathrm{m}}$ of $\sim 20 \AA^{2}$ at all pressures (Figure 5.9), much lower than the $29.0 \AA^{2}$ at onset indicated by the isotherms.

BAM images show the monolayer to consist of multi-domain islands (based on contrast differences), approximately 0.3 to $3.0 \mathrm{~mm}$ in size, the majority above $\sim 1 \mathrm{~mm}$ (Figure 5.10). The application of pressure leads these islands to collide and fracture 


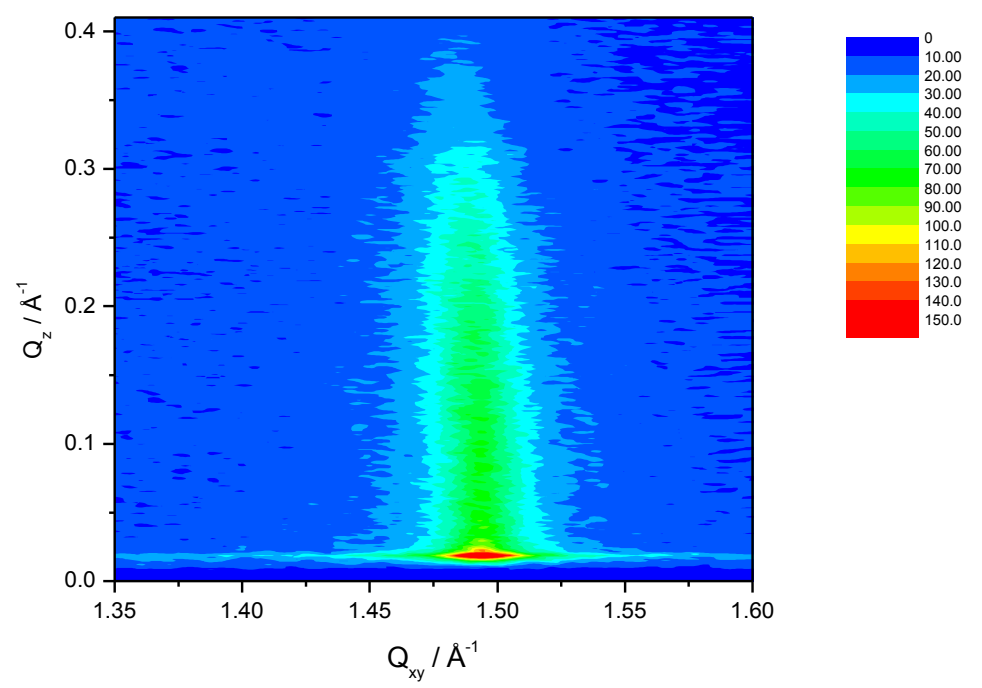

Figure 5.9 A 2-D contour plot of the GIXD pattern for 2-HSA on CCCS in the absence of applied pressure.

rather than merge, implying strongly bound rigid domains. It is only at higher pressures that there is sufficient force to lead to merging of islands. Thus the perturbated isotherm and large $\mathrm{A}_{\mathrm{m}}$ 's are more a consequence of macroscopic packing deficiencies rather than molecular structuring.

The rigid domains are a consequence of the strong $\mathrm{Ca}^{2+}$ binding by the hydroxy acids. A comparison of the calcium binding constants of 2-hydroxypropanoic acid (lactic acid) and 1-propanoic acid shows a greater than two-fold increase in binding strength upon addition of the hydroxy group[19]. This is associated with the preferential binding of the cation with the acid carbonyl and hydroxyl group oxygens rather than with the acid hydroxyl. For this to occur in 2-HSA monolayers, a molecular re-arrangement of the head group would be required. Such a conformation change (Figure 5.11) would be driven by the greater stability of fivemembered chelates over four-membered versions[20]. Both conformations have the capacity for intramolecular hydrogen bonding, which combined with a stoichiometry of $\mathrm{Ca}(2-\mathrm{HSA})_{2}$ would result in strongly bound domains. Evidence for this conformation change is scant, with the magnitude of the associated dimensional 


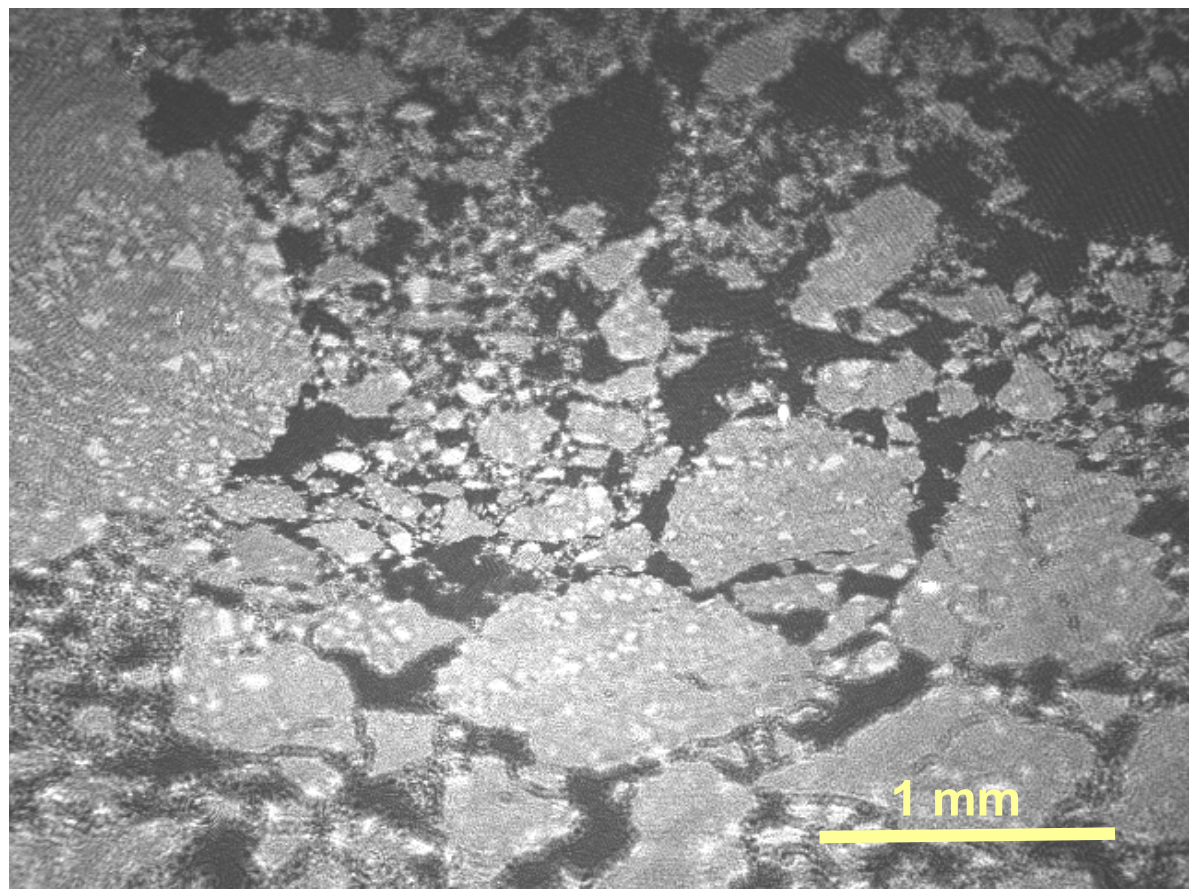

Figure 5.10 A BAM image of a 2-HSA monolayer on a $20 \mathrm{mM} \mathrm{CaCl}_{2}$ subphase at $0 \mathrm{mN} \mathrm{m}^{-1}$ pressure. The islands appear to be multi-domain based on the contrast and are very rigid.

changes falling within experimental error.

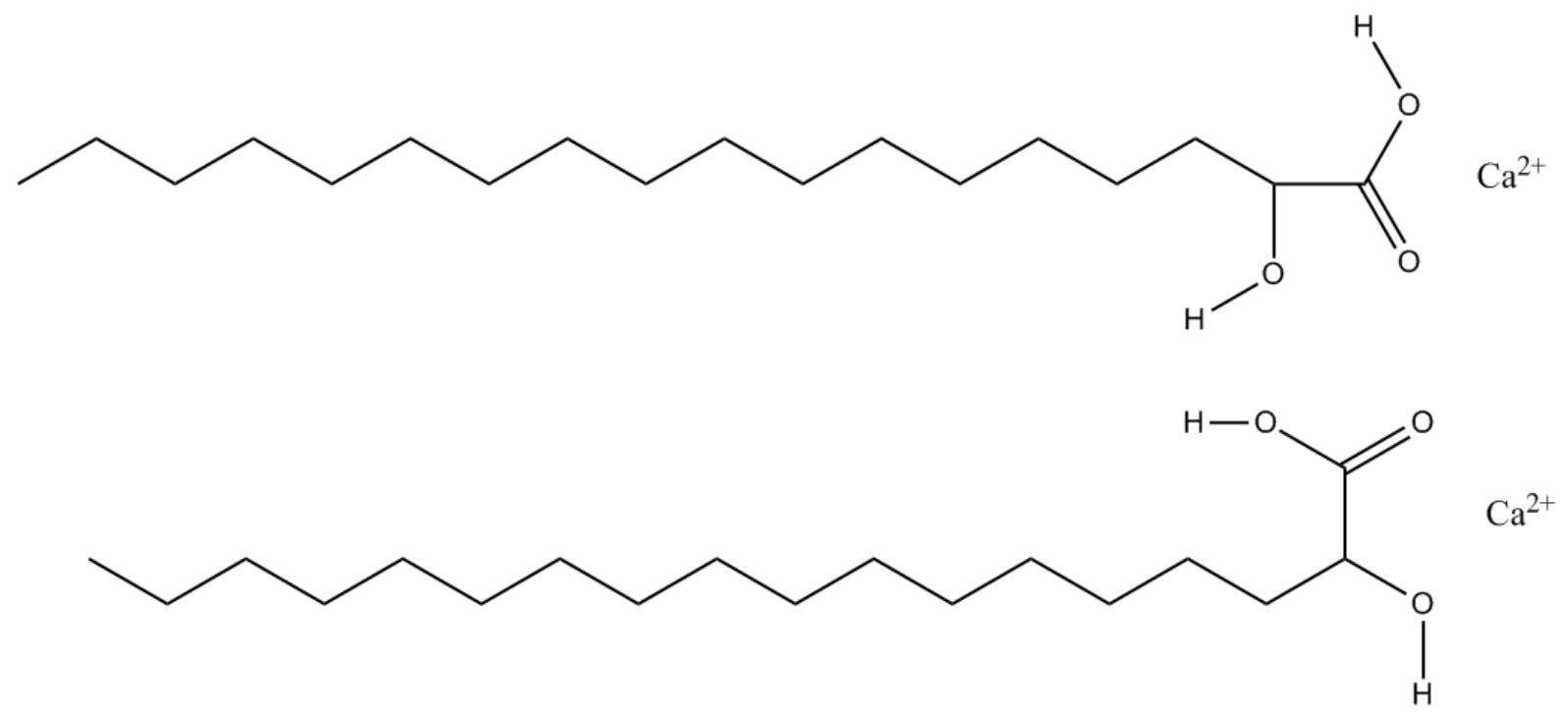

Figure 5.11 Indicative structures of 2-HSA head groups in response to cation binding, the precise conformation will vary with surface pressure and chelation. The top structure is consistent with a fourmembered ring chelation as opposed to the five-membered ring of the lower structure.

The observation of contrasting domains in the BAM images is somewhat surprising given the single untilted hexagonal phase evident in GIXD data. Further 
there is significant disparity in the domain size indicated by the smaller $(\sim 0.3 \mathrm{~mm})$ monochromic islands in BAM images and the GIXD-derived domain correlation length of $\sim 194 \AA$. Chirality may explain these observations. The 2-HSA used is a mix of $\mathrm{D}$ and $\mathrm{L}$ enantiomers and therefore the contrast may indicate phase separation. Neumann et al.[5] showed that the inclusion of divalent subphase cations can instigate chiral discrimination. Although $\mathrm{Ca}^{2+}$ was found, relative to $\mathrm{Pb}^{2+}$ and $\mathrm{Zn}^{2+}$, to be relatively poor at inducing increased phase separation, the capacity for phase separation remains. The reason for this discrimination is unclear but it is believed to be based around differences in the intra-complex bonding, leading to preferential homochiral (L:L or D:D) interactions as opposed to heterochiral (L:D) interactions[5].

With increasing pressure, the lattice parameters show a steady reduction, as expected. After reducing to an $\mathrm{A}_{\mathrm{m}}$ of $\sim 19.8 \AA^{2}$ at a pressure of $25 \mathrm{mN} \mathrm{m}^{-1}$, as determined by GIXD, the monolayer collapses. Collapse is characterised by a smooth inflection of the isotherm correlating with a similarly gradual increase in the nucleation of 3-D discontinuities as observed using BAM. The small spherical, highly reflecting nature of these defects is consistent with surfactant solidification. With a solid precipitate having been observed macroscopically on the air/water interface subsequent to the experiment.

On $\mathrm{CaCl}_{2}$ the surface potential profile (Figure 5.8) is very similar to that observed for the mixed systems. However, as the 2-HSA monolayer is untilted at all pressures, the rise in the potential cannot be attributed to a tilting transition. Rather this rise is consistent with a loss of small amounts of an expanded gaseous phase that remains in the inter-island regions beyond onset. The increasing pressure forces the rigid islands to pack together. The resulting rise in potential is then followed by a decline, again associated with the nucleation of 3-D phases. The magnitude of the potential is slightly higher than that observed for water ( $68.8 \mathrm{mV}$ compared to $52.6 \mathrm{mV}$ for water). This increase can mostly be attributed to the complete loss of tilt. 
The strong calcium/monolayer interaction is also evident in the XRR data. At both pressures $\left(\Pi=10\right.$ and $\left.25 \mathrm{mN} \mathrm{m}^{-1}\right)$, a 'three-box' model provides the best fit of the measured reflectivity profile (Figure 5.12 and Figure 5.13, respectively). Analysis of the electron density profile at $\Pi=10 \mathrm{mN} \mathrm{m}^{-1}$ shows the first box to account for the $\left[\mathrm{CH}_{3}\left(\mathrm{CH}_{2}\right)_{13}\right]$ tail group, the second box correlates with $\left[\left(\mathrm{CH}_{2}\right)_{2} \mathrm{CHOHCOOH}+0.5 \times\right.$ $\mathrm{Ca}$, and the third box can be attributed to four water molecules. The half a calcium reflects the expected $\mathrm{Ca}(2-\mathrm{HSA})_{2}$ charge-based stoichiometry. The grouping of four water molecules in the last box is consistent with the hydration of the cation, resulting in increased water packing and the disruption of water's favoured hydrogen-bonded structure.

At $\Pi=25 \mathrm{mN} \mathrm{m}^{-1}$ the profile reflects an increase in the tail length to include all the hydrocarbon $\mathrm{CH}_{3}$ and $\mathrm{CH}_{2}$ groups. The head group box includes the $[\mathrm{CHOH}]$ group, the acid functionality, half a calcium, and one water molecule. The third box again includes 5-6 water molecules.

The differences between the reflectivity at the two pressures is relatively small but potentially significant. The increase in pressure brings about four changes: 

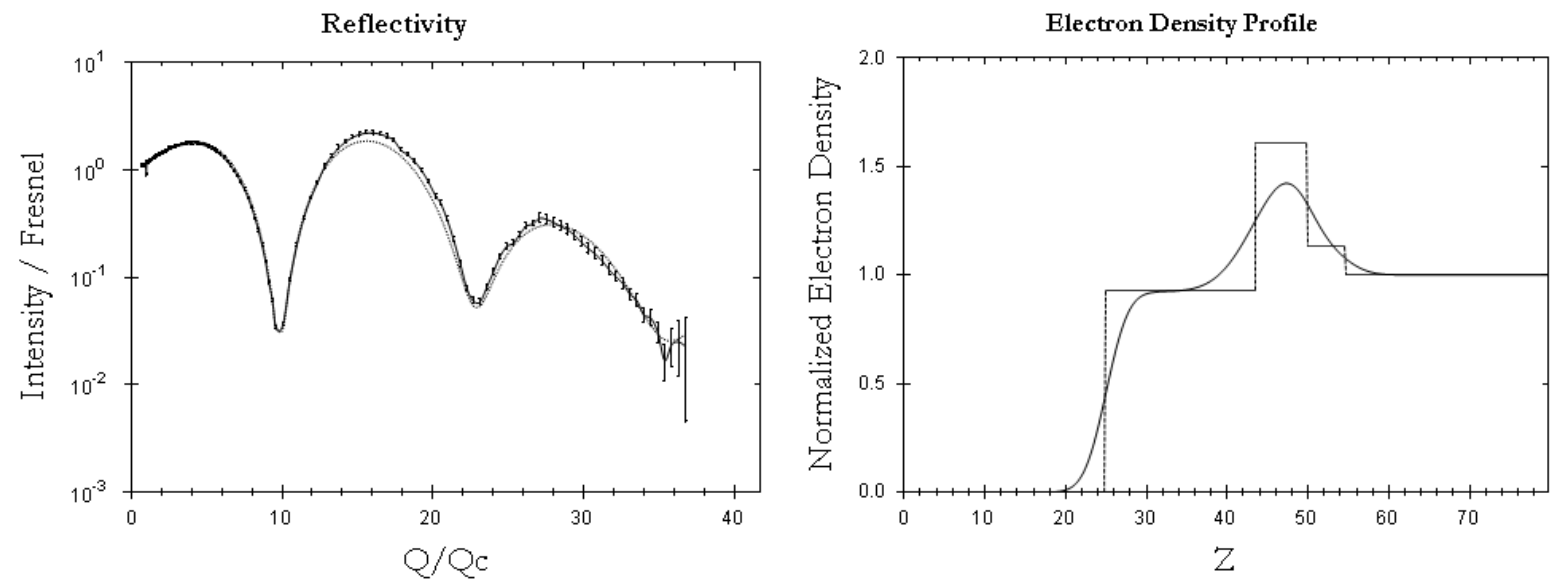

Figure 5.12 XRR reflectivity and electron density profiles for 2- $\mathrm{HSA}$ on $\mathrm{CaCl}_{2}$ at $\Pi=10 \mathrm{mN} \mathrm{m}{ }^{-1}$. In the reflectivity profile the solid line reflects the model fit of the experimentally determined data (points). In the electron density profile the dashed line represents the model boxes and the solid line is the smoothed box model.
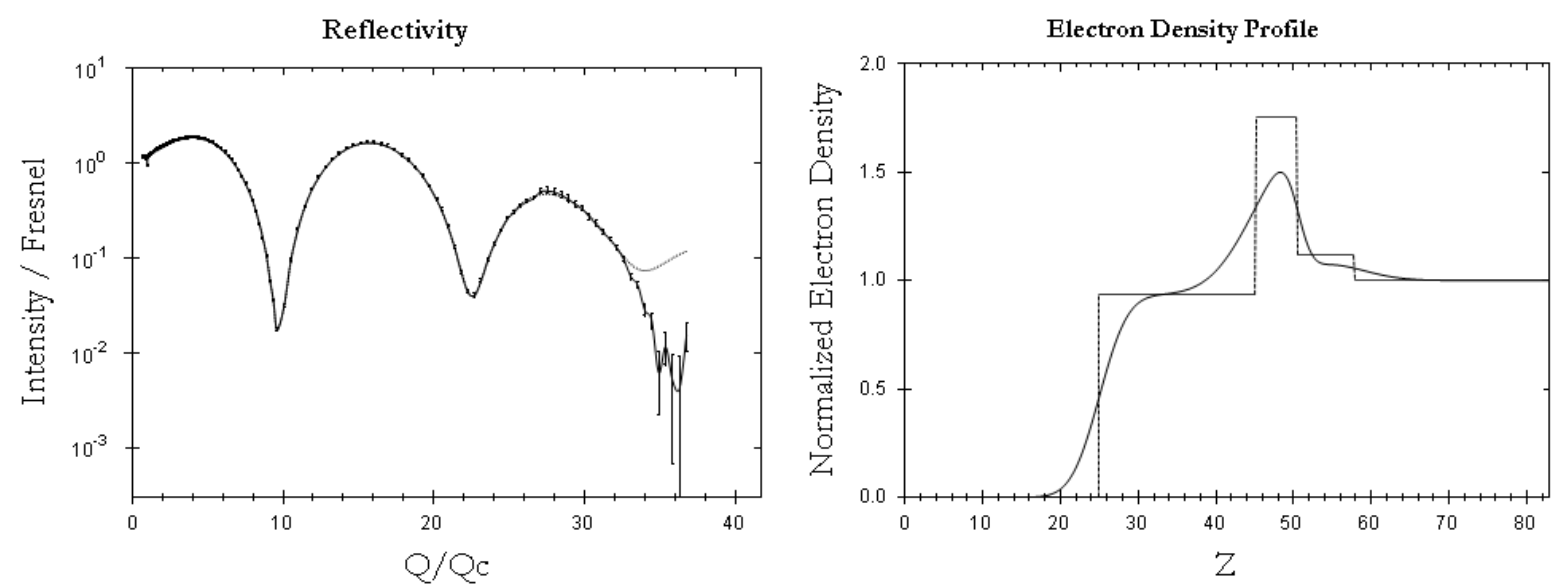

Figure 5.13 XRR data and electron density profiles for 2-HSA on $\mathrm{CaCl}_{2}$ at $\Pi=25 \mathrm{mN} \mathrm{m}^{-1}$. In the reflectivity profile the solid line reflects the model fit of the experimentally determined data (points). In the electron density profile the dashed line represents the model boxes and the solid line is the smoothed box model.

- the sterically-driven movement of the two $\mathrm{CH}_{2}$ groups from box two at $\Pi=10 \mathrm{mN} \mathrm{m}^{-1}$ to box-one at $\Pi=25 \mathrm{mN} \mathrm{m}^{-1}$;

- the reduction in $\mathrm{A}_{\mathrm{m}}$;

- an increase in the number of associated water molecules from four to six; and

- the increase in the combined $\left(\mathrm{L}_{1}+\mathrm{L}_{2}\right)$ box length.

These changes point to a possible disruption of the $\mathrm{Ca}^{2+}$-bridging chelation associated with the rigid domains. The higher applied pressures may reduce the capacity for 
the cation to bridge two surfactant molecules by removing the ability to rearrange (compare the two structures in Figure 5.11), as indicated by the loss of the two methylene groups from the head group box. The increased association of water with the head group at higher pressures could reflect the need to counter the calcium charge given the loss of the bridging capability.

In summary, the introduction of the calcium ion to the subphase sees a domination by electrostatic-based effects. This is very evident in the rigid domains observed, which perturb the Wilhelmy plate and therefore the surface potential measurements. However, XRR suggests that at high pressures steric effects begin to disrupt the $\mathrm{Ca}^{2+}$-bridging network.

\subsubsection{Sodium Bicarbonate $\left(\mathrm{NaHCO}_{3}\right)$}

2-HSA isotherm behaviour on $\mathrm{NaHCO}_{3}$ subphases is again quite different from that observed on water and calcium chloride subphases, Figure 5.2 shows a typical isotherm profile. For $A_{m}$ values greater than $30 \AA^{2}$, BAM images show a two phase coexistence with relatively rigid islands (Figure 5.14), where rigidity was gauged according to how readily the islands merged to form a coherent monolayer, for $\mathrm{NaHCO}_{3}$ based systems this does not occur until after onset. This behaviour is similar, but weaker, to that observed for $\mathrm{CaCl}_{2}$ pointing to a strong interfacial interaction. GIXD at $\Pi=0 \mathrm{mN} \mathrm{m}^{-1}$ shows two peaks at $Q_{x y} 1.406$ and $1.489 \AA^{-1}$, with the former out-of-plane and the latter in-plane. Based on integrated intensities, the two peaks are associated with two phases: a NN tilted phase and a stronger untilted

phase. An approximation based on the expected intensity ratio for a NN phase and the measured peak intensities indicates to a monolayer phase composition of 80:20 untilted to NN tilted (Figure 5.15). The existence of two phases is supported by the observation of two subtly different shades of grey in the BAM images (Figure 5.14).

Beyond onset the monolayer moves to much smaller $\mathrm{A}_{\mathrm{m}}$ yet the compressibility, as indicated by the isotherm slope, is not appreciably different from the $\mathrm{CaCl}_{2}$ 


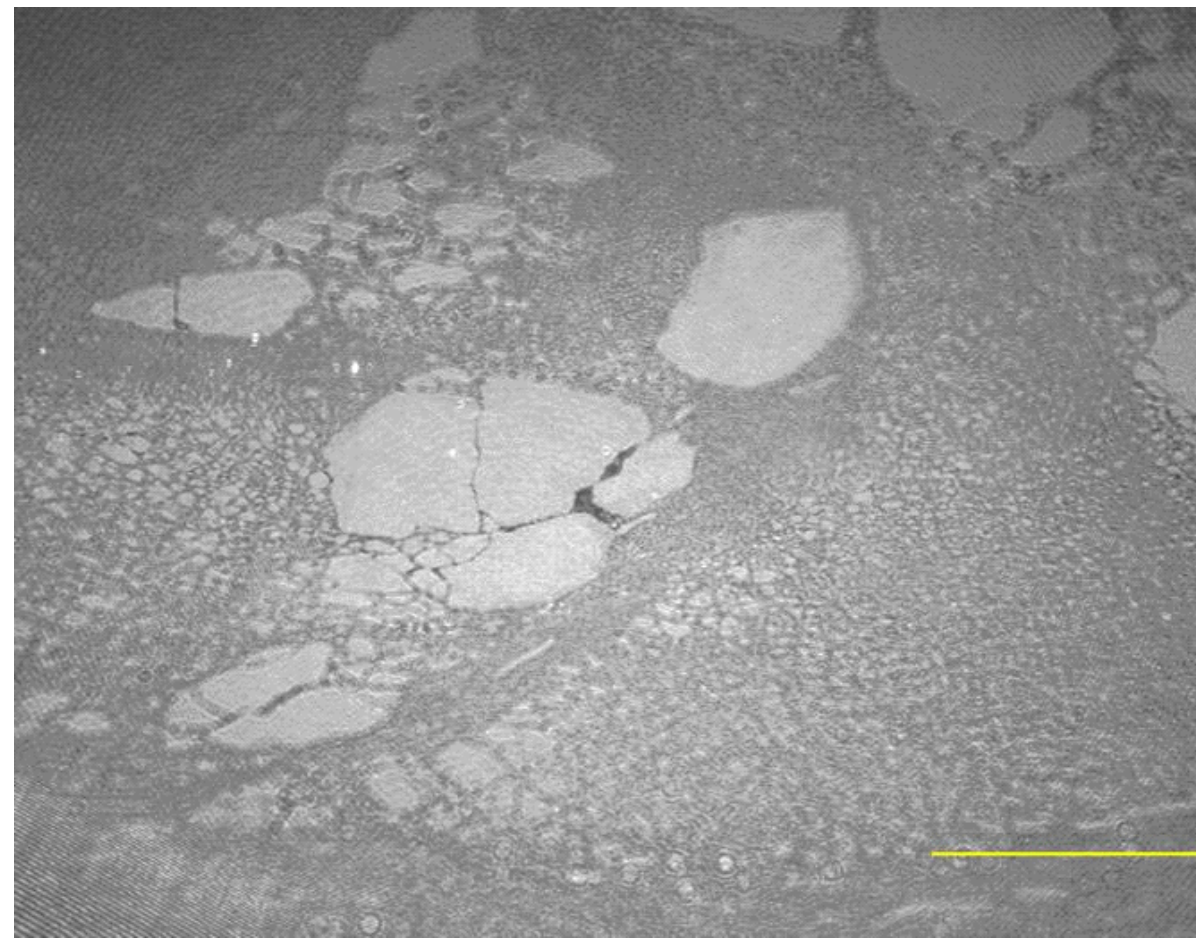

Figure 5.14 A BAM image illustrating the presence of two phases in a monolayer of 2-HSA on an aqueous $\mathrm{NaHCO}_{3}$ subphase. The scale bar represents $1 \mathrm{~mm}$.

subphase. The GIXD results show the lattice parameters and therefore the $A_{m}$ to change very little with increasing pressure up to $25 \mathrm{mN} \mathrm{m}^{-1}$ (Figure 5.16). These observations point to poor monolayer stability, with the reduction in $\mathrm{A}_{\mathrm{m}}$ due to slow collapse. This is reflected in the isotherm returning $A_{m}$ values of $<20 \AA^{2}$ at higher pressures which given the head group size is incorrect. 


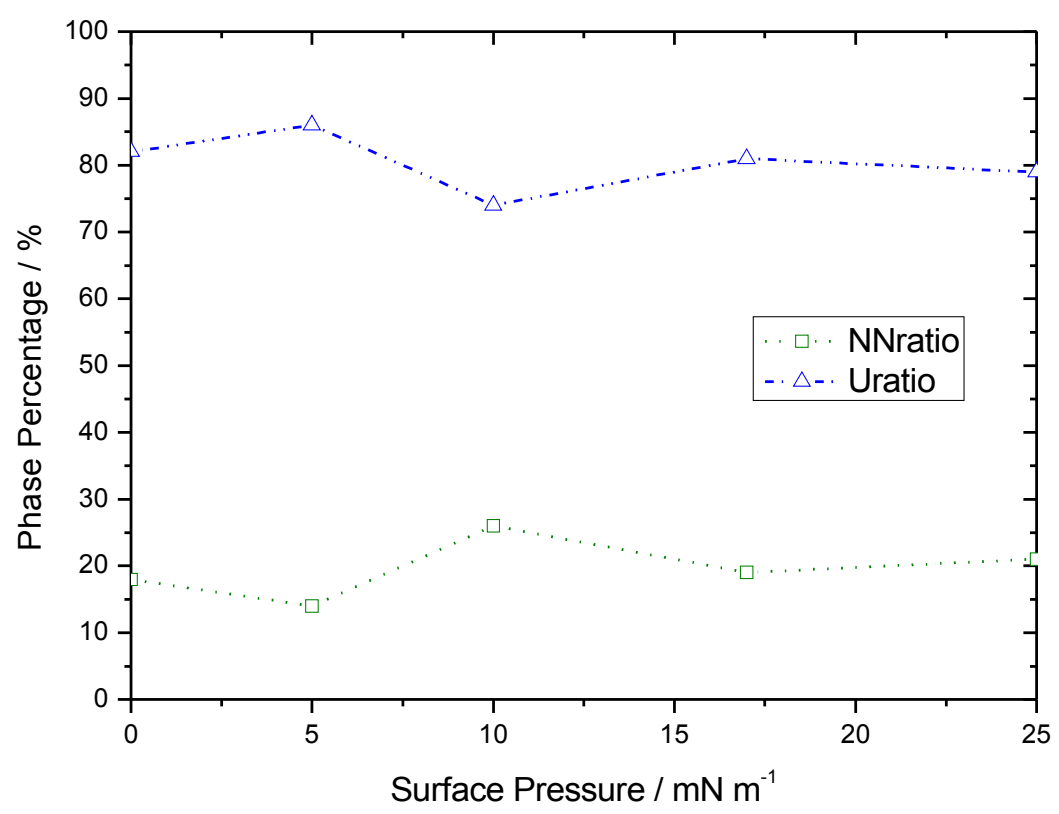

Figure 5.15 The ratio of NN-tilted and untilted (U) phases in a 2-HSA monolayer on a sodium bicarbonate subphase.

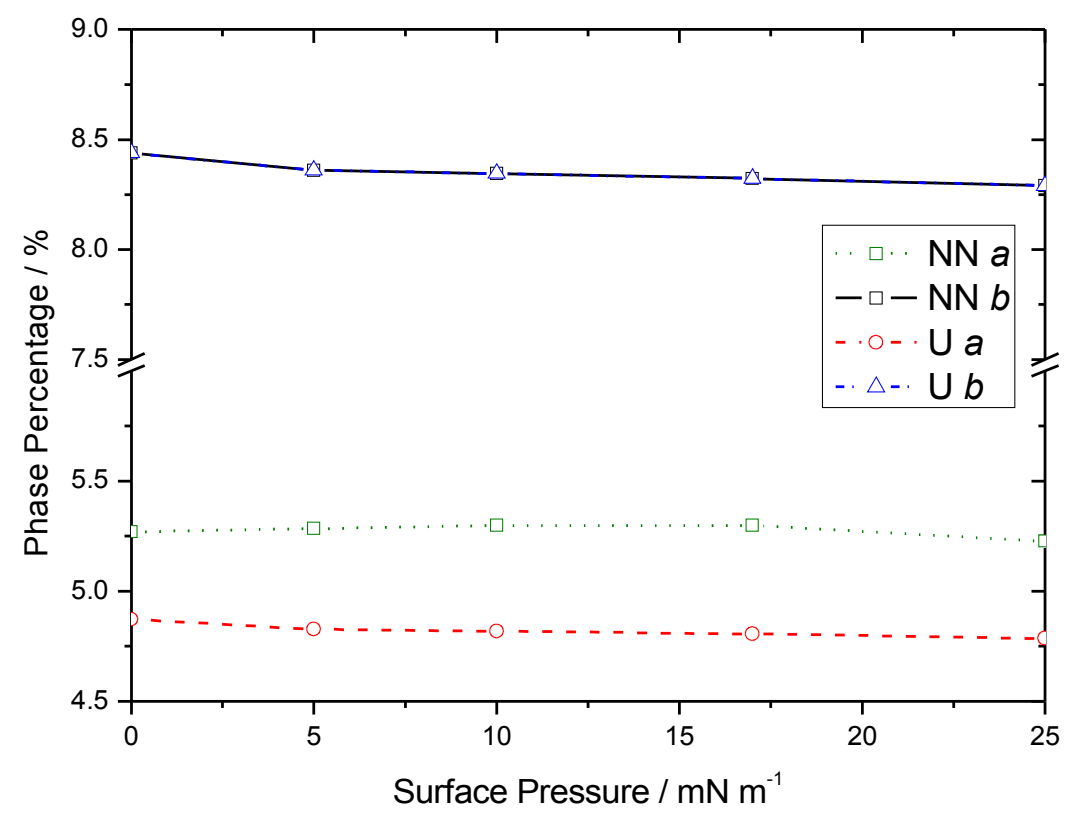

Figure 5.16 The variation of the lattice parameters with pressure. The $b$ lattice parameters coincide for the two phases. The lines are added to guide the eye. 
Between the pressures of 17 and $25 \mathrm{mN} \mathrm{m}^{-1}$ the isotherm shows an inflection and loss of contrast, consistent with a phase transition. This inflection is closely followed by brittle fracture collapse as shown in Figure 5.17. Given the already high percentage of untilted phase it is unclear what the inflection could indicate. The GIXD analysis performed at 17 and $25 \mathrm{mN} \mathrm{m}^{-1}$ includes both phenomena, inflection and collapse, therefore surmising the source of the inflection is not objective. At 17 $\mathrm{mN} \mathrm{m}^{-1}$ there is a weak but well defined peak associated with the $\mathrm{NN}$ tilted phase (Figure 5.18). However at $25 \mathrm{mN} \mathrm{m}^{-1}$ there is no clear out-of-plane peak, rather a very diffuse distribution of intensity across a large range of reciprocal space (Figure 5.19). Summing this data to a single $Q_{x y}$ profile produces two peaks in similar positions to those observed at lower pressures, however a small shift to higher $Q_{x y}$ was evident, accounting for some contraction of the cell with the increase in pressure. The important difference is the highly distributed nature of the out-of-plane diffraction. This leads to the proposition of a two step process, where the inflection is associated with the loss of tilt closely followed by fast collapse. The fast collapse is associated with extensive folding of the monolayer and it is this re-oriented surfactant that contributes to the diffuse scattering. BAM images (Figure 5.17) support this mechanism, with a complete loss of contrast at the inflection point implying a single untilted phase. The GIXD-based $A_{x y}$ decreases from $22.2 \AA^{2}$ at $\Pi=0$ $\mathrm{mN} \mathrm{m}^{-1}$ to $22.0 \AA^{2}$ at $\Pi=17 \mathrm{mN} \mathrm{m}^{-1}$, then from $\Pi=17$ to $25 \mathrm{mN} \mathrm{m}^{-1} \mathrm{~A}_{\text {xy }}$ drops from 22.0 to $21.7 \AA^{2}$. This decrease is statistically small but it does deviate from the very gradual trend shown at lower pressures. This drop is also consistent with the occurrence of the hypothesised phase transition. A comparison of the GIXD $A_{x y}$ and the isotherm-based $\mathrm{A}_{\mathrm{m}}\left(11.8 \AA^{2}\right)$ shows, much more strongly, the extent of collapse.

In isolation, the two-peak fit of the GIXD pattern indicates the continuation of the two-phase mixture, with a significant loss of order for the tilted phase but there remains a question as to whether the extent of folding is sufficient to account for the 
diffuse peak. Despite these issues the phase transition and collapse provides a better fit for all the results: GIXD, BAM and surface pressure.

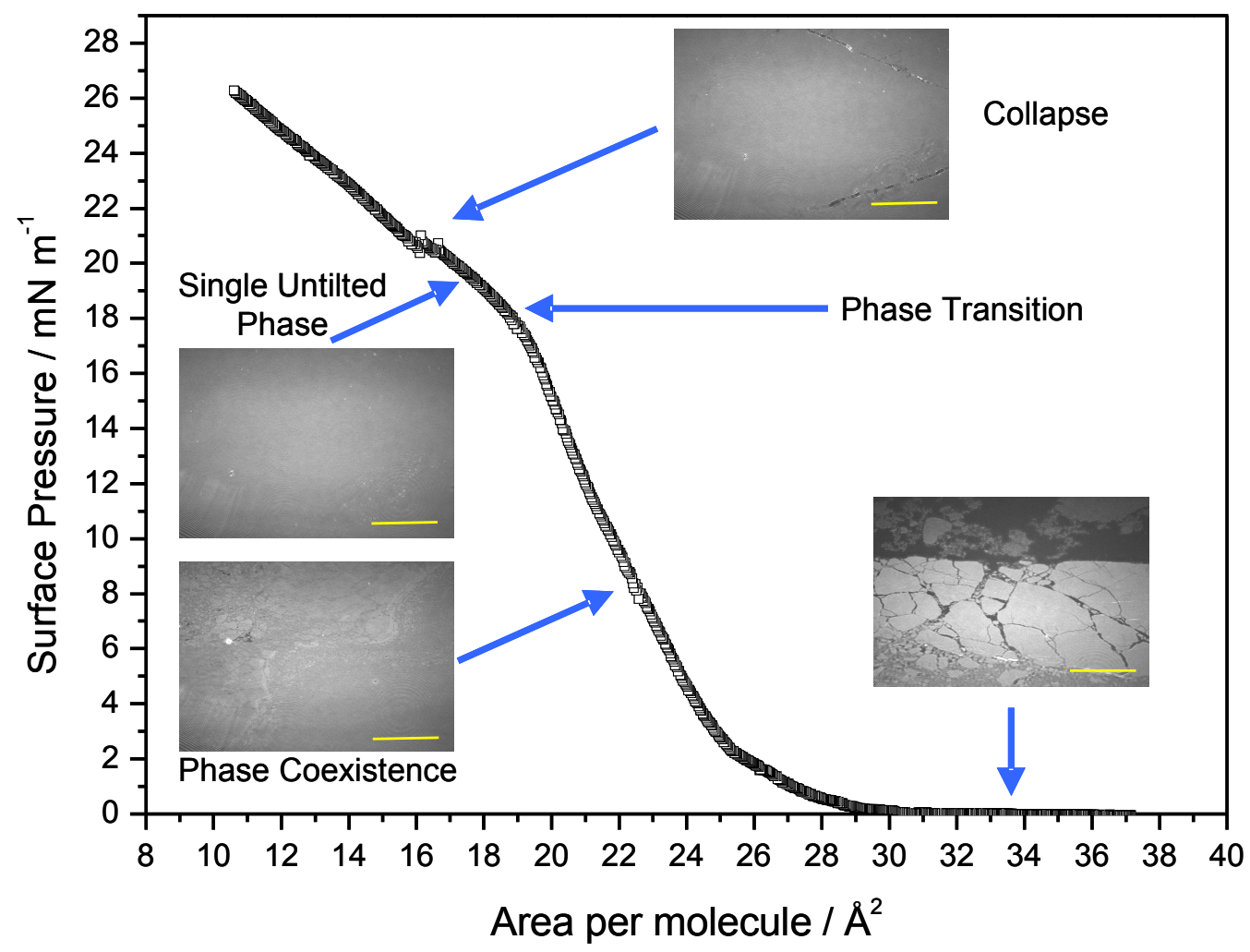

Figure 5.17 Isotherm of 2-HSA on a $\mathrm{NaHCO}_{3}$ subphase. Overlaid are BAM images illustrating the changes in monolayer structure that occur with the application of pressure. The first image at zero pressure, before onset, shows the phase coexistence that arises immediately on surfactant addition. As pressure is applied the relatively rigid domains come together to form a coherent monolayer. The second image shows the persistence of two phases, as indicated by the regions of differing contrast. The inflection in the isotherm is associated with a phase change and the loss of all phase contrast in the BAM images. Finally a small kink in the profile is associated with a brittle collapse event. The scale bar represents $1 \mathrm{~mm}$.

The measured surface potential peaked at $-30.6 \pm 12.0 \mathrm{mV}$; a significant deviation from that measured on any of the other subphases (Figure 5.8). In the mixed systems the surface potential on $\mathrm{NaHCO}_{3}$ was similar to that on water at $\sim 240$ $\mathrm{mV}$ for pure octadecanoic acid. The reduction from the undissociated ODA 
monolayer (at $\sim 400 \mathrm{mV}$ ) is attributed to structuring of subphase ions creating a potential that counteracted that of the monolayer. Here the negative potential suggests this subphase potential is greater than that of the monolayer. Understanding how this may occur requires consideration of the loss of surfactant from the monolayer. On the bicarbonate subphase the isotherm indicates the greatest amount of slow collapse and therefore the smallest $A_{m}$. Slow collapse is synonymous with the loss of film to a more stable 3-D phase via the formation of multi-layer islands[21]. The extent of the slow collapse can be approximated by comparing the average $A_{m}$ at collapse for the water and bicarbonate isotherms. This leads to a difference in $A_{m}$ of $\sim 5 \AA^{2}$, which equates to a loss of $20 \%$ or 1 in 5 of the molecules from the original monolayer. Given such a large number, it is reasonable to postulate that some combination of multi-layer formation and an enhanced double layer accounts for the surprisingly low surface potential.

The XRR data was found to be unreliable, the reflectivity profile was somewhat irregular and therefore, despite reasonable fits, the resulting electron density profiles at both pressures accounted for too few electrons.

In summary, the monolayer behaviour on sodium bicarbonate is consistent with a strong monolayer/subphase interaction. This is evident in the observation of a partially $(80 \%$ of the monolayer) untilted phase at all pressures investigated. However, the nature of this interaction is such that it promotes monolayer instability and therefore significant collapse. The overall behaviour of the system can be accounted for by the formation of a cation-mediated hydrogen-bonded network. For example, the monolayer instability, not observed for the mixed systems on $\mathrm{NaHCO}_{3}$, can be attributed to the increased propensity and strength of the monolayer/network hydrogen bonding brought about by the covalently bound hydroxyl group. 
(a)
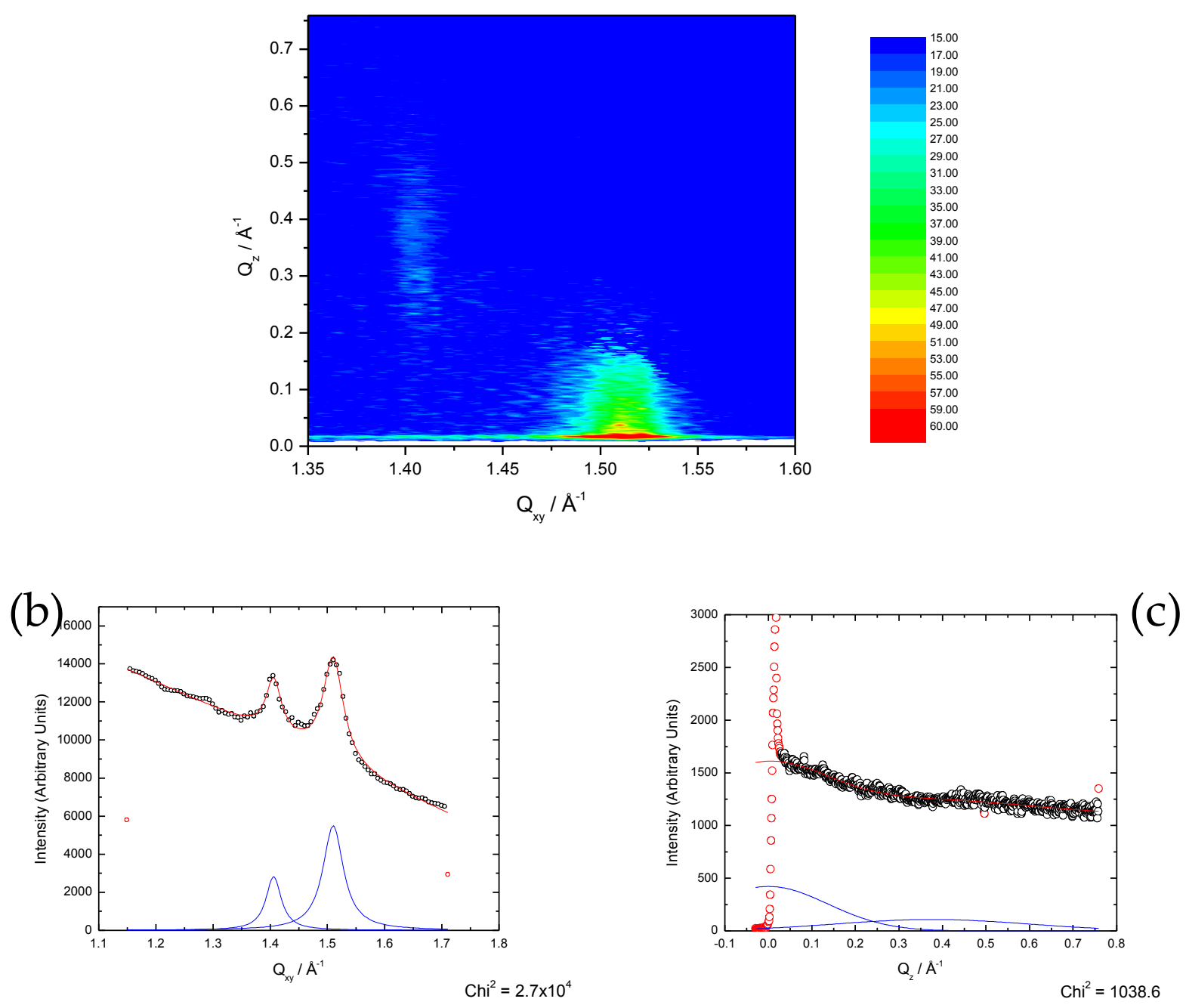

Figure 5.18 (a) 2-D contour plot of the GIXD diffraction pattern for a 2-HSA monolayer on a $\mathrm{NaHCO}_{3}$ subphase at a $\Pi=17 \mathrm{mN} \mathrm{m}^{-1}$. (b) The summed $Q_{x y}$ profile with a fitted 2-peak model. (c) The summed $Q_{z}$ profile also with a fitted 2-peak model. 
(a)
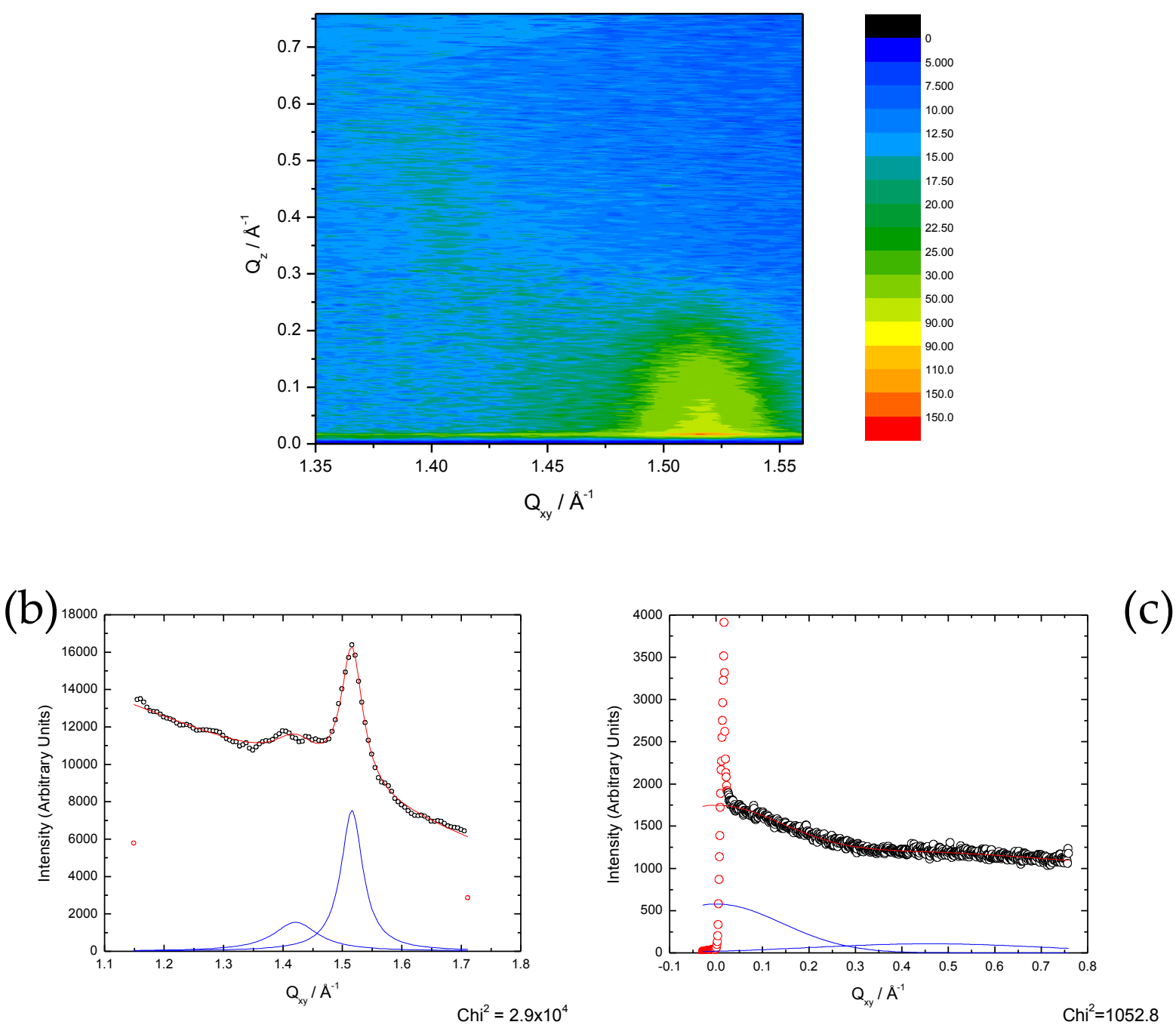

Figure 5.19 GIXD results for 2-HSA on $\mathrm{NaHCO}_{3}$ at $\Pi=25 \mathrm{mN} \mathrm{m}^{-1}$. (a) A contour plot of the diffraction pattern, interestingly diffuse scattering is evident at low $Q_{x y}$. (b) The summed $Q_{x y}$ profile with a two-peak model fit. (c) The equivalent summed $Q_{z}$ profile also with a two-peak model fit.

\subsubsection{Sodium Chloride}

The investigation of 2-HSA monolayers on sodium chloride was a cursory one aimed at confirming the role played by the bicarbonate anion. As such this discussion is limited to analysis of GIXD and XRR data.

At zero pressure there was no observable diffraction pattern suggesting that the 
subphase ions disturb the intramonolayer hydrogen bonding between the head groups. This observation highlights the role of the bicarbonate anion, which led to the presence of a partially untilted monolayer at $\Pi=0 \mathrm{mN} \mathrm{m}^{-1}$.

The application of pressure imposes significant order to the monolayer on $\mathrm{NaCl}$, with a classic $N N$ pattern observed at $\Pi=5 \mathrm{mN} \mathrm{m}^{-1}$ (Figure 5.20). There is no disorder as observed on water, and no two-phase coexistence, as the peak intensity ratio is consistent with a NN tilted phase. With increasing pressure the lattice parameters are reduced in-line with the reducing tilt angle. At $30 \mathrm{mN} \mathrm{m}^{-1}$ the tilt angle is reduced to $\sim 14^{\circ}$, from $\sim 29^{\circ}$ at $5 \mathrm{mN} \mathrm{m}^{-1}$. The gradual loss of tilt leads to a shift in the out-of-plane peak towards the in-plane peak.

Non-linear regression fitting of the XRR data at $\Pi=10$ and $25 \mathrm{mN} \mathrm{m}^{-1}$ resulted in realistic models. The models indicate a $C_{14}$ and $C_{15}$ hydrocarbon tails at low and high pressure, respectively, consistent with both the acid and hydroxyl groups being submerged in the liquid subphase. The fits at both pressures suggest extensive subphase electron density, with the best fit at $\Pi=25 \mathrm{mN} \mathrm{m}^{-1}$ consisting of four boxes, two of which were subphase related. Elucidation of this behaviour will require further experiments beyond the scope of this study.

In summary, 2-HSA on $\mathrm{NaCl}$ behaves significantly different from that on $\mathrm{NaHCO}_{3}$. Without external pressure $\mathrm{NaCl}$ is incapable of ordering the monolayer, in contrast to the high percentage of untilted phase present on $\mathrm{NaHCO}_{3}$. For pressures of $5 \mathrm{mN} \mathrm{m}^{-1}$ and above, the monolayer is much more ordered on $\mathrm{NaCl}$ than the equivalent $\mathrm{NaHCO}_{3}$ and water systems, highlighting the role of the sodium cation. This leads to the proposal that the disordering and multi-phase behaviour observed for water and $\mathrm{NaHCO}_{3}$ correlates to the propensity of the system to hydrogen bonding. The presence of a dominating cation (where $\mathrm{Ca}^{2+}$ is much more effective than $\mathrm{Na}^{+}$) appears to disrupt this hydrogen bonding, allowing electrostatics to dictate resulting in condensation and greater packing order. 
(a)

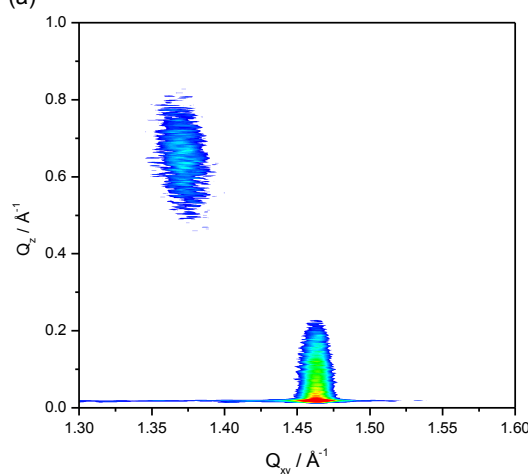

(c)

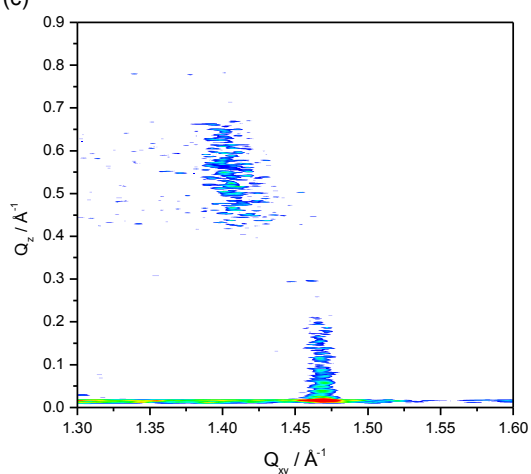

(e)

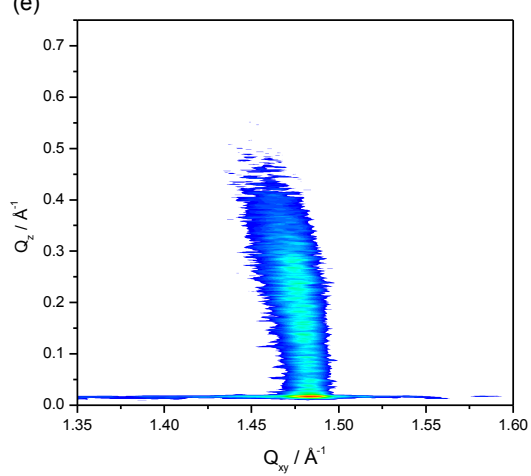

(b)
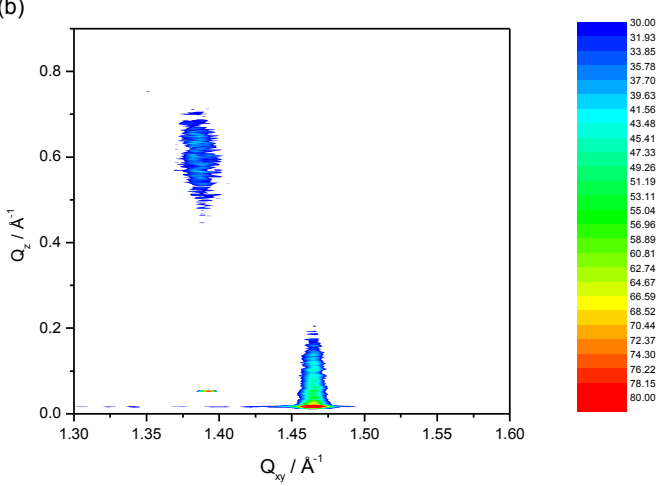

(d)
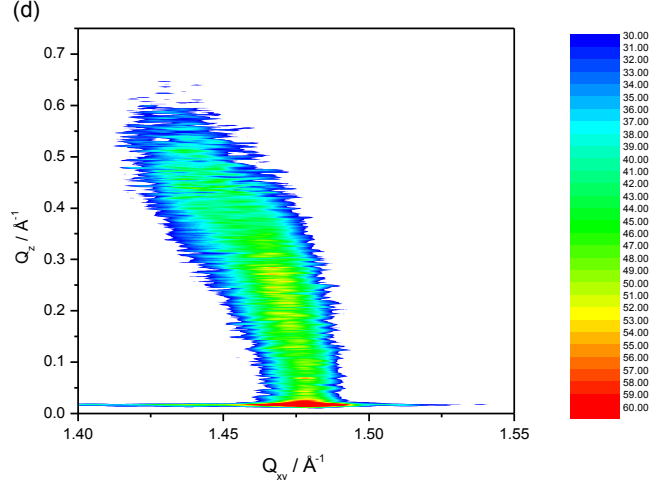

Figure 5.20 2-D contour plots illustrating the behaviour of 2-HSA on a $\mathrm{NaCl}$ subphase at different surface pressures: (a) 5, (b) 10, (c) 17, (d) 25, and (e) $30 \mathrm{mN} \mathrm{m}^{-1}$.

\subsubsection{Calcium Carbonate Crystallising Subphase (CCCS)}

The shift to CCCS based systems, containing both sodium bicarbonate and calcium chloride, results in intermediate properties for all the isotherm traits monitored. However the behaviour is weighted towards that of $\mathrm{CaCl}_{2}$, suggesting a dominance of cation-initiated electrostatic effects. 
As with the $\mathrm{CaCl}_{2}$ system, the monolayer is comprised of two coexisting phases from the outset (Figure 5.21). BAM images show the monolayer islands to be smaller than those observed on $\mathrm{CaCl}_{2}$, with a size range of 0.1 to $1.0 \mathrm{~mm}$, the majority being below $\sim 0.4 \mathrm{~mm}$. The islands, based on BAM contrast, are a mix of single and multidomain agglomerations. In general the smaller islands were single and the larger multi-domains, but this distinction was not universal. This compares with a GIXD derived domain correlation length of $130-200 \AA$. Given that the monolayer is untilted, the domain contrast observed with BAM is likely attributed to chiral-based discrimination. Therefore the disparity of the domain size may be related to a degree of chiral-based phase separation.

A comparison with $\mathrm{CaCl}_{2}$ systems, shows the lattice parameters and therefore the $A_{m}$ to be very similar. Thus on a molecular level at least, the bicarbonate anion is not impacting on the packing in quite the same manner as for the mixed systems. In the 2-HSA system, the strength of the cation binding appears to dominate the interfacial interaction.

This is again evident in BAM analysis of the collapse mechanism. Small localised bright spots consistent with the observation of surfactant solidification were observed, equivalent to collapse on $\mathrm{CaCl}_{2}$.

On CCCS the surface potential profile is similar to that observed on water (Figure 5.8). The presence of the cation appears to stabilise the bicarbonate interaction such that the monolayer is relatively stable. The reduced rigidity of the islands means that all gaseous domains are collapsed during the early stages of compression hence there is no rise in the potential profile with compression. Rather, as with water, the deviation from the plateau is negative associated with the initiation of collapse. The reduction in the magnitude of the maximum potential compared to water reflects the opposing potential of the subphase ions in the double layer .

The XRR data is again ambiguous, with the best fitting models exhibiting unusual 


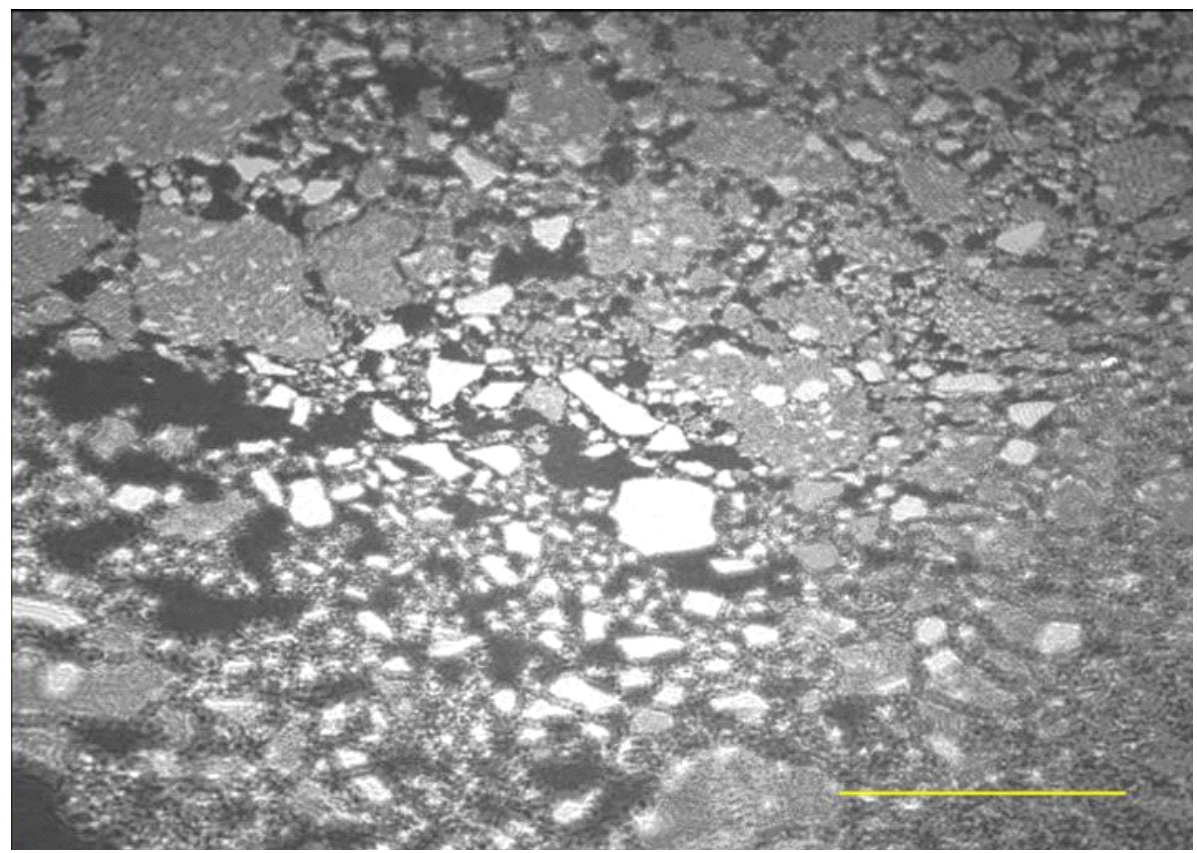

Figure 5.21 A BAM image of the phase coexistence that occurs for 2-HSA on CCCS immediately upon addition of the surfactant. The scale bar represents $1 \mathrm{~mm}$.

electron density profiles. Further there is very little in the way of subphase based electron density in a system where there should be the greatest. The difficulties in both the bicarbonate and CCCS based systems lies in the long collection times, the monolayers are simply not sufficiently stable.

Generally the behaviour of 2-HSA on CCCS is characterised by the domination of electrostatics. This effect is much greater than that observed for pure ODA, where there was a greater difference between $\mathrm{CaCl}_{2}$ and CCCS attributed to the bicarbonate. In the mixed systems, the introduction of the bicarbonate facilitated a significant condensation of the monolayer beyond that observed for the $\mathrm{CaCl}_{2}$. This does not occur for 2-HSA, which could be attributed to the larger head group and the strong Ca-binding locking in a spatially costly conformation. Thus the introduction of a strong five-membered chelation ring has disrupted the bicarbonate effect and therefore the formation of a cation-mediated hydrogen-bonded network. This is the key difference between covalently and intermolecularly bound hydroxyl groups, in the equivalent 50:50 mixed system the calcium binding was not as restrictive as the 2- 
HSA-calcium bond.

\subsubsection{Cation Mediated Hydrogen Bonding Network}

Reviewing the behaviour (see Table 5 for a summary of the GIXD data) of the 2-HSA monolayers on the different subphases presents two paradigms. The first on water and $\mathrm{NaHCO}_{3}$, reflects a hydrogen-bonding dominated system. On water the intra-monolayer hydrogen bonding leads to significant ordering of the monolayer. In contrast, on $\mathrm{NaHCO}_{3}$ hydrogen bonding is facilitated via a cation mediated network. In both cases the propensity for hydrogen bonding is greater than that observed for the equivalent mixed ODA/ODOH systems indicating the hydroxyl group as a key factor. The influence of the covalently bound hydroxyl group appears to be achieved through associated steric effects and the participatory capacity of the functional group.

In contrast, the $\mathrm{CaCl}_{2}$ and CCCS systems are dominated by ion-ion and ion-dipole electrostatic interactions. As observed in the mixed systems these electrostatic interactions dominate any hydrogen bonding activity. In fact for 2-HSA the electrostatic effect is so strong that it appears to eliminate hydrogen bonding, as suggested by the complete lack of a bicarbonate effect in the CCCS system.

In both cases the covalently bound hydroxyl group enhances the monolayer/subphase interactions whether they are electrostatically or hydrogenbonding dominated. This is achieved through a combination of induction and steric effects, the precise nature of which requires techniques such as infra-red and Raman spectroscopy to provide further insight. 
Table 5 A summary of the parameters of the different 2-HSA monolayers as determined by GIXD.

\begin{tabular}{|c|c|c|c|c|c|c|c|}
\hline Subphase & $\begin{array}{l}\text { No. of } \\
\text { Peaks }\end{array}$ & Symmetry & $\begin{array}{l}\mathbf{A}_{x y} \\
\left(\AA^{2}\right)\end{array}$ & $\Delta \mathbf{A}_{x y}$ & $\begin{array}{l}\text { Tilt Angle } \\
\text { (t) }\end{array}$ & $\Delta \mathrm{t}$ & $\begin{array}{c}\text { Tilt } \\
\text { Azimuth }\end{array}$ \\
\hline Water & 3 & $\begin{array}{l}\text { Rect. } \\
\text { Rect. } \\
\text { Hex. }\end{array}$ & $\begin{array}{l}22.9 \\
21.5 \\
22.6\end{array}$ & $\begin{array}{l}\downarrow \\
\downarrow \\
\downarrow\end{array}$ & $\begin{array}{c}26.1 \\
22.6 \\
-\end{array}$ & $\begin{array}{l}\downarrow \\
\downarrow \\
-\end{array}$ & $\begin{array}{c}\mathrm{NN} \\
\mathrm{NNN} \\
\mathrm{U}\end{array}$ \\
\hline $\mathrm{CaCl}_{2}$ & 1 & Hex. & 19.9 & $\downarrow$ & - & - & $\mathrm{U}$ \\
\hline $\mathrm{NaHCO}_{3}$ & 2 & $\begin{array}{l}\text { Rect. } \\
\text { Hex. }\end{array}$ & $\begin{array}{l}21.7 \\
19.8\end{array}$ & $\begin{array}{c}\text { Const. } \\
\downarrow\end{array}$ & $\begin{array}{c}21.0 \\
-\end{array}$ & $\begin{array}{c}\text { Var } \\
-\end{array}$ & $\begin{array}{c}\mathrm{NN} \\
\mathrm{U}\end{array}$ \\
\hline $\mathrm{NaCl}$ & 2 & Rect. & 21.6 & $\downarrow$ & 20.5 & $\downarrow$ & NN \\
\hline CCCS & 1 & Hex. & 19.9 & $\downarrow$ & - & - & $\mathrm{U}$ \\
\hline
\end{tabular}

( $A_{x y}$ and the tilt angle values represent the performance at a pressure of $25 \mathrm{mN} \mathrm{m}^{-1}$. The $A_{x y}$ and $t$ for cases where there are multiple phases is calculated as a weighted average. $\Delta A_{x y}$ reflects how $A_{x y}$ trends with increasing pressure, similar applies for $\Delta t$. Rect. = centred rectangular symmetry and Hex. = hexagonal symmetry. $\downarrow=$ decreases, Var = variable, and const. = reflects negligible change with pressure).

\subsection{Conclusions}

The interactions between 2-HSA monolayers and the subphase is a complex balance of hydrogen bonding, electrostatics, steric effects and entropy. The incorporation of the hydroxyl group into the acid molecule leads to greater enhancement of the hydrogen bonding or electrostatics associated behaviour traits compared to the equivalent mixed ODA/ODOH systems.

On water the absence of subphase ions results in a domination of hydrogen bonding. Thus the head group is largely associated with a conformation change to facilitate hydrogen bonding. The result is a tail-head group size mismatch which leads to an entropically driven disordering of the tail tilt direction. Hence the monolayer consists of three phases: tilted NN and NNN phases and an untilted phase.

Similarly on $\mathrm{NaHCO}_{3}$, hydrogen bonding dominates. However, the formation of a cation mediated network results in hydrogen bonding between the monolayer and 
network as opposed to the intramonolayer-based bonding on water. Network formation is also hypothesised to increase ordering, reflected in the observation of two phases: NN and an untilted phase.

The situation on calcium containing subphases is significantly different. The formation of a favoured five-membered chelation ring with calcium ions dominates the interfacial interaction. Monolayer domains become rigid, tilt is eliminated and hexagonal packing is observed. There is little evidence for the formation of a partially ordered network at the interface with the absence of an observable difference between the $\mathrm{CaCl}_{2}$ and $\mathrm{CaCO}_{3}$ systems that can be directly attributed to the bicarbonate anion.

\subsection{References}

[1] X. Wang, "A theory for the mechanism of action of the $\alpha$-hydroxy acids applied to the skin," Medical Hypotheses, vol. 53, Nov. 1999, pp. 380-382.

[2] B. Kellner and D. Cadenhead, "Monolayer studies of hydroxyhexadecanoic acids," Journal of Colloid and Interface Science, vol. 63, Mar. 1978, pp. 452-460.

[3] G. Weidemann, G. Brezesinski, D. Vollhardt, C. DeWolf, and H. Möhwald, "Disorder in Langmuir Monolayers: 2. Relation between Disordered Alkyl Chain Packing and the Loss of Long-Range Tilt Orientational Order," Langmuir, vol. 15, Apr. 1999, pp. 2901-2910.

[4] D. Vollhardt, S. Siegel, and D. Cadenhead, "Characteristic Features of Hydroxystearic Acid Monolayers at the Air/Water Interface," Journal of Physical Chemistry B, vol. 108, Nov. 2004, pp. 17448-17456.

[5] V. Neumann, A. Gericke, and H. Hühnerfuss, "Comparison of Enantomeric and Racemic Monolayers of 2-Hydroxyhexadecanoic Acid by External Infrared Reflection-Absorption Spectroscopy," Langmuir, vol. 11, 1995, pp. 2206-2212.

[6] G. Weidemann, G. Brezesinski, D. Vollhardt, and H. Möhwald, "Disorder in Langmuir Monolayers. 1. Disordered Packing of Alkyl Chains," Langmuir, vol. 14, Oct. 1998, pp. 6485-6492.

[7] A. Dhathathreyan, "Dissociation constants of long-chain hydroxy fatty acids in Langmuir-Blodgett films," Colloids and Surfaces A: Physicochemical and Engineering Aspects, vol. 318, Apr. 2008, pp. 307-314.

[8] D. Vollhardt, S. Siegel, and D. Cadenhead, "Effect of Hydroxyl Group Position and System Parameters on the Features of Hydroxystearic Acid Monolayers," Langmuir, vol. 20, 2004, pp. 7670-7677. 
[9] S. Siegel, D. Vollhardt, and D. Cadenhead, "Effect of the Hydroxy Group Position on the Monolayer Characteristics of Hydroxypalmitic Acids," Colloids and Surfaces A: Physicochemical and Engineering Aspects, vol. 256, 2005, pp. 9-15.

[10] D. Vollhardt and V. Fainerman, "Effect of the Hydroxyl Group Position on the Phase Behavior of Hydroxyoctadecanoic Acid Monolayers at the Air/Water Interface," Journal of Physical Chemistry B, vol. 108, Jan. 2004, pp. 297-302.

[11] M. Yazdanian, H. Yu, and G. Zografi, "Ionic Interactions of Fatty Acid Monolayers at the Air/Water Interface," Langmuir, vol. 6, 1990, pp. 1093-1098.

[12] V. Kaganer, H. Möhwald, and P. Dutta, "Structure and Phase Transitions in Langmuir Monolayers," Reviews of Modern Physics, vol. 71, 1999, pp. 779-819.

[13] I. Kuzmenko, H. Rapaport, K. Kjaer, J. Als-Nielsen, I. Weissbuch, M. Lahav, and L. Leiserowitz, "Design and Characterization of Crystalline Thin Film Architectures at the Air-Liquid Interface: Simplicity to Complexity," Chemical Reviews, vol. 101, 2001, pp. 1659-1696.

[14] J. Als-Nielsen, D. Jacquemain, K. Kjaer, F. Leveiller, M. Lahav, and L. Leiserowitz, "Principles and applications of grazing incidence $X$-ray and neutron scattering from ordered molecular monolayers at the air-water interface," Physics Reports, vol. 246, Oct. 1994, pp. 251-313.

[15] L. Wiegart and B. Struth, "Geometric boundary condition for the chain alignment in lipid monolayers," Physica B: Condensed Matter, vol. 357, Feb. 2005, pp. 126-129.

[16] L. Cristofolini, M. Fontana, C. Boga, and O. Konovalov, "Microscopic Structure of Crystalline Langmuir Monolayers of Hydroxysteric Acids by X-ray Reflectivity and GID: OH Group Position and Dimensionality Effect," Langmuir, vol. 21, 2005, pp. 11213-11219.

[17] B. Lin, M. Meron, J. Gebhardt, T. Graber, M.L. Schlossman, and P.J. Viccaro, "The liquid surface/interface spectrometer at ChemMatCARS synchrotron facility at the Advanced Photon Source," Physica B: Condensed Matter, vol. 336, Aug. 2003, pp. 75-80.

[18] S.M. Danauskas, D. Li, M. Meron, B. Lin, and K.Y.C. Lee, "Stochastic fitting of specular X-ray reflectivity data using \it StochFit," Journal of Applied Crystallography, vol. 41, Dec. 2008, pp. 1187-1193.

[19] R. Smith and A. Martell, Critical Stability Constants Vol. 6: Second Supplement, Plenum, 2005.

[20] F.P.J. Dwyer, Chelating Agents and Metal Chelates, New York: Academic Press, 1964.

[21] C. Ybert, W. Lu, G. Moller, and C.M. Knobler, "Collapse of a Monolayer by Three Mechanisms," The Journal of Physical Chemistry B, vol. 106, Feb. 2002, pp. 2004-2008. 


\section{Chapter 6: Calcite Crystallisation Under 2-HSA MONOLAYERS}

\subsection{Introduction}

Advancing our understanding of the molecular recognition processes fundamental to Nature's ability to achieve oriented inorganic growth in biomineralisation is the aim of this thesis. To this end, in this chapter we describe synchrotron-based experiments that add significantly to the corpus of knowledge on the nature of the interfacial interaction.

For the first time, rearrangement of the monolayer in response to inorganic nucleation at the interface has been observed in situ and in real-time. Additionally, using GIXD we have observed an intermediary state that is intimately associated with the monolayer and coincides with intense Bragg peaks in reciprocal space. This time-resolved study culminated in the observation of intense Bragg peaks correlating to the formation of calcite crystals.

Crystallisation under monolayers of 2-HSA provides an avenue for further investigation of the role of the alcohol group found to be significant in the mixed monolayer study in Chapters 3 and 4 . In Chapter 5 it was shown that the covalently bound alcohol group led to the formation of strong five-membered chelates with subphase calcium ions. Here we will show how this very strong binding influences the information transfer processes across the interface and therefore the subsequent calcium carbonate crystallisation. Furthermore, the strong electrostatic interaction at the interface allows an assessment of the role electrostatics has in FSN, in comparison to lattice matching, which should be compromised by the larger head group. 
Previous X-ray scattering investigations of calcium carbonate nucleation and growth under straight chain fatty acids[1-4] have sought to investigate the concepts of templated growth. Neither DiMasi et al.[3] or Kmetko[2] observed evidence for epitaxial growth, in fact Kmetko concluded that the electrostatic nature of the interaction was insufficient to observe using these techniques. However more recently studies[1,5] have begun to progress the field but much remains to be elucidated.

\subsection{Experimental}

The methodologies employed in this chapter have been previously described (Chapters 2 \& 5). However, two subtle, but critical modifications, were made when performing the GIXD experiments. Firstly, the position sensitive detector was change to a pinhole geometry. This facilitated faster data collection reducing the standard 10 minute scans to 3 minutes. This led to significantly improved monitoring of the dynamic monolayer and subphase. The second modification was procedural. In order to speed up nucleation and growth, the helium-filled chamber that housed the trough was opened for 30 to 60 minutes. It was believed that previous XRR experiments had failed to observe crystallisation because the $\mathrm{CO}_{2}$ degassing of the chamber was too slow. The opening of the chamber for a somewhat arbitrary period was found to rapidly advance nucleation and growth.

During this discussion the term 'monolayer' will refer to the surfactant monolayer, the term 'film' will be used solely to refer to the intermediate calcium carbonate film and 'crystal' will be used in reference to the calcium carbonate crystals.

\subsection{Results}

Studying crystallisation under Langmuir monolayers is extremely difficult, not only is there the complexity associated with probing a monomolecular interfacial 
interaction but the system is dynamic and heterogeneous. Beginning with the monolayer, in this section we will describe and discuss the in situ and ex situ examination of this interfacial interaction.

\subsubsection{Monolayer}

In order to provide a comparison with the mixed ODA/ODOH systems, crystals were grown under 2-HSA monolayers at two pressures, $\Pi=10$ and $25 \mathrm{mN} \mathrm{m}^{-1}$. While no structural changes arise between these two pressures, as the 2-HSA monolayers exhibit untilted hexagonal phases at all pressures, unit cell compression is evident. Moreover, in terms of monolayer behaviour, these pressures fall in the region of steepest isotherm ascent where the balance between the kinetics of collapse and the rate of compression is marginally in favour of compression. Consequently at these pressures there is evidence of collapse and monolayer instability, manifested as frequent large sudden drops in area. Due to the monolayer being very rigid, the effusion of carbon dioxide appears to involve the sudden release of large bubbles causing a sudden fracture and subsequent collapse of the monolayer. This perturbation is greatest at the higher pressure of $25 \mathrm{mN} \mathrm{m}^{-1}$. Aside from these abrupt drops in area, the monolayer was comparatively stable, in contrast to the 1:1 mixed ODA/ODOH monolayer.

In contrast to the mixed ODA/ODOH systems, where monolayer stability correlated with alcohol content and was the result of an increase in the monolayer equilibrium spreading pressure, here the stability of the monolayer (aside form the effervescence-induced collapse) arises from the strong, alcohol (hydroxyl)-facilitated, calcium binding. Therefore, the alcohol or hydroxyl group continues to play a significant role, however the mode of influence has changed reflecting the shift from intermolecular bonding to intramolecular covalent bonding between the acid and alcohol functional groups.

One manifestation of this difference is the disparity between the GIXD- 
determined molecular $A_{m}$ and the isotherm-determined physical $A_{m}$. GIXD measurements return an area per molecule of 20.2 and $19.9 \AA^{2}$ for $\Pi=10$ and $25 \mathrm{mN}$ $\mathrm{m}^{-1}$, respectively. In contrast, the isotherm-based $A_{m}$ is significantly different with values of 24.1 and $18.7 \AA^{2}$ for $\Pi=10$ and $25 \mathrm{mN} \mathrm{m}^{-1}$, respectively. This discrepancy points to important differences in how the monolayer is presented to any crystal nuclei. At the lower pressure, the larger $A_{m}$ reflects the heterogeneity of the monolayer, where areas of gas phase or absence of surfactant, brought about by the incomplete packing of the rigid surfactant islands remain. In contrast, the higher pressure monolayer presents a complete surface. However the small $\mathrm{A}_{\mathrm{m}}$ points to the expulsion of surfactant forming 3-D structures. It is not clear how these topographical features would specifically impact on nucleation and growth of calcium carbonate but these high energy features (island edges and 3-D precipitates) are likely to promote nucleation. The evidence for such structures was weak, a comparison of GIXD peak profiles for 2-HSA on $\mathrm{CaCl}_{2}$ and CCCS pointed to a subtle loss of order in the latter (Figure 6.1). The $Q_{x y}$ profiles (integrated over $Q_{z}$ ) show some broadening of the CCCS peak, consistent with a reduction in long-range order. The $Q_{z}$ profile (integrated over $Q_{x y}$ ) reflects the extension of the Bragg rod for the full $Q_{z}$ range investigated. A post-experiment examination of the monolayer provided further proof of partial collapse, with an accumulation of the surfactant aggregates at the moving barrier arm visible by eye.

In order to elucidate the nature of the dynamic monolayer/subphase interaction in situ real-time GIXD measurement were performed on the $\Pi=10 \mathrm{mN} \mathrm{m}^{-1}$ system. Figure 6.2 shows the in-plane diffraction peak(s) for the monolayer at intervals throughout the induction, nucleation and growth processes of calcium carbonate formation. The initial scattering pattern of the monolayer, on a $10 \mathrm{mM} \mathrm{CCCS}$ at a $\mathrm{pH}$ $\sim 5.8$, shows a single in-plane diffraction peak, consistent with an untilted hexagonal phase. Lorentzian peak fitting gives a peak position of $1.496 \AA^{-1}$, correlating to an 

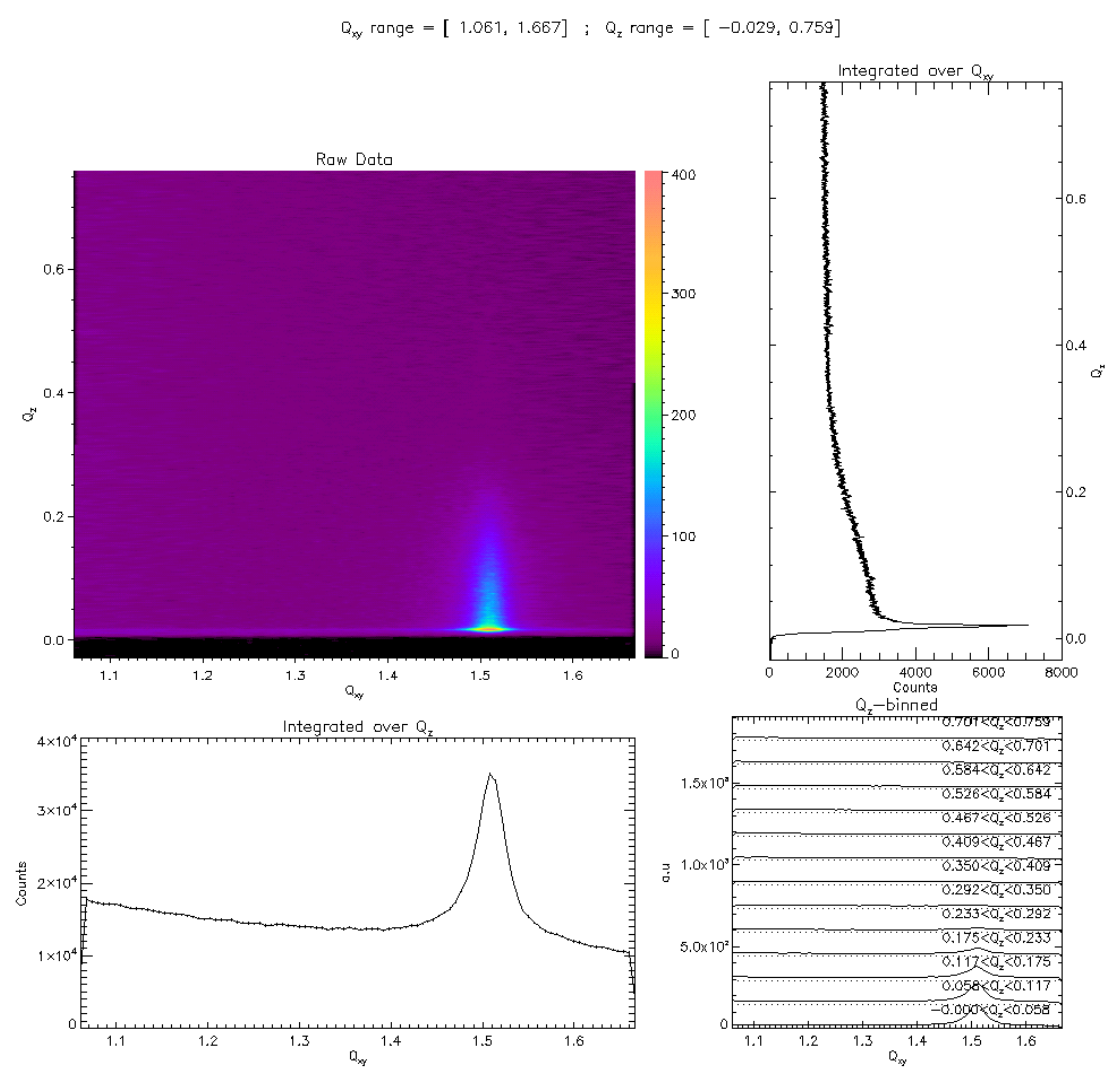

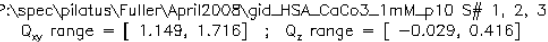
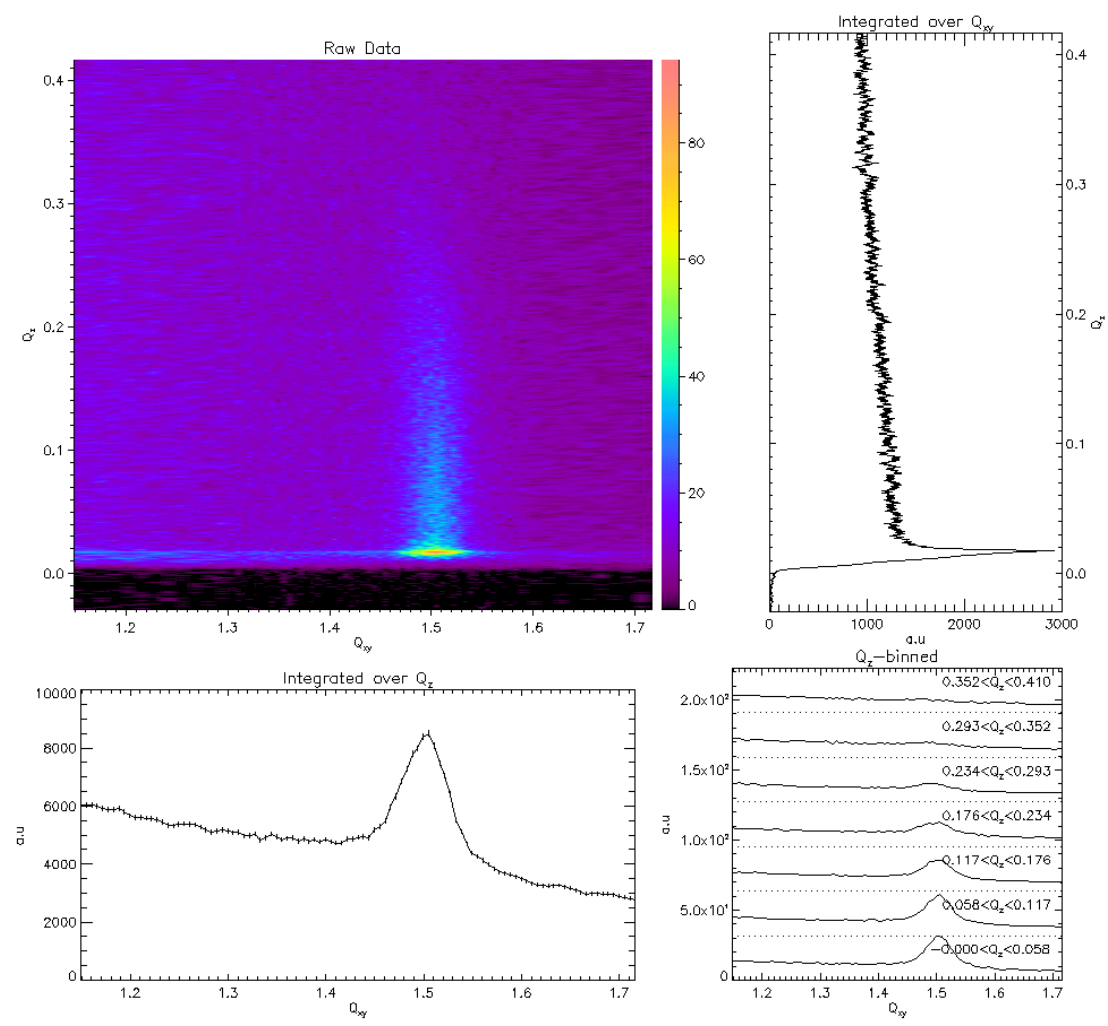

Figure 6.1 GIXD data for 2-HSA on $\mathrm{CaCl}_{2}$ (top) and CCCS (bottom). A comparison of the two systems shows a subtle loss of order and an extension in $Q_{z}$ for CCCS. 
equivalent centred rectangular cell with lattice parameters of $a=4.85 \AA$ and $b=8.40$ $\AA$, and an average molecular area of $20.37 \AA^{2} /$ molecule. The full width at half maximum (FWHM) indicates a correlation length in the order of $60 \pm 15 \AA$. The $Q_{z}$ dependence of the peak intensity is consistent with scattering from a layer of thickness $(2 \pi / 0.27) \approx 23 \AA$, and therefore can be attributed to the monolayer.

To facilitate calcium carbonate crystallisation the helium filled trough enclosure was opened to accelerate the carbon dioxide degassing of the subphase. After being open for 0.5 - 1.0 hour, and subsequent flushing with helium the monolayer was reexamined. The onset of nucleation (precipitation) is indicated by a gradual splitting of the triply degenerate hexagonal diffraction peak, as shown in Figure 6.2. Within one hour, two distinct monolayer peaks at $Q_{x y}=1.503$ and $1.626 \AA^{-1}$ are evident, consistent with two hexagonal phases. These peak positions give lattice parameters of $a=4.83 \AA(b=8.361 \AA)$, and $a=7.73 \AA(b=7.728 \AA)$, with the average molecular area of 20.2 and $17.2 \AA^{2} /$ molecule, respectively.

Both peaks are in-plane, however the higher $Q_{x y}$ peak cannot be fully characterised due to an overlapping broad band (vide infra). However, the peak appears to consist of a peak and a broad uniform band. This is confirmed in the $Q_{z}$ profile which shows the intense in-plane peak to have a $Q_{z}$ intensity dependence commensurate with a monolayer peak. That is the intensity in the $Q_{z}$ direction falls to a baseline level, associated with the broad band, at $Q_{z} \approx 0.25 \mathrm{~A}^{-1}$ consistent with scattering from a layer of thickness $(2 \pi / 0.25) \approx 25 \AA$.

Figure 6.3 illustrates the change in peak intensity and position with crystal growth time. Integrated intensity shows the new peak to immediately have a greater intensity than that of the original monolayer peak. The intensity ratio of the two peaks increases in favour of the new high $Q_{x y}$ peak, consistent with increasing nucleation, and very quickly exceeds the typical 2:1 ratio for a centred rectangular phase. Combined with the expected heterogeneity of the nucleation events, the 

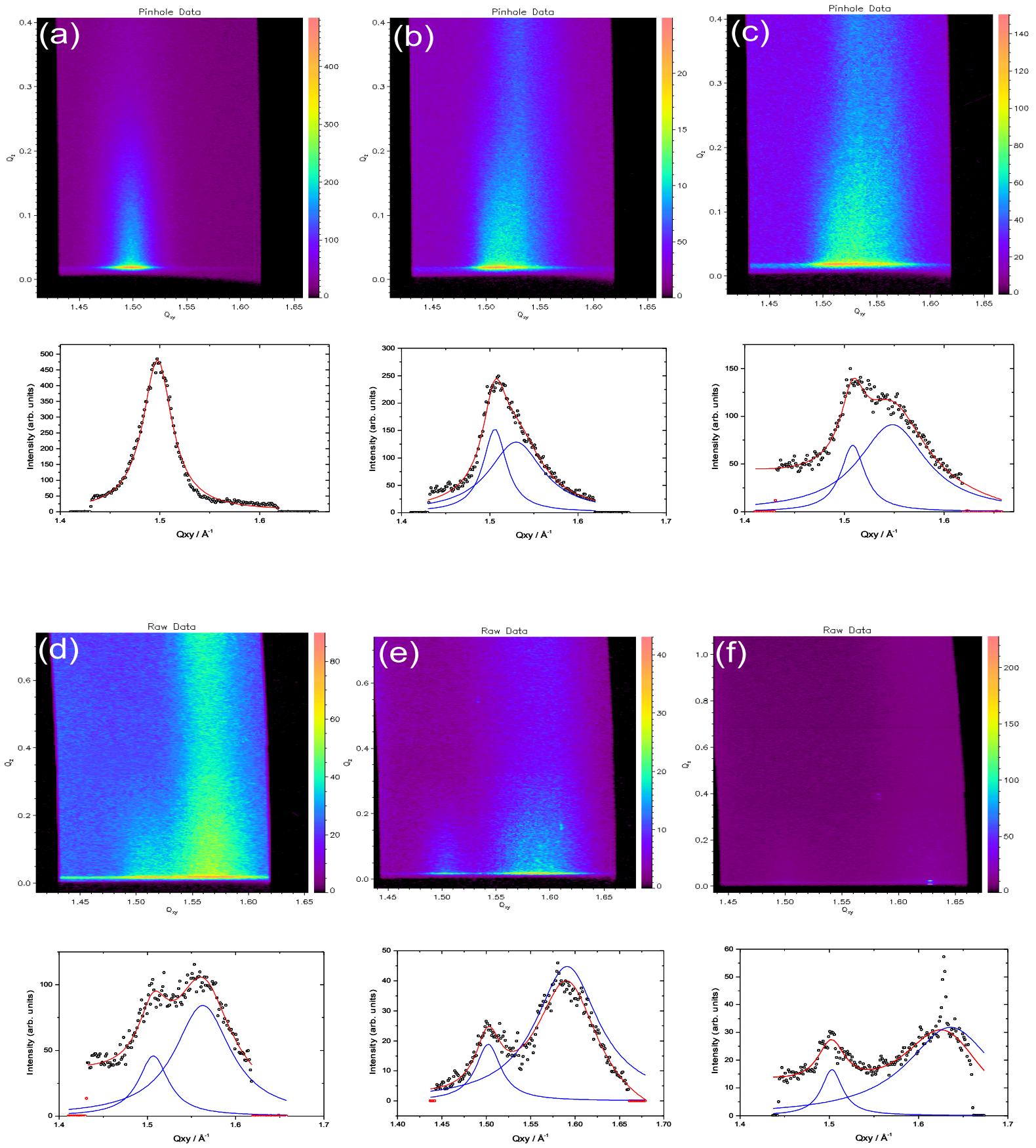

Figure 6.2 Monolayer rearrangement in response to subphase nucleation and growth events. The solid lines reflect the fitting of Lorentzian peaks to the scattered experimental data. The sequence of images (a) to (f) represents $\sim 1-1.5$ hours in experimental time, culminating in very small but intense Bragg spots in (f).

intensity data supports the proposition of two coexisting hexagonal phases. The two phases represent domains with (high $Q_{x y}$ ) and without (low $Q_{x y}$ ) interacting nuclei.

Originating at a common peak position the higher $Q_{x y}$ peak is shifted significantly. 


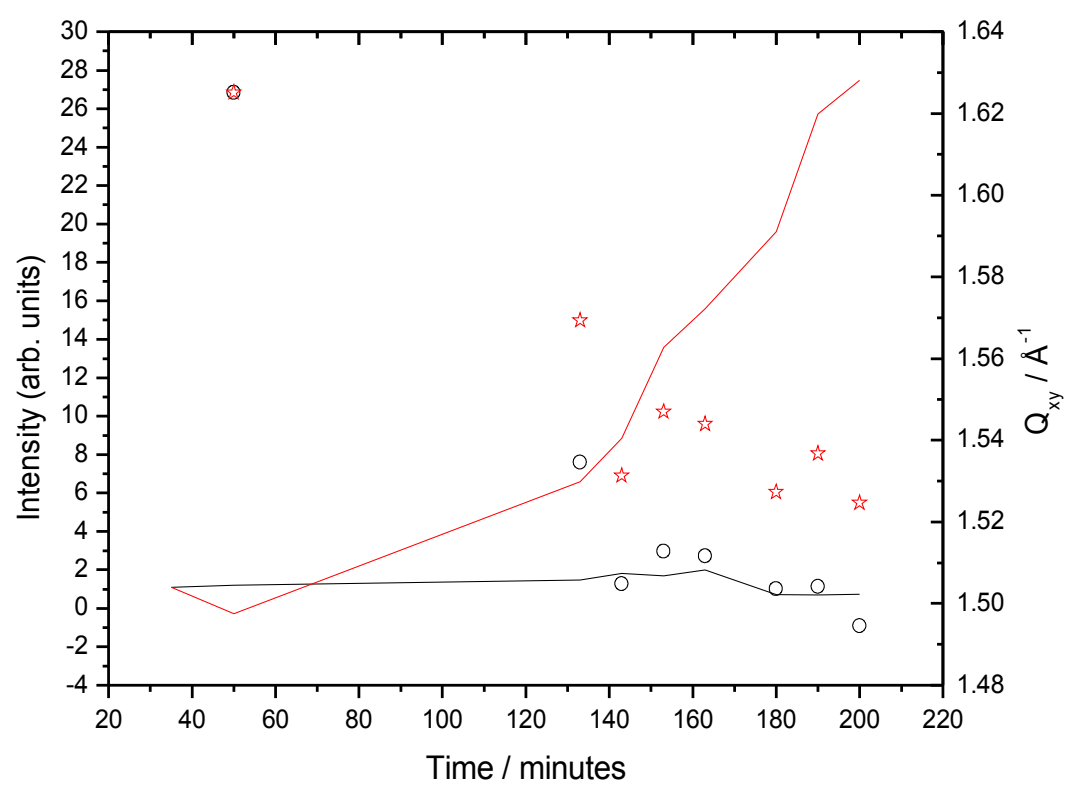

Figure 6.3 The change in peak properties with increasing crystallisation time. The symbols plot peak intensity variation and the solid lines plot the shift in $Q_{x y}$. (Note the large gap in the data between $T \sim 50$ and $T$ $\sim 130$ mins corresponds to the period the trough was opened to induce crystallisation.)

This movement of the second peak to higher $\mathrm{Q}_{\mathrm{xy}}$ corresponds to a 7.5\% reduction in the lattice parameters or a $14.5 \%$ reduction in $A_{m}$ as nucleation proceeds. The correspondingly small lattice reflecting significant condensation of the monolayer brought about by nucleation of an associated phase. In contrast, the lower $Q_{x y}$ peak remains effectively stationary (fluctuating about an average $Q_{x y}$ of $1.50 \AA^{-1}$ ) throughout the total data collection time.

The proposition of two hexagonal phases contrasts with the literature[3,4] which, based on static, 'before and after' studies had associated crystallisation with a centred rectangular lattice. In a study investigating crystal growth under C18 and C20 fatty acid films, DiMasi et al.[3] observed two lattice peaks which contrasted with a previously reported single peak. Based on the assumption of rectangular symmetry, lattice parameters were calculated that led to average molecular areas of 18.8 and $18.7 \AA^{2} /$ molecule on a calcium bicarbonate subphase. However we have shown using 
in situ GIXD that the monolayer restructures in response to nucleation events, which casts doubt on the findings based on a static model, such is the significance of the observation of monolayer rearrangement.

It would have been valuable to confirm the occurrence of nucleation events with XRR data however, as reported in the previous chapter the profiles obtained for 2HSA on CCCS are ambiguous and the measurements need to be repeated. DiMasi et al.[1] reported a similar inability to refine XRR data involving calcium carbonate crystallising subphases, which was attributed to mineralisation-induced roughening of the surface. Despite this set-back, this investigation has led to the first observation of calcium carbonate nucleation-initiated monolayer rearrangement using in situ, real-time GIXD. That is, we observe the mutual dynamic interaction of the monolayer and the nucleating crystal, potentially indicative of soft templation.

\subsubsection{Amorphous Film?}

Simultaneously with the first signs of monolayer peak splitting, a broad band (FWHM $=0.07442 \AA^{-1}$, correlating to a coherence length of $\sim 76 \AA$ ) of relatively uniform intensity (in terms of both $Q_{z}$ and $Q_{x y}$ ) was observed spanning the entire $Q_{z}$ range investigated $\left(0-1.1 \AA^{-1}\right)$. This band moves to higher $Q_{x y}$ in step with the monolayer peak and broadens to a maximum width of $F W H M=0.11357 \AA^{-1}$, which gives a coherence length of $\sim 50 \AA$. The broad nature of this band is consistent with an amorphous phase, an increasingly accepted transition phase for calcium carbonate nucleation. However the apparent lack of curvature tracing a Debye-Scherrer ring, within the $Q_{z}$ and $Q_{x y}$ range investigated, suggests otherwise.

The formation of 3-D multilayer structures is also reasonable, however the corresponding diffraction pattern would be expected to show undulations in the $Q_{z}$ direction, with a periodicity correlating to the layer thickness. However this does not appear to be the case, with a comparatively uniform intensity evident (Figure 6.1, bottom). 
The apparent coupling of this diffraction band to the higher $Q_{x y}$ monolayer peak implies intimate contact. Ostensibly the band extends in $Q_{z}$ as it moves in $Q_{x y}$ implying some extension of structure into the subphase.

After a time of $\sim 2-3$ hours Bragg peaks can be observed in the region of reciprocal space occupied by the broad band. The Bragg peaks were subsequently indexed to calcite (see below). Therefore the association of this band with both the monolayer and calcium carbonate crystallisation points to this being some intermediate phase.

Therefore until further experiments can be performed we will reservedly refer to this transitory band as an amorphous film. A film where the extension in $Q_{z}$ implies a thickness of $(2 \pi / 1.1)<6 \AA$ with a $d$-spacing of $3.85 \AA$ based on an in-plane $Q_{x y}$ value of $1.63 \AA^{-1}$.

\subsubsection{Crystallisation}

After reaching the final monolayer/film lattice we begin to see the appearance of discrete Bragg peaks consistent with crystallization of the mineral phase. The location, number and intensity of these Bragg spots varied across the monolayer, reflecting the heterogeneity of the monolayer and therefore the nucleation process (Figure 6.4). Based on $d$-spacings, the diffraction peaks were indexed to particular crystal faces of the five room temperature calcium carbonate crystalline polytypes: ikaite (calcium carbonate hexahydrate), monohydrocalcite, vaterite, aragonite and calcite (Table 6). Indexing attributed $90 \%$ of the peaks to either the calcite $\{012\}$ or ikaite $\{021\}$ plane, both having the same $d$-spacing. Figure 6.4 shows the overlay of the observed peaks on the $\{01.2\}$ calcite/ $\{021\}$ ikaite Debye-Scherrer ring. The distribution of these peaks along the ring points to a powder pattern and the absence of any preferential orientation, although the sample statistics are very low. The few remaining peaks were assigned to (110) and (020) vaterite reflections.

Interestingly, the crystal-assigned Bragg spots were paired (Figure 6.4). The 
origin of this peak pairing is unclear, but it could include refraction of the incident beam by the monolayer as described by Toney and Brennan[6], where the refraction of the incidence beam results in a shift to higher $Q_{z}$. Except for in-plane reflections, the outgoing beam generally has an exit angle $\alpha_{\mathrm{f}}>>\alpha_{\mathrm{c}}$, and the refraction of this beam is therefore less pronounced[7].

Analysis of the peak pairing gives a reasonably consistent peak splitting of $\Delta Q_{z}=$ $0.0171 \AA^{-1}$. Based on the linear relationship $\Delta Q_{z}=2 \alpha_{i, \text { eff }}$ the effective incidence angle $\alpha_{i, \text { eff }}=0.095^{\circ}$ is readily calculated. This value of $0.095^{\circ}$ compares with a setting for the incident angle of $0.1^{\circ}$ consistent with the peak splitting being a result of beam refraction.

Table 6 A list of the calcium carbonate d-spacings that lie in the vicinity of the observed GIXD crystal peaks.

\begin{tabular}{|c|c|c|c|c|}
\hline Phase & $\mathbf{h}$ & $\mathbf{k}$ & $\mathbf{l}$ & $d$-spacing \\
\hline Aragonite & 0 & 2 & 0 & 1.577 \\
\hline Ikaite & 2 & 0 & -2 & 1.583 \\
\hline Calcite & 0 & 1 & 2 & 1.630 \\
\hline Ikaite & 0 & 2 & 1 & 1.634 \\
\hline Vaterite & 1 & 0 & 1 & 1.696 \\
\hline Vaterite & 1 & 1 & 0 & 1.759 \\
\hline Vaterite & 0 & 2 & 0 & 1.759 \\
\hline Ikaite & 1 & 1 & 2 & 1.810 \\
\hline
\end{tabular}




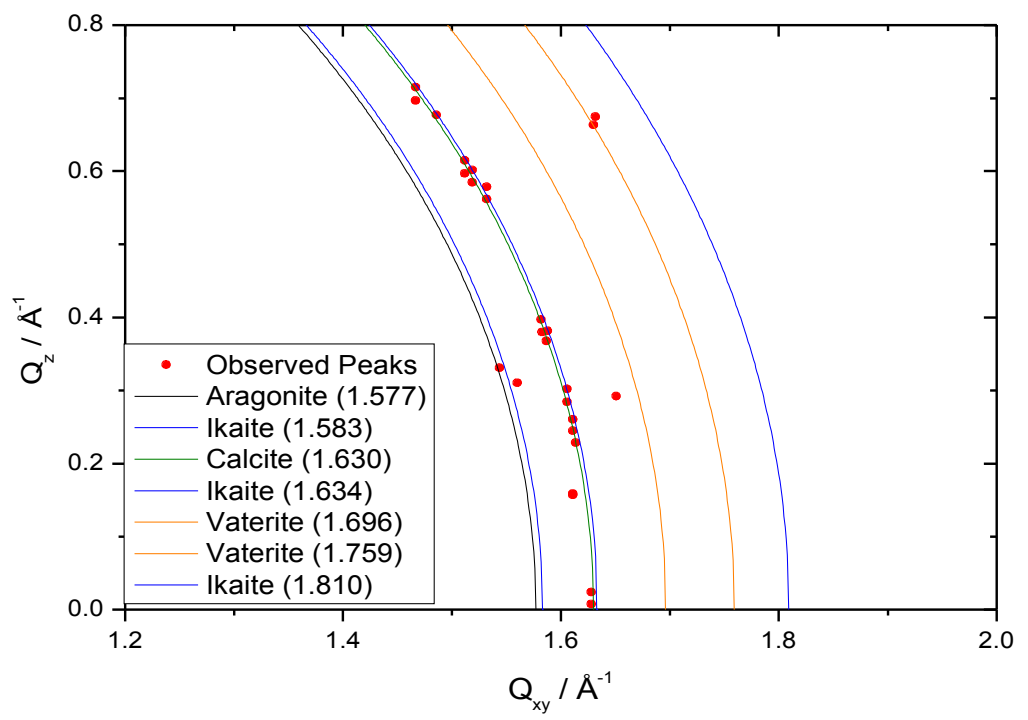

Figure 6.4 Debye-Scherrer rings for different d-spacings of calcite, aragonite, vaterite, ikaite and monohydrocalcite. Overlaid are the observed diffraction peaks. Note that the majority of peaks are paired reflecting the refraction of the incoming beam.

\subsubsection{Ex situ Crystallisation Studies.}

In accordance with the protocol employed in Chapter 4, crystals were grown under 2-HSA monolayers at pressures of 10 and $25 \mathrm{mN} \mathrm{m}^{-1}$ (Figure 6.5). In comparison to crystals grown under mixed ODA/ODOH monolayer, there was very little morphological or orientational variation in the 2-HSA derived crystals. There was no evidence of preferential orientation, with a random assortment of orientations. This absence of any orientational preference is consistent with the scattering of peaks along the Debye-Scherrer arc in the GIXD data, which is indicative of a powder pattern. There was no significant surface pressure effect, nor was there any correlation with monolayer stability. Crystal irregularity was absent and elongation was random. There was an increase in the number of crystal defects associated with diffusion-limited growth, but again there was no significant trend associating this phenomena with a particular mechanism.

The results suggest that the strong cation binding, brought about by the 
proximity of the covalently bound hydroxyl group to the acid group, disrupts the interfacial interaction that leads to FSN (this will be discussed in more detail in Chapter 7).

\subsection{Conclusions}

Real-time in situ experiments have shown the interrelationship between the monolayer and the growing crystal in the calcium carbonate and 2-HSA system. Beginning with a splitting of the monolayer diffraction peak associated with a contraction of the monolayer lattice in response to nucleation events. This is the first time that this has been observed in situ. Coinciding with the monolayer rearrangement is the appearance of a broad band of scattered intensity. While it remains unaccounted for, there is a suggestion that it may reflect a transition stage in the mineralisation process. Finally we observe intense Bragg peaks associated with crystallisation.

2-HSA binds calcium ions strongly resulting in a strong interfacial interaction which undoubtedly contributed to the observation of the monolayer rearrangement and the subsequent phenomena. However ex situ experiments, supported by GIXD data, show the absence of any preferential orientation. In fact the strong interfacial interaction appears to overshadow any bicarbonate effect and consequently result in a lack of orientational control. 

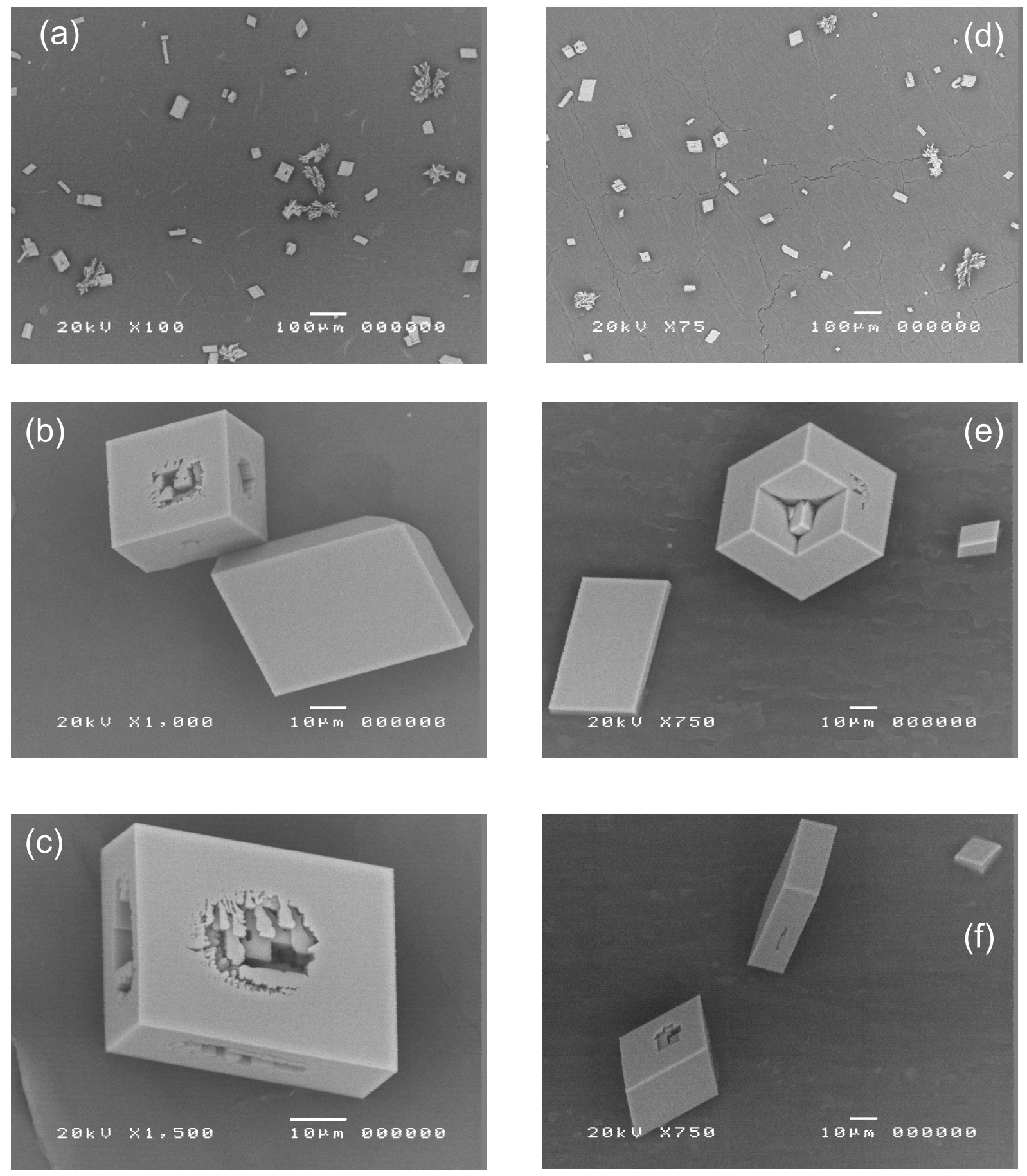

Figure 6.5 Representative SEM images of crystals grown under 2-HSA monolayers. (a)-(c) Were grown at a monolayer surface pressure of $10 \mathrm{mN} \mathrm{m}^{-1}$. (d)-(e) Were grown at a monolayer pressure of $25 \mathrm{mN} \mathrm{m}^{-1}$. 


\subsection{References}

[1] E. DiMasi, S. Kwak, B.P. Pichon, and N.A.J.M. Sommerdijk, "Structural adaptability in an organic template for $\mathrm{CaCO}_{3}$ mineralization," CrystEngComm, vol. 9, 2007, pp. 1192-1204.

[2] J. Kmetko, "Effects of Divalent Ions on Langmuir Monolayers: Synchrotron X-ray Scattering Studies," PhD Thesis, Northwestern University, Evanston, Illinois, USA. , 2002.

[3] E. DiMasi, M. Olszta, V. Patel, and L. Gower, "When is Template Directed Mineralization Really Template Directed?," CrystEngComm, vol. 5, 2003, pp. 346350.

[4] E. DiMasi, V. Patel, M. Sivakumar, M. Olszta, Y. Yang, and L. Gower, "PolymerControlled Growth Rate of an Amorphous Mineral Film Nucleated at a Fatty Acid Monolayer," Langmuir, vol. 18, 2002, pp. 8902-8909.

[5] Y. Lifshitz, O. Konovalov, N. Belman, A. Berman, and Y. Golan, “Template Growth of Nanocrystalline PbS, CdS, and ZnS on a Polydiacetylene Langmuir Film: An In-Situ Grazing Incidence X-ray Diffraction Study," Advanced Functional Materials, vol. 16, 2006, pp. 2398-2404.

[6] M.F. Toney and S. Brennan, "Observation of the effect of refraction on $x$ rays diffracted in a grazing-incidence asymmetric Bragg geometry," Phys.Rev.B, vol. 39, Apr. 1989, p. 7963.

[7] D.W. Breiby, O. Bunk, J.W. Andreasen, H.T. Lemke, and M.M. Nielsen, "Simulating X-ray diffraction of textured films," Journal of Applied Crystallography, vol. 41, Apr. 2008, pp. 262-271. 


\section{Chapter 7: Substituted Acids}

\subsection{Introduction}

In Chapters 5 and 6 the dramatic effect that the introduction of a hydroxyl group at the 2-position has on monolayer behaviour and subsequent calcium carbonate nucleation and growth was illustrated. This chapter emphasises the manipulation of the head- group electron density rather than explicitly the role of the alcohol moiety, by comparing monolayer behaviour and calcium carbonate crystal characteristics for monolayers of 2-methyloctadecanoic acid (2-MODA), 2-bromooctadecanoic acid (2BODA), octadecylmalonic acid (ODMA), and 3-hydroxyoctadecanoic acid (3-HSA). Data for 2-HSA is also included for ease of comparison.

Based on electronegativities, ( $\mathrm{H} \mathrm{2.2,} \mathrm{C} \mathrm{2.5,} \mathrm{Br} 2.7$ and $\mathrm{O} 3.5)$ and the proximity of the substituted functional group to the head group (2- vs 3-HSA) this comparative study provides an insight into the subtleties (or lack of) of the interfacial interaction. Using this approach additional understanding of the role electrostatics plays in templated nucleation of biominerals can be gained. As enunciated in Chapter 5, it is expected that the substituted acids should exhibit behaviour consistent with a single large polar head group. However, in Chapter 5 we also showed that the substitution of a second functional group alters more than just the head-group polarity. In particular, the addition of the hydroxyl group in the $\mathrm{C} 2$ position alters steric effects, the propensity for hydrogen bonding, the $\mathrm{pKa}$ and the subphase ion binding.

As with the previous systems the characteristics of the above surfactants have been investigated on four different subphases: water, $\mathrm{CaCl}_{2}, \mathrm{NaHCO}_{3}$ and CCCS. By studying the individual subphase components that comprise the CCCS, the influence of specific subphase ions on monolayer behaviour can be ascertained, which aids the 
elucidation of the interfacial interaction during calcium carbonate crystal growth.

\subsection{Results and Discussion}

Given the focus on the changing of the electron density of the head groups PM3 semi-empirical calculations were performed using HyperChem V. 7.5 (Hypercube, Inc. $)^{1}$. The calculations employed truncated $C_{6}$ molecules in a vacuum at $0 \mathrm{~K}$, and therefore their value is purely qualitative. Based on XRR electron density profiles reported in Chapter 5, where all the box-models accounting for the head group were comprised of four or less carbons $\left(-\mathrm{CH}_{2}-\mathrm{CH}_{2}-\mathrm{CHOH}-\mathrm{COOH}\right)$ it was felt adequate to use a $\mathrm{C}_{6}$ model.

These calculations clearly illustrate the effect of the substitution on the headgroup electron density (Figure 7.1). Ordering the molecules on increasing headgroup electron density gives: 2-MODA $<2-\mathrm{BODA}<\mathrm{ODOH}<2-\mathrm{HSA}<\mathrm{ODA}<\mathrm{ODMA}$ $<3$-HSA for the uncharged acids, which reorders to: $\mathrm{ODMA}^{-}<2-\mathrm{HSA}^{-}<3-\mathrm{HSA}^{-}<$ $\mathrm{ODA}^{-}<2-\mathrm{BODA}^{-}<2-\mathrm{MODA}^{-}<\mathrm{ODMA}^{2-}$ when deprotonation is considered. The reordering that occurs with deprotonation highlights the balance between head-group size and charge, which was found to be critical in terms of how these monolayers interacted with each other and the subphase. In terms of hydrogen-bonding capacity, the PM3 models show 3-HSA and 3-HSA ${ }^{-}$to form intramolecular hydrogen bonds. Structurally, this is more difficult for 2-HSA but the electron density distribution for 2-HSA does show a redistribution in favour of hydrogen bond formation. On changing the initial geometry of the hydroxyl group, energy minimisation always resulted in the hydroxyl hydrogen re-orienting towards the carbonyl oxygen. The tendency for such an orientation was increased on deprotonation.

$1 A b$ initio calculations were not performed as Hyperchem does not have the capacity to perform $a b$ initio calculations on molecules containing bromine. Since a straight comparison of all the molecules was required using the same calculation method PM3 was chosen. 

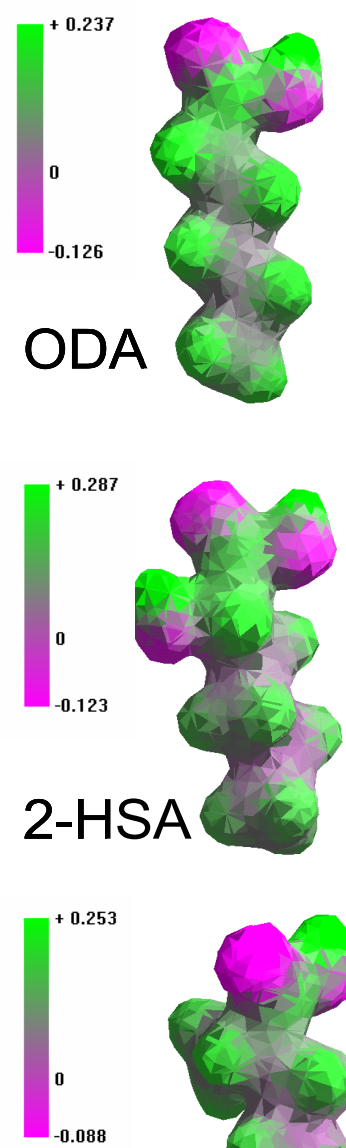

2-MODA
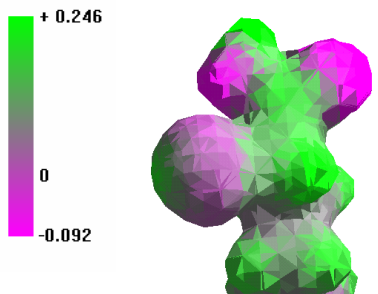

2-BODA
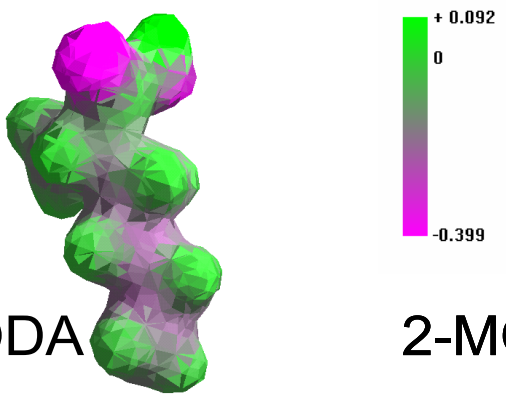

ODA ${ }^{1-}$
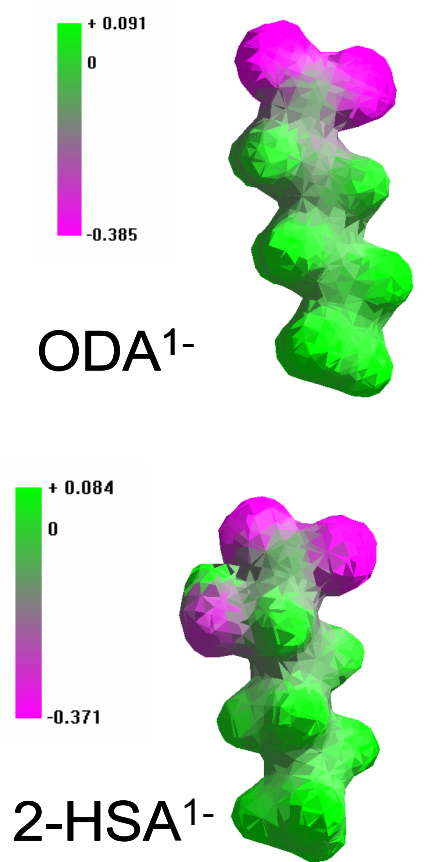

2-HSA ${ }^{1-}$

2-MODA ${ }^{1-}$
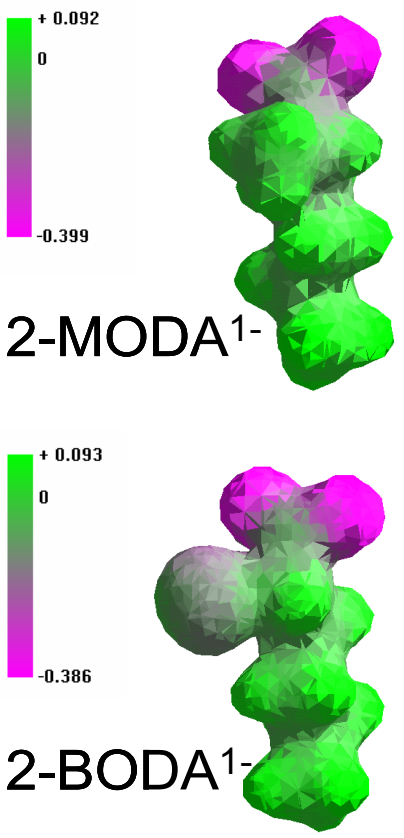

2-BODA ${ }^{1-}$

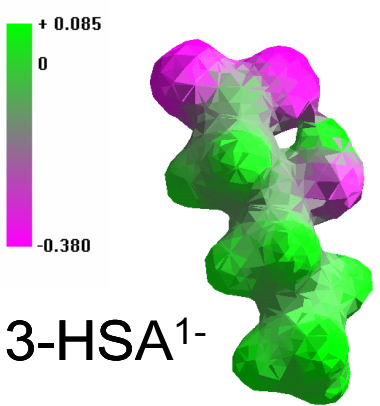

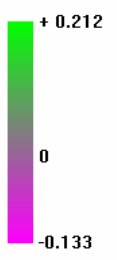

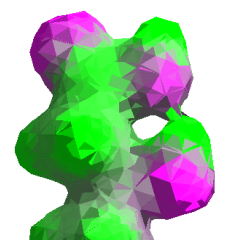

3-HSA

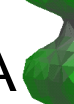

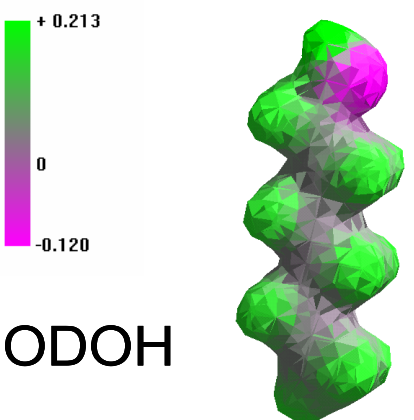
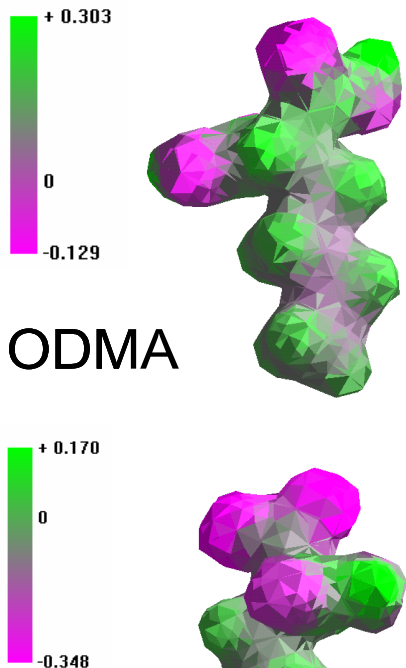

\section{ODMA $^{1}$

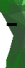
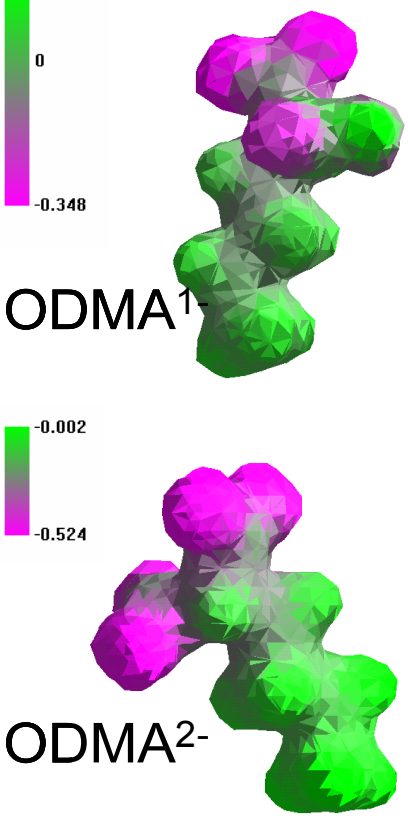
Assessment of conformation and steric implications is inappropriate given the absence of water, ions and temperature, however, anecdotally, these models show the variation of cross-sectional size. For example, the head group of $\mathrm{ODOH}$ is smaller than the hydrocarbon chain, in contrast to the large 2-BODA and ODMA head groups.

Gauging the differences between these molecules is valuable even if only on a qualitative basis. The interaction with the subphase is very much dependent on how the electron density is distributed around the surfactant head group. Similarly the size and conformation will alter the head group's ability to pack in a monolayer and interact with the subphase.

\subsubsection{Monolayer Behaviour}

\subsubsection{Water}

Figure 7.2 shows representative isotherms for the five substituted acids on a subphase of pure water at a $\mathrm{pH}$ of 5.6. Initial observations, which will be discussed in greater detail on a subphase by subphase basis below, include a shift in the onset $A_{m}$ to larger values than those observed for the mixed monolayers systems in Chapter 3. Also apparent is the curvilinear nature of the onset transition, indicating a loss of long-range order. Beyond the onset, the absence of any inflection indicates the absence of a tilted to untilted phase transition.

The insert in Figure 7.2 shows a very low pressure inflection in the 2-HSA isotherm. This phenomenon was evident to some degree for all the surfactant monolayers on all the subphases. Substituted acid systems all present with a coexistence of phases from the outset, and this inflection in the isotherm coincides with the movement of the condensed islands (Chapter 5) or film past the Wilhelmy plate. Thus the extent of the inflection varies for a single system and more so between systems, dependent on the addition of surfactant and the compression 
speed.

The surface potential profiles for the five substituted acids are shown in Figure 7.3. The variability in the profiles at large $A_{m}$ data reflects the phase coexistence. The formation of a coherent film coincides with the establishment of a plateau in the potential profile. At which point the profiles begin to show characteristics unique to the particular monolayer chemistry. As for previous systems, key characteristics were extracted from the surface pressure and potential isotherms. These data for the substituted octadecanoic acids on water are summarised in Figure 7.4.

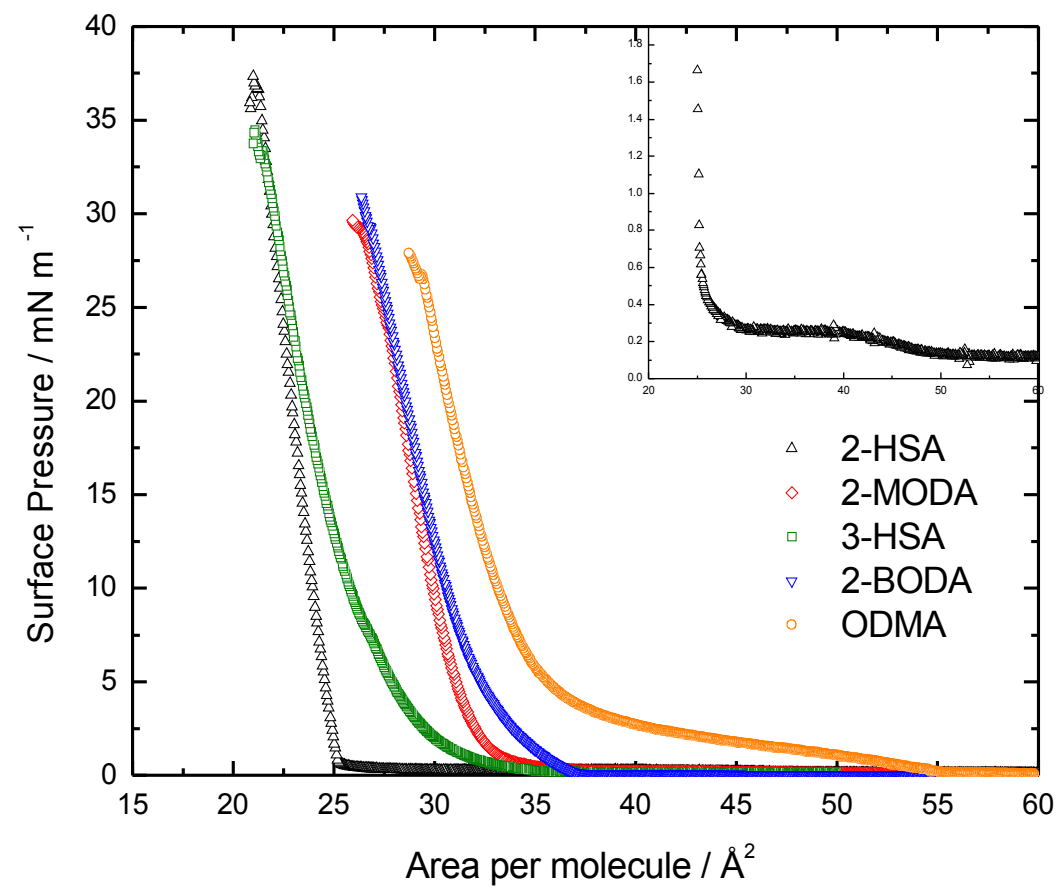

Figure 7.2 Surface pressure isotherms for the substituted octadecanoic acid monolayers on pure water at $p H=5.6$. The insert is a magnified view of a low-pressure inflection in the isotherm of the 2-HSA monolayer.

The surface pressure statistics reveal that the hydroxy acids have the smallest $A_{m}$ with the order being: 2-HSA $<3$-HSA $<2$-BODA $<2-$ MODA $<<$ ODMA. This ordering correlates well with the expected ranking of head-group size (Figure 7.1). However the presence of water and neighbouring molecules means that other interactions will also be influencing the different systems. The small $\mathrm{A}_{\mathrm{m}}$ of the 2-HSA monolayer is 
brought about by the increased capacity for intramonolayer hydrogen bonding, which leads to condensation of the film. At the other end of the scale the extremely large onset $\mathrm{A}_{\mathrm{m}}$ of ODMA can be attributed to a bulkier head group and increased like-charge repulsion. The $\mathrm{pKa}$ of these acids is subject to the specific conditions used, however, dicarboxylic acids often have a $\mathrm{pKa} \mathrm{a}_{1}$ lower than the $\mathrm{pKa}$ of a comparable mono-acid[1].

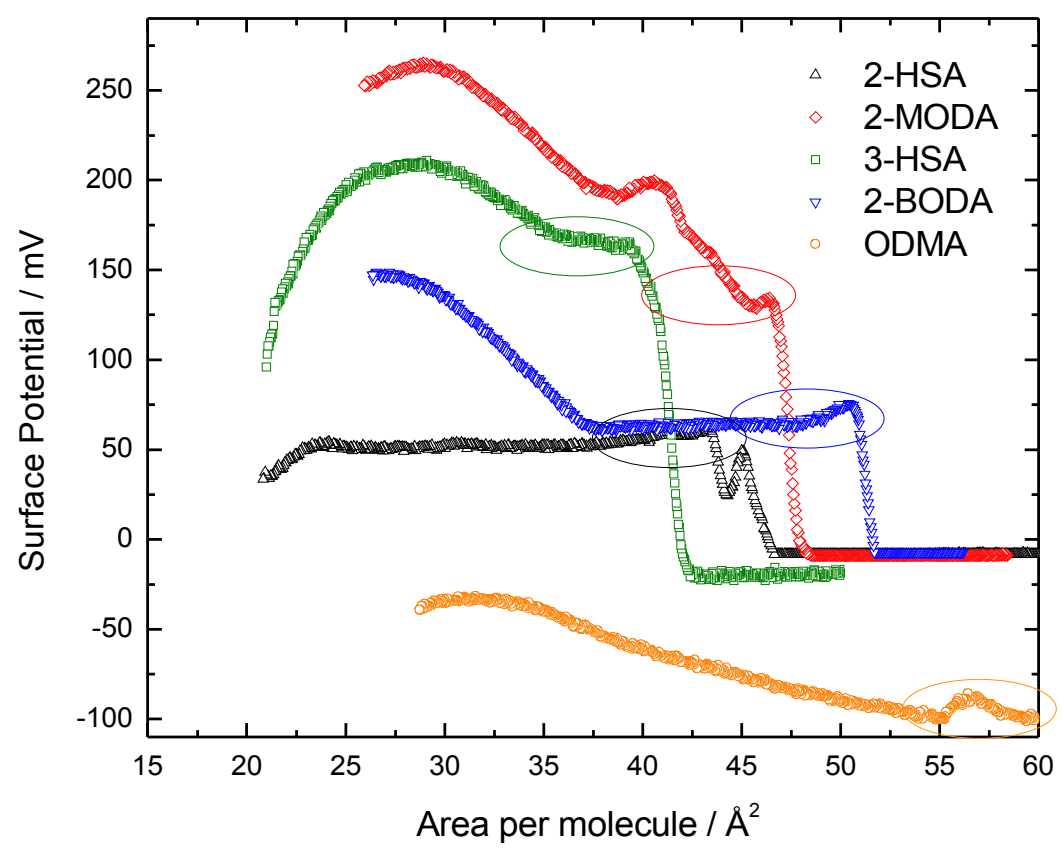

Figure 7.3 Surface potential profiles for the different substituted octadecanoic acids on water. The irregular profile at higher areas is consistent with a coexistence of two phases, one being gas-like. Colour coded ellipses highlight the plateau regions of the potential isotherm.

The order of the remaining three systems reflects principally steric or size effects. Shifting the hydroxyl group to the 3-position reduces the hydrogen bonding induced condensation of 3-HSA. Although the bromide in 2-BODA has the capacity to form $\mathrm{H} \cdots \mathrm{Br}$ dipole-dipole bonds, it is less effective at condensing the film as compared with a hydroxyl group. The relative lack of both inter- or intramolecular bonding ability in the 2-MODA system means that it is a good model system for probing the role of steric effects in monolayer and subphase interactions. 
The curvilinear transition evident in 3-HSA, 2-BODA and ODMA surface pressure isotherms is consistent with that observed for 2-HSA (Chapter 5), and points towards a disordered two phase system. In all cases, the substituted acid head groups would behave as a mono-polar entity as described for 2-HSA. This large mono-polar head group leads to size mismatch between the head and tail groups, the consequence being, to varying degrees specific to the head-group size, that the monolayer characteristics are consistent with a disordered tilt azimuth.

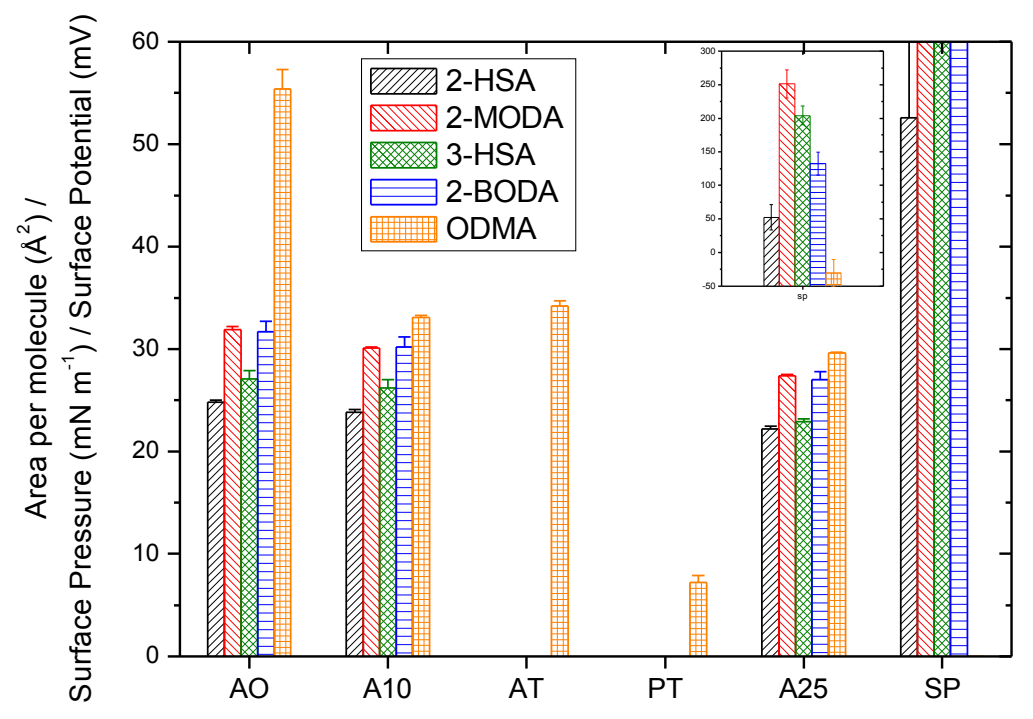

Figure 7.4 Isotherm characteristics for the substituted octadecanoic acids on a water subphase. Note, only the ODMA monolayer displayed a transition, hence the absence of AT and PT columns for the other surfactants. The negative surface potential for ODMA exceeds the ordinate scale, thus an insert is included to highlight the differences in $\Delta V_{\max }$ for the substituted acids. (Key: $A O=$ the $A_{m}$ at onset, $A_{10}=$ the $A_{m}$ at $\Pi=10 \mathrm{mN} \mathrm{m}{ }^{-1}, A T=$ the $A_{m}$ at the phase transition, $P T=\Pi$ at the phase transition, $A_{25}=$ the $A_{m}$ at $\Pi=25 \mathrm{mN} \mathrm{m}^{-1}$, and $S P=$ the maximum surface potential).

Beyond monolayer lift-off (onset), ODMA is unique in displaying a second low temperature transition. This general profile for ODMA is consistent with that reported by Vogel and Möbius[2]. The $\mathrm{A}_{\mathrm{m}}$ at onset and the $\mathrm{A}_{\mathrm{m}}$ at the phase transition suggest that this region is merely a high pressure equivalent of that shown for 2-HSA 
in the Figure 7.3 insert. The accentuation of this two-phase coexistence region to higher pressures reflects the higher degree of like-charge repulsion and steric effects for ODMA.

At lower $A_{m}$, the overall order is maintained but we begin to observe a 'pairing' in the behaviour. That is, the two hydroxy acids display similar behaviour, likewise 2MODA and 2-BODA converge in terms of having approximately equivalent $A_{m}$ at $\Pi=$ $25 \mathrm{mN} \mathrm{m}^{-1}$. This grouping is commensurate with similarities in the electron density and size between molecules within the pairs (Figure 7.1). This reflects a stericallyinduced reduction in the capacity to form hydrogen bonds at high pressures.

The surface potential trends differently than the $\mathrm{A}_{\mathrm{m}}$ data: ODMA $<<2-\mathrm{HSA}<2-$ BODA $<3-H S A<2-M O D A$. The negative potential for the ODMA film is consistent with literature[2], likely reflecting the dominance of the head group in the molecular dipole. In ODA the dipole associated with the hydrophobic tail is reported to dominate the oppositely polarised head-group dipole resulting in a positive potential[2]. However, here the addition of a second acid group shifts the molecular dipole in favour of the head group, hence the change in potential polarity. This same mechanism also explains the drop in the measured potential for the other substituted acids. With the order of the 2-substituted acids commensurate with the electronegativity of the substituted bonds. The shifting of the hydroxyl group one place further away from the acid group explains why 3-HSA is out of order based on electronegativity alone.

In terms of the profile of the surface potential plots, ignoring the high $\mathrm{A}_{\mathrm{m}}$ data, the behaviour generally parallels the mixed monolayer behaviour. Following their respective plateaus, 2-MODA, 2-BODA and 3-HSA all display a small rise starting at an $\mathrm{A}_{\mathrm{m}}$ approximately corresponding to the point of onset $\sim 35 \AA^{2}$, Figures 7.2 and 7.3. In the mixed systems this rise is associated with condensation (compaction of the monolayer associated with a phase change, resulting from either physical 
compression or intermolecular interactions), ordering and a reduction in tilt. However the substituted acid systems are already condensed from the outset. The absence of a rise in potential around onset in the 2-HSA potential profile occurs as a consequence of this early condensation (Chapter 5). As such the occurrence of a rise for the 2-MODA, 2-BODA and 3-HSA systems points to the initial condensation being only partial, with further ordering and reduction in tilt occurring with increasing pressure. This rise is greatly extended for the ODMA monolayers, coinciding with the low pressure phase coexistence, and reflecting a gradual restructuring of the monolayer with the application of pressure.

The subsequent fall in the surface potential for 2-MODA and 3-HSA is the result of the slow collapse of the monolayer, the data therefore indicates that 2-BODA does not experience significant slow collapse or more likely undergoes a different mechanism of slow collapse as compared to the other surfactants. The absence of slow collapse may reflect the duality of the bromide, where in general terms, its polar nature promotes dissolution, however its chaotropic characteristics counter this with a preference for residing at the surface.

The tendency for slow collapse is related to the ESP. Based on the surface potential data ESP increases (i.e., the monolayer is more stable) as: 3-HSA $<2-\mathrm{HSA}<$ 2-MODA < ODMA < 2-BODA. The relative stability of the ODMA monolayer is surprising and points to a favourable interaction with the subphase water.

Overall, on water there is clear evidence for larger $A_{m}$ in response to substitution. Further, this increase in area is specific to the chemistry of the additional functional group. Substitution appears to favour pressure-less condensation, such that twophase coexistence is common. In general the isotherm behaviour reflects the occurrence, extent and nature of the hydrogen bond formation. 2-HSA exhibits significant condensation consistent with strong intermolecular hydrogen bonding brought about by the presence of two participating functional groups. The tendency 
to form intramolecular hydrogen bonding means that 3-HSA behaves in a similar manner to ODA but with a larger head group that increases tilt disorder. The behaviour of 2-MODA and 2-BODA is not too dissimilar to that of 3-HSA, other than that the larger and more passive side groups result in a shift to larger $A_{m} s$. Finally ODMA, the combination of two carboxylate groups and a bulky head group generates a significant capacity for hydrogen bonding and tilt disorder.

\subsubsection{Calcium Chloride}

The introduction of calcium ions (and chloride ions) to the subphase has a varied response in terms of the surface pressure and potential behaviour (Figures 7.5, 7.6 and 7.7). The $A_{m}$ at onset now follows the order: 3-HSA $<2-B O D A<2-H S A<$ ODMA $<2-M O D A$, this is in stark contrast to that observed on water. The $A_{m}$ at onset reduced for all systems except 2-HSA which showed an $\sim 14 \%$ increase. Note that this increase is not real in terms of molecular packing but is related to the packing of the rigid islands as evidenced by BAM and described in Chapter 5.

In terms of reduction in area from that observed on water, ODMA displayed the greatest decrease with an approximately 50\% reduction. This dramatic decrease highlights the significant ordering effect of calcium binding. The di-acid is expected to create a 6-membered ring with the subphase calcium ions resulting in a coupling second only to the 5-membered ring of 2-HSA in strength. This is also reflected in the loss of the low pressure phase transition that was observed for ODMA on water. The remaining three acids show a more measured response with a reduction in $A_{m}$ at onset of $\sim 14 \%$ for $2-B O D A, ~ 11 \%$ for 3 -HSA and $\sim 4 \%$ for 2 -MODA.

The $A_{m}$ at $\Pi=10 \mathrm{mN} \mathrm{m}^{-1}$ shows a similar trend to that observed at onset: $3-\mathrm{HSA}<$ $2-\mathrm{HSA}<2-\mathrm{BODA} \approx \mathrm{ODMA}<2-\mathrm{MODA}$. A key difference is the 2-HSA result, here a greater reduction in area is seen at the higher pressure than for the other acids. This can be attributed to enhanced packing of the condensed islands associated with the 
higher pressures. As discussed in Chapter 5, GIXD does not show evidence supporting a rearrangement of the head group with pressure, but rather the reduction in $\mathrm{A}_{\mathrm{m}}$ limits the capacity for intramonolayer hydrogen bonding.

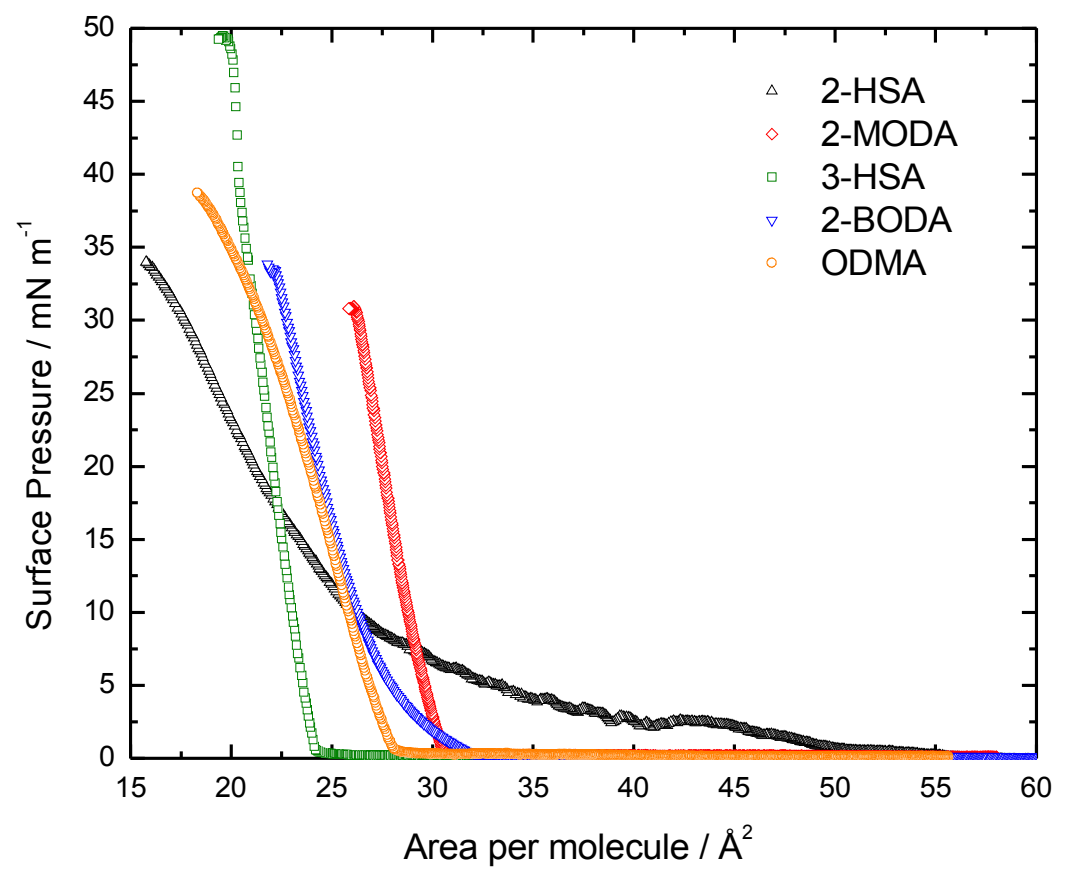

Figure 7.5 Surface pressure isotherms of the substituted octadecanoic acids on a $20 \mathrm{mM}$ calcium chloride subphase.

In contrast to the situation on water, only 3-HSA passes through a transition on $\mathrm{CaCl}_{2}$. The point of inflection occurs at high pressures $\left(\sim 37.8 \mathrm{mN} \mathrm{m}^{-1}\right)$ and is similar to the tilting to untilting transition of ODA. This similarity extends to the $A_{m}$, which suggests some loss of surfactant in the 3-HSA system considering the increased size associated with the hydroxy group. GIXD shows 2-HSA to be untilted from the outset. Given similarly strong calcium binding, ODMA is also likely to be untilted. In contrast, the absence of a transition, and the relatively large $A_{m}$, suggest that 2-MODA and 2-BODA remain tilted up to collapse under these conditions. BAM images show an absence of contrast throughout the isotherm for both 2-MODA and 2-BODA suggesting either an untilted monolayer or a disordered tilt. Both surface 
potential profiles display a gradual rise in potential coinciding with the onset point indicative of a gradual reduction in tilt (Figure 7.7). Collectively, the data points to a tilted lattice with a disordered tilt azimuth, similar to that observed for 2-HSA on water, which was confirmed by GIXD (Chapter 5).

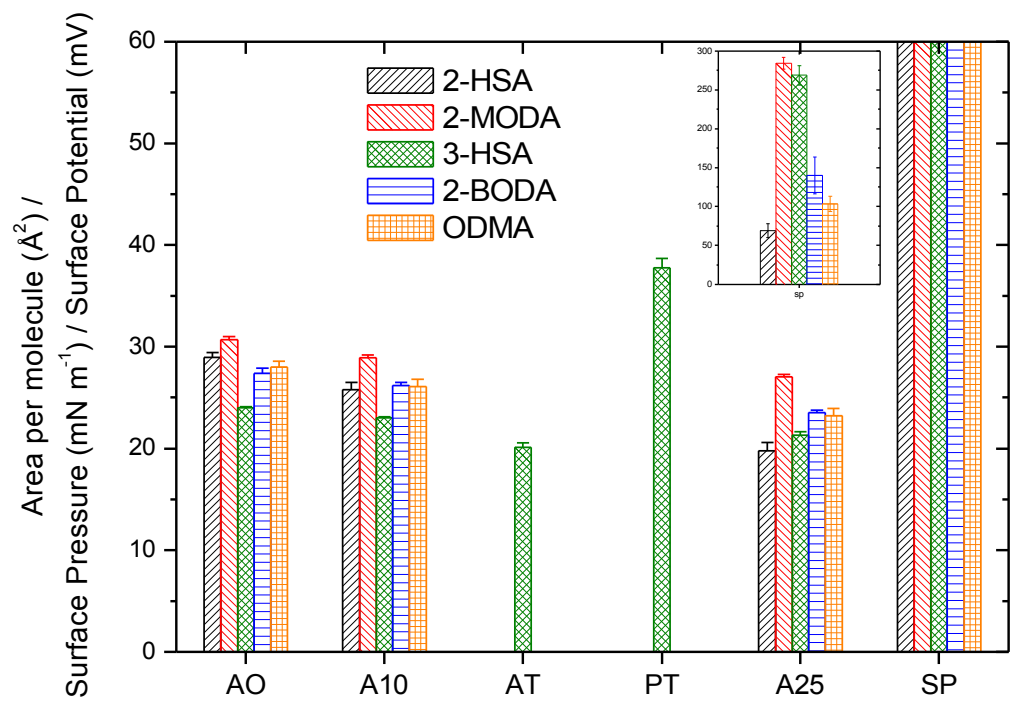

Figure 7.6 General isotherm trends for the substituted acids on a $20 \mathrm{mM}$ calcium chloride subphase. The insert summarises the surface potential results which exceed the ordinate-scale. (Key: $A O=$ the $A_{m}$ at onset, $A_{10}$ $=$ the $A_{m}$ at $\Pi=10 \mathrm{mN} \mathrm{m}^{-1}, A T=$ the $A_{m}$ at the phase transition, $P T=\Pi$ at the phase transition, $A_{25}=$ the $A_{m}$ at $\Pi=25 \mathrm{mN} \mathrm{m}^{-1}$, and $S P=$ the maximum surface potential).

Another significant drop in the $\mathrm{A}_{\mathrm{m}}$ of 2-HSA associated with slow collapse is evident in the data at $\Pi=25 \mathrm{mN} \mathrm{m}^{-1}$, while, 2-MODA again only showed a modest reduction in $A_{m}$. Other than these two observations, the order remains the same as that observed at $\Pi=10 \mathrm{mN} \mathrm{m}^{-1}$. The behaviour of these two systems (2-HSA and 2MODA) represents the spectrum of behaviour, with 2-HSA displaying the largest degree of slow collapse in contrast to 2-MODA, which shows the least. Not surprisingly, the $A_{m}$ at $\Pi=25 \mathrm{mN} \mathrm{m}^{-1}$ is dominated by slow collapse and the relationship of the monolayer ESP and the applied pressure.

Figure 7.7 shows representative surface potential profiles for the substituted acid 


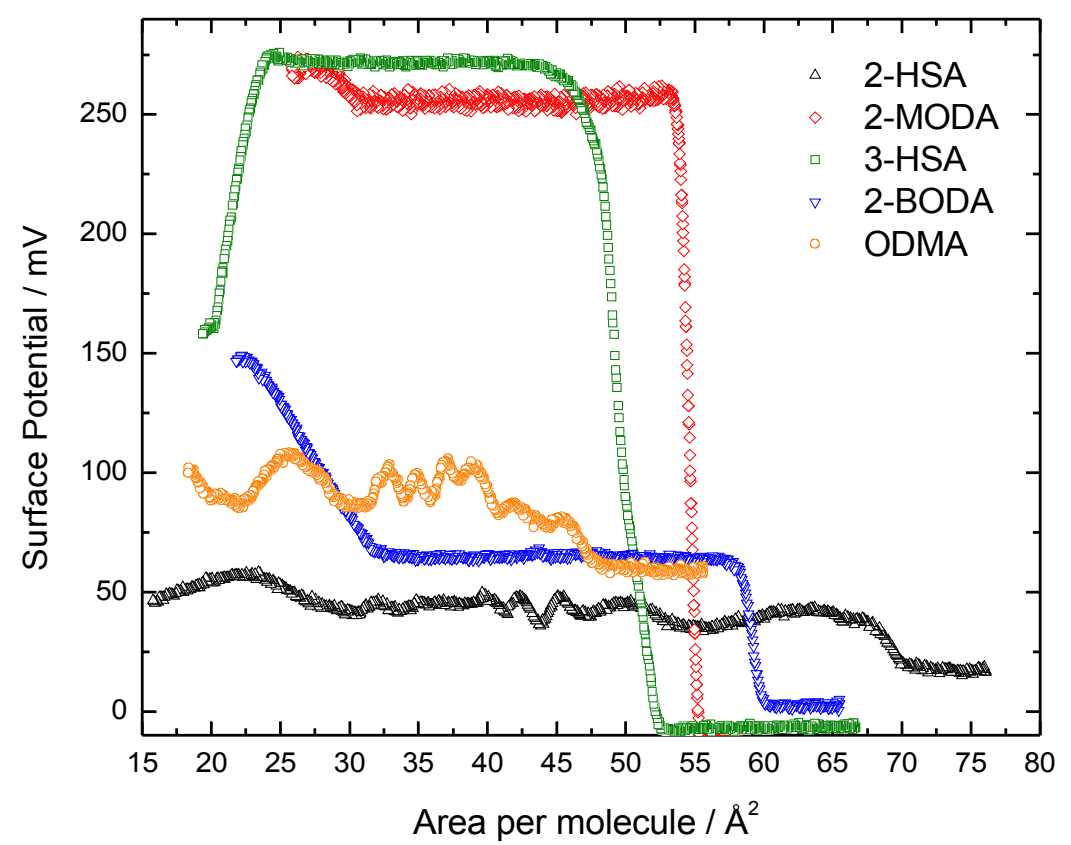

Figure 7.7 Surface potential profiles for the substituted acids on a $20 \mathrm{mM}$ calcium chloride subphase.

monolayers on calcium chloride. The strong interaction of ODMA and 2-HSA with the calcium containing subphase results in irregular profiles as expected. In contrast the 2-BODA profile changes very little with the introduction of calcium ions to the subphase. While, 2-MODA and 3-HSA continue to have higher potential profiles consistent with having less polar head groups. A comparison of the maximum surface potential on water and $\mathrm{CaCl}_{2}$ shows that all the acids exhibit an increase in potential with the addition of calcium to the subphase as expected; calcium binding offsetting the head-group polarity thereby enhancing the polarity of the tails.

Overall the $\mathrm{CaCl}_{2}$ system is exclusively dominated by electrostatic interactions between the monolayer and calcium ions, leading to increased order, condensation and reduction in head-group polarity. The influence of calcium being associated with the polarity of the head group and chelating capacity, with the greatest affect seen with ODMA and 2-HSA. 2-MODA, 3-HSA and 2-BODA all exhibited less behaviour modification, consistent with the substitution chemistry. Putting aside the 
$A_{m}$ differences, these latter systems are generally commensurate with ODA (Chapter 3).

\subsubsection{Sodium Bicarbonate}

As observed for the mixed monolayer systems (Chapter 3), the inclusion of sodium bicarbonate in the subphase results in a significant shift in the monolayer behaviour from that observed on water. Surface pressure isotherms show three distinct behaviours for the five substituted acids (Figure 7.8). 2-MODA and 3-HSA exhibit a well-defined onset transition, a shift to smaller $A_{m}$ and a more ordered and condensed profile. In contrast, 2-HSA and ODMA display a much more curvilinear profile with collapse at a lower pressure and at very small $A_{m}$ values. The third behaviour is exhibited by 2-BODA, which includes a shift to larger $A_{m}$ and a very pronounced curvilinear profile compared to that on water.

In terms of the surface potential, the profiles are atypical (Figure 7.8). In comparison with those obtained for the water subphase, only a reduced section of the full potential profile is seen, principally that from the plateau on, that is a coherent film always exists, we do not see its formation. The surface potential profile for 2HSA is effectively featureless, showing no change that correlates to the pressure isotherm. In contrast the potential profiles for 2-MODA, 2-BODA and ODMA exhibit weak features associated with events in the pressure isotherms. However, only 3HSA displays a profile commensurate with that observed on water. 

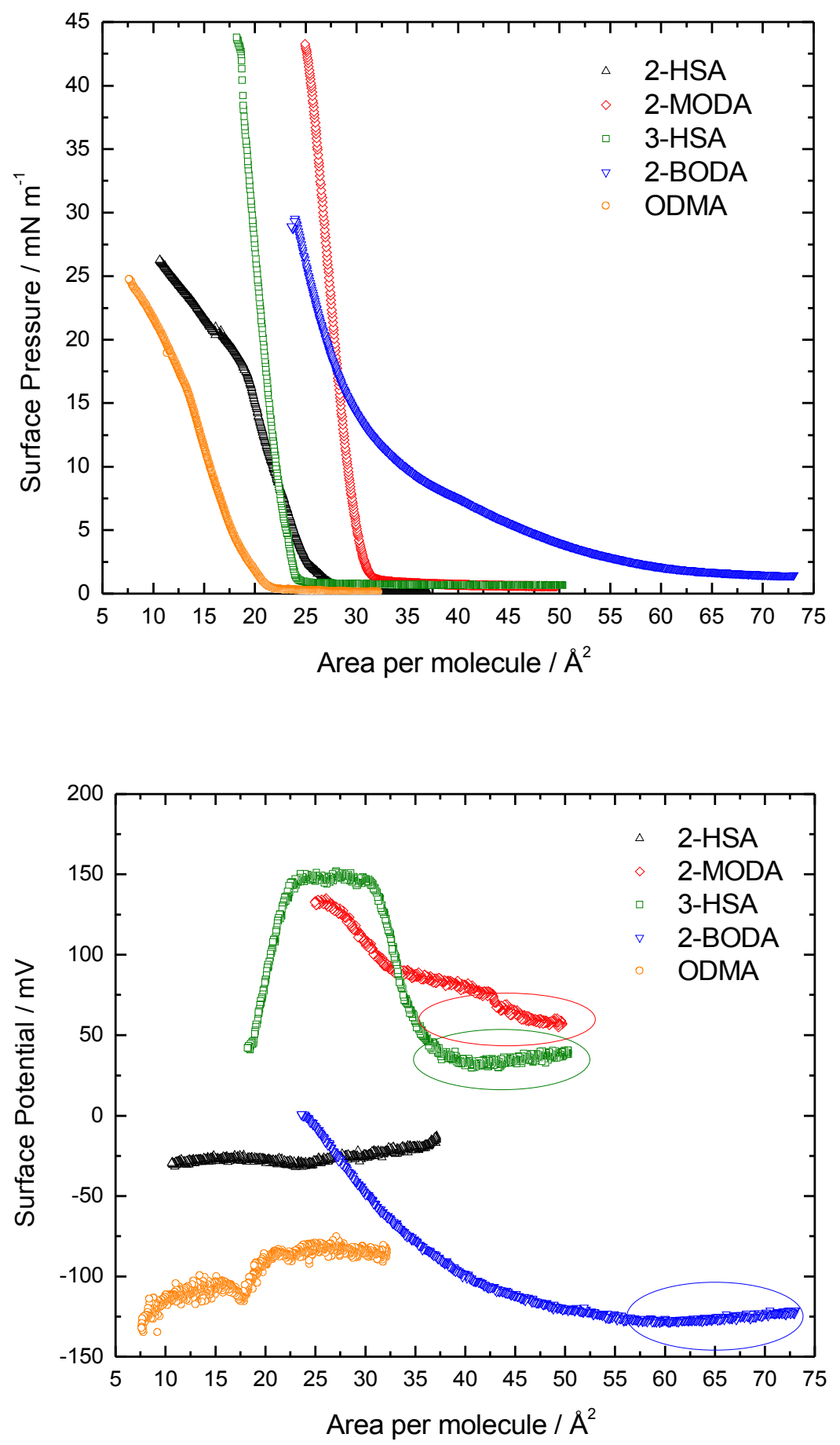

Figure 7.8 Surface pressure (top) and potential (bottom) isotherms for the substituted acids on $20 \mathrm{mM}$ sodium bicarbonate. The colour coded ellipses highlight the plateau region, absent in 2-HSA and ODMA profiles.

The key characteristics of the isotherms are summarised in Figure 7.9, which in 
terms of $\mathrm{A}_{\mathrm{m}}$ ODMA consistently returns the smallest values. The trend being ODMA $<3-H S A<2-H S A<2-M O D A<<2-B O D A$ for the onset $A_{m}$. Upon increasing pressure 2-HSA and 3-HSA exchange places, as do 2-MODA and 2-BODA at $\Pi=25 \mathrm{mN} \mathrm{m}^{-1}$. The maximum surface potential has a somewhat different order: $2-\mathrm{HSA}<\mathrm{ODMA}<2-$ BODA $<2-$ MODA $<3-H S A$.

The behaviour of the 2-MODA and 3-HSA systems is consistent with the establishment of a cation-mediated hydrogen-bonded network as discussed previously. Network formation accounts for the reduction in $A_{m}$, the minimisation of like-charge repulsion and the significant reduction in $\Delta \mathrm{V}_{\max }$ when compared to the behaviour on water. The reduction in area results in an increase in the ordering of the tail groups, which is reflected in the sharp onset transition. The inclusion of the surfactants in a network also accounts for the increased stability of the films, as evidenced by the higher collapse pressures than those recorded on water.

The shift to a $\mathrm{NaHCO}_{3}$ subphase also results in different behaviour for 2-HSA and ODMA. On water, the 2-HSA monolayer is ordered, with a sharp onset transition, it is stable, has a collapse pressure $>35 \mathrm{mN} \mathrm{m}^{-1}$, and is condensed with the smallest $\mathrm{A}_{\mathrm{m}}$ at onset. However, the introduction of $\mathrm{NaHCO}_{3}$ to the subphase yields a disordered and unstable film. This shift in behaviour points to the bicarbonate anion breaking the intramonolayer hydrogen bonding evident on water. The cation-mediated hydrogen-bonded network hypothesised in Chapter 3, is evidently enhanced by hydroxyl or methyl substitution at the 3- and 2-positions, respectively, as discussed above. In contrast hydroxyl substitution at the 2-position acts to move the balance of interactions, such that those between the monolayer and the subphase are now significantly enhanced. This is achieved at the expense of the intramonolayer hydrogen bonding, destabilising the monolayer and disrupting the chain-like cationmediated hydrogen-bonded network. This is supported by the large decrease in $\Delta \mathrm{V}_{\max }$ suggesting a significant subphase-based potential of opposite polarity to that 
of the molecular dipole. Since the cation-mediated hydrogen-bonded network may act as a conduit for structural control between the monolayer and a nucleating crystal, it would be expected that less facial control would arise in the 2-HSA system.

ODMA shows considerable condensation from that observed on water, consistent with significant reduction in like-charge repulsion. $\Delta \mathrm{V}_{\max }$ increases marginally, attributable to the reduced tilt associated with the condensation. Both 2-HSA and ODMA experience slow collapse consistent with a lowering of their respective ESP, brought about by an interaction with the bicarbonate. Consequently, in contrast to 2MODA and 3-HSA, the enhanced monolayer/subphase interaction has resulted in a decrease in the ESP and therefore a relatively unstable monolayer.

The behaviour of 2-BODA reflects a significant decrease in order in comparison to that observed on water. The reduction in order is a consequence of increased

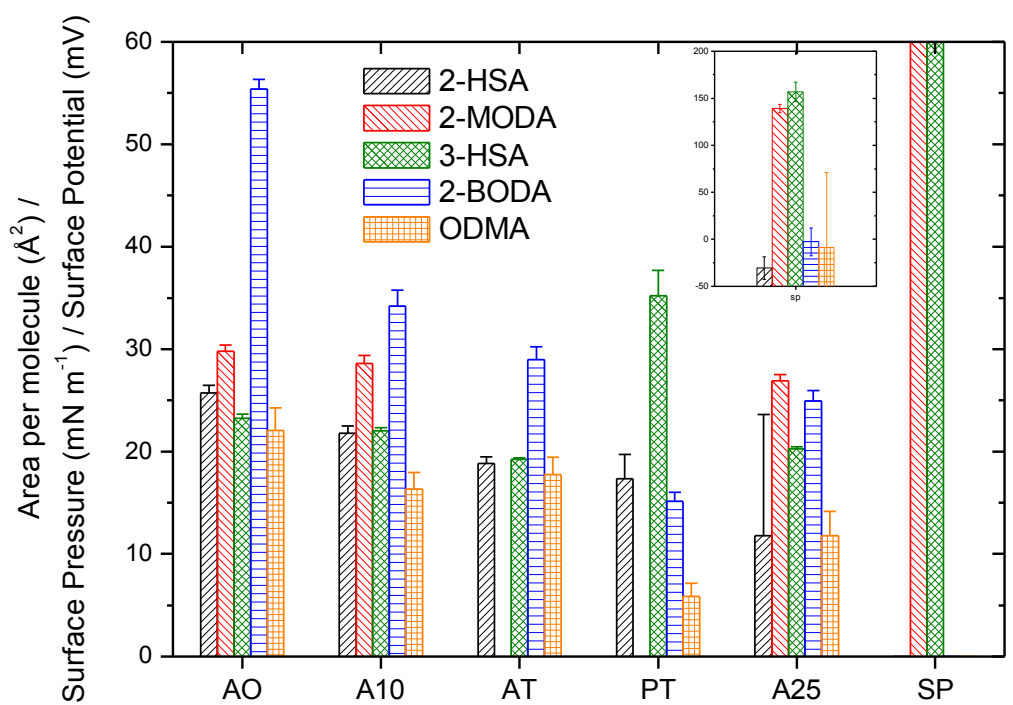

Figure 7.9 The overall characteristics of the substituted acids on a sodium bicarbonate aqueous subphase. The surface potential data exceeds the ordinate scale, thus an insert is included to highlight the differences in $\Delta V_{\max }$ for the substituted acids. (Key: $A O=$ the $A_{m}$ at onset, $A_{10}=$ the $A_{m}$ at $\Pi=10 \mathrm{mN} \mathrm{m}{ }^{-1}, A T=$ the $A_{m}$ at the phase transition, $P T=\Pi$ at the phase transition, $A_{25}=$ the $A_{m}$ at $\Pi=25 \mathrm{mN} \mathrm{m}^{-1}$, and $S P=$ the maximum surface potential). 
spacing, the mismatch in the size of the head group and tails leads to entropically driven disordering of the tails. This is supported by the surface potential profile which indicates a gradual loss of tilt with increasing pressure. The increase in the head-group size and therefore spacing may be associated with the inclusion of the carbon-bromide dipole-dipole interactions in the hydrogen-bonded network and for this set of surfactants the optimal cation-mediated hydrogen-bonded network formation. The large reduction in $\Delta \mathrm{V}_{\max }$ reflects the increased tilt, network formation and the rearrangement of the head group as the bromide shifts the system from intramonolayer hydrogen bonding to participating in the hydrogen-bonded network.

In summary, the data reflects three differing responses to the incorporation of sodium bicarbonate to the subphase. 2-BODA, 2-MODA and to a lesser extent 3-HSA display large decreases in potential in comparison with the water subphase. This indicates a strong interaction with the hypothesised subphase network. The extent of this change in potential with the introduction of sodium bicarbonate ions is much greater than that observed for ODA monolayers. Thus the addition of bulky and in terms of binding capacity, passive, functional groups results in an enhanced network formation. In comparison, the strong binding capacity of 2-HSA and ODMA results in a very strong and ordered monolayer/subphase interaction at the expense of intramonolayer interactions acting to destabilise the monolayer.

\subsubsection{Calcium Carbonate Crystallising Subphase}

The surface pressure and potential measurements for the substituted acids on CCCS are shown in Figure 7.10. In terms of the trends, as illustrated in Figure 7.11, the onset $\mathrm{A}_{\mathrm{m}}$ is in the order: 3-HSA $<2-\mathrm{HSA}<2-\mathrm{MODA} \approx 2$-BODA $<$ ODMA. The same order is present at $\Pi=10 \mathrm{mN} \mathrm{m}^{-1}$. Only at $\Pi=25 \mathrm{mN} \mathrm{m}^{-1}$ does the slow collapse of 2-HSA and ODMA propel them to the lowest $A_{m}$. 

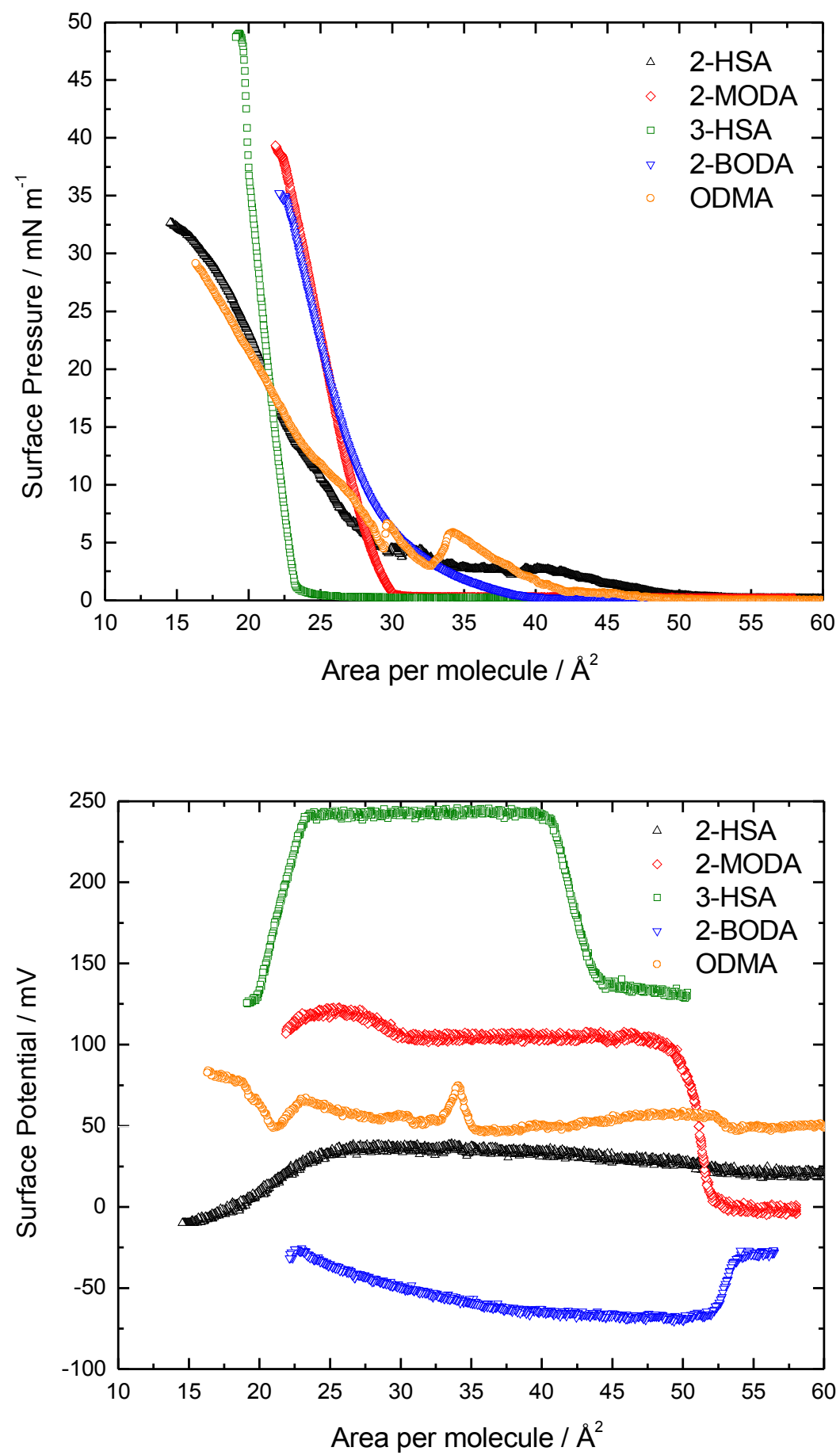

Figure 7.10 Surface pressure (top) and potential (bottom) isotherms of the substituted acids on CCCS.

2-MODA shows very little change with subphase in terms of the isotherm profile. There is a small shift to smaller $\mathrm{A}_{\mathrm{m}}$ moving from water, to $\mathrm{CaCl}_{2}, \mathrm{NaHCO}_{3}$ and finally to CCCS. On CCCS the monolayer is more compressible with a greater loss in $\mathrm{A}_{\mathrm{m}}$ at 
$\Pi=25 \mathrm{mN} \mathrm{m}^{-1}$. Although at a reduced magnitude, the surface potential profile for 2-MODA displays the typical features, including a small inflection at $\sim 30 \AA^{2}$ associated with the gradual loss of tilt. The suggestion of tilt is a deviation from ODA behaviour where in the mixed systems a corresponding inflection was only observed in systems containing $75 \%$ or more $\mathrm{ODOH}$.

Once again 3-HSA shows relatively little interaction with the subphase, as was the case on $\mathrm{CaCl}_{2}$ and $\mathrm{NaHCO}_{3}$. The $\mathrm{A}_{\mathrm{m}}$ at onset shows a consistent reduction moving from water, to $\mathrm{CaCl}_{2}, \mathrm{NaHCO}_{3}$ and finally to CCCS, with the largest change between water and $\mathrm{CaCl}_{2}$, which is attributed to the increased order brought about by the subphase ions. The phase transition at $\sim 20 \AA^{2}$ and a $\Pi$ of $\sim 36 \mathrm{mN} \mathrm{m}^{-1}$, is present on all the electrolyte subphases and neither the transition $A_{m}$ or pressure shifts with subphase. The surface potential profile (Figure 7.10) is also consistent with the other subphases. This apparent lack of interaction between 3-HSA and the subphases suggests that the movement of the hydroxy group by one position away from the acid group culminates in behaviour consistent with that of ODA, highlighting the importance of the 2-position.

In summary all the substituted acids show some affinity for $\mathrm{Ca}^{2+}$ binding. The extent of this binding and therefore the role electrostatics play in the interfacial interaction is dictated by the binding capacity of the head group, with 2-HSA and ODMA having the capacity for stable 5 or 6 -membered chelation rings. In general $\mathrm{Ca}^{2+}$ binding offsets the head group polarity thus leading to an increase in $\Delta \mathrm{V}_{\max }$. In contrast $\mathrm{NaHCO}_{3}$ enhances the head group polarity. The resulting interaction on CCCS is a mix of the cation and anion related effects in conjunction with steric and entropic effects. 


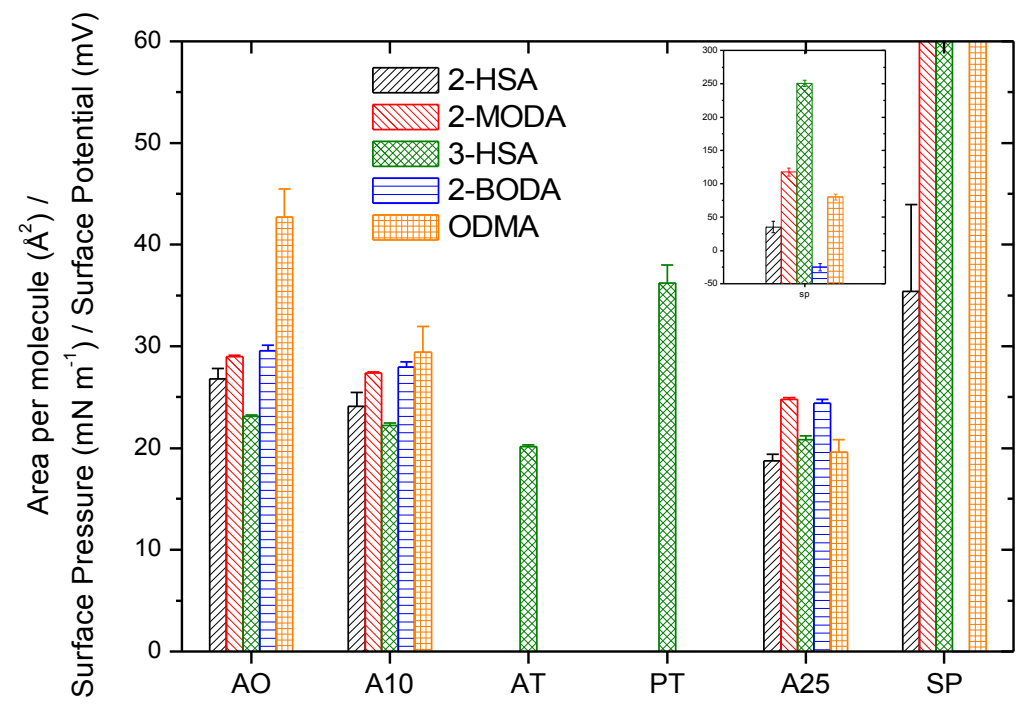

Figure 7.11 Substituted acid monolayer characteristics on CCCS. The surface potential data exceeds the ordinate scale, thus an insert is included to highlight the differences in $\Delta V_{\max }$ for the substituted acids. (Key: $A O$ $=$ the $A_{m}$ at onset, $A_{10}=$ the $A_{m}$ at $\Pi=10 \mathrm{mN} \mathrm{m}^{-1}, A T=$ the $A_{m}$ at the phase transition, $P T=\Pi$ at the phase transition, $A_{25}=$ the $A_{m}$ at $\Pi=25 \mathrm{mN} \mathrm{m}^{-1}$, and $S P=$ the maximum surface potential).

\subsubsection{Crystallisation}

Examination of the crystal properties identifies two significant changes from that observed for ODA monolayers 1) there is very little evidence of irregular growth in the substituted acid-derived crystals, and 2) the crystals exhibit a binary response to modification of the head group chemistry. The lack of irregularity points to this being a kinetic phenomenon, brought about by a reduction in the average monolayer charge density associated with the larger $A_{m} s$.

Notably all this variation in monolayer and subphase structure is not reflected in the morphology of the nucleated calcium carbonate crystals (Figure 7.12 and 7.13). Unlike the mixed monolayer systems there is little evidence for a surface pressure effect of crystal morphology. Further there is negligible variation associated with monolayer stability. In fact the results point to a rather bimodal morphological distribution, where oriented truncated rhombohedra predominate for 2-MODA and 
2-BODA monolayers, in contrast to more randomly oriented classical rhombohedra for ODMA and 2-HSA monolayers.

The occurrence of almost no facial orientational control under monolayers exhibiting the strongest monolayer/subphase interaction points to a lack of flexibility during nucleation. The monolayer crystal interaction is believed to be synergistic involving both mediums to accommodate defects. Therefore the inability of the strongly bound ODMA and 2-HSA monolayers to be moulded results in non-specific rhombohedral growth, an effect seen to the greatest extent for ODMA.

3-HSA is subtly, but importantly different from ODMA and 2-HSA, in that there is a weak propensity for oriented growth. This is an extension of the similarities between 3-HSA and ODA observed throughout this study. The inability of the hydroxyl group to actively participate in cation binding reduces its effect to a small reduction in head-group electron density (-0.380 c.f. -0.380 e for ODA, Figure 7.1) and a relatively small increase in head-group size, reduced by the propensity to form intramolecular hydrogen bonds.

The occurrence of highly oriented, truncated rhombohedra for 2-BODA and 2-MODA is supported by the assignment of $\sim 30 \%$ of the crystals to the $[1 \overline{1} .0]$ zone axis. This is an increase of $30-50 \%$ over what was achieved for the mixed systems. While these substitutions have not resulted in a change in the nucleated face from that observed for ODA (and/or ODA/ODOH), with the (11.15) face continuing to dominate the statistics, a subtle yet significant increase in the extent of preferential orientation occurs. This improvement in orientation is brought about by a balanced increase in the interfacial interaction, balanced in the sense that the propensity for preferential orientation is increased but not so that the interaction becomes dominated by cation-binding effects. That is, a more effective soap-like hydrogenbonded network is formed in these systems. 

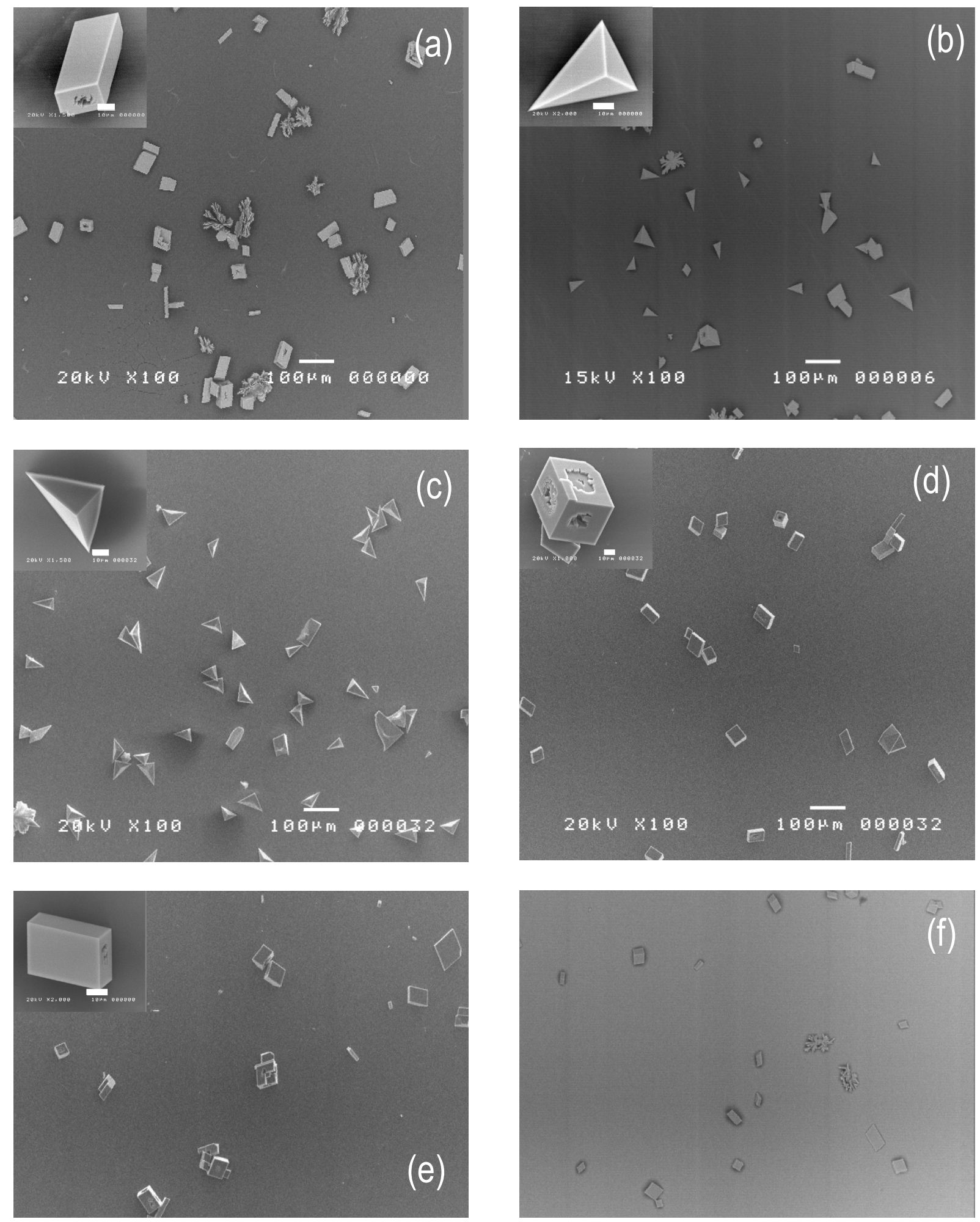

Figure 7.12 Representative SEM images of the crystals produced under the substituted acids at $\Pi=10 \mathrm{mN} \mathrm{m}^{-1}$. (a) 2-HSA, (b) 2-MODA, (c) 2-BODA, (d) 3-HSA, (e) ODMA, and (f) a surfactant free control. The inserts illustrate typical crystals where the scale bar is $1 \mu \mathrm{m}$. 

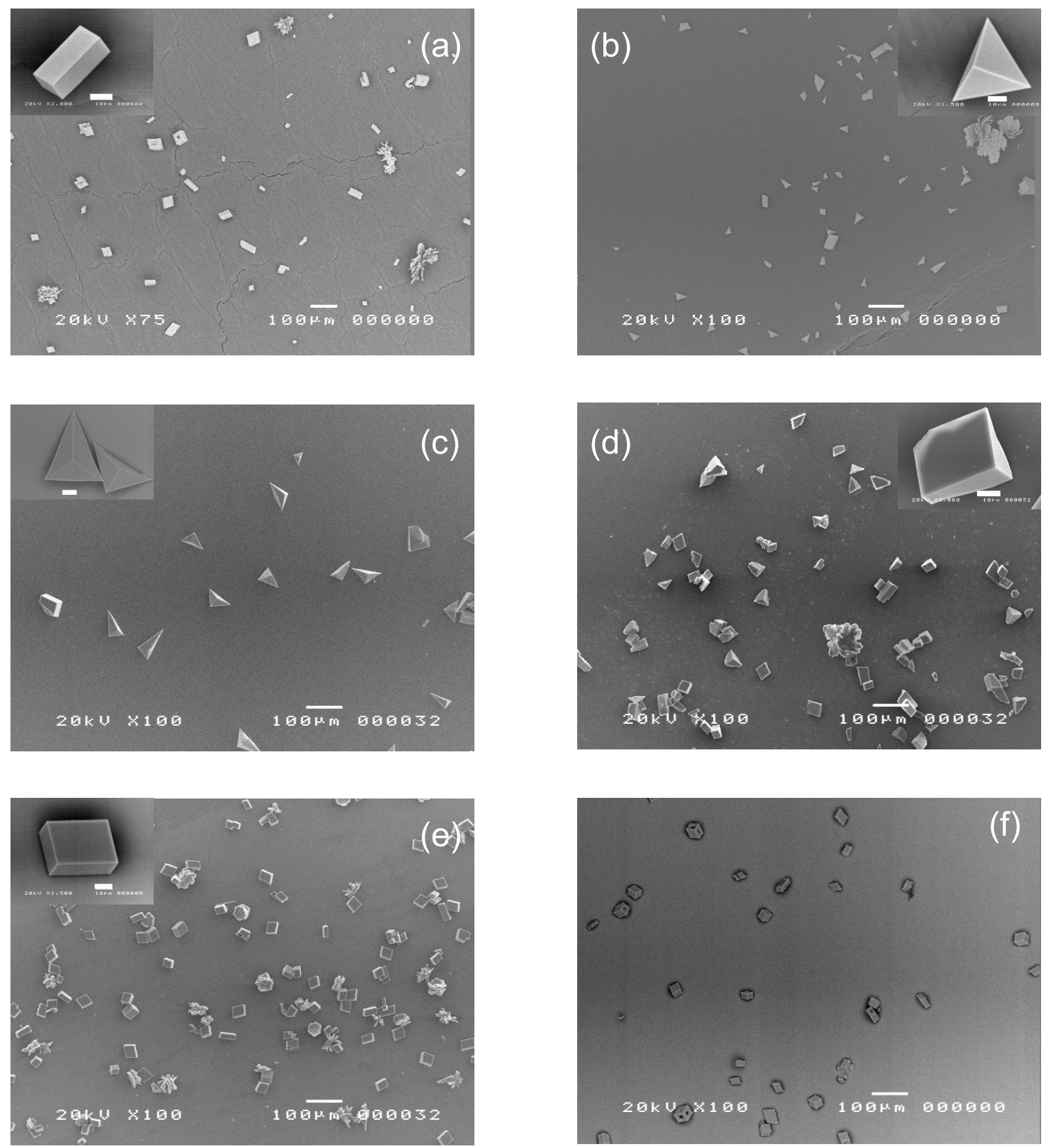

Figure 7.13 Representative crystal morphologies for the substituted acids at a constant surface pressure of $\Pi=25 \mathrm{mN} \mathrm{m}^{-1}$. (a) 2-HSA, (b) 2-MODA, (c) 2-BODA, (d) 3-HSA, (e) ODMA, and (f) a control free of surfactant. The inserts illustrate typical crystals where the scale bar is $1 \mu \mathrm{m}$.

The difference appears to lie in the mechanism by which the interfacial interaction is increased. Greater cation binding leads to inflexibility in the interface whereas 
increased bicarbonate interaction improves the interface interaction without dominating it.

\subsection{Conclusions}

The interaction between surfactant monolayers and subphase ions is a balance of many factors, including electron density, steric effects and ion binding. The polarity and cation binding strength play an important role in determining the nature of this interaction. 2-HSA and ODMA were found to exhibit strong calcium binding which led to an electrostatics-dominated interaction and subsequently random crystal growth. In contrast 2-MODA and 2-BODA, which were found to more favourably participate in the bicarbonate determined network, exhibited preferential orientation to a level much greater than that observed in the mixed systems. As for 3-HSA, the movement of the hydroxyl group one position led to a complete disruption of the interaction and consequently traits commensurate with ODA.

Unlike the mixed monolayer systems, there was little evidence of irregular growth and there was little variation in crystal morphology with surface pressure or monolayer stability.

\subsection{References}

[1] R. Aveyard, B. Binks, A. Cross, and P. Fletcher, "Dicarboxylic Acids: Stability of Insoluble Monolayers and Ionisation in Langmuir-Blodgett Multilayers," Colloids and Surfaces A: Physicochemical and Engineering Aspects, vol. 98, 1995, pp. 83-91.

[2] V. Vogel and D. Möbius, "Local Surface Potentials and Electric Dipole Moments of Lipid Monolayers: Contributions of the Water/Lipid and the Lipid/Air Interfaces," J.Colloid and Interface Sci., vol. 126 , 1988, pp. 408-420. 


\section{Chapter 8: Conclusions and Future Work}

There are many natural wonders in Nature, but from a materials stand point the design and fabrication of complex hierarchical biomineral structures has potentially the greatest appeal. The ability to control inorganic crystal orientation using an organic framework is awe-inspiring, especially given that this is achieved at ambient conditions using freely available materials. Before we could ever attempt such a complex design and fabrication process, we first must understand the basics. To this end, the work in this thesis has focused on improving our understanding of the molecular recognition process integral to the templation of biominerals. It is only through understanding the protocols or language of the interface, that we can begin to design similar structures ourselves.

The interfacial region is a very complex environment, involving many, often interdependent, phenomena. Therefore the use of a simplified Langmuir monolayer model has significant benefits. Perhaps the most important of these are its versatility. This study drew heavily on the ability to make subtle alterations to the surfactant chemistry and similarly the subphase, enabling a systematic comparative investigation of the interface.

The investigation of mixed ODA/ODOH monolayer behaviour on four different subphases: ultrapure water, calcium chloride, sodium bicarbonate and a CCCS, provided valuable insight into the interfacial interaction. From this study we hypothesised a cation-mediated hydrogen-bonded network, with parallels to the chain-like crystal structure of sodium bicarbonate. The intimate association of the monolayer and the subphase ions in a network, greatly facilitates the interfacial interaction. The bicarbonate anion is highly influential in terms of initiating this 
interfacial structure, and when present, should always be considered in any analysis of interfacial dynamics. The influence of the bicarbonate anion extends to the processes of templated crystal nucleation, where directionality, of some form, is required to achieve preferential orientation. We suggest that the formation of a hydrogen-bonded network provides the required degree of directionality necessary for preferential orientation of crystals.

Also of note, is how the introduction of alcohol molecules altered both monolayer behaviour and the interaction with the underlying subphase in the mixed monolayer systems. We found that alcohol-rich systems were not conducive to network formation, which we attributed to a reduction in charge density and head-group spacing brought about by the presence of the alcohol group. However at an optimum level (a 1:1 mix) the alcohol enhanced the network formation by mediating like-charge repulsion and participated in the hydrogen-bonding network.

In terms of the impact on calcium carbonate crystal nucleation and growth, the mixed monolayers display a spectrum of morphologies. The nature of this morphological manipulation was primarily two-fold. Firstly oriented growth, with increasing acid content there was a transition to truncated rhombohedra associated with nucleation on a face other than one of the $\{10.4\}$ family. The second phenomenon was elongated or lateral growth, which was found to be strongly associated with a good interfacial interaction. Evaluation and discussion of preferential orientation results leads to a number of conclusions regarding the role of charge density, lattice matching, symmetry and spatial geometry matching in faceselective nucleation. These include:

- the requirement of significant charge density to attain some level of oriented nucleation;

- the domination of face selectivity by electrostatics at high charge densities; but 
- more often face selectivity is achieved through some combination of spatial geometry, lattice matching, and electrostatics, in which case the concept of a cation-mediated hydrogen-bonded network may provide an improved understanding.

In terms of morphology, truncation, elongation, agglomeration and diffusion limited growth all contribute to the gross crystal shape. In addition the following points are of note:

- morphological irregularity and elongation are signs of a strong interfacial interaction, involving enhanced matching across the interface; and

- the strength of the interfacial interaction is also expressed in terms of monolayer stability.

Ultimately crystal templation beneath Langmuir monolayers is a complex interrelationship of many factors and to suggest that one factor (such as charge density) dominates is often too simplistic.

Exploring the role of the alcohol group further, involved the 2-HSA system, where the alcohol group is now covalently bonded to the fatty acid. As expected the combination of acid and hydroxyl groups led to enhanced cation binding. This electrostatic interaction was so dominant that it almost negated the influence of the bicarbonate anion completely. The result was an interfacial structure too rigid to promote oriented crystal growth.

The interactions between 2-HSA monolayers and the subphase is a complex balance of hydrogen bonding, electrostatics, steric effects and entropy. The incorporation of the hydroxyl moiety into the acid molecule leads to greater enhancement of the hydrogen bonding or electrostatic associated behaviour traits, depending on the subphase conditions, compared to the equivalent mixed ODA/ODOH systems. While on water or sodium bicarbonate the 2-HSA head group 
participates significantly in hydrogen bonding, upon addition of calcium ions the monolayer/subphase interactions are dominated by strong electrostatic binding interactions. Thus the covalently bound alcohol group plays a significantly greater role, considerably enhancing the dominate interaction: electrostatics.

Real-time in situ and ex situ crystallisation experiments have shown an interrelationship between the monolayer and the growing crystal in the calcium carbonate and 2-HSA system. Importantly this work has illustrated, for the first time, molecular rearrangement of the monolayer associated with crystal nucleation events. This rearrangement begins with a splitting of the monolayer diffraction peak, which is associated with a heterogeneous contraction of the monolayer lattice in response to nucleation events. Coinciding with the monolayer rearrangement is the appearance of a broad band of scattered intensity, while it remains unaccounted for, there is a suggestion that it may reflect a transition stage in the mineralisation process. Finally we observe intense Bragg peaks associated with crystallisation.

2-HSA binds calcium ions strongly resulting in a strong interfacial interaction which undoubtedly contributes to the observation of the monolayer rearrangement and the subsequent phenomena. However ex situ experiments, supported by GIXD data, do not show any preferential orientation. In fact the strong interfacial interaction appears to overshadow any bicarbonate anion effect and consequently results in a lack of orientational control of the nucleation event.

The interaction between surfactant monolayers and subphase ions is a balance of many factors, including electron density, steric effects and ion binding. The polarity and cation binding strength also play important roles in determining the nature of this interaction. 2-HSA and ODMA were found to exhibit strong calcium ion binding which led to an electrostatics-dominated interaction and subsequently random crystal growth. In contrast 2-MODA and 2-BODA, were found to more favourably participate in the bicarbonate anion determined network, and exhibited preferential 
orientation to a level much greater than that observed in the mixed systems. As for 3-HSA, the movement of the hydroxyl group one position away from the carboxylic acid group had a dramatic result. The shift led to a complete disruption of the interaction and consequently 3-HSA displayed traits commensurate with ODA.

\subsection{Future work}

In terms of future work there remains a lot of outstanding issues. Specific to this work, synchrotron-based examination of 2-MODA and 2-BODA, the two systems that produce the greatest orientation control would potentially offer the greatest insight. Having explored the 2-HSA system, comparing it with a system that does produce FSN should provide invaluable information regarding the process and the controlling factors. Associated with this the successful completion of XRR analysis of the crystallising systems. Data on the structure at the interface is important in terms of validating and expanding on our hypothesis.

Non-linear optic techniques such as sum frequency vibration spectroscopy has the capacity to probe the water structuring at the interface. The role of water is paramount to any true understanding of the interfacial interaction. And techniques like sum frequency vibration spectroscopy offer complementary information to that obtained from synchrotron based techniques. 


\section{Chapter 9: Appendix}

\section{Appendix A1 - NMR Data}

Proton and carbon 13 NMR of octadecylmalonic acid. 


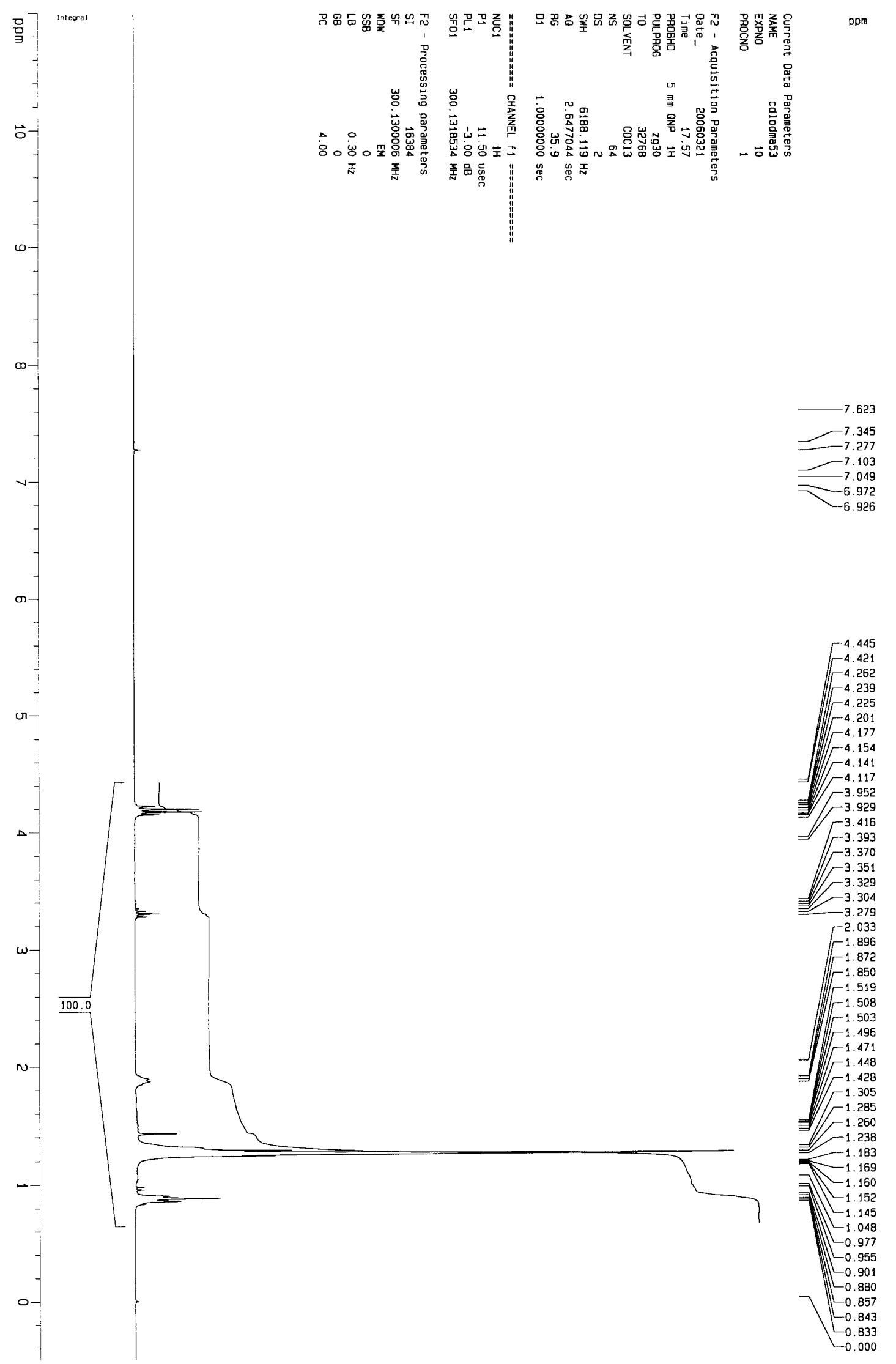




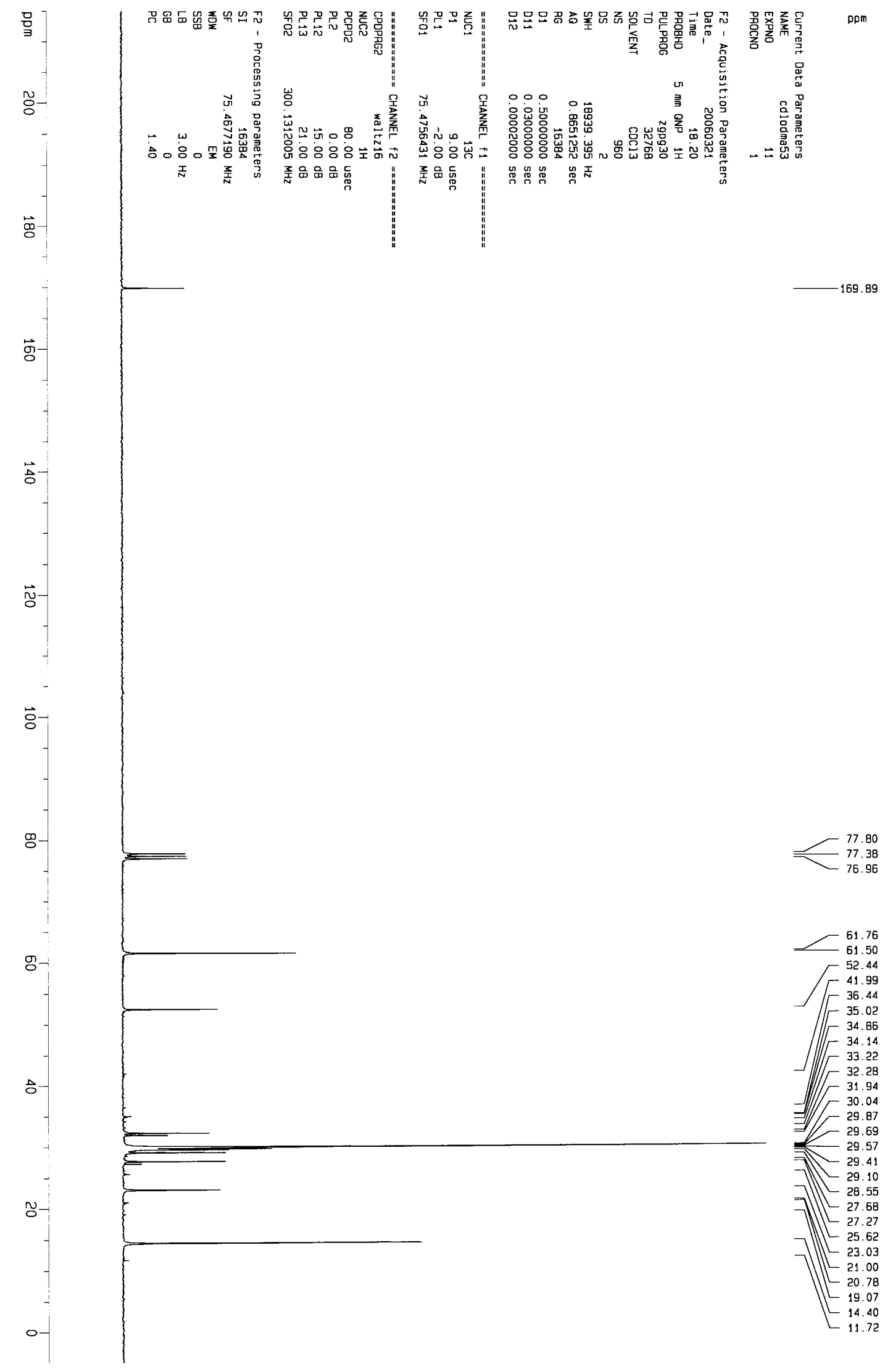




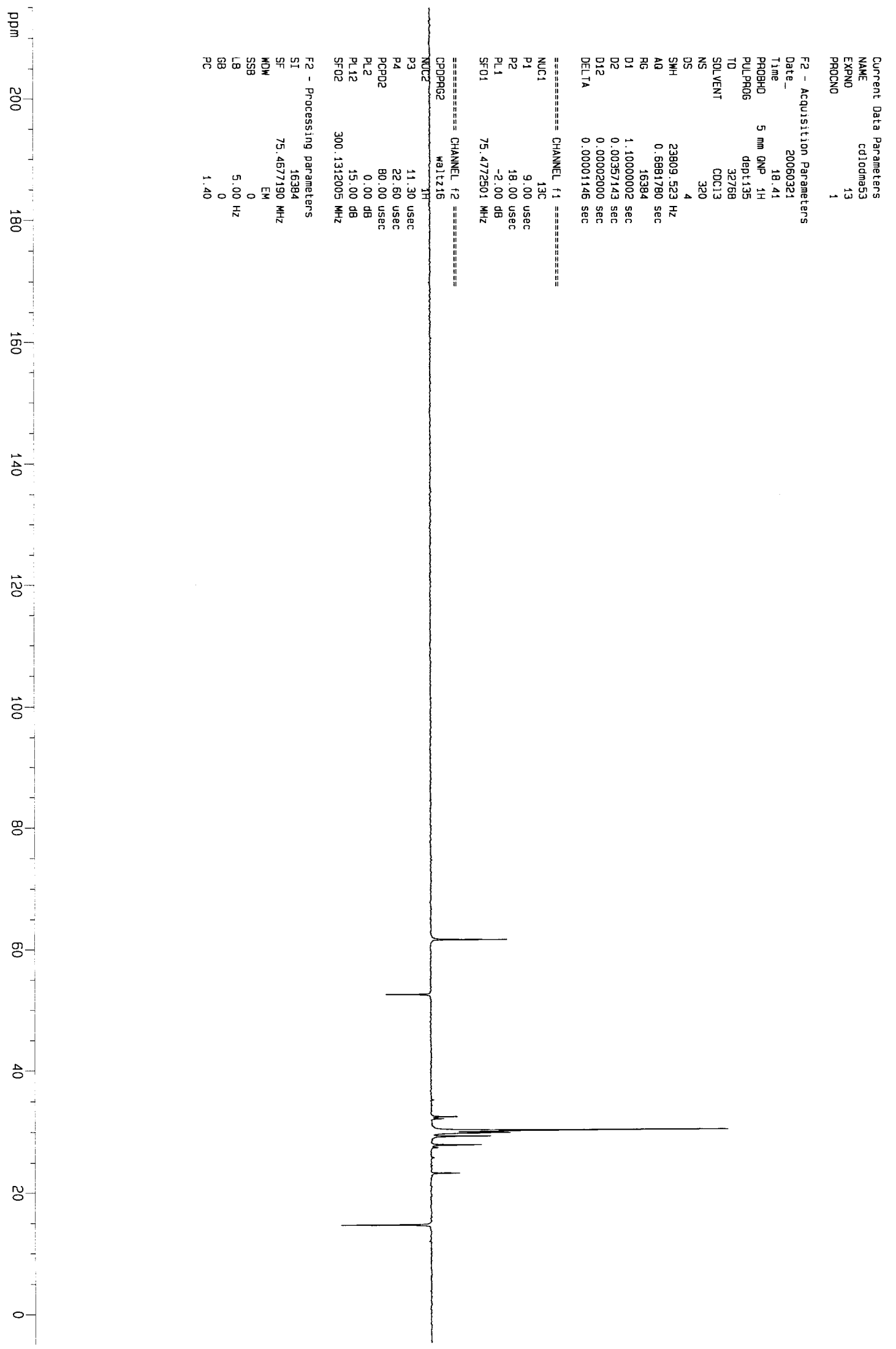




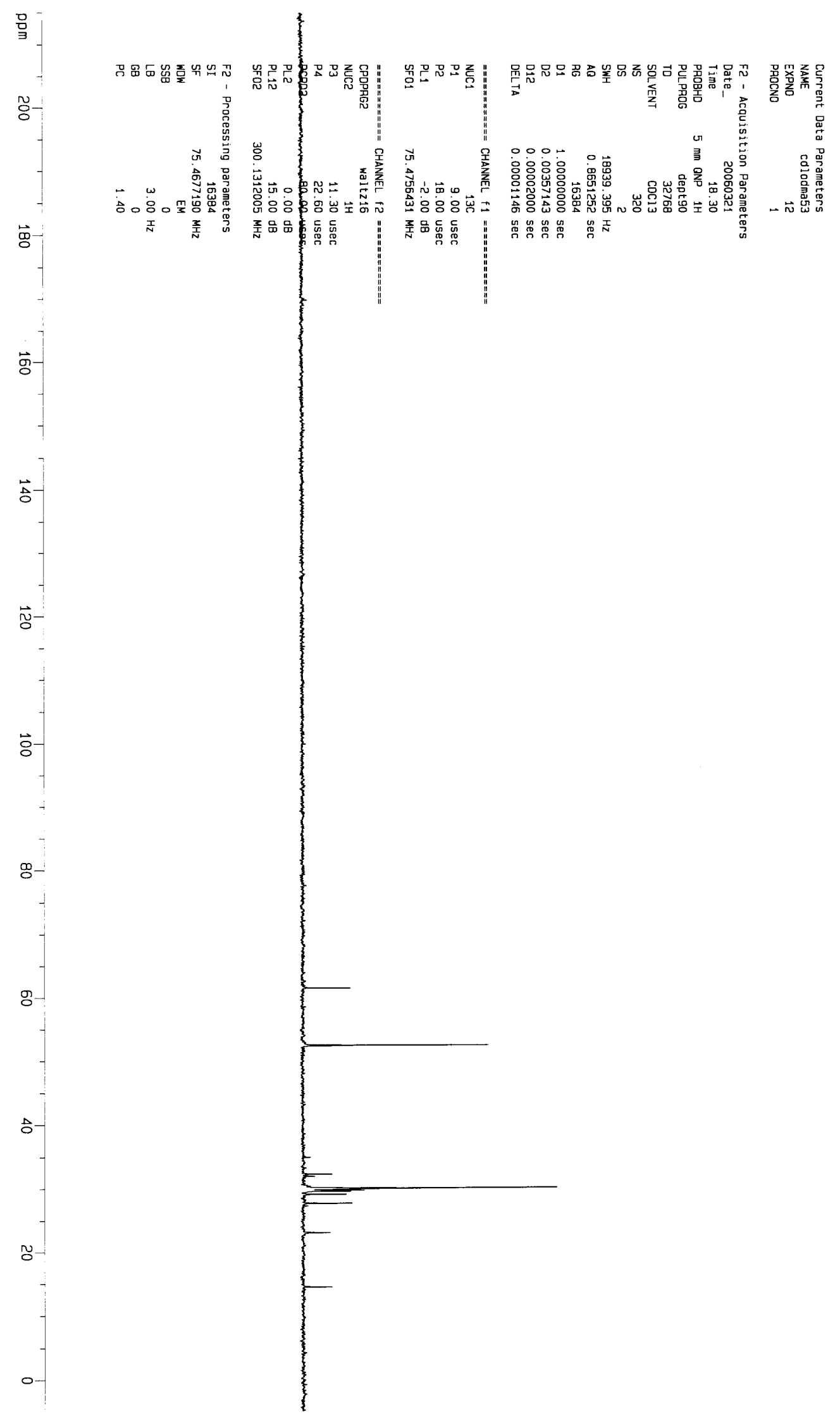




\section{Appendix A2 - Mixed Monolayer Trend Plots}

All 20 plots for the mixed monolayers on water, calcium chloride, sodium bicarbonate and the calcium carbonate crystallising subphase. 

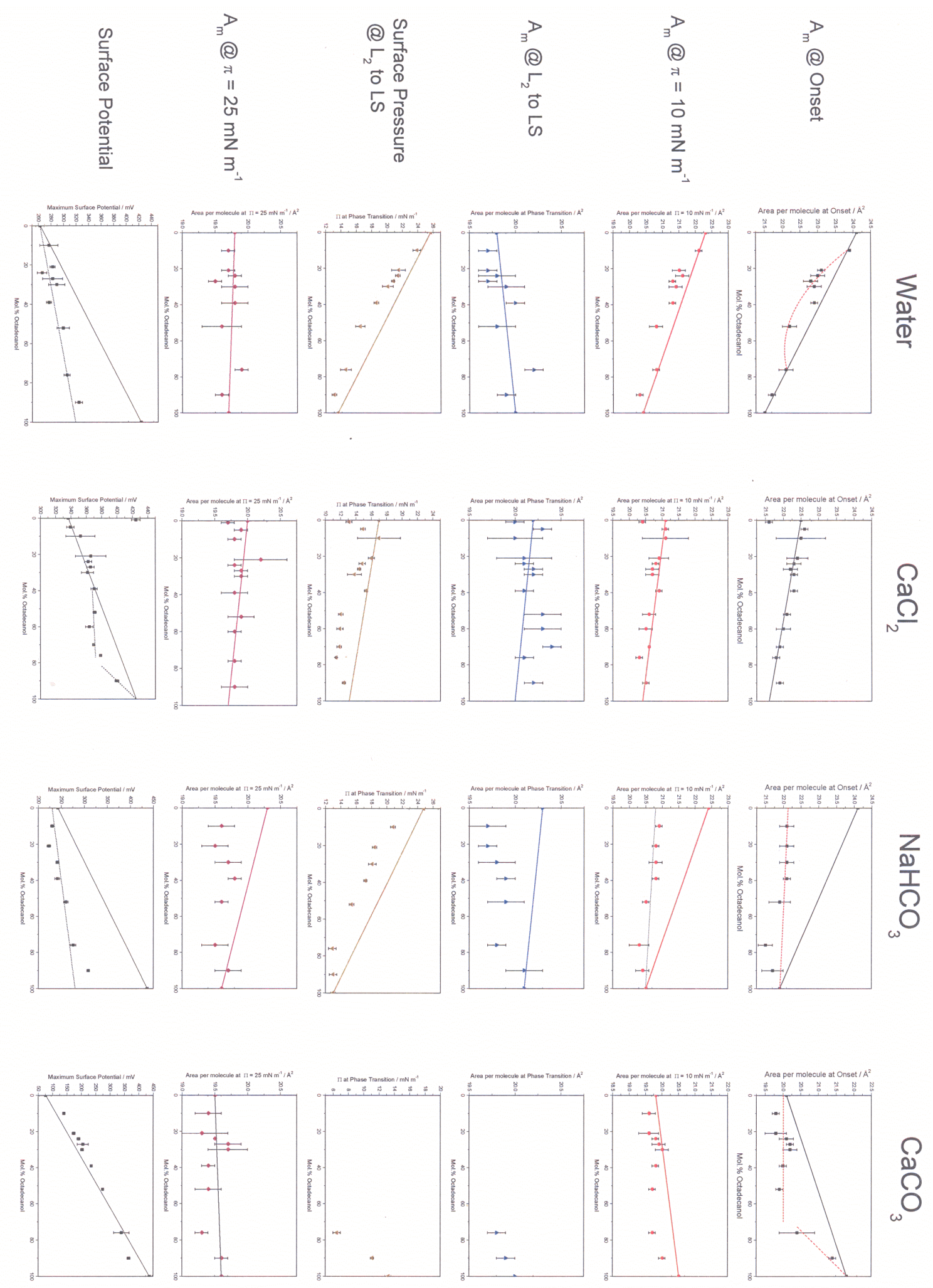


\section{Appendix A3 - GCS Models}

Table A1. Summary of the fitted parameters and the results of the numerical calculations. a) Water and calcium chloride, b) sodium bicarbonate and calcium carbonate.

\begin{tabular}{c|ccccc|} 
& \multicolumn{5}{|c}{ Water } \\
$\left(@ \Delta \mathrm{V}_{\mathrm{max}}\right)$ & $0 \%$ Alc & $25 \%$ Alc & $50 \%$ Alc & $75 \%$ Alc & $100 \%$ Alc \\
\hline $\mathrm{A}_{\mathrm{m}}(\AA)$ & 20.6 & 20.0 & 19.7 & 19.9 & 19.8 \\
$\kappa^{-1}(\AA)$ & 1915.3 & 1916.0 & 1916.8 & 1917.6 & 1918.4 \\
$\mathrm{~K}_{\mathrm{H}}$ & 5.400 & 5.400 & 5.400 & 5.400 & 1.979 \\
$\mathrm{~K}_{\mathrm{Na}}$ & -0.771 & -0.771 & -0.771 & -0.771 & -0.771 \\
$\mathrm{~K}_{\mathrm{Ca}}$ & 0.510 & 0.510 & 0.510 & 0.510 & 0.510 \\
$\mathrm{~K}_{\mathrm{OH}}$ & -50.00 & -50.00 & -50.00 & -50.00 & -50.00 \\
$K_{\mathrm{HCO}}$ & -50.00 & -50.00 & -50.00 & -50.00 & -50.00 \\
$K_{\mathrm{OHOH}}$ & -50.00 & -50.00 & -50.00 & -50.00 & -0.001 \\
$K_{\mathrm{OHOHCO}}$ & -50.00 & -50.00 & -50.00 & -50.00 & -0.452 \\
$\sigma_{\text {eqn5 }}$ & -0.002 & -0.002 & -0.002 & -0.001 & 0.000 \\
$\sigma_{\text {eqn6 }}$ & -0.002 & -0.002 & -0.002 & -0.001 & 0.000 \\
$\psi(0)(\mathrm{mV})$ & -162 & -158 & -151 & -139 & 0 \\
$\mathrm{pH} \mathrm{H}_{\mathrm{s}}$ & 2.9 & 2.9 & 3.0 & 3.2 & 5.6 \\
\cline { 2 - 6 } $\mathrm{A}_{\mathrm{c}}(\AA)(\mathrm{Fit})$ & 33.4 & 36.2 & 30.8 & 29.3 & 29.9 \\
$\mu_{1} / \varepsilon_{1}(\mathrm{Fit})$ & -122 & -157 & -95 & -74 & -113 \\
$\mu_{2}(\mathrm{mD})$ & 990.0 & 992.5 & 995.0 & 997.5 & 1000.0 \\
$\varepsilon_{2} @ \mathrm{LP}(\mathrm{Fit})$ & 78.5 & 76.1 & 6.6 & 78.5 & 78.5 \\
$\varepsilon_{2} @ \mathrm{HP}(\mathrm{Fit})$ & 4.1 & 3.3 & 4.7 & 5.5 & 6.8 \\
$\mu_{3}(\mathrm{mD})$ & 330.0 & 330.0 & 330.0 & 330.0 & 330.0 \\
$\varepsilon_{3} @ \mathrm{LP}(\mathrm{Fit})$ & 1.1 & 1.1 & 2.2 & 2.2 & 2.2 \\
$\mathcal{E}_{3} @ \mathrm{HP}(\mathrm{Fit})$ & 3.1 & 3.8 & 2.8 & 2.5 & 9.0 \\
$\mu_{1} / \varepsilon_{1}(\mathrm{mD})$ & -117 & -157 & -98 & -75 & -116 \\
$\mu_{2} / \mathcal{E}_{2}(\mathrm{mD})$ & 243 & 305 & 214 & 183 & 281 \\
$\mu_{3} / \varepsilon_{3}(\mathrm{mD})$ & 107 & 88 & 118 & 132 & 37 \\
$\mu \perp(\mathrm{mD})$ & 232 & 236 & 233 & 239 & 202 \\
$\mu^{\perp} / \mathrm{Aeo}$ & 425 & 443 & 446 & 453 & 385 \\
\cline { 2 - 5 }$\Delta \mathrm{V}_{\mathrm{calc}}(\mathrm{mV})$ & 262 & 285 & 295 & 314 & 385 \\
$\Delta \mathrm{V}_{\mathrm{Diff}}(\mathrm{mV})$ & -3.5 & -3.1 & 0.7 & 2.1 & -35.6
\end{tabular}




\begin{tabular}{c|ccccc|} 
& \multicolumn{5}{|c|}{ Calcium Chloride } \\
$\left(@ \Delta \mathrm{V}_{\text {max }}\right)$ & $0 \%$ Alc & $25 \%$ Alc & $50 \%$ Alc & $75 \%$ Alc & $100 \%$ Alc \\
\hline $\mathrm{A}_{\mathrm{m}}(\AA)$ & 20.2 & 19.3 & 19.9 & 19.7 & 19.5 \\
$\kappa^{-1}(\AA)$ & 12.4 & 12.4 & 12.4 & 12.4 & 12.4 \\
$\mathrm{~K}_{\mathrm{H}}$ & 5.400 & 5.400 & 5.400 & 5.422 & -67.135 \\
$\mathrm{~K}_{\mathrm{Na}}$ & -0.771 & -0.771 & -0.771 & -0.771 & -0.771 \\
$\mathrm{~K}_{\mathrm{Ca}}$ & 0.510 & 0.510 & 0.510 & 0.448 & -18.690 \\
$\mathrm{~K}_{\mathrm{OH}}$ & -50.00 & -50.00 & -50.00 & -50.00 & -50.00 \\
$K_{\mathrm{HCO}}$ & -50.00 & -50.00 & -50.00 & -50.00 & -50.00 \\
$K_{\mathrm{OHOH}}$ & -50.00 & -50.00 & -50.00 & -50.00 & -50.00 \\
$K_{\mathrm{OHOHCO}}$ & -50.00 & -50.00 & -50.00 & -50.00 & -50.00 \\
$\sigma_{\text {eqn5 }}$ & -0.027 & -0.026 & -0.025 & -0.022 & 0.000 \\
$\sigma_{\text {eqn6 }}$ & -0.027 & -0.026 & -0.025 & -0.022 & 0.000 \\
$\psi(0)(\mathrm{mV})$ & -33 & -32 & -31 & -29 & 0 \\
$\mathrm{pH}$ & 4.9 & 5.0 & 5.0 & 5.0 & 5.5 \\
\cline { 2 - 6 } $\mathrm{A}_{\mathrm{c}}(\AA)(\mathrm{Fit})$ & 36.0 & 44.8 & 48.0 & 42.1 & 45.9 \\
$\mu_{1} / \varepsilon_{1}(\mathrm{Fit})$ & -110 & -236 & -280 & -205 & -228 \\
$\mu_{2}(\mathrm{mD})$ & 990.0 & 992.5 & 995.0 & 997.5 & 1000.0 \\
$\varepsilon_{2} @ \mathrm{LP}(\mathrm{Fit})$ & 78.5 & 78.5 & 78.5 & 78.5 & 78.5 \\
$\mathcal{E}_{2} @ \mathrm{HP}(\mathrm{Fit})$ & 11.4 & 2.5 & 2.2 & 2.9 & 2.8 \\
$\mu_{3}(\mathrm{mD})$ & 330.0 & 330.0 & 330.0 & 330.0 & 330.0 \\
$\varepsilon_{3} @ \mathrm{LP}(\mathrm{Fit})$ & 1.1 & 5.0 & 4.3 & 1.1 & 1.1 \\
$\mathcal{E}_{3} @ \mathrm{HP}(\mathrm{Fit})$ & 1.5 & 8.4 & 10.0 & 4.6 & 3.8 \\
$\mu_{1} / \varepsilon_{1}(\mathrm{mD})$ & -109 & -242 & -281 & -207 & -232 \\
$\mu_{2} / \varepsilon_{2}(\mathrm{mD})$ & 87 & 399 & 456 & 346 & 361 \\
$\mu_{3} / \varepsilon_{3}(\mathrm{mD})$ & 215 & 39 & 33 & 71 & 87 \\
$\mu \perp(\mathrm{mD})$ & 192 & 196 & 208 & 209 & 215 \\
$\mu^{\perp} / \mathrm{AeO}$ & 358 & 383 & 393 & 400 & 416 \\
\cline { 2 - 6 }$\Delta \mathrm{V}_{\text {calc }}(\mathrm{mV})$ & 325 & 350 & 362 & 371 & 416 \\
$\Delta \mathrm{V}_{\text {expt }}(\mathrm{mV})$ & 339 & 360 & 370 & 378 & 424 \\
$\Delta \mathrm{V}_{\mathrm{Diff}}(\mathrm{mV})$ & -13.7 & -9.9 & -8.3 & -6.6 & -8.3
\end{tabular}




\begin{tabular}{|c|c|c|c|c|c|}
\hline \multirow[b]{2}{*}{ (@ $\left.@ \mathrm{~V}_{\max }\right)$} & \multicolumn{5}{|c|}{ Sodium Bicarbonate } \\
\hline & $0 \%$ Alc & $25 \%$ Alc & $50 \%$ Alc & $75 \%$ Alc & $100 \%$ Alc \\
\hline $\mathrm{A}_{\mathrm{m}}(\AA)$ & 19.4 & 19.6 & 19.0 & 19.7 & 19.2 \\
\hline$\kappa^{-1}(\AA)$ & 21.5 & 21.5 & 21.5 & 21.5 & 21.5 \\
\hline $\mathrm{K}_{\mathrm{H}}$ & 3.788 & 4.072 & 5.402 & 5.401 & 5.400 \\
\hline $\mathrm{K}_{\mathrm{Na}}$ & 1.102 & 1.092 & -0.586 & -0.772 & -0.771 \\
\hline $\mathrm{K}_{\mathrm{Ca}}$ & 0.510 & 0.510 & 0.510 & 0.510 & 0.510 \\
\hline $\mathrm{K}_{\mathrm{OH}}$ & -50.00 & -50.00 & -50.00 & -50.00 & -50.00 \\
\hline$K_{\mathrm{HCO} 3}$ & -50.00 & -50.00 & -50.00 & -50.00 & -50.00 \\
\hline$K_{\mathrm{OHOH}}$ & -50.00 & -50.00 & -50.00 & -50.00 & -50.00 \\
\hline КонОНсоз $_{\mathrm{OH}}$ & -50.00 & -50.00 & -50.00 & -50.00 & -50.00 \\
\hline$\sigma_{\text {eqn5 }}$ & -0.058 & -0.052 & -0.045 & -0.034 & 0.000 \\
\hline$\sigma_{\text {eqn6 }}$ & -0.058 & -0.052 & -0.046 & -0.034 & -0.001 \\
\hline$\psi(0)(\mathrm{mV})$ & -101 & -96 & -89 & -76 & -2 \\
\hline $\mathrm{pH}_{\mathrm{s}}$ & 4.3 & 4.4 & 4.5 & 4.7 & 6.0 \\
\hline $\mathrm{A}_{\mathrm{c}}(\AA)(\mathrm{Fit})$ & 47.6 & 39.8 & 42.7 & 46.6 & 43.7 \\
\hline$\mu_{1} / \varepsilon_{1}$ (Fit) & -145 & -86 & -157 & -187 & -239 \\
\hline$\mu_{2}(\mathrm{mD})$ & 990.0 & 992.5 & 995.0 & 997.5 & 1000.0 \\
\hline$\varepsilon_{2} @$ LP (Fit) & 78.5 & 78.3 & 78.5 & 78.5 & 6.2 \\
\hline$\varepsilon_{2} @ \mathrm{HP}$ (Fit) & 5.0 & 7.6 & 4.7 & 3.3 & 2.9 \\
\hline$\mu_{3}(\mathrm{mD})$ & 330.0 & 330.0 & 330.0 & 330.0 & 330.0 \\
\hline$\varepsilon_{3} @$ LP (Fit) & 1.1 & 1.1 & 2.2 & 3.8 & 2.2 \\
\hline$\varepsilon_{3} @ \mathrm{HP}$ (Fit) & 2.6 & 2.6 & 2.8 & 5.1 & 2.8 \\
\hline$\mu_{1} / \varepsilon_{1}(\mathrm{mD})$ & -148 & -89 & -164 & -190 & -247 \\
\hline$\mu_{2} / \varepsilon_{2}(\mathrm{mD})$ & 199 & 131 & 211 & 306 & 349 \\
\hline$\mu_{3} / \varepsilon_{3}(\mathrm{mD})$ & 126 & 128 & 117 & 64 & 117 \\
\hline$\mu^{\perp}(\mathrm{mD})$ & 177 & 169 & 164 & 180 & 218 \\
\hline$\mu^{\perp} /$ Aeo & 343 & 326 & 324 & 345 & 428 \\
\hline$\Delta \mathrm{V}_{\text {calc }}(\mathrm{mV})$ & 242 & 230 & 235 & 269 & 425 \\
\hline$\Delta \mathrm{V}_{\text {expt }}(\mathrm{mV})$ & 250 & 242 & 257 & 275 & 436 \\
\hline$\Delta \mathrm{V}_{\text {Diff }}(\mathrm{mV})$ & -7.7 & -11.7 & -22.3 & -6.5 & -10.1 \\
\hline
\end{tabular}




\begin{tabular}{|c|c|c|c|c|c|}
\hline \multirow[b]{2}{*}{$\left(@ \Delta \mathrm{V}_{\max }\right)$} & \multicolumn{5}{|c|}{ Calcium Carbonate } \\
\hline & $0 \%$ Alc & $25 \%$ Alc & $50 \%$ Alc & $75 \%$ Alc & $100 \%$ Alc \\
\hline $\mathrm{A}_{\mathrm{m}}(\AA)$ & 27.8 & 19.1 & 19.5 & 19.5 & 19.1 \\
\hline$\kappa^{-1}(\AA)$ & 15.2 & 15.2 & 15.2 & 15.2 & 15.2 \\
\hline $\mathrm{K}_{\mathrm{H}}$ & 5.397 & 5.399 & 5.401 & 5.400 & -4081.23 \\
\hline $\mathrm{K}_{\mathrm{Na}}$ & -0.760 & -0.717 & -0.748 & -0.771 & -28.334 \\
\hline $\mathrm{K}_{\mathrm{Ca}}$ & 0.508 & 0.512 & 0.510 & 0.510 & -1228.29 \\
\hline $\mathrm{K}_{\mathrm{OH}}$ & -10.00 & -50.00 & -50.00 & -50.00 & -10.00 \\
\hline$K_{\mathrm{HCO} 3}$ & -10.00 & -50.00 & -50.00 & -50.00 & -10.00 \\
\hline$K_{\mathrm{OHOH}}$ & -10.00 & -50.00 & -50.00 & -50.00 & -10.00 \\
\hline$K_{\mathrm{OHOHCO} 3}$ & -10.00 & -50.00 & -50.00 & -50.00 & -10.00 \\
\hline$\sigma_{\text {eqn5 }}$ & -0.030 & -0.030 & -0.029 & -0.026 & 0.000 \\
\hline$\sigma_{\text {eqn6 }}$ & -0.030 & -0.030 & -0.029 & -0.026 & 0.000 \\
\hline$\psi(0)(\mathrm{mV})$ & -41 & -42 & -41 & -38 & 0 \\
\hline $\mathrm{pH}_{\mathrm{s}}$ & 5.1 & 5.3 & 5.3 & 5.3 & 6.0 \\
\hline $\mathrm{A}_{\mathrm{c}}(\AA) \quad$ (Fit) & 46.3 & 46.5 & 44.1 & 44.4 & 42.7 \\
\hline$\mu_{1} / \varepsilon_{1}$ (Fit) & -64 & -162 & -176 & -223 & -218 \\
\hline$\mu_{2}(\mathrm{mD})$ & 990.0 & 992.5 & 995.0 & 997.5 & 1000.0 \\
\hline$\varepsilon_{2} @ \mathrm{LP}$ (Fit) & 78.5 & 78.5 & 19.2 & 78.5 & 78.5 \\
\hline$\varepsilon_{2} @ \mathrm{HP}$ (Fit) & 11.2 & 3.9 & 4.2 & 2.6 & 2.9 \\
\hline$\mu_{3}(\mathrm{mD})$ & 330.0 & 330.0 & 330.0 & 330.0 & 330.0 \\
\hline$\varepsilon_{3} @ \mathrm{LP}$ (Fit) & 5.0 & 5.0 & 1.1 & 3.9 & 2.2 \\
\hline$\varepsilon_{3} @ \mathrm{HP}$ (Fit) & 10.0 & 9.6 & 3.0 & 10.0 & 3.3 \\
\hline$\mu_{1} / \varepsilon_{1}(\mathrm{mD})$ & -50 & -167 & -180 & -227 & -227 \\
\hline$\mu_{2} / \varepsilon_{2}(\mathrm{mD})$ & 89 & 257 & 237 & 379 & 346 \\
\hline$\mu_{3} / \varepsilon_{3}(\mathrm{mD})$ & 35 & 34 & 108 & 33 & 98 \\
\hline$\mu^{\perp}(\mathrm{mD})$ & 74 & 123 & 164 & 184 & 217 \\
\hline$\mu^{\perp} /$ Aeo & 100 & 243 & 317 & 356 & 428 \\
\hline$\Delta \mathrm{V}_{\text {calc }}(\mathrm{mV})$ & 59 & 201 & 276 & 317 & 428 \\
\hline$\Delta V_{\text {expt }}(m V)$ & 65 & 203 & 276 & 326 & 433 \\
\hline$\Delta \mathrm{V}_{\text {Diff }}(\mathrm{mV})$ & -6.3 & -2.0 & 0.1 & -8.2 & -5.2 \\
\hline
\end{tabular}



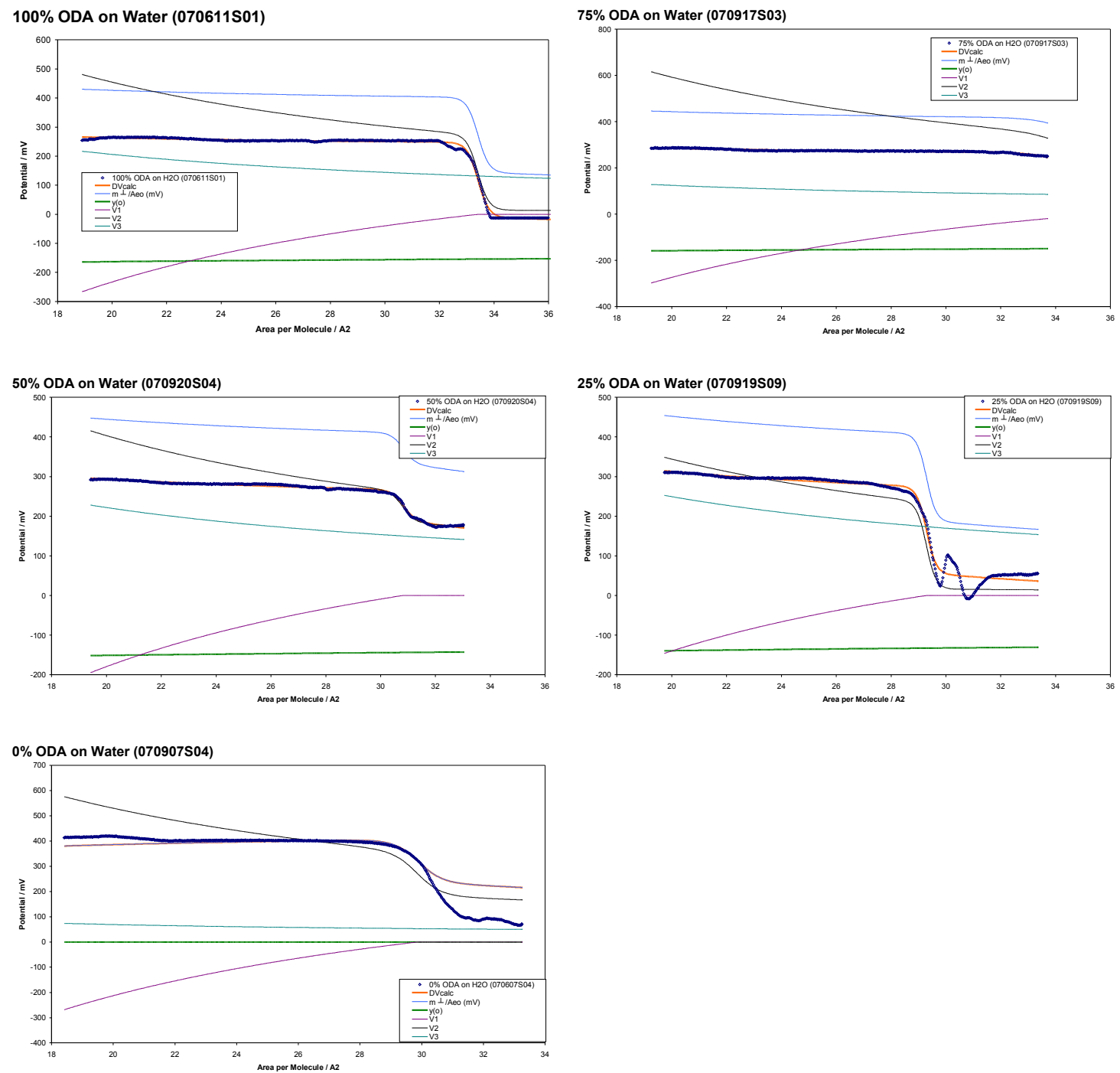

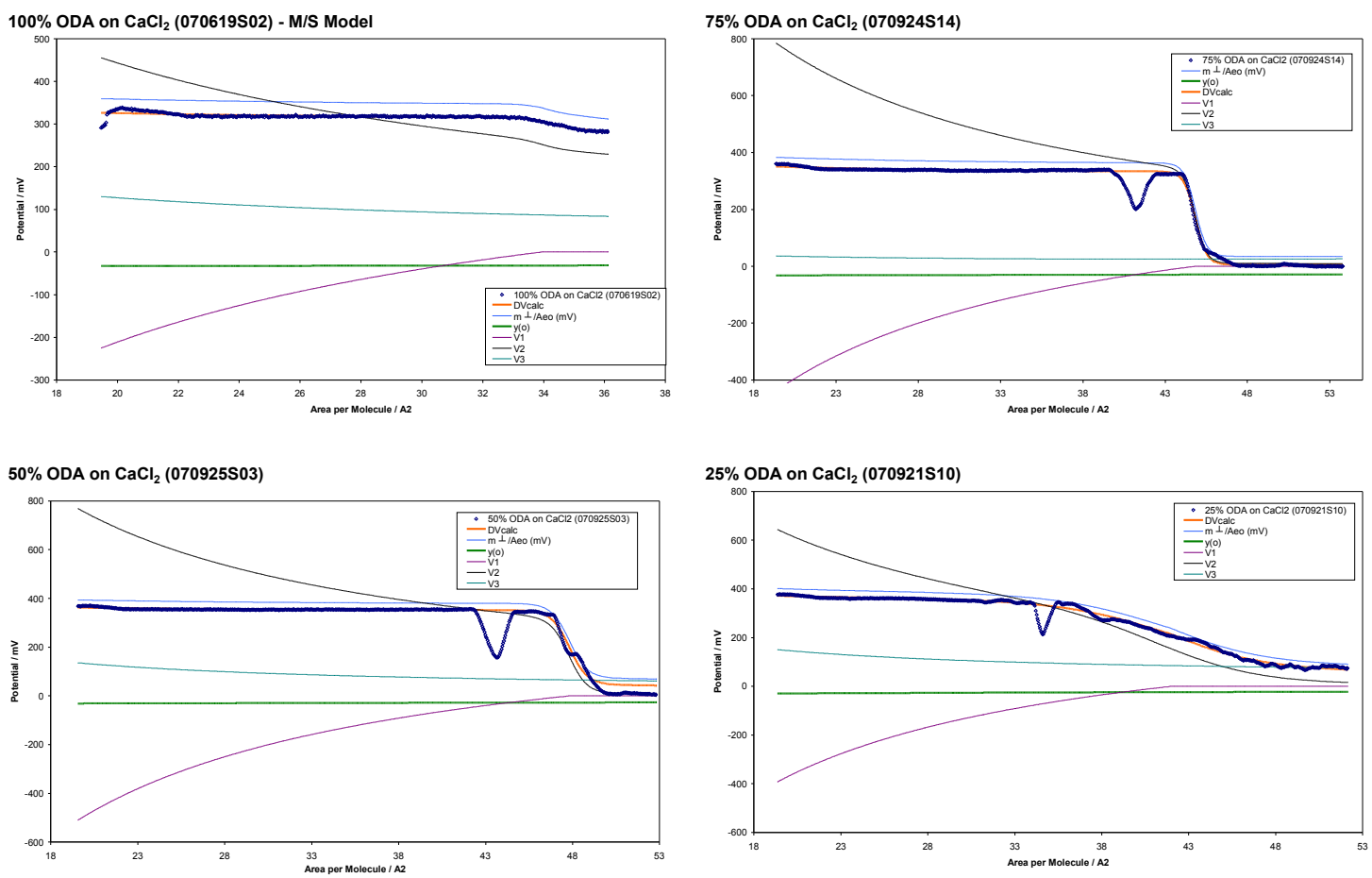

0\% ODA on $\mathrm{CaCl}_{2}(070612 \mathrm{~S} 04)$

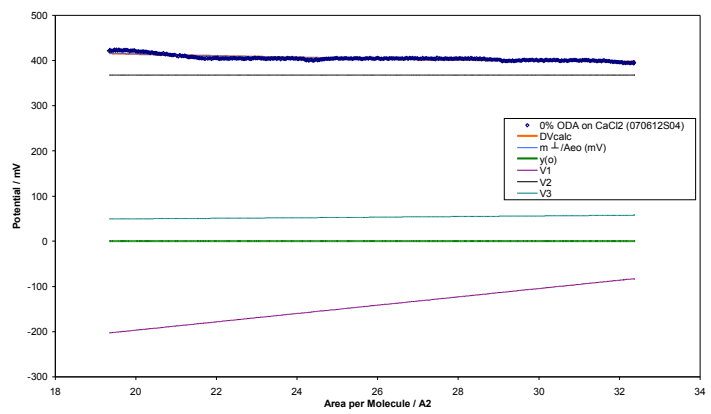



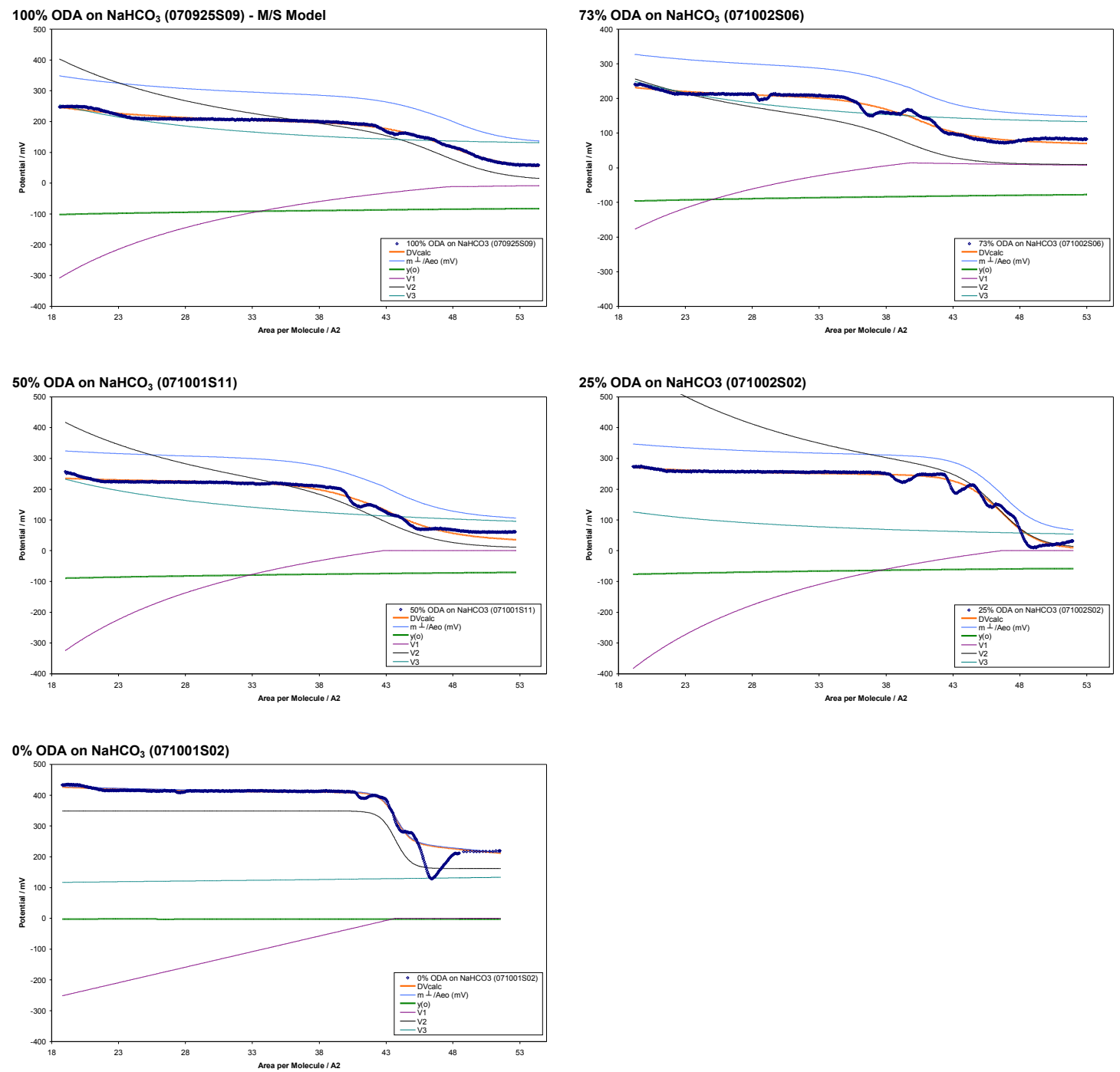

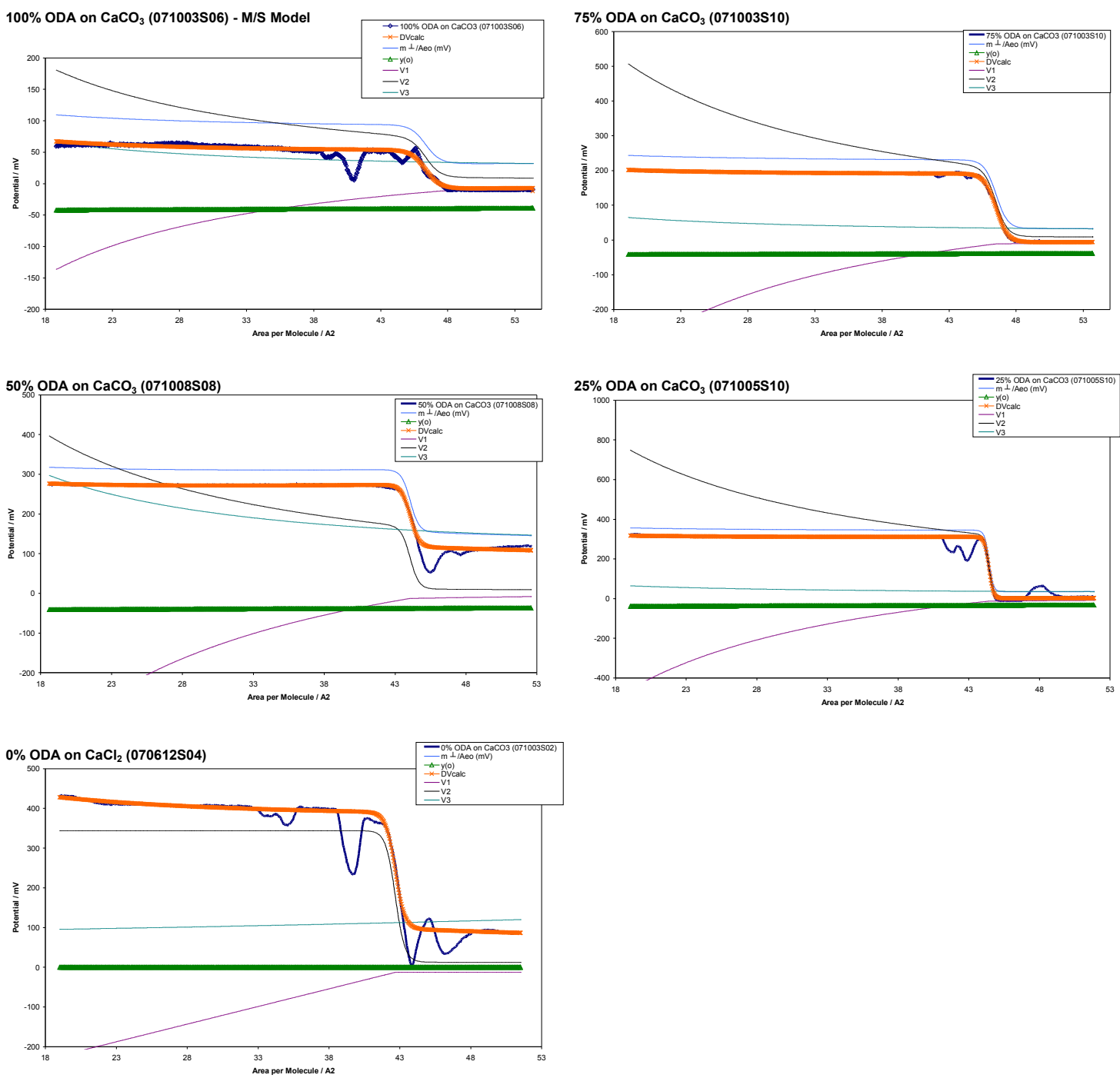


\section{Appendix A4 - Crystal Orientations}

Faces taken from:

- $\quad$ www.mincryst, ICDD (Calcite 5-586).

- Joint Committee on Powder Diffraction Standards - International Center for Diffraction Data, Swarthmore, U. K. File No. 5-586 Calcite. 


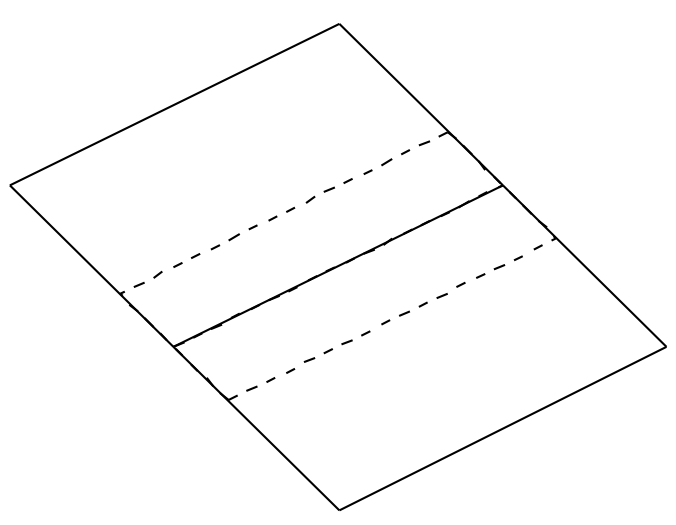

1. $<110>$

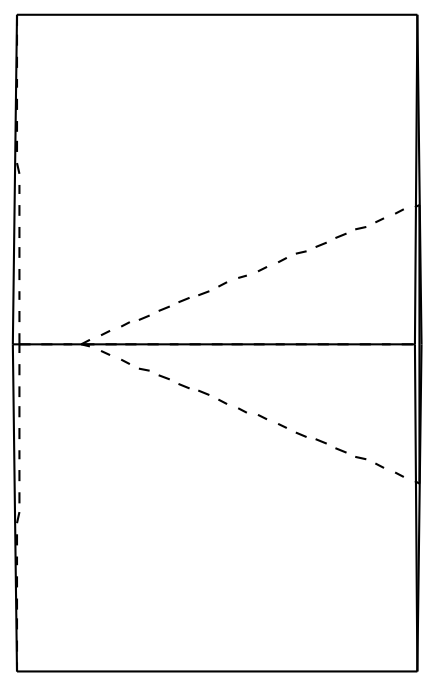

3. $<10-4>$

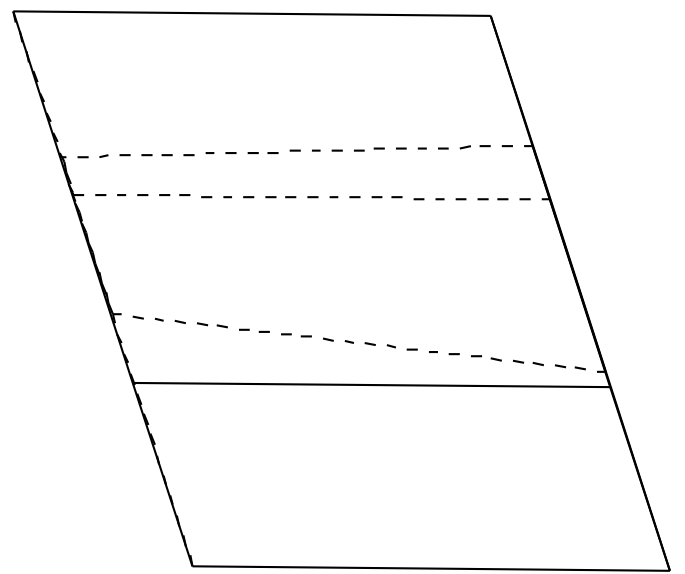

2. $<212>$

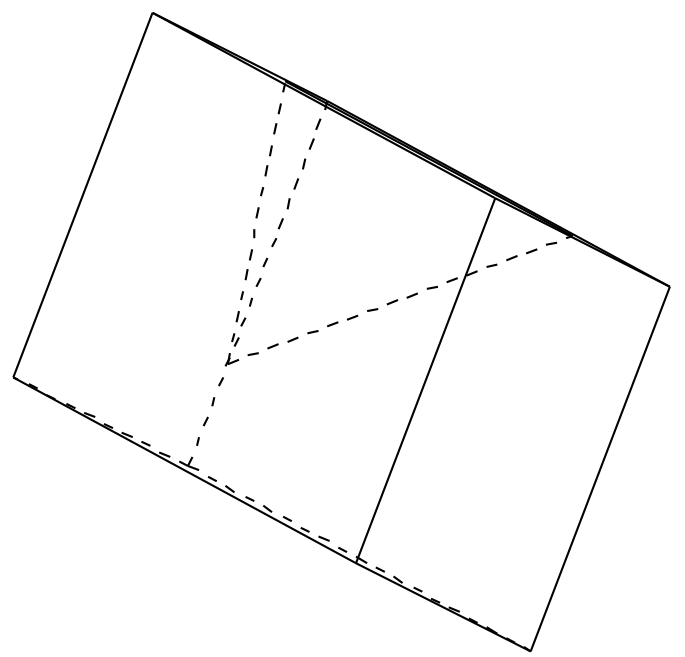

4. $<116>$ 


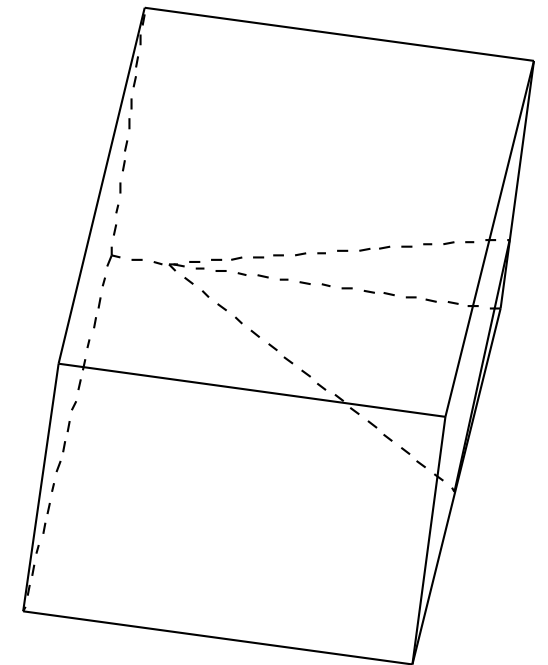

6. $<21-11>$

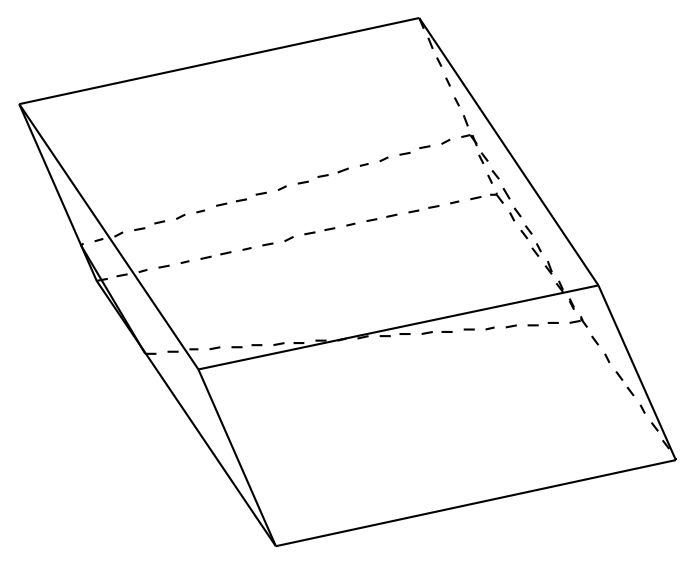

8. $<211>$

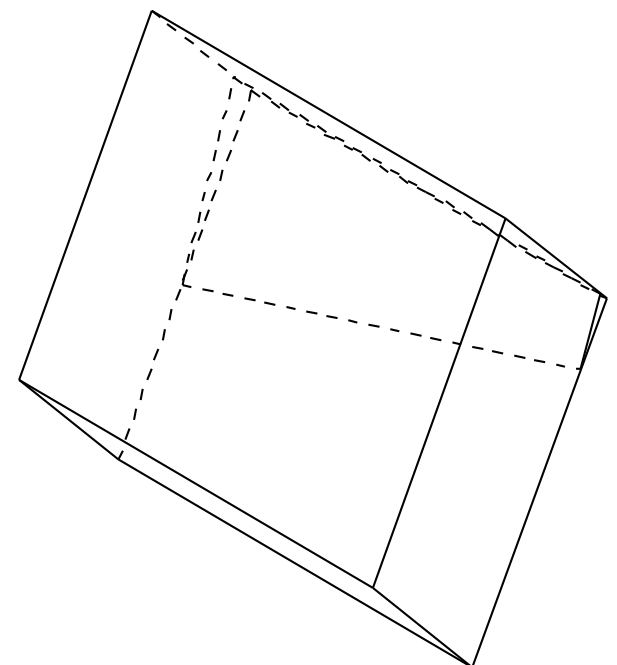

7. $<217>$

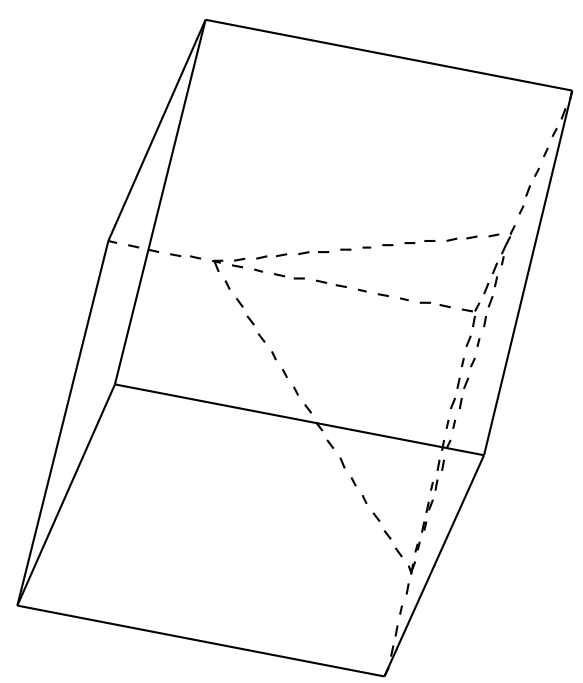

9. $<21-8>$ 


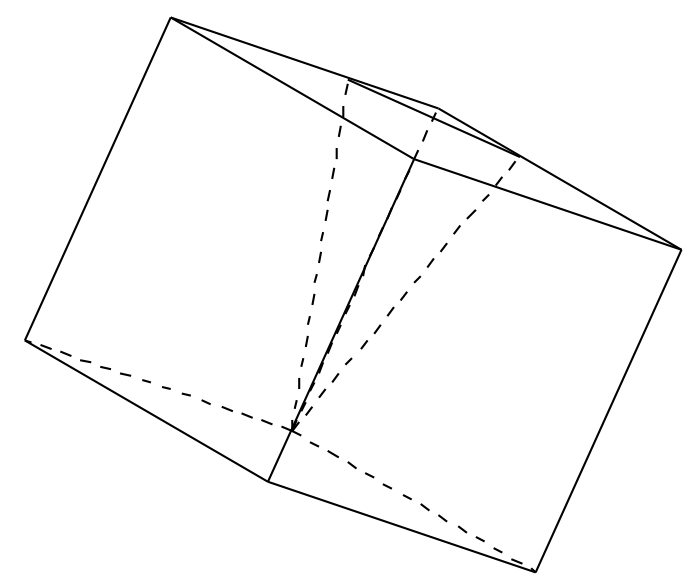

10. $<015>$

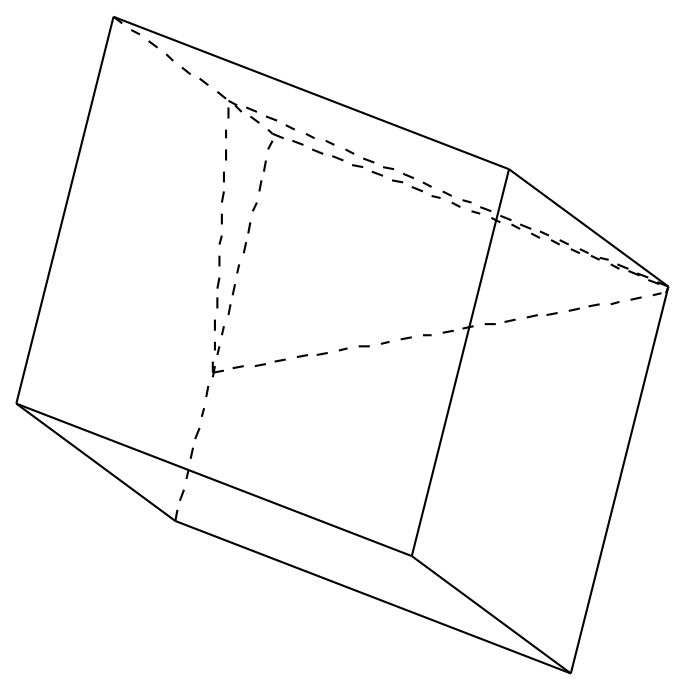

14. $<229>$

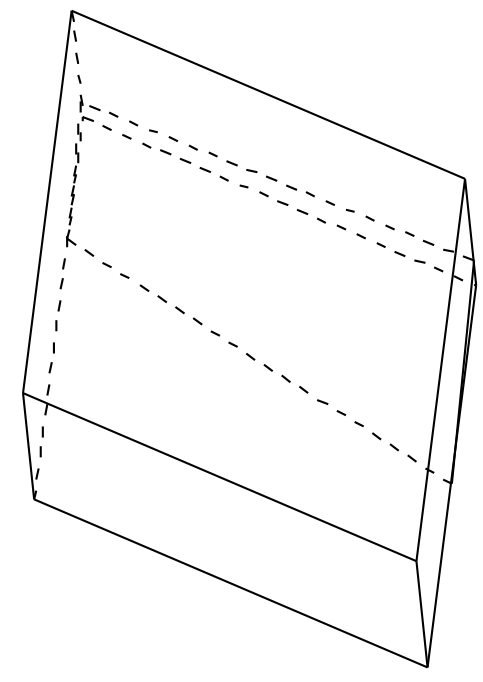

11. $<315>$ 


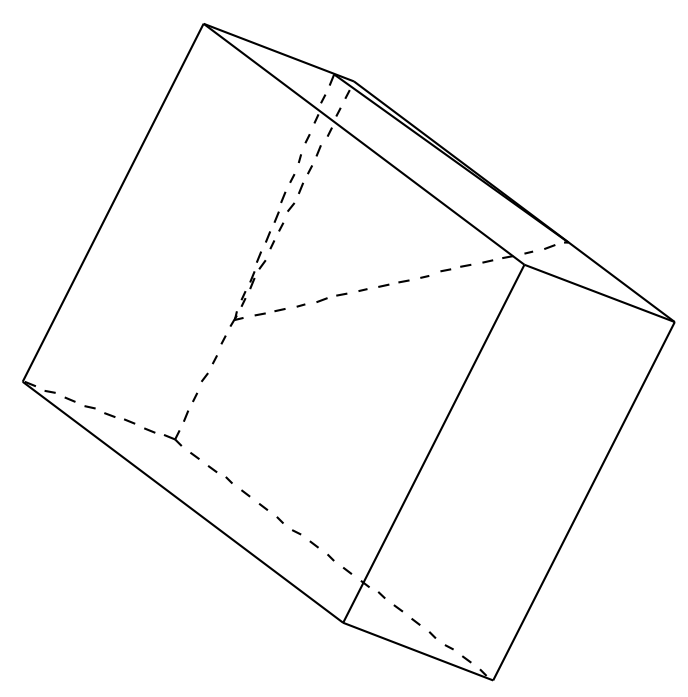

15. $<2110>$

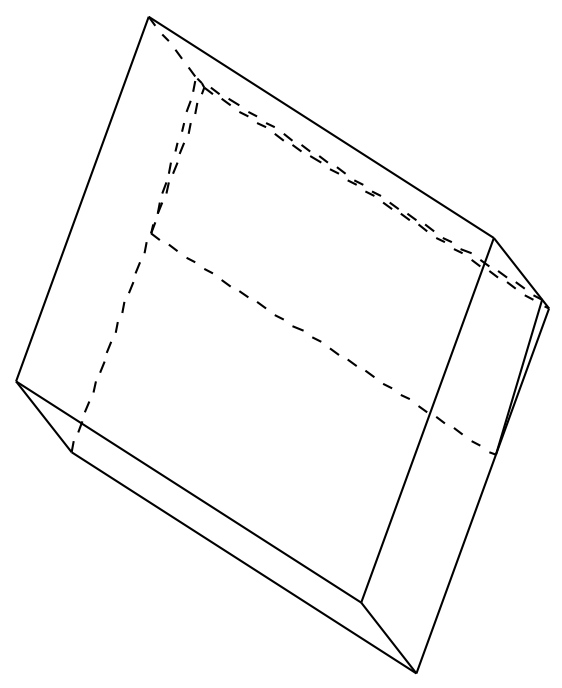

18. $<318>$

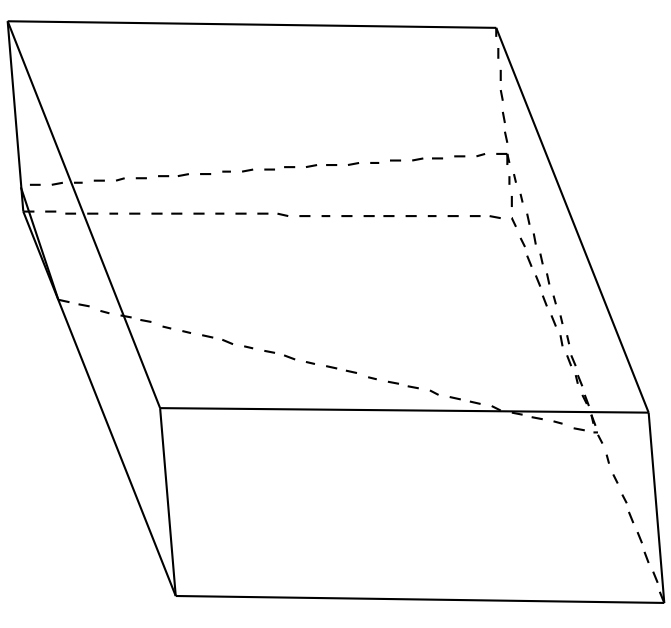

16. $<312>$ 

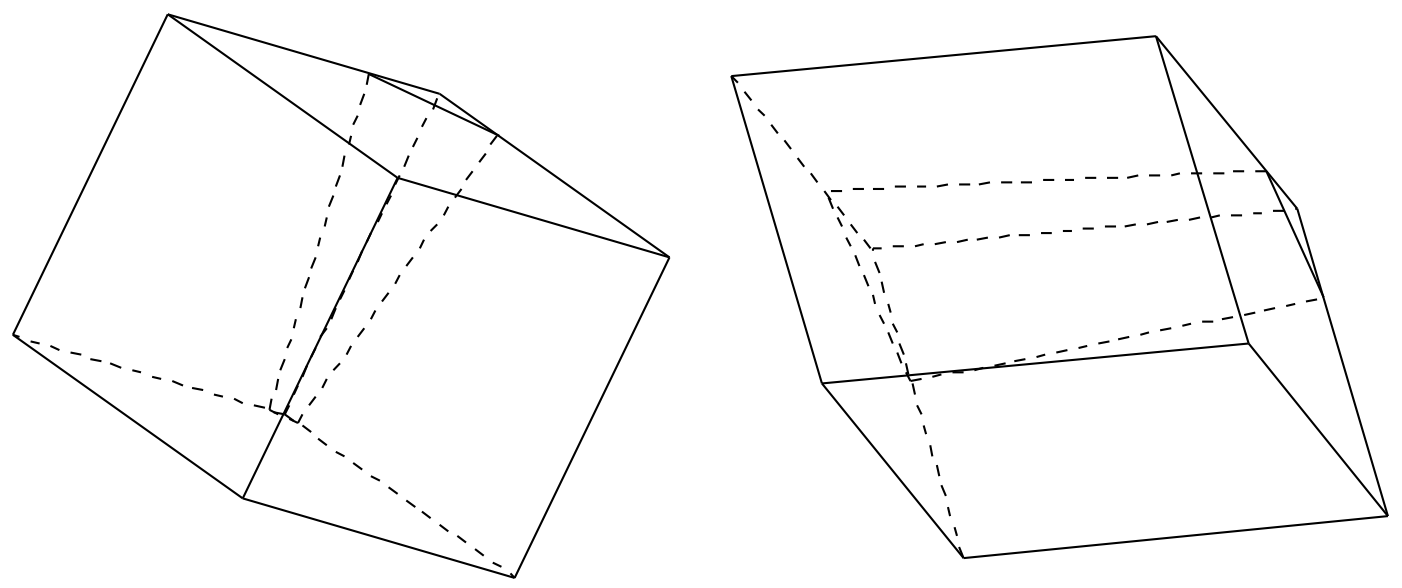

19. $<016>$

20. $<223>$

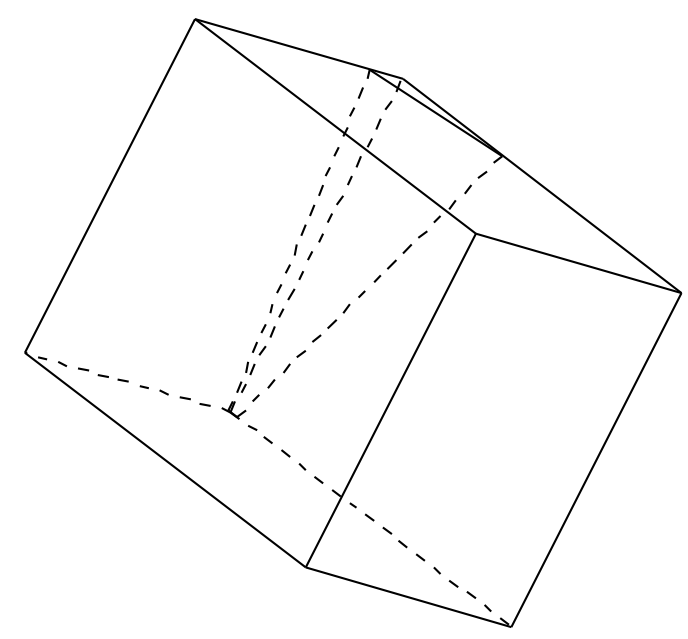

21. $<119>$

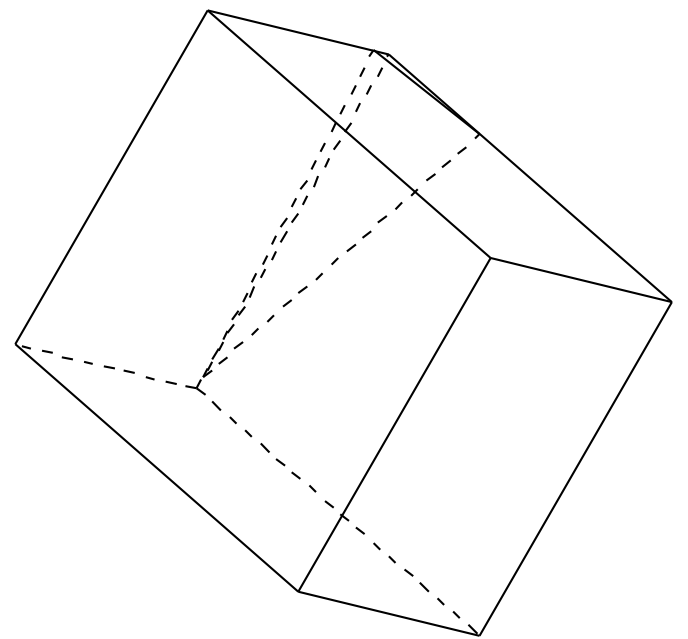

22. $<2113>$ 


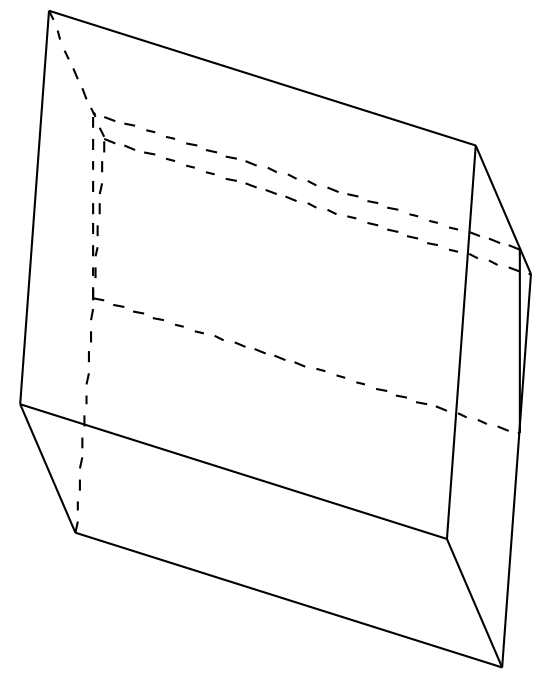

23. $<214>$

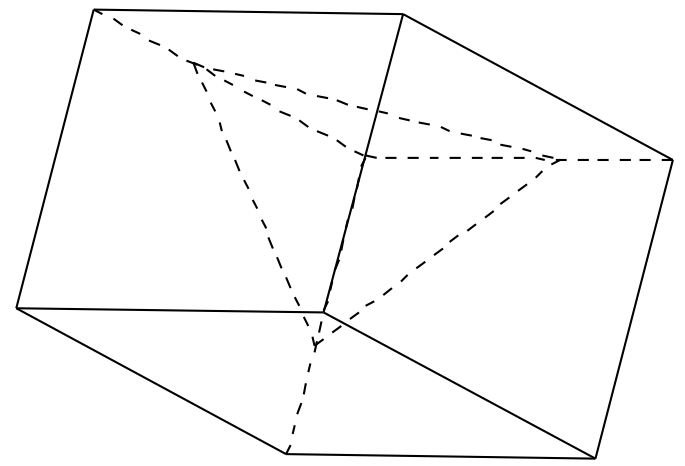

25. $<012>$

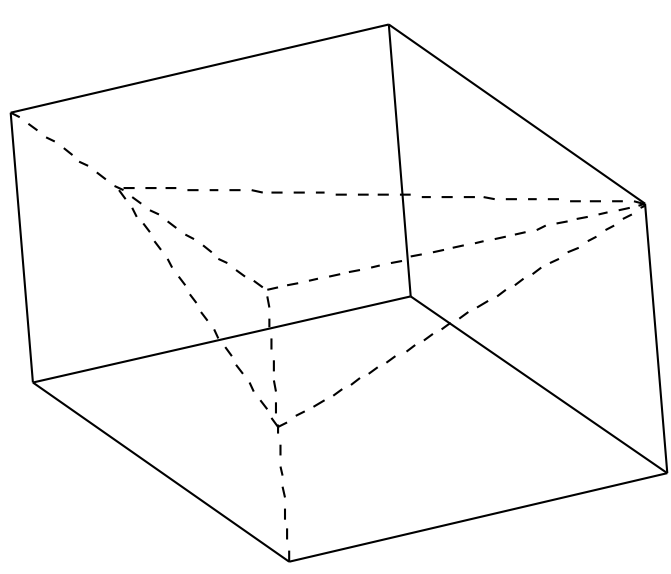

24. $<144>$

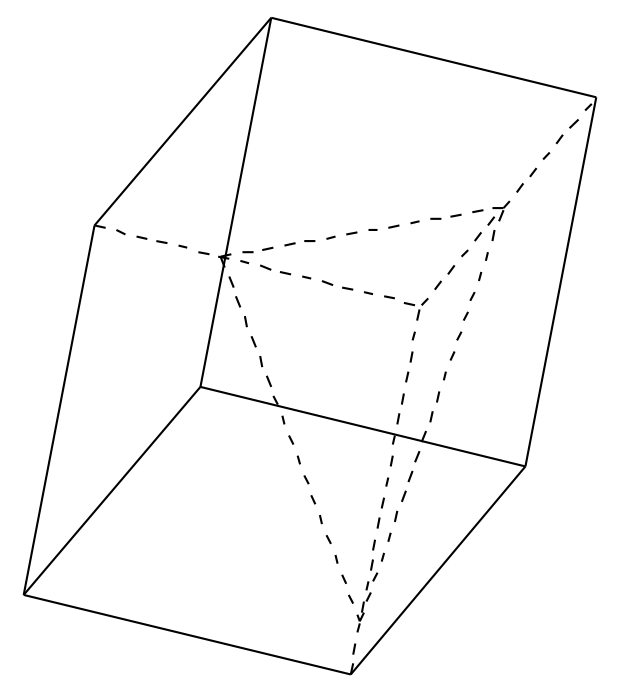

26. $<31-7>$ 


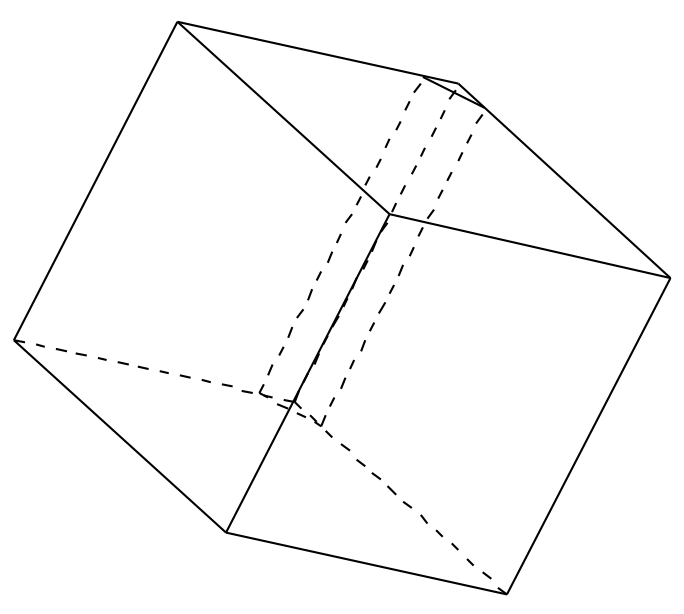

27. $<018>$

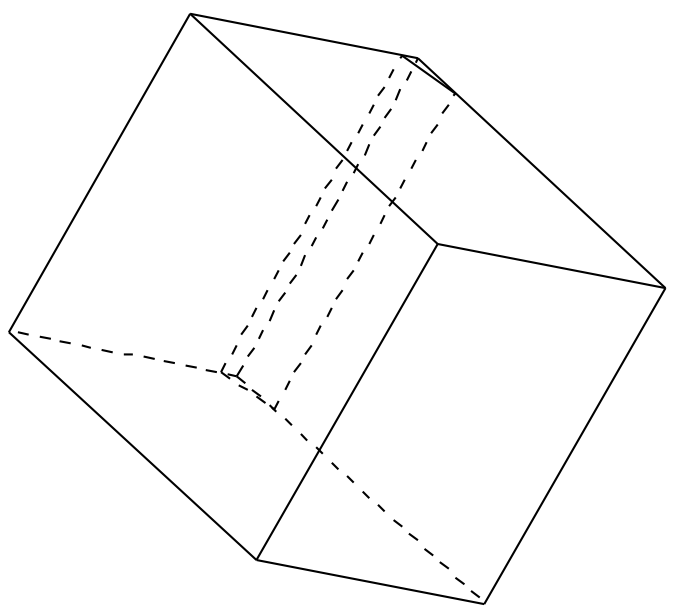

29. $<1112>$

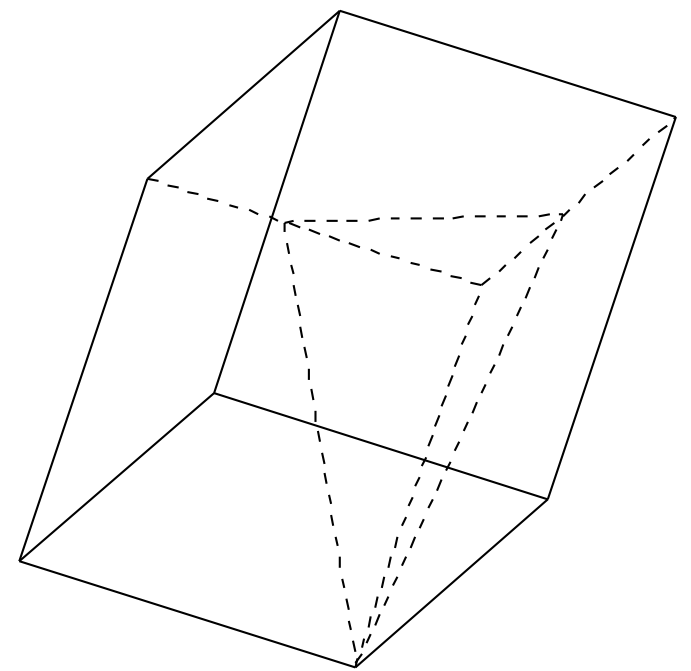

28. $<2$ - $1-5>$

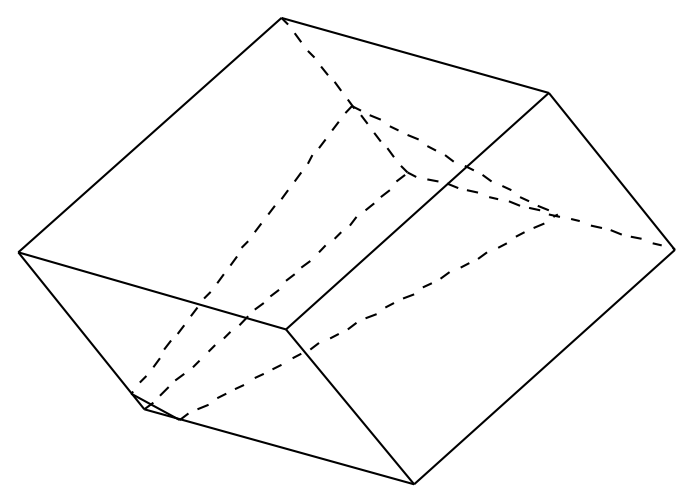

30. $<31-1>$ 


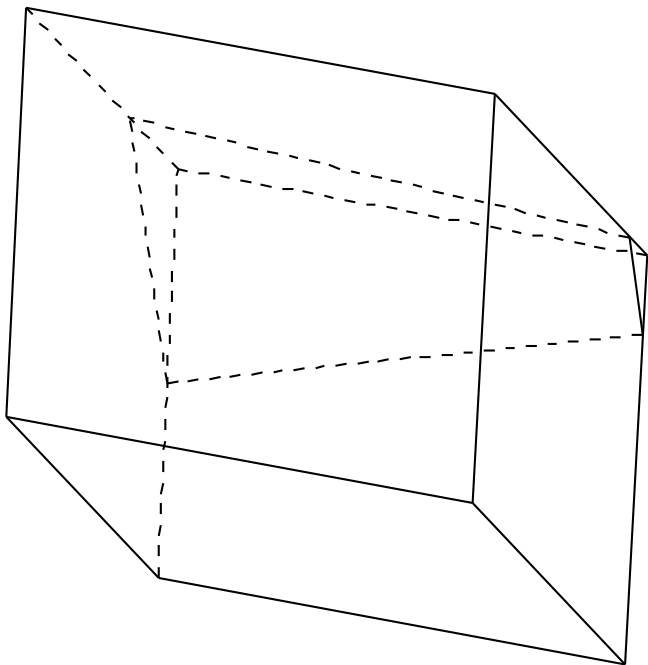

31. $<113>$

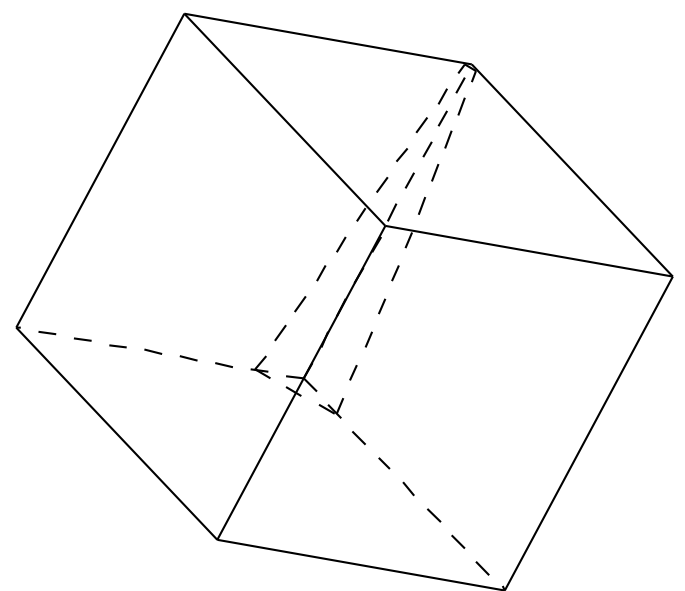

34. $<0110>$

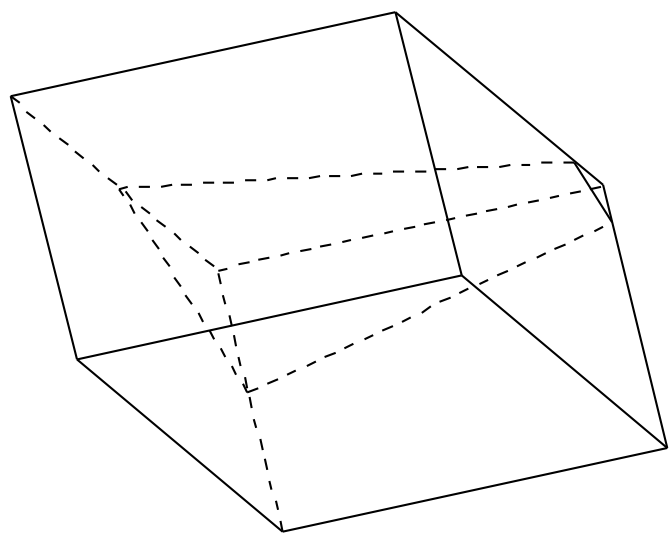

32. $<122>$

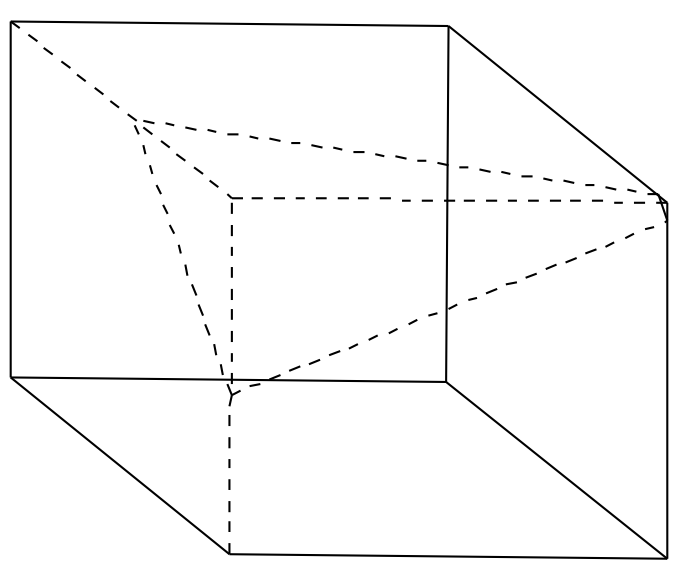

35. $<124>$ 

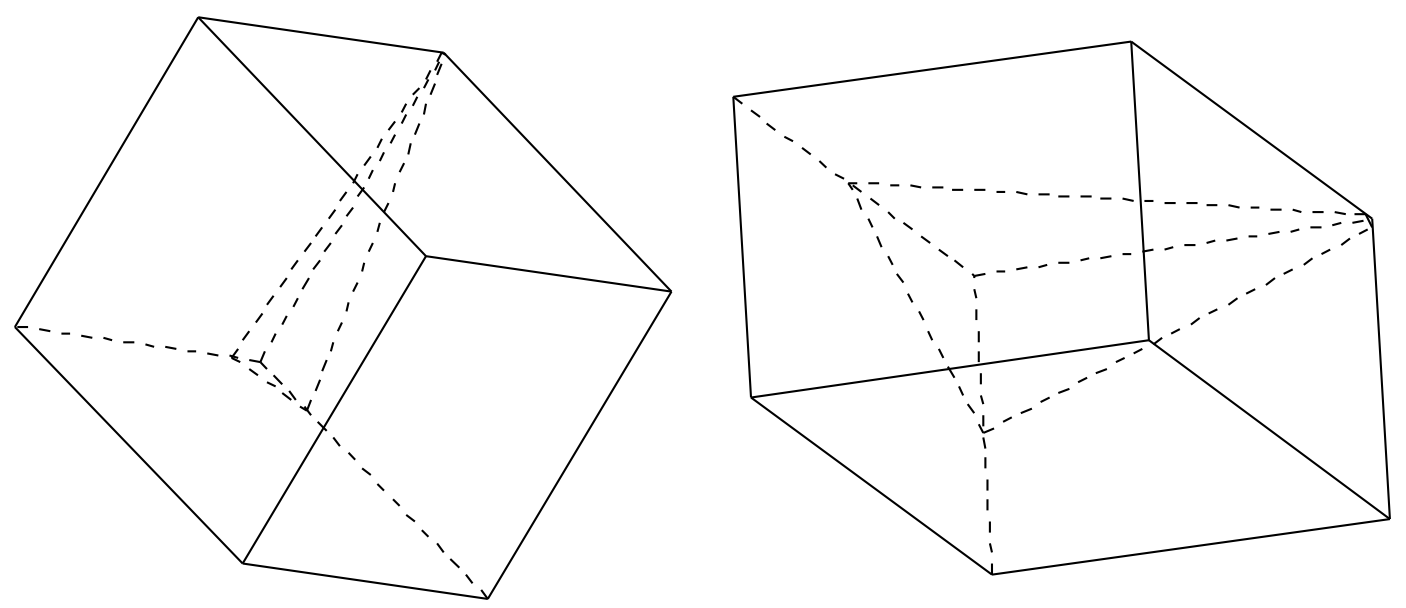

36. $<1115>$

37. $<134>$
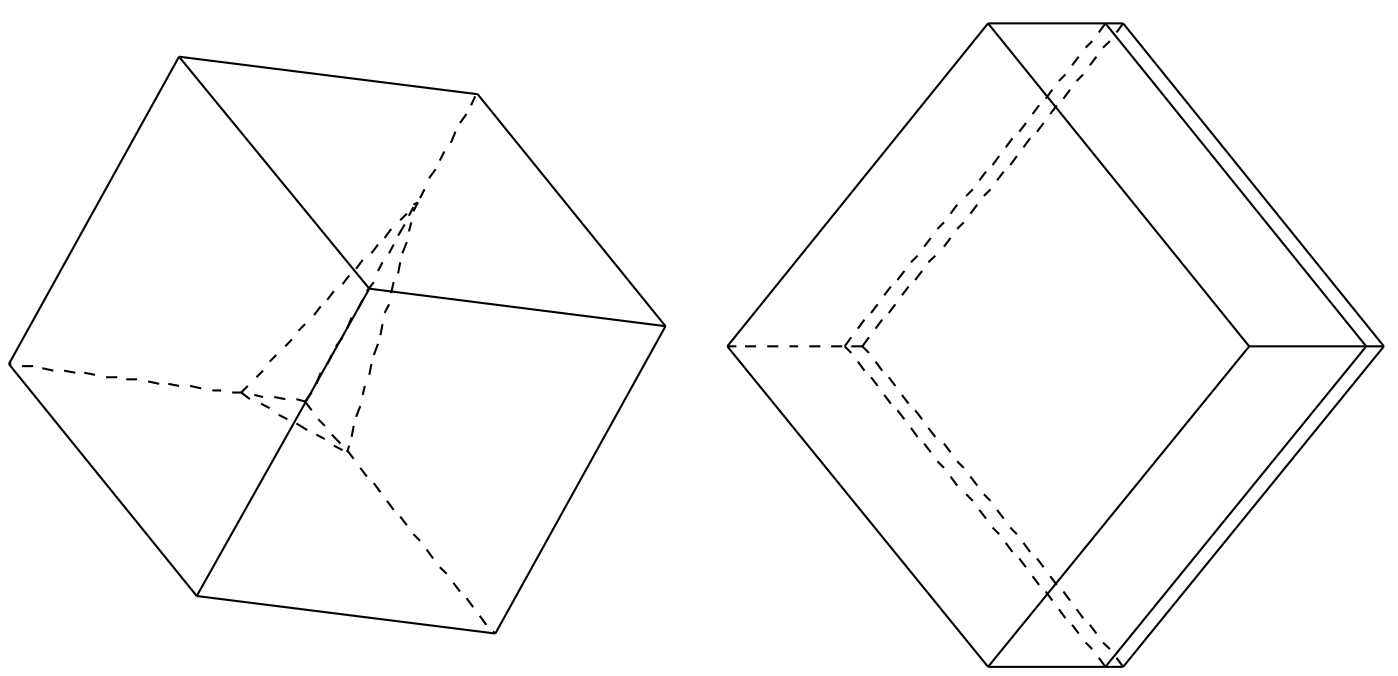

38. $<0114>$

39. $<104>$ 


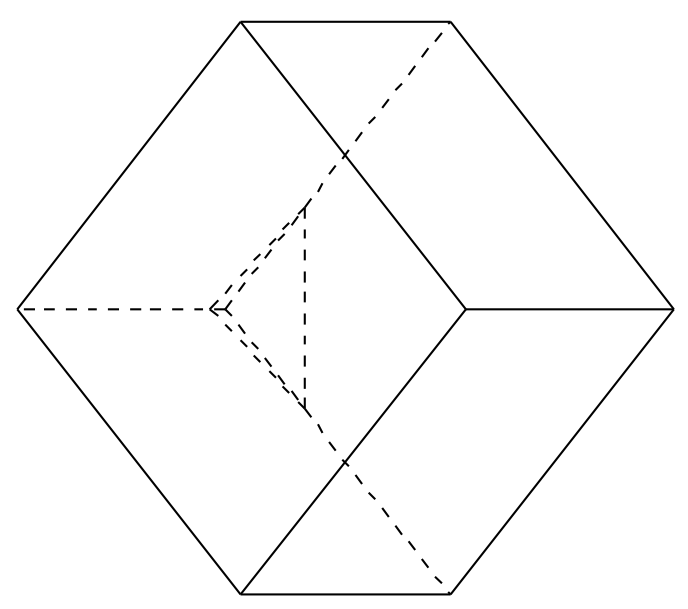

40. $<20$ 14>

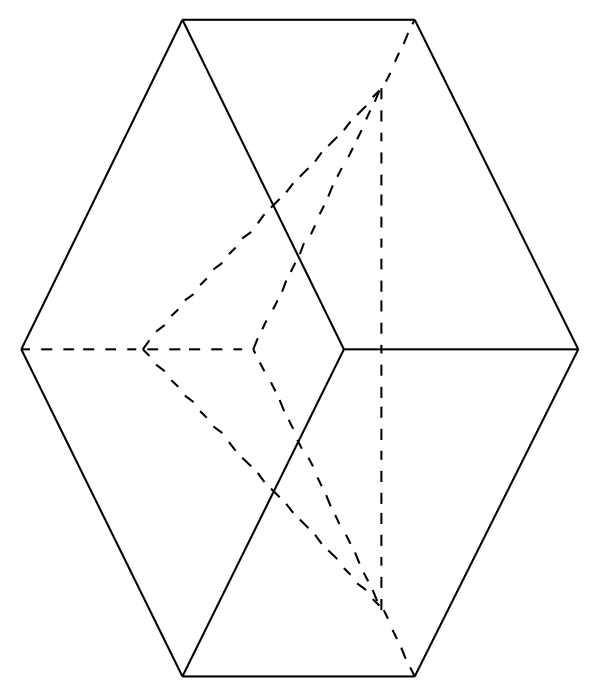

43. $<40-2>$

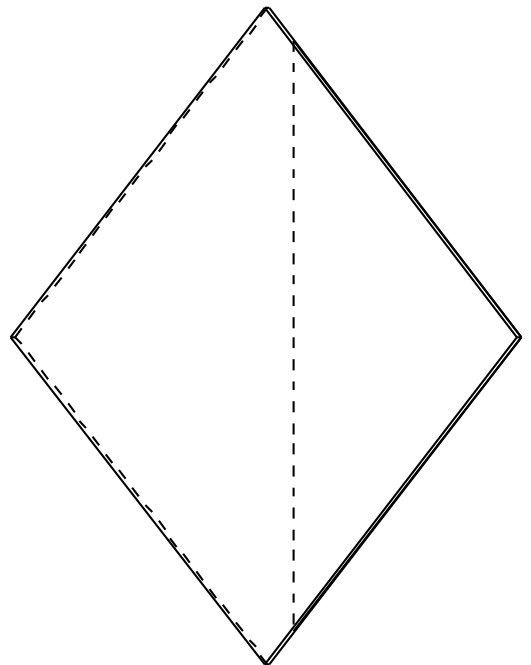

42. $<102>$

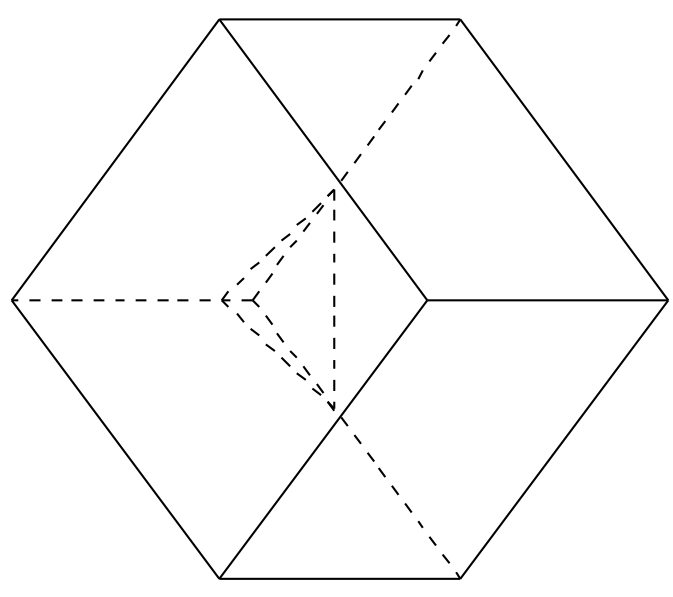

44. $<1010>$ 


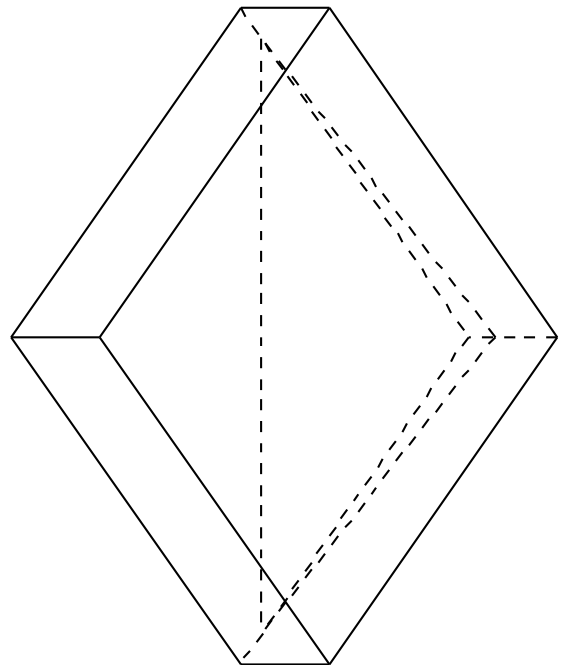

45. $<101>$

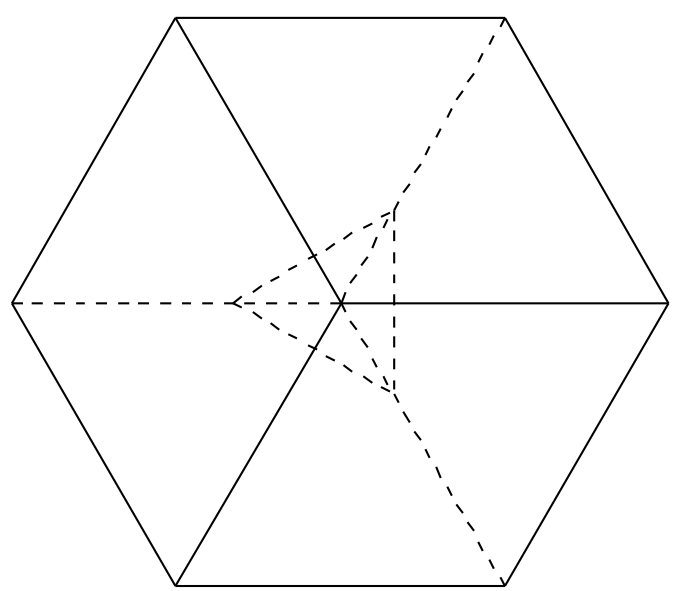

47. $<0001>$

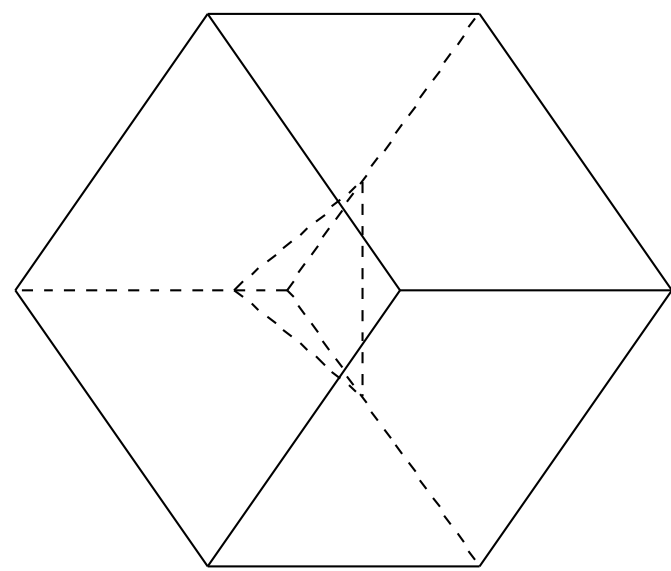

46. $<10$ 16>

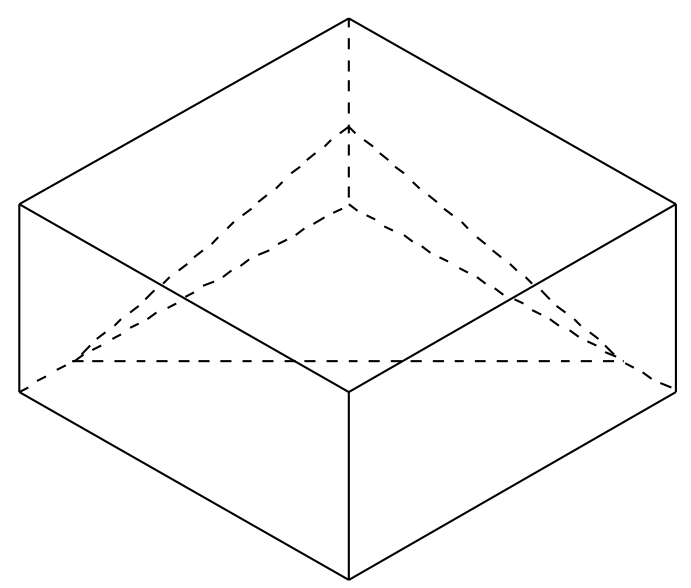

48. $<100>$ 

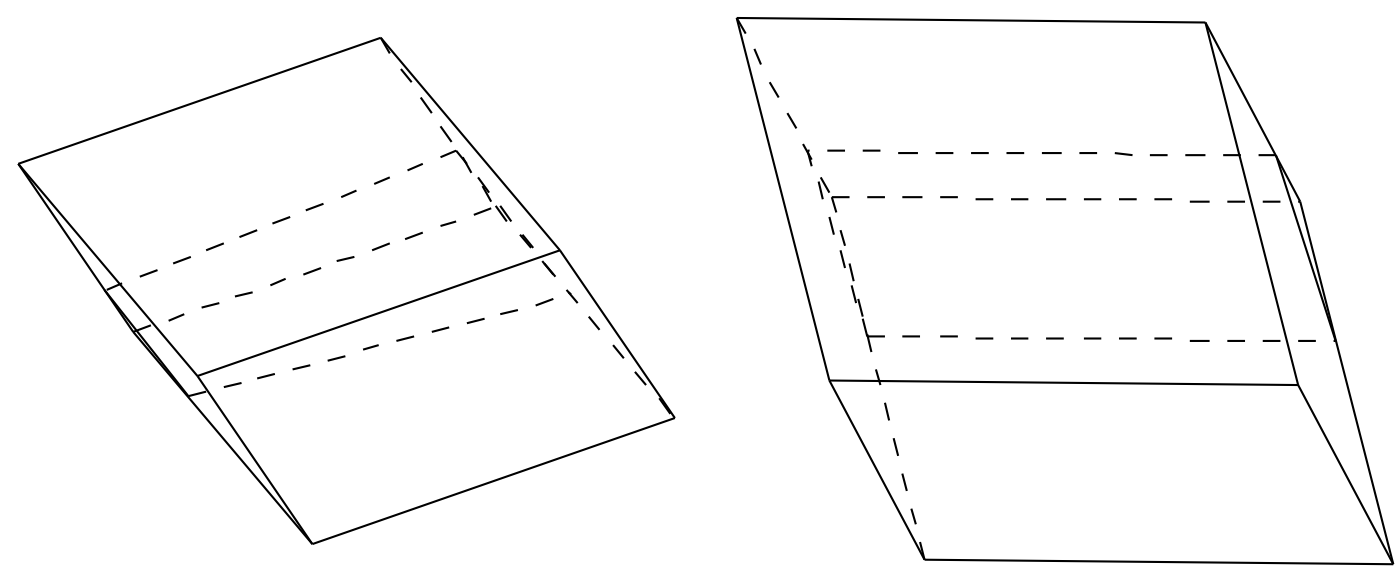

5. $<321>$

12. $<324>$

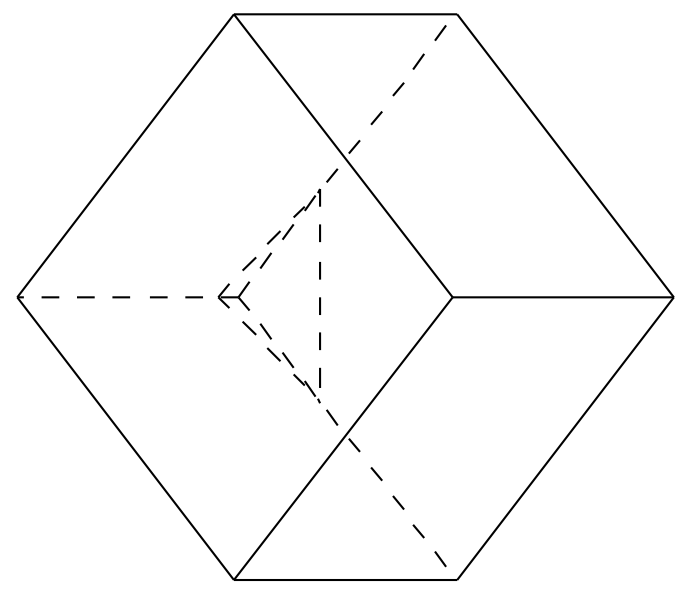

41. <2 0 16>

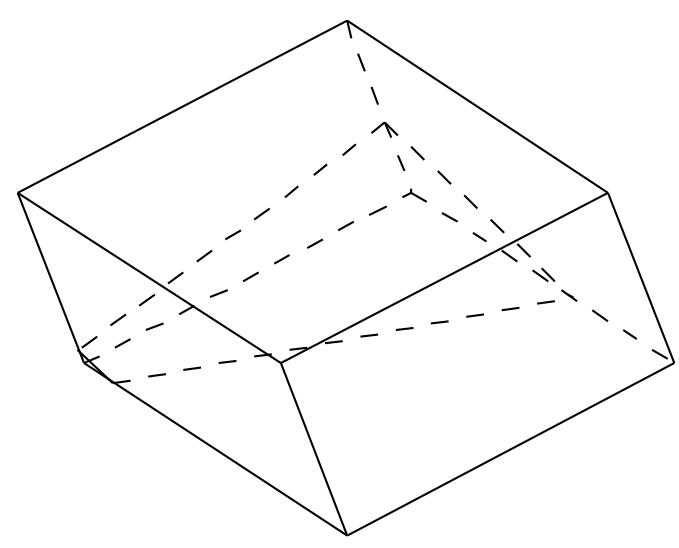

33. $<410>$ 


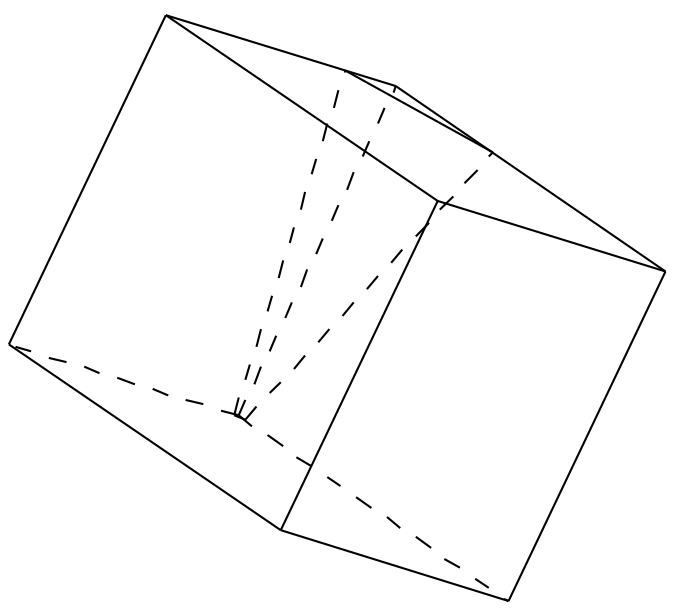

17. $<12$ 14>

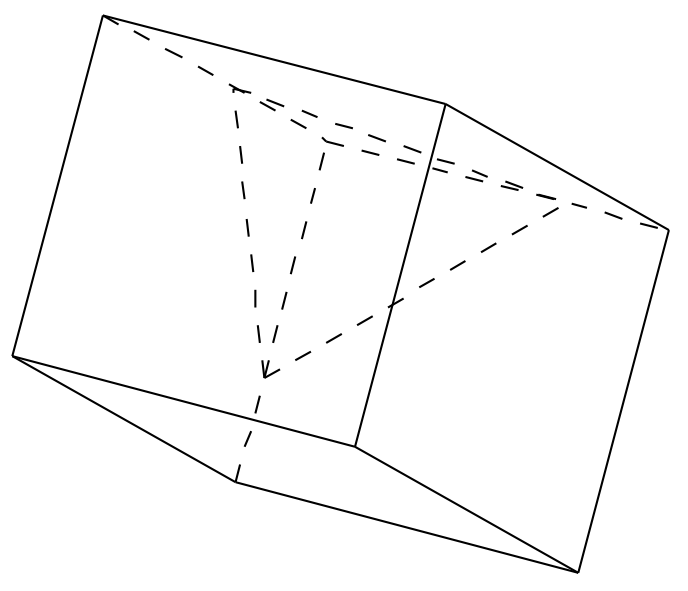

13. $<1310>$ 


\section{Appendix A5 - GIXD Analysis}

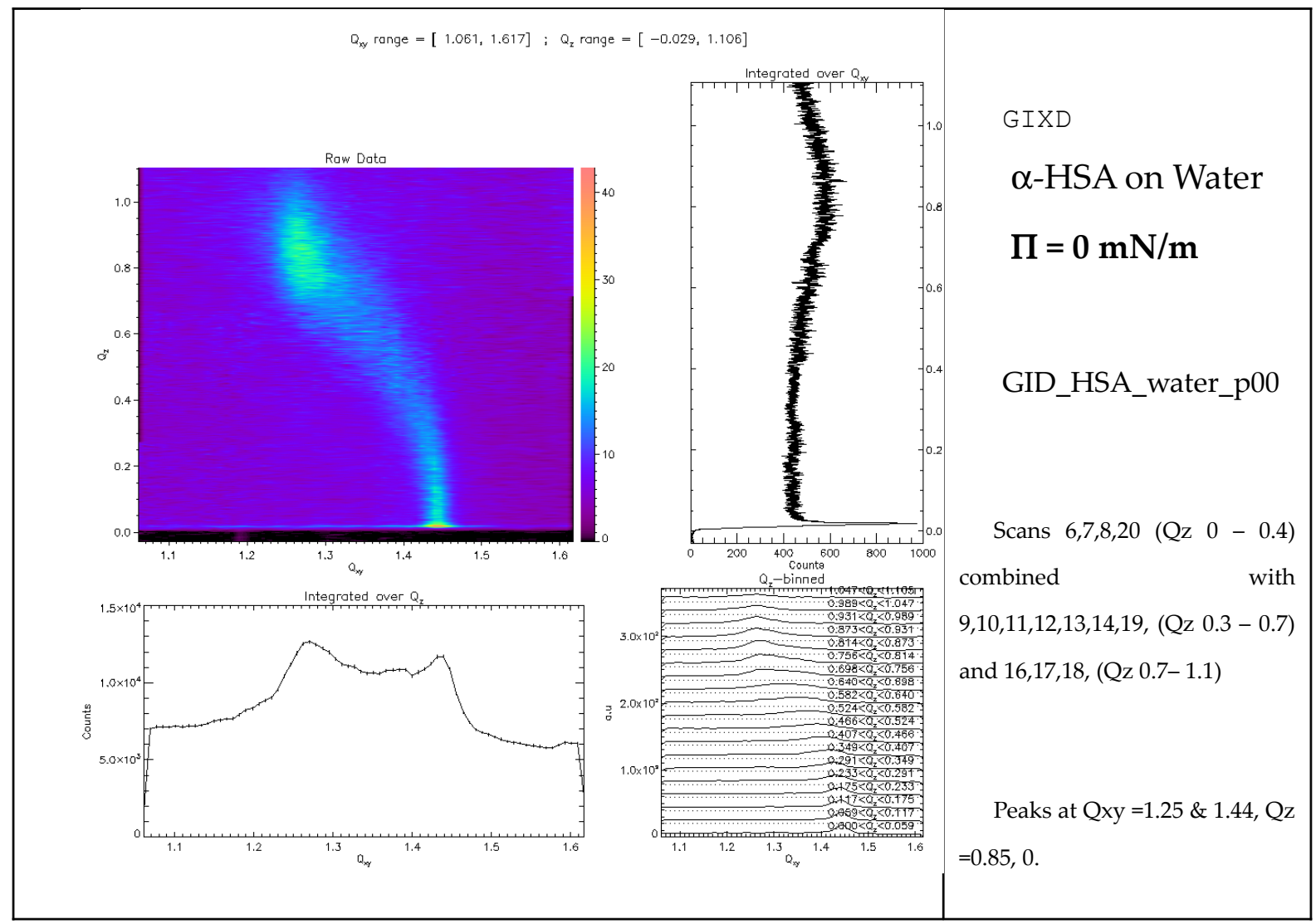




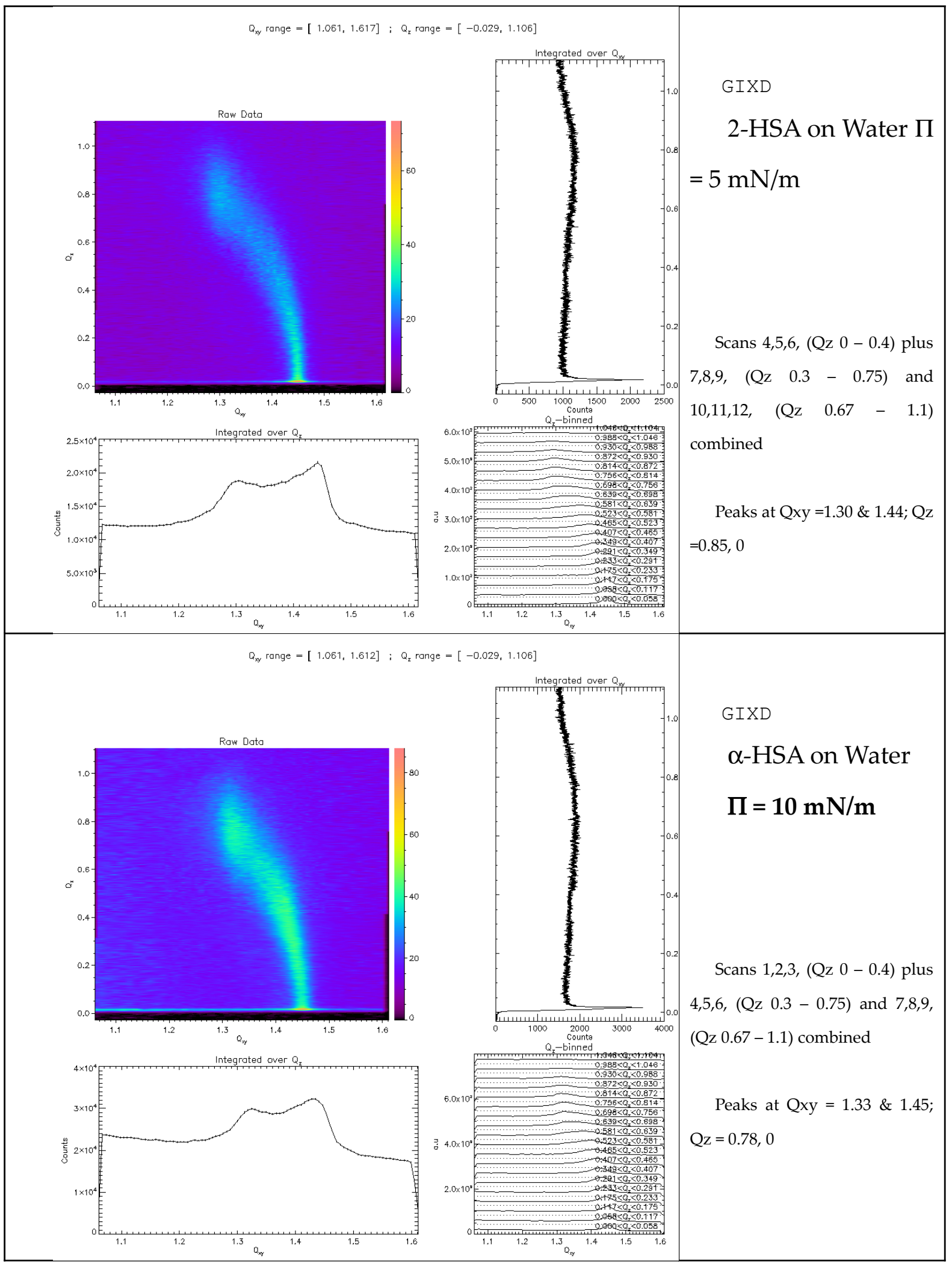




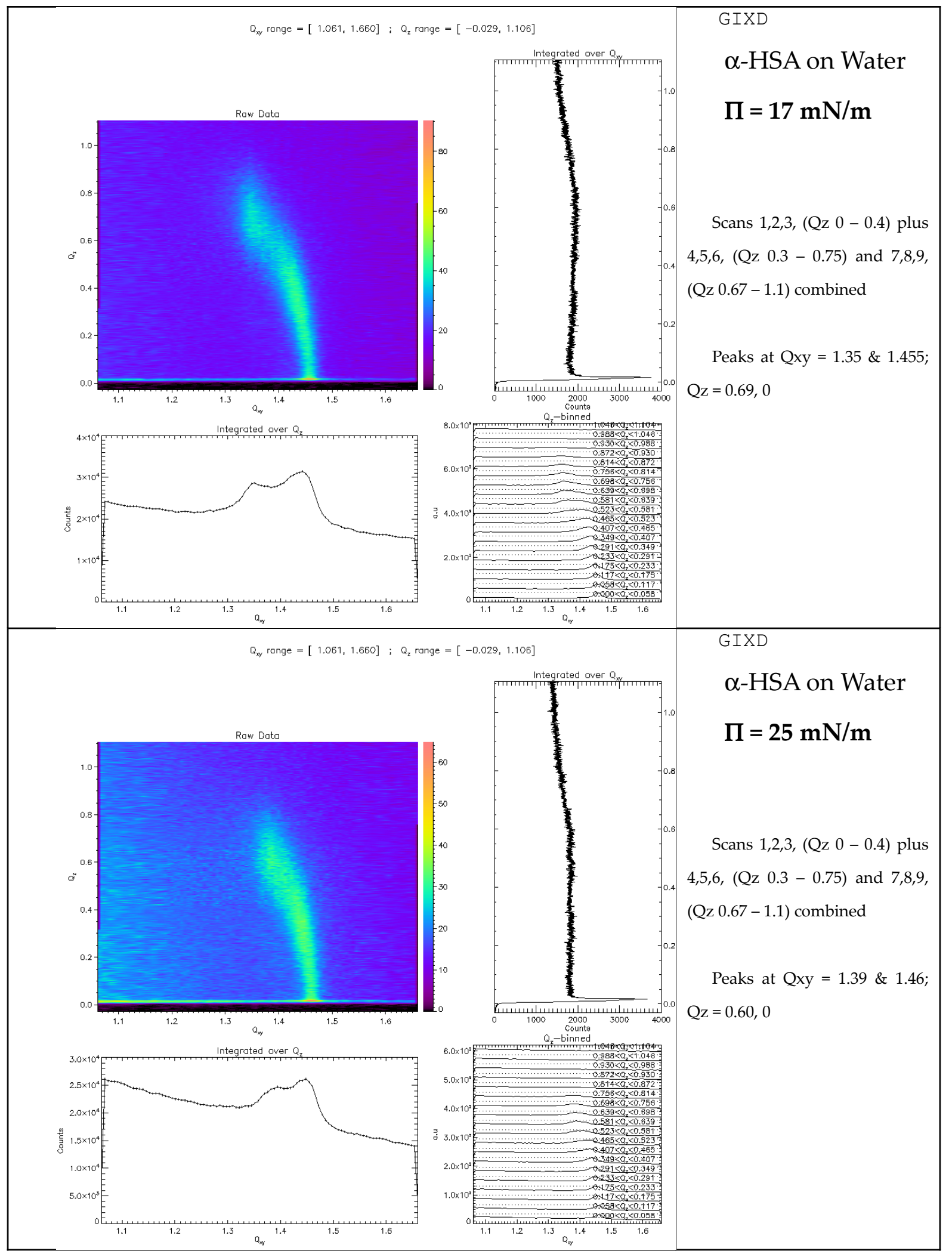


Chapter 9: Appendix

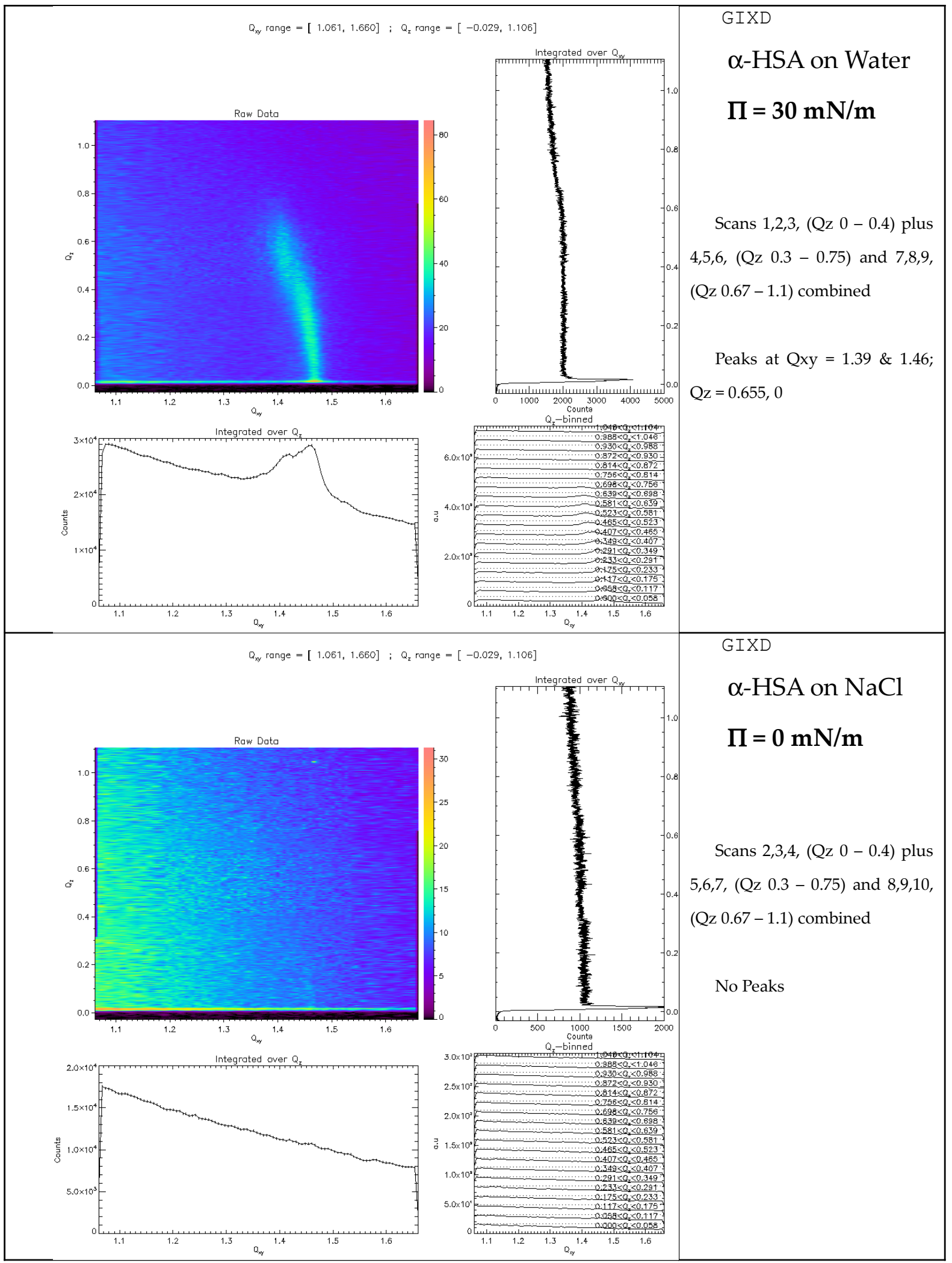




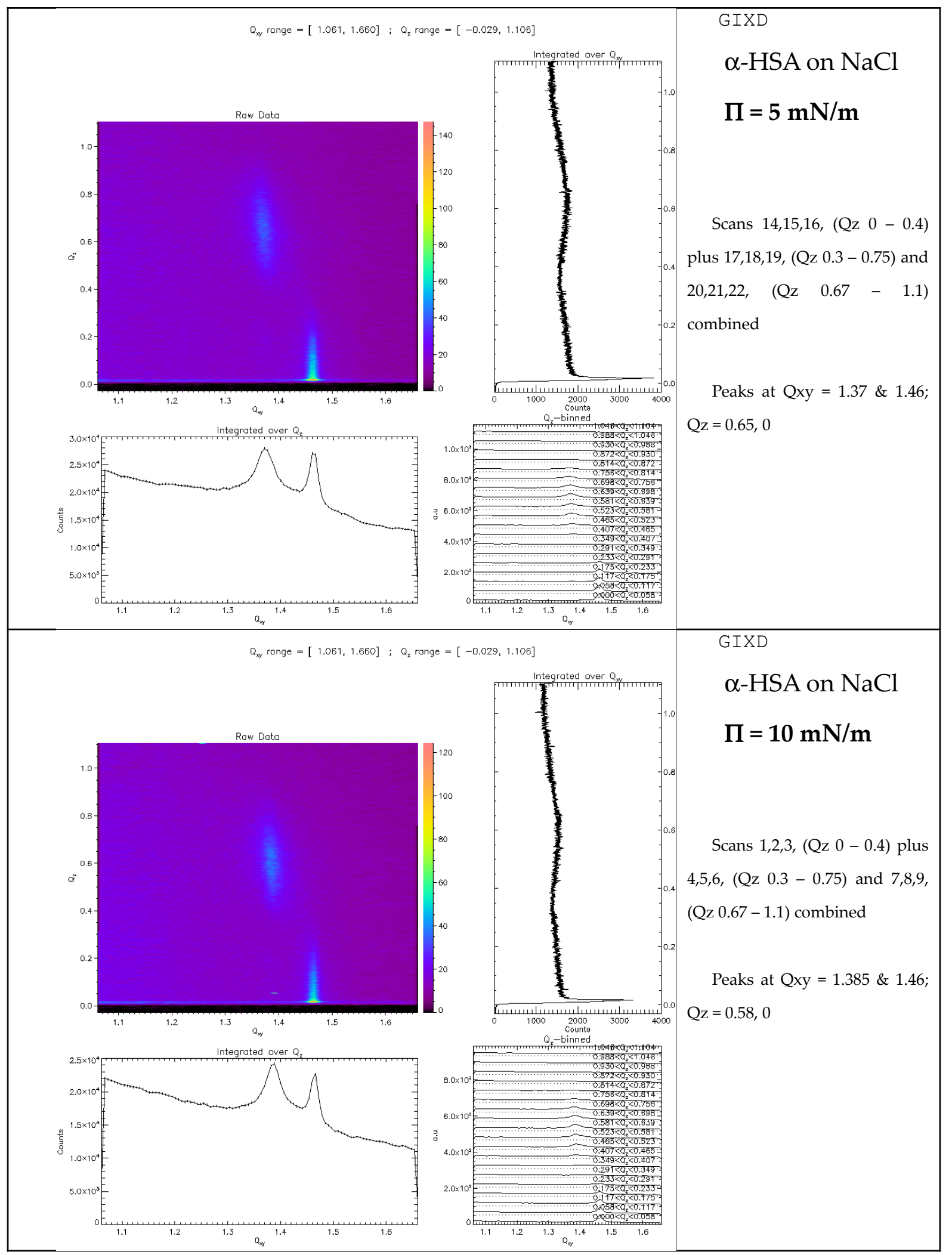


Chapter 9: Appendix

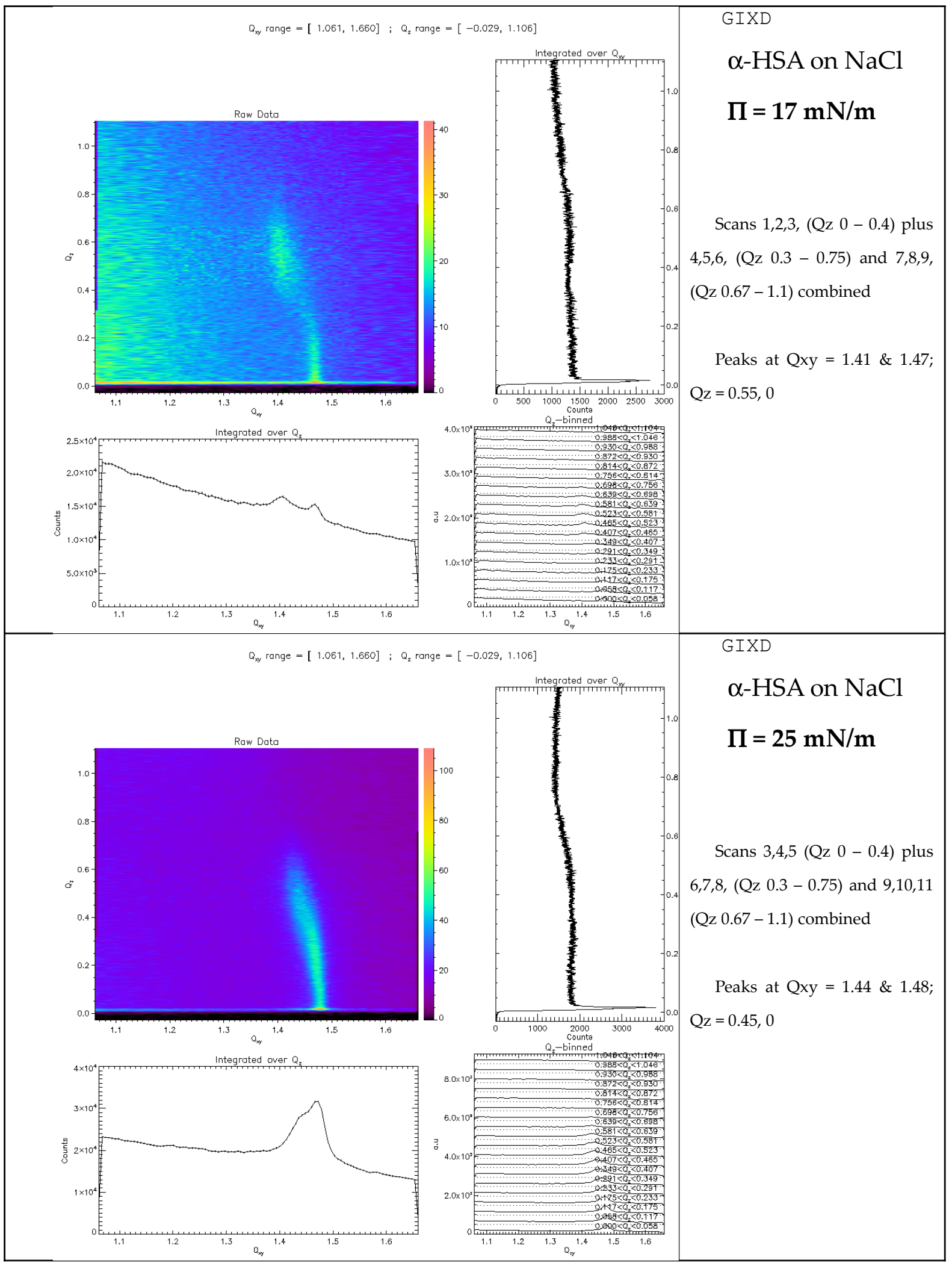




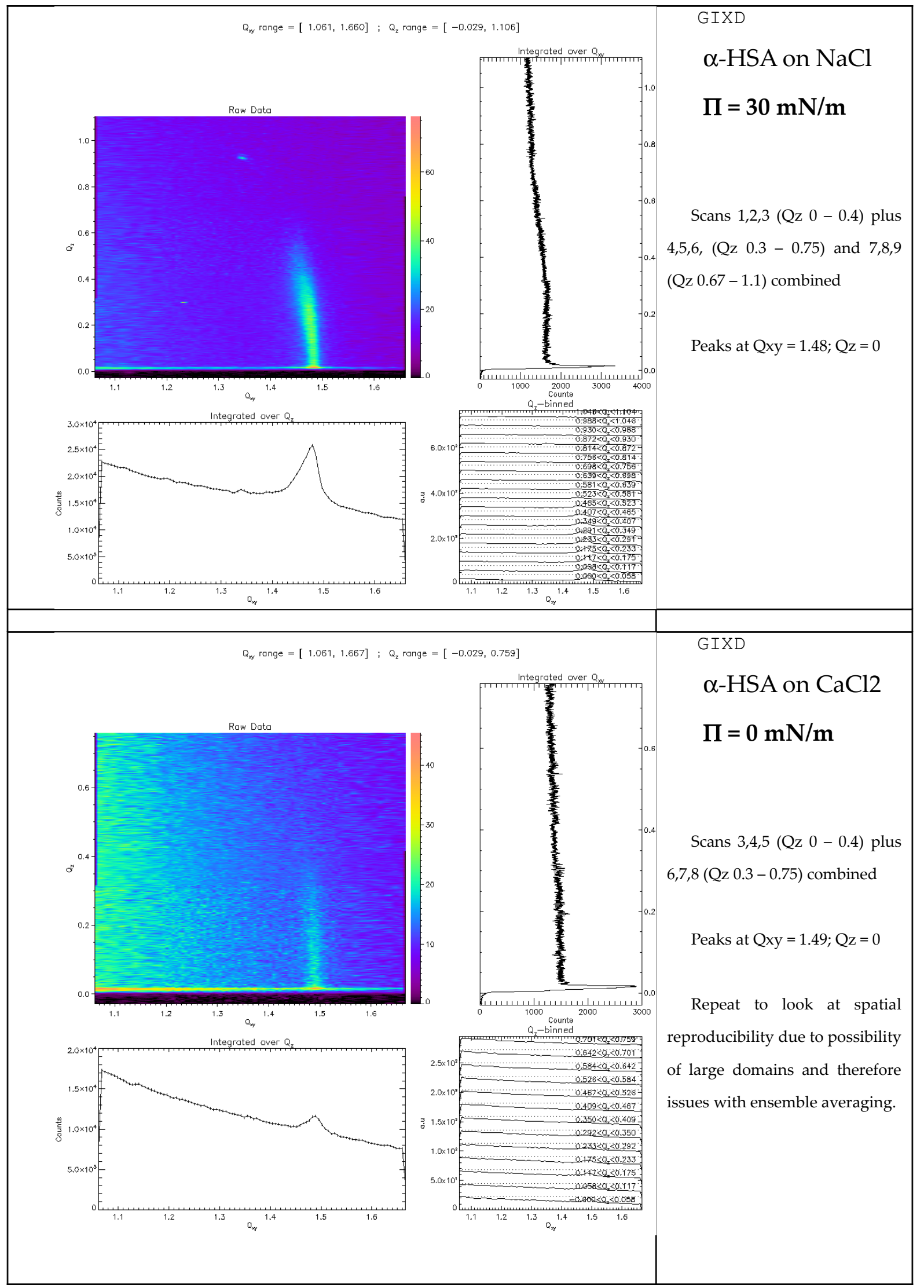


Chapter 9: Appendix

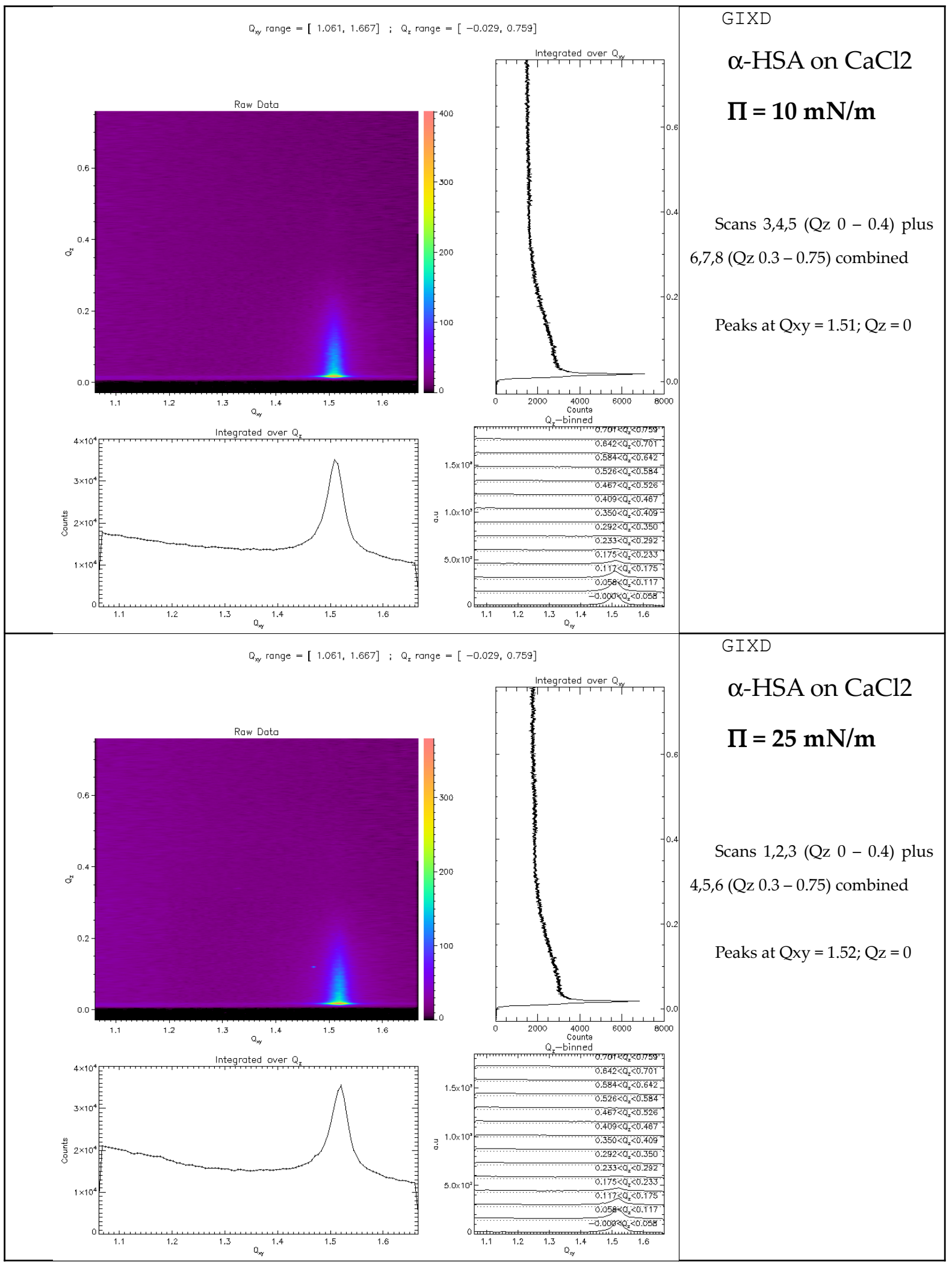




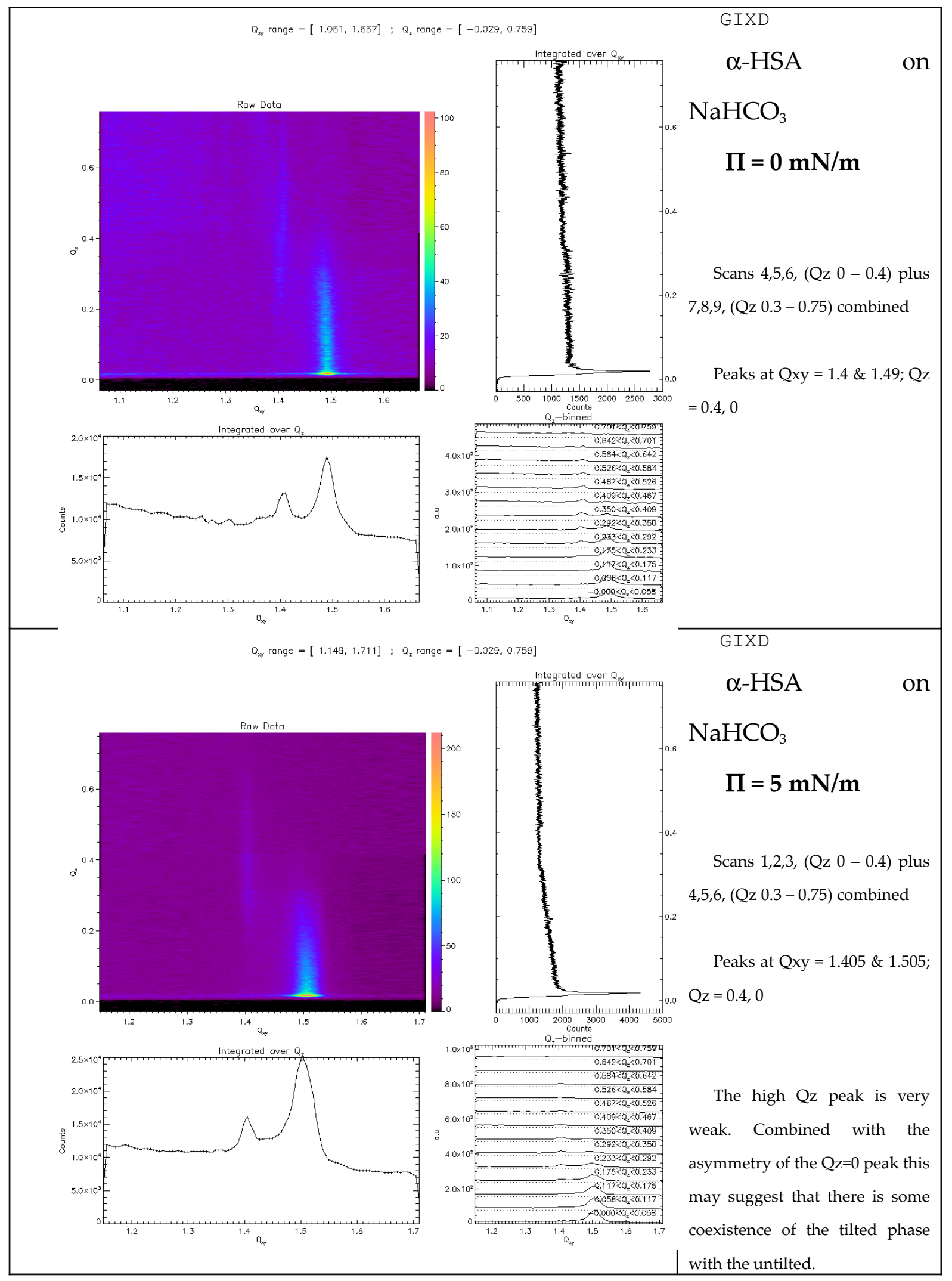


Chapter 9: Appendix

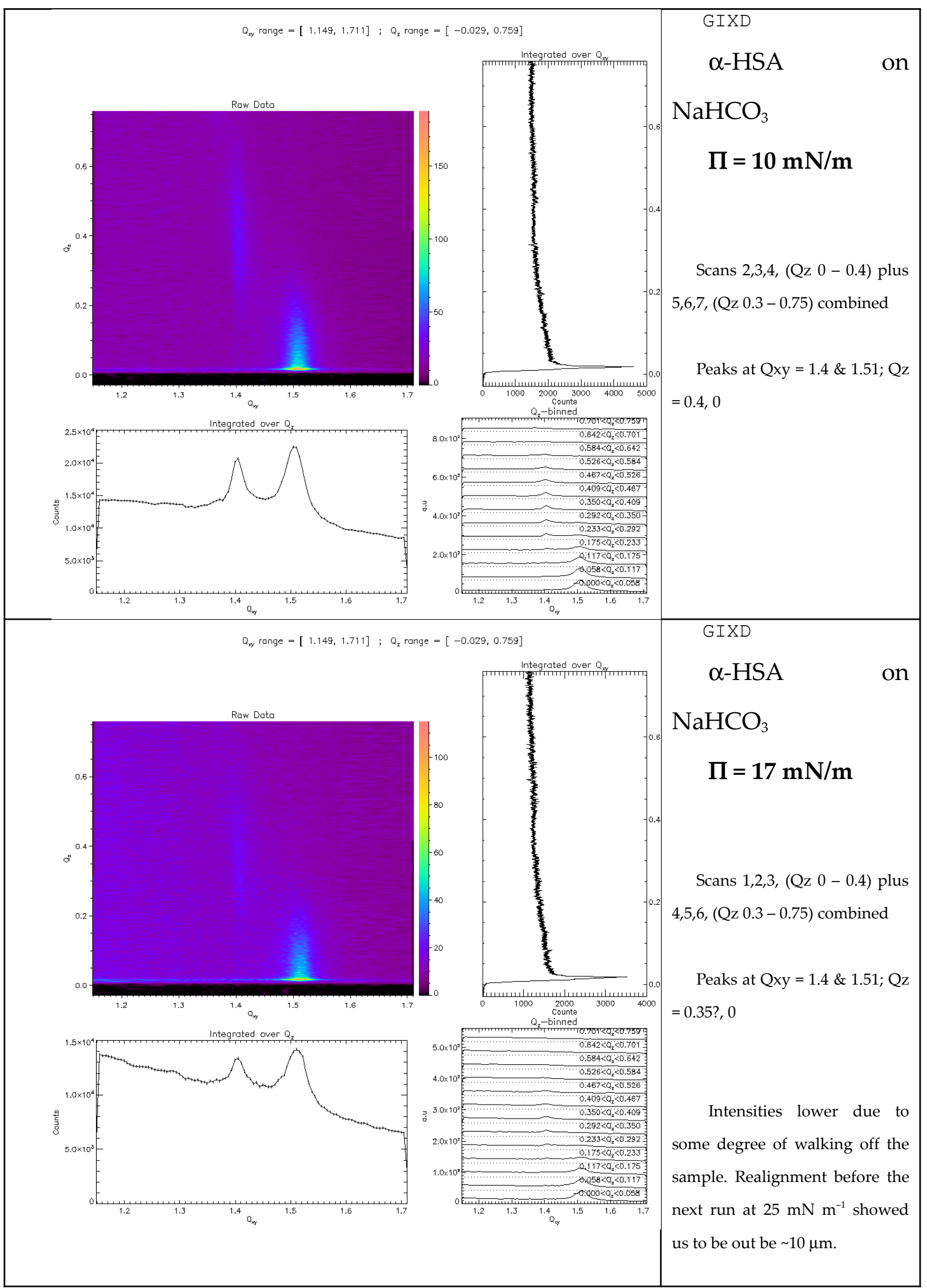




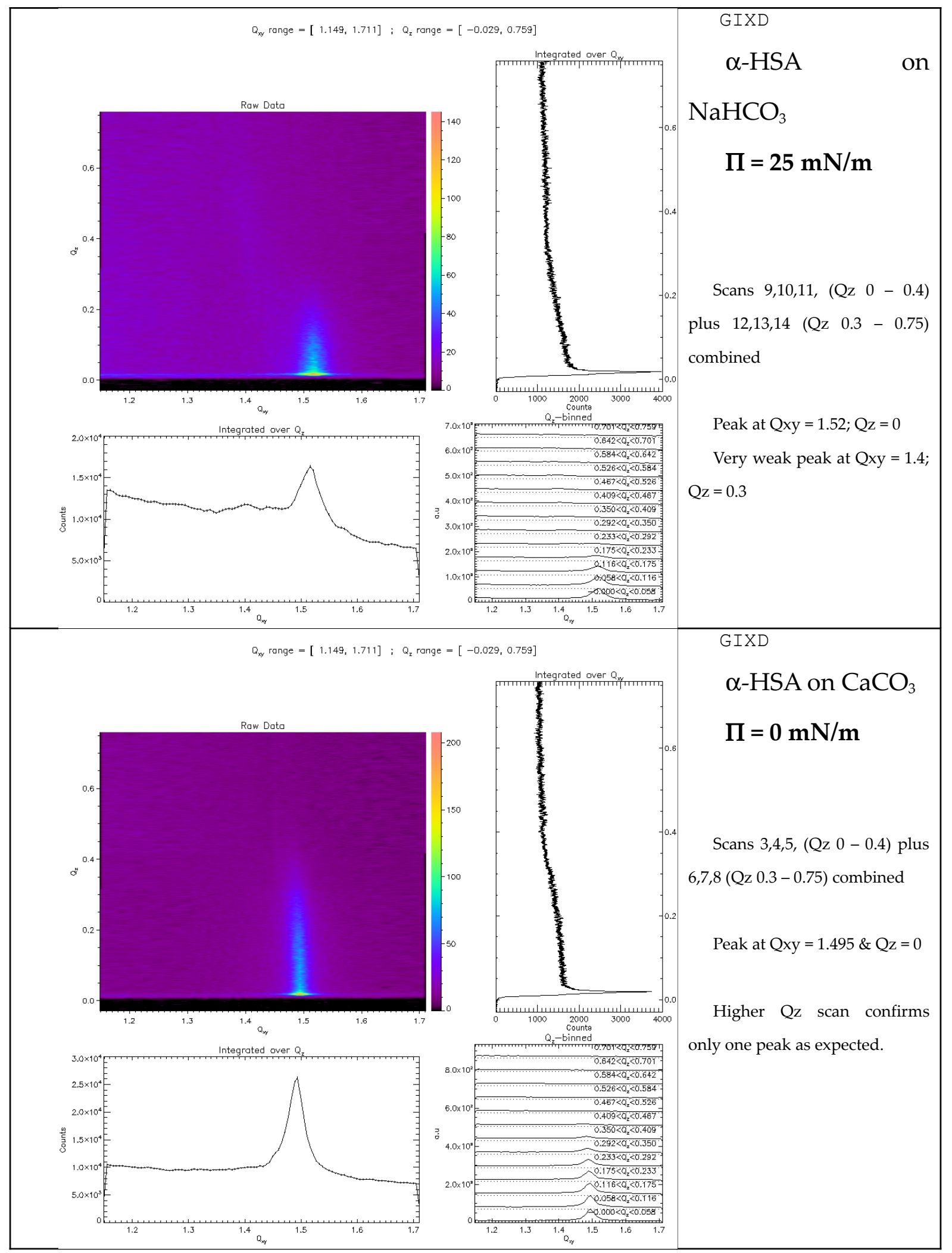


Chapter 9: Appendix

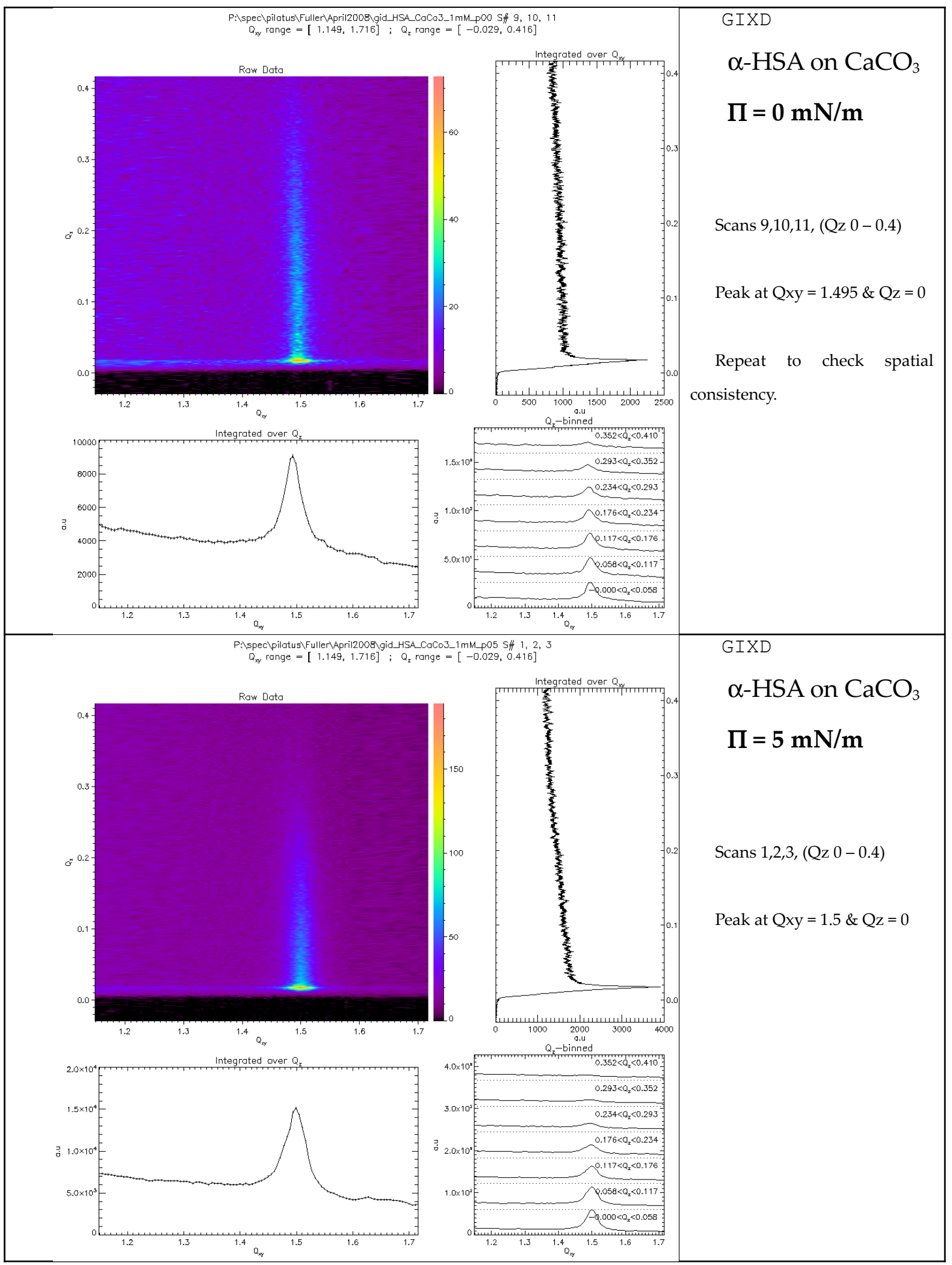




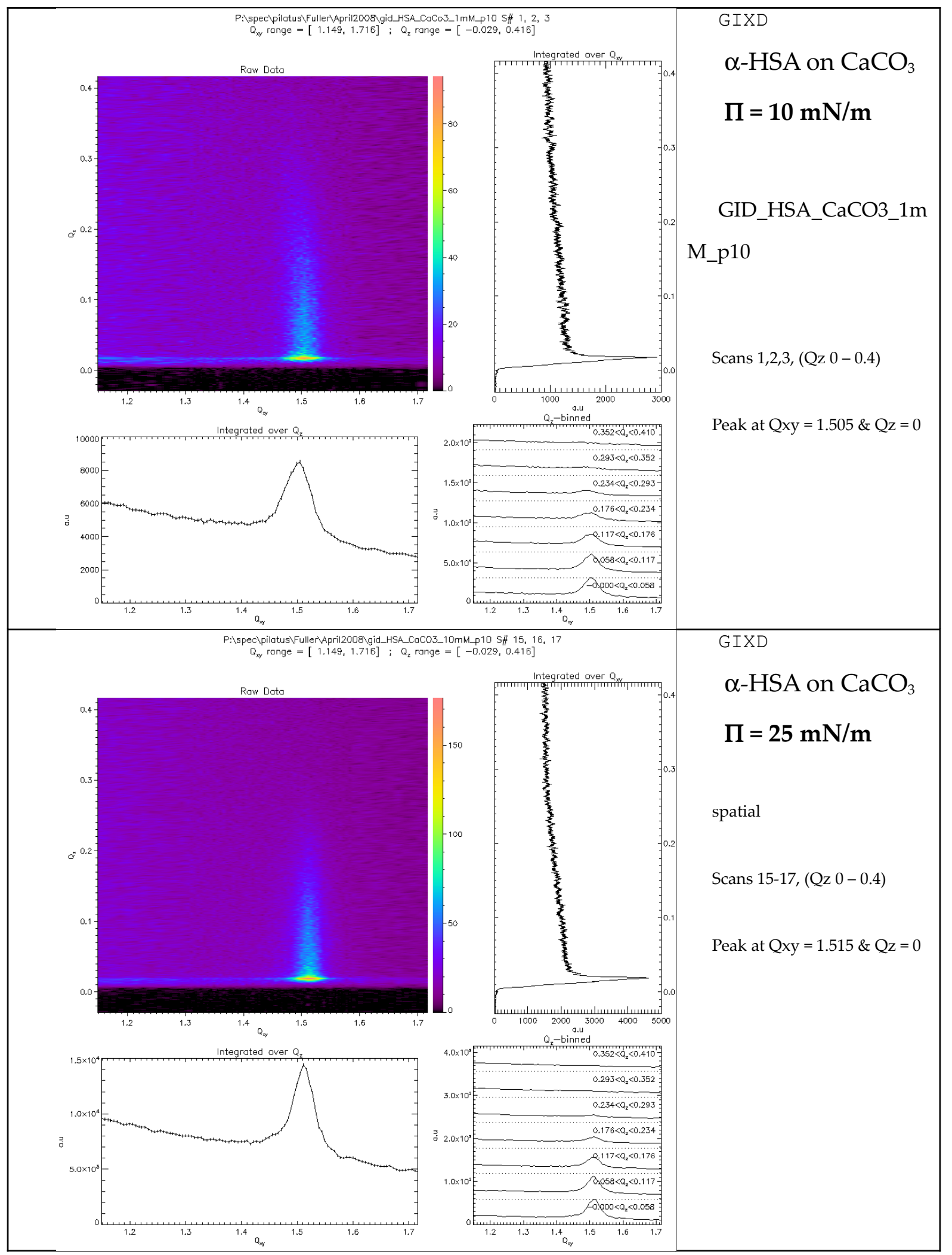




\section{Appendix A6 - XRR Analysis}

Reflectivity Non-Linear Regression Fit

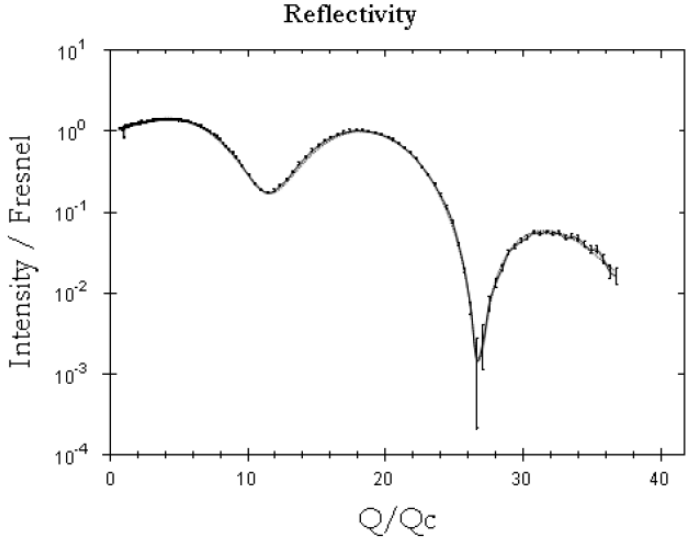

lectron Density Profile

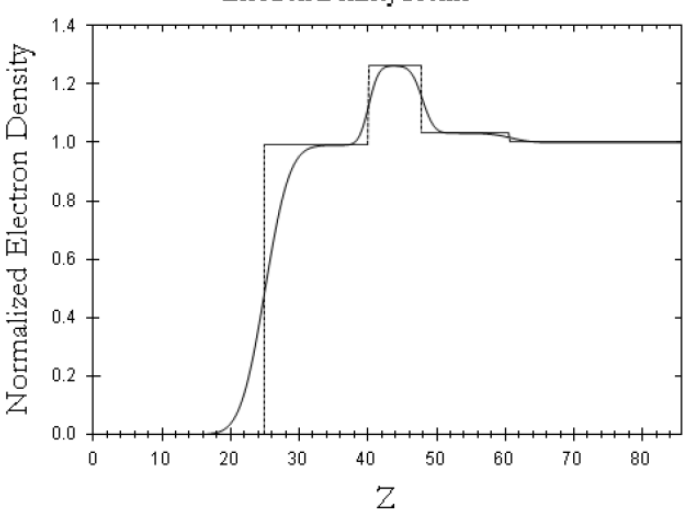

The reflectivity curve was fit with 4 roughness parameter

Percent Error in Q: 0

Normalization Constant 107984235324763

Critical Edge Offset: 0

High Q Offset: 0

Superphase SLD: 0

Subphase SLD: 9.409

Wavelength: 1.2244

Chi Square for reflectivity fit: $251 \mathrm{E}-002$

The subphase roughness was: $2.306 \mathrm{E} 0 \pm 2.739 \mathrm{E}-1$
Water

$\Pi=10 \mathrm{mN} / \mathrm{m}$

\begin{tabular}{|c|c|c|c|}
\hline Laver \# & Lenath & Rho/Rho(infinity) & Siama \\
\hline 1 & $1.51 \mathrm{E} 1 \pm 2.74 \mathrm{E}-2$ & $9.905 \mathrm{E}-1 \pm 5.34 \mathrm{E}-3$ & $2.816 \mathrm{E} 0 \pm 9.813 \mathrm{E}-3$ \\
\hline 2 & $7831 \mathrm{E} 0+7305 \mathrm{E}-2$ & $1.262 \mathrm{E} 0 \pm 7.922 \mathrm{E}-3$ & $1.32 \mathrm{E} 0 \pm 12 \mathrm{E}-1$ \\
\hline 3 & $1282 \mathrm{E} 1+3033 \mathrm{E}-1$ & $1032 \mathrm{E} 0+3.959 \mathrm{E}-3$ & $1335 \mathrm{E} 0+1079 \mathrm{E}-1$ \\
\hline
\end{tabular}


Reflectivity Non-Linear Regression Fit
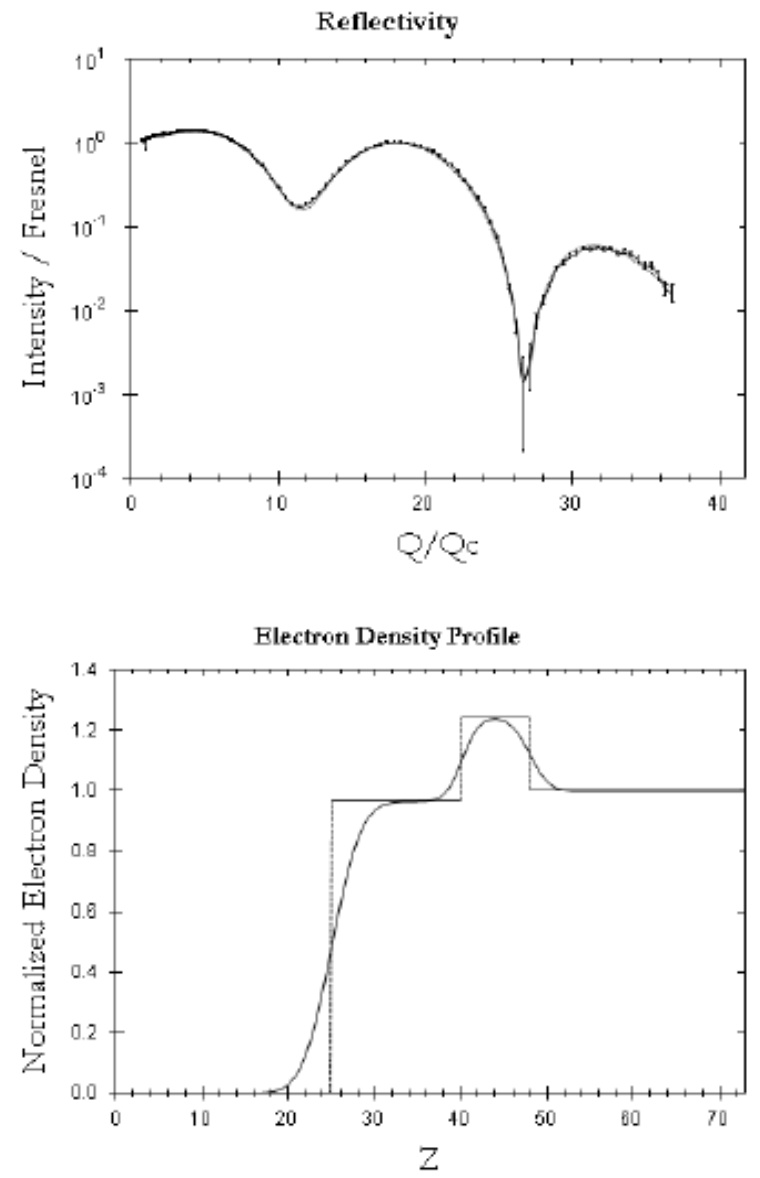

The reflectivity curve was fit wth 3 roughness parameters

Percent Error in Q: 0

Normalization Constant: 1.11281174802042

Critical Edge Offset: 0

High Q Offset: 0

Superphase SLD: 0

Subphase SLD: 9.408

Wavelength: 1.2244

Chi Square for reflectivity fit: 3.883 E-002

The subphase roughness was: $1.748 \mathrm{EO} \pm 7.823 \mathrm{E}-2$

\begin{tabular}{|c|c|c|c|}
\hline Laver_\# & Lenoth & Rho/Rholinfinity & Siama \\
\hline 1 & $1.513 \mathrm{E} 1 \pm 2889 \mathrm{E}-2$ & $9.652 \mathrm{E}-1 \pm 4.843 \mathrm{E}-3$ & $2.892 \mathrm{E} 0 \pm 9.608 \mathrm{E}-3$ \\
\hline 2 & $7.875 \mathrm{E} 0 \pm 7.219 \mathrm{E}-2$ & $1.241 \mathrm{E} 0 \pm 8.763 \mathrm{E}-3$ & $1.533 \mathrm{E} 0 \pm 8.452 \mathrm{E}-2$ \\
\hline
\end{tabular}

Water

$\Pi=10 \mathrm{mN} / \mathrm{m}$ 


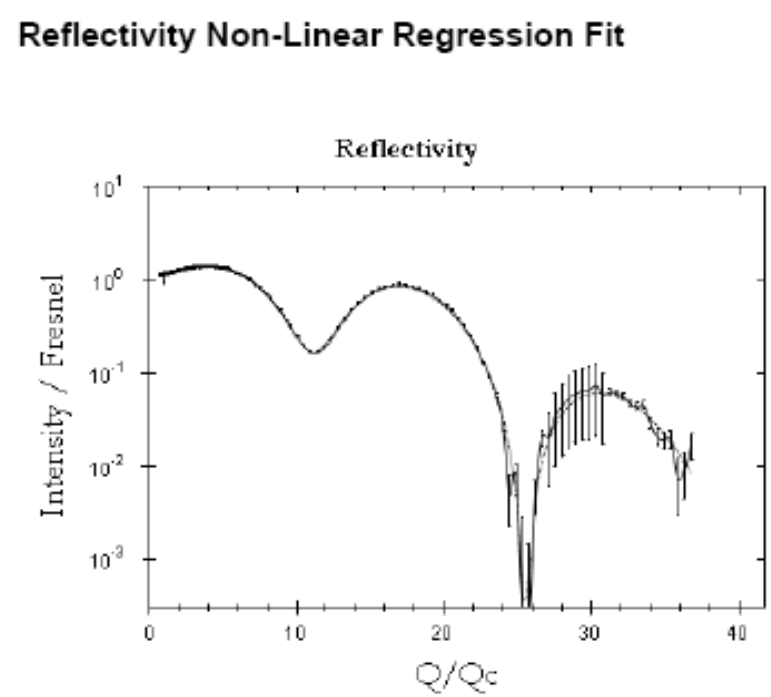

Water
$\Pi=25 \mathrm{mN} / \mathrm{m}$

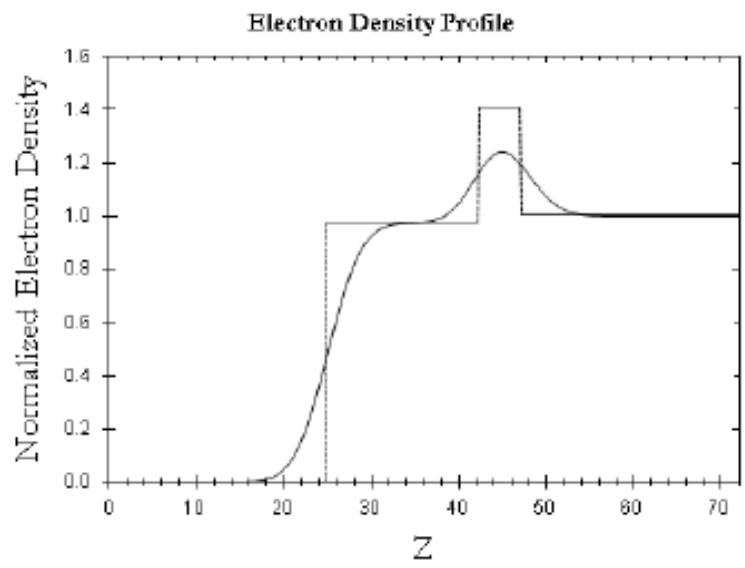

The reflectivity curve was fit with 3 roughness parameters

Percent Error in $Q$ : 0

Normalization Constant: 1.12007566704435

Critical Edge Offset: 0

High Q Offset: 0

Superphase SLD: 0

Subphase SLD: 9.409

Wavelength: 1.2244

Chi Square for reflectivity fit: $1.392 \mathrm{E}-002$

The subphase roughness was: $3.14 \mathrm{ED} \pm 1.129 \mathrm{EO}$

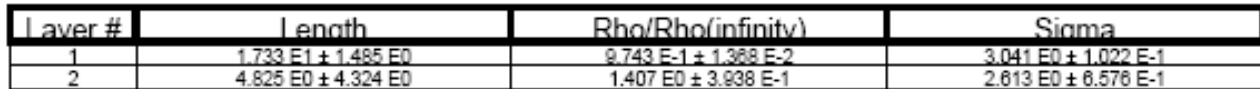




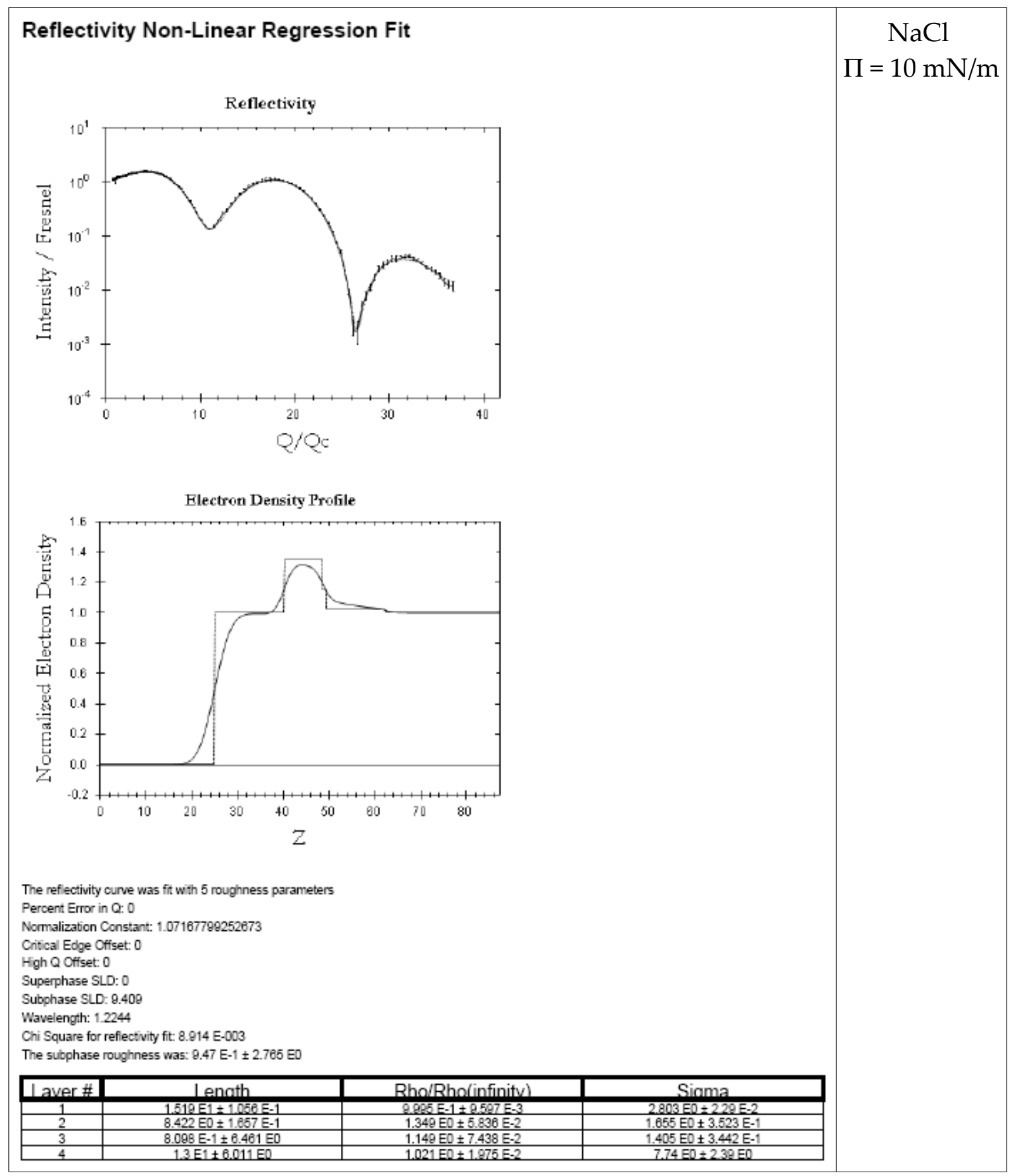




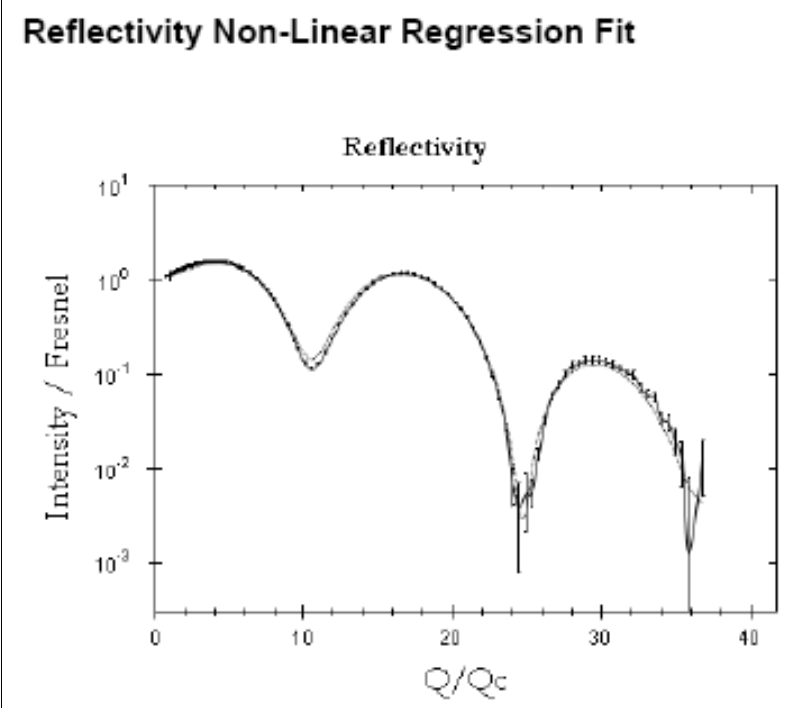

$\mathrm{NaCl}$
$\Pi=25 \mathrm{mN} / \mathrm{m}$

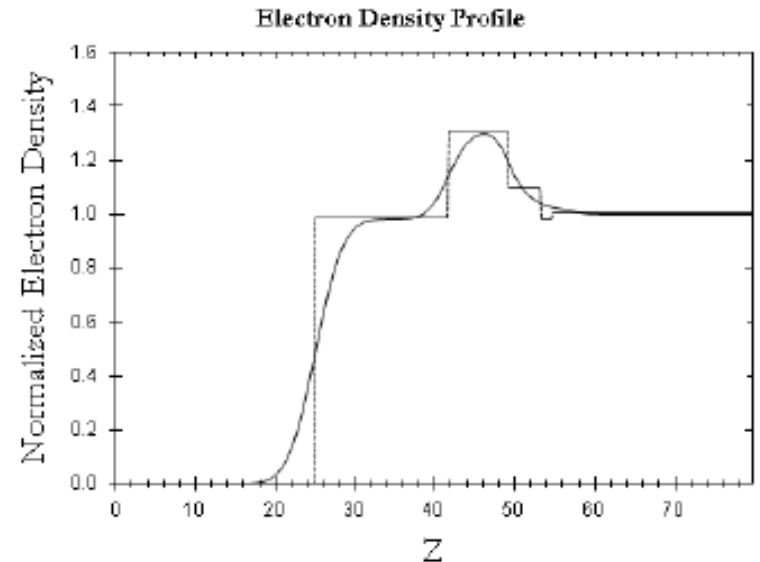

The reflectivity curve was fit with 5 roughness parameters

Percent Error in Q: 0

Normalization Constant: 1.09152281043862

Critical Edge Offset: 0

High Q Offset: 0

Superphase SLD: 0

Subphase SLD: 9.409

Wavelength: 1.2244

Chi Square for reflectivity fit: 6.645 E-003

The subphase roughness was: $1 \mathrm{ED} \pm 4.089 \mathrm{E} 2$

\begin{tabular}{|c|c|c|c|}
\hline Laver \# & Lenoth & Rho/Rho(infinity) & Sioma \\
\hline 1 & $1.67 E 1=1.311 E 0$ & $2.825 E-1 \pm 2.126 \mathrm{E}-2$ & $2727 E 0 \pm 3.994 E-2$ \\
\hline 2 & $7.479 \mathrm{EO} \pm 5.281 \mathrm{E} 1$ & $1.306 \mathrm{EO} \pm 1.305 \mathrm{E}-1$ & 2.054 EO $=1.108 \mathrm{EO}$ \\
\hline 3 & $4.148 E D \pm 2.84 E 2$ & $1.097 \mathrm{E} 0 \pm 1.344 \mathrm{E} 1$ & $1.387 \mathrm{EO}=4.814 \mathrm{E} 1$ \\
\hline 4 & $1.507 \mathrm{E} 00 \pm 2.687 \mathrm{E} 2$ & $2.781 \mathrm{E}-1 \pm 1.017 \mathrm{E} 1$ & $2.878 \mathrm{E0}=2.484 \mathrm{E1}$ \\
\hline
\end{tabular}




\section{Reflectivity Non-Linear Regression Fit}

Reflectivity
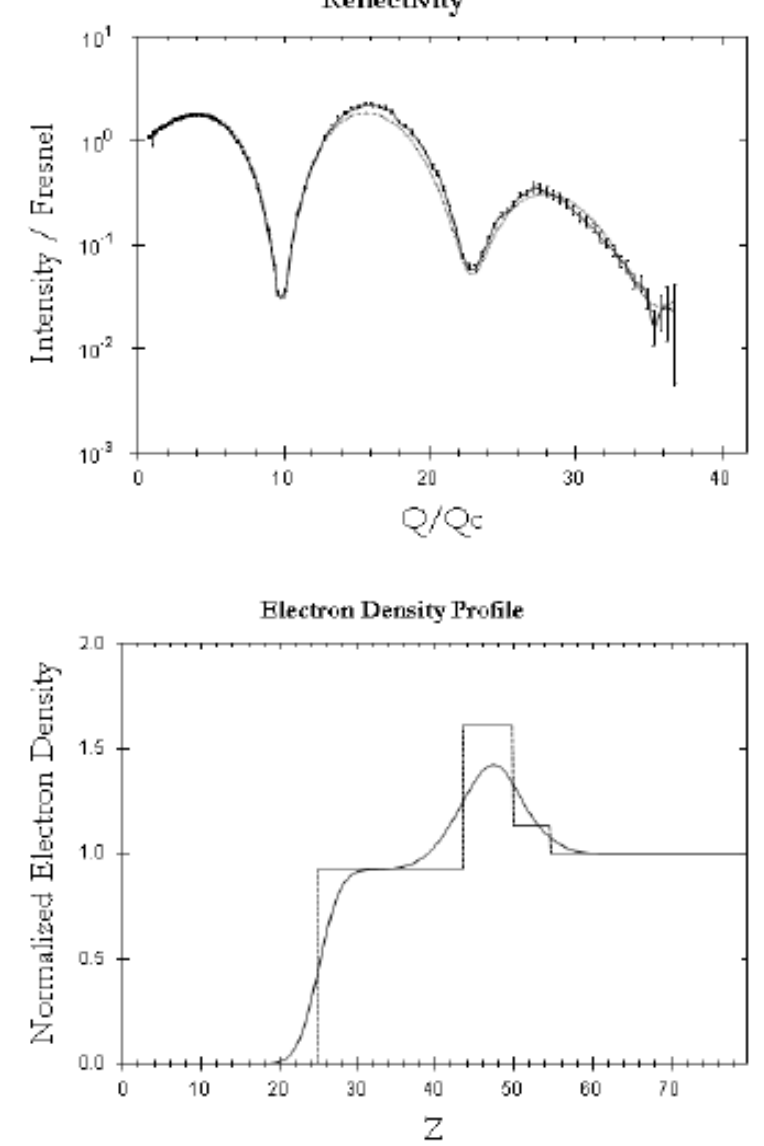

The reflectivity curve was fit with 4 roughness parameters

Percent Error in Q: 0

Normalization Constant: 1.153553887e0944

Critical Edge Offset: 0

High Q Offset: 0

Superphase SLD: 0

Subphase SLD: 9.409

Wavelength: 1.2244

Chi Square for reflectivity fit: 3.643 E-002

The subphase roughness was: $2.337 \mathrm{EO} \pm 2.382 \mathrm{ED}$

\begin{tabular}{|c|c|c|c|}
\hline Laver\# & Lenath & Rho/Rho(infinitv) & Siama. \\
\hline 1 & $1.828=1 \pm 1.144=0$ & $9.23 E-1 \pm 1.008 E-2$ & $2.177 E 0 \pm \pm 1765 E-2$ \\
\hline 2 & $6.288 \mathrm{EO} \pm 1.172 \mathrm{ED}$ & $1.601 \mathrm{ED} \pm 1.742 \mathrm{E}-1$ & $3.896 \mathrm{ED} \pm 5.605 \mathrm{E}-1$ \\
\hline 3 & $4.897 \mathrm{EO} \pm 4.779 \mathrm{ED}$ & $1.126 \mathrm{ED} \pm 3.496 \mathrm{E}-1$ & $2.337 E 0=1.285 E 0$ \\
\hline
\end{tabular}

\section{$\mathrm{CaCl}_{2}$}

$\Pi=10 \mathrm{mN} / \mathrm{m}$ 

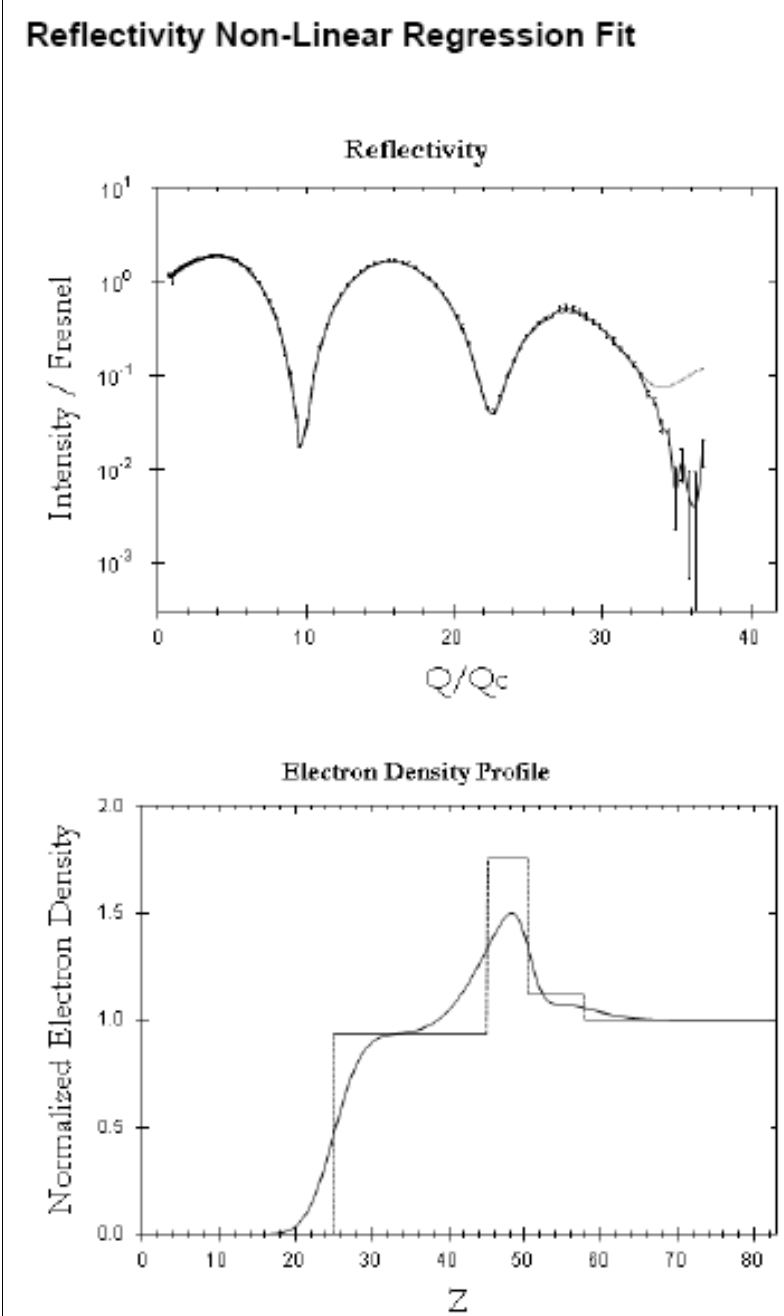

The reflectivity curve was fit with 4 roughness parameters

Percent Error in Q: 0

Normalization Constant: 1.14971056963775

Critical Edge Offset: 0

High Q Offset: 10

Superphase SLD: 0

Subphase SLD: 9.409

Wavelength: 1.2244

Chi Square for reflectivity fit: 1.857 E-002

The subphase roughness was: $4.397 \mathrm{EO} \pm 9.048 \mathrm{E}-1$

\begin{tabular}{|c|c|c|c|}
\hline Laver \# & Jenath & Rho/Rho(infinity) & Siama \\
\hline 1 & $2017 E 1 \pm 1.102 \mathrm{E0}$ & $9334 E-1 \pm 3.574 E-3$ & $2.873 E 0=1.58 E-2$ \\
\hline 2 & $5.356 \mathrm{EO} \pm 1.218 \mathrm{ED}$ & $1.749 \mathrm{ED} \pm 1.606 \mathrm{E}-1$ & $4.708 \mathrm{ED} \pm 5.066 \mathrm{E}-1$ \\
\hline 3 & $7.485 \mathrm{EO} \pm 1.847 \mathrm{ED}$ & $1.115 \mathrm{E} 0 \pm 5.087 \mathrm{E}-2$ & $1.477 \mathrm{ED} \pm 2.767 \mathrm{E}-1$ \\
\hline
\end{tabular}




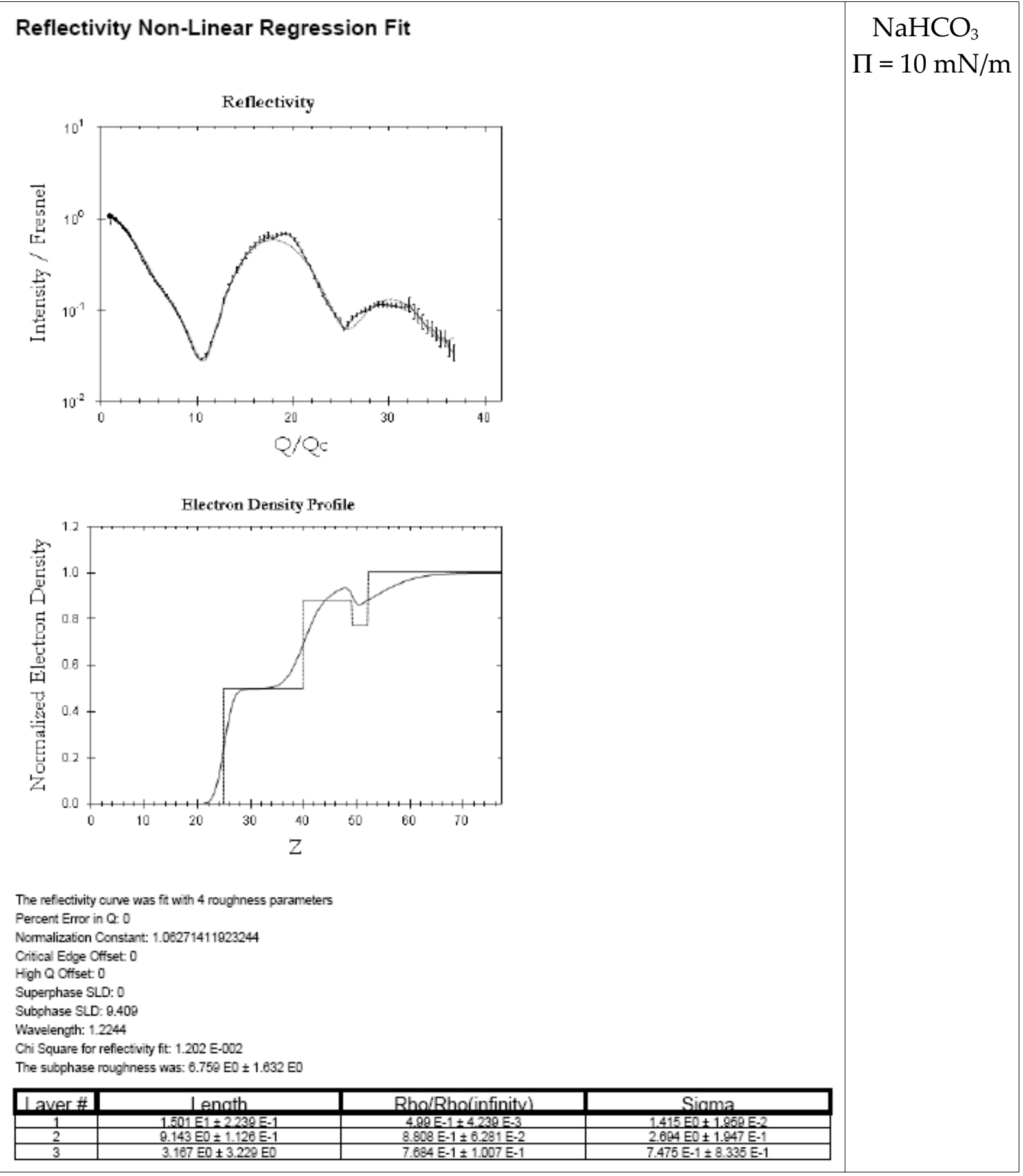



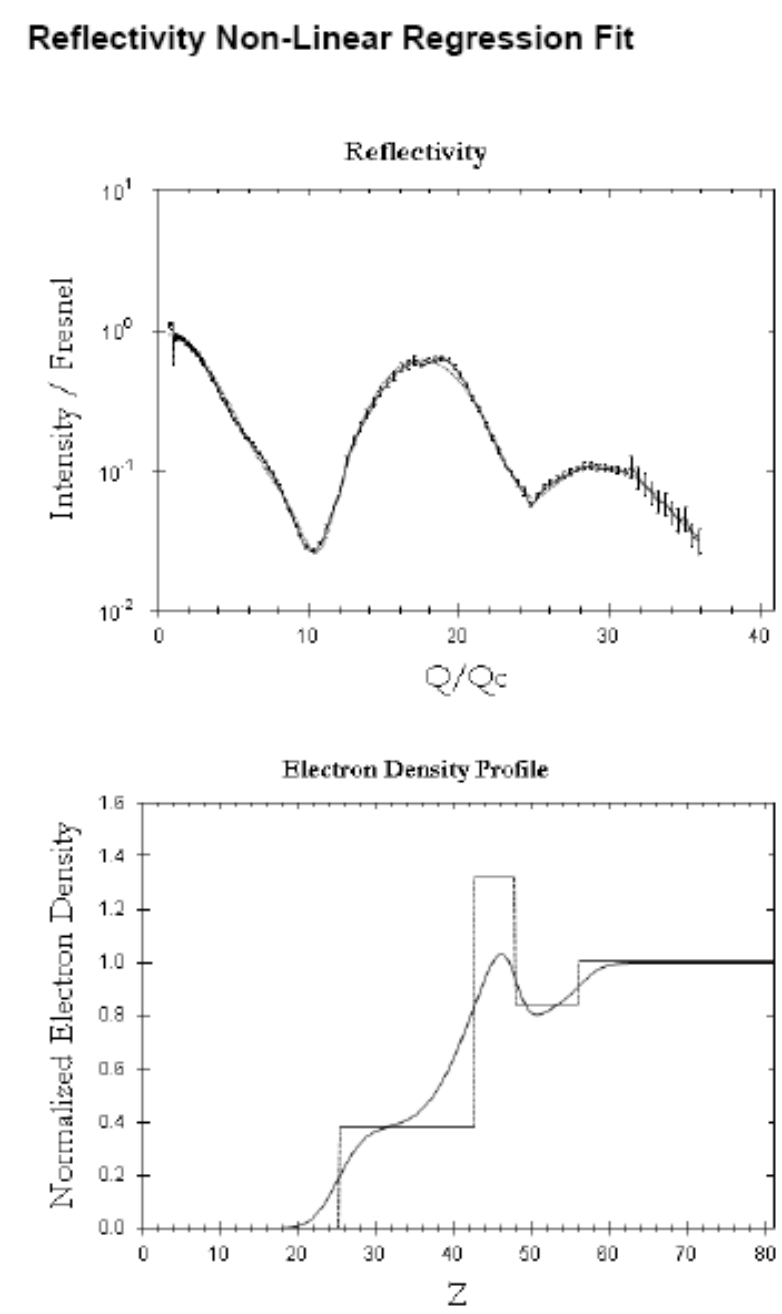

The reflectivity curve was fft with 4 roughness parameters

Percent Error in Q: 0

Normalization Constant: 0.833303070408436

Critical Edge Offset: 0

High Q Offset: 0

Superphase SLD: 0

Subphase SLD: 9.850

Wavelength: 1.2244

Chi Square for reflectivity fit: 2.127 E-001

The subphase roughness was: $2.334 \mathrm{EO} \pm 8.487 \mathrm{E}-1$

\begin{tabular}{|c|c|c|c|}
\hline Laver \# & Lenath & Rho/Rho(infinitv) & Sioma \\
\hline 1 & $1.758 E 1 \pm 2.055 E 0$ & $3756 \mathrm{E}-1 \pm 1.203 \mathrm{E}-2$ & $2.661 E 0 \pm 1.162 E-1$ \\
\hline$\frac{1}{2}$ & $5.112 \mathrm{EO}+2.147 \mathrm{EO}$ & $1.318 \mathrm{ED} \pm 3.206 \mathrm{E}-1$ & $4.836 \mathrm{EO}=1.047 \mathrm{EO}$ \\
\hline 3 & $8.3 \mathrm{ED} \pm 1.347 \mathrm{ED}$ & $8.37 \mathrm{E}-1 \pm 7.411 \mathrm{E}-2$ & $1.478 \mathrm{ED} \pm 5.634 \mathrm{E}-1$ \\
\hline
\end{tabular}

$\mathrm{NaHCO}_{3}$

$\Pi=25 \mathrm{mN} / \mathrm{m}$ 


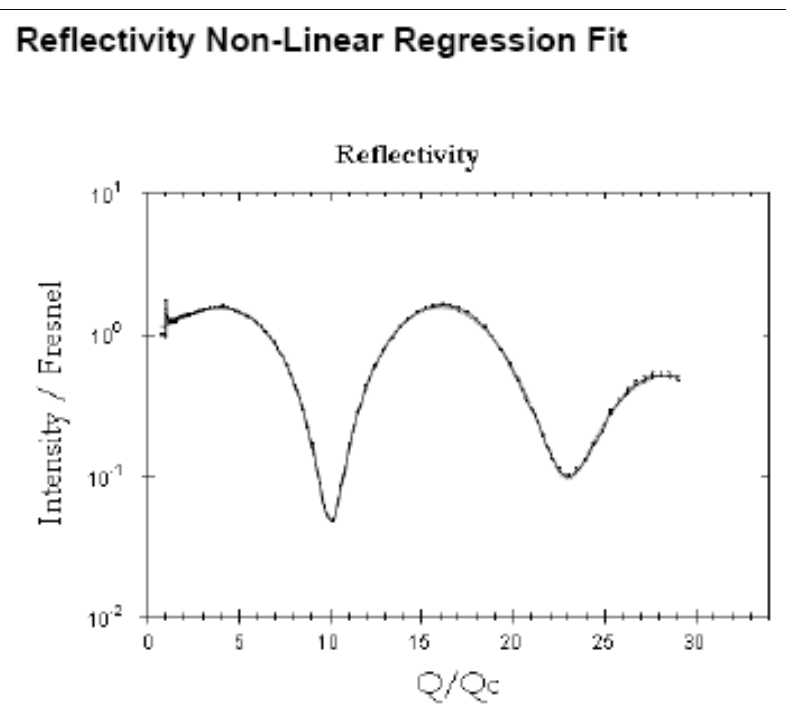

$\mathrm{CaCO}_{3}$
$\Pi=0 \mathrm{mN} / \mathrm{m}$

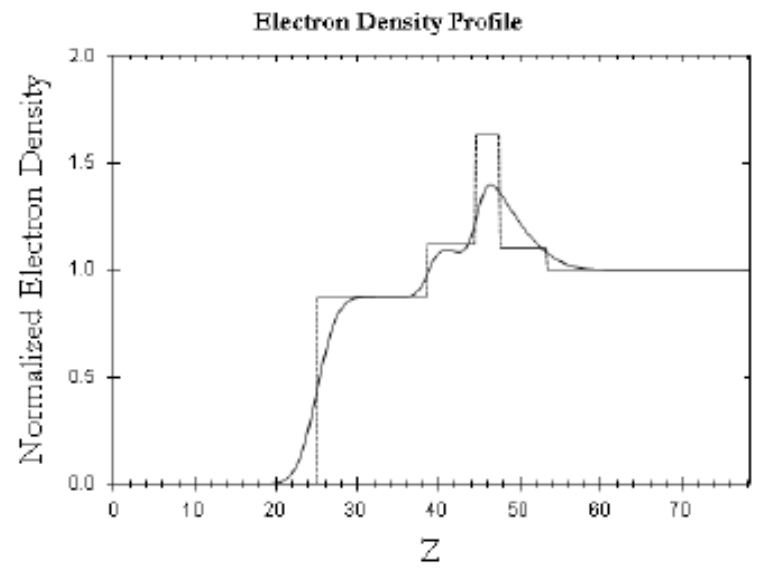

The refectivity curve was fit with 5 roughness parameters

Percent Error in Q: 0

Normalization Constant: 1.13193550574979

Critical Edge Offset: 0

High Q Offset: 0

Superphase SLD: 0

Subphase SLD: 9.38

Wavelength: 1.2244

Chi Square for reflectivity fit: 1.002 E000

The subphase roughness was: $2.842 \mathrm{EO} \pm 1.413 \mathrm{E2}$

\begin{tabular}{|c|c|c|c|}
\hline Laver \# & Lenath & Rho/Rho/infinitv) & Siama \\
\hline 1 & $1.371 \mathrm{E} 1 \pm 1.542 \mathrm{E} 2$ & $8.749 E-1 \pm 3.102 E 0$ & $1.858 E 0=34.22 E 1$ \\
\hline 2 & $6.000 \mathrm{ED} \pm 8.702 \mathrm{E1}$ & $1.114 \mathrm{ED} \pm 1.433 \mathrm{E} 1$ & $1.051 \mathrm{E} 0=6.151 \mathrm{E} 2$ \\
\hline 3 & $2.852 \mathrm{EO} \pm 6.242 \mathrm{E} 2$ & $1.622 \mathrm{EO} \pm 5.294 \mathrm{E} 1$ & $1.08 \mathrm{ED} \pm 2.055 \mathrm{E} 2$ \\
\hline 4 & $58 E 0=292 E 2$ & $1.102 \mathrm{E0} \pm 3.853 \mathrm{E} 1$ & $3.581 E 0=3457 \mathrm{E2}$ \\
\hline
\end{tabular}




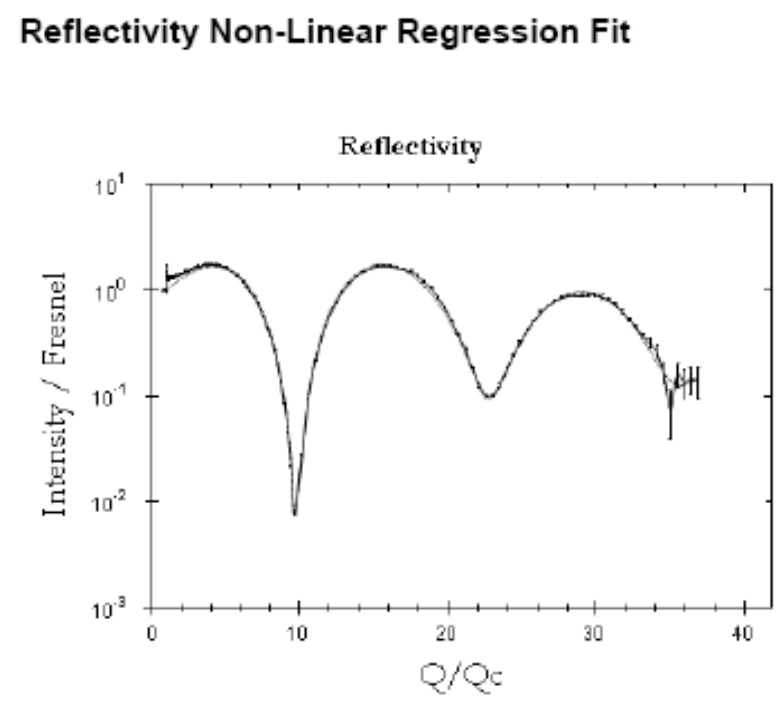

$\mathrm{CaCO}_{3}$
$\Pi=10 \mathrm{mN} / \mathrm{m}$

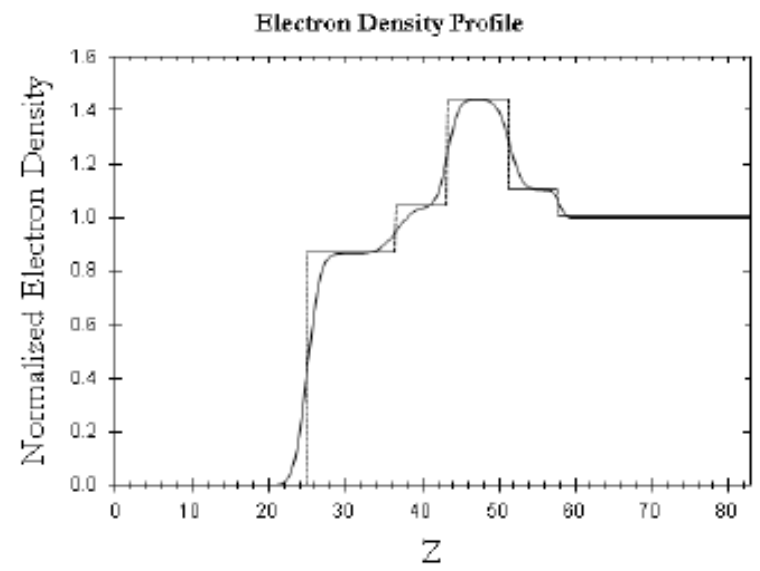

The reflectivity curve was fit with 5 roughness parameters

Percent Error in Q: 0

Normalization Constant: 1

Critical Edge Offset: 0

High Q Offset: 0

Superphase SLD: 0

Subphase SLD: 9.38

Wavelength: 1.2244

Chi Square for reflectivity fit: 4.888 E-001

The subphase roughness was: $5.923 \mathrm{E}-1 \pm 2.202 \mathrm{E} 1$

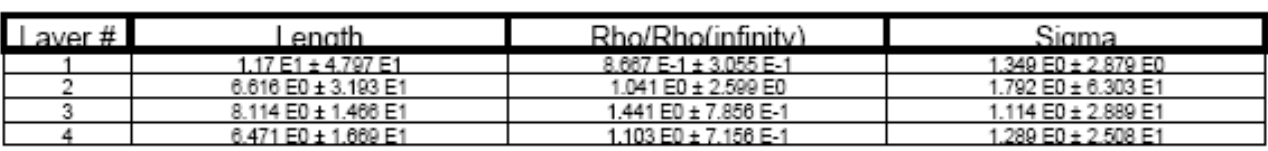




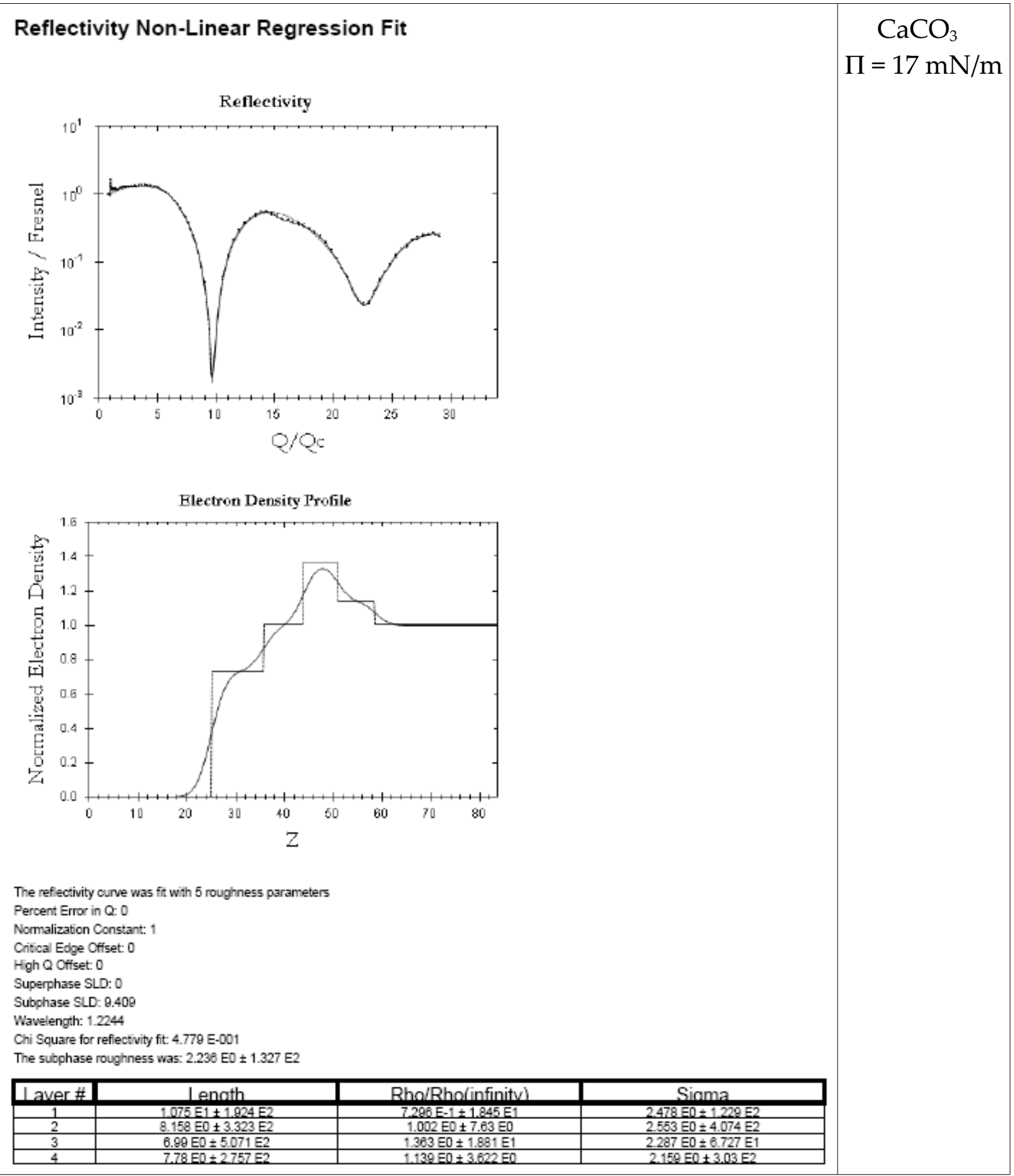




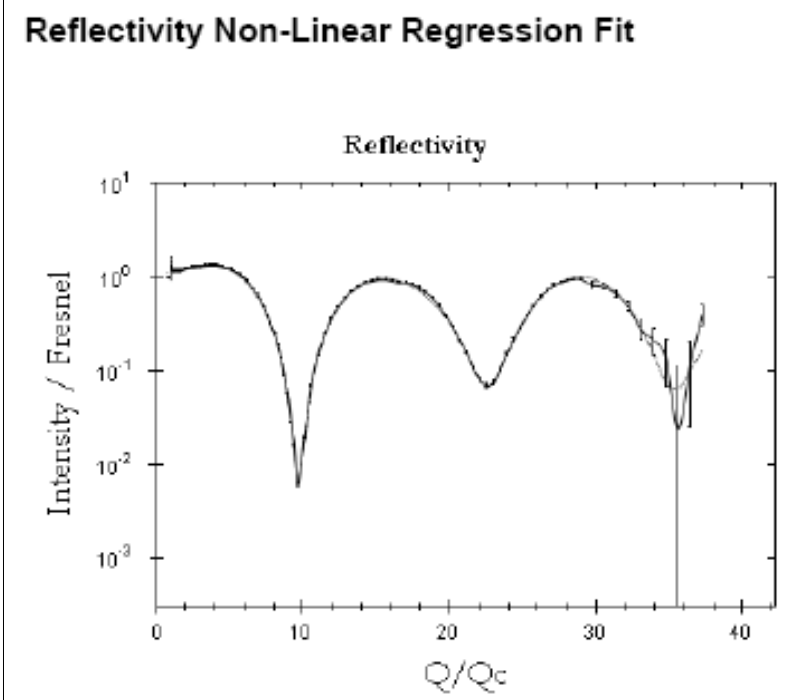

$\mathrm{CaCO}_{3}$
$\Pi=25 \mathrm{mN} / \mathrm{m}$

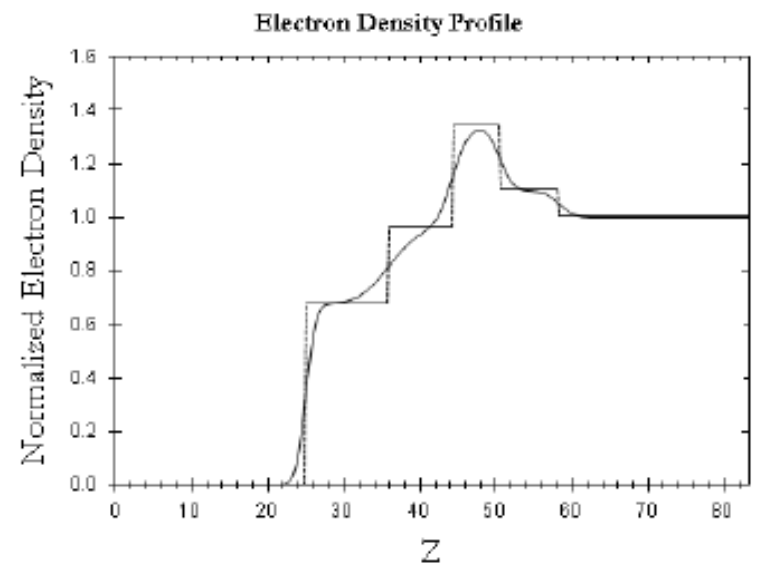

The reflectivity curve was fit with 5 roughness parameters

Percent Error in Q: 0

Normalization Constant: 1.08005181379243

Critical Edge Offset: 0

High Q Offset: 10

Superphase SLD: 0

Subphase SLD: 9.409

Wavelength: 1.2244

Chi Square for reflectivity fit: 7.001 E-001

The subphase roughness was: $1.560 \mathrm{EO} \pm 1.922 \mathrm{E2}$

\begin{tabular}{|c|c|c|c|}
\hline L aver \# & Lenoth & Rho/Rho(infinity) & Sioma \\
\hline 1 & 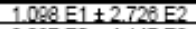 & $6.756 \mathrm{E}-1 \pm 2.303 \mathrm{E} 1$ & $1.043 E 0=2504=2$ \\
\hline 2 & $8.287 \mathrm{EO} \pm 4.147 \mathrm{E} 2$ & $9.632 \mathrm{E}-1 \pm 7.397 \mathrm{ED}$ & $3.18 \mathrm{ED} \pm 7.056 \mathrm{E} 2$ \\
\hline 3 & $6.256 \mathrm{EO} \pm 4.474 \mathrm{E} 2$ & $1.338 \mathrm{E} 0 \pm 2.097 \mathrm{E} 1$ & $1.682 \mathrm{E} 0=2.101 \mathrm{E} 2$ \\
\hline 4 & $7777 \mathrm{E0} \pm 2389 \mathrm{E} 2$ & $1009 E 0 \pm 4.293 E 0$ & $1383 \mathrm{E0}=2771 \mathrm{E2}$ \\
\hline
\end{tabular}




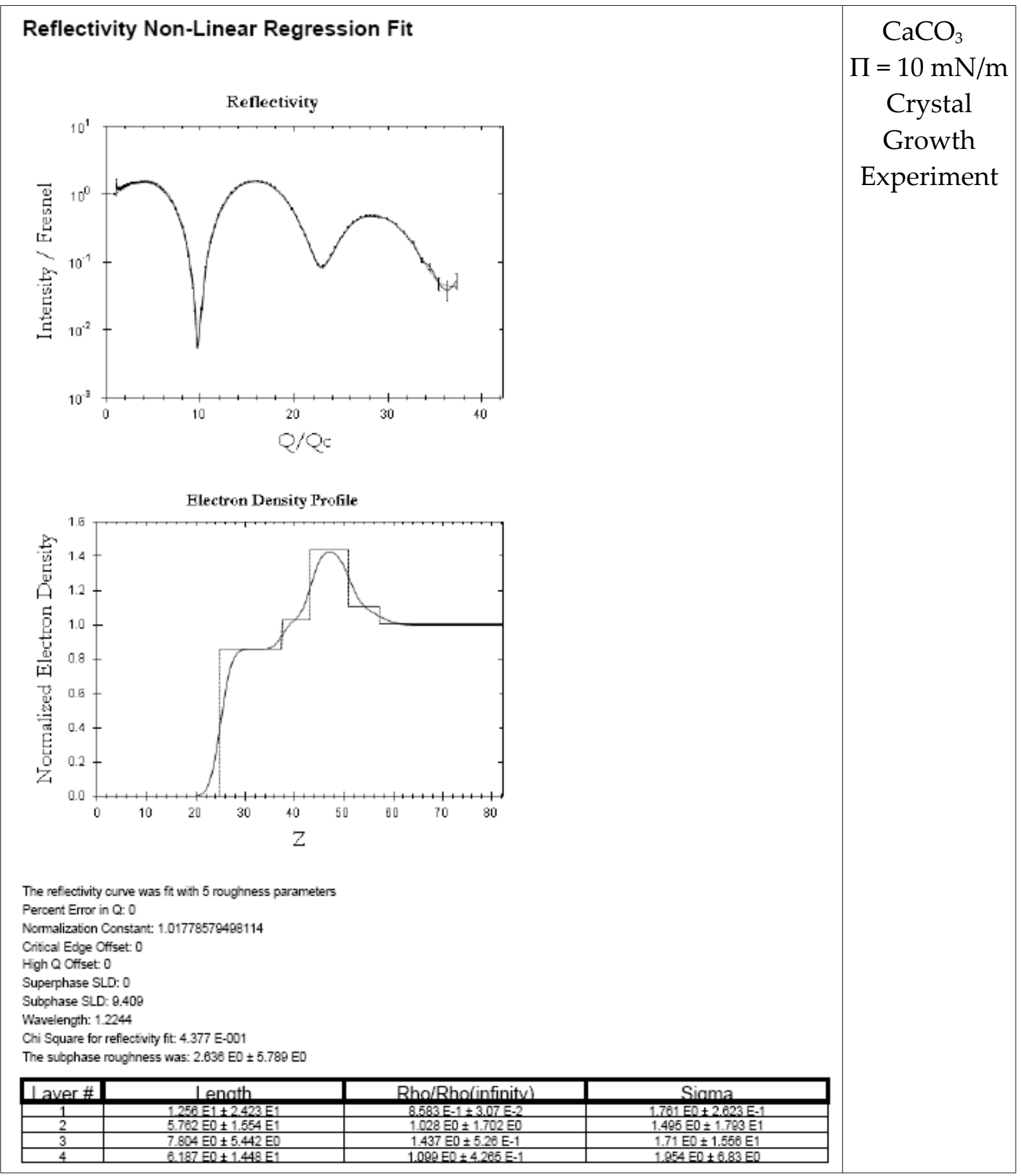

